Studien zum Physik- und Chemielernen H. Niedderer, H. Fischler, E. Sumfleth [Hrsg.]

259

Jan-Philipp Burde

Konzeption und Evaluation eines Unterrichtskonzepts zu einfachen Stromkreisen auf Basis des Elektronengasmodells

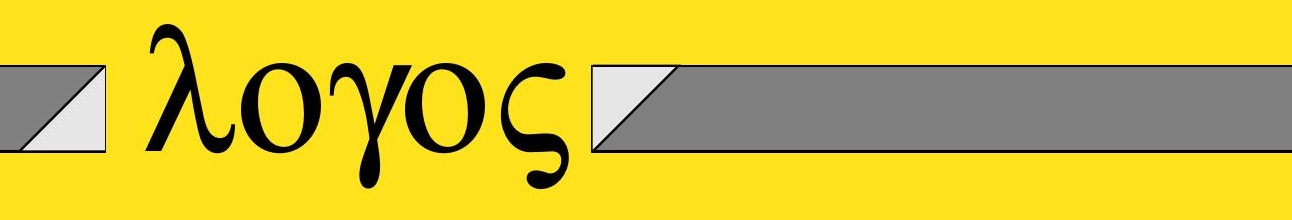




\section{Studien zum Physik- und Chemielernen}

Herausgegeben von Hans Niedderer, Helmut Fischler und Elke Sumfleth

Diese Reihe im Logos-Verlag bietet ein Forum zur Veröffentlichung von wissenschaftlichen Studien zum Physik- und Chemielernen. In ihr werden Ergebnisse empirischer Untersuchungen zum Physik- und Chemielernen dargestellt, z. B. über Schülervorstellungen, Lehr-/Lernprozesse in Schule und Hochschule oder Evaluationsstudien. Von Bedeutung sind auch Arbeiten über Motivation und Einstellungen sowie Interessensgebiete im Physik- und Chemieunterricht. Die Reihe fühlt sich damit der Tradition der empirisch orientierten Forschung in den Fachdidaktiken verpflichtet. Die Herausgeber hoffen, durch die Herausgabe von Studien hoher Qualität einen Beitrag zur weiteren Stabilisierung der physik- und chemiedidaktischen Forschung und zur Förderung eines an den Ergebnissen fachdidaktischer Forschung orientierten Unterrichts in den beiden Fächern zu leisten. 
Studien zum Physik- und Chemielernen

Band 259 

Jan-Philipp Burde

\section{Konzeption und Evaluation eines Unterrichtskonzepts zu einfachen Stromkreisen auf Basis des Elektronengasmodells}


Studien zum Physik- und Chemielernen

Hans Niedderer, Helmut Fischler, Elke Sumfleth [Hrsg.]

Bibliografische Information der Deutschen Nationalbibliothek

Die Deutsche Nationalbibliothek verzeichnet diese Publikation in der Deutschen Nationalbibliografie; detaillierte bibliografische Daten sind im Internet über http://dnb.d-nb.de abrufbar.

(C) Copyright Logos Verlag Berlin GmbH 2018

Alle Rechte vorbehalten.

ISBN 978-3-8325-4726-4

ISSN 1614-8967

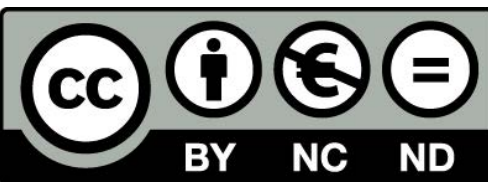

Logos Verlag Berlin GmbH

Comeniushof, Gubener Str. 47, D-10243 Berlin

Tel.: $+49(0) 30 / 42851090$

Fax: $+49(0) 30 / 42851092$

https://www.logos-verlag.de 


\title{
Konzeption und Evaluation \\ eines Unterrichtskonzepts zu einfachen Stromkreisen auf Basis des Elektronengasmodells
}

\author{
Dissertation \\ zur Erlangung des Doktorgrades \\ der Naturwissenschaften \\ vorgelegt beim Fachbereich Physik \\ der Johann Wolfgang Goethe-Universität \\ in Frankfurt am Main
}

von

Jan-Philipp Burde

aus Fulda

Frankfurt 2018

(D 30) 
vom Fachbereich Physik

der Johann Wolfgang Goethe-Universität

als Dissertation angenommen.

Dekan: Prof. Dr. Owe Philipsen

Gutachter: Prof. Dr. Thomas Wilhelm

Prof. Dr. Dr. Hartmut Wiesner

Prof. Dr. Martin Hopf

Datum der Disputation: 30.05.2018 


\section{Inhaltsverzeichnis}

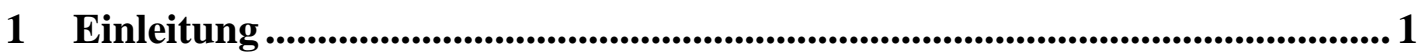

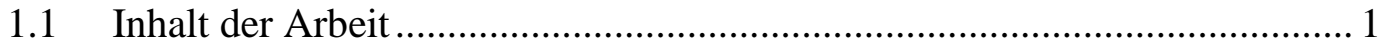

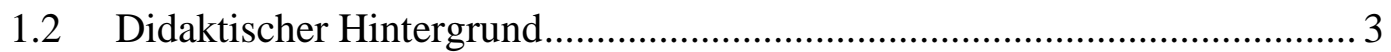

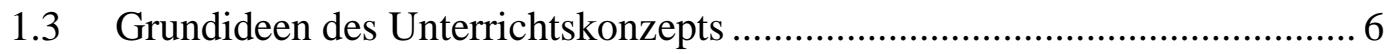

A) THEORETISCHE GRUNDLAGEN .............................................. 11

2 Die fachlichen Grundlagen der Elektrizitätslehre ......................................... 13

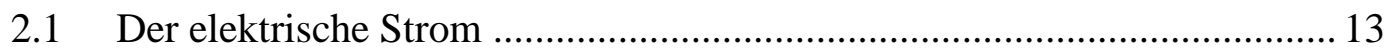

2.2 Elektrisches Feld, Spannung und Potenzial ........................................... 13

2.3 Grenzflächen- und Oberflächenladungen ................................................. 15

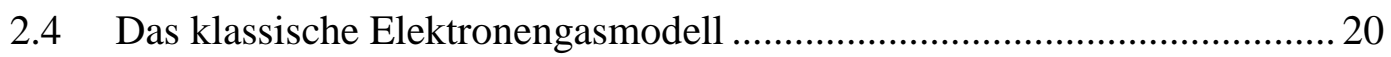

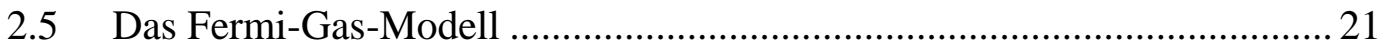

2.6 Das Bändermodell von Festkörpern....................................................... 22

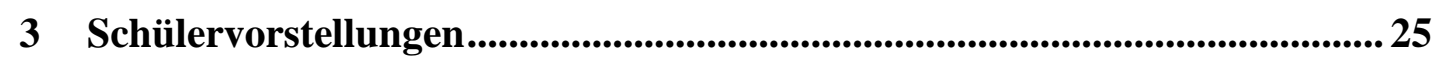

3.1 Generelle Aspekte zu Schülervorstellungen ........................................... 25

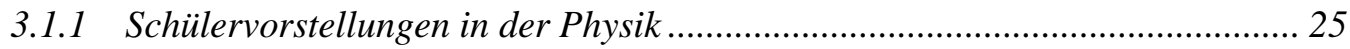

3.1.2 Das Lernen als aktiver Konstruktionsprozess .................................................. 26

3.1.3 Der Einfluss des Vorwissens auf das Lernen in der Physik............................. 28

3.1.4 Die Eigenschaften von Schülervorstellungen ................................................ 30

3.1.5 Theorien zur Wissensorganisation und Conceptual Change............................ 32

3.1.6 Unterrichtsstrategien zum Konzeptwechsel.................................................. 38

3.2 Schülervorstellungen zu einfachen Stromkreisen .................................... 41

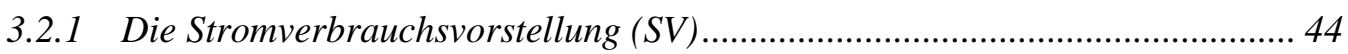

3.2.2 Der Stromverbrauch ist proportional zum Widerstand $(P R)$.......................... 46

3.2.3 Die Batterie als konstante Stromquelle (BS) ................................................. 46

3.2.4 Die Stromstärke ist unabhängig vom Widerstand $(U R)$.................................. 47

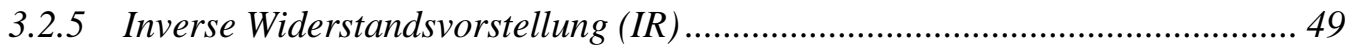

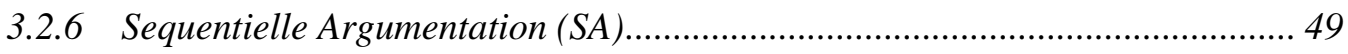

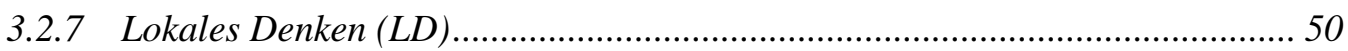

3.2.8 Probleme beim Erfassen von Parallelschaltungen $(P S)$................................. 51

3.2.9 Spannung als Eigenschaft des Stroms (UI) ................................................ 52 
4 Analogien und Modelle im Physikunterricht...................................................55

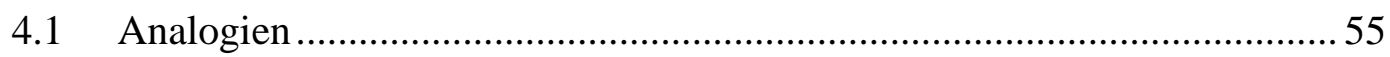

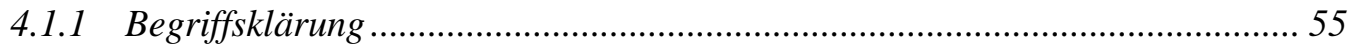

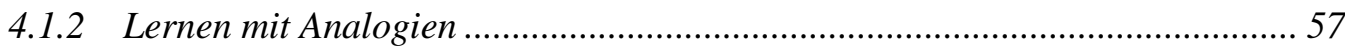

4.2 Fachliche und didaktische Aspekte von Modellen .................................... 59

4.3 Schulische Modelle zur Veranschaulichung von Stromkreisen.................. 62

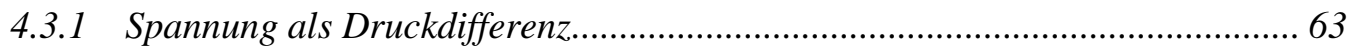

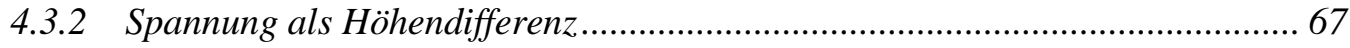

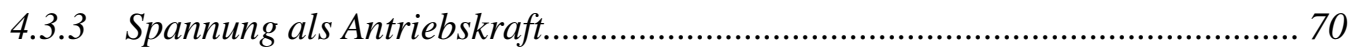

4.3.4 Spannung als transportierte Energie pro Ladung ......................................... 71

5 Analyse bedeutender Unterrichtskonzepte ..................................................73

5.1 Die Elektrizitätslehre nach dem IPN-Curriculum .................................... 73

5.2 Das Unterrichtskonzept von Wiesner und Jung ..................................... 75

5.3 Der Karlsruher Physikkurs (KPK) ……................................................... 77

5.4 Das CASTLE-Curriculum von Steinberg …......................................... 83

5.5 Das Unterrichtskonzept von Muckenfuß und Walz ................................. 88

5.6 Das Unterrichtskonzept von Schwedes und Dudeck ............................... 92

5.7 Das Münchener Stäbchenmodell von Gleixner ........................................ 95

5.8 Das Unterrichtskonzept von Koller und Späth ....................................... 98

5.9 Erkenntnisse aus den Unterrichtskonzepten ........................................ 101

B) KONZEPTERARBEITUNG .....................................................103

6 Entwicklung des Unterrichtskonzepts............................................................ 105

6.1 Didaktische Grundüberlegungen....................................................... 105

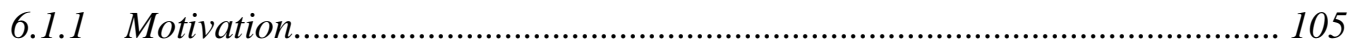

6.1.2 Zentrale Ideen hinter dem Unterrichtskonzept .............................................. 107

6.1.3 Elementarisierung des Elektronengasmodells.......................................... 112

6.1.4 Mögliche Darstellungsformen des elektrischen Potenzials .......................... 114

6.1.5 Vermittlung einer mikroskopischen Modellvorstellung ............................... 116

6.1.6 Didaktisch motivierte Begriffswahl zentraler Konzepte ............................... 118

6.1.7 Die Grenzen und die Anschlussfähigkeit des Elektronengasmodells ............. 120

6.1.8 Übersicht über die zu evaluierende grundlegende Sachstruktur................... 125 
6.2 Formative Evaluation der grundlegenden Konzeption ........................... 127

6.2.1 Die Methode der Teaching Experiments ................................................... 127

6.2.2 Beschreibung der Teaching Experiments ................................................. 129

6.2.3 Auswertung der Teaching Experiments ...................................................... 131

6.2.4 Zusammenfassung und Bewertung der Befunde ......................................... 148

6.2.5 Konsequenzen in Hinblick auf das Unterrichtskonzept ................................ 152

6.3 Die Sachstruktur des fertigen Unterrichtskonzepts................................ 160

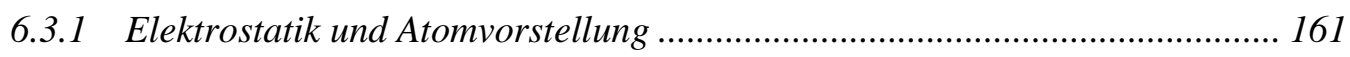

6.3.2 Luftströmungen durch Druckunterschiede .................................................. 161

6.3.3 Batterie, Potenzial und Spannung ............................................................ 162

6.3.4 Der elektrische Strom und Widerstand..................................................... 163

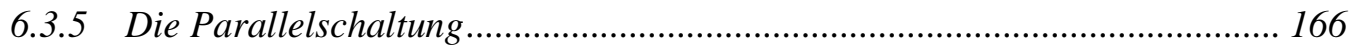

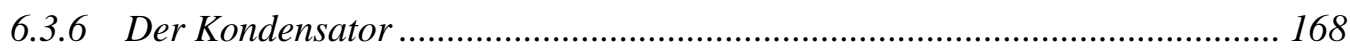

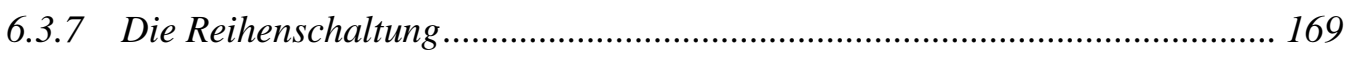

6.3.8 Messung und Berechnung von Stromstärke, Spannung und Widerstand ....... 171

C) SUMMATIVE EVALUATION ...................................................173

7 Summative Evaluation des Unterrichtskonzepts ......................................... 175

7.1 Die Studie als Design-Based-Research-Projekt....................................... 175

7.2 Forschungsfragen und Ablauf der Studie ........................................... 178

7.3 Konzeption der empirischen Studie zum Lernerfolg ............................. 181

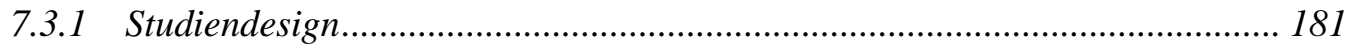

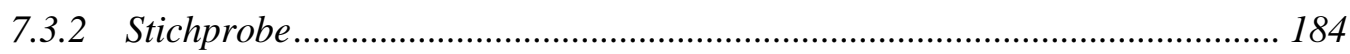

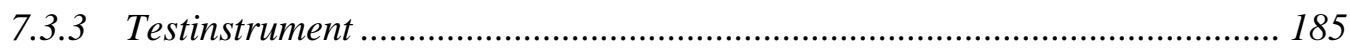

7.3.4 Erhebung der Schülervorstellungen ........................................................ 188

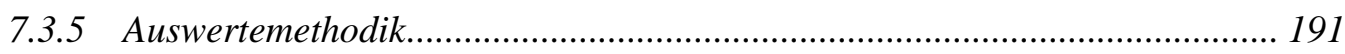

7.4 Ergebnisse der empirischen Studie zum Lernerfolg ............................... 198

7.4.1 Generelle Aspekte zum Testinstrument .......................................................... 198

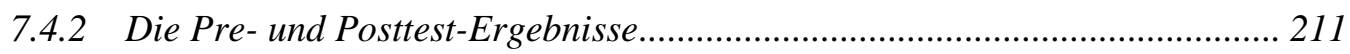

7.4.3 Analyse des absoluten Lernzuwachses ....................................................... 215

7.4.4 Analyse des relativen Lernzuwachses........................................................... 225

7.4.5 Kontrolle der Pretest-Unterschiede........................................................... 228

7.4.6 Vergleich von $K G$ und TG bei gleichen Lehrkräften .................................... 234

7.4.7 Der Lernzuwachs auf Klassenebene …………............................................ 238 
7.4.8 Analyse der Schülervorstellungen 244

7.5 Erstellung eines Hierarchisch Linearen Modells

7.5.1 Beschreibung des allgemeinen Vorgehens und der Kenngrößen ................... 249

7.5.2 Entwicklung eines Hierarchisch Linearen Modells..................................... 254

7.5.3 Darstellung des Gesamtmodells M2a ........................................................ 268

7.5.4 Mehrebenenanalytischer Vergleich der Schülervorstellungen ...................... 270

7.6 Schulpraktische Erfahrungen der Lehrkräfte ........................................ 273

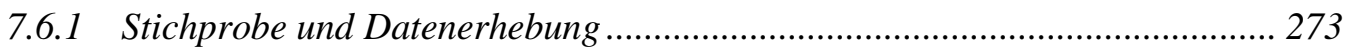

7.6.2 Förderung des Konzeptverständnisses ……………………………........... 274

7.6.3 Didaktische Einschätzung des Unterrichtskonzepts ..................................... 276

7.6.4 Detailanalyse zu einzelnen Aspekten des Unterrichtskonzepts...................... 277

7.6.5 Erfahrungen im Rahmen einer Pädagogischen Facharbeit ........................... 280

7.7 Zentrale Ergebnisse der summativen Evaluation.................................... 283

7.7.1 Zentrale Ergebnisse zu Forschungsfrage 1 ................................................ 283

7.7.2 Zentrale Ergebnisse zu Forschungsfrage 2 ................................................ 285

7.7.3 Zentrale Ergebnisse zu Forschungsfrage 3 ................................................ 289

7.7.4 Zentrale Ergebnisse zu Forschungsfrage 4 ………..................................... 291

D) SCHLUSSBETRACHTUNGEN ......................................................295

8 Die Design-Based-Research-Studie im Rückblick....................................... 297

8.1 Zentrale Erkenntnisse der Studie ........................................................ 297

8.1.1 Für die Unterrichtspraxis ........................................................................... 297

8.1.2 Für eine kontextspezifische Lerntheorie ………………………….............. 298

8.2 Kritische Reflexion der Studie ............................................................. 300

8.3 Ideen zur Weiterentwicklung und Ausblick........................................... 302

8.3.1 Verbesserungsvorschläge zum Unterrichtskonzept .................................... 302

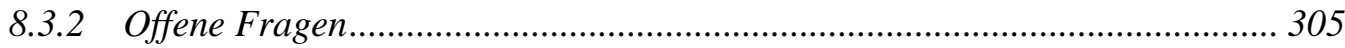

9 Zusammenfassung .......................................................................................... 307

10 Summary of the Doctoral Thesis....................................................................... 313

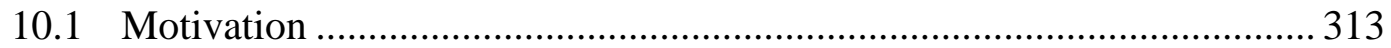

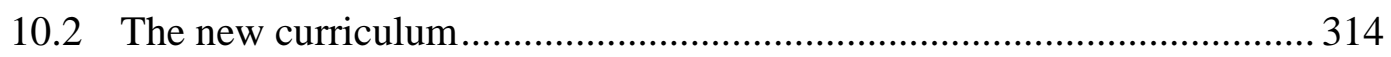

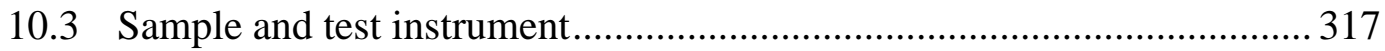

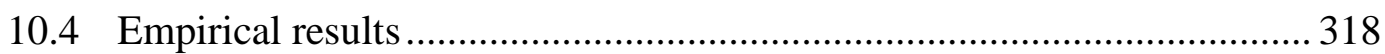


Inhaltsverzeichnis

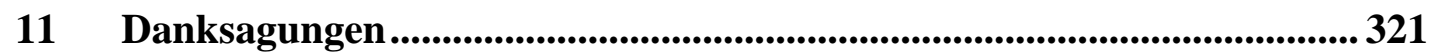

12 Literaturverzeichnis ............................................................................... 323

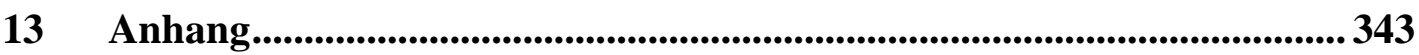




\section{Vorbemerkung zum Sprachgebrauch}

Zwar wurde in der vorliegenden Arbeit versucht, bei Personen sowohl die weibliche als auch die männliche Form zu benutzen, jedoch wurde aufgrund der leichteren Lesbarkeit teilweise auf eine separate Nennung der weiblichen Form verzichtet. Sofern sich nicht explizit auf das Geschlecht der Personen bezogen wird, sind mit der männlichen Form also immer beide Geschlechter gemeint. 


\section{Einleitung}

Kaum eine Entdeckung hat unsere Zivilisation so nachhaltig geprägt wie die Entdeckung der Elektrizität. Gleichzeitig haben die meisten Menschen aber nur sehr vage Vorstellungen von grundlegenden elektrischen Größen wie Spannung, Widerstand und Stromstärke und ihrer wechselseitigen Beziehung in einfachen Stromkreisen. Dies ist u.a. darauf zurückzuführen, dass es dem Physikunterricht seit Jahrzehnten trotz intensiver Bemühungen seitens der Lehrkräfte nicht gelingt, jungen Menschen angemessene physikalische Vorstellungen von den Vorgängen in sogenannten einfachen Stromkreisen zu vermitteln. Neben dem damit einhergehenden hohen Frustrationspotenzial für die Lernenden ist dieser Zustand insbesondere vor dem Hintergrund bedenklich, dass der Physikunterricht u.a. den Anspruch hat, den Schülerinnen und Schülern in einer zunehmend naturwissenschaftlich-technisch geprägten Welt eine angemessene gesellschaftliche und berufliche Teilhabe zu ermöglichen (,Scientific Literacy“). Aus physikdidaktischer Perspektive besteht deshalb eine Notwendigkeit darin, auf Basis einer ausführlichen Analyse typischer Lernschwierigkeiten und bisheriger Unterrichtsansätze in der Elektrizitätslehre einen umfassenden didaktischen Lösungsansatz zu erarbeiten. Das Ziel dieser Arbeit besteht deshalb in der theoriegeleiteten Entwicklung und empirischen Evaluation eines entsprechenden Unterrichtskonzepts zur Elektrizitätslehre für die ersten Jahre des Physikunterrichts in der Sekundarstufe I (im Folgenden „Anfangsunterricht“ genannt), um einen evidenzbasierten Beitrag zu dessen Verbesserung zu leisten.

\subsection{Inhalt der Arbeit}

Inhalt dieser Arbeit ist die Entwicklung und Evaluation eines Unterrichtskonzepts zur Elektrizitätslehre für den Anfangsunterricht im Schulfach Physik, das zum Ziel hat, den Lernenden ein qualitatives, konzeptionelles Verständnis der Grundbegriffe Potenzial, Spannung, Stromstärke und Widerstand sowie ihrer wechselseitigen Beziehung in einfachen Stromkreisen zu ermöglichen. Besonderer Wert wird dabei daraufgelegt, den Lernenden eine anschauliche Vorstellung der elektrischen Spannung und ihrer Bedeutung in Hinblick auf den elektrischen Strom zu vermitteln. Da hierzu auf eine didaktisch elementarisierte Form des klassischen Elektronengasmodells zurückgegriffen wird, werden in Kapitel 2 zunächst die fachlichen Grundlagen der Elektrizitätslehre dargestellt. Hier wird der Frage nachgegangen, wie es überhaupt zu einem Strom in einem einfachen Stromkreis kommt und welche Modelle zur Erklärung der Leitungsvorgänge existieren. Nach einer kurzen Klärung der Grundbegriffe der einfachen Elektrizitätslehre befasst sich dieses Kapitel insbesondere mit der Rolle von Grenzund Oberflächenladungen bei der Erzeugung eines elektrischen Feldes im Leiterinneren, das dort den elektrischen Strom bewirkt.

Da sich die Alltagserfahrungen vieler Menschen auf das Betätigen von Schaltern oder das Wechseln von Batterien beschränken, elektrische Prozesse im Allgemeinen unseren Sinnen verborgen bleiben und die Alltagssprache mit Begriffen wie „Stromverbrauch“ oder ,leeren Batterien" falsche Vorstellungen nahelegt, kommen viele Lernende bereits mit einem 
problematischen Vorverständnis zu den Vorgängen in elektrischen Stromkreisen in den Unterricht. In der Physikdidaktik sind solche Schülervorstellungen seit den 1970er Jahren Gegenstand intensiver Forschung, wobei sich diese Vorstellungen als äußerst stabil erwiesen haben und häufig der Übernahme einer physikalisch korrekten Vorstellung im Wege stehen. Kapitel 3 setzt sich daher mit dem großen Thema der Schülervorstellungen in der Physikdidaktik auseinander, wobei einerseits generelle Aspekte zu diesem Thema wie die Eigenschaften und Ursachen von Schülervorstellungen beleuchtet und andererseits die für diese Arbeit relevanten Schülervorstellungen zu einfachen Stromkreisen vorgestellt werden.

Vor dem Hintergrund, dass das hier entwickelte Unterrichtskonzept auf dem Modell des Elektronengases aufbaut, beschäftigt sich Kapitel 4 mit dem Analogie- und Modellbegriff und gängigen schulischen Modellen zur Veranschaulichung von einfachen Stromkreisen im Anfangsunterricht. Da Modelle und Analogien häufig unsystematisch und unreflektiert im Unterricht eingesetzt werden, findet zudem eine didaktische Auseinandersetzung mit Aspekten zum Lernen mit Analogien und Modellen statt. In Hinblick auf die Entwicklung eines didaktisch begründeten Unterrichtskonzepts ist zudem eine Analyse bisheriger Unterrichtskonzepte zu diesem Thema unerlässlich, die vor dem Hintergrund der Erkenntnisse der Schülervorstellungsforschung seit den 1970er Jahren entwickelt wurden. In Kapitel 5 werden deshalb bedeutende Unterrichtskonzepte der physikdidaktischen Forschung vorgestellt und kritisch reflektiert.

In dem sich anschließenden Kapitel 6 wird in einem ersten Teil die Entwicklung des hier vorgestellten Unterrichtskonzepts dokumentiert, wobei zunächst einmal dargelegt wird, weshalb aus didaktischer Sicht dringender Handlungsbedarf für ein innovatives Unterrichtskonzept zur Einführung in die Elektrizitätslehre besteht und worin die zentralen didaktischen Ideen hinter dem in dieser Arbeit entwickelten Konzept liegen. Darauf aufbauend wird die grundlegende Sachstruktur des angedachten Konzepts vorgestellt, die mit Hilfe der Methode der Teaching Experiments mit einzelnen Lernenden auf ihre Verständlichkeit hin überprüft wurde. Die Befunde dieser formativen Evaluation und ihre Konsequenzen für das fertige Unterrichtskonzept werden in der zweiten Hälfte dieses Kapitels dargelegt.

Auf Basis der Erfahrungen mit den Teaching Experiments wurde dann ein umfassendes Unterrichtskonzept inklusive Unterrichtsmaterialien entwickelt, dessen summative Evaluation Gegenstand des Kapitels 7 ist. Nach einer Verortung der Studie als Design-BasedResearch-Projekt werden die Forschungsfragen und die Konzeption der empirischen Studie beschrieben. Es schließt sich eine ausführliche Darstellung der Ergebnisse der empirischen Studie zur Evaluation der Lernförderlichkeit des Unterrichtskonzepts in der Schulpraxis an, wobei zur Analyse auf verschiedene in der didaktischen Forschung übliche statistische Methoden zurückgegriffen wird. Neben einer Betrachtung der nach dem Unterricht noch vorhandenen Schülervorstellungen in der Elektrizitätslehre findet auch die Entwicklung eines Hierarchisch Linearen Modells statt, um der Abhängigkeit des Lernerfolgs von der Klassenzugehörigkeit adäquat Rechnung zu tragen. Entsprechend der Forschungsphilosophie von Design- 
Based-Research wurden neben den Schülerleistungen auch die schulpraktischen Erfahrungen der Lehrkräfte mit dem Unterrichtskonzept erhoben, um einen umfassenderen Blick auf das Unterrichtskonzept sowie seine Stärken und Schwächen zu bekommen.

Den inhaltlichen Abschluss dieser Arbeit stellt Kapitel 8 dar, in dem zentrale Erkenntnisse zusammengefasst, das methodische und inhaltliche Vorgehen kritisch reflektiert und Ideen zur Weiterentwicklung des Unterrichtskonzepts auf Basis der in dieser Studie gewonnenen Erkenntnisse formuliert werden.

\subsection{Didaktischer Hintergrund ${ }^{1}$}

Für den enttäuschend niedrigen Lernerfolg im Elektrizitätslehreunterricht können sicherlich eine ganze Reihe an Gründen angeführt werden. Neben der prinzipiellen Unanschaulichkeit der Elektrizitätslehre, die mit hohen Anforderungen an das abstrakte Denkvermögen der Schülerinnen und Schüler einhergeht, haben sich in diesem Gebiet der Physik aber allen voran die stabilen Schülervorstellungen und die aus ihnen resultierenden Lernschwierigkeiten als besonderes Lernhemmnis erwiesen (siehe Kapitel 3). Es hat sich insbesondere gezeigt, dass viele Lernende dazu neigen, Stromkreise ausschließlich mit Hilfe des Strom- und Widerstandsbegriffs zu analysieren und die elektrische Spannung lediglich als Eigenschaft des Stroms wahrnehmen (Stichwort „Stromspannung“). Dies ist nicht nur bedauerlich, weil die elektrische Spannung an sich z.B. beim Umgang mit Batterien im Alltag eine relevante Größe darstellt, sondern insbesondere auch, weil ohne einen adäquaten Spannungsbegriff ein angemessenes Verständnis elektrischer Stromkreise nur schwer vorstellbar ist. Den Grund für die Dominanz des Strom- gegenüber des Spannungsbegriffs sehen Cohen et al. (1983) in der Tatsache, dass im Anfangsunterricht zur Elektrizitätslehre in der Regel zunächst das Stromkonzept ausführlich behandelt und die elektrische Spannung erst später thematisiert wird. Sie schreiben in diesem Zusammenhang, first impressions are strong and may impede a later, more rigorous, study of electricity" (ebd, S. 411) und fragen, ob es deshalb nicht ratsamer wäre, die elektrische Spannung vor dem elektrischen Strom einzuführen und insbesondere die Ursache-Wirkungs-Beziehung zwischen Spannung und Strom stärker in den Fokus zu rücken.

Da sich die physikalischen Vorgänge bei elektrischen Stromkreisen der direkten Wahrnehmung entziehen, ist es naheliegend, auf Analogien und Modelle zurückzugreifen, um den Lernenden das Verständnis der abstrakten Konzepte durch den Vergleich mit etwas Bekanntem zu erleichtern. Viele dieser Analogien und Modell haben sich jedoch als entweder didaktisch oder fachlich fragwürdig erwiesen (vgl. Abschnitt 4.3). Verschiedene didaktische Studien bescheinigen dem sogenannten Potenzialansatz jedoch eine vergleichsweise hohe Lernförderlichkeit zur Vermittlung eines angemessenen Verständnisses elektrischer Stromkreise und insbesondere der elektrischen Spannung. Dieser bis Anfang des 20. Jahrhunderts verbreitete Ansatz (vgl. Jochmann et al. 1914) wurde in den 1980er Jahren von Herrmann und Schmälzle (1984) wiederentdeckt, nachdem sich in der Zwischenzeit der Fokus im Unterricht

\footnotetext{
${ }^{1}$ Teile dieses Abschnitts wurden bereits in Burde et al. 2014 und Burde und Wilhelm 2016a veröffentlicht.
} 
aus historischen und nicht didaktischen Gründen vom Potenzialbegriff zum Strombegriff verschoben hatte (Gleixner 1998, S. 62). In den Folgejahren wurde der Potenzialansatz von diversen Unterrichtskonzepten aufgegriffen und in verschiedensten Formen umgesetzt (Steinberg und Wainwright 1993; Schwedes et al. 1995; Schumacher und Wiesner 1997; Gleixner 1998; Waltner et al. 2009). Gemeinsam haben diese Unterrichtskonzepte, dass sie das elektrische Potenzial explizit oder implizit zum Unterrichtsgegenstand machen und darauf aufbauend die elektrische Spannung als Potenzialdifferenz einführen. Gleixner (1998, S. 219) schreibt hierzu auf Basis seiner qualitativen Untersuchungen zum Stäbchenmodell: „Vor allem scheint die Einführung des Potentialbegriffes für die Schülerinnen und Schüler eine wesentliche Hilfe für das Verständnis und die korrekte Anwendung des Spannungsbegriff [sic] zu sein“. Im Gegensatz zu anderen Herangehensweisen wie z.B. der Einführung der elektrischen Spannung als Arbeitsfähigkeit $U=\frac{W}{Q}$ oder Antriebskraft (vgl. Abschnitt 4.3) wird beim Potenzialansatz also zunächst versucht, bei den Lernenden ein Verständnis für die der elektrischen Spannung zugrundeliegenden physikalischen Größe des elektrischen Potenzials herzustellen.

Ein solches Vorgehen ist nicht nur lerntheoretisch naheliegend, sondern hat insbesondere den Vorteil, dass das elektrische Potenzial im Gegensatz zur elektrischen Spannung keine Differenzgröße darstellt. Das elektrische Potenzial kann also im Gegensatz zur Spannung einem Leiterabschnitt lokal zugeordnet werden, was den Lernenden das Verständnis erleichtert. Als besonders hilfreich hat es sich dabei erwiesen, das elektrische Potenzial zu visualisieren, um den Lernenden den Aufbau eines angemessenen mentalen Modells zu ermöglichen, weshalb z.B. beim Stäbchenmodell (siehe Abb. 1) das Potenzial durch unterschiedliche Stäbchenhöhen dargestellt wird (Gleixner 1998). Auch Schwedes et al. (1995, S. 35) berichten darüber, dass die Verwendung einer Doppelwassersäule statt einer Pumpe zum Erzeugen von Druckunterschieden im geschlossenen Wasserstromkreis zu einem deutlich höheren Lernerfolg führt, weil mit den unterschiedlichen Wasserpegelständen die Wasserdruckdifferenz als Antrieb der Wasserströmung für die Lernenden klar erkennbar wird.

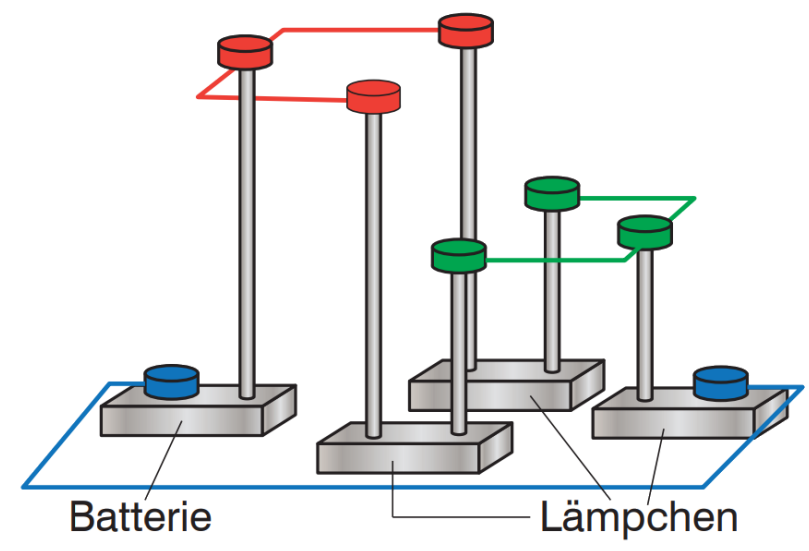

Abb. 1: Visualisierung des elektrischen Potenzials mit Hilfe des Stäbchenmodells nach Gleixner (1998) (Quelle: Burde und Wilhelm 2017c, S. 11; vgl. Koller 2008a, S. 122) 
Wie in Abschnitt 4.3 dieser Arbeit dargelegt, haben sich die bisherigen Ansätze jedoch lediglich zur Veranschaulichung einzelner Aspekte der Elektrizitätslehre bewährt. Das Stäbchenmodell von Gleixner liefert beispielsweise eine anschauliche Darstellung des Potenzials, lässt aber offen, was das Potenzial ist und gibt keine anschauliche Erklärung für den elektrischen Strom. Beim geschlossenen Wasserkreislauf mit Doppelwassersäule ist zwar der Potenzialunterschied an der Batterie über die unterschiedlichen Pegelhöhen visuell leicht wahrnehmbar, ein Verständnis für die Druck- bzw. Potenzialunterschiede an den Widerständen entwickeln aber nur wenige Lernende, da sich Wasser unter hohem Druck in nichts Sichtbarem von Wasser unter geringem Druck unterscheidet. Wird der Wasserdruck experimentell mit Hilfe von Druckmessern veranschaulicht, stellt dies für die Lernenden ein neues Phänomen dar, ,[...] dessen Verhalten bei komplexeren Schaltungen nicht vorhersagbar oder ableitbar ist, sondern von Fall zu Fall zur Kenntnis genommen und gelernt werden muss“ (Härtel 2012a, S. 24). Zudem hat sich dieses Modell grundsätzlich als problematische Lernhilfe erwiesen, da Lernende zu Wasserstromkreisen nicht nur die gleichen Schülervorstellungen besitzen wie zu elektrischen Stromkreisen, sondern der dieser Analogie zugrundeliegende Wasserdruck für sie intuitiv nicht zugänglich ist (Burde und Wilhelm 2016a). Andere Modelle wie das Fahrradkettenmodell bzw. der starre Elektronenring eignen sich hingegen gut gegen die Stromverbrauchsvorstellung, können aber nicht den Spannungsbegriff erklären oder wie sich der Strom an einer Parallelschaltung aufteilt. Es mangelt also bisher an einem anschaulichen und dennoch erklärungsmächtigen Modell für die vergleichsweise unanschauliche Elektrizitätslehre (vgl. Abschnitt 4.3).

Als besonders vielversprechend hat sich die von Steinberg und Wainwright (1993) in ihrem CASTLE-Curriculum verwendete Luftdruckanalogie erwiesen, bei der das elektrische Potenzial nicht mit dem Wasser- sondern mit dem Luftdruck verglichen wird. Gegenüber der Wasserdruckanalogie besteht der Vorteil der Luftdruckanalogie insbesondere darin, dass die Lernenden aus dem Alltag bereits eine anschlussfähige Luftdruckvorstellung mitbringen, wohingegen Wasser von den Schülern in der Regel als ein inkompressibles Kontinuum wahrgenommen wird. Insbesondere besitzen Lernende aus dem Alltag keinerlei Erfahrungen mit dem Wasserdruck in geschlossenen Wasserkreisläufen, wohingegen jedes Kind schon einmal die Erfahrung gemacht haben dürfte, dass in Luftmatratzen, Fußbällen oder Fahrradreifen die Luft komprimiert ist, ,unter Druck steht“ und deshalb ,gegen die Wände drückt“. Von hier aus ist es für sie nur ein kleiner Schritt zu erkennen, dass die Luft so lange zu Bereichen niedrigeren Luftdrucks strömt, bis sich die Drücke angeglichen haben und somit kein Druckunterschied mehr besteht. Auch wenn das CASTLE-Curriculum entgegen der Ideen von Cohen et al. (1983) zunächst ausführlich den elektrischen Strom thematisiert und erst später auf die ihn verursachenden „elektrischen Druckunterschiede“ eingeht, berichten Steinberg und Wainwright (1993) von einer gegenüber dem traditionellen Unterricht deutlich erhöhten Lernförderlichkeit des Curriculums und führen dies u.a. auf die dem Konzept zugrundeliegende intuitive und dennoch erklärungsmächtige Luftdruckanalogie zurück. 


\subsection{Grundideen des Unterrichtskonzepts ${ }^{2}$}

Die Grundidee des Elektronengasmodells besteht darin, dass sich in Metallen wie z.B. Kupfer die Elektronen in Teilchenform befinden und sich dort ähnlich einem Gas verhalten. In einem einfachen Stromkreis bestehend aus einer Batterie und einem Lämpchen sorgt eine Batterie im didaktisch vereinfachten Elektronengasmodell nun für eine Ungleichverteilung der Elektronen im Kupferleiter. Dabei wird die Batterie als Elektronenpumpe betrachtet, die Elektronen aus dem mit dem Pluspol verbundenen Leiterstück in das mit dem Minuspol verbundene Leiterstück pumpt. Entsprechend des Modells sinkt damit die Elektronendichte im mit dem Pluspol verbundenen Leiterstück, während sie im mit dem Minuspol verbundenen Leiterstück ansteigt. Mit diesen Grundannahmen weist die in dieser Arbeit verwendete Modellvorstellung nicht nur Ähnlichkeiten zum fachlichen Drude-Modell auf (vgl. Abschnitt 2.4), sondern auch zu früheren didaktischen Überlegungen zur Nutzung des Elektronengasmodells in der Schule u.a. von Kircher (1975, S. 75ff) sowie Rhöneck und Völker (1982). Im Gegensatz zu den damaligen Ansätzen, die mit vergleichsweise unintuitiven Elektronendichten bzw. Ladungsunterschieden arbeiten, wird im hier vorgeschlagenen Unterrichtskonzept versucht, ähnlich dem CASTLE-Curriculum von Steinberg und Wainwright (1993) auf dem intuitiven Luftdruckverständnis der Lernenden aufzubauen. Die Idee dabei ist, dass es im Leiter aufgrund der gegenseitigen Coulomb-Abstoßung der Elektronen zu einem von der Elektronendichte abhängigen „elektrischen Druck“ kommt. Im mit dem Minuspol verbundenen Leiterstück entsteht also ein hoher elektrischer Druck, wohingegen der elektrische Druck im Leiterstück, das mit dem Pluspol verbunden ist, absinkt. Durch Gleichsetzen des „elektrischen Drucks“ mit dem elektrischen Potenzial kann die elektrische Spannung im Elektronengasmodell so als ,elektrischer Druckunterschied" und Antrieb des elektrischen Stroms interpretiert werden. Die so bei den Lernenden etablierte Ankervorstellung des elektrischen Potenzials geht historisch auf Volta zurück, der aufgrund seiner Versuche mit Elektrometern um 1770 davon ausging, dass die freibeweglichen Ladungen in Leitern sich wie ein kompressibles Fluid mit druckähnlichen Eigenschaften verhielten (Steinberg 2008).

An dieser Stelle könnte man einwenden, dass die Dichte der Leitungselektronen in einem Stromkreis konstant ist und die Leitungselektronen damit kein kompressibles Fluid darstellen. Tatsächlich sind die Elektronen in Leitern aber in Folge von Ober- und Grenzflächenladungen nicht völlig homogen über den gesamten Stromkreis verteilt (vgl. Abschnitt 2.3). An einem Widerstand in einem einfachen Stromkreis entsteht beispielsweise an der einen Grenzfläche mit dem Leiter eine Elektronenanreicherung, während es an der anderen Grenzfläche zu einem Elektronenmangel kommt (Chabay und Sherwood 2011, S. 767). Das die Leitungselektronen antreibende elektrische Feld im Stromkreis ist dabei eine Folge dieser Grenzflächenladungen am Widerstand bzw. der Oberflächenladungen auf dem Leiter. Das dem hier vorgeschlagenen Unterrichtskonzept zugrundeliegende Elektronengasmodell unterscheidet im Rahmen der didaktischen Elementarisierung bewusst nicht zwischen Leitungselektronen und Grenz- bzw. Oberflächenladungen und beschränkt sich stattdessen darauf,

\footnotetext{
${ }^{2}$ Teile dieses Abschnitts wurden bereits in Burde und Wilhelm (2017b) veröffentlicht.
} 
lediglich die mittlere Elektronendichte innerhalb eines Leiterabschnitts zu betrachten (vgl. Abschnitt 6.1.3).

Im Sinne von Design-Based-Research (vgl. Abschnitt 7.1) wird gezielt versucht, die Erkenntnisse der bisherigen didaktischen Forschung u.a. zu Schülervorstellungen im Unterrichtskonzept zu berücksichtigen und sich an den Bedürfnissen des hiesigen Physikunterrichts $\mathrm{zu}$ orientieren. Besonderer Wert wird im Unterrichtskonzept darauf gelegt, dass die Schülerinnen und Schüler vor jeglicher Quantifizierung zunächst ein qualitativ fundiertes Verständnis der für die Elektrizitätslehre grundlegenden Konzepte „Spannung“, „Stromstärke“ und „Widerstand“" entwickeln.
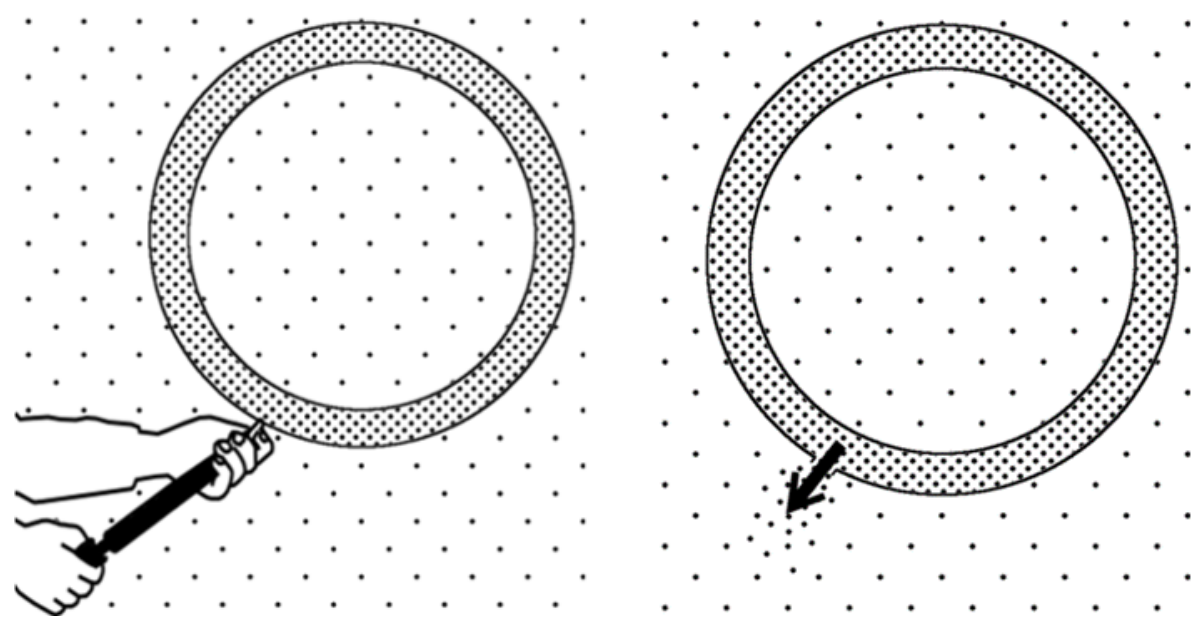

Abb. 2: Anhand eines Fahrradreifens wird erarbeitet, dass Strömungen die Folge von Druckunterschieden sind.

Um dieses Ziel zu erreichen, wird im Unterrichtskonzept an die alltägliche Luftdruckvorstellung der Schülerinnen und Schüler u.a. mit Luftmatratzen, Fußbällen und Fahrradreifen angeknüpft, wonach ,komprimierte Luft unter Druck steht, gegen die Wände drückt und das Bestreben hat, sich auszudehnen“, ohne jedoch ein fachlich korrektes Druckkonzept mit der in der Sekundarstufe I teils schwierigen Differenzierung zwischen skalarem Druckbegriff und vektoriellem Kraftbegriff vorauszusetzen oder dieses an dieser Stelle einzuführen. Stattdessen wird in Schülerexperimenten erarbeitet, dass Luft immer von Bereichen höheren Drucks zu Bereichen niedrigeren Drucks strömt. Angestrebt wird hier die allgemeine Erkenntnis, dass Strömungen immer eine Folge von Druckunterschieden sind und dass zwischen den beiden Größen Druck und Druckunterschied konzeptionell unterschieden werden muss.

Aufgrund der Bedeutung einer bildhaft-anschauungsorientierten Vorstellung des Potenzials für den Lernerfolg wird der in den Leitern herrschende elektrische Druck in Anlehnung an die Luftdruckbeispiele zunächst bei offenen Stromkreisen über eine Teilchendichtedarstellung eingeführt, im weiteren Verlauf dann aber insbesondere bei geschlossenen Stromkreisen mit Hilfe eines an alltägliche Konventionen angelehnten Farbschemas (z.B. in Hinblick auf hohe bzw. niedrige Temperaturen) direkt im Schaltplan visualisiert (rot $=$ hoher Druck, blau $=$ tiefer Druck). Durch Gleichsetzen des elektrischen Drucks mit dem elektri- 
schen Potenzial kann die Spannung dann in Analogie zu Luftdruckunterschieden als elektrischer Druckunterschied und Antrieb des elektrischen Stroms interpretiert werden.
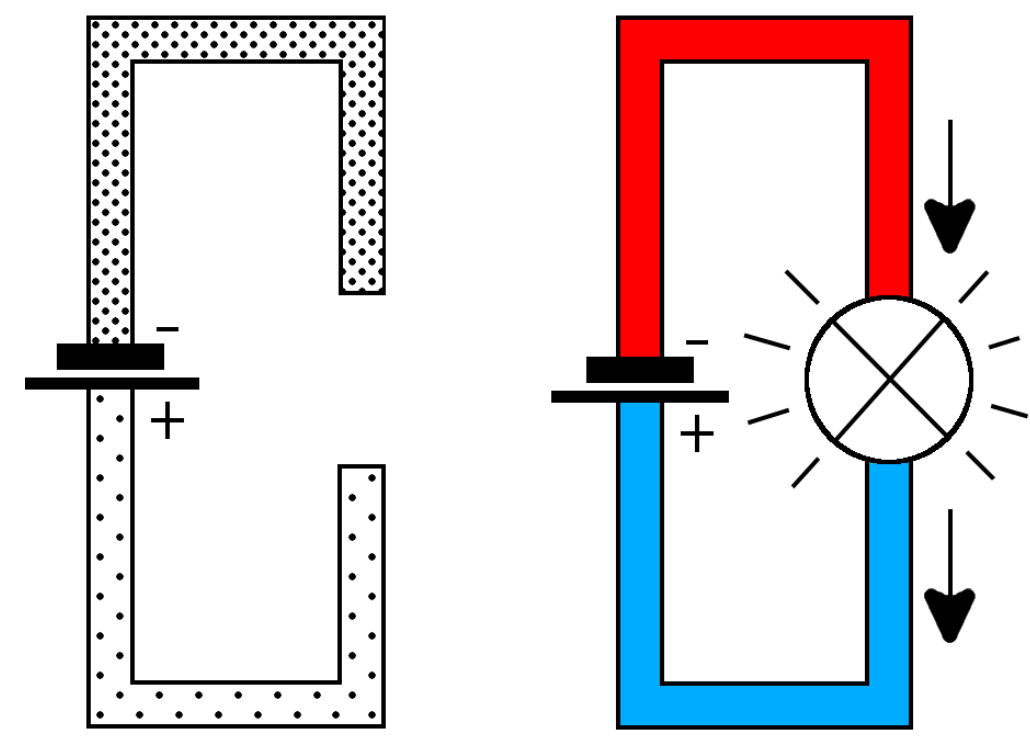

Abb. 3: Punktedichtedarstellung (links) und Farbdarstellung (rechts) des elektrischen Drucks bzw. Potenzials

Im Anschluss wird der Einfluss der Spannung auf die Stromstärke sowie vom Widerstand auf die Stromstärke halb-quantitativ über Je-desto-Beziehungen erarbeitet. Dabei ist das Ziel, bei den Lernenden ein qualitatives Verständnis der Wirkungszusammenhänge im Stromkreis zu erreichen, wonach die Stromstärke von der elektrischen Spannung kausal bewirkt und von dem Widerstand lediglich beeinflusst wird. Anschließend werden Parallel- und Reihenschaltungen diskutiert und eine mikroskopische Modellvorstellung des elektrischen Widerstands erarbeitet. Gegen Ende der Unterrichtseinheit wird dann der qualitative Wirkungszusammenhang in die quantifizierte Form $I=\frac{U}{R}$ überführt (siehe Abb. 4).

\section{Qualitativer Wirkungszusammenhang Quantitativer Wirkungszusammenhang}

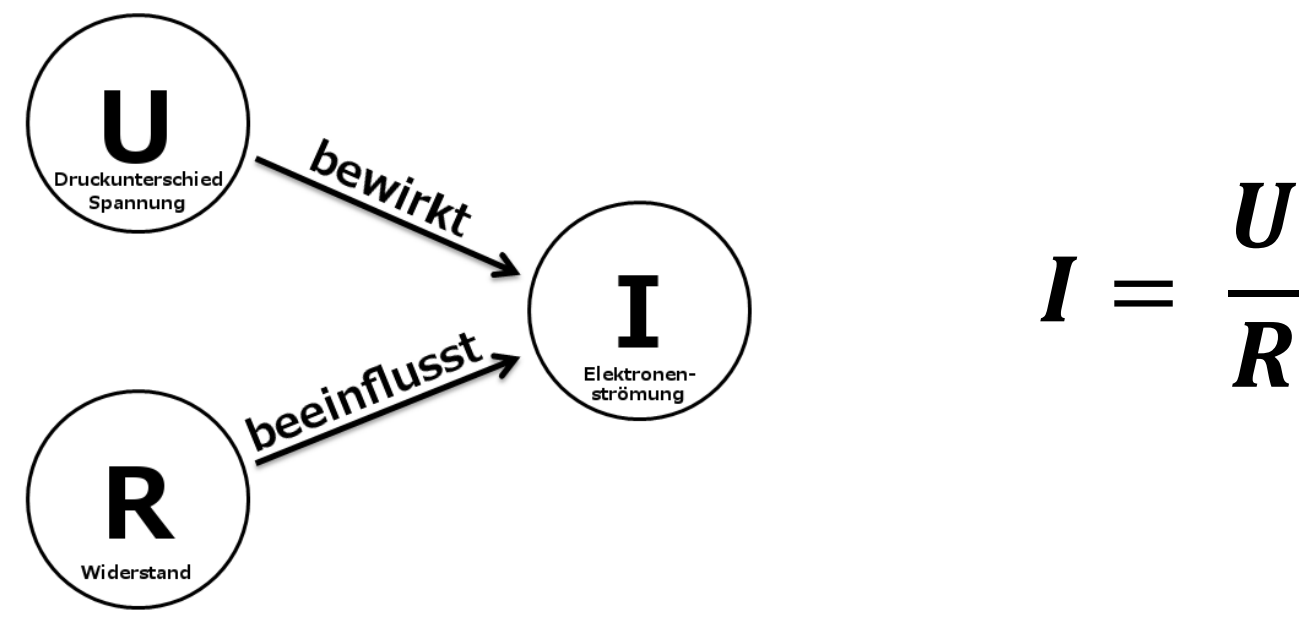

Abb. 4: Übergang vom qualitativen zum quantitativen Zusammenhang der Größen I, $U$ und $R$ 
Zusammenfassend versucht das hier vorgeschlagene Unterrichtskonzept auf Grundlage des Elektronengasmodells also an die Erfolge bisheriger Potenzialansätze (u.a. von Steinberg und Wainwright 1993, Gleixner 1998 und Waltner et al. 2009) anzuknüpfen und dabei insbesondere auf der von Steinberg und Wainwright (1993) im CASTLE-Curriculum genutzten Luftdruckanalogie aufzubauen, indem das elektrische Potenzial mit dem Luftdruck bzw. dem ,elektrischen Druck“ verglichen und entsprechend den Überlegungen von Cohen et al. (1983) noch vor der Stromstärke eingeführt wird. Um den Transfer der hier umrissenen Ideen in die Unterrichtspraxis zu erleichtern, wurden eine Reihe an Unterrichtsmaterialien erarbeitet, die auf der Internetseite JP-Bur.de/Dissertation heruntergeladen werden können. Für eine ausführlichere Beschreibung der Sachstruktur des Unterrichtskonzeptes sei an dieser Stelle zudem auf Abschnitt 6.3 dieser Arbeit verwiesen. 

A) Theoretische Grundlagen 



\section{Die fachlichen Grundlagen der Elektrizitätslehre}

\subsection{Der elektrische Strom}

Unter einem elektrischen Strom versteht man bewegte Ladung. Fließt eine Ladungsmenge $\mathrm{d} Q$ während der Zeit $\mathrm{d} t$ durch den Querschnitt eines Leiters wie beispielsweise eines Kupferdrahtes, so sagt man, es gibt einen Strom mit der Stromstärke

$$
I=\frac{d Q}{d t}
$$

Die SI-Einheit der Stromstärke ist das Ampere (A), welches definiert ist als $1 \mathrm{~A}=1 \mathrm{C} / \mathrm{s}$.

Auch wenn die Stromstärke in Illustrationen häufig mit richtungsangebenden Pfeilen dargestellt wird, ist die Stromstärke eine skalare und keine vektorielle Größe, da sowohl die Ladung als auch die Zeit skalare Größen sind. Die Pfeildarstellung soll in der Regel lediglich verdeutlichen, dass sich die Ladungen gerichtet bewegen oder gibt die Richtung der Stromdichte $\vec{\jmath}$ an. Dabei ist zu beachten, dass die eingezeichneten Vektoren aus historischen Gründen in der Regel die konventionelle Stromrichtung angeben, d.h. die Richtung, in die sich positive Ladungsträger bewegen würden.

Ist nicht der Ladungsstrom durch den gesamten Leiterquerschnitt von Interesse, sondern lediglich durch einen Teil des Leiterquerschnitts, wird dieser Fluss mit Hilfe der Stromdichte $\vec{j}$ beschrieben. Dabei ist die Stromdichte $\vec{j}$ definiert als das Verhältnis von Stromstärke $I \mathrm{zu}$ senkrecht durchflossener Querschnittsfläche $\vec{A}$ des Leiters. Die Stromstärke durch den gesamten Querschnitt eines Leiters berechnet sich dementsprechend wie folgt:

$$
I=\int \vec{\jmath} \cdot d \vec{A}
$$

Mit Hilfe der Stromdichte $\vec{\jmath}$ lässt sich ferner eine Beziehung zwischen der Strömungsgeschwindigkeit $\vec{v}$ der Ladungsträger und der Ladungsdichte $\varrho$ herstellen:

$$
\vec{\jmath}=\varrho \cdot \vec{v}
$$

Wie man an den beiden oberen Gleichungen erkennen kann, muss die Strömungsgeschwindigkeit $\vec{v}$ der Elektronen in einem unverzweigten Stromkreis mit überall konstanter Leiterquerschnittsfläche in dem Maße zunehmen, wie die Ladungsträgerdichte $\varrho$ abnimmt, damit die Stromdichte bzw. Stromstärke konstant bleibt.

\subsection{Elektrisches Feld, Spannung und Potenzial}

Elektrische Felder werden von Ladungen in dem sie umgebenden Raum erzeugt und haben neben der Feldstärke auch eine Richtung. Dabei hängt die Feldstärke an einem Punkt $P$ am Ort $\vec{r}$ sowohl von dem Betrag der felderzeugenden Ladung $Q$ als auch von der Entfernung von 
$P$ zur Ladung $Q$ ab. Die Richtung des Feldes wird einerseits durch das Vorzeichen der Ladung $Q$, andererseits durch die Richtung der Strecke von $Q$ nach $P$ festgelegt. Das elektrische Feld ist also ein Vektorfeld, das jedem Punkt im Raum um eine Ladung neben einer Größe auch eine Richtung zuweist.

Wird nun eine positive Probeladung $q_{0}$ in das von der Ladung $Q$ erzeugte Feld $\vec{E}$ gebracht, so wirkt auf diese Probeladung $q_{0}$ die elektrostatische Kraft $\vec{F}$. Der elektrische Feldstärkevektor $\vec{E}$ ist nun definiert als der Quotient der Kraft $\vec{F}$ und der Probeladung $q_{0}$ :

$$
\vec{E}=\frac{\vec{F}}{q_{0}}
$$

Möchte man also das elektrische Feld um einen geladenen Körper bestimmen, kann man mittels einer solchen positiven Probeladung $q_{0}$ den gesamten Raum auf die jeweils wirkende Kraft $\vec{F}$,,abtasten“ und so an jedem Ort den elektrischen Feldvektor $\vec{E}$ gemäß der obigen Definition ermitteln. Die SI-Einheit der elektrischen Feldstärke ist $[\mathrm{E}]=\frac{\mathrm{N}}{\mathrm{C}} \mathrm{bzw} \cdot \frac{\mathrm{V}}{\mathrm{m}}$. Die elektrostatische Kraft, die eine Ladung im elektrischen Feld $\vec{E}$ erfährt, ist dabei proportional zur elektrischen Feldstärke und zur Ladung $q$ selbst:

$$
\vec{F}=q \cdot \vec{E}
$$

Wird eine freie Ladung $q$ im elektrischen Feld $\vec{E}$ vom Punkt $\vec{r}_{A}$ zum Punkt $\vec{r}_{B}$ verschoben, so wird die Arbeit $W_{A B}$ verrichtet:

$$
W_{A B}=W\left(\vec{r}_{A}, \vec{r}_{B}\right)=\int_{\vec{r}_{A}}^{\vec{r}_{B}} \vec{F} \cdot d \vec{s}=q \int_{\vec{r}_{A}}^{\vec{r}_{B}} \vec{E} \cdot d \vec{s}
$$

Betrachtet man nun nicht die gesamte Arbeit, die das elektrische Feld an einer freien Ladung $q$ verrichtet, sondern nur die pro Ladungseinheit aufzubringende Arbeit, um diese entgegen der Feldrichtung von dem Punkt $\vec{r}_{A}$ zum Punkt $\vec{r}_{B}$ zu verschieben, so erhält man die Definition der elektrischen Spannung $U$ :

$$
U_{A B}=\frac{W_{A B}}{q}=-\int_{\vec{r}_{A}}^{\vec{r}_{B}} \vec{E} \cdot d \vec{s}
$$

Die Spannung ist unabhängig vom gewählten Integrationsweg, da es sich beim elektrostatischen Feld um ein wirbelfreies und somit konservatives Feld handelt:

$$
\vec{\nabla} \times \vec{E}=0
$$

Infolgedessen kann auch jedem Punkt des elektrostatischen Feldes eine feste Größe zugeordnet werden, die, wie die Spannung selber, nicht nur unabhängig von der Probeladung ist, sondern es durch Differenzbildung ermöglicht, die Spannung zwischen zwei Punkten zu bestim- 
men. Diese Größe ist das elektrische Potenzial $\phi$ und sie ist an einem beliebigen Punkt $\vec{r}_{A}$ wie folgt definiert, wobei $\vec{r}_{0}$ für den Ort des Nullpotenzials steht:

$$
\phi_{A}=-\int_{\vec{r}_{0}}^{\vec{r}_{A}} \vec{E} \cdot d \vec{s}
$$

Damit lässt sich die Spannung im Falle elektrostatischer Felder einfach als Potenzialdifferenz zwischen den Punkten A und B schreiben:

$$
U_{A B}=\phi_{B}-\phi_{A}
$$

So wie aus dem elektrischen Feld das elektrische Potenzial berechnet werden kann, lässt sich umgekehrt auch das elektrische Feld als Gradient des elektrischen Potenzials ausdrücken:

$$
\vec{E}=-\vec{\nabla} \phi
$$

\subsection{Grenzflächen- und Oberflächenladungen ${ }^{3}$}

Da mit Ausnahme von Supraleitern kein Leiter über eine ideale Leitfähigkeit verfügt, bedarf es eines elektrischen Feldes im Leiter, um einen konstanten Stromfluss zu ermöglichen. Wie durch das Ohm'sche Gesetz beschrieben, hängt die auf eine bestimmte Querschnittsfläche bezogene spezifische Stromdichte durch einen Leiter von dessen Leitfähigkeit $\sigma$ und der elektrischen Feldstärke $\vec{E}$ ab:

$$
\vec{\jmath}=\sigma \cdot \vec{E}
$$

Entsprechend des Ohm'schen Gesetzes muss sich die elektrische Feldstärke $\vec{E}$ in einem Leiter mit zwei Abschnitten unterschiedlicher Leitfähigkeit $\sigma$ ändern, damit weiterhin eine konstante Stromdichte $\vec{\jmath}$ vorliegt. Eine Antwort darauf, wie eine solche Änderung der elektrischen Feldstärke $\vec{E}$ bewirkt werden kann, geben die Maxwell-Gleichungen:

Tab. 1: Die differentielle Form der vier Maxwell-Gleichungen im materiefreien Raum für die elektrische Feldstärke $\vec{E}$ und die magnetische Flussdichte $\vec{B}$. Die Gleichungen, die die Quellen des elektrischen Feldes beschreiben, sind grau hinterlegt.

\begin{tabular}{c|l}
$\vec{\nabla} \cdot \vec{E}=\frac{\rho}{\varepsilon_{0}}$ & Gauß'sches Gesetz \\
\hline$\vec{\nabla} \cdot \vec{B}=0$ & Gauß'sches Gesetz für Magnetfelder \\
\hline$\vec{\nabla} \times \vec{E}=-\frac{\partial \vec{B}}{\partial t}$ & Faraday'sches Gesetz \\
\hline$\vec{\nabla} \times \vec{B}=\mu_{0} \vec{J}+\frac{1}{c^{2}} \frac{\partial \vec{E}}{\partial t}$ & (erweitertes) Ampère'sches Gesetz
\end{tabular}

Aus dem Gauß`schen und Faraday’schen Gesetz ergibt sich unmittelbar, dass ein elektrisches Feld entweder durch Ladungstrennung (z.B. Batterien bzw. Kondensatoren) oder Induktion

\footnotetext{
${ }^{3}$ Teile dieses Abschnitts wurden bereits in Burde et al. 2014 veröffentlicht.
} 
(z.B. Generatoren) erzeugt werden kann. Im beschriebenen Beispiel kommt als Erklärung nur das Gauß'sche Gesetz in Frage, was besagt, dass elektrische Ladungen die Quellen des elektrischen Feldes sind. Die erhöhte Feldstärke $\vec{E}$ im Leiter mit geringerer Leitfähigkeit $\sigma$ kann also nur mit zusätzlichen Ladungen erklärt werden, die im beschriebenen Fall an der Grenzfläche zwischen den beiden Leiterabschnitten unterschiedlicher Leitfähigkeit sitzen und deshalb auch Grenzflächenladungen genannt werden.

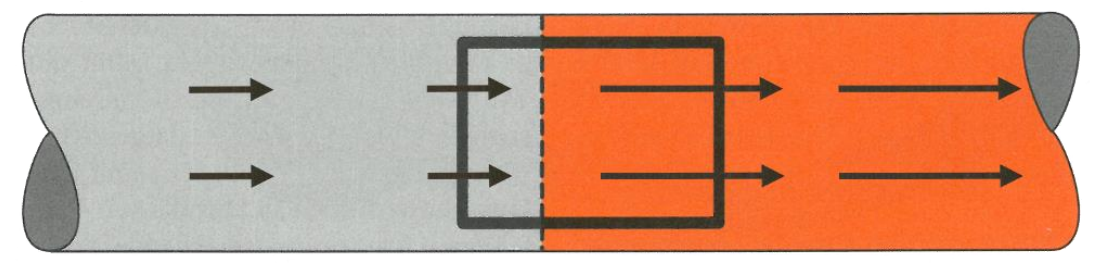

Abb. 5: Nach dem Gauß'schen Gesetz ist die positive Divergenz des elektrischen Feldes auf Ladungen innerhalb des eingeschlossenen Volumens bzw. hier der umrandeten Fläche zurückzuführen. Der Leiter mit hoher Leitfähigkeit ist grau dargestellt; der Leiter mit geringer Leitfähigkeit rot. Die Länge der Pfeile steht für die Feldstärke des elektrischen Feldes (nach Härtel 2012b, S. 26).

Nimmt man beispielsweise eine $1 \mathrm{~mm}^{2}$ große Kontaktfläche zwischen Kupfer und Konstantan an, so ergibt sich für eine Stromstärke von 1 A eine Grenzflächenladung von 27 Elementarladungen, was vor dem Hintergrund der etwa $6 \cdot 10^{18}$ Elektronen, die jede Sekunde die Grenzfläche durchströmen, verschwindend gering ist (Gleixner 1998, S. 11).

An den beiden Grenzflächen eines Widerstandselements in einem Stromkreis ist die Situation ganz ähnlich. Da die Leitfähigkeit eines Widerstands gegenüber einem Kupferdraht um viele Größenordnungen kleiner ist, die Stromstärke im Widerstand aber die gleiche sein muss wie im Draht, muss das die Elektronen im Widerstand antreibende elektrische Feld deutlich größer sein als im Kupferdraht.

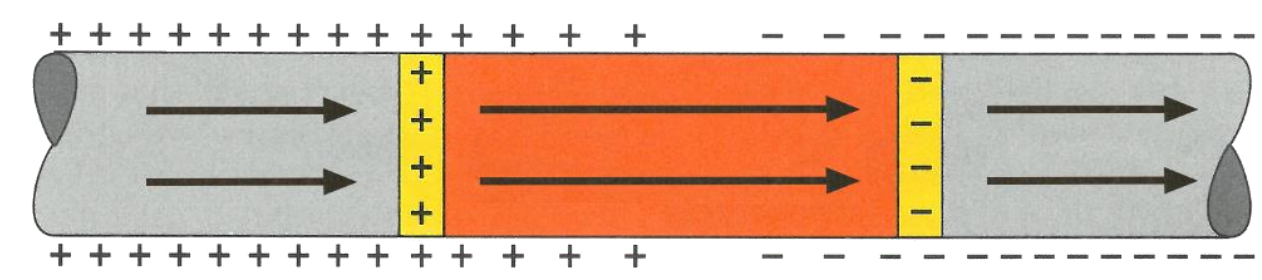

Abb. 6: Dargestellt ist die Ansammlung von Grenzflächenladungen (gelbe Bereiche) zwischen einem Leiter mit hoher Leitfähigkeit (grau) und einem Widerstand mit geringer Leitfähigkeit (rot). Die Länge der Pfeile steht für die Feldstärke des elektrischen Feldes (nach Härtel 2012b, S. 26).

Entsprechend des vorherigen Beispiels ist auch dieses elektrische Feld eine Folge von stationären Grenzflächenladungen, die sich nach dem Einschalten so lange an den Enden des Widerstands ansammeln, bis das durch sie erzeugte elektrische Feld im Widerstand groß genug ist, damit die Stromstärke innerhalb und außerhalb des Widerstands den gleichen Wert annimmt (Chabay und Sherwood 2011, S. 765; Härtel 2012b, S. 25). Ein starkes elektrisches Feld im Inneren des Widerstandes ist gleichbedeutend mit einer großen, am Widerstand anliegenden Spannung $U$, wie aus dem untenstehenden Zusammenhang unmittelbar folgt:

$$
U=\int \vec{E} \cdot d \vec{s}
$$


Offen bleibt bei der bisherigen Betrachtung die Frage, wie das im Leiter für den elektrischen Strom notwendige elektrische Feld überhaupt zustande kommt. Eine denkbare Erklärung wäre, dass das elektrische Feld im Leiter lediglich die Folge eines Ladungsüberschusses bzw. Ladungsmangels an den Batteriepolen ist. Solche extern erzeugten Felder alleine mögen als Ursache des Elektronenstroms zunächst plausibel erscheinen, denkt man aber an kompliziert gewickelte Drähte, die nicht den durch die externen Ladungen erzeugten dipolähnlichen Feldlinien folgen, wird schnell klar, dass die wahre Ursache für das elektrische Feld im Leiter selbst zu suchen sein muss (Chabay und Sherwood 2011, S. 757). Die Tatsache, dass das Drahtstück selber für die Feldrichtung verantwortlich sein muss, ist auch daran erkennbar, dass der Strom und damit das elektrische Feld immer der Geometrie des Drahtverlaufs folgen, unabhängig davon, wie dessen Krümmung verändert wird.

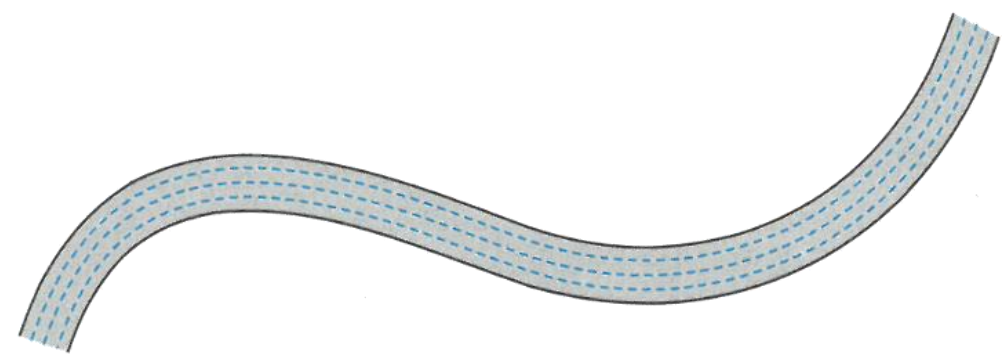

Abb. 7: Das elektrische Feld (gestrichelt) innerhalb eines stromdurchflossenen Leiters folgt der Geometrie des Drahtverlaufs unabhängig von dessen Krümmung (Quelle: Härtel 2012b, S. 26).

Wenn elektrische Felder entsprechend des Gauß`schen Gesetzes die Folge von Ladungen sind, es im Leiter selbst aber nicht zu Ladungsansammlungen kommen kann (Chabay und Sherwood 2011, S. 755) und die Batteriepole als alleinige Erklärung nicht ausreichen, bleibt die Frage, welche Ladungen das Feld innerhalb eines Leiters erzeugen.

Die Antwort sind Oberflächenladungen, also ein Elektronenüberschuss oder ein Elektronenmangel an der Oberfläche eines Leiters. Während im Inneren eines Leiters bei gleichmäßiger Verteilung von Ladungen an der Oberfläche Feldfreiheit herrscht, führt die ungleiche Verteilung von Oberflächenladungen zu der Herausbildung eines elektrischen Feldes im Leiter. Soll im Inneren des Leiters ein homogenes elektrisches Feld entstehen, kommen nach Walz (1985, S. 407) als einzige geeignete Anordnung aus Symmetrieüberlegungen gleichmäBig geladene, ringförmige Abschnitte von Ladungen an der Leiteroberfläche in Betracht. Denken wir uns hierzu ein langes, homogenes und zylindrisches Drahtstück mit einem nicht $\mathrm{zu}$ vernachlässigenden Widerstand, das an seinen Enden an die Pole einer Batterie angeschlossen ist. Aufgrund des Elektronenüberschusses an der Oberfläche des Minuspols und des Elektronenmangels an der Oberfläche des Pluspols der Batterie kommt es zu einer linear mit dem Abstand $x$ zum Minuspol abnehmenden Dichte von Oberflächenelektronen auf dem Leiterstück (siehe Abb. 8). 


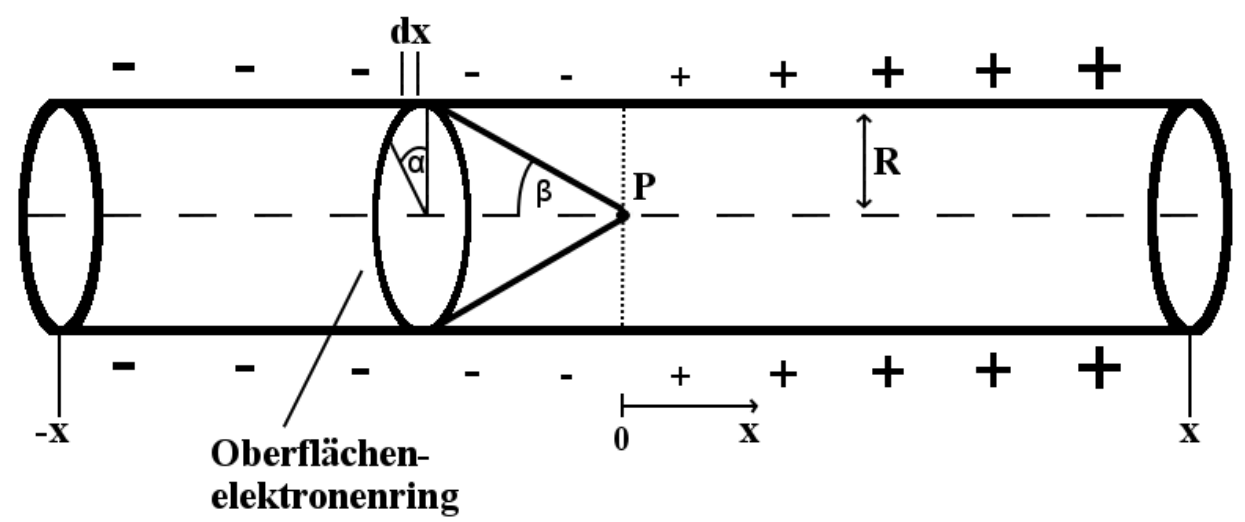

Abb. 8: Dargestellt sind die Oberflächenladungen bei einem langen, zylindrischen Draht mit homogenem Widerstand (nach Muckenfuß und Walz. 1997, S. 176).

Es ist dieser linear abfallende Dichtegradient $c$ der Oberflächenladungsdichte $\sigma$ zwischen Minus- und Pluspol der Batterie, der für die Entstehung eines homogenen elektrischen Feldes im Inneren eines solchen Leiters verantwortlich ist (Assis und Hernandes 2013, S. 26) und wie folgt beschrieben werden kann:

$$
\sigma=\mathrm{c} \cdot \mathrm{x}
$$

Für die durch eine Ladung im Flächenelement $d x \cdot d \alpha$ hervorgerufene Feldstärke am Punkt $P$ ergibt sich aufgrund des Coulomb'schen Gesetzes betragsmäßig folgender Zusammenhang:

$$
d E(x, \alpha)=\frac{1}{4 \pi \varepsilon_{0}} \frac{c \cdot x \cdot d x \cdot d \alpha}{R^{2}+x^{2}}
$$

Mit $d E_{x}=d E \cdot \cos \beta$ und $\cos \beta=\frac{x}{\sqrt{R^{2}+x^{2}}}$ erhält man für die Komponente in x-Richtung des elektrischen Feldes folgenden Zusammenhang:

$$
d E_{x}(x, \alpha)=\frac{1}{4 \pi \varepsilon_{0}} \cdot \frac{c \cdot x \cdot d x \cdot d \alpha}{R^{2}+x^{2}} \cdot \frac{x}{\sqrt{R^{2}+x^{2}}}=\frac{1}{4 \pi \varepsilon_{0}} \cdot \frac{c \cdot x^{2} \cdot d x \cdot d \alpha}{\left(R^{2}+x^{2}\right)^{\frac{3}{2}}}
$$

Integriert man nun über den Winkel $\alpha$, ergibt sich der Beitrag eines gesamten Kreisringes zu

$$
d E_{x}(x)=\frac{1}{4 \pi \varepsilon_{0}} \cdot \frac{c \cdot x^{2} \cdot d x \cdot 2 \cdot \pi \cdot R}{\left(R^{2}+x^{2}\right)^{\frac{3}{2}}}=\frac{c \cdot R}{2 \varepsilon_{0}} \frac{x^{2} \cdot d x}{\left(R^{2}+x^{2}\right)^{\frac{3}{2}}}
$$

Für das durch alle Oberflächenladungen des Leiters zwischen $-x$ und $x$ hervorgerufene elektrische Feld am Punkt $P$ ergibt sich damit (vgl. Muckenfuß und Walz 1997, S. 176):

$$
E_{P}=\frac{c \cdot R}{2 \varepsilon_{0}} \int_{-x}^{x} \frac{x^{\prime 2}}{\left(R^{2}+x^{\prime 2}\right)^{\frac{3}{2}}} d x^{\prime}
$$

Wie man sieht, ist die Feldstärke u.a. abhängig davon, wie stark sich die Dichte der Oberflächenladungen in Leitungsrichtung verändert, d.h. wie groß der Dichtegradient $c$ ist. Dieser ist 
bei einem Leiter mit homogenem Widerstand proportional zur angelegten Spannung (Chabay und Sherwood 2011, S. 761). Nach Muckenfuß und Walz (1992, S. 189) ist für die Erzeugung eines verhältnismäßig großen Stroms von $10 \mathrm{~A}$ in einem Kupferdraht von 1,5 mm² Querschnittsfläche aufgrund der großen Konstante

$$
\frac{1}{4 \pi \varepsilon_{0}}
$$

im Coulomb-Gesetz lediglich eine Änderung von wenigen hundert Oberflächenelektronen pro millimeterbreitem Oberflächenelektronenring nötig. Dies ist umso erstaunlicher, als dass auf ein Oberflächenelektron etwa $10^{16}$ Leitungselektronen im Leiterinneren kommen, die Oberflächenelektronen also nur einen verschwindend geringen Anteil an den Elektronen in einem Leiter haben (Muckenfuß und Walz 1997, S. 95). Auch ist das durch die wenigen Oberflächenladungen erzeugte elektrische Feld im Leiterinneren nur sehr schwach und kann trotzdem große elektrische Ströme bedingen (Rosser 1970, S. 265).

An Krümmungen von Leitern ist die Verteilung der Oberflächenladungen deutlich komplizierter und ist nicht mehr durch reine Symmetrieüberlegungen nachzuvollziehen. Nichtsdestotrotz gilt auch hier, dass die Oberflächenladungen sich so anordnen, dass das durch sie erzeugte elektrische Feld immer in Leiterrichtung zeigt. Qualitativ ist das so vorstellbar, dass die Elektronen in einer Krümmung so lange an der Leiteroberfläche hängenbleiben, bis die durch sie bewirkte Abstoßung von anderen anströmenden Elektronen so groß ist, dass sich durch das nun in Richtung des Leiters zeigende elektrische Feld keine weiteren Elektronen mehr an der Leiteroberfläche ansammeln (siehe Abb. 9). Eine Anleitung zur einfachen Konstruktion von Oberflächenladungen findet sich in Müller (2012, S. 12) sowie in Chabay und Sherwood (2011, S. 759).

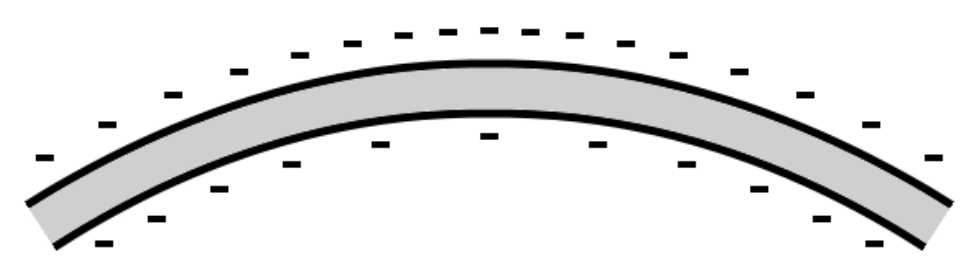

Abb. 9: Qualitative Darstellung der Verteilung von Oberflächenladungen im Falle eines gekrümmten Leiters (nach Härtel 2012b, S. 26)

Die Verteilung der Oberflächenladungen in Stromkreisen erfolgt etwa mit Lichtgeschwindigkeit, da die Leitungselektronen nahezu inkompressibel sind und zusätzliche Ladungen daher zu einer Verdichtung oder Verdünnung an der Leiteroberfläche führen (Härtel 2012b, S. 29). Wie bei elektrostatisch geladenen Leitern werden zusätzliche Elektronen also in Folge ihrer gegenseitigen Coulomb-Abstoßung an die Oberfläche gedrückt (Chabay und Sherwood 2011, S. 759). Muckenfuß und Walz (1992, S. 191) vergleichen diesen sehr schnell ablaufenden Anlaufvorgang zur passenden Verteilung der Oberflächenladungen mit einer Dichtewelle, welche mit der Lichtgeschwindigkeit des jeweiligen Mediums durch das El- 
ektronengas im Leiter läuft und so zur Ausbildung entsprechender Oberflächenladungen führt, die wiederum zur Ausbildung entsprechender elektrischer Felder in Leitungsrichtung führen.

Interessant ist in diesem Zusammenhang auch die weitgehend unbekannte Tatsache, dass die verschiedenen Leiterabschnitte eines stromführenden Leiters z.B. vor und nach einem Widerstand in Folge der unterschiedlichen Grenz- und Oberflächenladungen auch eine unterschiedliche elektrostatische Ladung aufweisen. Dies lässt sich bei hohen Spannungen ab ca. $10 \mathrm{kV}$ und der Verwendung von großen Widerständen über die daraus resultierende elektrostatische Abstoßung geladener Körper auch experimentell demonstrieren (siehe hierzu u.a. Chabay und Sherwood 1999, S. 777; Härtel 2012b, S. 29). Unterscheidet man nicht zwischen Leitungs- und Oberflächenladungen und betrachtet stattdessen nur die mittlere Elektronendichte, so kann diese in einem Stromkreis durchaus verschieden sein, auch wenn diese mittleren Dichteunterschiede aufgrund der sehr geringen Anzahl an Oberflächenladungen im Vergleich zur großen Anzahl an Leitungselektronen normalerweise zu vernachlässigen sind.

\subsection{Das klassische Elektronengasmodell}

Bisher offen geblieben ist die Frage, wie man sich die Leitungsvorgänge in einem elektrischen Leiter konkret vorstellen kann. Ein in der Schule häufig genutzter elektrischer Leiter ist Kupfer. Hierbei handelt es sich um einen metallischen Festkörper, der strukturell aus einem Metallgitter von Kupferatomen besteht. Entscheidend für die Leitfähigkeit des Kupfers ist, dass jedes der Kupferatome ein nur sehr leicht gebundenes Außen- bzw. Valenzelektron besitzt. Im Drude-Modell wird nun angenommen, dass die Valenzelektronen aufgrund ihrer nur sehr geringen Ionisierungsenergie nicht mehr einem bestimmten Atom angehören und sich daher frei durch das Gitter der Rumpfionen bewegen können, weshalb man von einem Elektronengas spricht (Meschede 2006, S. 923). Nichtsdestotrotz weisen Muckenfuß und Walz (1992, S. 183) darauf hin, dass das Elektronenkollektiv aufgrund der großen Rückstellkräfte, die bereits bei kleinen Dichteänderungen auftreten, eher mit einer Flüssigkeit zu vergleichen sei.

Verantwortlich für die Driftbewegung jedes einzelnen Elektrons im klassischen Drude-Modell ist das den Leiter durchziehende elektrische Feld $\vec{E}$, das im Falle eines gleichmäßig zylindrisch beschaffenen Leiters mit überall gleicher Leitfähigkeit homogen ist und auf jedes Elektron eine Kraft $\vec{F}$ parallel zur Leiterachse ausübt, wodurch es zu einer Elektronenbewegung in Leiterrichtung kommt:

$$
\vec{F}=q \cdot \vec{E}
$$

Wäre die vom elektrischen Feld auf die Elektronen ausgeübte Kraft die einzige wirkende Kraft, so würde dies zu einer permanenten Beschleunigung der Elektronen führen. In Folge der Stöße der Elektronen mit den Gitterrümpfen werden diese jedoch immer wieder abgebremst und in eine beliebige Raumrichtung gestreut. Bei diesen Stoßvorgängen geben die Elektronen ihre Energie an die Gitterrümpfe ab, wodurch diese in Schwingungen geraten, was 
mit einer Erwärmung des Materials einhergeht. Die Geschwindigkeitsänderung, welche die Elektronen im zeitlichen Mittel $\tau$ zwischen zwei Stößen erfahren, beträgt

$$
\Delta \vec{v}=\vec{a} \tau=\frac{\vec{F}}{m} \tau=\frac{q \vec{E}}{m} \tau
$$

In Folge des Wechselspiels zwischen Beschleunigung im elektrischen Feld und Abbremsung durch Stöße stellt sich ein konstanter Elektronenstrom mit einer konstanten durchschnittlichen Driftgeschwindigkeit ein. Bei einem Kupferdraht von $1 \mathrm{~mm}^{2}$ Querschnittsfläche und einem Strom von 10 A ist diese sehr gering und liegt typischerweise bei weniger als $1 \mathrm{~mm} / \mathrm{s}$ (Muckenfuß und Walz 1992, S. 184).

Diese gerichtete und sehr langsame Elektronendrift wird allerdings überlagert von einer sehr hohen, ungerichteten Bewegung der Elektronen, welche im Falle von Kupfer im Bereich von ca. $10^{6} \mathrm{~m} / \mathrm{s}$ liegt (Halliday et al. 2001, S. 570). Im Rahmen der klassischen Physik ist diese Bewegung nicht erklärbar, trägt allerdings auch nicht zum Nettostrom bei, da sie zufälliger Natur ist und keine Richtung bevorzugt. Nichtsdestotrotz können zufällige Abweichungen hiervon einen kurzzeitigen Nettostrom verursachen, was dann als Widerstandsrauschen messbar ist. Interessant ist, dass sich nur ca. $1 \%$ der Elektronen so frei bewegen können, wie man dies von einem idealen Gas erwarten würde, da die Bewegung der restlichen Elektronen aufgrund der positiv geladenen Gitteratome hoch korreliert ist. Weitere Modellgrenzen bestehen u.a. darin, dass das Elektronengas im Gegensatz zu einem idealen Gas kaum kompressibel ist (Muckenfuß und Walz 1992, S. 181), dass es im Gegensatz zu einem idealen Gas nahezu keinen Beitrag zur spezifischen Wärmekapazität liefert und dass der reale Widerstand deutlich geringer ist, als es das Modell vorhersagt. Nichtsdestotrotz besteht eine Leistung des Elektronengasmodells darin, die hohe Wärmeleitfähigkeit von Metallen über das Wiedemann-Franz-Gesetz mit ihrer hohen elektrischen Leitfähigkeit zu verknüpfen.

\subsection{Das Fermi-Gas-Modell}

Wären die Leitungsvorgänge in Leitern rein klassisch zu erklären, so müssten die Elektronen ähnlich einem Molekülgas der Maxwell-Boltzmann-Verteilung unterliegen, womit ihre mittlere Geschwindigkeit proportional zur Quadratwurzel der absoluten Temperatur wäre. Damit müsste die ungerichtete Bewegung der Elektronen nahe dem absoluten Nullpunkt zum Erliegen kommen. Dies ist aber nicht der Fall, da die Elektronen den Gesetzen der Quantenmechanik unterliegen und somit Welleneigenschaften sowie quantisierte Energiezustände besitzen und als Fermionen dem Pauli-Prinzip folgen. Aufgrund ihres Wellencharakters können Elektronen beispielsweise an dem Ionengitter gestreut werden, d.h. der Stoßvorgang am Ionengitter kann nicht mit einem einfachen mechanischen Stoß an einem Gitterrumpf verglichen werden. Sofern es sich um einen idealen Kristall mit periodischer Gitterstruktur handelt und die Wellenlänge der Elektronen deutlich größer ist als die Abstände der Kristallebenen, dürfte es zu keiner Streuung kommen, d.h. die Elektronenwellen müssten sich ungehindert ausbreiten können und es gäbe keinen elektrischen Widerstand. Zur Streuung von Elektronenwellen 
kommt es jedoch aufgrund der bei realen Gittern vorkommenden Gitterfehler, z.B. durch thermische Schwingungen der Atomrümpfe oder chemische Verunreinigungen (Tipler und Llewellyn 2003, S. 561; Meschede 2006, S. 946). Die Streuung der Elektronenwellen stellt in diesem Modell die Erklärung des elektrischen Widerstands dar, dessen Größe unter der Annahme des Wellencharakters der Elektronen deutlich besser vorhergesagt werden kann als im klassischen Elektronengasmodell, das von Elektronen als Teilchen ausgeht.

Nach dem Pauli-Prinzip kann es in einem System wie z.B. einem Atom aufgrund der Spin-Quantisierung ferner höchstens zwei Elektronen geben, die in allen räumlichen Quantenzahlen übereinstimmen. Daraus folgt unmittelbar, dass sich im Gegensatz zur klassischen Maxwell-Boltzmann-Verteilung lediglich zwei Elektronen eines Systems im untersten Energieniveau befinden können, während die restlichen Elektronen entsprechend des PauliPrinzips die nächst höheren Energieniveaus füllen und daher entsprechend höhere Energiezustände einnehmen müssen. Bei Temperaturen nahe dem absoluten Nullpunkt verlieren Elektronen in einem Leiter daher auch nicht ihre komplette Energie und führen weiterhin ungerichtete Bewegungen aus. Die Energieverteilung der Elektronen weicht in diesem Fall also stark von der klassisch zu erwartenden Maxwell-Boltzmann-Verteilung ab und lässt sich stattdessen mit der Fermi-Dirac-Statistik beschreiben, welche die Besetzungswahrscheinlichkeit $P(E)$ eines bestimmten Energieniveaus in einem System in Abhängigkeit von der FermiEnergie $E_{F}$ und der absoluten Temperatur $T$ angibt:

$$
P(E)=\frac{1}{e^{\frac{\left(E-E_{F}\right)}{k T}}+1}
$$

Die Fermi-Energie $E_{F}$ gibt dabei die Energie des höchsten besetzten bzw. halbbesetzten Energiezustandes bei $T=0$ an. Bei $T=0$ sind also unterhalb der Fermi-Energie alle Zustände besetzt, während alle Zustände über der Fermi-Energie unbesetzt sind. Für Kupfer liegt die Fermi-Energie bei $7 \mathrm{eV}$ und damit deutlich höher als die thermische Energie bei Zimmertemperatur, die bei $300 \mathrm{~K}$ lediglich ca. 0,04 eV beträgt (Tipler und Mosca 2006, S. 1229). Die Fermi-Energie ist im Wesentlichen von der Temperatur unabhängig und der Grund für die im klassischen Elektronengasmodell nicht erklärbare sehr hohe, aber ungerichtete Bewegung der Elektronen. Das Verhalten der freien Elektronen in Metallen ist aufgrund all dieser Eigenschaften nicht mit einem klassischen Gas zu vergleichen, weshalb man stattdessen häufig von einem Fermi-Gas spricht.

\subsection{Das Bändermodell von Festkörpern}

Betrachtet man wie bisher einzelne Atome, so befinden sich deren Elektronen auf diskreten Energieniveaus. Die Verteilung der 29 Elektronen eines isolierten Kupferatoms auf die verschiedenen diskreten Teilschalen wird durch dessen Elektronenkonfiguration beschrieben:

$$
1 s^{2} 2 s^{2} 2 p^{6} 3 s^{2} 3 p^{6} 3 d^{10} 4 s^{1}
$$


Die ersten sechs Teilschalen beim Kupfer sind also vollständig besetzt, während die äußere 4s-Schale nur halbbesetzt ist. Nähert man zwei Kupferatome einander an, so fangen deren Wellenfunktionen, beginnend mit den Wellenfunktionen der äußersten Elektronen, an sich zu überlagern. Da es sich ab diesem Zeitpunkt der Überlappung der Wellenfunktionen nicht mehr um zwei voneinander getrennte Wellenfunktionen handelt, spricht man von einem zweiatomigen System. Wie bei einem einatomigen System gilt aber auch für das zweiatomige System bestehend aus nun $2 \cdot 29=58$ Elektronen das Pauli-Prinzip, wonach alle 58 Elektronen unterschiedliche Quantenzustände besitzen müssen. Dies hat zur Folge, dass sich jedes Energieniveau der Einzelatome in zwei sehr nah beieinanderliegende Energieniveaus im zweiatomigen System aufspaltet. Werden nun weitere Atome dem System hinzugefügt, so spalten sich die Energieniveaus der zuvor einzelnen Atome im Gesamtsystem immer weiter auf. In einem Festkörper wie z.B. einem Kupferdraht bestehend aus $N$ Atomen spaltet sich dann jedes Niveau der einzelnen Atome in $N$ Energieniveaus für den Festkörper auf. Da die Anzahl an Atomen in einem solchen Festkörper immens groß ist und die Energieniveaus daher sehr nahe beieinanderliegen, spricht man nicht länger von diskreten Niveaus wie bei Einzelatomen, sondern von Energiebändern, die kontinuierlich erscheinen. Zwischen den Energiebändern besteht in der Regel eine Energielücke, d.h. ein Energiebereich, der von Elektronen nicht besetzt werden kann und der die einzelnen Energiebänder voneinander trennt. In Folge der größeren Überlappung der Wellenfunktionen äußerer Elektronen, kommt es bei diesen zu einer größeren Aufspaltung der Energieniveaus, was zu entsprechend breiteren Energiebändern führt (Halliday et al. 2001, S. 875).

Mit Hilfe der Energiebänder lässt sich auch die Frage beantworten, warum manche Stoffe gute Leiter und andere Stoffe gute Isolatoren sind. Für die Leitfähigkeit eines Kristalls ist entscheidend, wie das höchste mit Elektronen besetzte Energieband gefüllt ist. Dieses kann je nach Material entweder vollständig gefüllt, teilweise gefüllt oder leer sein. Bei Kupfer ist das höchste besetzte Energieband nur zur Hälfte mit Elektronen gefüllt, d.h. es gibt in diesem Band noch eine große Anzahl unbesetzter Zustände (siehe Teil (a) in Abb. 10). Wird nun eine Spannung und damit ein elektrisches Feld angelegt, so können die Elektronen eine Beschleunigung und damit Energiezufuhr erfahren. Sie können also leicht in einen der zuvor unbesetzten Zustände etwas höherer Energie desselben Bandes wechseln und so zum Stromfluss beitragen. Dieser einfache Wechsel in einen Zustand mit etwas höherer Energie ist bei Isolatoren hingegen nicht möglich, da bei ihnen das Valenzband, d.h. das höchste mit Elektronen gefüllte Band, bereits vollständig gefüllt ist. Zwischen dem Valenzband und dem nächsthöheren Energieband, dem Leitungsband, befindet sich eine Energie- bzw. Bandlücke $E_{b}$ von typischerweise 5 bis $10 \mathrm{eV}$, in dem sich keine zu besetzenden Energiezustände befinden (siehe Teil (b) in Abb. 10). Bei einer Zimmertemperatur von $300 \mathrm{~K}$ und der damit verbundenen thermischen Energie von $E_{t h}=\frac{3}{2} k T \approx 0,04 \mathrm{eV}$ gelingt es damit nahezu keinen Elektronen, die Bandlücke von $5 \mathrm{eV}$ zu überwinden und ins Leitungsband $\mathrm{zu}$ wechseln, was für einen Stromfluss aber nötig wäre. Materialien mit einer solchen Bandlücke stellen daher gute elektrische Isolatoren dar. 


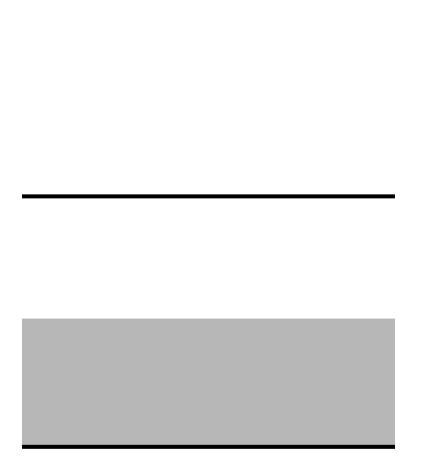

(a) Leiter

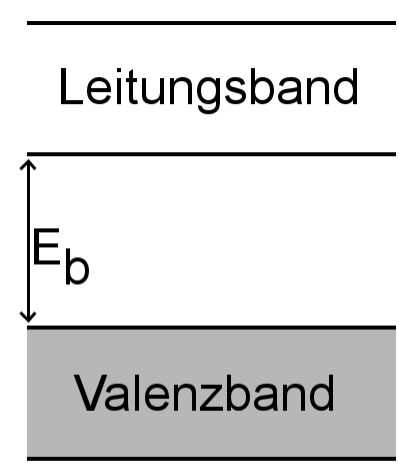

(b) Isolator

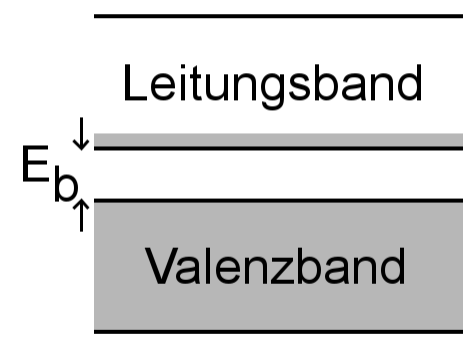

(c) Halbleiter

Abb. 10: Darstellung der Energiebänder eines Leiters, eines Isolators und eines Halbleiters. Die grauen Bereiche stehen für die durch Elektronen besetzten Zustände (nach Giancoli 2006, S. 1392).

Halbleiter unterscheiden sich von Isolatoren darin, dass bei ihnen das Valenzband vom ungefüllten Leitungsband nur durch eine Energielücke von etwa $1 \mathrm{eV}$ getrennt ist und einige Elektronen daher auch bei Raumtemperatur diese Energielücke überwinden können, um ins Leitungsband zu wechseln (siehe Teil (c) in Abb. 10). In Folge kommt es bei Halbleitern auch bei Zimmertemperatur zu einem kleinen Stromfluss, wenn eine Spannung angelegt wird. Im Gegensatz zu normalen, metallischen Leitern, deren Leitfähigkeit mit steigender Temperatur abnimmt, nimmt die Leitfähigkeit von Halbleitern aber mit steigender Temperatur zu. Der Grund besteht darin, dass mit steigender Temperatur die thermische Energie $E_{t h}$ der Elektronen entsprechend $E_{t h}=\frac{3}{2} k T$ zunimmt und somit mehr und mehr Elektronen vom Valenz- ins Leitungsband wechseln können (Giancoli 2006, S. 1391). 


\section{Schülervorstellungen}

\subsection{Generelle Aspekte zu Schülervorstellungen}

\subsubsection{Schülervorstellungen in der Physik}

Das Thema Schülervorstellungen löste in den 1970er Jahren einen Boom an internationalen Forschungsbemühungen aus und führte zu einem umfangreichen didaktischen Wissen in diesem Feld. Auch wenn die Vielzahl an Publikationen schwer zu überblicken ist, scheinen insbesondere die Themengebiete „Elektrizitätslehre“ und „Mechanik“ besonders gut untersucht und dokumentiert zu sein (Duit 2009b). Aufgrund der Größe und der Vielschichtigkeit der Schülervorstellungsforschung haben sich in der Didaktik verschiedene Begriffe zu diesem Thema etabliert. So finden sich in der Literatur abhängig von den jeweils vertretenen Sichtweisen und theoretischen Ansätzen neben dem Begriff „Schülervorstellungen“ u.a. auch die Begriffe „Fehlvorstellungen“, „Fehlkonzepte“, „Präkonzepte“, „Vorverständnis“, „Alltagstheorien“ oder „Alltagsvorstellungen“. Auch wenn die Begriffe leicht unterschiedlich konnotiert sind und mit ihnen unterschiedliche Wertungen einhergehen können, beschreiben sie doch alle den Umstand, dass Kinder nicht als unbeschriebene Blätter („Tabula rasa“) in den Unterricht kommen, sondern häufig aufgrund ihrer Alltagserfahrungen schon über gewisse, tief verankerte Vorstellungen zu physikalischen Phänomenen und physikalischen GesetzmäBigkeiten verfügen. Diese subjektiven Theorien der Schüler erweisen sich im Alltag trotz ihres in der Regel sehr eingeschränkten Geltungsbereiches oftmals als Hilfe, weil sie es ihnen ermöglichen, alltägliche Phänomene und Beobachtungen einzuordnen und sich in ihrer Umwelt zu orientieren.

Problematisch an diesen Vorstellungen ist jedoch insbesondere, dass sie in der Regel in wichtigen Aspekten nicht mit der akzeptierten wissenschaftlichen Erklärung übereinstimmen und physikalische Begriffe, Konzepte und Gesetze von den Schülern oftmals anders verstanden werden als von Physikern (Duit 1993a, 1993b). Dies führt in der Regel zu erheblichen Lernschwierigkeiten im Unterricht, da sich diese vorunterrichtlichen Vorstellungen aus Sicht der Schüler im Alltag zur Erklärung bestimmter Phänomene bzw. Beobachtungen bewährt haben und sie daher oftmals keinen Grund sehen, ihre bisherigen Erklärungen zugunsten einer für sie neuen wissenschaftlichen Sichtweise aufzugeben. Auch wenn das neue wissenschaftliche Konzept einen größeren Geltungsbereich besitzt und in sich konsistenter ist, sind die Schüler oftmals mit ihrem bisherigen Alltagskonzept zufrieden, zumal sie in der Regel keine hohen Ansprüche an die innere Konsistenz und die Verallgemeinerbarkeit von Theorien stellen. In der Folge erweist sich das Vorverständnis der Schüler gegenüber unterrichtlichen Bemühungen als sehr resistent und steht der Übernahme der physikalisch korrekten Vorstellung häufig im Wege. Hinzu kommt, dass gewisse Sprachbilder wie „Die Sonne geht auf“ oder „Der Strom wird verbraucht“ sowie Sinneserfahrungen wie z.B. „Die Zentrifugalkraft zieht mich in der Kurve nach außen“ die Ausbildung entsprechender Schülervorstellungen nicht nur fördern, sondern diese tagtäglich verstärken (Duit 2009a). Die häufig in Folge dieser 
Präkonzepte auftretenden Lernschwierigkeiten wurden in zahlreichen Studien zu unterschiedlichen Themengebieten gut dokumentiert (vgl. Maichle 1982; Shipstone 1984; Duit 1986, Wiesner 1986, Rhöneck 1986; Shipstone et al. 1988; McDermott und Shaffer 1992; etc.).

Für die oftmals noch nach dem Unterricht vorhandenen nichtwissenschaftlichen Vorstellungen werden teilweise die leicht abwertenden Begriffe „Fehlvorstellungen“ bzw. „Fehlkonzepte“ verwendet, wohingegen im schulischen Kontext die Begriffe „Präkonzepte“ und „Vorverständnis“ zur Beschreibung von Vorstellungen genutzt werden, die vor dem Unterricht zu finden sind. Die Begriffe „Alltagstheorien“ und „Alltagsvorstellungen“ stellen eine neutralere Bezeichnung von alternativen Erklärungsansätzen dar, da sie eine gewisse Würdigung der von den Lernenden erbrachten Denkleistung implizieren und im Gegensatz zum Begriff „Schülervorstellungen“ auch keinen direkten Bezug zur Schule aufweisen (Wilhelm 2005, S. 5). Gängige Begriffe im englischsprachigen Raum sind darüber hinaus noch „alternative frameworks“ (Driver et al. 1978), ,children's science“ (Osborne 1980) und ,intuitive

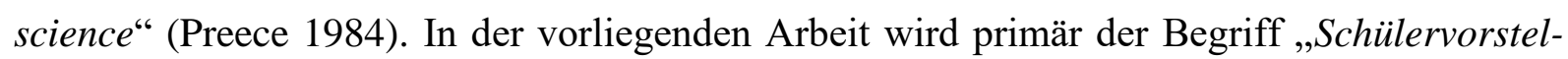
lungen" verwendet, da sich dieser Begriff einerseits im deutschsprachigen Raum etabliert hat und sich in dieser Arbeit andererseits vorrangig auf nichtwissenschaftliche Vorstellungen von Schülern nach dem Unterricht bezogen wird.

\subsubsection{Das Lernen als aktiver Konstruktionsprozess}

In der Lehr- und Lernforschung hat sich die konstruktivistische Sichtweise zur Erklärung von Lernprozessen spätestens seit den 1970er Jahren durchgesetzt. Zwar existiert keine einheitliche konstruktivistische Lerntheorie, den unterschiedlichen konstruktivistischen Ansätzen ist aber gemein, dass sie Lernen als einen aktiven Prozess verstehen, bei dem das Individuum auf Grundlage seiner Vorerfahrungen neues Wissen konstruiert und seiner sozialen bzw. natürlichen Umwelt auf diesem Wege Bedeutung verleiht (Steffe et al. 1995; Duit 1995b). Dem Subjekt und seinem individuellen Vorwissen bzw. Vorstellungen kommt aus konstruktivistischer Perspektive also eine zentrale Rolle zu, da neues Wissen vom Individuum nicht einfach passiv übernommen bzw. abgespeichert werden kann, sondern vom Lernenden immer aktiv und individuell in bereits bestehende kognitive Strukturen bzw. Schemata integriert werden muss. Auch wenn an dieser Stelle nicht im Einzelnen erläutert werden soll, wie Lernen in den verschiedenen konstruktivistischen Ansätzen im Einzelnen verstanden wird, ist offensichtlich, dass diese Perspektive auf das Lernen im klaren Gegensatz zum zuvor dominierenden behavioristischen Lernbegriff steht, bei dem das Subjekt als ein passives, von Umweltreizen zu beschreibendes, leeres Blatt gesehen wird.

Wie bereits angedeutet, handelt es sich beim Konstruktivismus jedoch nicht um eine kohärente erkenntnistheoretische bzw. lernpsychologische Theorie, sondern vielmehr um einen Oberbegriff für eine Vielzahl an theoretischen Ansätzen mit jeweils unterschiedlichen Annahmen und Akzentuierungen. Der z.B. von Glasersfeld (1993) vertretene radikale Konstruktivismus geht soweit, dass er die Möglichkeit eines vom Subjekt unabhängigen und somit objektiven Wissens ausschließt, da die gesamte vom Individuum wahrgenommene Reali- 
tät immer auf Basis der eigenen Sinneswahrnehmungen und des eigenen Vorwissens subjektiv konstruiert wird. Der radikale Konstruktivismus geht also mit einer relativierenden Sicht auf das Wissen selbst einher, da die Realität und somit das gesamte Wissen lediglich als Ergebnis einer subjektiven Wirklichkeitskonstruktion betrachtet wird (Reich 2001, S. 363). Eine für Lehrsituationen bedeutsame Strömung stellt ferner der soziale Konstruktivismus nach Vygotsky (1978) dar, der dem soziokulturellen Lernumfeld bei der Wissensvermittlung eine große Bedeutung beimisst. Die zentrale Annahme des sozialen Konstruktivismus besteht darin, dass Kinder ihr volles Entwicklungspotenzial (,Zone of Proximal Development") erst durch soziale Interaktionen voll ausschöpfen können. Entsprechend kommt nach dieser Schule des Konstruktivismus der aktiven Gestaltung von Lernarrangements eine besondere Bedeutung zu. Ein solches Lernarrangement hat nach Bruner (1986, S. 123) dabei die Funktion eines Gerüsts (,scaffolding“), dass den Lernenden vorrübergehend z.B. bei der Bearbeitung eines Problems konstruktiv unterstützt, jedoch schrittweise wieder entfernt wird, sobald der Lernende das Problem selbstständig lösen kann. Ein erster Schritt eines solchen Gerüstes zur Unterstützung von Lernenden kann beispielsweise eine Unterrichtssequenz mit einer sorgfältig ausgearbeiteten Sachstruktur darstellen, die versucht, auf optimale Weise an das Vorwissen der Schüler anzuknüpfen und auf diesem schrittweise aufzubauen.

Neben dem sozial-konstruktivistischen Ansatz hatte insbesondere der personalkonstruktivistische Ansatz, der maßgeblich auf die Arbeiten von Jean Piaget zurückgeht, erheblichen Einfluss darauf, wie Lernprozesse im Unterricht gestaltet und erklärt werden können. Nach Piaget (1954) ist der Lernprozess, d.h. die aktive Konstruktion neuen Wissens auf Basis bereits vorhandener kognitiver Strukturen, ein Wechselspiel von Assimilation und Akkommodation. Von Assimilation spricht er, sofern sich neue Informationen in die bereits vorhandenen kognitiven Strukturen integrieren lassen. Akkommodation hingegen beschreibt die Notwendigkeit auf Seiten der Lernenden, ihre bisherigen kognitiven Strukturen zu modifizieren oder neu aufzubauen, weil sich die neuen Informationen nicht in die bereits vorhandenen Wissensstrukturen assimilieren lassen. Assimilation und Akkommodation sind allerdings sehr eng miteinander verknüpft, da das Individuum nach Piaget das Bestreben hat, im Wechselspiel zwischen Assimilation und Akkommodation einen Ausgleich (,Äquilibration“) zwischen seinen Sinneseindrücken und den eigenen kognitiven Strukturen zu erzielen (Duit und Treagust 1998, S. 8).

In der Didaktik der Naturwissenschaften wird heute in der Regel eine moderate Sichtweise des Konstruktivismus vertreten, die einerseits davon ausgeht, dass menschliches Wissen grundsätzlich das Ergebnis eines Konstruktionsprozesses ist, andererseits aber die Existenz einer vom Subjekt unabhängigen Realität nicht anzweifelt. Auch wird in diesem gemäBigten Konstruktivismus sowohl die Bedeutung der individuellen Konstruktion von Wissen als auch der sozialen Dimension des Lernens für wichtig befunden (Gerstenmaier und Mandl 1995; Widodo und Duit 2004, S. 234). 


\subsubsection{Der Einfluss des Vorwissens auf das Lernen in der Physik}

Ein entscheidender Faktor für Lernprozesse aus konstruktivistischer Perspektive ist das Vorwissen, auf dessen Grundlage das Individuum neuen, durch Sinneswahrnehmungen aufgenommenen Informationen erst Bedeutung verleiht. Diese Einsicht spiegelt sich auch in dem berühmten Zitat von Ausubel (1968, vi) wider: „If I had to reduce all of educational psychology to just one principle, I would say this: The most important single factor influencing learning is what the learner already knows. Ascertain this and teach him accordingly". Das Problem dabei ist, dass dem Vorverständnis der Schüler im Physikunterricht eine ambivalente Rolle zukommt. Einerseits kann es grundsätzlich Anknüpfungspunkt für das weitere Lernen darstellen, andererseits erweist es sich oft als Lernhindernis, weil das aus physikalischer Sicht falsche Vorverständnis der Schüler die Aufnahme, Verarbeitung und Interpretation neuer Sinneseindrücke und Informationen maßgeblich beeinflusst.

Das Vorverständnis der Schüler ist in der Folge häufig die Ursache vieler Lernschwierigkeiten, weil die Schüler aufgrund ihrer Alltagsvorstellungen ,[...] das Neue nur durch die Brille des ihnen bereits Bekannten und Vertrauten ,sehen 'können" und in der Folge häufig gar nicht verstehen, ,[...] was sie im Unterricht hören oder sehen und was sie im Lehrbuch lesen“ (Duit 1993a, S. 7). Ein gutes Beispiel dafür, wie stark das Vorwissen ein korrektes Verständnis im Unterricht erschwert, stellt der Strombegriff dar. Während der Lehrer, wenn er im Unterricht von Storm spricht, den Ladungsträgerstrom meint, nehmen die Schüler vor dem Hintergrund des im Alltag aber energetisch verwendeten Strombegriffs oftmals an, der Lehrer spreche von einem „Energiestrom“. Nach Muckenfuß und Walz (1992, S. 21) könnte man sagen, dass „Lehrer und Schüler [...] u.U. verschiedene Sprachen trotz gleicher Wörter [sprechen]“. Eine an sich korrekte Erklärung des Lehrers kann von den Schülern also vor dem Hintergrund ihres Vorwissens völlig anders und oftmals falsch interpretiert werden. Sofern sich der Lehrer nicht über die Alltagsvorstellungen seiner Schüler bewusst ist, kann er die dann von seinen Schülern gegebenen Antworten in ähnlicher Weise aufgrund seines anderen Wissenshintergrundes ganz anders interpretieren, als sie von den Schülern gemeint waren und bestätigt den Schülern so ggf. fälschlicherweise die Korrektheit ihrer Aussage. In Folge dieses in der Pädagogik unter dem Begriff des ,hermeneutischen Zirkels“ bekannten Phänomens reden Lehrer und Schüler im Unterricht oft aneinander vorbei. Duit (2009a, S. 611) geht sogar so weit anzunehmen, dass Missverständnisse im Unterricht eher die Norm als die Ausnahme darstellen. Wenn Schüler aber die korrekten physikalischen Begriffe verwenden, jedoch weiterhin präkonzeptionell denken, werden nach Nachtigall (1992, S. 12) aus Präkonzepten durch den Unterricht Misskonzepte (vgl. Wilhelm 2005, S. 7).

Das Vorverständnis ist also mit einem Filter vergleichbar, der nicht nur bestimmt, wie Schüler Erklärungen im Unterricht verstehen, sondern auch, welche Aspekte sie beim Experimentieren wahrnehmen, da jede Beobachtung immer auch eine vom Vorwissen beeinflusste Interpretation darstellt (Duit 2009c). Da Experimente im Physikunterricht eine zentrale Rolle spielen und Lehrkräfte häufig davon ausgehen, Schüler mit den richtigen Experimenten (z.B. einem sogenannten „Entscheidungsexperiment“) von der physikalisch korrekten Sichtweise 
überzeugen zu können, ist es wichtig, sich darüber bewusst zu sein, wie stark die Wahrnehmung der Schüler letztlich durch ihre eigenen Überzeugungen geprägt wird. Oftmals ist es so, dass Schüler beim Experimentieren nicht das sehen, was der Lehrer bei einem Versuchsaufbau für klar und eindeutig zu erkennen hält, sondern nur das, ,[...] was ihnen ihre Vorstellungen zu sehen erlauben“ (Duit 1993b, S. 5), wie die folgenden Untersuchungen exemplarisch illustrieren.

In der Elektrizitätslehre der 10. Klasse eines Gymnasiums berichtet Schlichting (1991) von einem Experiment zur Untersuchung der Frage, warum die Wirkung des Stroms nach dem Einschalten im Prinzip auch an entfernten Stellen (nahezu) instantan einsetzt. Um den Schülern eine passende Fließvorstellung des elektrischen Stroms zu vermitteln, wurde ein Draht aufgespannt und gefragt, an welcher Stelle der Draht wohl zuerst zu glühen beginnt. Je nach angenommener Richtung des Stroms argumentierten die Schüler, der Draht müsse von links nach rechts bzw. von rechts nach links anfangen zu glühen. Eine andere Gruppe vertrat die Ansicht, das Glühen müsse sich von der Mitte aus zu den Rändern des Drahtes ausbreiten, da die beiden von den Polen der Batterie ausgehenden Ströme zuerst in der Mitte des Drahtes aufeinanderprallen würden und es dann zu einem Rückstau komme. Eine dritte Gruppe vertrat die physikalisch korrekte Annahme, dass der Draht an allen Stellen gleichmäßig anfängt zu glühen. Entgegen der Erwartung der Lehrkraft hatte das Experiment aber keine klärende Wirkung, sondern alle Schülergruppen sahen ihre ursprüngliche Vermutung durch das Experiment bestätigt. Eine Erklärung für diese zunächst paradox anmutende Situation liefern die mit dem Experiment verknüpften Erwartungen der Schüler, die hier ihren Blick auf den Versuchsaufbau im wahrsten Sinne des Wortes zu sehr verengt hatten: Da die Aufmerksamkeit der Schüler in Abhängigkeit ihrer Erwartungen jeweils auf einen Punkt des Drahtes fokussiert war, der Draht aber an allen Stellen gleichzeitig zu glühen beginnt, fühlten sich alle Schülergruppen in ihrer jeweiligen Sichtweise bestätigt.

In einer weiteren Untersuchung zur Elektrizitätslehre sollten Schüler in einem einfachen Stromkreis vorhersagen, inwiefern sich die mit Hilfe von zwei Amperemetern zu messende Stromstärke vor und nach einem Lämpchen unterscheidet. Anschließend wurde das Experiment durchgeführt und diskutiert. In einer Befragung drei Monate später zeigte sich, dass der wahre und beobachtete Ausgang des Experiments für einige Schüler völlig irrelevant gewesen zu sein scheint, da sie nicht nur an ihren ursprünglichen Präkonzepten festhielten, sondern darüber hinaus noch der Überzeugung waren, ihre Präkonzepte seien durch das Experiment bestätigt worden (Gauld 1986). Die Schülervorstellungen konnten durch das Experiment also nicht überwunden werden, sondern haben sich im Gegenteil als Deutungsrahmen als so stabil erwiesen, dass sich die Erinnerungen der Schüler an das Experiment im Nachhinein den Präkonzepten angepasst haben. Für weitere Beispiele dafür, wie stark die Wahrnehmung der Schüler beim Experimentieren auch in anderen Themengebieten der Physik durch ihre vorunterrichtlichen Vorstellungen beeinflusst wird, sei an dieser Stelle beispielhaft auf die Arbeiten von Tiberghien (1980), Champagne et al. (1985) und Wiesner (1994a) verwiesen. 


\subsubsection{Die Eigenschaften von Schülervorstellungen}

Schülervorstellungen basieren meist auf Alltagserfahrungen, besitzen eine hohe Plausibilität für Novizen und haben sich als außerordentlich stabil gegenüber unterrichtlichen Bemühungen erwiesen (Driver et al. 1985; Duit und Treagust 2003). Trotz ihrer Stabilität sind die Schülervorstellungen den Lernenden selbst oftmals nicht explizit bewusst und haben sich als sehr flexible kognitive Konstrukte erwiesen. Die Dualität von Stabilität und Flexibilität ist darauf zurückzuführen, dass man unter dem Begriff „Schülervorstellung“ einerseits grundlegende und festverwurzelte Überzeugungen der Schüler versteht, andererseits aber auch von Schülern spontan erzeugte „Ad-Hoc-Annahmen“ darüber, wie bestimmte Situationen zu erklären sind (vgl. Duit 1994, S. 4; Häußler et al. 1998, S. 177). Während Schülervorstellungen also Züge einer vorunterrichtlichen physikalischen Theorie haben können, die Konzepte und Regeln sinnstiftend miteinander verknüpft (Vosniadou und Skopeliti 2014), zeichnen sie sich gleichzeitig durch eine hohe Kontextabhängigkeit aus (Clough und Driver 1986; Wiesner 1994b; diSessa 2013). So kann eine aus physikalischer Sicht identische Situation bei den Schülern ganz unterschiedliche Vorstellungen aktivieren und zu teilweise widersprüchlichen Erklärungen führen, je nach dem in welchen Kontext sie eingebettet ist. Der Grund hierfür wird darin gesehen, dass Schüler sich in ihrem Denken primär an den Oberflächenmerkmalen einer Situation orientieren und nicht die ihr zugrundeliegende physikalische Struktur erkennen (vgl. Wodzinski 2004, S. 27). Gibt man Schülern genügend Zeit, artikulieren diese sogar ohne Veränderung des Kontexts verschiedene, sich teils widersprechende Erklärungen, wobei diesen Erklärungen sowohl diverse Schülervorstellungen als auch die physikalisch korrekte Vorstellung zugrunde liegen können. Da für Schüler auch physikalische Begriffe oftmals eher den Charakter eines allgemeinen Sammel- bzw. Clusterbegriffs haben, dessen konkrete Bedeutung sich erst aus der jeweiligen Situation ergibt (Schecker 1985, S. 270), ist bei Schülern oftmals eine enorme Erklärungsvielfalt bezüglich einer aus physikalischer Sicht identischen Situation zu beobachten (Hartmann 2004).

Da Schülervorstellungen sich einerseits durch eine hohe Kontextabhängigkeit auszeichnen, andererseits aber auch eine hohe Stabilität aufweisen, weil sie tiefverwurzelte und stabile Überzeugungen der Schüler widerspiegeln, schlagen Niedderer und Schecker (1992, S. 79) in Hinblick auf die Beschreibung der Eigenschaften von Schülervorstellungen eine Unterscheidung von „,eep structure“ und „current construction“ vor. Die „,deep structure“ spiegelt sich nach Niedderer und Schecker (1992, S. 80) vor allem darin wider, dass

- die Konzepte der Schüler sich als außerordentlich stabil und sehr resistent gegen Veränderungen gezeigt haben,

- die Schüler weltweit vergleichbare vorunterrichtliche Vorstellungen zu haben scheinen,

- eine begrenzte Anzahl grundlegender Schülervorstellungen genügt, um das Verhalten bzw. die Aussagen von Schülern in verschiedenen Situationen ausreichend zu erklären. 
Zu den Eigenschaften, die sie unter „current construction“ subsumieren, zählen sie hingegen die Forschungsbefunde zu Schülervorstellungen, wonach

- Schüler in Abhängigkeit der Situation unterschiedliche Konzepte aktivieren, auch wenn es sich aus physikalischer Sicht um die gleiche Situation handelt,

- Schüler ihre Konzepte sehr flexibel der jeweiligen Problemstellung anpassen und sich die konkrete Bedeutung eines Fachbegriffs für sie erst aus dem jeweiligen Kontext entwickelt,

- $\quad$ sich für Schüler bei sogenannten „Entscheidungsexperimenten“ keine eindeutigen Schlussfolgerungen ergeben, da sowohl ihre Beobachtung als auch ihre Interpretation stark kontextgeleitet ist.

Ein schönes Beispiel für diesen dualen Charakter von Schülervorstellungen stellt eine Untersuchung zur Wärmelehre von Tiberghien (1980) dar, bei der ein 12-jähriges Mädchen gefragt wurde, ob Eis schneller schmilzt, wenn es in Aluminiumfolie oder Wolle eingewickelt wird. Die Schülerin ist der Meinung, dass das Eis schneller schmelzen müsse, wenn es in Wolle eingewickelt ist, weil sie aus eigener Erfahrung weiß, dass Wolle ,warm macht“. Die experimentelle Evidenz, wonach das Eis in der Aluminiumfolie schneller schmilzt als in der Wolle, ist für das Mädchen wenig überzeugend (vgl. „,eep structure“). Statt ihre eigenen im Alltag bewährten Vorstellungen im Lichte des Versuchsergebnisses zu hinterfragen, lässt sich das Mädchen spontan unterschiedlichste Ad-Hoc-Erklärungen einfallen, warum ihre Vorhersage in dieser speziellen Situation nicht eingetreten sei (vgl. „,current construction“). Nach Duit (1993b, S. 5) ist dies ein typisches Beispiel für die hohe Stabilität von Schülervorstellungen, zu deren Überwindung oftmals mehrere gute Gegenbeispiele und vor allem eine einleuchtende neue Alternativvorstellung nötig sind, da ein empirisches Gegenbeispiel alleine die Schüler in der Regel nicht davon überzeugen kann, ihre bisherigen Vorstellungen zu Gunsten der wissenschaftlichen Sichtweise aufzugeben.

Selbst wenn die Schülerin aus dem obigen Beispiel die Unvereinbarkeit ihrer Vorstellung mit dem Ausgang des Experiments akzeptiert und die physikalische Erklärung verstanden hätte, heißt dies nicht, dass sie ihr neu erworbenes Wissen auch in anderen Kontexten oder im Alltag hätte nutzen können. Gerade wenn im Unterricht nicht aktiv auf das Vorverständnis der Schüler eingegangen wird, besteht die Gefahr, dass die Schüler das neue Wissen in einer separaten „mentalen Schublade“ abgelegen. Mandl et al. (1993) beschreiben diesen Umstand mit dem Begriff der Wissenskompartmentalisierung, worunter sie verstehen, dass Wissen über die gleiche Domäne aus verschiedenen, nicht miteinander verknüpften Teilen besteht. Bei den Schülern kann dies u.a. dazu führen, dass physikalisch korrekte Vorstellungen und Schülervorstellungen auch nach dem Unterricht ungestört und unbewusst nebeneinander existieren, da die neuen Konzepte nicht mit den bereits vorhandenen kognitiven Strukturen abgeglichen (,Akkommodation“), sondern lediglich in isolierten Wissensstrukturen abgelegt werden. Aufgrund dieser oftmals mangelnden Vernetzung des neuen Schulwissens mit den bisherigen Erfahrungen der Schüler tendieren diese bei der Erklärung von alltagsnahen 
Problemsituationen dann dazu, nicht auf ihr in der Schule gewonnenes Wissen, sondern auf ihre bisherigen Alltagsvorstellungen zurückzugreifen.

Das kognitive Nebeneinander von physikalisch korrekten und inkorrekten Konzepten ist auch ein Erklärungsansatz für die u.a. von Hartmann (2004) beobachtete Erklärungsvielfalt von Schülern. Hiermit ist die Beobachtung gemeint, dass Schüler zur Erklärung einer einzigen Situation mindestens zwei widersprüchliche Theorien vertreten und gerade in längeren Gesprächen gerne zwischen diesen hin- und herspringen (vgl. Wiesner 1994b). Mandl et al. (1993) weisen zudem darauf hin, dass Schüler vielfach überhaupt nicht die Relevanz der im Physikunterricht erlernten Erklärungen für ihr tägliches Leben erkennen und den Physikraum als künstliches Laborsystem erleben, das nichts mit der wirklichen Welt zu tun hat (Perkins und Simmons 1988). In der Folge findet das aus dem Physikunterricht stammende Wissen oftmals keine Anwendung in der Praxis, weshalb hierfür auch der Begriff des „trägen Wissens" geprägt wurde (Whitehead 1929; Renk1 1996). Dieses steht insbesondere im Gegensatz zum angestrebten ,intelligenten Wissen“, das von Weinert (1998, S. 25) als wesentliches Bildungsziel betrachtet wird und unter dem er ein ,[...] wohlorganisiertes, disziplinär, interdisziplinär und lebenspraktisch vernetztes System von flexibel nutzbaren Fähigkeiten, Fertigkeiten, Kenntnissen und metakognitiven Kompetenzen" versteht.

\subsubsection{Theorien zur Wissensorganisation und Conceptual Change}

Vor dem Hintergrund, dass die vorunterrichtlichen Vorstellungen zu einem so großen Maße die Lernprozesse der Schüler bestimmen, ist es wenig überraschend, dass das Themenfeld „Schülervorstellungen“ seit Ende der 1970er Jahre Gegenstand intensiver fachdidaktischer Forschung wurde. Nachdem anfänglich primär versucht wurde, die einzelnen Schülervorstellungen zu dokumentieren und zu katalogisieren, rückte im weiteren Verlauf zunehmend die Frage in den Mittelpunkt, welche Konsequenzen aus dem neuen Wissen über Schülervorstellungen für den Unterricht zu ziehen seien (Wodzinski 1996, S. 5). Während einerseits auf Basis der Erkenntnisse über Schülervorstellungen neue Sachstrukturen und Elementarisierungen für verschiedene Themengebiete der Physik entwickelt und evaluiert wurden, stellte sich andererseits auch die grundsätzliche Frage, wie die vorunterrichtlichen Vorstellungen der Schüler mental organisiert sind. Die Beobachtung, dass die Präkonzepte der Schüler gleichzeitig über einen längeren Zeitraum stabil zu sein scheinen und sich dennoch durch eine große Flexibilität und Kontextabhängigkeit auszeichnen, ließ insbesondere die Frage aufkommen, ob die Präkonzepte eher den Charakter einer stabilen und kohärenten Theorie haben oder von ihrer Struktur her eher mit unzusammenhängenden und unstrukturierten Wissenselementen zu vergleichen sind. Diese Frage ist deshalb von großer Bedeutung, weil sich abhängig von der Art und Weise, wie das Vorwissen organisiert ist, andere zu präferierende Strategien in Hinblick darauf ergeben, wie ein Konzeptwechsel, also der Übergang von den Alltagsvorstellungen hin zur physikalischen Vorstellung, im Unterricht erzielt werden kann. In dem in der physikdidaktischen Literatur weitverbreiteten Begriff „Konzeptwechsel“ deutet sich zudem bereits die zentrale Schwierigkeit beim Lernen von Physik an. Während Lernen in manchen anderen Inhaltsbereichen primär mit der Aneignung von völlig neuem Wissen einhergeht, 
bedeutet Lernen in der Physik häufig, dass Schüler einen Wechsel von ihren bereits vor dem Unterricht vorhandenen Alltagsvorstellungen hin zur physikalisch korrekten Sichtweise vollziehen müssen.

Der Begriff „Konzeptwechsel“ oder „,conceptual change“ ist allerdings aus heutiger Sicht missverständlich und primär auf die große Bedeutung der von Posner et al. (1982) formulierten klassischen Conceptual-Change-Theorie zurückzuführen. Während anfänglich davon ausgegangen wurde, die Präkonzepte der Schüler könnten vollständig durch die physikalisch korrekten Vorstellungen ausgetauscht werden, hat sich schnell gezeigt, dass ein solcher Austausch im Sinne eines Überschreibens weder möglich (Duit und Treagust 1998), noch erstrebenswert ist (Duit und Treagust 2003). Vielmehr geht es darum, den Schülern ein bewusstes Nebeneinander von Alltagsvorstellung und wissenschaftlicher Vorstellung zu ermöglichen und diese in die Lage zu versetzen, je nach Situation bewusst zwischen der wissenschaftlichen und alltäglichen Perspektive wechseln zu können, um beispielsweise auch reflektiert mit alltäglichen Begriffen wie Strom- oder Energieverbrauch umgehen zu können (Solomon 1983). In der didaktischen Literatur finden sich daher u.a. auch die alternativen Begriffe „,conceptual development“, „conceptual growth“ oder „conceptual reconstruction“ (Vosniadou 1999; Duit und Treagust 2003; Kattmann 2008). Bezüglich der Frage, wie das Vorwissen der Schüler organisiert ist und wie der Konzeptwechsel theoretisch beschrieben werden kann, dominieren im wissenschaftlichen Diskurs primär zwei konträre Sichtweisen.

Die eine Seite argumentiert, die Schüler entwickelten bereits in der frühen Kindheit ausgehend von ihren Alltagserfahrungen eine geringe Anzahl kohärenter Wissensstrukturen, die einen hohen Grad an Stabilität aufweisen und sinnstiftend miteinander vernetzt sind (McCloskey 1983; Carey 1991; Chi 1992; Vosniadou 1994; Vosniadou und Skopeliti 2014). McCloskey (1983) nimmt beispielsweise auf Basis seiner Untersuchungen zu Alltagsvorstellungen an, dass Schüler zu Bewegungsverläufen ähnlich kohärente und ausdifferenzierte Theorien besäßen, wie sie in der Wissenschaft z.B. mit der Impetus-Theorie in der Zeit vor Newton vertreten wurden. Eine theoretische Beschreibung dieser von Schülern vertretenen alternativen Theorien und wie sie das weitere Lernen beeinflussen können, liefert Vosniadou (1994) mit ihrem Rahmentheorieansatz (,Framework Theory“). Deren Grundannahme besteht darin, dass die Vorstellungen der Schüler in eine umfassende theoretische Struktur, die sogenannte „Framework Theory“, eingebettet sind, die neben ontologischen (z.B. Annahmen bzgl. der Eigenschaften von physikalischen Objekten) auch epistemologische Überzeugungen (z.B. Annahmen über den Charakter unseres Wissens über die Welt) beinhaltet. Entsprechend ihres Namens bestimmt diese Rahmentheorie bzw. Framework Theory den Rahmen, innerhalb dessen die Kinder Modelle bzw. Erklärungen zu unterschiedlichen Phänomenen entwickeln. Die physikalischen Erklärungen der Kinder über die sie umgebende Welt („naive physics“) werden diesem Ansatz entsprechend als das Ergebnis eines relativ kohärenten Erklärungssystems mit theorieähnlichem Charakter aufgefasst. Da nach Vosniadou und Skopeliti (2014) das Bestreben nach innerer Konsistenz und Kohärenz fest im kognitiven System verankert ist, entwi- 
ckeln bereits junge Kinder eine solche kohärente Rahmentheorie bzw. Framework Theory, die ihr weiteres Denken maßgeblich beeinflusst.

Im Gegensatz zur Annahme von McCloskey (1983) unterscheidet sich die Framework Theory der Kinder aber nach Vosniadou und Skopeliti (2014) in wesentlichen Punkten von einer wissenschaftlichen Theorie. Insbesondere weise ihre Framework Theory nicht den gleichen Grad an innerer Konsistenz und Erklärungsmächtigkeit auf wie wissenschaftliche Theorien und werde von den Kindern auch nicht in vergleichbarem Maße systematisch überprüft. Auch wenn die eigene Rahmentheorie den Lernenden meist nicht explizit bewusst ist, stellt sie doch eine stabile und kohärente kognitive Struktur dar, die maßgeblichen Einfluss auf ihre Interpretation von Beobachtungen, ihre Modellvorstellungen und ihre Vorhersagen und Erklärungen hat. Die Tatsache, dass Schüler oft inkonsistente und hochgradig kontextabhängige Erklärungen liefern, erklären Vosniadou und Skopeliti (2014, S. 1433) damit, dass die Framework Theory selber nur einen Rahmen darstellt, innerhalb dessen die Schüler durchaus noch einen gewissen Variationsspielraum haben, ihre Erklärungen der jeweiligen Situation anzupassen.

Da sich die Framework Theory bereits in der frühen Kindheit auf Basis von Alltagserfahrungen bildet, sich im alltäglichen Leben aus Sicht der Lernenden bestens bewährt und als fundamentales Erklärungssystem für viele weitere Annahmen dient, ist sie außerordentlich stabil. Gelingt es den Lernenden nicht, die wissenschaftliche Sichtweise mit der eigenen Rahmentheorie zu vereinbaren, führt dies zu einem kognitiven Konflikt, in dessen Folge sie sogenannte synthetische Modelle entwickeln. Vosniadou und Brewer (1992) zeigen dies am Beispiel der Vorstellungen von jüngeren Kindern, die auf Basis ihrer Alltagserfahrung die Erde zunächst als Scheibe betrachten (,initiales Modell“), dann aber in Folge der wissenschaftlich akzeptierten Sichtweise der Erde als Kugel ein Hohlkugel-Modell konstruieren, wonach sich die flache Erde in einer hohlen Kugel befindet (,synthetisches Modell“), da sie die wissenschaftliche Sicht zunächst nicht mit ihrer Rahmentheorie vereinbaren können. Durch Kontakt mit der wissenschaftlichen Erklärung wird so die Kohärenz der bisherigen Framework Theory zerstört und im Bestreben nach mentaler „Äquilibration“ entsteht aus einem Präkonzept ein sogenanntes Misskonzept (vgl. Nachtigall 1992, S. 12). Wie in diesem Beispiel illustriert, kann ein Konzeptwechsel nach der Theorie von Vosniadou nur gelingen, wenn nicht an einzelnen Wissenselementen, sondern an den grundlegenden Annahmen der Rahmentheorie angesetzt wird, da sonst lediglich die Entstehung von Misskonzepten in Folge misslungener Äquilibration gefördert wird.

Die Modellvorstellungen der Schüler sind also in die übergeordnete Rahmentheorie eingebettet und werden ganz wesentlich durch diese beeinflusst, was im Falle einer zugrundeliegenden inadäquaten Rahmentheorie das physikalische Lernen im Sinne eines Konzeptwechsels signifikant erschwert. Im Gegensatz zum klassischen Conceptual-ChangeModell von Posner et al. (1982), bei dem sich in Folge eines kognitiven Konfliktes ein rascher Konzeptwechsel ähnlich einem Paradigmenwechsel nach Kuhn (1962) vollzieht, weil sich die 
wissenschaftliche Perspektive als unvereinbar mit den bisherigen Überzeugungen erweist, wird der Übergang zum neuen wissenschaftlichen Verständnis von Vosniadou und Skopeliti (2014) als ein schwieriger und langwieriger Prozess betrachtet. Der Grund hierfür liegt in der zentralen Annahme, dass die Vorstellungen der Schüler nicht als separate Wissenselemente aufgefasst werden können, sondern Teil eines umfassenden und komplexen kognitiven Netzwerks sind. Wird ein Konzeptwechsel angestrebt, muss hierfür nicht nur ein einzelnes Wissenselement geändert werden, sondern ein ganzes Netz an tief verwurzelten konzeptionellen, epistemologischen und ontologischen Überzeugungen. In Folge tendieren viele Lernende oftmals dazu, sogar gegenteilige empirische Evidenz nicht zu akzeptieren oder für ungültig zu halten (vgl. Abschnitt 3.1.3 und 3.1.4), um ihre fest verankerte Framework Theory nicht in Frage stellen zu müssen (Chinn und Brewer 1993). Mit ihrer Framework Theory liefert Vosniadou also einen fundierten Erklärungsansatz für die Persistenz von Schülervorstellungen, die aus dieser Sicht in gefestigte und hochgradig vernetzte Wissensstrukturen eingebettet sind.

Eine zu Vosniadou ähnliche Position wird von Chi (1992) im Rahmen ihres ontologischen Ansatzes vertreten, wonach Schülervorstellungen hauptsächlich die Folge einer falschen Zuordnung von physikalischen Konzepten zu ontologischen Kategorien sind. Beispielsweise halten viele Lernende den elektrischen Strom zunächst für eine Substanz und ordnen ihn deshalb der Gegenstandskategorie zu. Die Aufgabe des Unterrichts besteht nach dieser Sichtweise darin, bei den Lernenden eine Umkategorisierung des Strombegriffs von der Gegenstands- zur Prozesskategorie zu erreichen. Der Konzeptwechsel wird also als ontologischer Kategorienwechsel aufgefasst, der nicht ohne Lernschwierigkeiten zu bewältigen ist. Ein sehr ähnlicher Ansatz wurde zudem bereits von Jung (1979) mit seinen vier Kategorien „Körper“, „Prozess", „Eigenschaft“ und „Beziehungen“" verfolgt. Wird die jeweils angenommene ontologische Kategorisierung als implizite Rahmentheorie aufgefasst, kommt eine Veränderung dieser Kategorisierung einer revidierten Rahmentheorie gleich, womit die beiden Ansätze von Vosniadou und Chi gewisse Parallelen aufweisen (Stark 2003, S. 136). Der wesentliche Unterschied zwischen den beiden besteht nach Vosniadou und Skopeliti (2014, S. 1431) darin, dass mit Hilfe der Framework Theorie erklärt werden kann, warum Schüler physikalische Konzepte so häufig der falschen ontologischen Kategorie zuordnen und warum sich diese falschen ontologischen Zuordnungen als so robust erweisen, während der rein ontologische Ansatz hier lediglich auf einer deskriptiven Ebene verbleibt.

Neben den Ansätzen von Vosniadou und Chi findet in jüngerer Vergangenheit aber zunehmend eine andere Sichtweise Beachtung (vgl. Linn 2006), die davon ausgeht, dass das Vorwissen der Schüler auf der untersten Ebene eine lose Ansammlung einer großen Anzahl von isolierten und unstrukturierten Wissensbausteinen darstellt (Minstrell 1982; diSessa 1988; Minstrell 1992; diSessa 1993, 2013). Einer der ersten Vertreter dieser Position war Minstrell, der mit seinen ,facets of knowledge unzusammenhängende und unstrukturierte Argumentationsmuster und Strategien von Schülern beschreibt, die sich trotz ihrer begrenzten Erklärungskraft aus deren Sicht zum alltäglichen Verständnis physikalischer Fragestellungen bewährt haben. Dabei betrachtet Minstrell (1992) diese ,facets of knowledge“ nicht als Hindernis für 
einen Konzeptwechsel, sondern vielmehr als Ausgangsmaterial für den Aufbau eines angemessenen physikalischen Konzeptes.

Eine im Vergleich zu Minstrell elaboriertere Theorie liefert diSessa (1993, S. 112), der davon ausgeht, dass jeder (Alltags-)Vorstellung der Schüler eine ganze Reihe unabhängiger und für sich gültiger Grundbausteine, ,perhaps as atomic and isolated a mental structure as one can find“, zugrunde liegt, die er als „phenomenological primitives“ oder „p-prims“ bezeichnet. Der Name leitet sich davon ab, dass diese p-prims die Grundbausteine der Kognitionsstruktur auf der Basis minimal abstrahierter Erklärungen zu alltäglichen Phänomenen mit einem lediglich sehr engen Geltungsbereich darstellen oder wie diSessa (1988, S. 52) es ausdrückt: ,p-prims [...] can be understood as simple abstractions from common experiences that are taken as relatively primitive in the sense that they generally need no explanation; they simply happen". Der Grund für die mangelnde Kohärenz der eigenen Vorstellungen wird in metakognitiven Defiziten auf Seiten der Lernenden gesehen, in deren Alltag keine Notwendigkeit zur kohärenten Theoriebildung besteht: „Indeed, perhaps the most fundamental problem is the simple fact that students come to physics classes with no theory at all, but instead are used to dealing with the world on a catch-as-catch-can basis, where it is quite fair to change tactics whenever the problem is minutely varied" (diSessa 1988, S. 61). Statt auf Basis der eigenen Alltagserfahrungen nach einer schlüssigen Theorie zu streben und diese systematisch auf ihre logische Konsistenz zu überprüfen, geht diSessa also davon aus, dass den Kindern gar nicht bewusst ist, dass ihr Alltagswissen lediglich aus bruchstückhaften und teils widersprüchlichen Elementen besteht.

Auch wenn die gegebenen Erklärungen der Kinder den Anschein erwecken können, ihnen läge eine kohärente Theorie zugrunde, ist es nach diSessa falsch, hieraus auf eine Kohärenz der zugrundeliegenden mentalen Strukturen zu schließen. Vielmehr geht er davon aus, dass sich die Konzepte und Vorstellungen der Schüler aus einer großen Anzahl diskreter Wissenselemente zusammensetzen und sich Schüler ihre „Theorien“ je nach Kontext auf Basis dieser Wissenselemente spontan konstruieren (diSessa 2013, S. 35). Die Theorien der Schüler folgen also aus der gemeinsamen Verwendung einer ganzen Reihe von p-prims, wobei der Kontext jeweils darüber entscheidet, welche p-prims aktiviert und verwendet werden. Gestützt wird diese These u.a. durch die Beobachtung, dass sich die Argumentation von Schülern oftmals durch eine starke Kontextabhängigkeit auszeichnet (vgl. Wiesner 1994b). Darüber hinaus kann aber auch die Persistenz von Schülervorstellungen auf der Basis von p-prims erklärt werden, wenn man davon ausgeht, dass der gleiche Kontext, ohne den Einfluss von Instruktion, immer zur Aktivierung der gleichen Kombination an p-prims und somit gleichen Vorstellungen führt.

In Bezug auf das schulische Lernen plädiert diSessa daher dafür, im Unterricht an die Erfahrungen der Schüler anzuknüpfen statt diese mit einer übermäßigen Systematik, die ihrer bisherigen Denkweise widerspricht, zu überfordern: „Students have a richness of conceptual resources to draw on. Attend to their ideas and help them build on the best of them" (diSessa 
2013, S. 41). Diese Sichtweise stellt somit eine Gegenposition zur oben dargestellten Framework Theory von Vosniadou dar, wonach die Vorstellung der Schüler über die Welt von einer geringen Anzahl kohärenter Wissensstrukturen geprägt ist, die sich letztlich als Hindernis auf dem Weg zu einem erfolgreichen Konzeptwechsel erweisen. Die maßgeblich von diSessa geprägte „knowledge-in-pieces“ Sichtweise betrachtet das Vorwissen hingegen als wertvolle Ressource, an die es in einem konstruktivistischen Sinn anzuknüpfen gilt. Diese ausgesprochen positive und nutzbringende Perspektive auf die Rolle des Vorwissens auch im Kontext des Physiklernens leitet sich daraus ab, wie der Lernprozess theoretisch erklärt wird. Für diSessa bedeutet Lernen von Konzepten im Wesentlichen, dass die zuvor losen und unstrukturierten p-prims in eine umfassende und kohärente mentale Struktur, ggf. in Kombination mit neuen p-prims, überführt werden. Nach diSessa (2013, S. 45) eignen sich zum Aufbau eines physikalischen Konzepts auch solche p-prims, die phänomenologisch von Lernenden zunächst überhaupt nicht mit dem zu erlernenden Konzept assoziiert werden, aber für dessen Entwicklung eine entscheidende Rolle einnehmen können (,out of the Shadows“). Ein denkbares Beispiel hierfür besteht darin, an die p-prims der Lernenden zum Thema Luftdruck anzuknüpfen, um ihnen eine anschauliche Vorstellung des elektrischen Potenzials bzw. der elektrischen Spannung zu ermöglichen. Eines der fundamentalsten und überzeugendsten Beispiele für ein p-prim stellt nach diSessa (1988, S. 53) das Ohm'sche Gesetz dar: „It is really an enlarged version of the 'more effort begets more results' primitive [...] [in] that increased effort begets increased result; increased resistance begets reduced result; and so on".

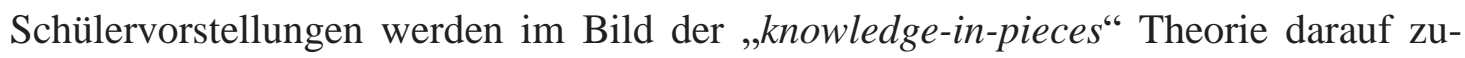
rückgeführt, dass in bestimmten Kontexten bei den Lernenden eine ungeeignete Kombination an p-prims aktiviert wird. Ein Konzeptwechsel lässt sich folglich dadurch erzielen, dass die Aktivierungspriorität ungeeigneter p-prims verringert und die Aktivierungspriorität geeigneter und ggf. neuer p-prims erhöht wird. Hier argumentiert diSessa insbesondere, der Unterricht dürfe nicht direkt das Ziel haben, ganze Vorstellungen oder Konzepte zu verändern, sondern müsse vielmehr den Fokus auf die diesen Konzepten zugrundeliegenden p-prims und ihrer Aktivierungsprioritäten legen (diSessa 2013). Auch wenn die Änderung der Aktivierungspriorität der p-prims keine leichte Aufgabe darstellt, erscheint der hierfür zu beschreitende Weg doch praktikabler als bei Vosniadou, da im Unterricht an das Vorwissen der Schüler angeknüpft werden kann. Dementsprechend wird im Zusammenhang mit der „knowledge-inpieces" Perspektive auch gerne von Konzept- bzw. Begriffsentwicklung statt von Konzeptbzw. Begriffswechsel gesprochen (vgl. Wilhelm et al. 2012). Vielversprechend ist auch, dass es durch diesen konstruktivistischen Ansatz besser gelingen sollte, den Aufbau von parallelen, unvernetzten Wissensstrukturen zu vermeiden und so einer Wissenskompartmentalisierung und der Entstehung trägen Wissens vorzubeugen (vgl. Whitehead 1929; Renkl 1996).

Die Aufgabe der Fachdidaktik kann im Rahmen der „knowledge-in-pieces“ Sichtweise deshalb darin gesehen werden, durch die gezielte Auswahl geeigneter Kontexte und Sachstrukturen bei den Lernenden solche p-prims zu aktivieren, die in Hinblick auf die Entwicklung eines angemessenen physikalischen Konzepts anknüpfungsfähig sind. Auf Basis 
des Wissens über Schülervorstellungen kann so versucht werden, Lernumgebungen zu entwickeln, die einerseits Lernschwierigkeiten gezielt vermeiden, indem ungeeignete Vorstellungen möglichst erst gar nicht aktiviert werden, während andererseits die physikalische Konzeptentwicklung durch die gezielte Aktivierung anknüpfungsfähiger vorunterrichtlicher Vorstellungen gefördert wird. Charakteristisch für einen solchen Lernprozess ist dabei der angenommene kontinuierliche Verlauf von den bisherigen Alltagsvorstellungen hin zur physikalischen Vorstellung, der im klaren Gegensatz zum anfänglich angenommenen diskontinuierlichen Konzeptwechsel nach Posner et al. (1982) steht.

\subsubsection{Unterrichtsstrategien zum Konzeptwechsel}

Aufgrund ihres Einflusses und ihrer Bedeutung soll im Folgenden kurz auf die klassische Theorie des Konzeptwechsels nach Posner et al. (1982) eingegangen werden. Der Kerngedanke dieser bekannten Conceptual-Change-Theorie besteht darin, dass sich der Konzeptwechsel ähnlich einem Paradigmenwechsel nach Kuhn (1962) vollzieht, weil sich die bisherigen Überzeugungen als unvereinbar mit der wissenschaftliche Perspektive erweisen. Dabei wird im Gegensatz zu neueren Theorien zu Conceptual Change angenommen, dass der Wechsel von einer falschen zu einer richtigen Vorstellung plötzlich passiert. Es werden vier Bedingungen aufgeführt, die erfüllt sein müssen, damit „Conceptual Change“, also der Wechsel von den Alltagsvorstellungen hin zur wissenschaftlichen Vorstellung, stattfinden kann:

1. Die Lernenden müssen mit ihren bisherigen Vorstellungen unzufrieden sein (,dissatisfaction“).

2. Das neue Konzept muss für die Lernenden nachvollziehbar und verständlich sein (,intelligibility“).

3. Das neue Konzept muss für die Lernenden insofern plausibel sein, als dass sie selbst von dessen Angemessenheit überzeugt sind (,plausibility“).

4. Das neue Konzept muss sich in der Anwendung auf verschiedenste Situationen als fruchtbar erweisen (,fruitfulness" ${ }^{6}$ ).

In ihrem klassischen Modell postulieren Posner et al. (1982) also, dass ein erfolgreicher Konzeptwechsel nur dann stattfinden kann, wenn einerseits die bisherigen Vorstellungen von den Lernenden als unbefriedigend angesehen werden und andererseits das neue Konzept von ihnen als einsichtig, plausibel und fruchtbar eingeschätzt wird. Damit konzentrieren sich Posner et al. (1982) mit ihrer Theorie zu Conceptual Change vor allem auf kognitive Aspekte. Aufgrund der Vernachlässigung affektiver und sozialer Komponenten beim Lernen wurde das ursprüngliche Modell umfassend kritisiert. Insbesondere wurde von Pintrich et al. (1993) gefordert, affektive Merkmale bei Conceptual Change nicht nur implizit einzubeziehen, sondern Faktoren wie Motivation und Interesse und ihre Rolle beim Konzeptwechsel explizit zu berücksichtigen. Neuere Ansätze tendieren dazu, kognitiven und affektiven Merkmalen in Hinblick auf den Konzeptwechsel die gleiche Bedeutung zuzuschreiben (Zembylas 2005). 
Wie in diesem Kapitel bereits dargelegt, besteht das Problem im Unterricht aber häufig darin, dass auf Seiten der Schüler keine Unzufriedenheit mit den bisherigen Alltagskonzepten besteht, da sich diese im Alltag ja augenscheinlich bewährt haben. Auch kann nicht davon ausgegangen werden, dass die neuen Konzepte den Schülern ohne Weiteres verständlich, plausible und fruchtbar erscheinen. Es drängt sich daher die Frage auf, wie durch die Gestaltung des Unterrichts ein erfolgreicher Konzeptwechsel gezielt gefördert werden kann. Neben der Berücksichtigung grundlegender emotionaler Aspekte wie z.B. der Sicherstellung eines vertrauensvollen Unterrichtsklimas, in dem Schüler ihre persönlichen Vorstellungen in einer von gegenseitigem Verständnis geprägten Atmosphäre frei äußern können (vgl. Duit 1993b, S. 9), wird in der didaktischen Diskussion hierzu vor allem auf die Unterrichtsstrategien nach Jung (1986) verwiesen. Hierbei kann man grundsätzlich zwischen Aufbaustrategien (Anknüpfen, Umdeuten und Überbrücken bzw. Umgehen) sowie der Konfrontationsstrategie unterscheiden.

Die Konfrontationsstrategie entspricht ihrem Geiste nach der klassischen ConceptualChange-Theorie nach Posner et al. (1982) und zielt darauf ab, das Vorverständnis der Schüler zu aktivieren und es ihnen bewusst zu machen, indem die Lernenden ihre vorunterrichtlichen Vorstellungen zu bestimmten Phänomenen explizit verbalisieren. Die Vorstellungen können dann z.B. an der Tafel festgehalten werden und anschließend anhand eines Experiments überprüft und der physikalischen Sichtweise gegenübergestellt werden. Im Idealfall diskutieren die Lernenden untereinander und tauschen Argumente über die Vor- und Nachteile der neuen wissenschaftlichen Perspektive aus (Wiesner 2008). Die Hoffnung besteht bei diesem insbesondere in den 1980er und 1990er Jahren weit verbreiteten Vorgehen (Baser 2006) darin, bei den Schülern einen kognitiven Konflikt auszulösen und somit in Anlehnung an das klassische Conceptual-Change-Modell bei ihnen Unzufriedenheit über ihre bisherigen Vorstellungen zu generieren. Dahinter steht die auch in der klassischen Conceptual-Change-Theorie vertretene Annahme, dass ein Konzeptwechsel nur gelingen könne, wenn sich die Lernenden über ihre eigenen Alltagsvorstellungen bewusstwerden. Mit der Konfrontationsstrategie wird also ein diskontinuierlicher Lernweg verfolgt, der in der Terminologie Piagets der „Akkommodation“ entspricht.

Die Wirksamkeit der Konfrontationsstrategie ist allerdings umstritten. Einerseits wird berichtet, dass ein solcher diskontinuierlicher Weg zu besseren Lernergebnissen führt und insbesondere den Konzeptwechsel erleichtere (Limón und Carretero 1999; Mason 2001), andererseits gibt es zahlreiche Hinweise darauf, dass ein einfacher kognitiver Konflikt alleine nicht genügt, um die Lernenden von der wissenschaftlichen Sichtweise zu überzeugen. Insbesondere scheinen jüngere Schüler häufig nicht den Unterschied zwischen den auf ihren Alltagsvorstellungen basierenden Vorhersagen und dem Ausgang des Experiments zu erkennen oder sehen sogar ihre eigenen Präkonzepte durch das Experiment bestätigt (Duit et al. 2008), wie u.a. am bereits in Abschnitt 3.1.3 erläuterten Beispiel von Schlichting (1991) deutlich wird. Es kann also insbesondere nicht davon ausgegangen werden, dass die Lernenden die Nichtvereinbarkeit von Theorie und Experiment zwangsläufig als kognitiven Konflikt und 
damit als Anlass zur Revision ihrer bisherigen Vorstellungen wahrnehmen (Chan et al. 1997). Da der Wechsel hin zur physikalischen Sicht auf Seiten der Lernenden die metakognitive Bereitschaft erfordert, tief verwurzelte und scheinbar bewährte Vorstellungen aufzugeben, tendieren Lernende zudem oftmals dazu, sogar gegenteilige empirische Evidenz nicht zu akzeptieren oder sie für ungültig zu halten, um ihre fest verankerte Überzeugungen nicht in Frage stellen zu müssen (Chinn und Brewer 1993); ein Verhalten, das u.a. im Beispiel von Tiberghien (1980) in Abschnitt 3.1.4 beschrieben ist. Die Tendenz, auch im Lichte gegenteiliger experimenteller Evidenz an den vorhandenen Ansichten festzuhalten, ist in der Psychologie unter dem Begriff „Belief Perseverance“ bzw. „Perseverationstendenz“" bekannt (Anderson 2007, S. 109). Der Erfolg der Konfliktstrategie ist also entscheidend davon abhängig, ob die Lernenden über die metakognitiven Fähigkeiten verfügen, den kognitiven Konflikt als solchen überhaupt wahrzunehmen. Wiesner (2008, S. 5) weist zudem darauf hin, dass das „Hervorlocken" von Schülervorstellungen problematisch sein kann, weil durch dieses Vorgehen aus vagen Ad-Hoc-Annahmen explizite Fehlvorstellungen werden können (vgl. rückblickende Kritik an ihrem Modell von Strike und Posner 1992). Im weiteren Unterrichtsverlauf tendieren Schüler dann dazu, ihre Ad-Hoc-Annahmen zu verteidigen, was insofern problematisch ist, als dass deren Widerlegung oftmals aufgrund von mangelnden Experimenten schwer ist und zudem unnötig viel Unterrichtszeit benötigt (vgl. Duit 2009a, S. 618). Hinzu kommt, dass die Konfliktstrategie zwar motivierend auf leistungsstarke Schüler wirken kann, die unentwegte Erfahrung, dass die eigenen Vorstellungen letztlich falsch sind, aber insbesondere auf leistungsschwächere Schüler oftmals demotivierend wirkt und bei ihnen ein negatives Selbstbild fördert (Dreyfus et al. 1990). Zusammenfassend kann gesagt werden, dass es keine überzeugenden Hinweise darauf gibt, dass sich die Konfliktstrategie im Vergleich zu Aufbaustrategien besser dazu eignet, einen Konzeptwechsel bei den Lernenden zu bewirken (Harlen 1999).

Der Konfrontationsstrategie stehen die Aufbaustrategien des Anknüpfens, Umdeutens und Überbrückens bzw. Umgehens gegenüber. Das Ziel der Anknüpfungsstrategie besteht darin, einen möglichst bruchlosen Übergang von den vorunterrichtlichen Vorstellungen hin zur physikalischen Vorstellung zu erzielen, indem versucht wird, an solche Alltagserfahrungen anzuknüpfen, zu denen Lernende Vorstellungen haben, die nicht im Widerspruch zum wissenschaftlichen Verständnis stehen. Indem an diese ausbaufähigen Vorstellungen angeknüpft wird, sollen die Lernenden oftmals Schritt für Schritt durch direkte Instruktion in Kombination mit gezielten eigenen Experimentierphasen zum physikalischen Konzept geführt werden (Wiesner 2008; Duit 2009a). Dieses Vorgehen entspricht der alten didaktischen Maxime von Ausubel (1968, vi), wonach erfolgreiches Lernen nur dann gelingt, wenn an geeignetes Vorwissen der Schüler angeknüpft wird und korrespondiert mit Piagets Konzept der „Assimilation“, da ein kontinuierlicher Lernweg verfolgt wird. Eine theoretische Begründung für die Anknüpfungsstrategie kann insbesondere in der in Abschnitt 3.1.5 beschriebenen Theorie von diSessa gesehen werden, für den das Lernen von physikalischen Konzepten die mentale Reorganisation loser, elementarer und oftmals schon bestehender Wissenselemente darstellt. Eine interessante Variante der Anknüpfungsstrategie stellt die von Clement (1993) vor- 
geschlagene Bridging-Strategie dar, bei der über Analogien zu sogenannten Ankervorstellungen versucht wird, die Schüler über Brückenbeispiele hin zur physikalischen Vorstellung zu führen. Ausgehend vom Luftdruck als Ankervorstellung lässt sich so beispielsweise die Druckvorstellung auf elektrische Stromkreise übertragen und somit letztlich das elektrische Potenzial in elementarisierter Form als elektrischer Druck einführen.

Eine besondere Form der Aufbaustrategie stellt darüber hinaus die Umdeutungsstrategie dar, bei der versucht wird, alltägliche Aussagen von Schülern physikalisch umzuinterpretieren. Hierzu wird den Schülern mitgeteilt, dass sie im Prinzip schon über richtige Konzepte verfügen, in der Physik aber zur Beschreibung dieser Konzepte andere Begriffe genutzt werden als es in der Alltagssprache üblich ist. Entsprechend der Umdeutungsstrategie kann in der Elektrizitätslehre beispielsweise die tief verwurzelte Überzeugung, dass ein Lämpchen im Stromkreis den Strom verbraucht, so umgedeutet werden, dass man den Schülern sagt, ihre Vorstellung treffe prinzipiell schon zu, nur würde man das, was im Lämpchen verbraucht wird, in der Physik nicht Strom sondern elektrische Energie nennen (Duit 1995a, S. 17). In ähnlicher Form könnte man auch argumentieren, in einer Batterie sei kein Strom gespeichert, sondern Energie oder dass die von Schülern oftmals in Bewegungsrichtung eines Körpers angenommene „Kraft“ in der Physik als „Impuls“ bezeichnet wird.

Bei der Überbrückungs- oder Umgehungsstrategie werden potenziell problematische Alltagsvorstellungen der Schüler am Anfang des Unterrichts gezielt vermieden, um diese nicht unnötig zu aktivieren und so Lernschwierigkeiten zu provozieren. Erst gegen Ende des Unterrichts, wenn die Schüler bereits über ein gefestigtes physikalisches Konzept verfügen, wird dieses mit den vorunterrichtlichen Vorstellungen verglichen, um ein unbewusstes $\mathrm{Ne}$ beneinander der beiden Vorstellungen zu vermeiden. Um problematische Assoziationen zu verhindern, kann in der Mechanik beispielsweise von „Impulsrate“ statt „Kraft“ gesprochen werden (Herrmann 2003). In der Elektrizitätslehre wurde vorgeschlagen, zunächst von „Elektrogeräten“ und der „Intensität des Stroms“ statt von „Verbrauchern“ und „Stromstärke“ zu sprechen (vgl. Abschnitt 6.1.6), da z.B. letzterer Begriff von den Schülern häufig mit der elektrischen Spannung assoziiert wird, die sie als Stärke des Stroms verstehen (Gleixner 1998, S. 83).

\subsection{Schülervorstellungen zu einfachen Stromkreisen}

Der alltägliche Kontakt vieler Lernenden mit Elektrizität beschränkt sich in der Regel auf das Betätigen von Schaltern oder das Wechseln von Batterien. Hinzu kommt, dass elektrische Prozesse normalerweise unseren Sinnen verborgen bleiben, also geräuschlos, geruchlos und unsichtbar sind. Nichtsdestotrotz verfügen Kinder in Folge gewisser Sprachbilder (,Stromverbrauch“, „leere Batterien“, etc.) und Alltagserfahrungen bereits über vorunterrichtliche Vorstellungen, wie Stromkreise „funktionieren“. Diese mit in den Unterricht gebrachten Schülervorstellungen haben sich als äußerst stabil erwiesen und überdauern oftmals den Physikunterricht. In der Elektrizitätslehre konnten Tiberghien und Delacôte (1976), Evans (1978) sowie Fredette und Lochhead (1980) beispielsweise zeigen, dass sowohl Grund-, Sekundar- 
stufen- als auch Oberstufenschüler selbst nach dem Unterricht in Folge ihrer schwerwiegenden konzeptionellen Fehlvorstellungen zu etwa 50\% nicht in der Lage waren, mit Hilfe einer Batterie und Drähten ein Lämpchen zum Leuchten zu bringen.

Die in der Elektrizitätslehre anzutreffenden Schülervorstellungen sind heute ausführlich dokumentiert, wobei sich gezeigt hat, dass es eine Vielzahl unterschiedlicher und gleichzeitig widersprüchlicher Vorstellungen zu den Vorgängen in Stromkreisen gibt (Duit 1993b). Ihre Kategorisierung ist nicht einfach, da die verschiedenen Schülervorstellungen sich oftmals gegenseitig bedingen. Nichtsdestotrotz existiert eine Reihe von Grundvorstellungen, die sich in verschiedenen internationalen Untersuchungen immer wieder gezeigt haben. In dieser Arbeit wird auf die im E-Lehre-Test von Urban-Woldron und Hopf (2012, S. 207) verwendete Kategorisierung der Schülervorstellungen zurückgegriffen, die um zwei Schülervorstellungen zur Spannung (UI und KS) erweitert wurde. Im Folgenden werden diese Schülervorstellungen zunächst zur besseren Übersicht tabellarisch aufgeführt und anschließend ausführlicher erläutert. 
Tab. 2: Übersicht über typische Schülervorstellungen in der einfachen Elektrizitätslehre in Anlehnung an Urban-Woldron und Hopf (2012, S. 207); erweitert um die Schülervorstellungen UI und KS

\begin{tabular}{|c|c|c|}
\hline Schï & Abk. & Beschreibung \\
\hline $\begin{array}{l}\text { Stromverbrauchs- } \\
\text { vorstellung }\end{array}$ & SV & $\begin{array}{l}\text { Vorstellung, dass der Strom in der Batterie gespeichert ist, } \\
\text { dann zur Lampe fließt und dort schließlich zumindest teil- } \\
\text { weise verbraucht wird. }\end{array}$ \\
\hline $\begin{array}{l}\text { Stromverbrauch ist } \\
\text { proportional } \mathrm{zu} R\end{array}$ & PR & $\begin{array}{l}\text { Typische Schülervorstellung, dass die Stromstärke bei Ver- } \\
\text { größerung des Widerstands kleiner wird, da ein größerer } \\
\text { Widerstand mehr Strom verbraucht. }\end{array}$ \\
\hline $\begin{array}{l}\text { Batterie als konstan- } \\
\text { te Stromquelle }\end{array}$ & $\mathrm{BS}$ & $\begin{array}{l}\text { Die Batterie wird als Quelle für einen konstanten Strom und } \\
\text { nicht einer konstanten Spannung gesehen. }\end{array}$ \\
\hline $\begin{array}{l}\text { Stromstärke ist } \\
\text { unabhängig von } R\end{array}$ & UR & $\begin{array}{l}\text { Die Veränderung eines Widerstands wirkt sich nicht auf die } \\
\text { Stromstärke aus. }\end{array}$ \\
\hline $\begin{array}{l}\text { Inverse Wider- } \\
\text { standsvorstellung }\end{array}$ & IR & $\begin{array}{l}\text { Bei einer Vergrößerung des Widerstands in einer Schaltung } \\
\text { erhöht sich auch die Stromstärke durch diesen Widerstand. }\end{array}$ \\
\hline $\begin{array}{l}\text { Sequentielle } \\
\text { Argumentation }\end{array}$ & SA & $\begin{array}{l}\text { Wird in einer Reihenschaltung ein Widerstand geändert, so } \\
\text { wirkt sich das auf die Stromstärke „vor“ diesem Widerstand } \\
\text { nicht aus, sondern nur auf die Stromstärke „nach“ dem Wi- } \\
\text { derstand. }\end{array}$ \\
\hline Loka & LD & $\begin{array}{l}\text { An einem Verzweigungspunkt wird eine Aufspaltung des } \\
\text { Stromes in gleiche Teile erwartet. Damit wird die Strom- } \\
\text { stärke nicht als Folge der vorhandenen Widerstände und der } \\
\text { anliegenden Spannungen gesehen. }\end{array}$ \\
\hline $\begin{array}{l}\text { Erfassen von } \\
\text { Parallelschaltungen }\end{array}$ & PS & $\begin{array}{l}\text { Parallel geschaltete Widerstände müssen im Schaltbild auch } \\
\text { tatsächlich parallel zueinander gezeichnet sein, damit sie als } \\
\text { solche erkannt werden. }\end{array}$ \\
\hline $\begin{array}{l}\text { Spannung als Eigen- } \\
\text { schaft des Stroms }\end{array}$ & UI & $\begin{array}{l}\text { Eine elektrische Spannung kann es nur geben, wenn Strom } \\
\text { fließt. Wird ein Stromkreis durch Öffnen eines Schalters } \\
\text { unterbrochen, liegt deshalb am Schalter keine Spannung an. }\end{array}$ \\
\hline $\begin{array}{l}\text { Konzeptionelle } \\
\text { Schwierigkeiten mit } \\
\text { der Spannung }\end{array}$ & KS & $\begin{array}{l}\text { Es wird geglaubt, dass zwischen zwei Punkten eines nicht } \\
\text { unterbrochenen idealen Leiters eine Spannung herrsche. }\end{array}$ \\
\hline
\end{tabular}




\subsubsection{Die Stromverbrauchsvorstellung (SV)}

Die Stromverbrauchsvorstellung zählt zu den bekanntesten und meist verbreitetsten Schülervorstellungen in der Elektrizitätslehre. In diversen internationalen Studien hat sich die Vorstellung, der elektrische Strom müsse in irgendeiner Form im Stromkreis verbraucht werden, gegenüber unterrichtlichen Bemühungen als äußerst robust erwiesen. So wird diese Schülervorstellung nicht nur von jungen Schülern vor dem Physikunterricht vertreten (Tiberghien und Delacôte 1976; Osborne 1983), sondern auch noch von über 60\% der 13 bis 14-jährigen Schüler (Maichle 1982; Licht et al. 1990) bzw. 17-jährigen Schüler nach dem Physikunterricht (Andersson et al. 1979; Shipstone et al. 1988) und sogar von Ingenieursstudenten an der Universität (Fredette und Lochhead 1980). Gemein ist allen Varianten der Stromverbrauchsvorstellung, dass die Batterie als Quelle und das Elektrogerät als (teilweiser) Verbraucher bzw. Konsument des Stroms angesehen wird.

Bei jüngeren Schülern tritt die Stromverbrauchsvorstellung insbesondere in Kombination mit einer fehlenden Stromkreisvorstellung auf („Einwegzuführungsvorstellung“), wonach zum Betrieb eines Elektrogerätes nur eine Hinleitung, jedoch keine Rückleitung nötig ist (Tiberghien und Delacôte 1976). Sie besitzen also eine Einstoffverbrauchsvorstellung, wonach der elektrische Strom in der Batterie gespeichert sei wie Öl in einem Fass und von dort aus zum Lämpchen fließt, wo er verbraucht oder umgewandelt wird (Fredette und Lochhead 1980; Osborne 1981; Arnold und Millar 1987). Vor dem Hintergrund, dass Elektrogeräte im Normalfall lediglich mit einem Kabel, d.h. in den Augen der Kinder einer Leitung, an die Steckdose angeschlossen werden, ist die Entwicklung einer solchen Vorstellung wenig verwunderlich. Wiesner (2008, S. 4) weist in diesem Zusammenhang darauf hin, dass der Alltag der Schüler geprägt ist von Situationen, die eine Verbrauchsvorstellung nahelegen. Denkt man z.B. an Verbrennungsmotoren bei PKWs oder den eigenen „Verbrauch“ von Nahrungsmitteln, muss sich Schülern nahezu unvermeidlich der Gedanke aufdrängen, auch in einem Stromkreis müsse der Strom vom Lämpchen verbraucht werden. Hinzu kommt, dass man im Alltag von „vollen“ bzw. „leeren“ Batterien spricht, was die Verbrauchsvorstellung vermutlich ebenfalls fördert. In einer Untersuchung von Maichle (1982, S. 384) mit 272 Realschülern im Alter von 13 und 14 Jahren gaben beispielsweise 84\% der Schüler nach dem Elektrizitätslehreunterricht an, in jeder neuen Batterie sei eine gewisse Menge Strom gespeichert und 82\% waren der Überzeugung, der elektrische Strom einer Batterie würde mit der Zeit durch die angeschlossenen Geräte aufgebraucht werden. Die Schüler sehen im elektrischen Strom also eine brennstoffähnliche Substanz, die in Elektrogeräten verbraucht und in Batterien gelagert wird wie Öl in einem Fass - eine Vorstellung, die eng mit der alltäglichen Energievorstellung vieler Schüler korrespondiert, wonach Energie sowohl verbraucht als auch erzeugt werden kann (Duit 1993a).

Wird Schülern demonstriert, dass ein Lämpchen nicht durch den Anschluss einer Leitung alleine, sondern nur durch den Anschluss einer weiteren Rückleitung zum Leuchten gebracht werden kann, so erklären sich Schüler dies häufig mit der sogenannten Zweiwegezuführungsvorstellung. Nach dieser Variante der Einstoffverbrauchsvorstellung liefert ein Batte- 
riepol alleine nicht genügend „Elektrizität“, erst beide Pole zusammen ermöglichten einen ausreichend hohen Fluss gleicher Elektrizitätsteilchen hin zum Lämpchen, um dieses zum Leuchten zu bringen (Stork und Wiesner 1981). Eine weitere Variante der Zweiwegezuführungsvorstellung stellt das Zweistoffverbrauchsmodell dar, wonach aus dem Plus- bzw. Minuspol der Batterie positive bzw. negative Ladungsträger strömen und sich im Lämpchen treffen, wo die beiden Ladungsträgerarten zusammenstoßen (,clashing currents") bzw. dort „,chemisch“ miteinander reagieren und das Lämpchen so zum Leuchten bringen (Osborne 1981, 1983). In einer Untersuchung von Shipstone (1984) hat sich jedoch gezeigt, dass diese Vorstellung primär bei jüngeren Schülern zu finden ist und nur noch von weniger als zehn Prozent der 17-jährigen Schüler vertreten wird.

Auch wenn die Schüler die Stromkreisvorstellung nach dem Physikunterricht grundsätzlich akzeptieren, sind insbesondere jüngere Schüler weiterhin der Überzeugung, dass der Strom in einem Lämpchen dennoch zumindest teilweise verbraucht werden würde (Osborne 1983, S. 74; Shipstone et al. 1988, S. 305). Bei dieser als ,attenuation model“ bezeichneten Variante der Stromverbrauchsvorstellung wird von den Schülern also hingenommen, dass der Strom im Kreis fließt, jedoch gleichzeitig geglaubt, dass der Strom von jedem Lämpchen abgeschwächt werden würde, weshalb z.B. durch das letzte Lämpchen in einer Reihenschaltung am wenigsten Strom fließen müsse (vgl. Shipstone 1985). Möglicherweise wird die Stromverbrauchsvorstellung unbeabsichtigt auch dadurch gefördert, dass Elektrogeräte in Physikbüchern und im Unterricht häufig als „Verbraucher“ bezeichnet werden.

Einen Grund für die Stabilität der Stromverbrauchsvorstellung sieht Rhöneck (1986, S. 13) in der Dominanz des Strombegriffs in der Mittelstufe, in dessen Folge ein ,übermächtiger Strombegriff" entstehe, dem auch energetische Aspekte zugeordnet werden (vgl. Closset 1983; Shipstone und Gunstone 1985; Gott 1985; McDermott und Shaffer 1992). Konkret konnte beispielsweise Rhöneck (1981b) zeigen, dass es Schülern schwerfällt, den Strom- und Energiebegriff konzeptionell voneinander zu trennen und dementsprechend viele Schüler der Aussage „Strom ist Energie“ zustimmen. Zu ähnlichen Ergebnissen kam auch Duit (1983), der Schüler der sechsten Klasse fragte, was sie mit dem Begriff „Energie“ assoziieren und von knapp der Hälfte der Schüler als Antwort den Begriff „Strom“ genannt bekam. Allgemein scheinen Schüler große Schwierigkeiten damit zu haben, zwischen den unterschiedlichen Konzepten und Begriffen „Strom“, „Energie“, „Leistung“ und „Spannung“ zu unterscheiden und verwenden diese teilweise synonym (McDermott und van Zee 1985; Gott 1985).

Muckenfuß und Walz (1992) sehen den Grund für die konzeptionelle Nähe des Stromund Energiebegriffs in der Diskrepanz, wie der Strombegriff in Alltags- und Fachsprache genutzt wird. Während unter „Strom“ in der Alltagssprache gemeinhin ,fließende Energie“ bzw. konkreter das physikalische Konzept des ,elektrischen Energiestroms“ ( $P=\Delta W / \Delta t)$ verstanden wird, ist in der Fachsprache mit „Strom“ ein fließender zirkulierender Ladungsträgerstrom $(I=\Delta Q / \Delta t)$ gemeint. Beispiele dafür, dass sich im Alltag der Strombegriff nicht auf eine Ladungs- sondern Energieströmung bezieht und auch von Experten entsprechend ver- 
wendet wird, begegnen einem alltäglich in Form von „Stromrechnungen“, „Stromerzeugern“ oder des „Stromsparens“. In Anbetracht der tiefen Verwurzlung dieses „Alltags-StromKonzepts“ ist es daher wenig verwunderlich, dass den Schülern das Erlernen der physikalischen Stromvorstellung große Schwierigkeiten bereitet (Niedderer und Goldberg 1995, S. 77).

\subsubsection{Der Stromverbrauch ist proportional zum Widerstand (PR)}

Bei einer Reihenschaltung aus identischen Lämpchen wird sowohl von jüngeren als auch älteren Schülern mit dem ,sharing model" eine besondere Form der Stromverbrauchsvorstellung vertreten, wonach die Lämpchen deshalb gleich hell leuchten, weil sich der elektrische Strom gleichmäßig auf alle Lämpchen aufteile und dort auch zu gleichen Teilen verbraucht werden würde (Shipstone 1984). Besitzen die in Reihe geschalteten Lämpchen einen unterschiedlich großen Widerstand, wird oftmals angenommen, dass der dann auftretende Stromverbrauch in den Lämpchen proportional zu ihrem Widerstand sein müsse. Der Widerstandsbegriff wird also mit Verbrauch statt Hemmung assoziiert (Cohen et al. 1983). Wird beispielsweise ein kleiner Widerstand $\mathrm{R}_{1}$ in einem einfachen Stromkreis wie in Abb. 11 durch einen größeren Widerstand $\mathrm{R}_{2}$ ersetzt, so wäre hier eine typische Erklärung, dass die Stromstärke im Stromkreis deshalb abnehme, weil ein größerer Widerstand mehr Strom verbrauche als ein kleinerer Widerstand. An diesem Beispiel wird auch deutlich, wie wichtig es ist, sich nicht mit einfachen Antworten zufriedenzugeben, sondern diese auch immer begründen zu lassen, weil die falsche Vorstellung, die hinter der zunächst korrekt klingenden Antwort steht, sonst unbemerkt bleiben würde.
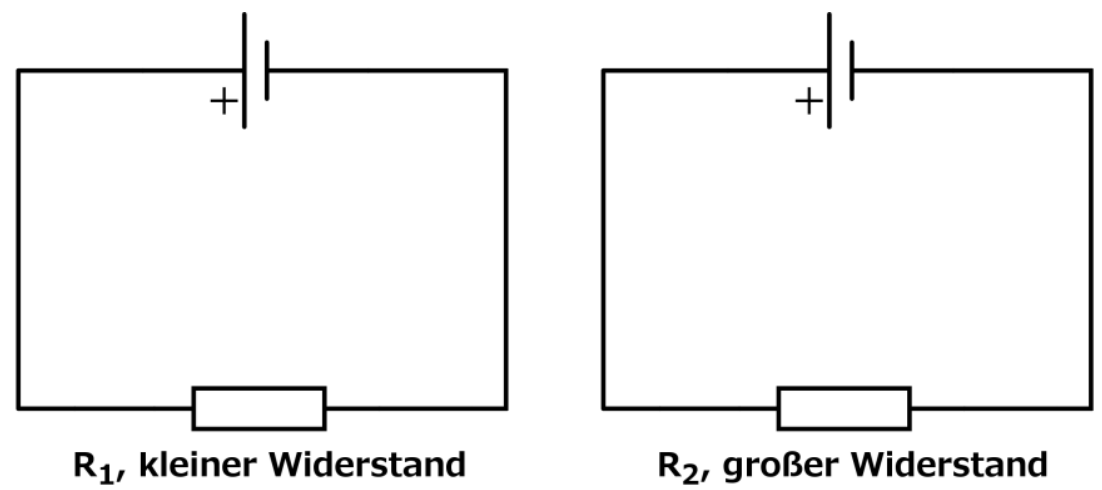

Abb. 11: Nach einer speziellen Variante der Stromverbrauchsvorstellung (PR) sinkt die Stromstärke im Stromkreis, wenn ein größerer Widerstand eingebaut wird, weil ein größerer Widerstand auch mehr Strom verbrauchen müsse.

\subsubsection{Die Batterie als konstante Stromquelle (BS)}

Eine weitere sehr verbreitete und resistente Schülervorstellung betrifft die Rolle der Batterie in Gleichstromkreisen, die für viele Schüler eine Quelle konstanten Stroms und nicht konstanter Spannung ist (McDermott und Shaffer 1992, S. 997). Sie verstehen also nicht, dass eine ideale Batterie unabhängig vom angeschlossenen Stromkreis stets für eine konstante Potenzialdifferenz zwischen ihren Polen sorgt und glauben stattdessen, eine Batterie liefere stets einen konstanten Strom (Cohen et al. 1983; McDermott und van Zee 1985; Dupin und Johsua 1987). Unbeabsichtigt gefördert wird diese Batterie-als-konstante-Stromquelle-Vorstellung im 
Unterricht zudem oftmals durch die leichtfertige Verwendung des Begriffs „Stromquelle“ anstatt „Spannungsquelle“ sowie ein oftmals fehlendes Verständnis für die elektrische Spannung auf Seiten der Schüler. In einer Reihenschaltung zeigt sich diese Schülervorstellung teilweise auch in Form des ,sharing models“ (Shipstone 1985), wonach die Helligkeit von in Reihe geschalteten Lämpchen mit jedem zusätzlichen Lämpchen weiter abnimmt, weil der konstante Batteriestrom sich auf immer mehr Lämpchen aufteilen müsse und folglich jedem Lämpchen weniger „Strom“ zur Verfügung stünde. In einer Untersuchung von Licht et al. (1990) in den Niederladen glaubten beispielsweise 40\% der der 13 bis 14-jährigen Schüler und sogar 44\% der 15 bis 16-jährigen Schüler, dass die Batterie eine Konstantstromquelle sei. $\mathrm{Zu}$ ähnlichen Ergebnissen sind auch Cohen et al. (1983) in Israel sowie Dupin und Johsua (1987) in Frankreich gekommen.

Bei der Analyse einer Parallelschaltung argumentieren Schüler entsprechend der Konstantstromvorstellung, dass die Stromstärke im Hauptzweig der Schaltung unabhängig von der Anzahl der parallelgeschalteten Widerstände immer konstant bleiben müsse. Es wird also davon ausgegangen, dass von der Batterie aus ein immer konstanter Batteriestrom in den Hauptzweig der Parallelschaltung fließt, der sich dann auf die jeweilige Anzahl parallelgeschalteter Lämpchen aufteile. Schaltet man beispielsweise wie in Abb. 12 zu einer Parallelschaltung aus zwei gleichen Widerständen einen dritten identischen Widerstand $\mathrm{R}_{3}$ parallel, so würden Schüler davon ausgehen, dass die Gesamtstromstärke mit 6 A konstant bliebe, sich jetzt aber gleichmäßig auf die drei parallelgeschalteten Widerstände aufteilen müsse und dort demnach nicht mehr 3 A sondern 2 A betrage.

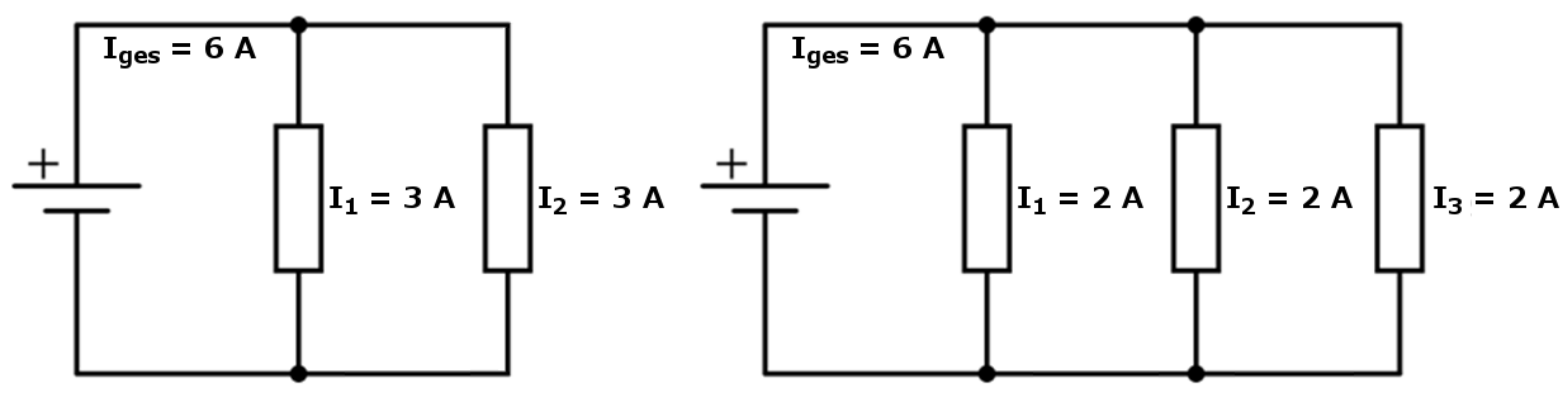

Abb. 12: In Folge der Konstantstromvorstellung wird geglaubt, dass sich der konstante Batteriestrom auf die einzelnen Zweige der Parallelschaltung aufteilen müsse. Im Beispiel soll es sich um gleiche Batterien und Widerstände handeln.

Eine weitere Folge der Batterie-als-konstante-Stromquelle-Vorstellung zeigt sich auch in Form einer Kompensationsvorstellung bei Parallelschaltungen (Rhöneck und Grob 1989). Würde in der linken Parallelschaltung von Abb. 12 beispielsweise der rechte Widerstand vergrößert werden, so argumentieren Schüler, dass dann in Folge des konstanten Batteriestroms der Strom durch den linken unveränderten Widerstand ansteigen müsse (Rhöneck 1986).

\subsubsection{Die Stromstärke ist unabhängig vom Widerstand (UR)}

Eine weitere Schülervorstellung stellt die Konstantstromvorstellung dar, die ihren Ursprung oftmals in der im Unterricht eingeführten Regel „I= konst.“ hat. Diese Regel wird von vielen Schülern insofern übergeneralisiert, als dass sie die Regel auf den Batteriestrom beziehen und 
glauben, die in einem Stromkreis befindlichen Widerstände hätten grundsätzlich keinen Einfluss auf die Stromstärke (Rhöneck 1986). Wird beispielsweise in einer Reihenschaltung aus zwei Widerständen und einem Lämpchen einer der beiden Widerstände vergrößert, so sind Schüler mit der Konstantstromvorstellung der Überzeugung, dass die Helligkeit des Lämpchens trotz des nun größeren Widerstands nicht abnehmen würde, weil die Stromstärke nach der Regel „I= konst.“ ja konstant bleibt (siehe Abb. 13).

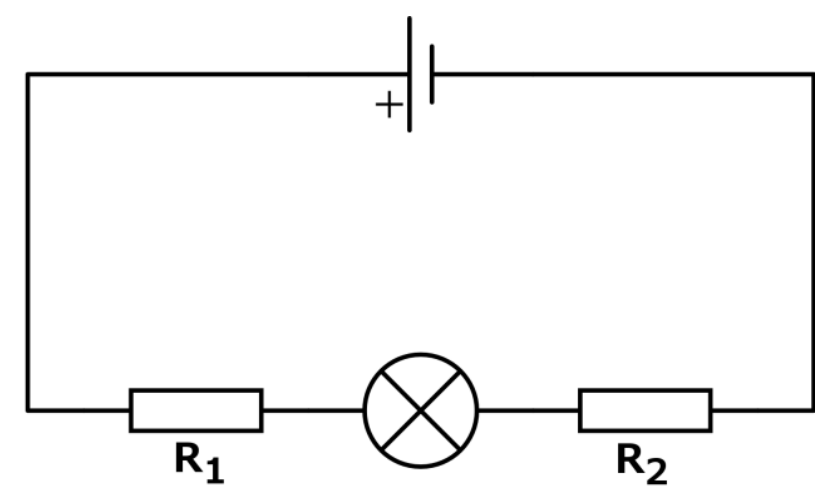

Abb. 13: Nach der Konstantstromvorstellung hat eine Vergrößerung oder Verkleinerung der Widerstände $R_{1}$ oder $R_{2}$ keinen Einfluss auf die Stromstärke durch das Lämpchen.

In ähnlicher Weise kann die Konstantstromvorstellung auch bei Parallelschaltungen auftreten. Hier würden die Schüler, wie in Abb. 14 dargestellt, argumentieren, dass die Stromstärke entsprechend der Regel „I= konst.“ in allen Teilen des Stromkreises gleich sei. Da die Konstantstromvorstellung ihren Ursprung oftmals im Unterricht selber hat, kann sie als lehrinduzierte Fehlvorstellung betrachtet werden.

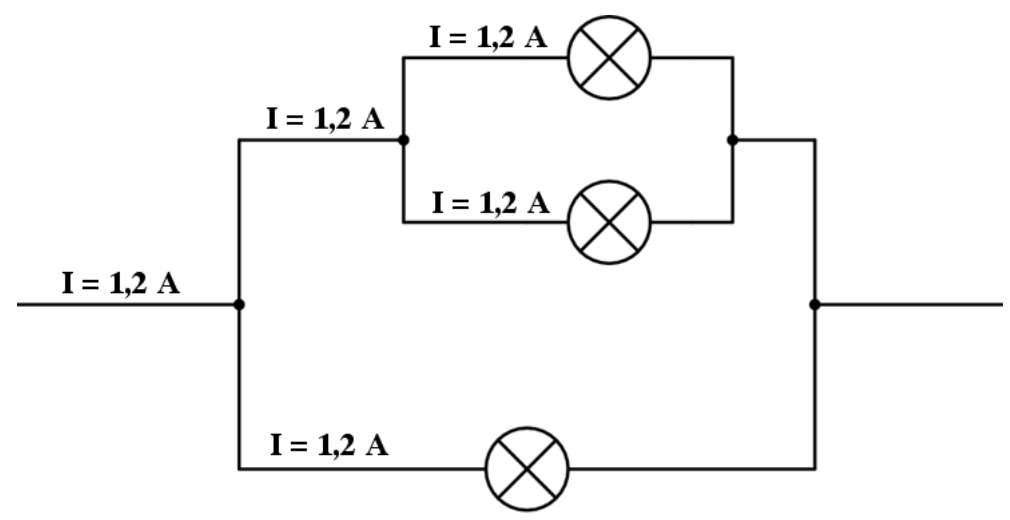

Abb. 14: Konstantstromvorstellung bei einer Parallelschaltung

McDermott und Shaffer (1992, S. 998) berichten von einer weiteren Variante der Konstantstromvorstellung, wonach manche Schüler bei der Analyse der Helligkeit von Lämpchen nicht zwischen Reihen- und Parallelschaltungen unterscheiden. Stattdessen argumentieren sie, es spiele keine Rolle, wie beispielsweise zwei Lämpchen an eine Batterie angeschlossen würden - solange sie irgendwie mit der Batterie verbunden sind, würden sie mit dem gleichen Strom versorgt und müssten deshalb auch gleich hell leuchten. 


\subsubsection{Inverse Widerstandsvorstellung (IR)}

Neben der Schülervorstellung, dass die Größe der Widerstände keinen Einfluss auf die Stromstärke hätte, existiert auch die Vorstellung, dass eine Vergrößerung des Widerstands zu einer höheren Stromstärke führe. In einer Untersuchung von Rhöneck (1986, S. 12) mit 414 Zehntklässlern wurde diese hier als inverse Widerstandsvorstellung bezeichnete Schülervorstellung von $14 \%$ der Lernenden vertreten. Konkret wurde von diesen Schülern angenommen, dass eine Vergrößerung des Widerstands $R_{2}$ im Schaltplan von Abb. 15 von $40 \Omega$ auf $50 \Omega$ eine höhere Stromstärke durch diesen zur Folge hätte, weil ein größerer Widerstand mehr Strom braucht und deshalb auch mehr Strom von der Quelle erhalte.

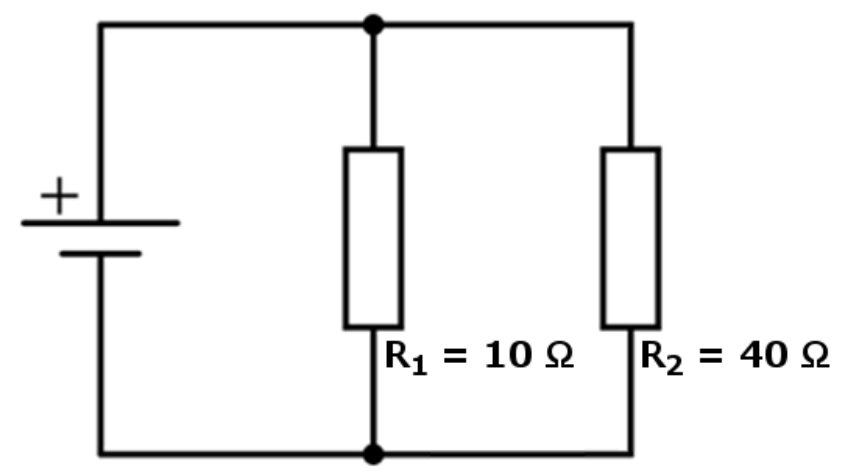

Abb. 15: Nach der inversen Widerstandsvorstellung glauben Schüler, dass eine Vergrößerung des Widerstands $R_{2}$ mit einer Erhöhung der Stromstärke durch diesen einherginge.

\subsubsection{Sequentielle Argumentation (SA)}

Eine sehr verbreitete und stabile Schülervorstellung stellt auch die sequentielle Argumentation dar, wonach der elektrische Strom von einem Batteriepol ausgehend den Stromkreis Lämpchen für Lämpchen sequentiell durchläuft (Maichle 1982; Closset 1984; Shipstone 1984). Die Lernenden erkennen also nicht den Systemcharakter des Stromkreises und analysieren ihn stattdessen aus Sicht des Stroms, der in ihrer Vorstellung ein Bauteil nach dem anderen passiert. In der Folge glauben sie, dass in einem Stromkreis nur Änderungen vor einem Lämpchen sich auf dieses auswirken würden, nicht jedoch Änderungen „nach“ einem Lämpchen, weil dann der Strom schon an diesem vorbeigeflossen sei. Ungewollt gefördert wird diese Schülervorstellung im Unterricht oftmals dadurch, dass der Verlauf des Stroms vom einen zum anderen Batteriepol im Unterricht gemeinsam nachvollzogen wird (Rhöneck 1986; Härtel 2012a). 


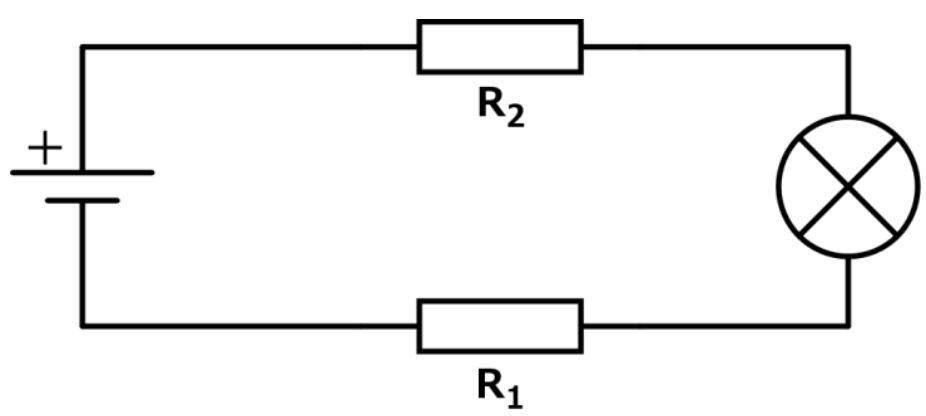

Abb. 16: Je nach Stromrichtung hat eine Veränderung des Widerstands keinen Einfluss auf die Helligkeit des Lämpchens, da der Strom dieses der sequentiellen Argumentation folgend schon passiert hat.

Wird in Abb. 16 beispielsweise der Widerstand $\mathrm{R}_{2}$ vergrößert, so gehen sequentiell denkende Schüler davon aus, dass die Stromstärke durch das Lämpchen sinkt und es somit an Helligkeit verlieren würde, weil der Widerstand $\mathrm{R}_{2}$ bezogen auf die technische Stromrichtung vor dem Lämpchen liegt. Wird hingegen der Widerstand $\mathrm{R}_{1}$ vergrößert, so erwarten die Schüler, dass hierdurch die Stromstärke durch das Lämpchen nicht beeinflusst werden würde, weil der Strom unter der Annahme der technischen Stromrichtung in ihrer Vorstellung schon am Lämpchen vorbeigeflossen ist. In einer Untersuchung von Rhöneck (1986) mit 414 Zehntklässlern vertraten 30\% der Schüler die sequentielle Argumentation. Häufig sind Schüler mit einer solchen Vorstellung auch geneigt, die Stromaussendevorstellung zu vertreten, wonach der elektrische Strom in der Batterie wie Öl in einem Ölfass gespeichert ist und von einem Pol der Batterie aus in den Stromkreis strömt.

\subsubsection{Lokales Denken (LD)}

Auch das lokale Denken bzw. die lokale Argumentation ist eine Folge davon, dass es Schülern oftmals nicht gelingt, ein Verständnis für den Systemcharakter von elektrischen Stromkreisen zu entwickeln. Stattdessen beschränken sie sich bei der Analyse von Stromkreisen auf einen Punkt in der Schaltung und ignorieren völlig den Einfluss der anderen Bauteile im Stromkreis (Rhöneck 1986). Insbesondere wird von den Schülern ausschließlich aus Sicht des Stroms argumentiert, dem im Verlaufe des Durchströmens des Stromkreises „Erlebnisse“ zustoßen und der nicht wissen könne, was für Bauteile weiter hinten im Stromkreis verbaut seien und sich an jeder Verzweigung neu entscheiden müsse, wie er sich aufteilt (Closset 1984). Nach Heller und Finley (1992) existieren hier verschiedene Vorstellungen davon, wie der Strom sich an den Verzweigungspunkten aufteilen müsse, wobei u.a. angenommen wird, der Strom müsse sich immer zu gleichen Teilen an den Verzweigungspunkten aufteilen oder der Zweig, der sich näher an der Batterie befinde, müsse von mehr Strom durchflossen werden. Die elektrische Spannung, als die den elektrischen Strom verursachende Größe, sowie die im Stromkreis verbauten Widerstände werden bei diesen Argumentationen jedoch völlig ignoriert. In umfangreichen internationalen Studien hat sich gezeigt, dass die lokale Argumentation sehr schwer zu überwinden ist und von mehr als der Hälfte der 15 bis 16-jährigen Schüler auch noch nach dem Physikunterricht verwendet wird (Rhöneck 1986; Shipstone et al. 1988). Ein klassisches Beispiel für die lokale Argumentation ist in Abb. 17 illustriert. Bei einer solchen Parallelschaltung wird häufig geglaubt, dass sich der Strom an den Verzwei- 
gungspunkten unabhängig von den sich im Stromkreis befindlichen Widerständen jeweils zur Hälfte aufteilen müsse, da er nicht wissen könne, was ihn weiter hinten im Stromkreis erwartet. In der Folge nehmen Schüler an, dass die beiden oberen Lämpchen in der Parallelschaltung in Abb. 17 weniger hell leuchten müssten als das untere Lämpchen, weil sie von weniger Strom durchflossen würden.

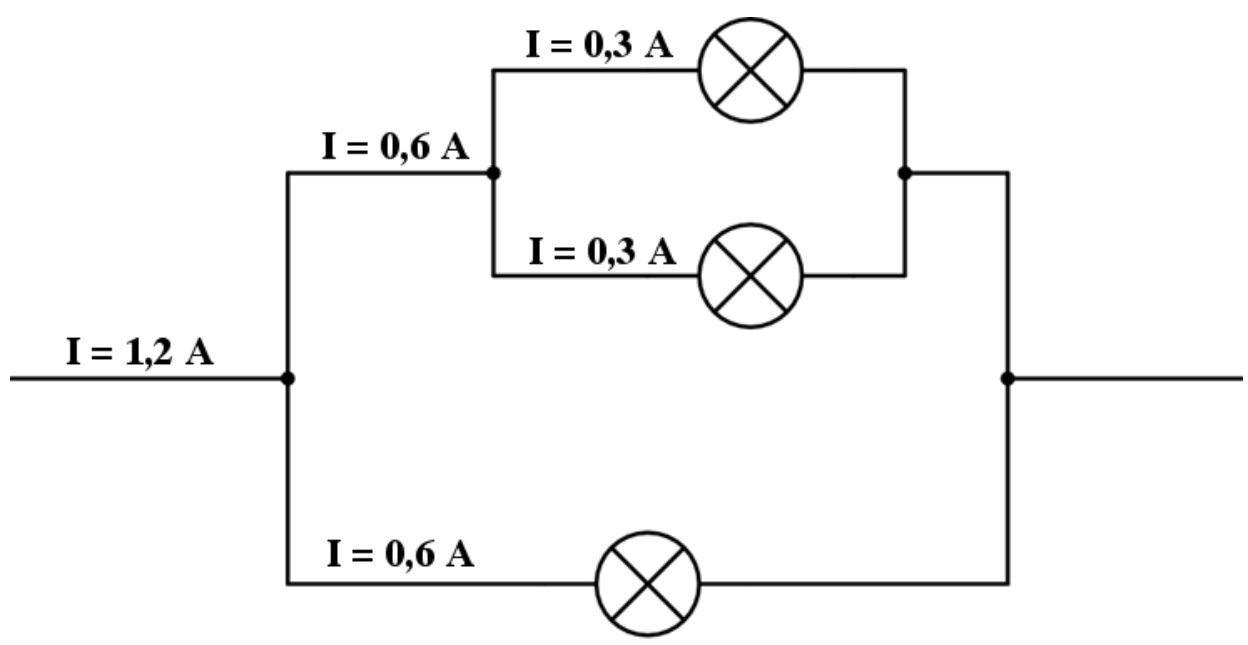

Abb. 17: Dargestellt ist die Aufteilung der Stromstärke nach der lokalen Argumentation. In dem Beispiel soll es sich um baugleiche Lämpchen handeln.

\subsubsection{Probleme beim Erfassen von Parallelschaltungen (PS)}

Oftmals fällt Schülern auch die Unterscheidung bzw. Identifikation von Reihen- und Parallelschaltungen schwer. McDermott und Shaffer (1992, S. 999) berichten beispielsweise davon, dass Schüler häufig nicht zwischen Parallel- und Reihenschaltungen unterscheiden können, wenn sich im Stromkreis mehr als zwei Widerstände befinden. Wird z.B. zu einer Parallelschaltung aus zwei Widerständen ein einzelner weiterer Widerstand in Reihe geschaltet, gingen Schüler oftmals davon aus, es läge eine Reihenschaltung aus dem einzelnen Widerstand mit einem der beiden Widerstände der Parallelschaltung vor.

Aber auch die Geometrie des Stromkreises führt oftmals zu Identifikationsproblemen bzgl. Reihen- und Parallelschaltungen. Schüler tendieren insbesondere dazu, von den geometrischen Eigenschaften des Schaltplans wie z.B. der Parallelität der Widerstände auf die Art der Schaltung zu schließen (Caillot 1985; McDermott und Shaffer 1992). Das heißt aber auch, dass Parallelschaltungen nur dann als solche erkannt werden, wenn die Lämpchen bzw. Widerstände im Schaltplan auch parallel zueinander angeordnet sind. Da der Stromkreis also nicht auf Grundlage physikalischer Gesetzmäßigkeiten analysiert wird, sondern die reine geometrische Anordnung der Widerstände in einer Schaltung ausschlaggebend ist, haben Schüler beispielsweise Schwierigkeiten mit der Identifikation der rechten Parallelschaltungen in Abb. 18. Zwar würde die linke Schaltung in Abb. 18 als Parallelschaltung erkannt werden, nicht jedoch die rechte, weil hier die beiden Widerstände $\mathrm{R}_{1}$ und $\mathrm{R}_{2}$ geometrisch gesehen nicht parallel zu einander angeordnet sind. 

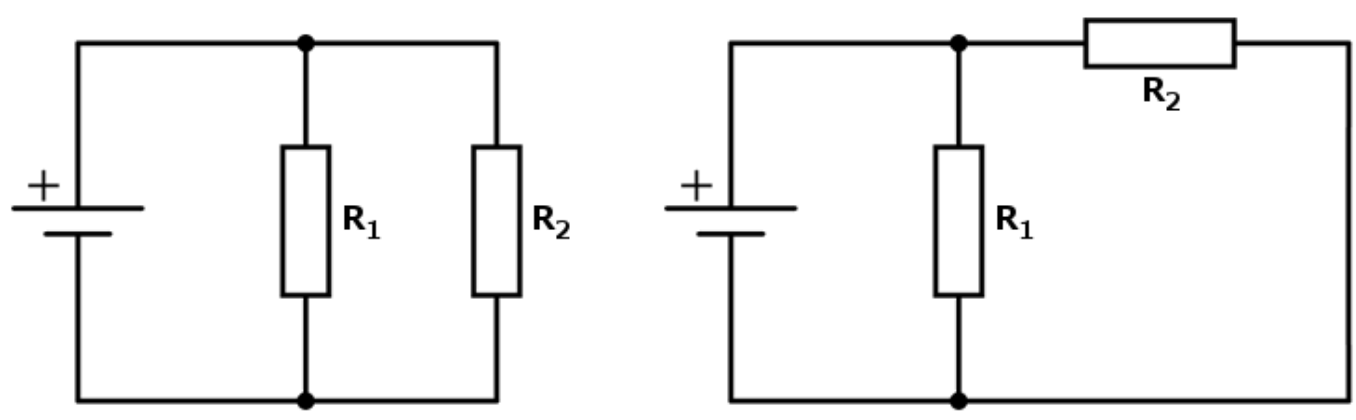

Abb. 18: Abhängig von der Geometrie des Schaltplans werden Parallelschaltungen nur als solche erkannt, wenn die Widerstände parallel zueinander angeordnet sind (wie in der linken Schaltung).

\subsubsection{Spannung als Eigenschaft des Stroms (UI)}

Trotz teils intensiver Unterrichtsbemühungen gelingt es vielen Schülern auch nach dem Unterricht nicht, ausreichend zwischen Strom und Spannung zu differenzieren (Rhöneck 1981a; Maichle 1982; Shipstone 1984; Shipstone et al. 1988; Rhöneck und Völker 1985). Stattdessen wird die Spannung häufig als „Kraft“ oder ,Stärke“ des Stroms wahrgenommen, was möglicherweise auch mit dem Begriff der „Stromstärke“ zusammenhängt (Rhöneck und Völker 1984). Statt ein eigenständiges Spannungskonzept zu entwickeln, wird die Spannung also oftmals als Eigenschaft des elektrischen Stroms gesehen. Eine Konsequenz hiervon ist, dass von Schüler angenommen wird, ohne Strom könne es auch keine Spannung geben (Rhöneck 1981a; Maichle 1982).

Nach Rhöneck (1986) ist die mangelnde Differenzierung zwischen dem elektrischen Strom und der elektrischen Spannung, die sich auch in der Verwendung des Begriffs der „Stromspannung“ widerspiegelt, eine Folge der Dominanz des Strombegriffs in der Mittelstufe. Die im Unterricht häufig vorkommende Fokussierung auf den elektrischen Strom ohne dabei hinreichend auf die Ursache-Wirkungs-Beziehung zwischen Spannung und Strom einzugehen, hat nach Cohen et al. (1983) auch zur Konsequenz, dass Schüler versuchen, elektrische Stromkreise ausschließlich aus Sicht des für sie intuitiven elektrischen Stroms zu analysieren und dabei die entscheidende Rolle der elektrischen Spannung als Ursache des elektrischen Stroms völlig verkennen.

In einer Untersuchung von Rhöneck (1981a) zeigte sich, dass Schüler zwar dann eine Spannung an einem Schalter vermuten, wenn ein Strom fließt, jedoch glauben, es läge keine Spannung an einem offenen Schalter an, wenn kein Strom fließt (vgl. Kersten 1990). In einer anderen Untersuchung von Maichle (1982, S. 384) mit 272 Realschülern im Alter von 13 bis 14 Jahren verneinten $63 \%$ der Schülerinnen und Schüler die Aussage, eine elektrische Spannung könne auch ohne elektrischen Strom auftreten, 70\% waren der Ansicht, dass der elektrische Strom aus Stromstärke und Spannung bestehe und ganze 22\% gaben explizit an, man könne den elektrischen Strom auch elektrische Spannung nennen. 


\subsubsection{Konzeptionelle Schwierigkeiten mit der elektrischen Spannung (KS)}

Unglücklicherweise ist die elektrische Spannung zudem eine vergleichsweise komplexe physikalische Größe, da sie die Differenz zweier Potenzialwerte darstellt und sich immer auf zwei Punkte in einem Stromkreis bezieht. Alleine aufgrund ihres Differenzcharakters ist sie somit schwieriger zu verstehen als das elektrische Potenzial, das einem Punkt bzw. einem Leiterabschnitt lokal zugeordnet werden kann. Paradoxerweise wird von den Schülern aber oftmals im Anfangsunterricht erwartet, ein Verständnis für die Spannung zu entwickeln, ohne die dahinterstehende Größe selbst, nämlich das elektrische Potenzial, zu kennen, geschweige denn zu verstehen (Herrmann und Schmälzle 1984, S. 477).

In der Folge gelingt es Schülern oftmals nicht, ein Verständnis für den Differenzcharakter der Spannung zu entwickeln. Stattdessen wird die Spannung als lokale Größe aufgefasst, die ähnlich dem Potenzial einem Punkt im Stromkreis zugeordnet werden kann. Erschwert wird den Schülern ein Verständnis der Spannung zusätzlich durch den oftmals synonymen Gebrauch des Spannungs- und Potenzialbegriffs. Dieser zeigt sich beispielsweise im gängigen Begriff des „Spannungsabfalls“ an einem Widerstand, wodurch den Schülern sprachlich suggeriert wird, in den einzelnen Leiterabschnitten vor und nach einem Widerstand existiere eine lokal messbare „Spannung“, obwohl hier eigentlich das Potenzial gemeint ist. Ähnlich sieht es auch aus, wenn davon die Rede ist, ein bestimmter Punkt im Stromkreis liege auf einer Spannung von 230 V, wobei Experten im Gegensatz zu Schülern klar ist, dass sich hier auf die „Masse“ bzw. „Erde“ als Referenzpunkt bezogen wird (Gleixner 1998, S. 49). 



\section{Analogien und Modelle im Physikunterricht}

\subsection{Analogien}

\subsubsection{Begriffsklärung}

Der Begriff „Analogie“ leitet sich aus dem Altgriechischen ab, wo er so viel bedeutet wie „gemäß dem Verhältnis“ (Kuhn 1994, S. 2). Erklärungen mit Hilfe von Analogien spielen sowohl im Alltag als auch in der Wissenschaft eine bedeutende Rolle. Nach Kircher (2015a, S. 125) spricht man umgangssprachlich von einer Analogie, ,[...] wenn man aufgrund von Ähnlichkeiten mit Bekanntem oder durch einen Vergleich einen bis dahin unbekannten Sachverhalt erkennt und versteht". Auch wenn der Begriff im Alltag also verhältnismäßig vage verwendet wird und offenbleibt, worin genau diese Ähnlichkeiten bestehen, wird deutlich, dass Analogien genutzt werden, um den Erkenntnisprozess zu erleichtern. Besonders in der Wissenschaftsgeschichte wurden Analogien immer wieder zur Problemlösung verwendet (Willer 1992; Kuhn 1994). So wurde nicht nur das Coulomb-Gesetz aus dem Gravitationsgesetz und die Wellentheorie des Lichtes aus der Wellentheorie des Schalls aufgrund der jeweils hohen strukturellen Ähnlichkeiten entwickelt, sondern auch Ohm fand die Gesetze über strömende Elektrizität über die Analogie zur Wärmeleitung (Ohm 1827; Kircher 2015a). Wenn Analogien also so bedeutend für den wissenschaftlichen Fortschritt waren und auch im Alltag allgemeinhin Verwendung finden, liegt die Vermutung nahe, dass Analogien auch im naturwissenschaftlichen Unterricht eine geeignete Lernhilfe darstellen müssten. Auf der anderen Seite ist aber auch bekannt, dass manche Vergleiche hinken. Vor der Klärung der Frage, inwiefern Analogien also im schulischen Kontext Lernprozesse erleichtern können, ist zunächst eine genauere Auseinandersetzung mit dem Analogiebegriff notwendig.

Duit und Glynn (1995, S. 4) verstehen unter einer Analogie allgemein ,[...] Ähnlichkeiten zweier Bereiche hinsichtlich gewisser Eigenschaften“ bzw. konkreter „eine Relation zwischen diesen Bereichen, [...] [die] sich auf unterschiedliche Arten der Übereinstimmung beziehen [kann]". Zur sinnvollen Nutzung einer Analogie muss dabei einer dieser Bereiche den Lernenden vertrauter sein als der andere. Nach Mikelskis-Seifert und Kasper (2011, S. 6) haben sich hier zur Unterscheidung die Begriffe „Analogbereich“, „Ausgangsbereich“ bzw. „analoger Lernbereich“ für den vertrauten Bereich und „Zielbereich“ bzw. ,primärer Lernbereich" für den neuen, zu lernenden Bereich etabliert. Wie in Abb. 19 schematisch dargestellt, müssen Ausgangs- und Zielbereich jedoch nicht in jeder Hinsicht übereinstimmen. So kann der Ausgangsbereich Strukturen und Eigenschaften enthalten, in diesem Fall dargestellt durch die beiden zusätzlichen Kreise, die sich so nicht im Zielbereich finden lassen. Auch andersherum kann der Zielbereich Strukturen und Eigenschaften enthalten, in diesem Fall dargestellt durch die beiden zusätzlichen Quadrate, die kein Äquivalent im Ausgangsbereich haben (,Eigengesetzlichkeit der Lernbereiche"). Erfasst der Ausgangsbereich wie hier lediglich eine 
Teilmenge des Zielbereichs, so spricht man im Allgemeinen von den „Grenzen einer Analogie $^{6}$.

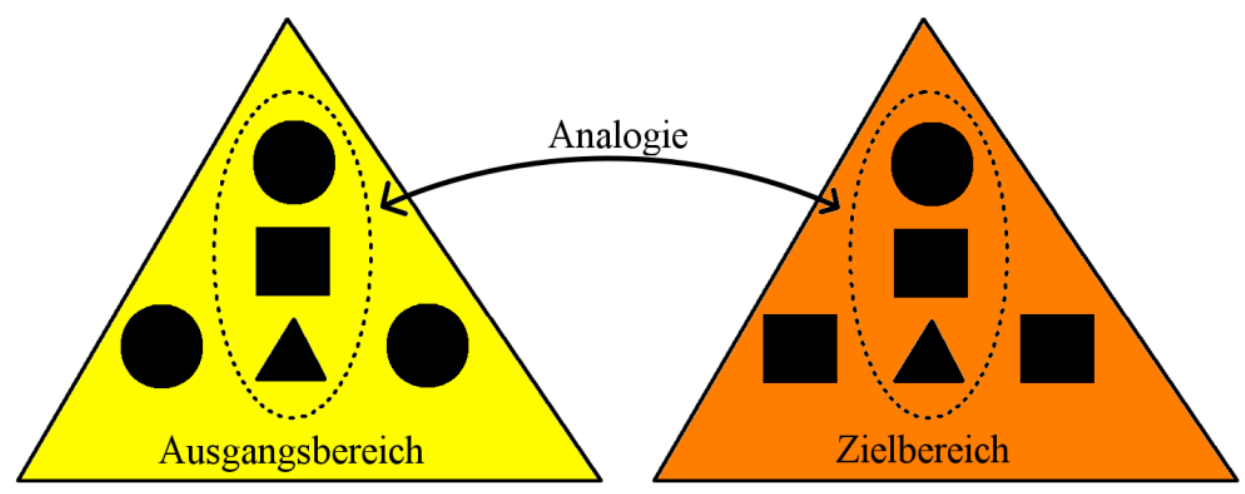

Abb. 19: Eine Analogie beschreibt eine Ähnlichkeit zweier Bereiche in Bezug auf bestimmte Eigenschaften (nach Duit und Glynn 1995, S. 4)

Entscheidend dafür, dass von einer Analogie zwischen Ausgangs- und Zielbereich gesprochen werden kann, ist aber die Tatsache, dass diese beiden Bereiche hinsichtlich gewisser Strukturen und Eigenschaften übereinstimmen, d.h. ein Teil der Strukturen und Eigenschaften aus dem Ausgangsbereich analog ist zu denen im Zielbereich - in Abb. 19 jeweils dargestellt durch die Merkmale innerhalb der oval-gestrichelten Umrandung. Eine Analogie verknüpft nun den Ausgangs- mit dem Zielbereich, indem sie eine symmetrische Relation zwischen den Strukturen und Eigenschaften der übereinstimmenden Teile herstellt. In der Literatur wird das Identifizieren und Herstellen von solchen Relationen als „Analogieschluss“ bezeichnet und erklärt, dass ein solcher Analogieschluss nie mehr als eine erste Hypothese über den Zielbereich auf Grundlage des vertrauten Ausgangsbereiches darstellen kann (Hesse 1963; Tiemann 1993). Entsprechend eignen sich Analogien auch prinzipiell nur zur Illustration eines Sachverhalts, nie aber zu dessen Erklärung (Kircher 2015a, S. 126).

Dabei können zwischen dem Ausgangs- und dem Zielbereich ganz unterschiedliche Analogierelationen bestehen. Zu unterscheiden ist nach Kircher et al. (2001, S. 127) zwischen gegenständlichen, begrifflichen bzw. strukturellen und experimentellen Analogien:

1. Gegenständliche Analogien: Bei gegenständlichen Analogien steht der Vergleich der optischen Gestaltähnlichkeit (,Oberflächenstruktur“) zwischen dem Ausgangs- in den Zielbereich mit Hilfe von greifbaren Modellen im Vordergrund. Ein Beispiel wäre hier die qualitative Illustration des Fließvorgangs im elektrischen Stromkreis mit Hilfe einer Wasseranalogie.

2. Begriffliche bzw. strukturelle Analogien: Zwischen Ausgangs- und Zielbereich können dann die begrifflichen und strukturellen Analogien erarbeitet werden, um ein Verständnis der physikalischen Gesetze, Theorien und Modelle (,Tiefenstruktur") des Zielbereichs zu ermöglichen. Im Fall der Wasseranalogie kann z.B. auf die Entsprechung von Wasserstromstärke und elektrischer Stromstärke bzw. Wasserdruckunterschied und elektrischer Spannung eingegangen werden. 
3. Experimentelle Analogien: Durch anschließende Analogversuche am Wasserkreislaufmodell und dem elektrischen Stromkreis können dann aufgestellte Hypothesen bzgl. der physikalischen Gesetzmäßigkeiten experimentell untersucht und Gemeinsamkeiten und Unterschiede zwischen Ausgangs- und Zielbereich anschaulich herausgearbeitet werden.

Unabhängig von der jeweiligen Analogierelation wird mit Hilfe von Analogien also immer versucht, an das Vorwissen der Lernenden im Ausgangsbereich anzuknüpfen, um das Verständnis für den Zielbereich zu erleichtern. Der Analogienutzung liegt somit im Prinzip eine konstruktivistische Vorstellung zu Grunde, wonach neue Informationen grundsätzlich in bereits vorhandene kognitive Schemata integriert und nur vor diesen interpretiert und verstanden werden können (vgl. Abschnitt 3.1.2).

\subsubsection{Lernen mit Analogien}

Um Schülern das Verständnis von abstrakten und unanschaulichen Themen in der Physik zu erleichtern, liegt es nahe, im Unterricht auf Analogien und damit Vergleiche mit Bekanntem zurückzugreifen. Entgegen dieser zunächst plausiblen Annahme ist der Einsatz von Analogien im Unterricht jedoch nicht unproblematisch, da Analogien ,zweischneidige Schwerter" sind, die zwar durchaus „Brücken zum Verständnis“ schlagen können, aber auch Verständnisschwierigkeiten provozieren und so gesehen in die Irre leiten können (Duit und Glynn 1995, S. 4). Grundsätzlich können Analogien auf ganz unterschiedliche Art und Weise im Unterricht als Lernhilfe eingesetzt werden. Eine häufige Form der Analogienutzung stellen prägnante sprachliche oder bildhafte Vergleiche mit bereits Bekanntem dar, die lediglich nebenbei erwähnt werden. In anderen Fällen kann auch eine ganze Unterrichtsreihe auf einer Analogie basieren, um auf bereits vorhandenen Wissensstrukturen aufzubauen und den neu zu erlernenden Bereich auf diese Weise systematisch zu erschließen. Bei der systematischen Nutzung von Analogien im Unterricht ist aber zu bedenken, dass das Lernen über Analogien immer einen gewissen Umweg darstellt, da statt des Zielbereichs zunächst der Ausgangsbereich thematisiert und gelernt werden muss.

Die Gefahr dabei ist, dass unter erheblichem zeitlichem Aufwand der Ausgangsbereich der Analogie zum primären Lernobjekt im Unterricht erhoben wird, weil Analogien nur lernwirksam sind, wenn die Lernenden mit dessen Ausgangsbereich und seinen physikalischen Gesetzen ausreichend vertraut sind. Nach Kircher (2015a, S. 128) wäre ein solches Vorgehen aber problematisch, da sich der Physikunterricht hauptsächlich mit dem primären Lernbereich befassen sollte. Insbesondere wenn Schüler den Ausgangsbereich aus dem Alltag zwar kennen, jedoch falsche physikalische Vorstellungen von ihm haben, besteht die Gefahr, dass viel Unterrichtszeit auf einen entsprechenden Vorkurs verwendet werden muss, wie dies beispielsweise bei der geschlossenen Wasserkreislaufanalogie der Fall ist (vgl. Schwedes und Dudeck 1993a; Herrmann 2003; Burde und Wilhelm 2016a). Unter lernökonomischen Gesichtspunkten stellt sich dann aber die Frage, inwiefern ein solches Vorgehen im realen Unterricht insbesondere unter Lehrplanaspekten sinnvoll erscheint und ob das direkte Erlernen des 
Zielbereichs nicht sogar zielführender wäre (vgl. Kircher 1984, S. 55; Kircher et al. 2001, S. 127). Der Nutzen von Analogien im Physikunterricht ist deshalb umstritten und ,[...] sowohl von den lerntheoretischen Grundlagen her als auch von der empirischen Feldforschung in den Schulen noch als offen zu betrachten" (Kircher 2015b, S. 804).

Doch selbst wenn es gelingt, den Schülern ein angemessenes Verständnis des Ausgangsbereichs zu vermitteln, sind insbesondere jüngere und lernschwächere Schüler mit dem für eine erfolgreiche Analogieübertragung nötigen Anspruch an das formale Denken oftmals überfordert (Tenney und Gentner 1985). Statt die formalen Entsprechungen, d.h. die Tiefenstruktur, zu erkennen, tendieren solche Schüler dazu, lediglich die optische Gestaltähnlichkeit, d.h. die Oberflächenstruktur der Analogie, wahrzunehmen. In der Folge kann es bei ihnen zu einem Akzeptanzproblem kommen, das Kircher (1984, S. 58) als „ontologisches Problem“ bezeichnet, da ihnen der grundsätzliche Sinn der Analogienutzung verborgen bleibt. Sie können nicht nachvollziehen, wie ein Wasserkreislauf die Vorgänge im elektrischen Stromkreis erklären soll, weil es sich für sie augenscheinlich um grundverschiedene Phänomene handelt (Kircher 1984, S. 58; Wilkinson 1972).

Voraussetzung für eine didaktisch sinnvolle Nutzung von Analogien ist deshalb insbesondere in niedrigeren Klassenstufen, dass eine möglichst große Oberflächenähnlichkeit zwischen Ausgangs- und Zielbereich der Analogie existiert, damit diese von den Lernenden grundsätzlich akzeptiert wird (Kircher 2015a, S. 128). Darüber hinaus sollten die Lernenden aber auch physikalisch korrekte Vorstellungen vom Ausgangsbereich besitzen und über eine ausreichende Fähigkeit des formalen Denkens verfügen, um den anschließenden Analogieschluss leisten zu können. Sind diese Voraussetzungen nicht gegeben, besteht beim Einsatz von Analogien die Gefahr, dass Schüler die Analogie ablehnen, Schülervorstellungen aus dem Ausgangs- in den Zielbereich übertragen oder mit dem Analogietransfer überfordert sind (Wilkinson 1972; Kircher 1984; Tenney und Gentner 1985; Gentner 1989; Duit und Glynn 1995). 


\subsection{Fachliche und didaktische Aspekte von Modellen}

Modelle sind von Menschen zu einem bestimmten Zweck geschaffene Konstrukte, die prinzipiell eine Vereinfachung der Realität darstellen und diese nie in vollem Umfang abbilden können (Mikelskis-Seifert und Kasper 2011). Die mit dem Modellbegriff umgangssprachlich häufig verbundenen Eigenschaften der „Vorläufigkeit, Unvollkommenheit, Unzulänglichkeit“ stimmen damit im Prinzip mit dem wissenschaftstheoretischen Modellverständnis überein, wonach ,[...] unser Wissen über die Realität prinzipiell hypothetisch ist und dass die Physik nur einen ganz bestimmten Aspekt dieser Realität beschreiben und erklären kann" (Kircher 2015b, S. 784). Ein schönes Beispiel für diesen grundsätzlich hypothetischen Charakter von Modellen stellen die Grenzen der klassischen Mechanik dar. Auch wenn sich die uns unmittelbar zugängliche Welt durch die von Newton im 17. Jahrhundert entwickelte Mechanik bestens beschrieben werden kann, zeigte sich Anfang des 20. Jahrhunderts, dass dieses Modell letztlich nur einen Teilaspekt der Wirklichkeit beschreibt und in großen bzw. kleinen Dimensionen durch die Relativitätstheorie bzw. die Quantenmechanik ergänzt werden muss.

Vor dem Hintergrund der Vielfalt an unterschiedlichen Erscheinungsformen von Modellen, angefangen bei Modelleisenbahnen als Spielzeug bis hin zu wissenschaftlichen Modellen wie dem Standardmodell der Teilchenphysik, findet häufig der Versuch einer Kategorisierung statt, wobei grundsätzlich zwischen gegenständlichen Modellen, bildhaften Modellen und mathematischen Modellen unterschieden werden kann. Für alle Modelle sind nach Stachowiak (1973) jedoch die folgenden Merkmale konstitutiv:

- Abbildungsmerkmal: Modelle sind Abbildungen eines Originals, z.B. in Bezug auf seine Struktur oder seine Funktionsweise.

- Verkürzungsmerkmal: Modelle enthalten im Allgemeinen nur solche Attribute des Originals, die für den jeweiligen Zweck relevant erscheinen.

- Pragmatisches Merkmal: Modelle werden von Menschen für einen bestimmten Zweck geschaffen und sind deshalb nicht per se richtig oder falsch.

Werden Modelle im Unterricht verwendet, so sollten diese nach Kircher (2015b, S. 792) ,anschaulich, einfach, transparent und [den Lernenden] vertraut" sein. Weiterhin sollte den Lernenden bewusst sein, dass ein Modell ein menschengemachtes Konstrukt ist, das gezielt für einen bestimmten Zweck geschaffen und verwendet wird und stets eine Vereinfachung bzw. Idealisierung der Realität darstellt (Mikelskis-Seifert et al. 2005, S. 35). Didaktische Modelle sollten einerseits ähnlich fachlichen Modellen einen möglichst großen Geltungsbereich und Korrektheitsgrad aufweisen, andererseits aber eben auch für die Lernenden leicht verständlich sein, indem z.B. versucht wird, an ihr Vorwissen anzuknüpfen. Nach Kircher und Duit (1975, S. 19) treten deshalb bei didaktischen Modellen ,der fachliche Aspekt und der didaktische Aspekt [...] in Konkurrenz zueinander", da das Bestreben nach didaktischer Vereinfachung häufig mit einer Reduktion von fachlicher Komplexität und damit in der Regel auch des Geltungsbereichs des Modells einhergeht. In Anlehnung an Überlegungen von 
Jung (1973) führen Kircher und Rhöneck (1975, 14f) deshalb einige Merkmale auf, die ein aus fachdidaktischer Sicht gutes Modell auszeichnen:

1. Fachliche Relevanz: Die Zweckmäßigkeit eines Modells für die jeweiligen im Unterricht verfolgten Ziele ist wichtiger als eine möglichst große Abdeckung aller Merkmale des ,realen“ Objekts durch das Modell.

2. Entwicklungsfähigkeit: Die in einem Modell aus didaktischen Gründen vorgenommenen Elementarisierungen sollten nach Möglichkeit einer späteren fachlichen Erweiterung des Modells nicht im Wege stehen.

3. Assoziabilität: Aus didaktischen Gründen vorgenommene Elementarisierungen sollten bei den Schülern keine fachlich gesehen falschen Vorstellungen provozieren, wobei sie einschränken, dass ,vermutlich viele Phänomene in der Schule nicht erklärt werden können, wenn die fachliche Richtigkeit der einzige Bezugspunkt ist".

Verschiedene Studien konnten zeigen, dass Schüler häufig grundsätzliche Schwierigkeiten hinsichtlich des korrekten Umgangs mit Modellen haben. Aufgrund der Bedeutung von Modellen für die Physik und auch den Physikunterricht wird daher oft als Konsequenz gefordert, dem Denken und Arbeiten mit Modellen im Unterricht über alle Jahrgangsstufen hinweg eine zentralere Stellung zukommen zu lassen (Mikelskis-Seifert und Leisner 2003; Manthei 1992; Leisner 2005), auch wenn dies aufgrund der geringen Stundenzahlen des Physikunterrichts nicht unumstritten ist (Kircher 2015a, S. 128). Neben der Tatsache, dass viele Schüler mit dem Modellbegriff primär gegenständliche Modelle (Weerda 1982) assoziieren, besteht eine wesentliche Schwierigkeit darin, dass sie oftmals nicht ausreichend zwischen Modellvorstellung und Realität differenzieren (Mikelskis-Seifert 2002). Dies ist insofern problematisch, als dass für ein angemessenes Modellverständnis die erkenntnistheoretische Unterscheidung zwischen dem naiven und dem hypothetischen Realismus Voraussetzung ist. Die Mehrheit der Schüler vertritt jedoch ein Modellverständnis im Sinne des naiven Realismus, hält also Modelle für Eins-zu-eins-Abbildungen der Realität und ist in Folge häufig mit dem Umgang der Vielfalt an Modellen und möglicherweise nötigen Modellwechseln im Unterricht überfordert (Mikelskis-Seifert und Kasper 2011, S. 7; Grosslight et al. 1991).

Aufgrund der Bedeutung einer angemessenen Modellvorstellung sowie der konzeptionellen Nähe von Analogien und Modellen, erscheint eine tiefergehende, formale Auseinandersetzung mit dem Modell- bzw. Analogiebegriff angebracht. Im Folgenden soll daher die hypothetisch-realistische Perspektive nach Vollmer (1998), wie sie in Abb. 20 in Form einer Subjekt-Modell-Objekt-Relation in Anlehnung an Stachowiak (1980) schematisch dargestellt ist, erläutert und der in Abschnitt 4.1.1 eingeführte Analogiebegriff in dieses Bild integriert werden. 


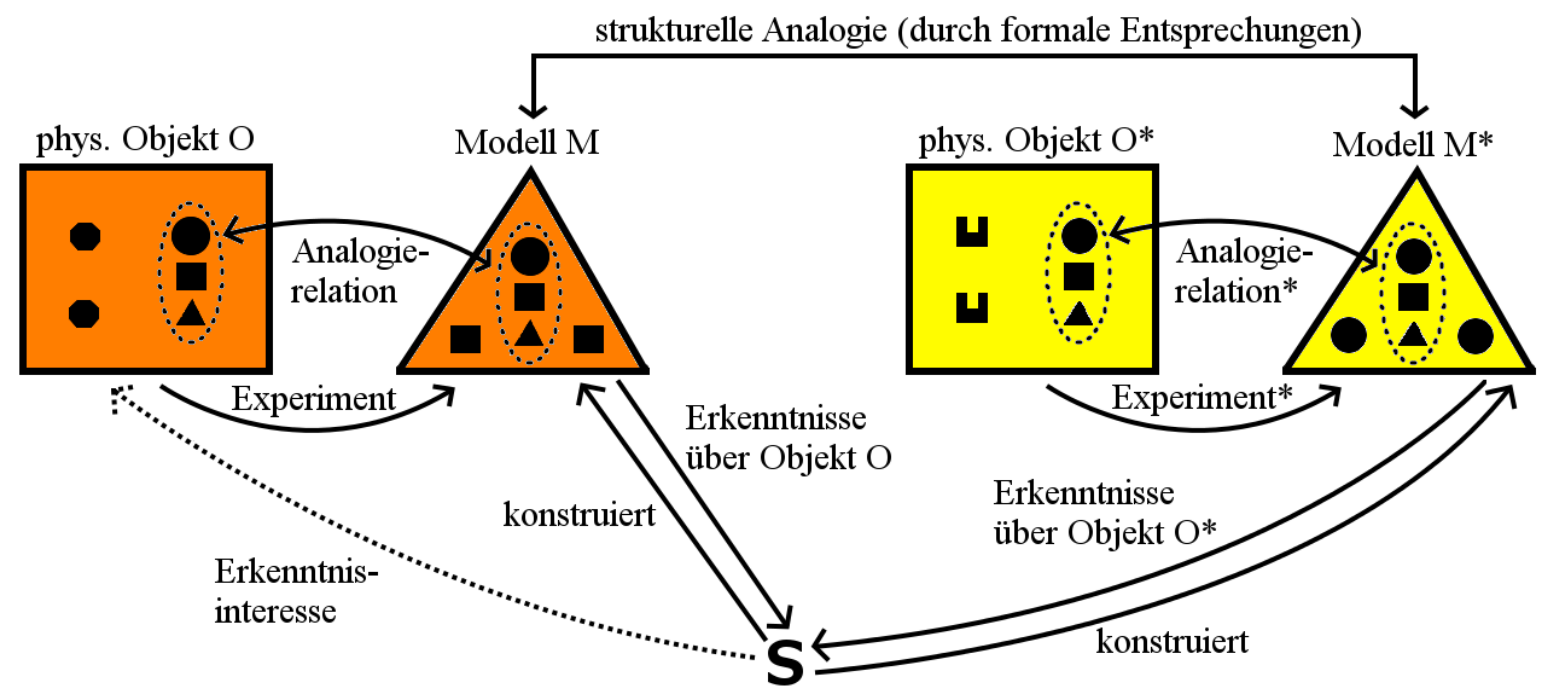

Abb. 20: Der Prozess der physikalischen Modellbildung durch das Subjekt S mit Bezug zum Analogiebegriff. Eigene Adaption in Anlehnung an Kircher (1984, S. 49), Duit und Glynn (1995, S. 4) und Mikelskis-Seifert et al. (2005, S. 35). Orange: Zielbereich der Analogie; Gelb: Ausgangsbereich der Analogie (vgl. Abschnitt 4.1.1)

Zunächst muss zwischen den drei Einheiten physikalisches Objekt O, Modell $\mathrm{M}$ und Subjekt S unterschieden werden, wie in der linken Hälfte von Abb. 20 dargestellt. Grundlegende Annahme des hypothetischen Realismus ist nun, dass das Subjekt S ein Erkenntnisinteresse am physikalischen Objekt $\mathrm{O}$ hat, es ihm aber nicht möglich ist, unmittelbare, direkte Erkenntnisse über das Objekt O zu erlangen. Stattdessen kommt es durch den Prozess der Modellbildung zur Abbildung der Realität bzw. des physikalischen Objekts $\mathrm{O}$ auf ein vom Subjekt konstruiertes Modell M (O $\rightarrow \mathrm{M})$ mit Hilfe von Experimenten und begründeten Hypothesen. Wichtig unter erkenntnistheoretischen Gesichtspunkten ist, dass das so konstruierte Modell M sich nicht eindeutig aus den experimentellen Daten ergibt, sondern das Ergebnis einer Interpretationsleistung dieser Daten durch das Subjekt $S$ in Abhängigkeit der jeweiligen Annahmen und Ziele darstellt. Hinsichtlich der Relation O-M lässt sich also sagen, dass das Modell M die Realität bzw. das physikalische Objekt O nicht eindeutig, vollständig bzw. endgültig darstellen kann, da es in Folge des Konstruktionsprozesses immer Merkmale im Objekt $\mathrm{O}$ geben kann, die nicht durch das Modell M erfasst werden (in Abb. 20 dargestellt durch die beiden zusätzlichen Achtecke im Objekt $\mathrm{O}$, die sich nicht im Modell $\mathrm{M}$ wiederfinden). Diese in der Wissenschaft als „Verkürzungsmerkmal“ (Stachowiak 1973, S. 130) bezeichnete Tatsache, dass das Modell M nur eine Teilmenge der Merkmale des Objekts O erfasst, wird auch als „Grenze eines Modells“ bezeichnet. Andersherum kann es aber auch in Modell M bestimmte Merkmale geben, die nicht mit dem Objekt O übereinstimmen, weshalb man bzgl. der Analogierelation zwischen Objekt $\mathrm{O}$ und Modell $\mathrm{M}$ von einer partiellen Isomorphie spricht. Ein fachlich gesehen gutes Modell M zeichnet sich u.a. dadurch aus, dass es möglichst viele Merkmale des physikalischen Objekts $\mathrm{O}$ abbildet, dass also eine möglichst gute Analogierelation zwischen Objekt $\mathrm{O}$ und konstruiertem Modell M existiert, mit deren Hilfe das Subjekt S Erkenntnisse über das physikalische Objekt $\mathrm{O}$ gewinnen kann. 
Angenommen die linke Bildhälfte bestehend aus physikalischem Objekt O und Modell M beschreibt den Prozess der Modellbildung beim elektrischen Stromkreis (Zielbereich), so besteht zunächst eine Analogierelation zwischen den Merkmalen von O und M. Das Modell $\mathrm{M}$ beschreibt also nur bestimmte Merkmale des eigentlichen zugrundeliegenden physikalischen Objekts O, weil z.B. im schulischen Kontext im Zusammenhang mit Stromkreisen nur ein Modellverständnis der Beziehung von Strom, Spannung und Widerstand angestrebt wird, nicht aber von Oberflächenladungen. Da der Stromkreis als primäres Lernobjekt relativ unanschaulich ist, kann es ratsam sein, zunächst ein Modellverständnis $\mathbf{M}^{*}$ für ein anderes physikalisches Objekt O*, in diesem Fall z.B. den ebenen geschlossenen Wasserkreislauf, aufzubauen, von dem man annimmt, dass es für die Lernenden anschaulicher ist (Ausgangsbereich). Dies ist in der rechten Bildhälfte in Abb. 20 dargestellt, wobei auch hier zunächst eine Analogierelation zwischen den Merkmalen von $\mathrm{O}^{*}$ (dem geschlossenen Wasserkreislauf) und $M^{*}$ (dem angestrebten Modellverständnis) vorliegt. Das angestrebte Modellverständnis $M^{*}$ des geschlossenen Wasserkreislaufs beinhaltet also auch hier nicht alle Merkmale des eigentlichen physikalischen Objekts, sondern nur solche, die zweckmäßig erscheinen. Im vorliegenden Beispiel wäre z.B. die genaue Funktionsweise der Wasserpumpe irrelevant, die Beziehung zwischen Wasserdruckunterschied und Wasserstromstärke hingegen zentral für das angestrebte Modellverständnis $\mathrm{M}^{*}$ beim geschlossenen Wasserkreislauf. Eine (strukturelle) Analogie verknüpft nun den Ausgangs- mit dem Zielbereich, da zwischen Modell $\mathrm{M}^{*}$ (im Ausgangsbereich) und Modell M (im Zielbereich) große formale Entsprechungen bestehen. Wird diese Tiefenstruktur der Analogie von den Lernenden erkannt und gelingt es ihnen, ihr Verständnis vom Modell $\mathrm{M}^{*}$ auf das Modell $\mathrm{M}$ zu übertragen, so stellt dies einen erfolgreichen Analogieschluss dar.

\subsection{Schulische Modelle zur Veranschaulichung von Stromkreisen ${ }^{4}$}

Der elektrische Stromkreis stellt Schülerinnen und Schüler in der Sekundarstufe I vor große Herausforderungen. Einerseits besitzen die Lernenden eine Reihe stabiler vorunterrichtlicher Vorstellungen zu Stromkreisen, die der physikalisch korrekten Sichtweise häufig widersprechen und sich im Unterricht nur mühsam korrigieren lassen. Andererseits sind die Konzepte der Elektrizitätslehre überaus abstrakt und es handelt sich um ein sehr unanschauliches Gebiet der Physik, da sich die physikalischen Vorgänge wie die Elektronenbewegung der direkten Wahrnehmung entziehen. Insbesondere gelingt es vielen Schülerinnen und Schülern trotz intensiver unterrichtlicher Bemühungen häufig nicht, eine Systemvorstellung vom Stromkreis und ein angemessenes Spannungskonzept zu entwickeln. Stattdessen analysieren die Schüler Stromkreise häufig aus Sicht des Stroms, der ihre Vorstellung von Stromkreisen dominiert und diese in ihrer Vorstellung von der Batterie ausgehend sequentiell Lämpchen für Lämpchen durchströmt, wobei er zumindest teilweise verbraucht wird. Die elektrische Spannung erscheint den Lernenden in diesem Zusammenhang nicht als eigenständige physikalische Größe, sondern wird von ihnen häufig lediglich als Eigenschaft bzw. als Bestandteil des

\footnotetext{
${ }^{4}$ Teile dieses Abschnitts wurden bereits in Burde und Wilhelm 2016a bzw. Burde und Wilhelm 2017c veröffent-
} licht. 
elektrischen Stroms wahrgenommen (vgl. Abschnitt 3.2). Aus didaktischer Sicht ist dies problematisch, weil die elektrische Spannung die Ursache und nicht lediglich eine Eigenschaft des elektrischen Stroms ist und außerdem der Systemcharakter eine zentrale Eigenschaft von Stromkreisen darstellt. Ein angemessenes Verständnis elektrischer Stromkreise setzt also bei den Schülern zwingend voraus, dass diese den Systemcharakter verstehen und über ein adäquates Spannungskonzept verfügen.

Da eine rein fachliche Einführung der elektrischen Spannung über den Feldbegriff wenig anschaulich ist und somit für den Anfangsunterricht unangemessen erscheint, hat sich in der Sekundarstufe I im Laufe der Jahrzehnte eine Vielzahl an unterschiedlichen Modellen etabliert. Die dahinterstehende Idee besteht darin, im Unterricht auf Analogien zwischen Modell und Stromkreis zurückzugreifen, um den Schülern das Verständnis der abstrakten Konzepte der Elektrizitätslehre durch den Vergleich mit etwas Bekanntem zu erleichtern. Während gute Modelle hierzu grundsätzlich geeignet sind, können inadäquate Elementarisierungen bei den Schülern Fehlvorstellungen fördern und verstärken. Die gängigen Modelle und Analogien in der Elektrizitätslehre sind zwar grundsätzlich dazu geeignet, einzelne Aspekte des elektrischen Stromkreises anschaulicher und damit verständlicher zu machen, jedoch existiert kein Modell, das vorbehaltslos für den Unterricht empfohlen werden kann. Dies liegt einerseits daran, dass jedes Modell seine Grenzen hat, da es eine vereinfachte und damit unvollständige Abbildung der Realität darstellt (vgl. Abschnitt 4.2), es andererseits aber bisher auch an umfassenden empirischen Vergleichsuntersuchungen $\mathrm{zu}$ ihrer Lernförderlichkeit mangelt. Werden Modelle im Unterricht eingesetzt, ist es daher grundsätzlich wichtig, sich der Schwächen des jeweiligen Modells bewusst zu sein und die Schüler auf die Modellgrenzen hinzuweisen, um möglichen Verständnisproblemen vorzubeugen. Im Folgenden wird daher ein Überblick über gängige schulische Stromkreismodelle sowie ihre jeweiligen Vor- und Nachteile gegeben, wobei die Modelle danach unterschieden werden, wie die elektrische Spannung dargestellt wird.

\subsubsection{Spannung als Druckdifferenz}

Ein Ansatz, Schülern eine Vorstellung von der elektrischen Spannung zu vermitteln, besteht in Druckanalogien, wie sie dem ebenen geschlossenen Wasserkreislaufmodell und dem Elektronengasmodell zugrunde liegen. Da das Elektronengasmodell Gegenstand dieser Arbeit ist, wird für eine detailliertere Beschreibung dieses Modells auf Abschnitt 6.1 verwiesen. Sowohl beim ebenen geschlossenen Wasserkreislaufmodell als auch beim Elektronengasmodell entspricht der Druck jedoch dem elektrischen Potenzial und der Druckunterschied der elektrischen Spannung.

\subsubsection{Der ebene geschlossene Wasserkreislauf}

Der ebene, geschlossene Wasserkreislauf ist ein sehr weittragendes und wahrscheinlich auch das bekannteste Modell für den elektrischen Stromkreis (siehe Abb. 21). Unter einem geschlossenen Wasserkreislauf versteht man ein System, bei dem dichte Wasserrohre mit immer gleichem Querschnitt in einer Ebene zu einem geschlossenen Kreis oder einer verzweigten 
Schaltung aufgebaut sind. An einer Stelle ist eine Pumpe (in einer modifizierten Version zur besseren Veranschaulichung der konstanten Druckdifferenz eine Doppelwassersäule (Schwedes und Dudeck 1993a)) eingebaut und an einer anderen Stelle ein Wasserrad bzw. eine Turbine. Nun entsprechen die Bauteile Rohre, Pumpe, Turbine, Manometer und Wasserstromstärkemesser den Bauteilen Kabel, Batterie, Widerstand/Glühbirne, Voltmeter und Amperemeter im elektrischen Stromkreis. Der Druck entspricht dem elektrischen Potenzial, die Druckdifferenz der Spannung, das Wasservolumen der Ladung, die Wasserstromstärke $I=\Delta V / \Delta t$ (bzw. die Durchflussgeschwindigkeit) der elektrischen Stromstärke $I=\Delta \mathrm{Q} / \Delta t$.
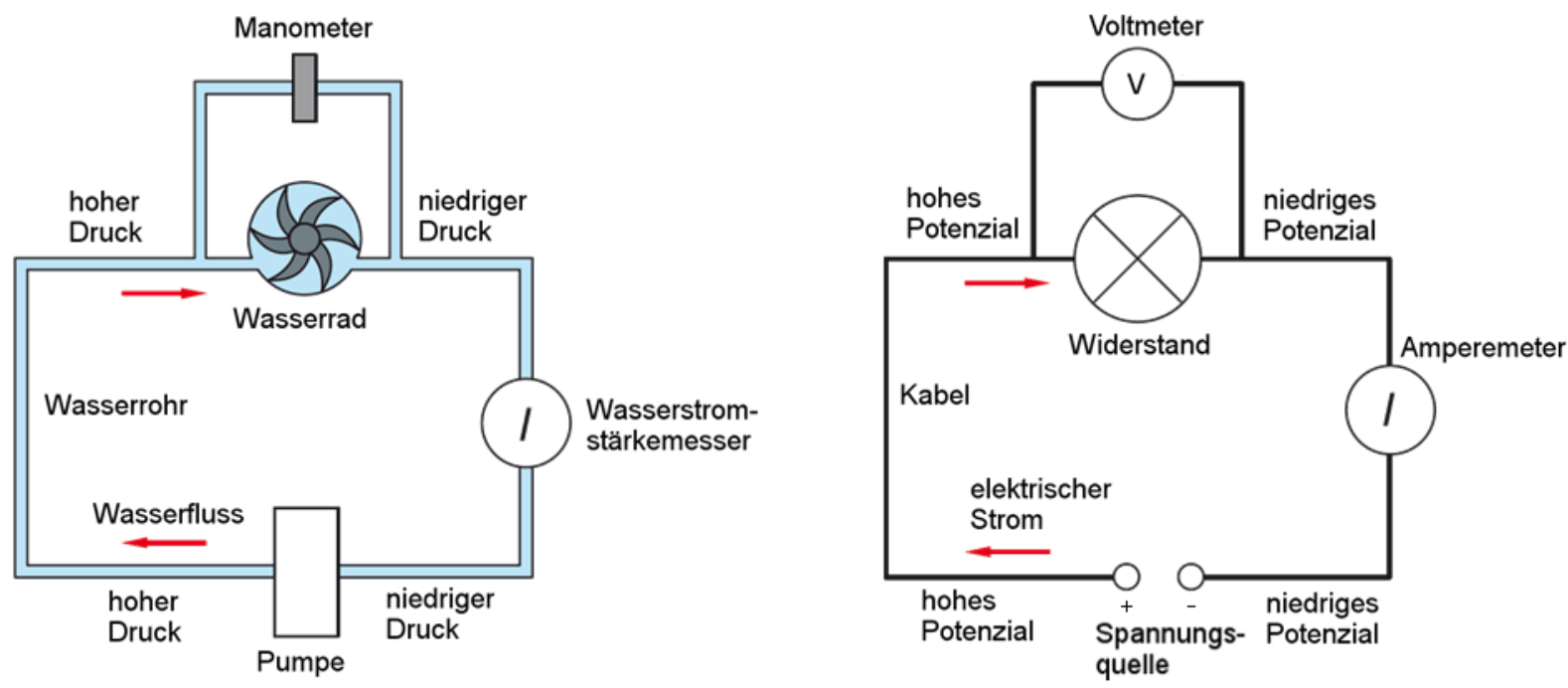

Abb. 21: Gegenüberstellung vom Modell des geschlossenen Wasserstromkreises und einem elektrischen Stromkreis (nach Burde und Wilhelm 2017c, S. 8)

Man kann nun sehen: So wie die Pumpe zunächst nicht den Wasserfluss bewirkt, sondern nur eine Druckdifferenz, bewirkt die Batterie nicht den Stromfluss, sondern nur eine Spannung (Potenzialdifferenz). So wie die Turbine kein Wasser verbraucht, sondern dem Kreislauf Energie entnimmt (die sie in elektrische Energie umwandelt), verbraucht die Glühbirne keinen Strom, sondern entnimmt dem Stromkreis Energie (die sie in Wärme und Licht umwandelt). So wie die Wasserstromstärke durch den mechanischen Widerstand des Wasserrades gemäß $I=\Delta p / R$ begrenzt wird, wird die elektrische Stromstärke durch den elektrischen Widerstand gemäß $I=\Delta \varphi / R$ bzw. $I=U / R$ begrenzt.

Offensichtlich sind die formalen Entsprechungen zwischen dem Wasserstromkreis und dem elektrischen Stromkreis sehr hoch (die sogenannte Tiefenstruktur), was den Wasserstromkreis auf den ersten Blick als prädestiniertes Modell für elektrische Stromkreise erscheinen lässt. Muckenfuß und Walz (1997, S. 96) sehen schon aufgrund der Begriffe ,fließen“, „Strom“ oder „Quelle“ eine ,zwangsläufige Gedankenverbindung“ zwischen dem Wassermodell und dem elektrischen Stromkreis. Ein weiterer Vorteil des geschlossenen Wasserkreislaufmodells wird oft in der Nähe zur Lebenswelt der Lernenden gesehen. So seien Wasserstromkreise nicht nur anschaulicher als elektrische Stromkreise, sondern böten auch viele Anknüpfungspunkte an die Erfahrungen der Lernenden und eigneten sich daher, Schülervorstel- 
lungen zum Stromkreis wie das Verbrauchskonzept zu überwinden (Dudeck und Schwedes 1990). Aufgrund der hohen Akzeptanz und Vertrautheit der Schüler mit Wasserstromkreisen gelänge Lernenden dann problemlos der Transfer der Konzepte vom Wasserstromkreis zum elektrischen Stromkreis. In der physikdidaktischen Forschung konnten sich diese mit dem geschlossenen Wasserkreislaufmodell verknüpften Hoffnungen aber nicht bewahrheiten möglicherweise, weil geschlossene Wasserkreisläufe, die vollständig in einer Ebene liegen, im Alltag der Schüler nicht vorkommen, denn meist spielen auch noch Höhenunterschiede eine Rolle. Stattdessen hat sich der geschlossene Wasserkreislauf als problematisch erwiesen, da er den Schülern aus ihrem Alltag nur wenig vertraut ist und bei ihnen die gleichen Schülervorstellungen provoziert wie bei elektrischen Stromkreisen (vgl. Burde und Wilhelm 2016a).

Ein Grund für die Probleme mit ebenen, geschlossenen Wasserkreisläufen könnte darin liegen, dass das Wasser von vielen Schülern als Kontinuum und als inkompressibel angesehen wird. Damit ist der Wasserdruck jedoch anschaulich nicht vorstellbar, da sich dann Wasser unter hohem Druck in nichts Sichtbarem von Wasser unter geringem Druck unterscheidet. Auch die experimentelle Demonstration des Wasserdrucks mit Hilfe von Druckmessern wie z.B. einer Wassersäule ändert an diesem Problem wenig, da es für die Schüler ein neues Phänomen darstellt, „[...] dessen Verhalten bei komplexeren Schaltungen nicht vorhersagbar oder ableitbar ist, sondern von Fall zu Fall zur Kenntnis genommen und gelernt werden muss" (Härtel 2012a, S. 24). Solange den Schülern jedoch das Konzept des Wasserdrucks unklar und die Druckverteilung in einem Wasserstromkreis nicht bewusst ist, werden sie die Pumpe für den unmittelbaren Antrieb des Wasserstroms bzw. die Batterie für den unmittelbaren Antrieb des elektrischen Stroms halten. Damit besteht für sie aber auch keine Notwendigkeit mehr für einen Konzeptwechsel hin zu einer physikalisch korrekten Sichtweise, wonach die Wasserpumpe bzw. Batterie lediglich einen Druck- bzw. Potenzialunterschied an den Widerständen bewirkt, infolgedessen es dann zu einem (Wasser-)Strom kommt. In dem Fall sind jedoch Zweifel angebracht, inwiefern die geschlossene Wasserstromkreisanalogie geeignet ist, ein angemessenes Spannungskonzept in Stromkreisen zu fördern. Auch argumentieren die Schüler hinsichtlich der Druckverteilung in Wasserkreisen sequentiell, d.h. sie glauben eine Erhöhung des Widerstands durch eine Verengung des Schlauches führe nur zu einem Druckabfall nach dem Widerstand, nicht aber zu einem Druckanstieg davor, da die Strömung den Widerstand ja bereits passiert habe. Die Schüler begreifen den ebenen, geschlossenen Wasserkreislauf also nicht als zusammenhängendes System, sondern legen wie bei Stromkreisen eine sequentielle Argumentation an den Tag. 


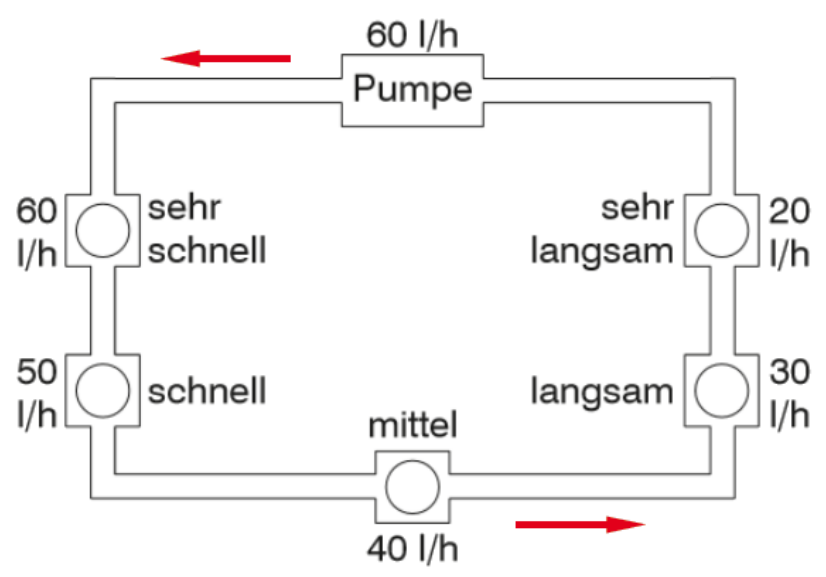

Abb. 22: Stromverbrauchsvorstellung beim geschlossenen Wasserstromkreis (nach Burde und Wilhelm 2017c, S. 9; vgl. Schwedes und Schilling 1983)

Ein weiteres Problem besteht darin, dass Lernende trotz ihrer Alltagserfahrungen mit Flüssigkeiten in der Regel keine korrekte und konsistente Vorstellung von strömenden Flüssigkeiten besitzen. Insbesondere haben sie keine Kontinuitätsvorstellung zu Flüssigkeitsströmungen entwickelt. Die Untersuchungen von Schwedes und Schilling 1983 zeigen beispielsweise, dass sehr viele Schüler davon ausgehen, dass in einem unverzweigten Wasserkreis die Wasserstromstärke mit zunehmendem Abstand von der Pumpe abnimmt, was bedeuten würde, dass mehr Wasser von der Pumpe weg- als zurückfließt (siehe Abb. 22). Ein weiteres Problem besteht darin, dass eine Verengung an einer Stelle des Wasserschlauchs, also eine Erhöhung des Widerstands, bei Schülern häufig nicht wie erhofft dazu führt, dass diese von einer im gesamten Wasserstromkreis gleichmäßig verminderten Wasserstromstärke ausgehen. Stattdessen argumentieren manche Schüler z.B. einem sequentiellen Denkmuster folgend, dass das Wasser nur nach, aber nicht vor der Engstelle langsamer sei (Dudeck und Schwedes 1990).

Didaktisch sinnvoll ist ein Modell dann, wenn der Analogbereich den Lernenden ausreichend vertraut ist und ihr Wissen über den Analogbereich fruchtbare und zielführende Anknüpfungspunkte für den elektrischen Stromkreis bietet (vgl. Abschnitt 4.2). Beim geschlossenen Wasserkreislaufmodell ist es jedoch so, dass die Schüler weder eine korrekte Stromstärkevorstellung oder ein korrektes Druckverständnis noch ein ausreichend entwickeltes Systemdenken besitzen. Soll das Modell des geschlossenen Wasserkreislaufs also die Grundlage für ein Verständnis der elementaren Elektrizitätslehre darstellen, so besteht die große Gefahr, dass Schüler diese Fehlvorstellungen auf den elektrischen Stromkreis übertragen. Doch selbst wenn wie bei Schwedes und Dudeck (1993a) die physikalischen Gesetzmäßigkeiten des geschlossenen Wasserkreislaufmodells im Rahmen eines langen Hydraulikvorkurses mit einem Umfang von zwölf Doppelstunden, d.h. sage und schreibe 24 Unterrichtsstunden, gründlich erarbeitet und von den Schülern verstanden werden, bedeutet diese nicht zwangsläufig, dass die Lernenden ihr so gewonnenes Verständnis ohne Weiteres auf elektrische Stromkreise übertragen können (Tenney und Gentner 1985). Der Grund hierfür liegt darin, dass Schüler eher die optische Gestaltähnlichkeit („Oberflächenstruktur“) der Analogie wahrnehmen statt 
die Ähnlichkeit der physikalischen Gesetzmäßigkeiten („Tiefenstruktur ${ }^{6}$ ) zu erkennen und in Folge den Transfer vom analogen Bereich auf den primären Lernbereich nicht leisten können. Ohne eine gezielte Metakognition der Analogienutzung im Unterricht gelingt in einer Studie von Wilkinson (1972) nur etwas mehr als der Hälfte der Schüler nach einer kurzen Einführung in Wasserstromkreise überhaupt eine Ähnlichkeit zum elektrischen Stromkreis zu erkennen, weniger als einem Drittel die Analogie in Problemsituationen zu nutzen und lediglich sechs Prozent die Analogie auch korrekt zu verwenden. Abhängig von der geistigen Entwicklung scheinen insbesondere jüngere Schüler in der Sekundarstufe I mit dem für einen erfolgreichen Analogietransfer nötigen Anspruch an das formale Denken überfordert zu sein. Kircher (1984, S. 58) identifiziert bei diesen Schülern ein ontologisches Problem, wonach diese „keinen Zusammenhang und damit keinen Sinn in den Analogmodellen und insbesondere in den Analogversuchen" sähen und fordert deshalb, der Behandlung des Wasserstromkreises im Unterricht nicht mehr als drei bis vier Unterrichtsstunden zu widmen, damit die Analogie nicht zu einem Selbstzweck im Unterricht verkommt. Auch Muckenfuß und Walz (1997, S. 98) sehen das Modell des geschlossenen Wasserkreislaufs kritisch, da ,geschlossene Wasserstromkreise, in denen Stromstärke und Druckdifferenz ermittelt werden, [...] schnell eine Gestalt annehmen, die in ihrer Komplexität elektrischen Stromkreisen kaum nachsteht", wodurch sie ihre ,heuristische Funktion“" verlören.

Das Dilemma der Analogie des geschlossenen Wasserstromkreises liegt also darin, dass Kinder kein physikalisch korrektes Vorwissen über geschlossene Wasserstromkreise mit in den Unterricht bringen und eine Erarbeitung der Gesetzmäßigkeiten des geschlossenen Stromkreises eine intensive Auseinandersetzung unter hohem Zeitaufwand erfordert (vgl. Schwedes und Dudeck 1993a). Zusätzlich muss auch noch ausreichend Zeit eingeplant werden, Lernende bei dem Transfer ihres Wissens über Wasserstromkreise auf elektrische Stromkreise zu unterstützen. Setzt die Lernwirksamkeit des Modells aber voraus, dieses unter erheblichem zeitlichem Aufwand zum primären Lernobjekt im Unterricht zu erheben, stellt sich die Frage, inwiefern dieses Vorgehen noch lernökonomisch und im realen Unterricht unter Lehrplanaspekten praktikabel ist. Insbesondere vor dem Hintergrund, dass der geschlosse Wasserkreislauf im Vergleich zu anderen Modellen im Allgemeinen zu schlechteren Lernleistungen führt (Gentner und Gentner 1983), stellt sich nicht zuletzt die Frage, „ob das Lernen des Wassermodells schwieriger ist als das Lernen der Stromkreise selbst" (Kircher 1984, S. 58).

\subsubsection{Spannung als Höhendifferenz}

Zur Veranschaulichung der elektrischen Spannung eignen sich auch verschiedenste Höhenanalogien wie der offene Wasserkreislauf oder das Münchener Stäbchenmodell, bei denen eine Höhe einem elektrischen Potenzial entspricht und somit ein Höhenunterschied einer elektrischen Spannung. 


\subsubsection{Der offene Wasserkreislauf}

Im Gegensatz zu Wasserströmungen in ebenen geschlossenen Wasserkreisläufen, dürfte offen fließendes Wasser in Folge von Höhenunterschieden den meisten Schülern aus ihrem Alltag her bereits bekannt sein. In diesem Modell entspricht die Wasserhöhe dem elektrischen Potenzial, der Höhenunterschied der elektrischen Spannung und die Wasserströmung der elektrischen Stromstärke (siehe Abb. 23). Die Spannungsquelle wird im Modell des offenen Wasserkreislaufs in der Regel mit einer Pumpe verglichen und der elektrische Widerstand wird häufig mit einem Wasserrad dargestellt, das von der Wasserströmung angetrieben wird.
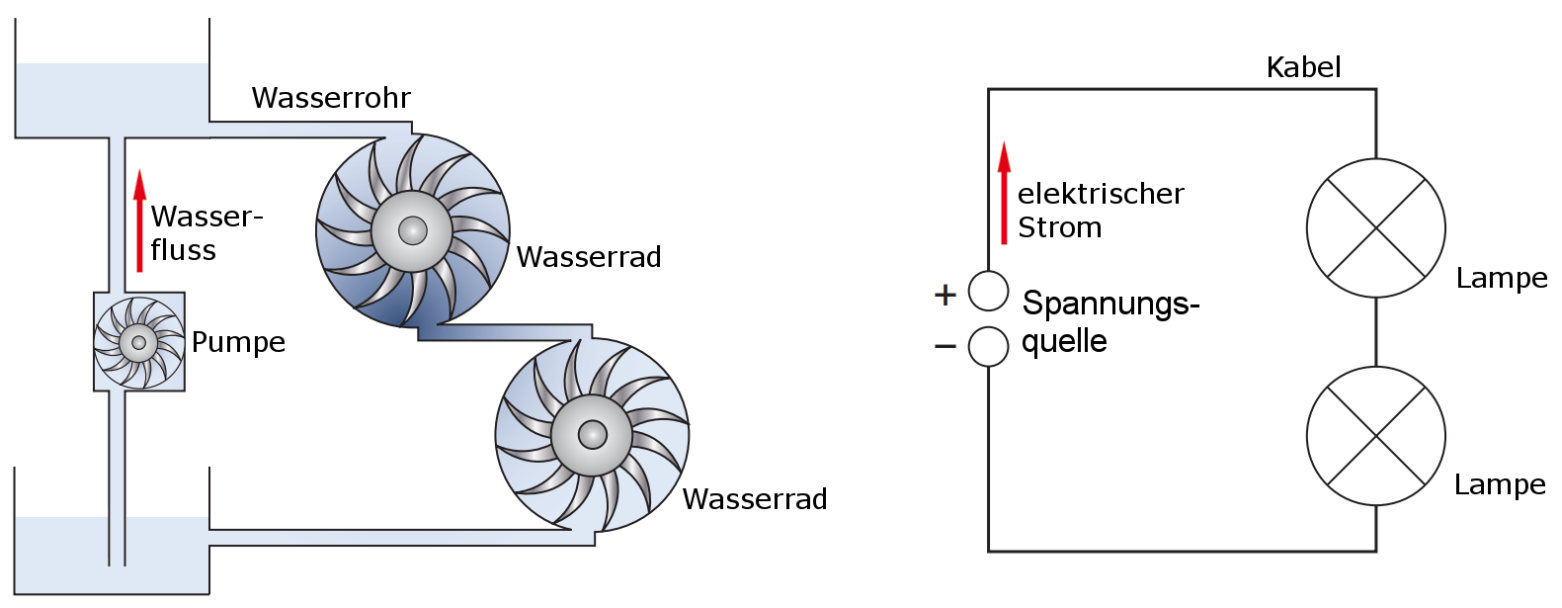

Abb. 23: Gegenüberstellung vom offenen Wasserstromkreismodell und einem einfachen elektrischen Stromkreis (nach Burde und Wilhelm 2017c, S. 11)

Positiv am Modell des offenen Wasserkreislaufs ist zunächst einmal, dass unmittelbar klar ist, dass es ohne Höhenunterschied keinen Wasserstrom gibt - dass die elektrische Spannung also den elektrischen Strom bewirkt. Auch haben sich visuelle Darstellungen des Potenzials in der physikdidaktischen Forschung als lernförderlich erwiesen (Gleixner 1998). Darüber hinaus ist bei diesem Modell der Wasserstrom für die Schüler sichtbar und es lassen sich sowohl Reihen- als auch Parallelschaltungen darstellen.

Problematisch ist hingegen, dass die Vorerfahrungen der Schüler bei diesem Modell leicht Fehlvorstellungen bzgl. elektrischer Stromkreise provozieren können, da offen fließendes Wasser im Alltag üblicherweise nicht im Kreis strömt. Stattdessen dürften die Schüler schon einmal beobachtet haben, dass sich fließendes Wasser bei den meisten Verzweigungen zu gleichen Teilen aufteilt, hinter einem Wasserrad eine geringere Strömungsgeschwindigkeit besitzt als davor und sich natürlich streckenweise auch anstaut (vgl. Wodzinski 2013). Werden diese Erfahrungen unreflektiert auf den elektrischen Stromkreis übertragen, so dürften sie insbesondere die lokale und sequentielle Argumentation unbeabsichtigt fördern. Auch ist der offene Wasserkreislauf nicht geeignet, Schülern den Einschalt- bzw. Ausschaltvorgang bei Stromkreisen verständlicher zu machen, da das Wasser im Modell nach dem Einschalten erst mit einer merklichen Verzögerung beim Wasserrad ankommt bzw. auch nach dem Abschalten der Pumpe das Wasserrad noch einige Zeit antreiben kann. Dieses Verhalten steht nicht nur im Gegensatz zur Physik elektrischer Stromkreise, sondern fördert darüber hinaus noch die 
Stromaussendevorstellung, wonach der elektrische Strom zunächst in der Batterie gespeichert ist wie Öl in einem Ölfass und den Stromkreis dann ausgehend von einem Batteriepol Lämpchen für Lämpchen durchströmt. Grundsätzlich problematisch wird die Verwendung des offenen Wasserkreislaufmodells von Muckenfuß und Walz (1997, S. 98) gesehen, da ihrer Einschätzung nach das Vorstellen von Höhenunterschieden in einer elektrischen Schaltung eine Abstraktion voraussetzt, die das Abstraktionsvermögen insbesondere jüngerer Schüler - an die sich das Modell ja primär richtet - übersteigen dürfte.

Während das Modell des offenen Wasserkreislaufs also prinzipiell die elektrische Spannung gut veranschaulichen kann, erscheint es eher ungeeignet, den Systemcharakter von Stromkreisen zu vermitteln. Dies gilt auch für das Rutschbahnmodell, bei dem die Stromstärke durch die Geschwindigkeit von Schülern auf Schwimmreifen in einer Wasserrutsche dargestellt wird. Ihre Geschwindigkeit wird durch die Höhendifferenz und die Anzahl von Steinen im Rutschweg bestimmt (vgl. Grob et al. 1988).

\subsubsection{Das Stäbchenmodell}

Das Münchener Stäbchenmodell von Gleixner (1998) (bzw. in einer modifizierten Version das Mauermodell, siehe Bierwirth 2014) stellt eine abstraktere Variante der Höhenanalogie dar, hat sich in Untersuchungen aber als verhältnismäßig lernförderlich erwiesen (Koller et al. 2008). Beim Stäbchenmodell bekommt jedes Bauteil des elektrischen Stromkreises zwei Stäbchen zugewiesen, deren Höhe das elektrische Potenzial vor und nach dem Bauteil veranschaulicht (siehe Abb. 24). Da die Spannung bzw. Potenzialdifferenz stets einer Höhendifferenz entspricht, lassen sich so leicht Spannungen an Parallel-, Reihen- und gemischten Schaltungen analysieren.
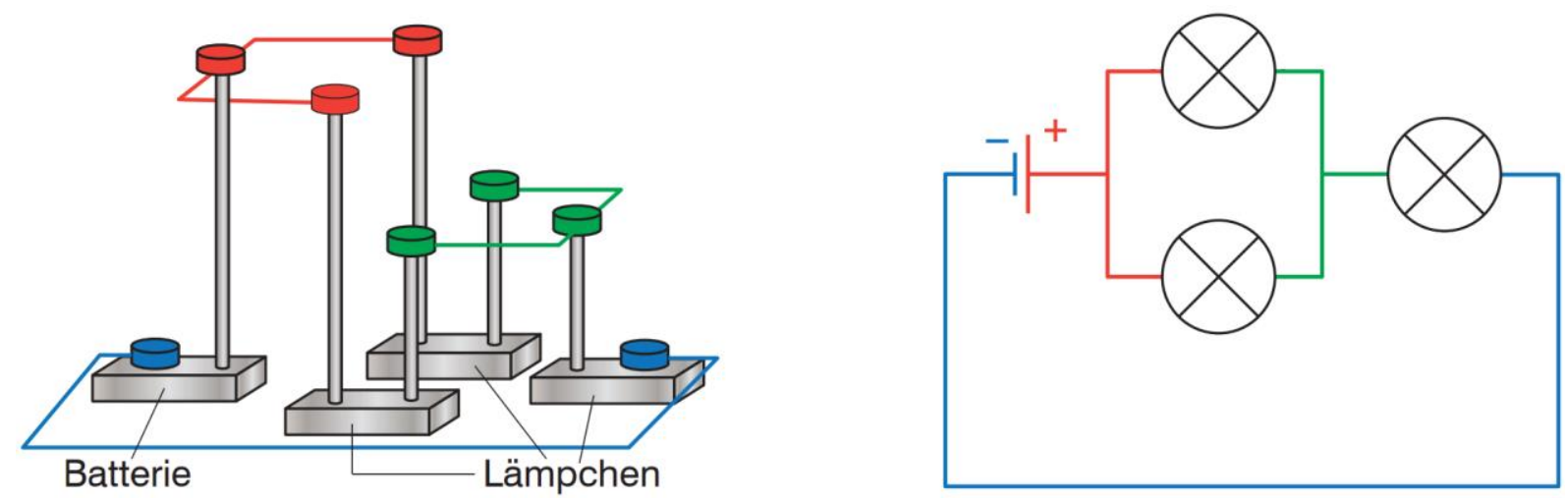

Abb. 24: Gegenüberstellung von Stäbchenmodell und Schaltplan (nach Burde und Wilhelm 2017c, S. 11; vgl. Koller 2008a, S. 122)

Anders als der offene Wasserkreislauf eignet sich dieses Modell aber nicht zur Veranschaulichung des elektrischen Stroms, sondern lediglich zur Veranschaulichung des elektrischen Potenzials bzw. der elektrischen Spannung. Eine tiefergehende Erklärung des elektrischen Potenzials liefert das Modell ebenso nicht, weshalb offenbleibt, was man unter „elektrisch hoch“ bzw. „elektrisch tief“ zu verstehen hat (Gleixner 1998, S. 220). Insbesondere bei jüngeren Schülern ist abhängig von deren kognitiven Entwicklung zu befürchten, dass 
sie mit abstrakten Modellen überfordert sind und in Folge „,keinen Zusammenhang und damit keinen Sinn in den Analogmodellen" sehen (Kircher 1984, S. 58).

\subsubsection{Spannung als Antriebskraft}

\subsubsection{Das Fahrradkettenmodell}

Wie der Name bereits andeutet, vergleicht das Fahrradkettenmodell den elektrischen Stromkreis mit dem Kreislauf der Kettenglieder einer Fahrradkette. Dabei entspricht die konstante Antriebskraft an den Pedalen der elektrischen Spannung und die an anderer Stelle an der Kette reibenden Bremsklötze den in Reihe geschalteten elektrischen Widerständen (siehe Abb. 25). In Folge der unrealistischerweise angenommenen Geschwindigkeitsabhängigkeit der Reibungskraft der Bremsklötze $F_{\text {Reib }}=\gamma v$ stellt sich so bei der Fahrradkette eine Gleichgewichtsgeschwindigkeit der Kettenglieder $v_{G}=F_{\text {Antrieb }} / \gamma$ ein, die im elektrischen Stromkreis der Stromstärke $I=U / R$ entspricht.
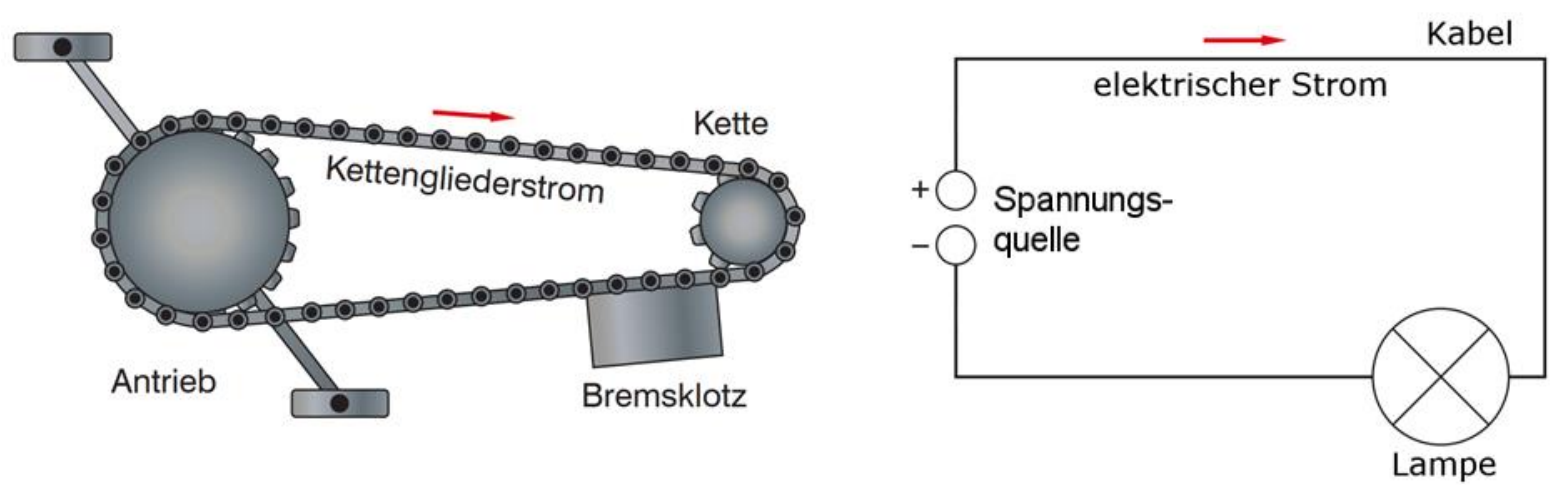

Abb. 25: Gegenüberstellung von Fahrradkettenmodell und Stromkreis (nach Burde und Wilhelm 2017c, S. 12)

Die Fahrradkette als gut sichtbarer Bestandteil von Fahrrädern ist den meisten Kindern aus ihrer Alltagswelt vertraut und dürfte sich daher hervorragend eignen, um zentrale Konzepte des elektrischen Stromkreises zu veranschaulichen. Dies gilt insbesondere auch deshalb, weil Masse und Geschwindigkeit der Kettenglieder verhältnismäßig klein sind, was der Schülervorstellung vorbeugen kann, dass die kinetische Energie der Kettenglieder bzw. Elektronen der entscheidende Faktor für die Energieübertragung im System darstellt. Insbesondere scheint das Fahrradkettenmodell geeignet zu sein, der Stromverbrauchsvorstellung zu begegnen, denn so wie der Bremsklotz keine Kettenglieder verbraucht, sondern dem Kreislauf lediglich Energie entnimmt, verbraucht auch die Glühbirne keinen Strom, sondern entnimmt dem Stromkreis nur Energie. In einer anderen Variante verbindet ein Keilriemen zwei Wellräder, so dass deutlich wird, dass trotz umlaufendem Keilriemen die Energie von einem Wellrad zum anderen wandert (Muckenfuß und Walz 1997). Das Modell ist also dazu prädestiniert, den Unterschied zwischen Ladungsträgerstrom und Energiestrom in Stromkreisen zu illustrieren (Härtel 2012a). Auch kann Schülern mit Hilfe des Fahrradkettenmodells der Systemcharakter von Stromkreisen in besonderer Weise nähergebracht werden, da z.B. das Bremsen der Fahrradkette an einer Stelle in Analogie zur Stromstärke im Stromkreis auch immer die Geschwindigkeit der gesamten Fahrradkette reduziert. 
Die Grenzen des Fahrradkettenmodells liegen einerseits darin, dass sich Parallelschaltungen mit ihm nicht adäquat repräsentieren lassen, es andererseits aber zunächst auch nur ein sehr rudimentäres und für die Sekundarstufe I nicht angemessenes Konzept der elektrischen Spannung als „Antrieb des elektrischen Stroms“ liefert (vgl. Rhöneck und Völker 1982, S. 409). Zwar könnte man die mechanische Spannung in der Kette mit dem elektrischen Potenzial vergleichen, womit die Differenz der mechanischen Spannung an zwei Punkten der Kette der elektrischen Spannung entspräche, jedoch bliebe dieser fachlich gesehen korrekte Vergleich für die Lernenden vermutlich unanschaulich und schwierig. Auch findet im Fahrradkettenmodell die Energieübertragung ausschließlich über die stramme Zugseite der Kette statt, während eine Spannungsquelle in symmetrischer Weise Kräfte auf beide Leitungen im Stromkreis auswirkt. Ein steifer Ring wie z.B. ein Hula-Hoop-Reifen, an dem in einem Schülerversuch sowohl gezogen als auch geschoben werden kann, bietet sich daher als mögliche Alternative an (Härtel 2012a, S. 20).

\subsubsection{Spannung als transportierte Energie pro Ladung}

\subsubsection{Das Rucksackmodell}

Manchmal sind im Unterricht Modelle des elektrischen Stromkreises anzutreffen, bei denen einzelne Ladungsträger die Energie von der Batterie zum Lämpchen transportieren, dort abgeben und sich anschließend ohne Energie zurück zur Batterie bewegen. Bekannte Beispiele für diese Ladungsträgermodelle sind das Rucksackmodell, das Energiehutmodell und das Bienchenmodell (Wilhelm 2015). Beim Rucksackmodell beispielsweise laufen Männchen in einer Reihe auf einem vorgegebenen Weg im Kreis und bekommen an der Spannungsquelle ihren Rucksack mit einer gewissen Energieportion $\Delta E$ gefüllt und laufen von dort aus zum Lämpchen, an dem sie ihren Rucksack leeren und anschließend mit leerem Rucksack wieder zurück zur Spannungsquelle laufen (siehe Abb. 26). Im Rucksackmodell entspricht die elektrische Stromstärke also der Anzahl der an einer Stelle vorbeilaufenden Männchen pro Zeit, während die Spannung gemäß der Gleichung $U=\Delta E / Q$ der Energieportion $\Delta E$ entspricht, die ein Männchen in seinem Rucksack mit sich trägt.

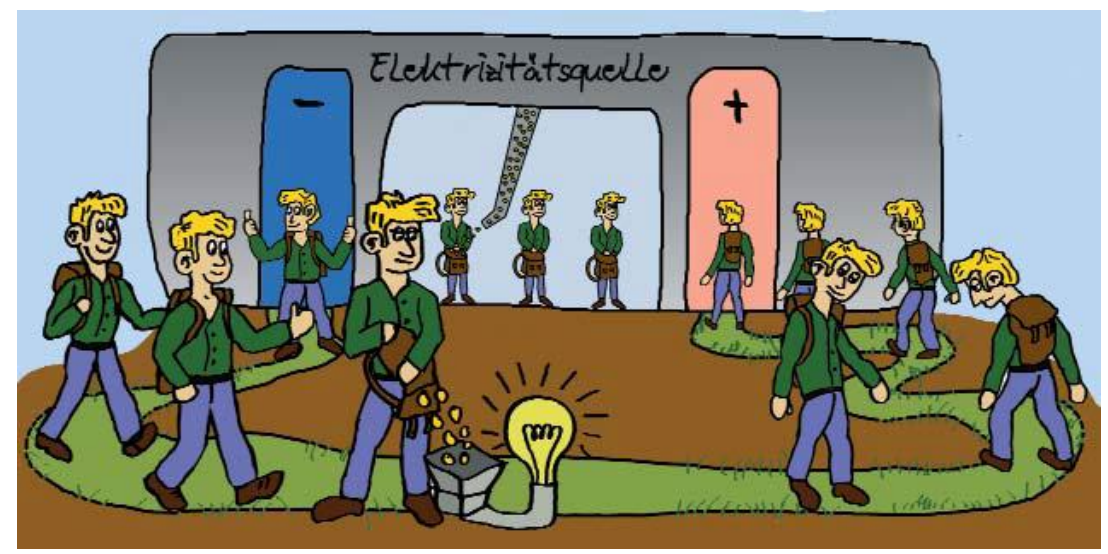

Abb. 26: Darstellung eines einfachen elektrischen Stromkreises im Rucksackmodell (Quelle: Wilhelm 2015) 
Neben der visuell gut einprägsamen Darstellung von Spannung als Energie pro Ladung bzw. Männchen dürfte eine weitere Stärke des Modells darin liegen, dass der Stromverbrauchsvorstellung durch die im Kreis laufenden Männchen klar entgegengewirkt wird, da den Kindern leicht ersichtlich ist, dass die Männchen in Elektrogeräten nicht verschwinden bzw. verbraucht werden. Auch wenn dieses Modell auf den ersten Blick also einleuchtend erscheint sowie Stromstärke (Männchen pro Zeit) und Spannung (Rucksackfüllung pro Männchen) konzeptionell klar voneinander trennt, hat es aus fachlicher und didaktischer Sicht eine Reihe gravierender Schwächen (Wilhelm 2015). So wird die Energie fachlich gesehen nicht durch die Elektronen übertragen, sondern durch das elektrische und magnetische Feld, wobei es hier entgegen der Modelldarstellung aus energetischer Sicht auch keinen Unterschied zwischen der Hin- und Rückleitung gibt (Backhaus 1987). Didaktisch problematisch ist besonders, dass das Modell bekannte Schülerfehlvorstellungen unterstützt statt ihnen entgegenzuwirken, da die Männchen von der Quelle aus den Stromkreis Bauteil für Bauteil durchlaufen. Das Modell verleitet die Schüler somit dazu, sich in die Lage der Männchen zu versetzen und fördert so zwangsläufig die lokale und sequentielle Denkweise bei elektrischen Stromkreisen statt bei den Schülern ein Systemdenken zu etablieren. Damit bleibt dann aber offen, woher die Männchen bei Reihenschaltungen wissen, an welchem Widerstand sie welche Energieportion abzugeben haben bzw. wie sie sich bei Parallelschaltungen an den Verzweigungspunkten aufteilen sollen. Da der Antrieb für die kreisförmige Bewegung in den einzelnen Männchen liegt, stößt das Modell auch bei Ein- und Ausschaltvorgängen sowie Wechselspannungen an seine Grenzen. Nimmt man eine Driftgeschwindigkeit der Elektronen in Kupfer von ca. $1 \mathrm{~mm} / \mathrm{s}$ an, ist es im Modell unerklärlich, warum eine Lampe nach dem Einschalten sofort angeht. Auch suggeriert das Modell fälschlicherweise, dass sich die Elektronen vor und nach einem Widerstand unterscheiden bzw. dass sich nach dem Ausschalten noch Energie auf der Hinleitung befinden müsse. Bei Wechselstromkreisen ist es zudem völlig unerklärlich, wie die Männchen immer wieder Energie am Lämpchen abgeben können, obwohl sie sich doch im Modell immer nur einen Schritt vor und zurück bewegen, ohne jemals wieder zurück zur Batterie zu kommen. Im Rucksackmodell besteht zudem die Gefahr, dass die Spannung von den Schülern nicht als Differenzgröße, sondern als Eigenschaft der Batterie aufgefasst wird und konzeptionell mit der elektrischen Energie verwechselt wird. Muckenfuß und Walz (1992, S. 44) machen ferner darauf aufmerksam, dass das Modell auch zu einer falschen Vorstellung hinsichtlich des Zusammenhangs zwischen Stromstärke und Spannung bei konstantem Widerstand führt, weil man leicht vermutet, dass Männchen mit doppelt so vollem Rucksack nicht doppelt so schnell laufen, sondern höchstens gleich schnell bzw. vermutlich eher langsamer. Damit wird auch eine falsche Energieabgabe am Lämpchen nahegelegt, denn es gilt: $\Delta E=U \cdot I \cdot \Delta t=\left(U^{2} / R\right) \cdot \Delta t$. Wird beispielsweise die Spannung verdoppelt, so verdoppelt sich demnach im Modell nur die Energieportion, welche die einzelnen Männchen mit sich tragen, nicht jedoch ihre Laufgeschwindigkeit und damit Stromstärke. 


\section{Analyse bedeutender Unterrichtskonzepte ${ }^{5}$}

\subsection{Die Elektrizitätslehre nach dem IPN-Curriculum}

Das nach dem „Sputnik-Schock“ in Kiel etablierte Institut für die Pädagogik der Naturwissenschaften entwickelte vor allem in den 1970er und 1980er Jahren nach didaktischen Überlegungen umfassende Curricula inklusive Unterrichtsmaterialien für die Fächer Physik, Chemie und Biologie und hatte dabei einen Auftrag für ganz Deutschland. Der Einstieg in die Elektrizitätslehre wird im IPN-Curriculum für das 7. und 8. Schuljahr über einen Vergleich verschiedener Energieübertragungssysteme motiviert, von denen angenommen wird, dass sie den Schülern aus ihrem Alltag vertraut sind (Härtel et al. 1981, S. 63).
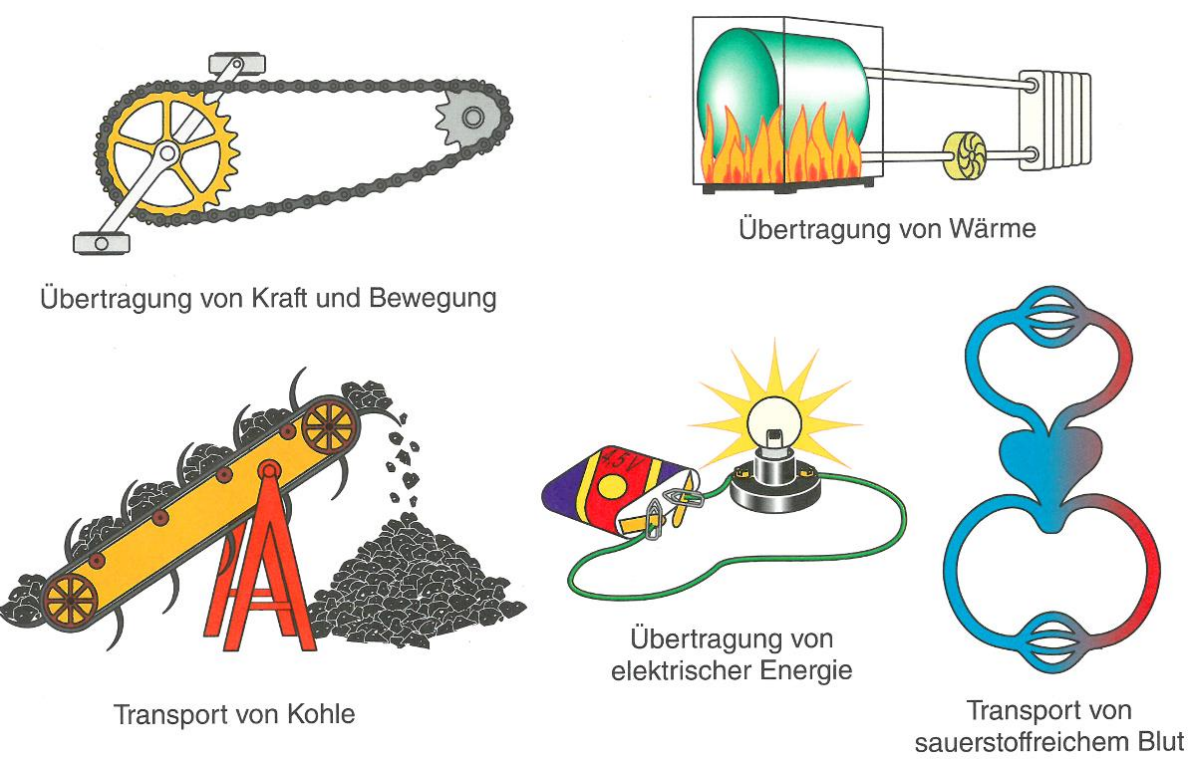

Abb. 27: Beispiele für verschiedene Energieübertragungssysteme im IPN-Curriculum (Quelle: Härtel 2012a, S. 19)

Mit dem Ziel, der Stromverbrauchsvorstellung schon frühzeitig entgegenzuwirken, wird zu Beginn ausführlich der Unterschied zwischen offenen Energieübertragungssystemen (Kohletransport, Pipeline, Lastzüge), bei denen energiereiche Materie transportiert und „verbraucht" wird, und geschlossenen Energieübertragungssystemen (Fahrradkette, Transmissionsriemen oder Blutkreislauf), bei denen das Trägermedium lediglich dem „Kraftschluss“ dient und nicht verbraucht wird, diskutiert. Anschließend wird zunächst am Beispiel des unverzweigten Stromkreises herausgearbeitet, dass auch der Stromkreis ein geschlossenes System zur Energieübertragung darstellt, bei dem die Energie in Analogie zum Fahrradkettenmodell durch die Bewegung eines ,steifen Elektronenrings“ im Leiter übertragen wird. Dabei werden die folgenden Beziehungen zwischen den verschiedenen physikalischen Größen hergestellt: Antrieb $\leftrightarrow$ Spannung; in sich geschlossener Materiefluss $\leftrightarrow$ elektrischer Strom; Behinderung $\leftrightarrow$ elektrischer Widerstand.

\footnotetext{
${ }^{5}$ Ein Vergleich diverser Unterrichtskonzepte zur Elektrizitätslehre findet sich auch in Gleixner (1998, 63ff).
} 
Aufgrund der Tatsache, dass das Fahrradkettenmodell bei der Erklärung von Parallelschaltungen und der Spannung schnell an seine Grenzen stößt, findet im weiteren Verlauf des IPN-Curriculums eine Variante des Wasserkreislaufmodells Verwendung, bei der die Schüler durch das Drücken gegen den Kolben einer Spritze selber den Antrieb der Wasserströmung darstellen und es ihnen so ermöglicht wird, durch eigene Krafteinwirkung den unterschiedlichen Strömungswiderstand von Reihen- und Parallelschaltungen am eigenen Körper zu erfahren (,Kolbenprobermodell $\left.{ }^{\circ}\right)$. Hier wird die Spannung dann als Wasserdruckunterschied eingeführt und an Parallel- und Reihenschaltungen entsprechende Regeln erarbeitet. Nach der Formulierung halbquantitativer Beziehungen der physikalischen Größen Spannung, Widerstand und Stromstärke (,je größer - bei konstanter Behinderung - der Antrieb wird, desto größer wird die Drehgeschwindigkeit“) wird das Ohm'sche Gesetz erarbeitet. Am Ende der Unterrichtsreihe steht eine Diskussion des spezifischen Widerstands, der Temperaturabhängigkeit des Widerstands sowie der Gefahren des elektrischen Stroms.

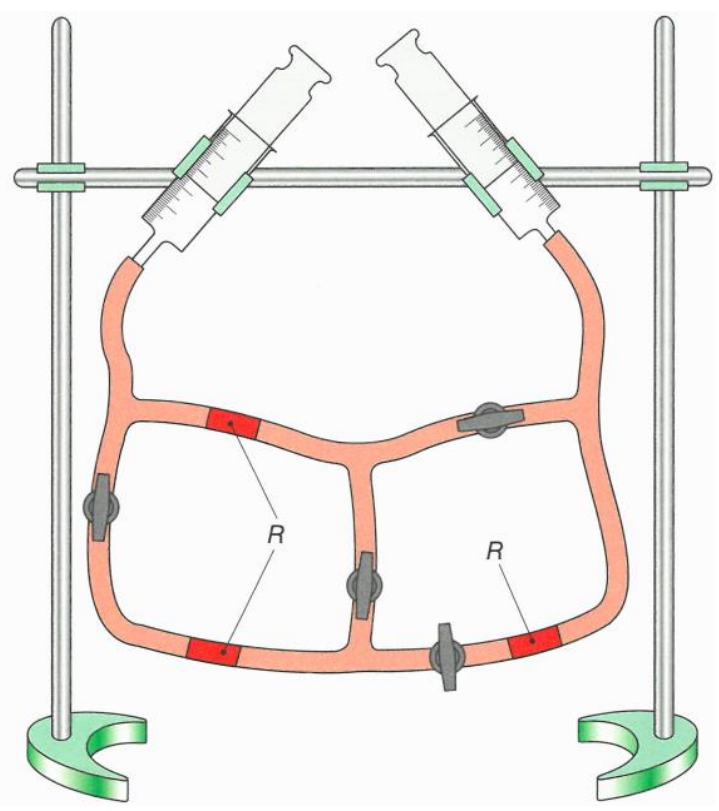

Abb. 28: Das im IPN-Curriculum vorgeschlagene Kolbenprobermodell (Quelle: Härtel 2012a, S. 22)

\section{Kritische Betrachtung}

Positiv ist zunächst die anfängliche Kontextorientierung des Unterrichtsansatzes, wonach die Bedeutung der Elektrizität für die zivilisatorische Entwicklung den Ausgangspunkt für den weiteren Unterrichtsverlauf darstellt und versucht wird, bei den Schülern das Interesse für eine tiefere Auseinandersetzung mit dem Thema zu wecken (vgl. Muckenfuß 1995). So wird den Kindern zunächst die historische Bedeutung verschiedener Energieübertragungssysteme (z.B. dem Transmissionsriemen zur Energieübertragung bei Dampfmaschinen, Pipelines zum Transport von Öl sowie dem Stromnetz zur Übertragung elektrischer Energie) für die gesellschaftliche Entwicklung vor Augen geführt, bevor eine systematische Erarbeitung der Regeln des elektrischen Stromkreises im traditionellen Sinne stattfindet. 
Im Gegensatz zu anderen Unterrichtsentwürfen setzt das IPN-Curriculum zur Vermittlung der Elektrizitätslehre nicht auf eine einzelne Analogie, sondern illustriert unterschiedliche Aspekte des elektrischen Stromkreises mit Hilfe unterschiedlicher Analogien. Positiv ist hier insbesondere der am Anfang der Unterrichtsreihe gezogene Vergleich zwischen Fahrradkette und dem elektrischen Stromkreis mit seinem ,starren Elektronenring“ hervorzuheben, da diese Analogie sowohl den Systemcharakter elektrischer Stromkreise betont als auch der Stromverbrauchsvorstellung und dem sequentiellen Denken entgegenwirkt (Härtel 2012a, S. 19).

Kritisch ist aus heutiger physikdidaktischer Perspektive hingegen die intensive Nutzung der Wasserstromkreisanalogie im weiteren Verlauf der Unterrichtsreihe zu sehen, da sich insbesondere der geschlossene Wasserkreislauf später immer wieder als problematisch erwiesen hat (Gentner und Gentner 1983; Tenney und Gentner 1985; Wilkinson 1972; Dudeck und Schwedes 1992; Schwedes und Schilling 1983). Auch wenn das Thema Elektrizitätslehre zu Beginn der Unterrichtseinheit über den Energietransport motiviert wird, geht das IPN-Curriculum im weiteren Verlauf nicht mehr auf das Thema „Energie“ ein. Gleixner (1998, S. 65) kritisiert deshalb zu Recht, dass so bei den Schülern vermutlich falsche Erwartungen bzgl. des kommenden Unterrichts geweckt werden. Ebenfalls problematisch ist die starke Betonung des Ohm'schen Gesetzes bei der Einführung des elektrischen Widerstands, da dies dazu führen kann, dass Schüler die Definition des elektrischen Widerstands mit dem Ohm'schen Gesetz gleichsetzen und womöglich glauben, es handele sich um ein und dasselbe physikalische Konzept (Gleixner 1998, S. 65).

\subsection{Das Unterrichtskonzept von Wiesner und Jung}

Das in Frankfurt entwickelte und auf Vorarbeiten von Jung (1981) zurückgehende Unterrichtskonzept nach Wiesner et al. (1982, S. 388) kommt ohne Analogien bzw. Modelle zum elektrischen Stromkreis aus und versucht stattdessen ausschließlich mit Hilfe von ,beobachtbaren Phänomenen“ die Elektrizitätslehre in 10 Doppelstunden zu erklären - oder um die Worte Jungs (1981, S. 94) zu nutzen: „Modelle stehen hier [...] nicht im Vordergrund, sondern die Phänomene, das heißt der harte Kern der physikalischen Fakten“. Zu den erklärten Zielen des Unterrichtskonzepts gehört u.a., der Stromverbrauchs- und Konstantstromvorstellung entgegenzuwirken und den Schülern die Unterscheidung zwischen der elektrischen Spannung und der elektrischen Stromstärke zu erleichtern. Analog zu der von Jung (1980) bereits in seinem Mechanikkonzept vorgenommenen Unterteilung in die vier Grundkategorien „Körper“, „Prozess", ,Eigenschaft" und „Beziehung“ wird im vorliegenden Unterrichtskonzept zur Elektrizitätslehre daher vorgeschlagen, die Spannung als Eigenschaft der Spannungsquelle und die Stromstärke im Gegensatz hierzu als Prozess bzw. Vorgang in einem Elektrogerät einzuführen.

Ausgehend von der Beobachtung, dass klassische Versuche mit Amperemetern in unverzweigten Stromkreisen nicht in einem befriedigenden Maße zu einer Entkräftung der Stromverbrauchsvorstellung bei Schülern führen, setzen Wiesner et al. (1982, S. 389) von 
Anfang an auf Kompassnadeln als alternative Stromstärkeindikatoren auf Basis der magnetischen Wirkung des elektrischen Stroms (siehe Abb. 29).

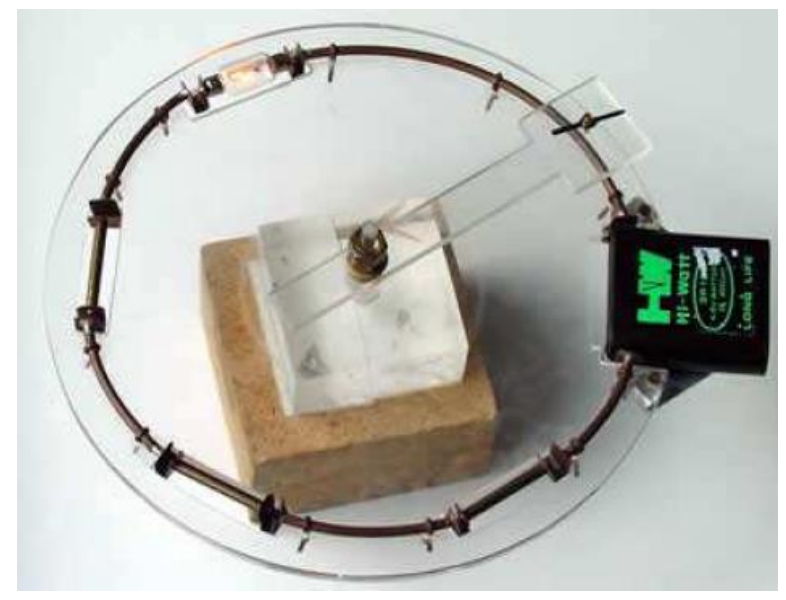

Abb. 29: Stromkreis mit Kompassnadel zum Nachweis des elektrischen Stroms (Quelle: Koller 2008a, S. 15)

Auf diese Weise demonstrieren sie die Konstanz der Stromstärke in verschiedenen Versuchen, bevor sie anschließend mit Hilfe eines einfachen Demonstrationsexperiments zu einer Reihenschaltung, die um ein in Reihe geschaltetes Lämpchen erweitert wird, der Konstantstromvorstellung begegnen. Nachdem auch in Parallelschaltungen die Stromstärke besprochen und die Knotenregel formuliert wurde, wird in der zweiten Hälfte des Unterrichtskonzepts dann die Spannung als Stärke einer Spannungsquelle eingeführt, wobei im Unterrichtskonzept der Begriff „Generator“ als Überbegriff für Batterien und Netzgeräte verwendet wird. Motiviert wird die Einführung der Spannung als Stärke des Generators damit, dass verschiedene Generatoren beim gleichen Elektrogerät unterschiedliche Stromstärken verursachen. Die Spannung als Eigenschaft eines Generators wird im Konzept dann durch die Wirkung auf ein „Standardgerät“ gemessen. Zur Spannungsmessung insbesondere bei Reihenschaltungen wird das Konzept des Ersatzgenerators genutzt, wobei der Ersatzgenerator die Spannungsquelle darstellen soll, die nötig wäre, um den gleichen Stromfluss durch den Widerstand zu erreichen, wenn alle anderen Komponenten des Stromkreises zusammengefasst werden würden. Abschließend wird die Brücke zu den Kirchhoff'schen Regeln geschlagen und die Begriffe Leitfähigkeit und Widerstand werden eingeführt. Auf eine Thematisierung des Ohm'schen Gesetzes wird bewusst verzichtet, da es für die im Unterrichtskonzept eingesetzten Geräte ohnehin nicht zutrifft.

\section{Kritische Betrachtung}

Durch die ausschließliche Orientierung an beobachtbaren Phänomenen ohne jeglichen Rückgriff auf Modelle oder Analogien zur Erklärung wesentlicher physikalischer Größen kommt dem Unterrichtskonzept nach Wiesner et al. (1982) eine Sonderrolle zu. Dieses Vorgehen ist aus konstruktivistischer Sichtweise zu kritisieren, da nicht versucht wird, an das Vorwissen der Schüler anzuknüpfen und ihnen eine anschauliche Erklärung für den Strom- oder für den Spannungsbegriff an die Hand zu geben. Die Elektrizitätslehre stellt aber ein sehr unanschauliches Gebiet der Physik dar, weil sich die physikalischen Vorgänge wie z.B. die Elektronen- 
bewegung der direkten Wahrnehmung entziehen. Gerade bei abstrakten Konzepten wie der elektrischen Spannung wäre es aber sinnvoll, den Lernenden durch das Anknüpfen an bestehenden kognitiven Strukturen den Aufbau eines angemessenen mentalen Modells zu erleichtern. Wie Wiesner et al. (1982, S. 391) selber schreiben, haben die meisten Schüler auch nach der Unterrichtsreihe kein tragfähiges Spannungskonzept entwickelt und differenzieren nicht ausreichend zwischen den Konzepten „Spannung“ und „Stromstärke“. Neben dem Verzicht auf jegliche Modellvorstellung könnte ein Grund hierfür auch in dem verhältnismäßig abstrakten Konzept des Ersatzgenerators liegen, das insbesondere für lernschwache Schüler schwer zu verstehen sein dürfte.

Positiv ist hingegen die im Unterrichtskonzept vorgeschlagene Stromstärkemessung mit Hilfe von Kompassnadeln zu beurteilen. Der wesentliche Vorteil dieses Ansatzes liegt darin, dass der Stromkreis zur Messung der Stromstärke sowie Stromrichtung im Gegensatz zu Messungen mit Amperemetern nicht verändert werden muss. So lässt sich beispielsweise die örtliche Invarianz des elektrischen Stroms in einem unverzweigten Stromkreis ganz leicht durch „Abfahren“ desselben mit einer Kompassnadel gut sichtbar demonstrieren, ohne hierzu durch den Einbau von Amperemetern für die Schüler potenziell verwirrende Änderungen am Stromkreis selber vornehmen zu müssen. Dies kann insbesondere zu Beginn des Elektrizitätslehreunterrichts zum Nachweis der Stromstärkekonstanz von großem Vorteil sein, da Schüler so nicht unnötig von den Anschlussbedingungen der Amperemeter abgelenkt werden. Ferner lässt sich mit Hilfe der Kompassnadel die Stromstärke an beliebigen Orten des Stromkreises messen, was explizit auch die Batterie selbst miteinschließt. Wiesner et al. (1982, S. 390) berichten, dass sich der Ansatz im Unterricht als fruchtbar erwies, da den Schülern die Tatsache, dass die magnetische Wirkung und damit der elektrische Strom überall im Stromkreis gleich groß sind, in Erinnerung geblieben ist. Aufgrund der Eleganz der qualitativen Stromstärkemessung mit Hilfe von Kompassnadeln und der damit erzielten Erfolge bzgl. der Überwindung der Stromverbrauchsvorstellung hat der Ansatz auch Eingang in diverse andere Unterrichtskonzepte gefunden (vgl. Gleixner 1998; Koller 2008a; Späth 2009). Aufbauend auf der magnetischen Wirkung des elektrischen Stroms ließen sich auch quantitative Messungen vornehmen, indem der Stromkreis statt mit einer Kompassnadel mit einer empfindlichen Stromzange ,abgefahren“ wird (Girwidz 1995).

\subsection{Der Karlsruher Physikkurs (KPK)}

Der Karlsruher Physikkurs (KPK) ist das Ergebnis langjähriger Entwicklungsforschung an der Universität Karlsruhe und hat den Anspruch, den bisherigen Physikunterricht an Schule und Hochschule mit seinem historisch gewachsenen Inhaltskanon umfassend zu reformieren. Die Grundidee des Karlsruher Physikkurses ist es, dem gesamten Physikunterricht eine grundlegend neue Sachstruktur zu geben, indem Analogien zwischen unterschiedlichen physikalischen Teilgebieten geschickt genutzt werden und auf sogenannte ,historische Altlasten“, wie z.B. die Verwendung unterschiedlicher Energiebegriffe in jedem Teilgebiet der Physik, verzichtet wird (Herrmann 1995). 
Im Mittelpunkt des maßgeblich von Herrmann und Falk entwickelten Karlsruher Physikkurses steht die Idee, extensive Größen wie die Energie, den Impuls, die Entropie oder die elektrische Ladung als mengenartige Größen, d.h. wie Stoffmengen, zu behandeln. Konkret versteht Herrmann (1995, S. 43) unter mengenartigen Größen solche physikalischen Größen, die folgende Kontinuitätsgleichung erfüllen:

$$
\frac{d}{d t} X=I_{x}+\Sigma_{\mathrm{X}}
$$

Hierbei steht $d X$ / $d t$ für die zeitliche Änderung der mengenartigen Größe $X$ innerhalb eines bestimmten Raumgebietes, $I_{X}$ für die Stromstärke der Größe $X$ durch die Oberfläche des Raumgebietes und $\Sigma_{X}$ stellt ein Maß für die Erzeugung bzw. Vernichtung von $X$ innerhalb des Raumgebietes dar. Aus didaktischer Sicht sieht Herrmann (1998, S. 830) den entscheidenden Vorteil der Verwendung von mengenartigen Größen darin, dass die Schüler sich von diesen Größen leicht eine Anschauung bilden können und auch über sie sprechen können, wie über allägliche Stoffe wie Wasser oder Luft. Entsprechend wird im KPK beispielsweise im Zusammenhang mit den physikalischen Größen Energie, Impuls oder Entropie davon gesprochen, dass diese „,verdünnt“, ,angehäuft“ oder „,verteilt“ würden. Das hinter diesen Begriffen stehende Strömungskonzept der mengenartigen Größen findet im gesamten Karlsruher Physikkurs in Form des SAW-Konzepts Verwendung, wonach jede Strömung unter den Gesichtspunkten $\underline{\text { Strom, }}$ Antrieb und $\underline{\text { Widerstand betrachtet wird. }}$

Konzeptionell stellt die Strömungsmechanik den Ausgangspunkt des KPKs dar, deren Konzepte und Begriffe sich wie ein roter Faden durch die gesamte Unterrichtskonzeption ziehen. Im Rahmen eines Hydraulikvorkurses wird zunächst auf Basis der Alltagserfahrungen der Schüler mit Wasserströmungen bzw. dem Luftdruck qualitativ geklärt, dass Flüssigkeiten bzw. Gase immer von Bereichen höheren zu Bereichen niedrigeren Drucks strömen und Druckunterschiede den Antrieb einer Strömung darstellen.

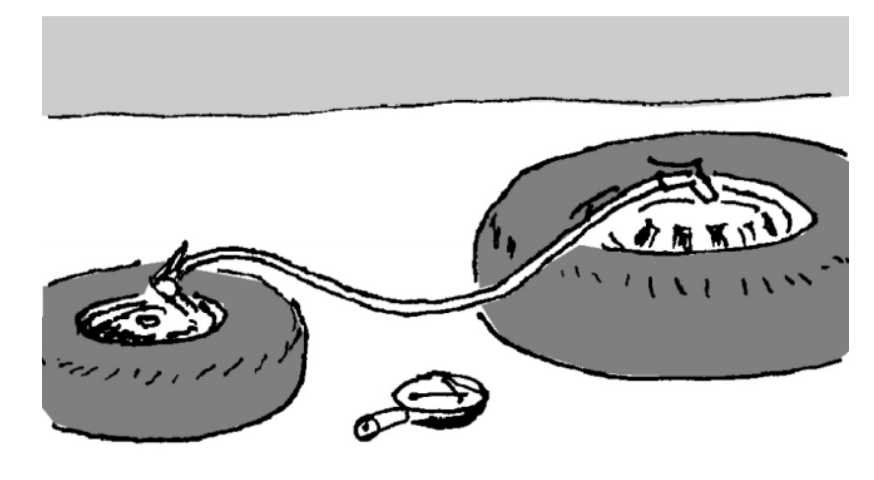

Abb. 30: Die Luft strömt vom Reifen mit höherem Druck in den Reifen mit niedrigerem Druck (Quelle: Herrmann et al. 2014a, S. 18)

Hinter diesem Vorgehen steht die Idee, dass zwischen den verschiedenen physikalischen Teilgebieten eine strukturelle Analogie besteht und dass die Schüler deshalb ihr in der Strömungsmechanik entwickeltes Verständnis auf andere Gebiete wie z.B. die Mechanik oder 
die Elektrizitätslehre übertragen können. Die dem KPK zugrundeliegenden formalen Entsprechungen zwischen den verschiedenen Teilgebieten der Physik und Chemie sind in Tab. 3 dargestellt.

Tab. 3: Zuordnung verschiedener physikalischer Größen zu den Teilgebieten der Physik und Chemie

\begin{tabular}{|llll|}
\hline \multirow{2}{*}{ Mechanik } & Extensive Größe & Stromstärke & Intensive Größe \\
\hline Elektrizitätslehre & Empuls $\vec{p}$ & Kraft $\vec{F}$ & Geschwindigkeit $\vec{v}$ \\
\hline Wärmelehre & Entropie $S$ & Entropiestromstärke $I_{S}$ & Temperatur $T$ \\
\hline Chemie & Stoffmenge $n$ & Stoffstromstärke $I_{N}$ & Chemisches Pot. $\mu$ \\
\hline
\end{tabular}

In Analogie zu Fluidströmungen, bei denen das Fluid immer vom Bereich höheren zum Bereich niedrigeren Drucks strömt, wird in der Mechanik z.B. postuliert, dass die men-

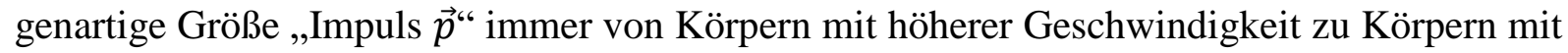
niedrigerer Geschwindigkeit $\vec{v}$ strömt und die Kraft $\vec{F}$ dementsprechend als Impulsstromstärke eingeführt. In der Wärmelehre gilt beispielsweise, dass die mengenartige Größe „Entropie $S$ “ immer von Bereichen höherer Temperatur zu Bereichen niedrigerer Temperatur strömt und sich deshalb eine Entropiestromstärke $I_{S}$ einstellt.

Die im traditionellen Physikunterricht vorgenommen Unterscheidung verschiedener Formen des Energietransports wie z.B. der elektrischen Energie, der Wärme oder der chemischen Energie wird im KPK als eine historisch gewachsene Altlast der Physik kritisiert, da sie nahelegt, ,[...] daß es sich bei den Energieformen um verschiedene physikalische Größen handelt, mit der merkwürdigen Eigenschaft, daß man eine in die andere umwandeln kann" (Herrmann 1995, S. 45). Da seit der speziellen Relativitätstheorie aber bekannt ist, dass die Energie eine eigenständige physikalische Größe und keine abgeleitete Rechengröße darstellt, sei eine Unterscheidung zwischen verschiedenen Energieformen in den unterschiedlichen Teilgebieten der Physik aus heutiger Sicht nicht zielführend. Auf Grundlage der Gibbs ‘schen Fundamentalgleichung

$$
d E=T d S+\varphi d Q+\vec{v} d \vec{p}+\mu d n
$$

wird argumentiert, dass bei jeder Energieänderung mindestens noch eine der mengenartigen Größen $S, Q, p$ oder $n$ ihren Wert ändert. Betrachtet man die Energieänderung, so erhält man

$$
\frac{d}{d t} E=T \frac{d}{d t} S+\varphi \frac{d}{d t} Q+\vec{v} \frac{d}{d t} \vec{p}+\mu \frac{d}{d t} n
$$

und damit die dem KPK zugrundeliegende Gleichung des „Energiestroms $P^{\text {“: }}$

$$
P=T I_{S}+\varphi I+\vec{v} \vec{F}+\mu I_{n}
$$


Der Energiestrom $P$ ist also immer mit einer Strömung der mengenartigen Größen $S, Q, \vec{p}$ oder $n$ verbunden, die im Karlsruher Physikkurs Energieträger genannt werden. Die in der Gleichung mit den extensiven Größen direkt verbundenen intensiven Größen $T, \varphi, \vec{v}$ und $\mu$ sind in diesem Bild ein Maß dafür, wie stark sich die mengenartige Größe ändert und damit auch, wie viel Energie pro Zeit über das jeweilige Trägermedium übertragen wird.

In der Einheit des KPKs zur Elektrizitätslehre entspricht die mengenartige Größe der elektrischen Ladung $Q$, die Stromstärke entspricht der elektrischen Stromstärke $I$ und die zugehörige intensive Größe ist das elektrische Potenzial $\varphi$. Im Gegensatz zum auf der Wasserstromkreisanalogie aufbauenden Unterrichtskonzept von Schwedes et al. (1995) wird im Karlsruher Physikkurs allerdings weniger die direkte Korrespondenz der physikalischen GröBen und Regeln zwischen Wasserstromkreis und elektrischem Stromkreis betont. Stattdessen wird entsprechend der Konzeption des KPKs versucht, auf den formalen Entsprechungen zwischen Stromkreisen und Strömungsmechanik aufzubauen und an das im Hydraulikvorkurs aufgebaute Verständnis von Strömungen anzuknüpfen. So wird z.B. in Analogie zu einem Hydraulikstromkreis, bei dem eine Pumpe für einen zirkulären Materiestrom aber damit linearen Energiestrom sorgt, die Batterie als Elektrizitätspumpe eingeführt, die für einen zirkulären Elektrizitätsstrom und linearen Energiestrom im Stromkreis sorgt.
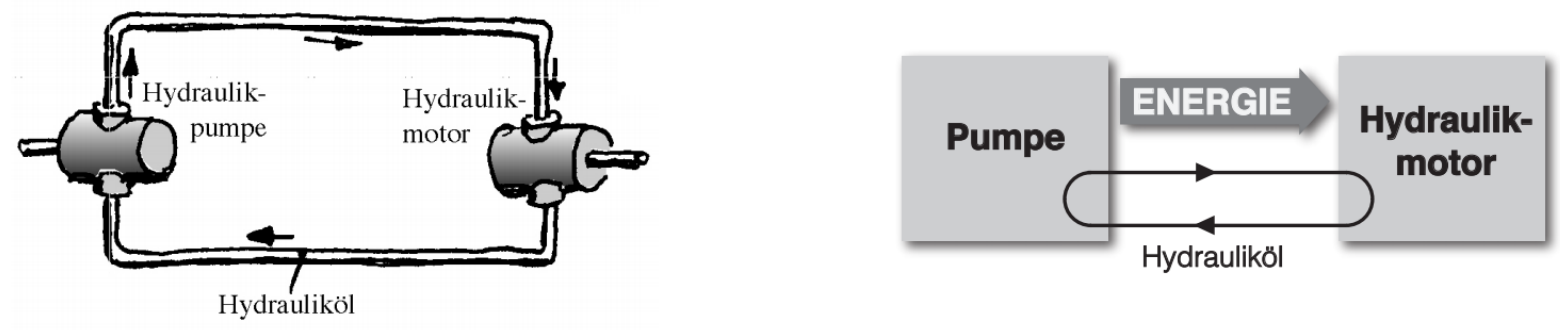

Abb. 31: Hydraulikstromkreis (links) und Flussbild des Hydraulikstromkreises (rechts) (Quelle: Herrmann et al. 2014b, S. 18)

Im weiteren Verlauf rückt der Energieaspekt dann aber zunächst in den Hintergrund, weil auf die Grundgrößen Stromstärke, elektrisches Potenzial und elektrischer Widerstand näher eingegangen wird. Nach der Einführung der Stromstärke und der Knotenregel wird das elektrische Potenzial und der elektrische Potenzialunterschied - im Gegensatz zu Schwedes et al. (1995) - explizit thematisiert und als Antrieb für den elektrischen Strom eingeführt:

„Das elektrische Potential in einem elektrischen Stromkreis entspricht dem Druck in einem Hydraulikstromkreis. Eine Batterie erzeugt einen Potentialunterschied, und dieser Potentialunterschied stellt den Antrieb für einen Elektrizitätsstrom dar “ (Herrmann 2003, S. 28).

Im folgenden Verlauf wird dann die Spannung als Potenzialunterschied definiert, die Erde als Potenzialnullpunkt eingeführt und die Wechselwirkung zwischen Spannung, Widerstand und Stromstärke im Rahmen des für den KPK typischen SAW-Konzepts thematisiert. Auch lernen Schüler, bei der Analyse von Stromkreisen in einem ersten Schritt alle Leitungen 
in Abhängigkeit von ihren jeweiligen Potenzialwerten farblich zu markieren, wobei die Regel gilt, ,[...] dass alle Leitungen, die dasselbe Potential haben, auch dieselbe Farbe bekommen" (Herrmann 2003, S. 32). Durch die Abgrenzung der Widerstandskennlinie eines langen Drahtes gegenüber den Widerstandskennlinien von Dioden, Elektromotoren und Glühbirnen wird die Sonderstellung von Ohm'schen Widerständen herausgearbeitet. Anschließend wird auf die Gefahren des elektrischen Stroms im Alltag hingewiesen und qualitativ auf das Thema Wechselstrom eingegangen. In einer abschließenden Einheit wird das Thema Energieübertragung bei Herrmann (2003, S. 41) wieder aufgegriffen und über das Konzept der Energiestromstärke $P=E / t$ auch quantitativ behandelt.

\section{Kritische Betrachtung}

Ähnlich dem IPN-Curriculum findet auch beim Karlsruher Physikkurs der Einstieg in das Thema Elektrizitätslehre zunächst über die Betrachtung des elektrischen Stromkreises als System zur Energieübertragung statt, was vor dem Hintergrund der Bedeutung der Elektrizität für die Energieversorgung im Alltag durchaus sinnvoll erscheint. Hierzu wird der elektrische Stromkreis, bei dem ein zirkulärer Elektrizitätsstrom für einen linearen Energiestrom sorgt, mit einem Hydraulikstromkreis verglichen, wobei davon ausgegangen wird, dass die Schüler ein ausreichendes Verständnis der Strömungsmechanik aus einem Vorkurs zu Beginn des Karlsruher Physikkurses besitzen. Damit wird in der Elektrizitätslehre an ein Flussbild angeknüpft, dass im KPK auch bei anderen physikalischen Größen Anwendung findet (z.B. „Impulsstrom" und „Entropiestrom“).

Anders als bei den bisher vorgestellten Unterrichtskonzepten wird im KPK die elektrische Spannung explizit als Potenzialdifferenz eingeführt, wobei das elektrische Potenzial mit dem Druck in einem Hydraulikstromkreis verglichen und dieser Druck- bzw. Potenzialunterschied als Antrieb des Elektrizitätsstroms eingeführt wird. Dieses Vorgehen ist insofern problematisch, als dass sich gezeigt hat, dass insbesondere die Entwicklung eines angemessenen Verständnisses der Druckverteilung in Wasserstromkreisen für die Schüler eine große Herausforderung darstellt (Schwedes et al. 1995, S. 29). Wie in Abschnitt 4.3.1 sowie Burde und Wilhelm (2016a) erläutert, könnte dies u.a. darin liegen, dass sich viele Schüler den Druck in einer Flüssigkeit nur schwer vorstellen können, da sich eine Flüssigkeit unter hohem Druck nicht sichtbar von einer Flüssigkeit unter geringem Druck unterscheidet und Flüssigkeiten von Schülern oft als Kontinuum bzw. als inkompressibel angesehen werden. Hinzu kommt, dass im KPK mit einer schlichten Hydraulikpumpe gearbeitet wird (siehe Abb. 32), obwohl sich gezeigt hat, dass die Nutzung einfacher Pumpen die Entwicklung eines angemessenen Spannungskonzeptes erschwert. Als problematisch hat sich bei der Verwendung einer einfachen Pumpe insbesondere erwiesen, dass die Schüler die Pumpe selbst und nicht den durch die Pumpe erzeugten Druckunterschied für den Antrieb der Strömung halten und damit nur ein unzureichendes Konzept von der Spannung als Antrieb der Strömung entwickeln (Schwedes et al. 1995, S. 35). 


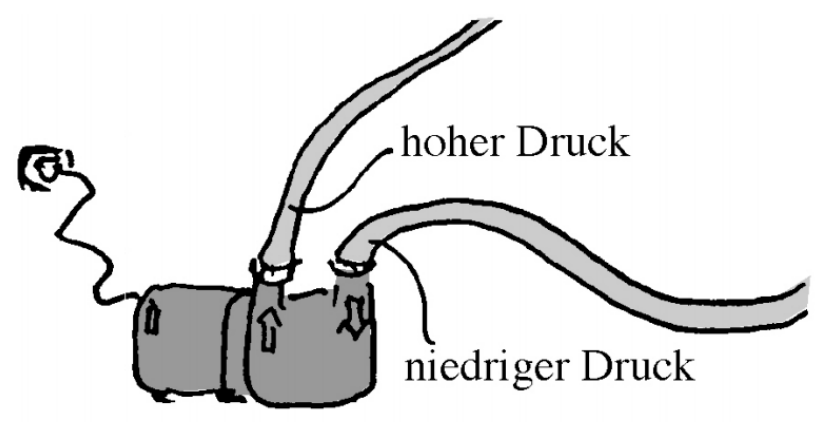

Abb. 32: Einfache Hydraulikpumpe im KPK (Quelle: Herrmann et al. 2014b, S. 22)

Auf der anderen Seite dürfte das im KPK vorgeschlagene farbige Kennzeichnen von Leitern mit gleichen Potenzialwerten die Entwicklung eines Potenzial- und Spannungskonzepts erleichtern, da sich die Potenzial- und Spannungsverhältnisse in Schaltskizzen mit diesem Verfahren übersichtlich darstellen lassen und dieses Verfahren von Schülern gerne genutzt wird (Herrmann und Schmälzle 1984, S. 482).

Gegenüber der Unterrichtskonzeption des IPN-Curriculums ist beim Karlsruher Physikkurs positiv anzumerken, dass die Sonderrolle von Ohm'schen Widerständen klar herausgearbeitet wird, damit die Schüler das Ohm'sche Gesetz nicht mit der Definition des Widerstands verwechseln. Dennoch ist fraglich, inwiefern der hierzu gewählte Weg über Widerstandskennlinien von Dioden oder Elektromotoren didaktisch geschickt ist, da gerade lernschwächere Schüler womöglich mit der Interpretation von Graphen überfordert sind. Weiterhin ist positiv anzumerken, dass der KPK im Gegensatz zum IPN-Curriculum die Energieübertragung mit Hilfe von Stromkreisen nicht nur zum Einstieg in die Unterrichtsreihe nutzt, sondern auch gegen Ende das Thema Energieübertragung mit Hilfe des Konzepts der Energiestromstärke wieder aufgreift und vertieft (Herrmann 2003, S. 41).

In der bisher einzigen Evaluation des KPKs durch Starauschek (2001) in Form einer quasiexperimentellen Treatment-Kontrollgruppen-Untersuchung in Baden-Württemberg mit ca. 2.000 Schülern der Klassen 8 bis 10 hat sich gezeigt, dass es bzgl. der Veränderung der Alltagsvorstellungen der Schüler keine großen Unterschiede zwischen dem traditionellen Physikunterricht und dem Karlsruher Physikkurs gibt. In der Elektrizitätslehre gelang es weder traditionell noch nach dem Karlsruher Physikkurs unterrichteten Schülern ein vom elektrischen Strom unabhängiges, eigenständiges Spannungskonzept zu entwickeln oder den elektrischen Stromkreis als zusammenhängendes System zu begreifen. Darüber hinaus existierten auch keine Unterschiede bzgl. des Energieverständnisses in Stromkreisen, da die Schüler beider Gruppen den elektrischen Strom konzeptionell nicht vom Energiestrom bzw. der elektrischen Energie trennen konnten (Starauschek 2002, S. 14). Auch wenn damit insgesamt die mit dem KPK verbundenen Erwartungen enttäuscht wurden, lobten insbesondere die Mädchen die Verständlichkeit der im Karlsruher Physikkurs gegebenen Erklärungen und besaßen ein etwas höheres Selbstkonzept in Physik als traditionell unterrichtete Schülerinnen. Ferner zeigte sich auch, dass die nach dem KPK unterrichteten Schülerinnen und Schüler eine tendenziell konsistentere Sprache verwendeten (Starauschek 2001). 


\subsection{Das CASTLE-Curriculum von Steinberg}

Das von Steinberg am Smith College, Massachusetts, im Jahre 1990 für den Anfangsunterricht in US-amerikanischen High Schools entwickelte CASTLE-Curriculum, ein Akronym für „Capacitor-Aided System for Teaching and Learning Electricity“, versucht durch den Vergleich des elektrischen Potenzials mit dem Luftdruck bzw. dem in Leitern herrschenden „elektrischen Druck“ den Schülern ein qualitatives Verständnis für die wechselseitige Beziehung der Grundgrößen Spannung, Stromstärke und Widerstand in elektrischen Stromkreisen zu vermitteln (Steinberg 2004, S. 42). Das Konzept verfolgt somit einen Potenzialansatz und baut auf dem intuitiven Druckverständnis der Schüler (z.B. mit Luftballons) auf, wonach ein komprimiertes Gas ,unter Druck“ steht und in seinem Bestreben, den Druck abzubauen, gegen die Außenwände drückt (Clement und Steinberg 2002, S. 397). Zentraler Unterrichtsgegenstand sind Versuche zu Lade- und Entladevorgängen an Kondensatoren mit großer Kapazität, um zu demonstrieren, dass eine Ladungsströmung immer eine Folge von elektrischen Druck- bzw. Potenzialunterschieden ist, wobei argumentiert wird, dass Ladungen in einem Leiter ähnlich frei beweglich sind wie „Luftteilchen“ in einem Schlauch.

Insgesamt umfasst das CASTLE-Curriculum zehn Einheiten, wobei sich nur die ersten sechs Einheiten mit der elementaren Elektrizitätslehre auseinandersetzen, während in den letzten vier Einheiten auf weitergehende Themen wie Elektromotoren, Wechselstrom und Halbleiter eingegangen wird. Die erste Einheit geht der Frage nach, was eigentlich in den Leitungen passiert, wenn ein Strom fließt. In einem ersten Schritt untersuchen die Schüler, was für Bedingungen erfüllt sein müssen, damit ein Strom in einfachen Stromkreisen überhaupt fließen kann und welche Materialien Leiter bzw. Nicht-Leiter sind. Der Nachweis des elektrischen Stroms und seiner Richtung erfolgt wie bei Wiesner et al. (1982) mit Hilfe einer Kompassnadel, die aufgrund der magnetischen Wirkung des elektrischen Stroms in Abhängigkeit seiner Intensität unterschiedlich stark ausschlägt. Die zweite Einheit beschäftigt sich mit der Frage, welchen Einfluss Glühlampen auf bewegte Ladungen in einem Stromkreis haben. $\mathrm{Zu}$ diesem Zweck wird zunächst erarbeitet, dass die Stromstärke, im CASTLE-Curriculum „charge flow" bzw. Ladungsströmung genannt, mit einer zunehmenden Anzahl an in Reihe geschalteten Widerständen abnimmt. Anschließend wird das Widerstandsthema vertieft, indem die Schüler Luft durch unterschiedlich dicke bzw. lange Strohhalme blasen, um auf diese Weise ein Gefühl dafür zu entwickeln, wie eine Strömung durch die Geometrie eines Widerstands beeinflusst wird. In der dritten Einheit wird der Frage nachgegangen, wo die bewegten Ladungen in einem Stromkreis herkommen. Hierzu wird zunächst mit Hilfe einer Kompassnadel untersucht, welchen Einfluss die Polung einer Batterie auf die Stromrichtung hat, bevor anschließend am Beispiel des Lade- bzw. Entladevorgangs eines Kondensators mit einer großen Kapazität von $25.000 \mu \mathrm{F}$ experimentell geprüft wird, wo jeweils die Ladungen herkommen, die die Lämpchen zum Leuchten bringen (siehe Abb. 33). Auf diese Weise wird der Stromaussendevorstellung begegnet und den Schülern eine Vorstellung der Batterie als eine Art Elektronenpumpe nahegelegt. 

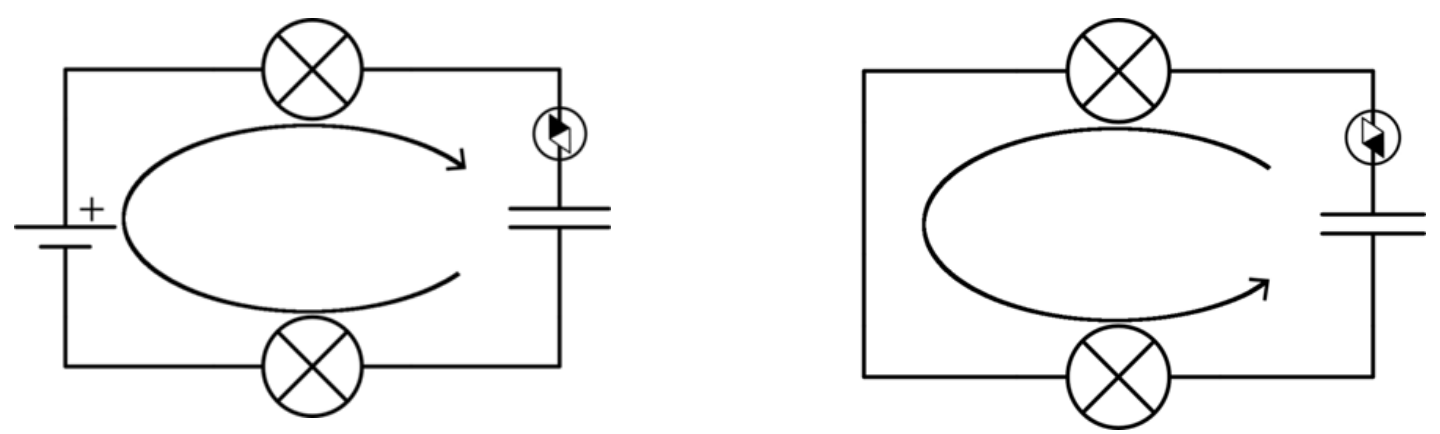

Abb. 33: Untersuchung des Lade- bzw. Entladevorgangs an einem Kondensator mit großer Kapazität (nach Steinberg und Wainwright 1993, S. 353)

Mit Hilfe eines handgetriebenen Generators („Genecon“) machen die Schüler ferner die Erfahrung, dass es anstrengend ist, die Rolle der Batterie in einem Stromkreis zu übernehmen und die Ladungsströmung anzutreiben, wobei in diesem Zusammenhang auch kurz auf den Unterschied zwischen zirkulärer Ladungsströmung und linearer Energieübertragung hingewiesen wird. Nachdem in der dritten Einheit geklärt wurde, dass die Ladungsströmung nicht aus der Batterie entspringt, ist das Ziel der vierten Einheit zu zeigen, dass es auch ohne Batterie zu einer Ladungsströmung kommen kann. Hierzu werden wieder Lade- und Entladevorgänge eines Kondensators in Kombination mit zwei Lämpchen analysiert, wobei erarbeitet wird, dass sich in den Kondensatorplatten in Folge des Ladungsüberschusses bzw. des Ladungsmangels ein „elektrischer Druck“ einstellt, der mit dem Luftdruck verglichen wird: „Air pressure is the name given to the effort to expand by compressed air. The name electric pressure is the same effort by compressed charge" (Steinberg 2004, S. 42). Anhand der Entladung eines Kondensators wird dann demonstriert, dass der elektrische Strom eine Folge von elektrischen Druckunterschieden ist und nicht zwangsläufig einer Batterie bedarf. Zur qualitativen Visualisierung des elektrischen Drucks in Stromkreisen wird ähnlich zu Herrmann (2003, S. 32) vorgeschlagen, Leiterabschnitte in Abhängigkeit ihres elektrischen Drucks farblich zu markieren. Primärer Unterrichtsgegenstand der fünften Einheit sind Reihenschaltungen und die Frage, auf welche Weise sich der elektrische Druck in den einzelnen Leiterabschnitten einer Reihenschaltung einstellt.

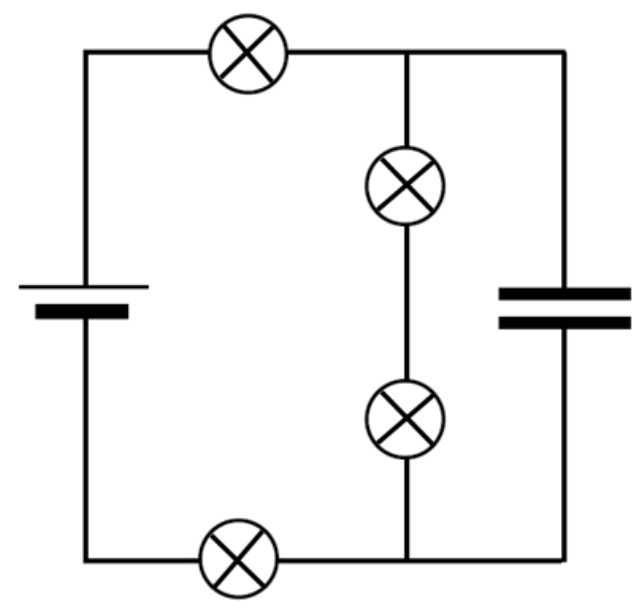

Abb. 34: In Reihe geschaltete Lämpchen mit einem parallelgeschalteten Kondensator (nach Steinberg 2004, S. 54) 
Wie in Abb. 34 dargestellt, wird hierzu ein Kondensator einer Reihenschaltung aus Lämpchen parallelgeschaltet, um zu zeigen, wie sich die elektrischen Drücke bei gleichen und ungleichen Widerständen einstellen und die allgemeine Regel formuliert, dass in einer Reihenschaltung an einem größeren Widerstand auch ein größerer elektrischer Druckunterschied anliegt (Steinberg 2004, S. 59). In der sechsten Einheit werden die Anschlussbedingungen für Volt- und Amperemeter besprochen und der elektrische Widerstand eingeführt, wobei der Sondercharakter des Ohm'sche Gesetzes betont wird. Ebenfalls Unterrichtsgegenstand sind in dieser Einheit das Konzept des Ersatzwiderstands und das Thema elektrische Energie und Leistung. Die verbleibenden vier Einheiten des CASTLE-Curriculums gehen über die elementare Elektrizitätslehre hinaus und behandeln weiterführende Themen wie Halbleiter und Elektromotoren, weshalb sie an dieser Stelle nicht näher vorgestellt werden.

\section{Kritische Betrachtung}

Das Unterrichtskonzept von Steinberg (2004) versucht den Schülern durch Fokussierung auf ein qualitatives Modelldenken ein tieferes Verständnis der kausalen Zusammenhänge in Stromkreisen zu ermöglichen, damit diese Stromkreise analysieren und die Folgen von Parameteränderungen qualitativ vorhersagen können. Hierfür ist es notwendig, dass die Schüler ein eigenständiges und weittragendes Spannungskonzept besitzen und verstehen, dass die Spannung die kausale Ursache des elektrischen Stroms ist. Um den Schülern ein solches Verständnis zu ermöglichen, führt Steinberg (2004) zunächst das Konzept des in Leitern herrschenden ,elektrischen Drucks“ ein, das an die Alltagserfahrungen der Schüler zum Luftdruck anknüpft und ihnen so eine intuitive Vorstellung des elektrischen Potenzials vermittelt. Ohne die Begriffe Potenzial, Potenzialdifferenz oder Spannung anfänglich explizit zu nutzen, wird die elektrische Spannung dann als ,elektrischer Druckunterschied“" und Antrieb des Ladungsträgerstroms eingeführt. Neu gegenüber bisherigen Vorschlägen, die Spannung als Potenzial- bzw. Ladungsunterschied einzuführen (vgl. Rhöneck und Völker 1982, S. 407, Herrmann und Schmälzle 1984, S. 478 und Kircher 1975, 75ff) ist die verwendete Luftdruckanalogie, die nach Steinberg und Wainwright (1993, S. 355) gegenüber der Wasserstromkreisanalogie den Vorteil bietet, dass die Kompressibilität des Mediums von den Schülern erfahren werden kann und den Antrieb des elektrischen Stroms im Stromkreis selbst verortet:

„Air is a more attractive analogy for charge flow than water for at least two reasons. Besides the palpable compressibility of air compared with water, there is the fact that a charged capacitor discharges spontaneously in a complete circuit. This provides evidence of an internal causal agent - a property of the compressed charge itself - whereas water must be driven externally by gravity in order to obtain perceptible flow."

Anders als beim geschlossenen Wasserkreislauf, dem mit Wasser ein häufig als inkompressibel wahrgenommenes Kontinuum zu Grunde liegt, ist den Schülern die Kompressibilität der Luft in der Regel aus ihrem Alltag hinreichend bekannt (vgl. Burde und Wilhelm 2016a). Unverständlicherweise wird im CASTLE-Curriculum aber auf eine Visualisierung 
unterschiedlicher Druckzustände mit Hilfe eines Teilchenbilds verzichtet, obwohl auf diese Weise der Teilchencharakter der Analogie verdeutlicht und der Zusammenhang zwischen Kompression und Druck für die Schüler sichtbarer gemacht werden könnte.

Nichtsdestotrotz finden sich in Bezug auf die Visualisierung von Potenzial und Stromstärke im CASTLE-Curriculum einige interessante Ideen. Fließt beispielsweise in einem Stromkreis mit Lämpchen ein Strom, so wird deren Leuchtstärke über unterschiedlich viele ikonische Lichtstrahlen rund um die Lämpchen visualisiert. Bei Batterien, Widerstandselementen und anderen elektrischen Bauteilen wird die Größe der Stromstärke über Pfeile dargestellt, wobei die Pfeile je nach Stromstärke unterschiedlich viele parallele Schäfte haben und so ein qualitativer Vergleich von Stromstärken ermöglicht werden soll (vgl. Steinberg 2004, S. 13).

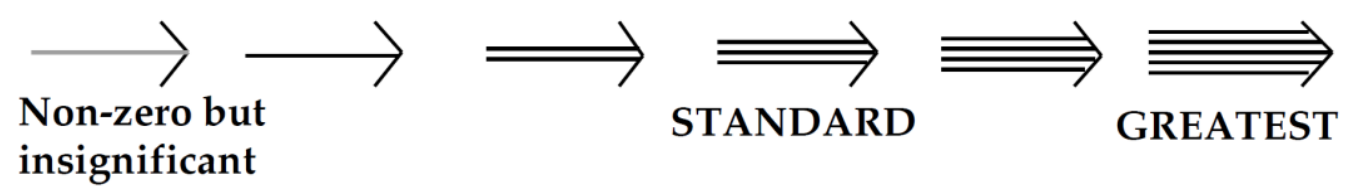

Abb. 35: Qualitative Darstellung der Stromstärke im CASTLE-Curriculum (Quelle: Steinberg 2004, S. 14)

Der in den unterschiedlichen Leiterabschnitten herrschende „elektrische Druck“ wird ähnlich wie bei Herrmann und Schmälzle (1984) über eine Farbkodierung dargestellt, wobei sich die dabei verwendete Systematik an den Farben des Farbkreises orientiert (siehe Tab. 4). Hier stellt sich die Frage, inwiefern Schüler mit der dem Farbkreis zugrundeliegenden Systematik vertraut sind und insbesondere die Wahl der Farbe Grün für einen geringen elektrischen Unterdruck (,,below normal“) intuitiv nachvollziehen können.

Tab. 4: Das Farbschema zur Kodierung des elektrischen Drucks im CASTLE-Curriculum (Steinberg 2004, S. 45)

\begin{tabular}{|l|l|}
\hline Color & Electric Pressure \\
\hline Orange & Highest Above Normal \\
\hline Yellow & Above Normal \\
\hline Green & Normal \\
\hline Blue & Below Normal \\
\hline
\end{tabular}

Wie Steinberg und Wainwright (1993, S. 356) berichten, hat sich der gewählte Ansatz in einer von Brown (1992) durchgeführten Vergleichsstudie mit über 600 Schülern in der Praxis als sehr erfolgreich erwiesen. Während der Lernzuwachs klassisch unterrichteter Schüler vernachlässigbar klein war, erzielten Schüler, die nach dem CASTLE-Curriculum unterrichtet wurden, bedeutende Lernzuwächse. Genauere Ergebnisse der Studie wurden leider nie veröffentlicht. Auch in einer von Clement und Steinberg (2002) durchgeführten Akzeptanzbefragung zeigte sich, dass das vermittelte Spannungskonzept auf Basis der Luftdruckanalogie von den Lernenden intuitiv verstanden wird und ihnen ein tieferes qualitatives Verständnis für die wechselseitige Beziehung der Größen Spannung, Stromstärke und Widerstand in einfa- 
chen Stromkreisen ermöglicht. Mit Hilfe ihrer dynamischen Modellvorstellung waren die Schüler beispielsweise in der Lage, bei Widerstandsänderungen in einer Reihenschaltung Schritt für Schritt zu erklären, wie sich die an den Widerständen anliegende Spannung bis zur Einstellung eines dynamischen Gleichgewichtszustandes ändern muss. Dies ist insbesondere deshalb bemerkenswert, weil viele Schüler bei traditionellen Unterrichtsansätzen keinen eigenständigen Spannungsbegriff entwickeln und häufig nicht ausreichend zwischen Spannung und Stromstärke differenzieren können (Rhöneck 1986). Bedenkt man, dass sich in der physikdidaktischen Forschung Potenzialansätze allgemein als vergleichsweise lernförderlich bzgl. eines besseren Verständnisses der Spannung und der Vorgänge in Stromkreisen allgemein erwiesen haben (vgl. Schumacher und Wiesner 1997; Gleixner 1998; Koller 2008b; Späth 2009), erscheint der gewählte Weg, das elektrische Potenzial über das intuitive Luftdruckverständnis verständlich zu machen, sehr vielversprechend.

Aus didaktischer Sicht ungeschickt ist hingegen der gewählte Einstieg in das Thema Stromkreise über die elektrische Stromstärke, da die Stromstärke ohnehin die Vorstellung vieler Schüler von elektrischen Stromkreisen dominiert und sie die Spannung oft lediglich als Eigenschaft und nicht Ursache des elektrischen Stroms begreifen (Rhöneck 1986; Cohen et al. 1983). Auch verzichtet das CASTLE-Curriculum im Gegensatz zu anderen auf Analogien aufbauenden Unterrichtskonzepten (vgl. insbesondere Schwedes et al. 1995 und Herrmann 2003) auf einen eigenen Vorkurs zu Luftdruckphänomenen, um beispielsweise gemeinsam zu erarbeiten, dass Luftströmungen immer eine Folge von Luftdruckunterschieden sind. Vor dem Hintergrund der hohen Bedeutung des Verständnisses des Analogbereichs für die Fähigkeit, überhaupt von der Analogie profitieren zu können (vgl. Schwedes et al. 1995), erscheint der frühzeitige Aufbau eines solchen Grundverständnisses aber durchaus angebracht. Stattdessen beschränkt sich Steinberg im CASTLE-Curriculum auf kurze experimentelle oder sprachliche Vergleiche mit Luftdruckphänomenen, um einzelne Aspekte wie den „elektrischen Druck“ besser veranschaulichen zu können. Die von Brown (1992) und Clement und Steinberg (2002) dokumentierte hohe Lernwirksamkeit des CASTLE-Curriculums deutet zwar darauf hin, dass ein solches Vorgehen - vermutlich aufgrund der hohen Vertrautheit der Schüler mit Luftdruckphänomenen - möglich ist, jedoch wird durch den Verzicht eines kurzen Vorkurses über die Bedeutung von Druckunterschieden für Strömungen und eine darauf folgende Thematisierung von Potenzial und Potenzialdifferenzen in Stromkreisen die Chance vertan, in der Vorstellung der Schüler die elektrische Spannung schon frühzeitig konzeptionell als Ursache für den elektrischen Strom zu etablieren.

Das gesamte Unterrichtskonzept von Steinberg ist sehr phänomenologisch orientiert. Um den Schülern beispielsweise plausibel zu machen, wie ein elektrischer Widerstand von seiner Querschnittsfläche beeinflusst wird, blasen Schüler Luft durch unterschiedlich dicke Strohhalme, um so am eigenen Körper zu erfahren, dass der Luftstrom umso stärker behindert wird, je dünner der Strohhalm ist. Anders als z.B. bei Muckenfuß und Walz (1992, S. 137) bekommen die Schüler im CASTLE-Curriculum allerdings keine weitergehende mikroskopische Modellvorstellung von Widerständen auf Basis von Atomrümpfen oder Elektronen als 
Vorstellungshilfe an die Hand. Damit bleibt für die Schüler aber völlig unklar, auf welche Weise die Elektronen in einem elektrischen Widerstand gehemmt werden und wie sie sich beispielsweise die Legierungs- und Temperaturabhängigkeit eines Widerstands vorstellen können. Einerseits kann dies auf die Schüler demotivierend wirken, da den meisten die Begriffe „Atom“ und „Elektron“ aus dem Alltag bereits bekannt sein dürften, andererseits besteht aber auch die Gefahr, dass die Schüler eigene unangemessene Modellvorstellungen von den Vorgängen in Stromkreisen entwickeln (Muckenfuß und Walz 1997, S. 131).

Methodisch verfolgt das CASTLE-Curriculum einen Ansatz, der als angeleitetes entdeckendes Lernen bezeichnet werden kann, da Schüler vorrangig mit Hilfe eines „Student Manuals" Arbeitsaufträge bearbeiten und selbstständig Experimente durchführen. Mit Hilfe der Konfrontationsstrategie werden auf diesem Weg eine Reihe von Schülervorstellungen systematisch abgearbeitet. Indem die Schüler so immer wieder mit Phänomenen konfrontiert werden, die sie mit Hilfe ihrer bisherigen Modellvorstellung nicht erklären können (,small discrepant events"), versucht Steinberg kognitive Konflikte gezielt zu nutzen, um Schüler einerseits zu motivieren, ihre bisherige Modellvorstellung weiterzuentwickeln und bei ihnen andererseits ein Bewusstsein für den vorläufigen, hypothetischen Charakter von Modellen zu fördern. Die gewählte Methode ist dabei einerseits dem wissenschaftlichen hypothesengeleiteten Vorgehen nachempfunden, bei dem sich eine Modellvorstellung erst durch das Wechselspiel von Modell, Hypothese und Experiment entwickelt, erinnert andererseits aber auch an zentrale Aspekte der konstruktivistischen Lehr- und Lerntheorie, wonach das Subjekt interne kognitive Strukturen u.a. durch den Prozess der Akkommodation an neue Erfahrungen anpasst und auf diese Weise neues Wissen konstruiert (vgl. Piaget 1967). In Hinblick auf das deutsche Schulsystem mit seinen Unterrichtstunden von 45 Minuten und den oftmals eher dürftigen und nicht auf Schülerexperimente mit Kondensatoren ausgelegten Sammlungen erscheint der im CASTLE-Curriculum gewählte methodische Ansatz hierzulande jedoch nur sehr eingeschränkt umsetzbar.

\subsection{Das Unterrichtskonzept von Muckenfuß und Walz}

Dem an der PH Weingarten entwickelten Unterrichtskonzept von Muckenfuß und Walz (1992) liegt das erklärte Ziel eines allgemeinbildenden Elektrizitätslehreunterrichts zugrunde, der nicht die Vermittlung von Handlungs- bzw. Verfügungswissen für zukünftige Physiker und Ingenieure zum Ziel hat (vgl. Muckenfuß 1982), sondern vielmehr lebenspraktische Zusammenhänge wie die Energieübertragung mit Hilfe von Elektrizitätsnetzen zur pädagogischen Leitidee erklärt. Um den besonders in der Sekundarstufe I auftretenden Motivationsproblemen entgegenzuwirken und den Unterricht für die Schüler relevanter zu gestalten, soll nach Muckenfuß und Walz (1992, S. 15) für die Lernenden daher stets erkennbar sein, dass „nicht physikalisches Expertenwissen die Richtschnur des Unterrichts bildet, sondern der Aufbau einer ,Alltagskompetenz für Laien “. Ziel des Unterrichtskonzepts ist es daher, den Kindern eine Vorstellung der elektrischen Größen zu vermitteln und sie zu befähigen, infor- 
miert und kritisch Stellung zu bedeutenden gesellschaftlichen Fragen wie dem Energieproblem zu beziehen.

Ausgehend von einer Betrachtung der zivilisatorischen Bedeutung der Elektrizität für unsere gesellschaftliche Entwicklung, wird im Unterrichtsgang nach Muckenfuß und Walz (1992) der Stromkreis als ein System zur Energieübertragung behandelt. Entsprechend wird noch vor der Thematisierung von Stromstärke und Spannung das Konzept des Energiestroms eingeführt, was dem oftmals energetisch geprägten Vorverständnis der Schüler zu Stromkreisen entgegenkommt. Mit Hilfe eines handgetriebenen Generators („DynaMot") bekommen die Schüler einen physiologischen Eindruck davon, was es heißt, den Energiestrom für unterschiedliche Geräte wie z.B. eine $30 \mathrm{~W}$ Lampe selber zu generieren.

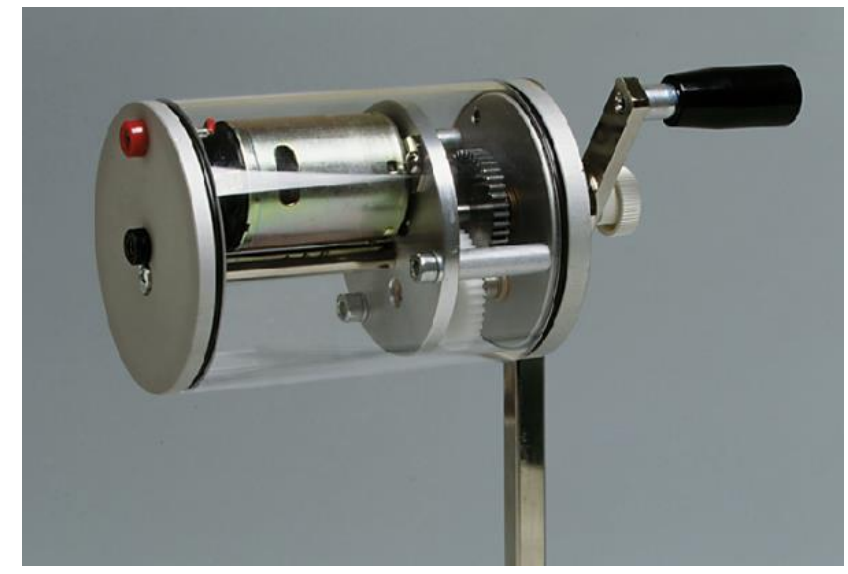

Abb. 36: Praktische Umsetzung des handgetriebenen Generators „,DynaMot“ (Quelle: winlab.de 2018)

In Analogie zur Energieübertragung mit mechanischen Transmissions- bzw. Keilriemen wird dann für Stromkreise die Hypothese eines im Kreis strömenden Elektronen- bzw. Ladungsstroms als elektrischer Riemen formuliert, der einen linearen Energiestrom erzeugt (vgl. Abb. 37). Ähnlich zum IPN-Curriculum von Härtel et al. (1981) wird auch hier ein konstanter elektrischer Strom als Folge eines Gleichgewichtszustands zwischen Antrieb (Spannungsquelle) und Hemmung (Widerstand, Verbraucher) gesehen.
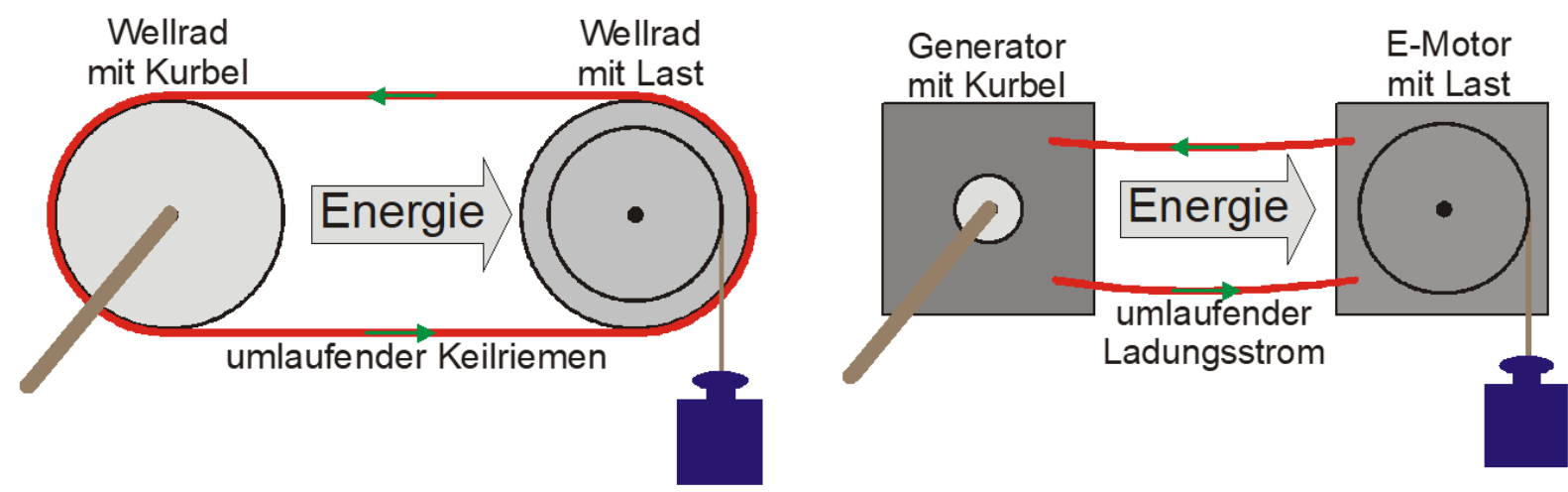

Abb. 37: Umlaufender Keilriemen, aber linearer Energiestrom (links) und umlaufender Ladungsstrom, aber linearer Energiestrom (rechts) (Quelle: T. Wilhelm, unveröffentlicht) 
Über einen kurzen Exkurs in die Elektrostatik wird die Elektrizitätslehre in eine einfache atomistische Modellvorstellung eingebettet und die Stromstärke (,Elektrizitätsstrom“) als $\mathrm{Maß}$ für die pro Zeiteinheit durch einen Leiterquerschnitt fließende Ladungsmenge eingeführt. Auf Basis des Wissens von Atomrümpfen und Elektronen wird den Schülern ferner eine erste mikroskopische Modellvorstellung von Widerständen als Vorstellungshilfe an die Hand gegeben, die veranschaulicht, auf welche Weise der Elektrizitätsstrom z.B. durch die Geometrie oder die Temperatur des Widerstands gehemmt wird (Muckenfuß und Walz 1997, S. 128).

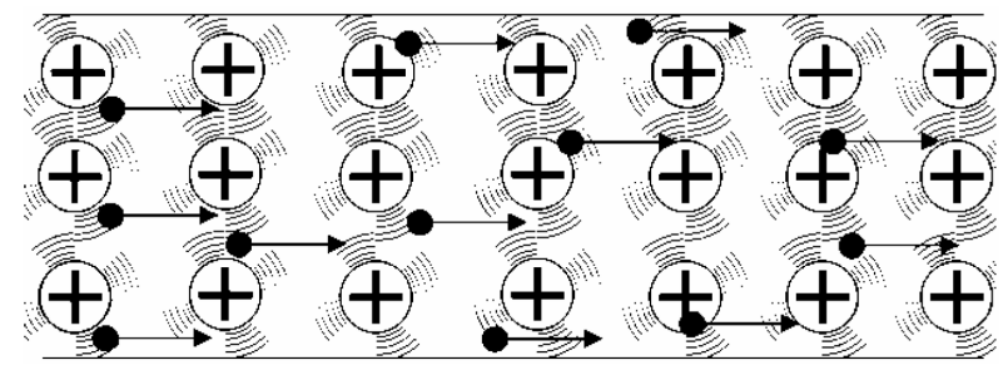

Abb. 38: Mikroskopische Modellvorstellung der Temperaturabhängigkeit des elektrischen Widerstands (Quelle: Muckenfuß und Walz 1997, S. 128)

Anhand der Beobachtung, dass bei gleicher Stromstärke ein unterschiedlich großer Energiestrom vorhanden sein kann, wird dann die Notwendigkeit einer weiteren Größe zur vollständigen Beschreibung des Energiestroms motiviert. Folglich wird die elektrische Spannung einerseits als ,[...] ein Maß für den von der Quelle zum Verbraucher fließenden Energiestrom, der durch einen zirkulierenden Ladungsträgerstrom von 1 Ampere erzeugt wird" (Muckenfuß und Walz 1997, S. 44) und andererseits ,[...] als Maß dafür, wie stark die einzelnen Elektronen angetrieben werden“" (Muckenfuß und Walz 1997, S. 68) eingeführt. Entsprechend dem Antriebs-Hemmungs-Konzept wird ein Voltmeter als ein Instrument vorgestellt, , [...] das mißt, wie sehr die Elektronen zwischen den Anschlußstellen des Spannungsmessers angetrieben werden" (Muckenfuß und Walz 1997, S. 122). Quantitativ wird die elektrische Spannung der Argumentation über den Energiestrom folgend über die Gleichung $U=P / I$ definiert.

\section{Kritische Betrachtung}

Die zentrale Idee des Unterrichtskonzepts, den elektrischen Stromkreis unter dem Aspekt der Energieübertragung zu behandeln, erinnert an den Einstieg in die Elektrizitätslehre bei Härtel et al. (1981). Im Gegensatz zum IPN-Curriculum, bei dem jedoch lediglich der Einstieg in die Elektrizitätslehre über die Energieübertragung motiviert wird, stellt der Energieübertragungsaspekt von Stromkreisen bei Muckenfuß und Walz (1992) aufgrund anderer didaktischer Ziele den zentralen Unterrichtsgegenstand dar. Bei der Auswahl und Reihenfolge der Inhalte orientieren sich Muckenfuß und Walz (1992) darüber hinaus weniger an der Fachsystematik als deren Bedeutung für den Alltag der Schüler. Insbesondere der Alltagsbezug zieht sich wie ein roter Faden durch die inhaltliche und methodische Konzeption des Unterrichtsgangs, was vor dem Hintergrund, dass Muckenfuß zum Inbegriff der Kontextorientierung wurde, wenig überrascht (vgl. Muckenfuß 1995). Entsprechend wird im Unterrichtskonzept eine vielschichtige 
Verankerung der Konzepte in das bisherige Erfahrungswissen angestrebt, um einerseits ein tiefergehendes Verständnis für die physikalischen Zusammenhänge zu erleichtern und andererseits das Interesse der Kinder an den Inhalten zu fördern. Das Unterrichtskonzept beschränkt sich aber nicht auf rein phänomenologische Erklärungen, sondern gibt den Schülern auch eine mikroskopische Modellvorstellung des elektrischen Stroms und von Widerständen über Atomrümpfe und Elektronen. Vor dem Hintergrund, dass die meisten Schüler in ihrem Alltag oder im Fach Chemie zumindest schon von Atomen und Elektronen gehört haben, erscheint es sinnvoll, an dieses Vorwissen anzuknüpfen. Der Grund hierfür ist, dass die Schüler auf diesem Weg eine Vorstellungshilfe davon bekommen, auf welche Weise die Elektronen in einem elektrischen Widerstand gehemmt werden, warum der Widerstandswert von der Länge und dem Querschnitt des Widerstands abhängt und wie sie sich beispielsweise die Legierungs- und Temperaturabhängigkeit eines Widerstands vorstellen können (Muckenfuß und Walz 1997, S. 128).

Ein bedeutsames Beispiel für die ,[...] ausgeprägte Handlungsorientierung als Maxime der methodischen Gestaltung“" (Muckenfuß und Walz 1992, S. 63) im Unterrichtskonzept stellt die Idee dar, mit Hilfe handgetriebener Generatoren das an sich abstrakte Konzept des Energiestroms für die Schüler physiologisch erfahrbar zu machen. Entscheidend ist dabei für Muckenfuß und Walz (1992, S. 27), dass der Weg der Begriffsbildung hier nicht über abstrakte Definitionen, sondern über konkrete Erfahrungen verläuft, da ein unreflektiertes Faktenund Regelwissen die Entwicklung von Schülervorstellungen provoziert. Heute kann der handgetriebene Generator, der auch als Elektromotor verwendbar ist, bei Lehrmittelfirmen unter dem Produktnamen „Dynamot“ ohne Probleme bezogen werden und stellt ein bewährtes Mittel dar, um den Schülern ein Gefühl für Energieströme bzw. Energieumsätze in Stromkreisen zu vermitteln.

Wie Gleixner (1998, S. 68) jedoch anmerkt, könnte dieses Vorgehen im vorliegenden Unterrichtskonzept aufgrund des verwendeten Antriebs-Hemmungs-Konzepts die Gefahr bergen, dass die Schüler nicht in ausreichendem Maße zwischen dem Spannungs- und Kraftbegriff differenzieren lernen. Auch kommt der Differenzcharakter der Spannung nicht zur Geltung, weshalb die Schüler die elektrische Spannung leicht als lokale Absolutgröße wahrnehmen könnten. Insbesondere die gegen Ende des Unterrichtskonzeptes vorgenommene quantitative Definition der Spannung über $U=P$ / I suggeriert den Schülern, die Spannung sei lediglich eine Eigenschaft des Stroms und könne daher nur in Kombination mit diesem auftreten (Stichwort „Stromspannung“). Wie Muckenfuß und Walz (1992, S. 52) selber anmerken, findet die vorgeschlagene Sinnbildung des Spannungsbegriffs daher spätestens bei der Analyse von Spannungen an Batteriepolen oder offenen Stromkreisen ihre Grenzen. Interessant ist ferner, dass Muckenfuß und Walz (1997, 191ff) für stark verzweigte Stromkreise selber die Einführung einer Potenzialvorstellung in Form eines Höhenmodells vorschlagen, da sich ihr Riemenmodell nicht gut zur Veranschaulichung solcher Schaltungen eignet. 


\subsection{Das Unterrichtskonzept von Schwedes und Dudeck}

Ausgangspunkt des 18 Doppelstunden umfassenden Unterrichtskonzepts, das an der Universität Bremen von Mitte der 1980er bis Mitte der 1990er Jahre für die Sekundarstufe I entwickelt und evaluiert wurde (vgl. Schwedes und Schilling 1983, 1984; Dudeck und Schwedes 1992, 1990; Schwedes und Dudeck 1993b; Schwedes et al. 1995), stellt das Modell des ebenen, geschlossenen Wasserkreislaufs dar, wobei die Auseinandersetzung mit dem Wasserstromkreis und seinen Gesetzmäßigkeiten alleine 12 Doppelstunden in Anspruch nimmt (Schwedes et al. 1995). Der elektrische Stromkreis ist nach diesem Ansatz also lediglich Unterrichtsgegenstand von sechs Doppelstunden. Der Umstand, dass sich ein Unterrichtskonzept für die Elektrizitätslehre vorwiegend mit Wasserstromkreisen auseinandersetzt, wird damit begründet, dass die Schüler in früheren Untersuchungen erhebliche Schwierigkeiten bzgl. des Verständnisses von Wasserstromkreisen gezeigt haben und zu geschlossenen Wasserkreisläufen vergleichbare Fehlvorstellungen besitzen wie zu elektrischen Stromkreisen (Schwedes und Schilling 1983). Ein erfolgreicher späterer Analogietransfer setzt daher zunächst eine intensive Auseinandersetzung mit dem Modell des geschlossenen Wasserkreislaufs voraus. Im vorliegenden Ansatz wurde hierzu eine schülerorientierte Unterrichtsstrategie gewählt, bei der alle Experimentieraufträge bzw. Forschungsfragen trotz des erhöhten Zeitaufwands von den Schülern selbstständig in Kleingruppen bearbeitet werden. Der anschließende Analogietransfer zum elektrischen Stromkreis stellt die Schüler vor keine großen Probleme, sofern es ihnen aufgrund des intensiven Vorkurses zu geschlossenen Wasserkreisen gelungen ist, ein angemessenes Verständnis für die dortigen Gesetzmäßigkeiten zu entwickeln (Schwedes et al. 1995, S. 28).

Anhand der Betrachtung eines einfachen Wasserstromkreises mit Doppelwassersäule wird zunächst herausgearbeitet, dass der Wasserdruckunterschied den Antrieb des Wasserstroms darstellt. Hier ist es entscheidend, zur Erzeugung des Druckunterschieds statt einer normalen Tauchpumpe eine Doppelwassersäule zu verwenden, um den Schülern über die unterschiedliche Höhe der Wasserpegel in den beiden Säulen vor Augen führen zu können, dass die Wasserströmung von dem durch die unterschiedlichen Wasserhöhen verursachten Druckunterschied angetrieben wird (Schwedes et al. 1995, S. 35; Schwedes und Dudeck 1993b).

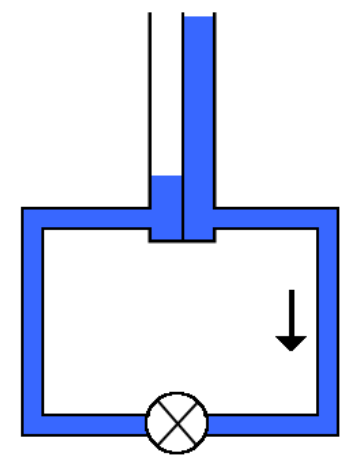


Anschließend wird die Erkenntnis formuliert, dass die Wasserströmung umso größer ist, desto größer der Wasserhöhen- bzw. Wasserdruckunterschied ist und der Wasserwiderstand als Behinderung der Wasserströmung eingeführt. In den beiden folgenden Doppelstunden wird die Wasserstromstärke als Wasservolumen pro Zeit definiert und der Zusammenhang zwischen Wasserstromstärke, Schlauchquerschnitt und Wassergeschwindigkeit erarbeitet. Von der fünften bis zur achten Doppelstunde wird dann näher auf die Gesetzmäßigkeiten bei Reihen- und Parallelschaltungen in Wasserstromkreisen eingegangen. Hier wird bei Reihenschaltungen u.a. der Gesamtwiderstand thematisiert, während bei Parallelschaltungen der Fokus u.a. auf der Unterscheidung zwischen Teil- und Gesamtstromstärke liegt. Im weiteren Verlauf soll der Druckbegriff dann auf eine solide Basis gestellt werden, weshalb er anhand von einfachen mechanischen Beispielen als Kraft pro Fläche eingeführt wird. Darüber hinaus wird der Unterschied zwischen den beiden Größen Druck und Druckunterschied besprochen und in der 10. Doppelstunde mit Hilfe von Druckmessern die Druckdifferenzverteilung an einem Widerstandsschlauch analysiert, um u.a. auch auf den Systemcharakter von Wasserstromkreisen näher eingehen zu können. Am Beispiel von ungleichen Widerständen bei Parallel- und Reihenschaltungen wird dann der Wasserwiderstand definiert als $R_{w}=\Delta p / I_{w}$. Abgeschlossen wird der Abschnitt zu Wasserstromkreisen mit der Analyse von Reihen- und Parallelschaltungen der Doppelwassersäule sowie einer Reihe von Übungsaufgaben.

$\mathrm{Zu}$ Beginn des zweiten Abschnitts werden die unterschiedlichen Elemente von Wasserkreisen ihrem entsprechenden Analogon bei Stromkreisen zugeordnet (z.B. Wasserkreisel $\rightarrow$ Lämpchen; Schlauch $\rightarrow$ Draht; Doppelwassersäule $\rightarrow$ Batterie) und der elektrische Strom als Ladungsstrom eingeführt, der das Ergebnis des Ausgleichsbestrebens von durch die Batterie getrennter positiver und negativer Ladungen darstellt. Hieran schließt sich eine Phase des freien Experimentierens an, bei dem die Schüler Stromkreise ihrer Wahl untersuchen und mit ihren Kenntnissen über Wasserstromkreise vergleichen sollen. Im Anschluss findet dann die Begriffsbildung zu den elektrischen Größen Stromstärke, Widerstand und Spannung statt und es werden die Regeln des elektrischen Stromkreises besprochen. Die letzte Doppelstunde der Unterrichtsreihe befasst sich u.a. mit der Frage der Bestimmung von Widerständen, Potentiometern, Kondensatoren sowie Ohm'schen und Nicht-Ohm'schen Widerständen (Schwedes et al. 1995, S. 29).

\section{Kritische Betrachtung}

Wesentlicher Bestandteil des Unterrichtskonzepts nach Schwedes et al. (1995) ist das Modell des ebenen geschlossenen Wasserstromkreises. Aus fachlicher Sicht erscheint der geschlossene Wasserstromkreis aufgrund der hohen formalen Entsprechungen zwischen den beiden Systemen als prädestiniertes Modell für den elektrischen Stromkreis. Nahegelegt wird diese Annahme auch durch die große sprachliche Ähnlichkeit zur Beschreibung beider Systeme (,Von der Quelle aus fließt ein Strom...“) (vgl. Muckenfuß und Walz 1997, S. 96). Insbesondere mit Blick auf den Physikunterricht wurde lange Zeit argumentiert, der Wasserstromkreis sei nicht nur besonders anschaulich, sondern böte auch viele Anknüpfungspunkte an die Erfahrungen 
der Schüler. Aus didaktischer Sicht haben sich diese Hoffnungen aber nicht erfüllt, da die Schüler im Alltag keine Erfahrungen mit ebenen, geschlossenen Wasserkreisläufen machen und sogar oftmals schon eine Reihe von Schülervorstellungen zu diesen besitzen (Schwedes und Schilling 1983). Insbesondere hat sich gezeigt, dass Schüler keine adäquate Kontinuitätsvorstellung von Flüssigkeitsströmungen besitzen und darüber hinaus große Schwierigkeiten mit den in Wasserkreisläufen herrschenden Druckverhältnissen haben (Schwedes und Schilling 1983; Schwedes et al. 1995). Die Gefahr ist dann, dass die Schüler ihre bereits existierenden Schülervorstellungen zu ebenen, geschlossenen Wasserkreisläufen auf den elektrischen Stromkreis übertragen. Für eine ausführliche Auseinandersetzung mit den grundsätzlichen Schwächen des Modells des geschlossenen Wasserkreislaufs sei an dieser Stelle auf Abschnitt 4.3.1 sowie Burde und Wilhelm (2016a) verwiesen.

In Hinblick auf die Konzeption ihres Unterrichtskonzepts sind sich Schwedes et al. (1995, S. 28) der Probleme des Wasserkreislaufmodells durchaus bewusst und warnen explizit davor, für die Analogiebildung relevante Kenntnisse über den Wasserstromkreis bei den Schülern vorauszusetzen oder zu glauben, diese ließen sich durch eine kurze Demonstration eines Wasserkreislaufs im Unterricht vermitteln. Um sicherzustellen, dass die Schüler dennoch ein angemessenes Verständnis geschlossener Wasserkreisläufe entwickeln, verwenden sie deshalb etwa zwei Drittel der gesamten Unterrichtszeit auf die Auseinandersetzung mit dem geschlossenen Wasserkreislauf und seinen physikalischen Gesetzmäßigkeiten. Wie sie selber berichten, stellt insbesondere die Entwicklung eines Verständnisses für die Druckverteilung in Wasserstromkreisen keine leichte Aufgabe dar (Schwedes et al. 1995, S. 29). Ein Grund hierfür liegt vermutlich darin, dass Wasser von vielen Schülern als Kontinuum und als inkompressibel angesehen wird. Damit ist der Wasserdruck für sie anschaulich nicht vorstellbar, da sich dann Wasser unter hohem Druck in nichts Sichtbarem von Wasser unter geringem Druck unterscheidet. Auch die Unterscheidung zwischen der Absolutgröße „Druck“ und der Relativgröße „Druckunterschied“" sowie das Erkennen des Druckunterschieds als Ursache für die Strömung bereitet den Schülern große Schwierigkeiten. Dies ist in Übereinstimmung mit Erkenntnissen der Schülervorstellungsforschung, die gezeigt hat, dass Schüler häufig aus Sicht der vorkommenden Bewegungen argumentieren statt die Bewegung über die aus physikalischer Sicht entscheidenden Druckunterschiede zu erklären (Séré 2004; Dudeck und Schwedes 1992). Zur besseren Veranschaulichung der Rolle des Druckunterschieds als Antrieb der Wasserströmung setzen Schwedes et al. (1995, S. 35) deshalb auf eine Doppelwassersäule, was den Lernerfolg gegenüber der Verwendung einer regulären Tauchpumpe deutlich verbessern und der Konstantstromquellenvorstellung entgegenwirken konnte. Offensichtlich begünstigt eine gute Visualisierung der Druckverhältnisse, im vorliegenden Fall mit Hilfe der Höhenunterschiede der beiden Wassersäulen, die Entwicklung eines Verständnisses für Druckdifferenzen als Strömungsantrieb (vgl. Gleixner 1998, S. 70). Der hohe Anteil von Schülerexperimenten im Unterrichtskonzept ist einerseits positiv, andererseits stellt sich aber auch die Frage, inwiefern in der Unterrichtspraxis davon ausgegangen werden kann, dass für jeden Schüler ausreichend solcher Geräte zum selbstständigen Experimentieren zur Verfügung stehen. 
Zusammenfassend lässt sich sagen, dass das Dilemma dieses Unterrichtskonzepts primär darin besteht, dass die Schüler ohne ein physikalisch korrektes Vorwissen über geschlossene Wasserstromkreise in den Unterricht kommen und die Erarbeitung seiner Gesetzmäßigkeiten deshalb unter erheblichem zeitlichem Aufwand zum zentralen Unterrichtsgegenstand erhoben werden muss. Setzt die Lernwirksamkeit des Modells aber einen zwölf Doppelstunden umfassenden Vorkurs zu Wasserstromkreisen voraus, stellt sich die Frage, inwiefern dieses Vorgehen noch lernökonomisch bzw. im realen Unterricht unter Lehrplanaspekten praktikabel ist und inwiefern das Erlernen des Wasserstromkreises für die Kinder nicht schwieriger ist als das direkte Erlernen der elektrischen Stromkreise selbst (Kircher 1984, S. 55).

\subsection{Das Münchener Stäbchenmodell von Gleixner}

Das von Gleixner (1998) an der LMU München entwickelte Stäbchenmodell verfolgt auch einen Potenzialansatz, setzt im Gegensatz zum KPK aber nicht auf eine Druck- sondern eine einfache Höhenanalogie. Beim Stäbchenmodell bekommt jedes Bauteil des elektrischen Stromkreises zwei Stäbchen zugewiesen, deren Höhe das elektrische Potenzial vor und nach dem Bauteil veranschaulicht. Die elektrische Spannung kann dementsprechend als Höhenunterschied zwischen den Stäbchen interpretiert werden, was sich als verhältnismäßig lernförderlich erwiesen hat (Gleixner 1998, S. 221). Anders als bei Wasserstromkreisen sollen so die Grundlagen der einfachen Elektrizitätslehre vermittelt werden können, ohne einen Umweg über einen Vorkurs nehmen zu müssen.

Der von Gleixner (1998, S. 227) vorgeschlagene Unterrichtsgang gliedert sich in die beiden übergeordneten Unterrichtsblöcke „Elektrischer Strom, Stromstärke, Widerstand“ und „Elektrisches Potenzial und elektrische Spannung“. Ausgehend von einer kurzen Einführung in die Modell- und Theoriebildung in der Physik, lernen die Schüler zwischen Elektrogeräten und Generatoren (= Spannungsquellen) zu unterscheiden und erfahren, dass man einen Stromfluss immer an seiner Wirkung erkennen kann (z.B. Wärme- und Leuchtwirkung sowie magnetische Wirkung). Mit Hilfe von handgetriebenen Generatoren (vgl. Muckenfuß und Walz 1992) können die Schüler dann am eigenen Körper die Erfahrung machen, dass Arbeit verrichtet werden muss, um ein Lämpchen zum Leuchten zu bringen. Über die Abhängigkeit der Stromrichtung von der Polung der Batterie wird dann demonstriert, dass ein Unterschied zwischen den beiden Polen einer Batterie existieren muss. Die Größe der Auslenkung einer Kompassnadel aufgrund der magnetischen Wirkung des elektrischen Stroms wird im Folgenden zur qualitativen Begriffsbildung der elektrischen Stromstärke sowie des elektrischen Widerstands genutzt und der Aufbau eines Amperemeters diskutiert und anschließend in Reihenund Parallelschaltungen erprobt.

Ausgehend von der Erfahrung, dass der Strom je nach Polung der Batterie in eine andere Richtung fließt, wird im zweiten Unterrichtblock dann der Potenzialbegriff über die Argumentation eingeführt, dass die Pole einer Batterie auf einem hohen bzw. niedrigen Potenzial lägen. Es wird demonstriert, dass unterschiedliche Generatoren in einem Stromkreis zu unterschiedlichen Stromstärken führen und erklärt, dass der „elektrische Unterschied“ zwi- 
schen dem hohen bzw. niedrigen Potenzial der elektrischen Spannung entspricht. Zur Veranschaulichung der elektrischen Potenziale bzw. der elektrischen Spannung wird dann das Stäbchenmodell eingeführt, bei dem die Potentiale über die Höhe der Stäbchen und die Spannung entsprechend als Höhenunterschied der Stäbchen modelliert werden. Dabei wird dem Minuspol die Farbe Blau sowie eine niedrige Stäbchenhöhe und dem Pluspol die Farbe Rot sowie eine hohe Stäbchenhöhe zugeordnet.
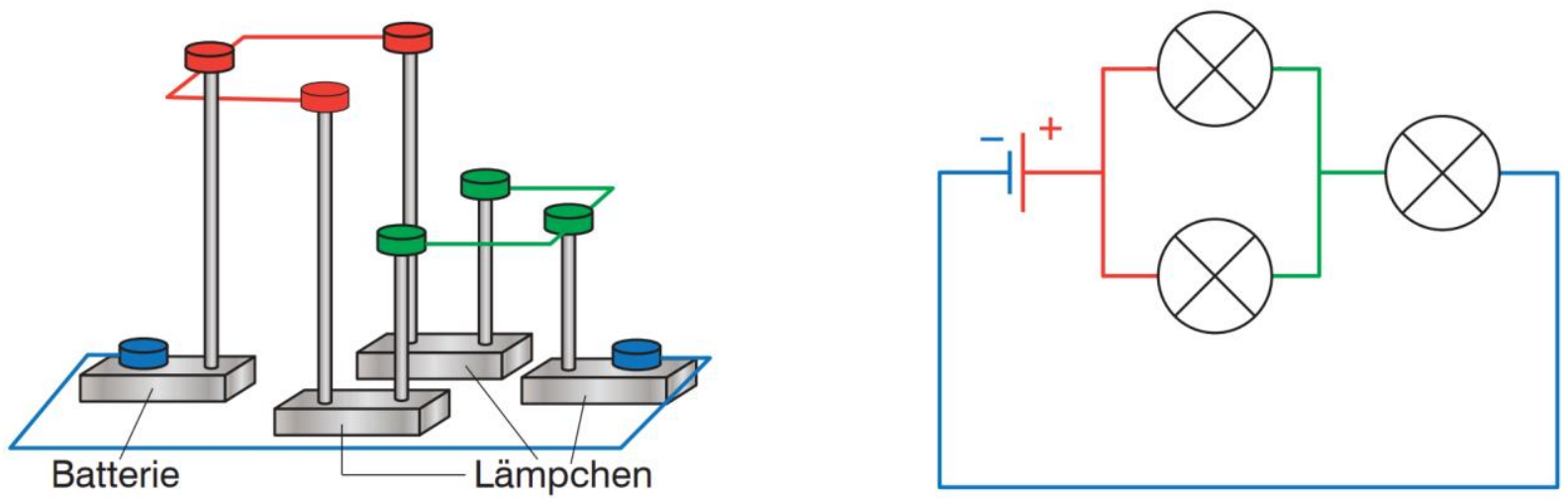

Abb. 40: Das Stäbchenmodell von Gleixner (nach Burde und Wilhelm 2017c, S. 11; vgl. Koller 2008a, S. 122)

Am Beispiel des Hintereinander- bzw. Gegeneinanderschaltens von Batterien findet ferner eine qualitative Auseinandersetzung mit dem Stäbchenmodell statt, bevor das Modell im Anschluss mit Hilfe quantitativer Messungen der Spannung überprüft wird. Im weiteren Verlauf werden die Potenzialunterschiede bei Reihenschaltungen untersucht und eine simplifizierte Form der Maschenregel formuliert. Indem in einer Reihenschaltung von zwei Elektrogeräten eines gegen ein Elektrogerät mit größerem Widerstand ausgetauscht wird, lernen die Schüler, dass an einem größeren Widerstand auch ein größerer Potenzialunterschied anliegt. Gegen Ende der Unterrichtsreihe wird dann auf Potentialunterschiede bei Parallel- und gemischten Schaltungen eingegangen und der Zusammenhang zwischen Stromstärke, Spannung und Widerstand auch quantitativ behandelt.

\section{Kritische Betrachtung}

Positiv ist das mit dem Unterrichtskonzept verbundene Ziel zu beurteilen, bei den Schülern ein fundiertes qualitatives Verständnis von elektrischen Stromkreisen aufzubauen, das ihnen in Kombination mit der vermittelten Modellvorstellung erlaubt, begründete qualitative Vorhersagen zu Spannung und Stromstärke in einem Stromkreis machen zu können. Gleixner (1998, S. 78) begründet die Fokussierung auf ein qualitatives Verständnis damit, dass in der Elektrizitätslehre häufig

„[...] zwar die mathematische Beschreibung eines physikalischen Zusammenhangs den Schülerinnen und Schülern bekannt ist, daß aber die Anwendung dieses Wissens auf ein konkretes Problem nicht erfolgt. [...] [da es] an Umgang mit ,qualitativer Physik' mangelt, d.h. am gedanklichen oder realen Durchspielen physikalischer Situationen [...]". 
Um Fehlvorstellungen zu bestimmten Begriffen in Folge unterschiedlicher Bedeutung in Fach- und Alltagssprache zu vermeiden, verfolgt Gleixner (1998, S. 83) einen interessanten sprachlichen Ansatz. Statt die regulären Fachbegriffe zu nutzen, ersetzt er zumindest anfänglich didaktisch problematische Fachausdrücke durch semantisch weniger missverständliche Begriffe wie beispielsweise Verbraucher durch Elektrogerät, Strom- bzw. Spannungsquelle durch Generator, Stromstärke durch Intensität des Stroms (wie das Formelzeichen I) und Spannung durch den elektrischen Unterschied (wie das Formelzeichen $U$ ). Ferner greift Gleixner an verschiedenen Stellen auf bereits bewährte Ansätze zurück. So nutzt er beispielsweise zur qualitativen Heranführung an die Stromstärke- und Stromrichtungsmessung den von Wiesner et al. (1982) entwickelten Magnetnadelansatz, der auf der magnetischen Stromwirkung beruht. Der Vorteil dieses Ansatzes liegt darin, dass der Stromkreis selber zur Messung baulich nicht verändert werden muss und der Messvorgang dadurch für Schüler besser nachvollziehbar wird. Auch lässt sich die örtliche Invarianz des elektrischen Stroms in einem unverzweigten Stromkreis ganz leicht durch „Abfahren“ desselben mit einer Kompassnadel gut sichtbar demonstrieren. Um die Schüler am eigenen Körper ein Gefühl dafür entwickeln zu lassen, dass Arbeit verrichtet werden muss, um ein Lämpchen leuchten zu lassen, greift Gleixner auf die bewährte Idee des handgetriebenen Generators von Muckenfuß und Walz (1992) zurück.

Vor dem Hintergrund, dass viele Schüler die elektrische Spannung lediglich als eine Eigenschaft des elektrischen Stroms auffassen, aber Gleixner mit seinem Unterrichtskonzept insbesondere ein unabhängiges Spannungskonzept fördern möchte, stellt sich die Frage, warum die Spannung nicht von Beginn an konsequent als Antrieb des elektrischen Stroms behandelt wird. Stattdessen wird in der ersten Hälfte des Konzepts zunächst ausführlich auf die Themen elektrischer Strom und Widerstand eingegangen, was die Schüler möglicherweise in der weit verbreiteten Fehlvorstellung bestärkt, Stromkreise aus Sicht des Stroms zu analysieren, ohne sich dabei der wichtigen Rolle der elektrischen Spannung bewusst zu sein. Problematisch ist ferner, dass das Stäbchenmodell über die Höhe der Stäbchen lediglich das Potenzial bzw. die elektrische Spannung visualisieren kann, den Schülern bzgl. Stromstärke und Widerstand aber keinerlei Modellvorstellung an die Hand gibt. Insbesondere in Hinblick auf den elektrischen Widerstand wäre die Vermittlung einer mikroskopischen Modellvorstellung aber wünschenswert, um den Schülern eine Vorstellungshilfe davon zu geben, auf welche Weise der elektrische Strom z.B. durch die Geometrie oder die Temperatur eines Widerstands gehemmt wird (Muckenfuß und Walz 1992, S. 137). Auch hinsichtlich des elektrischen Potenzials liefert das Modell keine tiefergehenden kausalen Erklärungen und bleibt sehr abstrakt. Zum Beispiel bleibt die Frage völlig offen, was man sich unter „elektrisch hoch“ bzw. „elektrisch tief“ vorstellen kann und wie diese Zustände erreicht werden, was für Schüler sehr unbefriedigend sein kann, wie Gleixner (1998, S. 220) selber anmerkt: 
Kapitel 5: Analyse bedeutender Unterrichtskonzepte

„[Es zeigt sich], daß eine relativ abstrakte Vorstellung wie ,elektrisch hoch' oder ,elektrisch tief" den Schülerinnen und Schülern zunächst sehr suspekt erscheint. Es bestand an dieser Stelle ein auffälliges Bedürfnis nach ,tiefgreifenden 'Erklärungen “

Insbesondere bei jüngeren Schülern ist abhängig von deren kognitiver Entwicklung zu befürchten, dass sie mit dem für das Modell benötigten Abstraktionsvermögen überfordert sind und keinen Zusammenhang zwischen Analogmodell und elektrischem Stromkreis sehen (vgl. Kircher 1984, S. 58).

Trotz der angesprochenen kritischen Punkte des Konzepts, berichtet Gleixner davon, mit seinem auf dem Potenzialansatz aufbauenden Unterrichtskonzept bei den Schülern eine Trennung der Konzepte Stromstärke und Spannung erreicht und ein Verständnis des Spannungsbegriffs erzielt zu haben. Insbesondere konnte bei den Schülern durch die Fokussierung auf ein qualitatives Verständnis der Vorgänge in Stromkreisen ,[...] ein Bedürfnis nach einer Konkretisierung der Zusammenhänge zwischen den einzelnen physikalischen Größen [...]““ (Gleixner 1998, S. 222) geweckt werden. Ähnliche Erfahrungen von Clement und Steinberg (2002) deuten darauf hin, dass ein auf dem Potenzialansatz aufbauendes und auf ein qualitatives Verständnis abzielendes Unterrichtskonzept erfolgsversprechend ist.

\subsection{Das Unterrichtskonzept von Koller und Späth}

Das an der LMU München ursprünglich von Koller (2008b) entwickelte und von Späth (2009) überarbeitete und evaluierte Unterrichtskonzept für die 7. Jahrgangsstufe stellt ein weiteres Beispiel für einen Unterrichtsgang auf Grundlage des Potenzialansatzes dar. Gegenüber der ersten Version von Koller (2008b) hat Späth (2009) den Anteil textlastiger Aufgaben verringert und den Anteil qualitativer Aufgabenstellungen erhöht. Gleichzeitig wurde in der überarbeiteten Fassung durch das Streichen einer Reihe nicht grundlegender bzw. lehrplanrelevanter Themen (z.B. Diskussion der Funktionsweise eines Drehspulinstruments sowie das Hintereinander- und Gegeneinanderschalten von Batterien) der Umfang der Unterrichtsreihe von 17 auf 14 Unterrichtsstunden reduziert (Späth 2009, S. 26). Das Unterrichtskonzept besteht aus acht Einheiten, wobei das elektrische Potenzial und die elektrische Spannung erst gegen Ende in der sechsten und siebten Einheit thematisiert werden.

$\mathrm{Zu}$ Beginn der Unterrichtsreihe werden zunächst die Anschlussbedingungen von Elektrogeräten (Verbrauchern) und Generatoren (Spannungsquellen) in einem Stromkreis erarbeitet und neben der Leucht- und Wärmewirkung auch die magnetische Wirkung des elektrischen Stroms demonstriert. Mit Hilfe bewährter Ansätze wie dem handgetriebenen Generator und der Fahrradkettenanalogie (Muckenfuß und Walz 1992) sowie dem Wiesner'schen Stromkreis (Wiesner et al. 1982) wird der Stromverbrauchs- bzw. Zweizuführungsvorstellung entgegengewirkt und der Systemcharakter des Stromkreises betont. Wie bei Gleixner (1998) wird auf eine mikroskopische Veranschaulichung des elektrischen Stroms verzichtet und stattdessen allgemein von ,fließender Elektrizität“ gesprochen, die sich vom 
Plus- zum Minuspol bewegt. Über die Änderung der Helligkeit eines Lämpchens je nach Stromstärke wird eine Quantifizierung der ,fließenden Elektrizität“ motiviert und der Messvorgang am Beispiel von Drehspulinstrumenten vertieft. Abgeschlossen wird das Thema Stromstärke mit der Diskussion von Reihen- und Parallelschaltungen und hier insbesondere der Regel „I= konst.“ für Reihenschaltungen und der Knotenregel für verzweigte Schaltungen.
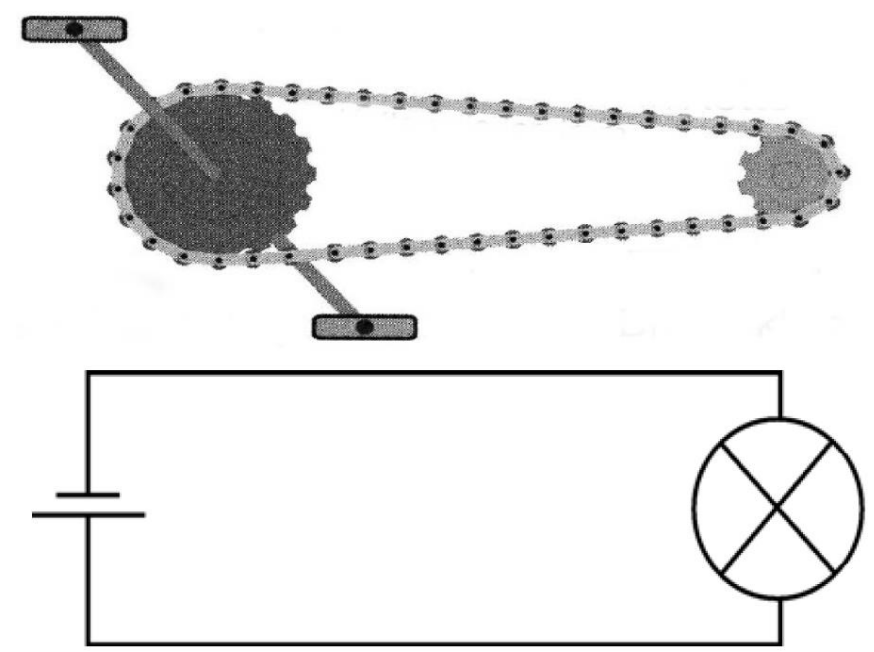

Abb. 41: Fahrradkettenmodell eines einfachen Stromkreises (Quelle: Koller 2008a, S. 34)

Das elektrische Potenzial wird zunächst in Analogie zum Wasserdruck eingeführt, indem die Analogie zwischen dem elektrischen Stromkreis und dem geschlossenen Wasserkreislauf aufgezeigt wird. Anschließend wird das Wasserkreislaufmodell mit dem Stäbchenmodell von Gleixner (1998) verglichen, der Potenzial- bzw. Druckunterschied als Voraussetzung für den elektrischen Strom herausgearbeitet und die elektrische Spannung als Potenzialdifferenz eingeführt. Im weiteren Verlauf des Unterrichtskonzepts verwenden Koller (2008b) und Späth (2009) ferner die von Herrmann und Schmälzle (1984) vorgeschlagene Farbkodierung, um Potenziale in Schaltskizzen und im Stäbchenmodell zu visualisieren, wobei die Schüler in jedem Stromkreis die einem Potenzial zugeordnete Farbe willkürlich festlegen können. Ebenfalls in Anlehnung an Herrmann und Schmälzle (1984) werden fünf Regeln formuliert, welche die Kernaussagen zu elektrischen Potenzialen in Stromkreisen zusammenfassen und den Schülern helfen sollen, sich leichter die Strom- und Spannungsverhältnisse in neuen Stromkreisen zu erschließen (Späth 2009, S. 275). Den Abschluss der Unterrichtsreihe stellt eine zusammenfassende Übungseinheit zu Spannung, Stromstärke und Widerstand in einfachen Stromkreisen dar.

\section{Kritische Betrachtung}

Das von Koller (2008b) und Späth (2009) entwickelte Unterrichtskonzept versucht bisherige in der physikdidaktischen Forschung bewährte Ansätze u.a. von Wiesner et al. (1982), Herrmann und Schmälzle (1984), Muckenfuß und Walz (1992), Schwedes et al. (1995) sowie Gleixner (1998) aufzugreifen. Positiv hervorzuheben ist bei dem Unterrichtskonzept insbesondere 
- die Verwendung möglichst unmissverständlicher Begriffe, die keine Schülervorstellungen provozieren (z.B. „Elektrogerät“ statt „Verbraucher“, ,Intensität der Elektrizität"statt „Stromstärke"),

- der Rückgriff auf die Fahrradkettenanalogie und den Wiesner'schen Stromkreis mit Kompassnadeln zur Entkräftung der Stromverbrauchsvorstellung,

- die Nutzung des handgetriebenen Generators, um die Energieübertragung mit Stromkreisen für die Schüler physiologisch erfahrbar zu machen,

- das Anknüpfen an die Erfolge bisheriger Potenzialansätze, wie z.B. das Stäbchenmodell und die farbliche Kodierung von Potenzialen,

- das Formulieren von klaren Regeln zur Erarbeitung der Strom- und Spannungsverhältnisse in Stromkreisen, um lernschwächere Schüler zu unterstützen und

- das Anbieten einer Vielzahl unterrichtsnaher Arbeits- und Merkblätter inklusive einer zusammenfassenden Einheit mit vielen Übungsaufgaben am Ende der Unterrichtsreihe (vgl. Bedeutung von Vertiefungsphasen bei Gleixner 1998, S. 233).

Aus didaktischer Sicht zu kritisieren ist auch bei diesem Konzept, dass die ersten fünf Unterrichtseinheiten von der Auseinandersetzung mit dem elektrischen Strom dominiert werden und die Potenzialdifferenz als Ursache des elektrischen Stroms erst ganz am Ende des Unterrichtskonzepts behandelt wird. Ein solches Vorgehen birgt die Gefahr, Schüler in ihrer Überzeugung zu stärken, die Stromstärke sei die zentrale Größe in Stromkreisen und die Spannung lediglich eine Eigenschaft derselben, was sie häufig dazu veranlasst, elektrische Stromkreise aus Sicht des elektrischen Stroms zu analysieren und dabei die zentrale Rolle der elektrischen Spannung als Ursache des elektrischen Stroms zu verkennen (vgl. Cohen et al. 1983 und Rhöneck 1986). Die farbliche Kodierung von Leiterabschnitten gleichen Potenzials erfolgt im vorliegenden Konzept im Gegensatz zum CASTLE-Curriculum von Steinberg (2004) nicht regelgeleitet. Auch wenn die willkürliche Farbwahl in Anbetracht der beliebigen Festlegung des Potenzialnullpunktes fachlich gesehen nicht zu beanstanden ist, korrespondiert sie didaktisch nicht mit der zuvor etablierten Systematik der Höhenanalogie. Ein konsequenterer und für die Schüler nachvollziehbarer Ansatz bestünde darin, die Systematik der Höhenanalogie aufzugreifen und den Zuständen „elektrisch hoch“ bzw. „elektrisch tief“ bestimmte Farben zuzuordnen, die z.B. in Abhängigkeit des Potenzialwertes in ihrer Intensität variiert werden könnten. In einer Treatment-Kontrollgruppen-Studie mit 16 Klassen hat sich das Konzept mit seinem hohen Grad an Strukturiertheit in der Unterrichtspraxis im Vergleich zum traditionellen Unterricht dennoch bewährt und führte zu einem signifikant höheren Lernerfolg (Späth 2009, S. 76). 


\subsection{Erkenntnisse aus den Unterrichtskonzepten}

Wie in diesem Abschnitt dargelegt, enthalten die diskutierten Unterrichtskonzepte eine Reihe fruchtbarer Ideen und teils bewährter Ansätze, die es sich in Hinblick auf die Entwicklung eines Unterrichtskonzepts auf Basis des Elektronengasmodells lohnt aufzugreifen und weiterzuentwickeln. Im Folgenden sollen für jedes der vorgestellten Unterrichtskonzepte die potenziell gewinnbringenden Aspekte daher knapp zusammengefasst werden:

Tab. 5: Übersicht über die gewinnbringenden Aspekte der verschiedenen Unterrichtskonzepte

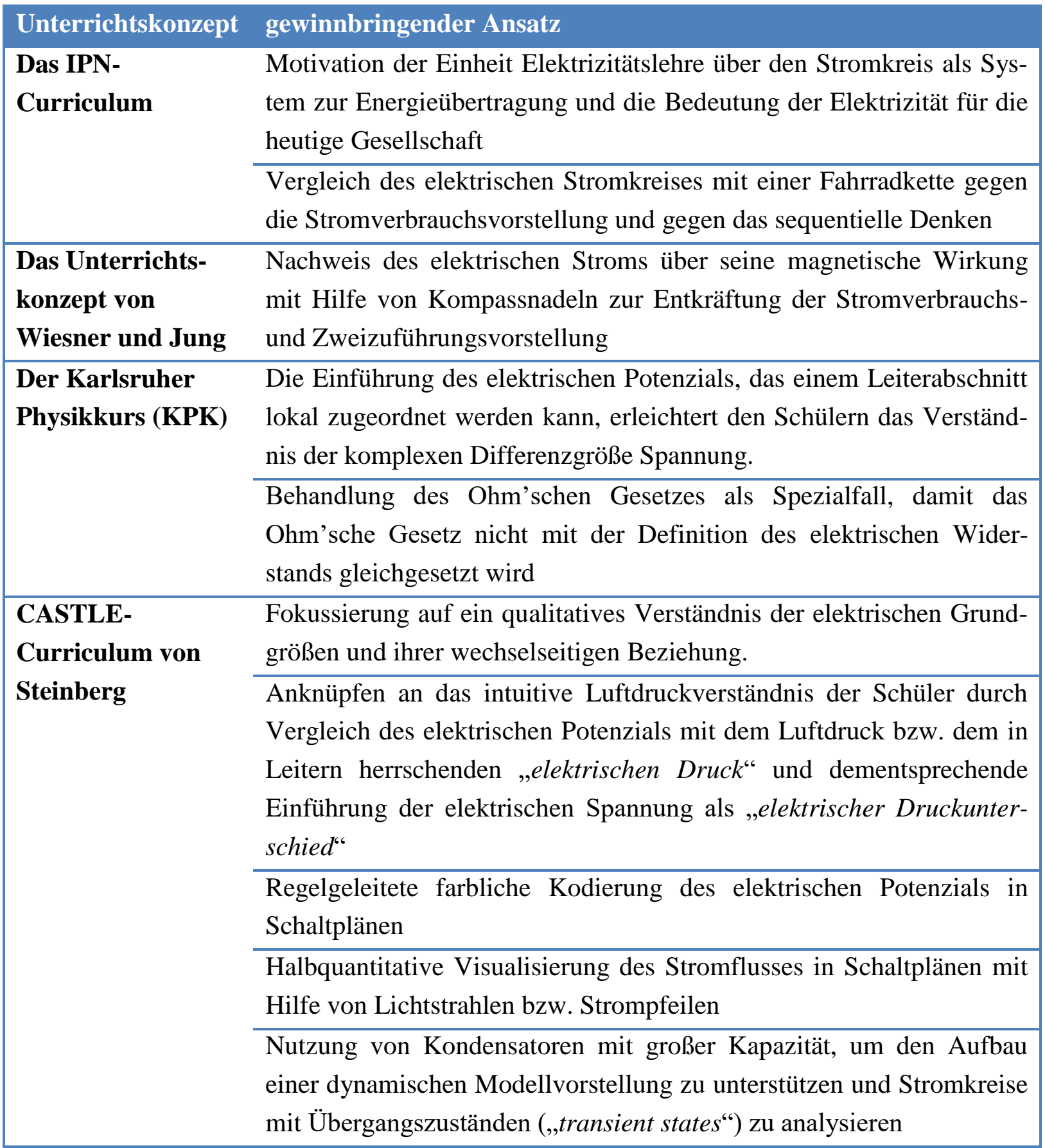




\begin{tabular}{|c|c|}
\hline \multirow{2}{*}{$\begin{array}{l}\text { Das Unterrichts- } \\
\text { konzept von } \\
\text { Muckenfuß und } \\
\text { Walz }\end{array}$} & $\begin{array}{l}\text { Nutzung eines handgetriebenen Generators, um die Energieübertra- } \\
\text { gung über Stromkreise für die Schüler physiologisch erfahrbar zu ma- } \\
\text { chen }\end{array}$ \\
\hline & $\begin{array}{l}\text { Anbieten einer mikroskopischen Modellvorstellung von Widerständen } \\
\text { auf Basis von Atomrümpfen und Elektronen, um den Schülern eine } \\
\text { Vorstellungshilfe davon zu geben, auf welche Weise die Elektronen in } \\
\text { Widerständen behindert werden (z.B. in Abhängigkeit der Geometrie, } \\
\text { Legierung oder Temperatur) }\end{array}$ \\
\hline \multirow{2}{*}{$\begin{array}{l}\text { Das Unterrichts- } \\
\text { konzept von } \\
\text { Schwedes und } \\
\text { Dudeck }\end{array}$} & $\begin{array}{l}\text { Herausstellung der Bedeutung des Verständnisses des Analogbereichs } \\
\text { für die Fähigkeit, überhaupt von der Analogie profitieren zu können }\end{array}$ \\
\hline & $\begin{array}{l}\text { Herausstellung der Bedeutung einer anschaulichen Visualisierung der } \\
\text { Druckverhältnisse in (Wasser-)Stromkreisen für die Entwicklung eines } \\
\text { konzeptionellen Verständnisses der elektrischen Spannung (vgl. Dop- } \\
\text { pelwassersäule) }\end{array}$ \\
\hline \multirow[t]{3}{*}{$\begin{array}{l}\text { Das Münchener } \\
\text { Stäbchenmodell } \\
\text { von Gleixner }\end{array}$} & $\begin{array}{l}\text { Vermeidung der Förderdung von Fehlvorstellungen in Folge der miss- } \\
\text { verständlichen Semantik von Fachbegriffen (z.B. „Elektrogerät“ statt } \\
\text { „Verbraucher“ bzw. „Intensität der Elektrizität“ statt „Stromstärke“) }\end{array}$ \\
\hline & $\begin{array}{l}\text { Mnemotechnische Verknüpfung der Formelbuchstaben: } U \text { wie } \\
\text { „elektrischer Unterschied“" und I wie ,Intensität der Elektrizität““ }\end{array}$ \\
\hline & $\begin{array}{l}\text { Verzicht auf eine mathematische Beschreibung der Physik, solange } \\
\text { kein qualitatives Verständnis der zugrundeliegenden Konzepte aufge- } \\
\text { baut wurde }\end{array}$ \\
\hline \multirow[t]{2}{*}{$\begin{array}{l}\text { Das Unterrichts- } \\
\text { konzept von Koller } \\
\text { und Späth }\end{array}$} & $\begin{array}{l}\text { Formulierung klarer Regeln zur Analyse von Strom- und Spannungs- } \\
\text { verhältnissen in Stromkreisen, um insbesondere lernschwächere Schü- } \\
\text { ler zu unterstützen }\end{array}$ \\
\hline & $\begin{array}{l}\text { Anbieten einer Vielzahl unterrichtsnaher Arbeits- und Merkblätter } \\
\text { inklusive einer zusammenfassenden Übungseinheit am Ende, um eine } \\
\text { höhere Strukturiertheit des Unterrichtskonzepts zu erreichen }\end{array}$ \\
\hline
\end{tabular}


B) Konzepterarbeitung 



\section{Entwicklung des Unterrichtskonzepts}

\subsection{Didaktische Grundüberlegungen}

\subsubsection{Motivation}

Die fachdidaktische Forschung hat gezeigt, dass es vielen Lernenden nicht gelingt, in der Mittelstufe ein angemessenes Verständnis elektrischer Stromkreise zu entwickeln (vgl. Abschnitt 3.2). Insbesondere neigen sie dazu, Stromkreise ausschließlich mit Hilfe des Strom- und Widerstandsbegriffs zu analysieren und die elektrische Spannung lediglich als Eigenschaft des Stroms wahrzunehmen (Stichwort „Stromspannung“) (Cohen et al. 1983; Rhöneck 1986). Aus didaktischer Sicht besteht ein dringender Handlungsbedarf darin, einen Weg zu finden, der es den Schülerinnen und Schülern ermöglicht, ein eigenständiges Spannungskonzept zu entwickeln, wie Härtel in seinem Vorwort zur Monografie von Assis und Hernandes (2013, vvi) zur Rolle von Oberflächenladungen in elektrischen Stromkreisen schreibt:

„Wie die Erfahrung zeigt, gelangen nur wenige Schüler zu einem tieferen Verständnis des Spannungsbegriffs. Dagegen führt bei vielen Schülern ein solches Scheitern gerade an einem so grundlegenden Begriff wie dem der Spannung zur Aufgabe des Interesses an physikalischen Inhalten. Die Frage ist, wie viele vor allem jüngere Schüler mit noch schwach entwickeltem Selbstvertrauen ein solches Scheitern sich selbst und dem eigenen Unvermögen zuschreiben und deshalb ihre Lernbereitschaft untergraben wird? Physik ist ein allgemein bildendendes und wichtiges Schulfach und da hiervon größere Schülerpopulationen betroffen sind, stellt die Suche nach weniger abstrakten und damit verständlicheren Alternativen eine dringende didaktische Aufgabe dar."

Der Grund dafür, warum die Schülerinnen und Schüler in der Sekundarstufe I kein angemessenes Konzept der elektrischen Spannung entwickeln, wird einerseits in ihrem vergleichsweise komplexen Charakter als Differenzgröße, andererseits aber auch in der im traditionellen Physikunterricht oftmals verfolgten Sachstruktur gesehen (Cohen et al. 1983; Muckenfuß und Walz 1997; Gleixner 1998). Erschwert wird den Lernenden die Entwicklung eines eigenständigen Spannungskonzepts oftmals auch dadurch, dass im traditionellen Unterricht sehr oft Beispiele betrachtet werden, bei denen Spannung und Stromstärke nur gemeinsam auftreten und bei denen die Stromstärke und Spannung ein proportionales Verhältnis aufweisen. Insbesondere eine ausgiebige quantitative Auseinandersetzung mit der elektrischen Spannung in der Form $U=R \cdot I$ ist insofern problematisch, als dass hier Stromstärke und Spannung definitionsgemäß immer nur gemeinsam auftreten können. Schüler können so leicht den Eindruck bekommen, die Spannung sei eine Art Eigenschaft des Stroms und könne immer nur gemeinsam mit diesem in Erscheinung treten, wie von der Formel $U=R \cdot I$ mit $I=0$ hinsichtlich der Spannung $U$ nahegelegt wird (Rhöneck 1988). Auch Muckenfuß und Walz 
(1997, 32f) sehen in einem solchen Vorgehen ein Problem, da es das Denkmuster, die Spannung sei eine Eigenschaft des Stroms, stärkt.

Als Potenzialdifferenz bezieht sich die elektrische Spannung immer auf zwei Punkte in einem Stromkreis und ist somit schwieriger zu verstehen als das elektrische Potenzial, das einem Punkt bzw. einem Leiterabschnitt zugeordnet werden kann. In Schulbüchern wird das elektrische Potenzial zur Erklärung der Spannung in aller Regel aber gar nicht thematisiert und stattdessen häufig auf die mathematische Definition der elektrischen Spannung als Arbeitsfähigkeit $U=\frac{W}{Q}$ zurückgegriffen, die aber wenig anschaulich und vor allem ungeeignet ist, die wechselseitige Beziehung der Grundgrößen in Stromkreisen qualitativ zu erklären (Härtel 2012b, S. 25). Von den Lernenden wird also insbesondere im Anfangsunterricht oftmals erwartet, ein Verständnis für die elektrische Spannung zu entwickeln, ohne die dahinterstehende Größe selbst, nämlich das elektrische Potenzial, zu kennen, geschweige denn zu verstehen (Herrmann und Schmälzle 1984, S. 477). Ein solches Vorgehen kann nach Clement und Steinberg (2002, S. 393) nicht von Erfolg gekrönt sein, da ,physicists and engineers are generally agreed that effective reasoning about electric circuits requires a robust conception of electric potential“. Es verwundert daher wenig, dass sich Schülerinnen und Schüler in der Abwesenheit eines anschaulichen konzeptionellen Verständnisses der Grundgrößen der Elektrizitätslehre in ihrer Not bei nahezu jeder Frage an den vermeintlichen Rettungsanker $U=R \cdot I$ klammern, der ihnen von ratlosen Lehrkräften nur zu oft als Ersatz für ein qualitatives Verständnis verfrüht in den Unterricht geworfen wird. In der Folge einer solchen verfrühten Mathematisierung verfügen die Lernenden auf diesem Gebiet oftmals über reines Formelund Faktenwissen, ohne jedoch die zugrundeliegenden Konzepte zu verstehen, was maßgeblich zur Entstehung von Schülervorstellungen beitragen kann (Cohen et al. 1983).

Hinzu kommt, dass der Physikunterricht der Mittelstufe aus historischen, nicht jedoch didaktischen Gründen maßgeblich vom Strombegriff geprägt ist (Gleixner 1998, S. 62), obwohl bis Anfang des 20. Jahrhunderts die Einführung in die Elektrizitätslehre vorrangig über die Elektrostatik und das elektrische Potenzial stattfand (vgl. Jochmann et al. 1914). Bereits in den 1980er Jahren wurde deshalb die Hypothese aufgestellt, dass die oftmals im Physikunterricht verfolgte Sachstruktur zur Entstehung eines „übermächtigen Strombegriff“ (Rhöneck 1986, S. 13) beiträgt. Kritisiert wird insbesondere, dass im Anfangsunterricht in der Regel zunächst der elektrische Strom ausführlich thematisiert wird und erst danach die elektrische Spannung zum Unterrichtsgegenstand wird. Ein solches Vorgehen ist nach Cohen et al. (1983) insofern problematisch, als dass der elektrische Strom hierdurch zum Primärkonzept der Lernenden gemacht wird, obwohl der elektrischen Spannung als Ursache des elektrischen Stroms eine viel bedeutendere Rolle für ein angemessenes Verständnis von einfachen Stromkreisen zukommt. Sie schreiben in diesem Zusammenhang, first impressions are strong and may impede a later, more rigorous, study of electricity" (ebd, S. 411) und fragen, ob eine bessere Sachstruktur nicht darin bestünde, zunächst die elektrische Spannung im Unterricht zu behandeln, weil hierdurch die Ursache-Wirkungs-Beziehung zwischen der elektrischen Span- 
nung dem elektrischen Strom deutlich würde. Hierzu schlagen sie eine Analogie vor, in der die Batterie einen gewissen „Druck“ auf die elektrischen Ladungen im Draht ausübt, um dann mit Hilfe von Druckunterschieden erklären zu können, wie es zu einem elektrischen Strom kommt.

Auch wenn eine solche Druckanalogie von Steinberg und Wainwright (1993) in ihrem CASTLE-Curriculum in Form des Luftdrucks aufgegriffen wurde und sie davon berichten, dass sie sich als vielversprechend erwiesen hat, wurden genaue empirische Ergebnisse zur Lernförderlichkeit des CASTLE-Curriculums nie veröffentlicht. Ein wesentlicher Kritikpunkt an dem Unterrichtskonzept aus hiesiger Sicht besteht darin, dass es hierzulande mit seiner Fokussierung auf selbstständig zu bearbeitende Experimentieraufträge u.a. zu den Lade- und Entladevorgängen von Kondensatoren mit großer Kapazität nur bedingt unter den curricularen und institutionellen Rahmenbedingungen des Physikunterrichts umsetzbar ist. Hinzu kommt, dass im CASTLE-Curriculum entgegen der Kritik von Cohen et al. (1983) zunächst ausführlich der elektrische Strom thematisiert wird, statt mit der elektrischen Spannung als Ursache des elektrischen Stroms zu beginnen und damit zu versuchen, die Spannung statt den Strom zum Primärkonzept der Lernenden zu machen. Ein solches Vorgehen wäre jedoch wünschenswert, da die Stromstärke ohnehin die Vorstellung vieler Schüler von elektrischen Stromkreisen dominiert und sie die Spannung oft lediglich als Eigenschaft und nicht Ursache des elektrischen Stroms begreifen (Rhöneck 1986). Weitere dem hier vorgeschlagenen Unterrichtskonzept von ihrem Charakter her ähnliche Ansätze wie z.B. der Einführung der Spannung mittels Elektronengasmodell (Kircher 1975), mittels Ladungsunterschied (Rhöneck und Völker 1982) und mittels Elektronendrucks (Niedderer und Goldberg 1995) wurden aus heutiger Sicht ebenfalls nur unzureichend empirisch evaluiert.

\subsubsection{Zentrale Ideen hinter dem Unterrichtskonzept}

Vor dem Hintergrund der konstruktivistischen Lerntheorie (vgl. Abschnitt 3.1.2) und insbesondere der von diSessa geprägten „knowledge-in-pieces“ Perspektive auf das Lernen (vgl. Abschnitt 3.1.5) wird der Sachstruktur und dem Vorwissen der Schüler im Unterrichtskonzept eine besondere Bedeutung beigemessen und entsprechend eine Anknüpfungsstrategie verfolgt. Durch die gezielte Auswahl geeigneter Kontexte und einer passenden Abfolge der Inhalte wird versucht, an solche Vorstellungen bei den Lernenden anzuknüpfen, die einer physikalischen Begriffsentwicklung förderlich sind. Der Terminologie von diSessa entsprechend besteht das Ziel also darin, mit Hilfe der u.a. auf Basis der Schülervorstellungsforschung entwickelten Sachstruktur genau solche p-prims bei den Lernenden zu aktivieren, die in Hinblick auf die Entwicklung eines angemessenen physikalischen Konzepts anknüpfungsfähig sind und gleichzeitig die Aktivierung von ungeeigneten p-prims, die zu Schülervorstellungen und damit Lernschwierigkeiten führen, zu vermeiden. Es wird also aktiv versucht, an das Vorwissen der Schüler anzuknüpfen und durch eine sorgfältige Auswahl der Kontexte und der Sachstruktur die zuvor losen und unstrukturierten p-prims in eine umfassende und kohärente mentale Struktur zu überführen. Ein solches Vorgehen wird nach Wilhelm et al. (2012, S. 35) ,in- 
struktionsinduzierte Begriffsentwicklung“ genannt und kann als Gegenpol zu offenen Unterrichtsformen wie dem selbstentdeckenden Lernen gesehen werden.

Die Strategie der instruktionsinduzierten Begriffsentwicklung eignet sich in besonderem Maße zur Vermittlung komplexer Inhalte wie z.B. dem konzeptionell schwierigen Spannungskonzept und hat sich in der Vergangenheit bei einer Vielzahl an physikdidaktischen Studien bewährt. Wiesner et al. (2010, 8f) berichten beispielsweise davon, dass dieser Ansatz zu etwa doppelt so hohen Lernerfolgen im Bereich der Optik (Herdt 1989), Energie (Bader 2001), Wärmelehre (Bader 2001) und der Elektrizitätslehre (Späth 2009) geführt hat und die instruktionsinduzierte Begriffsentwicklung zudem durch die ,[...] zunehmende Einsicht in die Bedeutung der Instruktion für erfolgreiches Lernen gestützt [wird] (z.B. Klahr und Nigam 2004; Mayer 2004)“. Das auf Basis einer systematisch ausgearbeiteten Sachstruktur stattfindende inhaltsbezogene Lernen, bei dem die Aktivierung falscher Vorstellungen gezielt vermieden wird, stellt für Weinert (2000, S. 6) die ideale Voraussetzung dar, intelligentes Wissen auf dem anspruchsvollen Gebiet der Physik zu erwerben, das letztlich Voraussetzung für alle darauf aufbauenden Kompetenzen ist:

„Intelligentes Wissen zu erwerben, ist und bleibt die wichtigste Aufgabe des Bildungssystems, des Ausbildungssystems und des lebenslangen Lernens. Es gibt keine herausragende Kompetenz auf anspruchsvollen Gebieten ohne ausreichendes inhaltliches Wissen. [...] Sowohl Voraussetzung als auch Resultat ist ein sachlogisch aufgebautes, systematisches, inhaltsbezogenes Lernen, das [...] falsche Wissenselemente vermeidet. Intelligentes Wissen [...] muss in systematischer Weise erworben werden. Daher kann es nicht in die Beliebigkeit des einzelnen Schülers gestellt sein, dieses Wissen zu erwerben, sondern es erfordert die Verantwortlichkeit auf Seiten des Lehrers. Es erfordert eine Unterrichtsmethode, die lehrergesteuert, aber schülerzentriert ist."

In einer früheren Veröffentlichung beschreibt Weinert (1998, S. 25) die Methode der direkten Instruktion bzw. Unterweisung als hierfür zweckmäßigste Unterrichtsform. Diese sei

,[...] weder der zurecht kritisierte Frontal- oder Paukunterricht noch eine lehrerzentrierte und schülerrezeptive Didaktik, sondern eine lehrergesteuerte, aber schülerzentrierte, die Lernenden aktivierende, Verständnis fördernde und auf die permanente Beseitigung von Wissensdefiziten bei einzelnen Schülern ausgerichtete Unterrichtsform. “

In Bezug auf die eingesetzten Methoden gilt es aus Sicht des Autors dieser Arbeit die richtige Balance zwischen direkter Instruktion, die sich insbesondere zur Einführung schwieriger Konzepte eignet, und „offeneren“ Unterrichtsmethoden, die eher für eine horizontale Vernetzung der gelernten Inhalte geeignet sind, zu finden. Da jede Lehrkraft andere pädagogische Überzeugungen besitzt und die genaue methodische Ausgestaltung auch immer an den Bedürfnissen der jeweiligen Klasse orientiert sein sollte, werden im Unterrichtskonzept be- 
wusst keine expliziten methodischen Vorgaben gemacht. Eine zu enge Führung wie z.B. auch die Vorgabe der Phasen einzelner Unterrichtsstunden würde vermutlich auch die Akzeptanz des Unterrichtskonzepts bei den Lehrkräften mindern, was nicht mit den Zielen von DesignBased-Research (vgl. Abschnitt 7.1) vereinbar ist.

Das hier zu entwickelnde Unterrichtskonzept auf Grundlage des Elektronengasmodells hat nicht nur den Anspruch, den Lernenden ein qualitatives Verständnis für die wechselseitige Beziehung der Grundgrößen Spannung, Stromstärke und Widerstand in einfachen elektrischen Stromkreisen zu vermitteln, sondern ihnen insbesondere ein angemessenes Spannungskonzept zu ermöglichen. Besonderer Wert soll darauf gelegt werden, Schülervorstellungen zu vermeiden und vor jeglicher Quantifizierung zunächst ein qualitativ fundiertes Verständnis der Grundgrößen der Elektrizitätslehre zu erreichen, weil im regulären Physikunterricht „,verfrühte Mathematisierungen und, exakte 'Definitionen [...] oftmals das Denken in Anschauungen [verstellen], ohne dieses wirklich ersetzen zu können“ (Muckenfuß und Walz 1997, S. 161). Der Weg der Begriffsbildung soll also nicht über abstrakte Definitionen verlaufen, sondern über konkrete Erfahrungen und das Vorwissen der Lernenden, um eine vielschichtige Verankerung der neu zu erlernenden physikalischen Konzepte zu erleichtern. Hierzu wird gezielt versucht, an die Erfolge bisheriger Potenzialansätze (u.a. von Steinberg und Wainwright 1993, Gleixner 1998 und Waltner et al. 2009) anzuknüpfen und auf der von Steinberg und Wainwright (1993) im CASTLE-Curriculum genutzten Luftdruckanalogie aufzubauen, indem das elektrische Potenzial mit dem Luftdruck bzw. dem ,elektrischen Druck“ verglichen und entsprechend den Überlegungen von Cohen et al. (1983) noch vor der Stromstärke eingeführt wird. Das konzeptionelle Anknüpfen an die Luftdruckvorstellung der Lernenden und die Verwendung derselben als Ankervorstellung für eine elementarisierte Vorstellung des elektrischen Potenzials hat dabei gewisse Ähnlichkeiten mit der von Clement (1993) vorgeschlagenen „Bridging-Strategie“. Die Vorstellung des elektrischen Potenzials als elektrischer Druck geht historisch auf Volta zurück, der aufgrund seiner Versuche mit Elektrometern um 1770 davon ausging, dass die freibeweglichen Ladungen in Leitern sich wie ein kompressibles Fluid mit druckähnlichen Eigenschaften verhielten (Steinberg 2008). Die Betonung der für die Lernenden intuitiven Luftdruckvorstellung im Gegensatz zur bloßen Argumentation mit abstrakten Elektronendichten ist ein wesentlicher Unterschied zu den von z.B. Kircher (1975, 75ff) und Rhöneck und Völker (1982) gemachten Vorschlägen zur Thematisierung der Elektrizitätslehre mit Hilfe des Elektronengasmodells.

Die Grundidee des angedachten Unterrichtskonzepts besteht dementsprechend darin, an die alltägliche Luftdruckvorstellung der Schülerinnen und Schüler u.a. zu Luftmatratzen, Fußbällen und Fahrradreifen anzuknüpfen, wonach ,komprimierte Luft unter Druck steht, gegen die Wände drückt und das Bestreben hat, sich auszudehnen“. Entsprechend der Terminologie von diSessa (2013) stellt dieses grundlegende Verständnis einen in Hinblick auf die Begriffsentwicklung des elektrischen Potenzials anknüpfungsfähigen ,p-prim“ dar (vgl. Abschnitt 3.1.5). Wichtig ist an dieser Stelle zu betonen, dass dieser p-prim (,phenomenological primitive، $)$ entsprechend der Theorie von diSessa keinem ausdifferenzierten physikalischen 
Begriffsverständnis entspricht, sondern lediglich ein isoliertes, auf Alltagserfahrungen basierendes, kognitiv noch nicht weiter vernetztes Wissenselement darstellt. Dementsprechend wird ein fachlich korrektes Druckkonzept mit der in der Sekundarstufe I teils schwierigen Differenzierung zwischen skalarem Druckbegriff und vektoriellem Kraftbegriff weder vorausgesetzt noch eingeführt. Die Vorstellung vom Luftdruck soll dann auf den in Leitern herrschenden elektrischen Druck übertragen werden, womit die elektrische Spannung in Analogie zu Luftdruckunterschieden als elektrischer Druckunterschied und Antrieb des elektrischen Stroms eingeführt werden kann. Qualitativ soll in Stromkreisen dann die wechselseitige Beziehung der Größen Spannung, Widerstand und Stromstärke als Vorbereitung der quantitativen Beziehung $I=\frac{U}{R}$ erarbeitet werden, wobei gerade diese Beziehung nach diSessa (1988, S. 53) eines der fundamentalsten und überzeugendsten Beispiele für ein anknüpfungsfähigen p-prim darstellt: ,It [Ohm's Law] is really an enlarged version of the 'more effort begets more results' primitive [...] [in] that increased effort begets increased result; increased resistance begets reduced result; and so on."

Allerdings scheint die Einführung der Spannung über den Druckbegriff nicht ohne didaktische Schwierigkeiten, wie aus den folgenden beiden Zitaten vorangegangener Arbeiten zu dem Thema deutlich wird:

„, [...] Die Einführung des Potentialbegriffes ist hilfreich, wenn auch nicht ohne didaktische Schwierigkeiten, die hauptsächlich in einer mangelnden Unterscheidungsfähigkeit zwischen Absolut- und Differenzgrößen seitens der Schülerinnen und Schüler begründet liegt. “(Gleixner 1998, S. 192)

„,Die eigentliche Vorstellung von Druck und Druckdifferenz als Antrieb des Elektronenstromes [...] findet demgegenüber nicht die erhoffte Resonanz. Insbesondere bereitet den Studentinnen die Vorstellung vom Gleichgewicht von Druck und Gegendruck und von der zentralen Bedeutung der Druckdifferenz Schwierigkeiten. " (Niedderer und Goldberg 1995, S. 84)

Vor dem Hintergrund dieser Erfahrungen, die elektrische Spannung als Druckunterschied einzuführen und den in diesem Zusammenhang dokumentierten Schwierigkeiten der Lernenden, zwischen Absolut- und Differenzgröße unterscheiden zu können, wird im angedachten Unterrichtskonzept eine kurze anfängliche Auseinandersetzung mit Luftdruckphänomenen für nötig erachtet. Dabei wird angestrebt, den Lernenden auf möglichst direktem Wege ein Verständnis der elementaren Elektrizitätslehre zu ermöglichen, ohne hierzu auf einen zeitlich umfangreichen Vorkurs wie zu geschlossenen Wasserstromkreisen angewiesen zu sein (vgl. Schwedes et al. 1995). Aufgrund der großen Bedeutung des Verständnisses des Ausgangsbereichs für die effektive Nutzung einer Analogie (vgl. Abschnitt 4.1.2) und den angesprochenen Lernschwierigkeiten kann aber auch nicht völlig darauf verzichtet werden, den Lernenden an Luftdruckbeispielen zunächst eine korrekte physikalische Vorstellung davon zu vermitteln, dass Luftströmungen immer eine Folge von Druckunterschieden sind (vgl. Herrmann et al. 
2014a, S. 18). Den theoretischen Unterbau des hier angedachten Vorgehens bildet das Konzept „out of the Shadows“ von diSessa (2013, S. 45), wonach sich zum Aufbau eines physikalischen Konzepts auch solche p-prims eignen, die phänomenologisch von den Lernenden zunächst überhaupt nicht mit dem zu vermittelnden Konzept assoziiert werden, aber für dessen Entwicklung eine entscheidende Rolle einnehmen können. Das zunächst für das Verständnis von Stromkreisen völlig irrelevant zu scheinende Thema des Luftdrucks wird so genutzt, um den Lernenden eine anschauliche Vorstellung des elektrischen Potenzials bzw. der elektrischen Spannung zu ermöglichen und insbesondere ein Verständnis dafür zu vermitteln, warum Potenzialunterschiede einen elektrischen Strom verursachen. Hierzu erscheint es wichtig, dass die Lernenden eigene Erfahrungen („Primärerfahrungen“) mit Luftströmungen in Folge von Luftdruckunterschieden machen. Um den zunächst isolierten p-prim zum Luftdruck in eine angemessene kognitive Struktur einbetten zu können, sollten kurze Schülerexperimente durchgeführt und am Beispiel von gängigen Alltagsobjekten erarbeitet werden, dass Luft immer von Bereichen höheren Drucks zu Bereichen niedrigeren Drucks strömt. Nur wenn dieses Verständnis im Ausgangsbereich der Analogie vorhanden ist, kann es auf den Zielbereich, d.h. den elektrischen Stromkreis, erfolgreich übertragen und der Differenzcharakter der elektrischen Spannung von den Schülern in seiner Bedeutung erkannt werden.

Eine intuitive Vorstellung vom elektrischen Widerstand soll im Rahmen der Luftdruckanalogie dadurch erzielt werden, dass die Schülerinnen und Schüler Luft durch ein Stück Stoff (z.B. einen Kragen oder einen Ärmel) blasen. Dabei kann die Erfahrung gemacht werden, dass die Hemmung bzw. Behinderung der Luftströmung spürbar von der Dicke bzw. Dichte des Stoffes abhängt, was im Sinne von Clement (1993) als Ankervorstellung in Hinblick auf den elektrischen Widerstand dienen soll. Es wird hier also zunächst rein phänomenologisch argumentiert, bevor den Lernenden später auch eine mikroskopische Modellvorstellung des elektrischen Widerstands vermittelt werden soll. Dies hat insbesondere den Vorteil, dass ihnen eine Vorstellungshilfe davon gegeben werden kann, wie die Elektronen in einem elektrischen Widerstand gehemmt werden und wie die Legierungs- und Temperaturabhängigkeit eines Widerstands innerhalb des Modells erklärt werden kann (Muckenfuß und Walz 1997, S. 128). Vor dem Hintergrund, dass die meisten Schüler in ihrem Alltag oder im Fach Chemie bereits in Kontakt mit der Atomvorstellung gekommen sind, erscheint es sinnvoll, an dieses Vorwissen anzuknüpfen und ihre epistemologische Neugier zu befriedigen (vgl. Abschnitt 6.1.5).

Ein wesentliches Ziel des Unterrichtskonzepts besteht auch darin, der Entstehung bekannter Schülervorstellungen zu elektrischen Stromkreisen durch konzeptionelle, lerntheoretische und sprachliche Maßnahmen entgegenzuwirken. Während konzeptionell im Unterrichtskonzept eine Anknüpfungsstrategie verfolgt wird, die einen möglichst bruchlosen Übergang von den vorunterrichtlichen Vorstellungen hin zur physikalischen Vorstellung ermöglichen soll, findet bzgl. der verwendeten Begriffe eine Überbrückungs- bzw. Umgehungsstrategie Anwendung (vgl. Abschnitt 6.1.6). Im Sinne von Design-Based-Research (vgl. Abschnitt 7.1) sollen bei der Entwicklung des Konzepts zudem die Erkenntnisse der bisherigen didaktischen 
Forschung u.a. zu Schülervorstellungen und bereits existierenden Unterrichtskonzepten genauso berücksichtigt werden wie die Bedürfnisse des hiesigen Physikunterrichts. Damit das Unterrichtskonzept zu einer nachhaltigen Verbesserung der Unterrichtspraxis beitragen kann, ist es nämlich nicht nur Voraussetzung, dass es eine hohe Lernförderlichkeit besitzt, sondern, dass es insbesondere auch von den Lehrkräften angenommen und im eigenen Unterricht umgesetzt wird. Explizit nicht angestrebt wird im Rahmen des hier angestrebten Unterrichtskonzepts eine Auseinandersetzung mit Aspekten, die vom eigentlichen Lernziel, die grundlegenden Zusammenhänge in der Elektrizitätslehre verständlich zu machen, abbringen oder sogar neue Verständnisschwierigkeiten provozieren. Da beispielsweise die chemische Funktionsweise einer Batterie und ihres Innenwiderstands, der Aufbau eines Volt- und Amperemeters und ihr Innenwiderstand, die nicht ideale Leitfähigkeit realer Kupferleitungen, die Beliebigkeit des Potenzialnullpunkts und die Funktionsweise von Elektromotoren und Generatoren für ein grundlegendes konzeptionelles Verständnis der Größen Spannung, Stromstärke und Widerstand sowie ihrer wechselseitigen Beziehungen zweitrangig sind und ggf. mehr Fragen aufwerfen, als sie beantworten, werden sie im Sinne eines Spiralcurriculums hier bewusst ausgeklammert und dem weiterführenden Unterricht vorbehalten.

\subsubsection{Elementarisierung des Elektronengasmodells ${ }^{6}$}

Modelle sind von Menschen zu einem bestimmten Zweck geschaffene Konstrukte, die prinzipiell eine Vereinfachung der Realität darstellen und diese nie in vollem Umfang abbilden können (Mikelskis-Seifert und Kasper 2011). Insbesondere hängt die genaue Ausgestaltung eines Modells wesentlich davon ab, welches Ziel mit ihm verfolgt wird. Ist ein Modell primär dazu gedacht, das Erlernen bestimmter physikalischer Zusammenhänge in der Schule zu erleichtern, ist es normal, dass ,der fachliche Aspekt und der didaktische Aspekt [...] in Konkurrenz zueinander [treten]" (Kircher und Duit 1975, S. 19), da das Bestreben nach didaktischer Vereinfachung mit einer Reduktion von fachlicher Komplexität des Modells einhergeht. Soll die elementare Elektrizitätslehre in der Sekundarstufe I anhand des Elektronengasmodells eingeführt werden, so müssen aufgrund der in Abschnitt 2.3 erläuterten fachlichen Komplexität der Thematik einige didaktische Elementarisierungen vorgenommen werden. Angestrebt wird dabei, auf der einen Seite auf der Erklärungsmächtigkeit der Grenz- bzw. Oberflächenladungen bzgl. des Spannungs- und Potenzialbegriffs aufzubauen, auf der anderen Seite aber die dazu nötigen fachlichen Erklärungen möglichst einfach zu halten.

Wie in Abschnitt 2.3 dargelegt, ist die Driftbewegung der Leitungselektronen die Folge eines den Leiter durchziehenden elektrischen Feldes, das aufgrund von Grenz- und Oberflächenladungen entsteht. Bei einem idealen Leiter ohne Widerstand kommt es vor und nach einem Material mit geringer Leitfähigkeit (z.B. einem Widerstand) zu einer unterschiedlichen Grenz- und Oberflächenladungsverteilung (siehe Abb. 42). Das hier vorgeschlagene Elektronengasmodell verzichtet im Rahmen der didaktischen Elementarisierung auf eine Unterscheidung zwischen Leitungs- und Grenz- bzw. Oberflächenladungen sowie die Einführung des

\footnotetext{
${ }^{6}$ Teile dieses Abschnitts wurden bereits in Burde et al. 2014 veröffentlicht.
} 
elektrischen Feldbegriffs und beschränkt sich stattdessen auf die Betrachtung der unterschiedlichen mittleren Elektronendichten innerhalb der Leiterabschnitte (siehe Abb. 43). Im Gegensatz zu Modellen der Fachwissenschaft sollen didaktische Modelle nämlich nicht nur einen großen Geltungsbereich besitzen, sondern für Lernende auch möglichst einfach zu verstehen sein - ein nicht leicht zu lösender Zielkonflikt. Diese Entscheidung wurde u.a. vor dem Hintergrund getroffen, dass in der bisherigen didaktischen Literatur die Einführung insbesondere des elektrischen Feldbegriffs im Anfangsunterricht nicht für sinnvoll erachtet wird (vgl. Kircher 1975, S. 81 sowie Rhöneck und Völker 1982, S. 407). Es wird befürchtet, dass die Thematisierung des elektrischen Feldes die fachliche Komplexität des Modells in einem Maße erhöhen würde, welches das Bedürfnis der Lernenden nach einem anschaulichen, intuitiven und leicht verständlichen Modell konterkariert. Andererseits steht eine systematische Untersuchung der Frage, ob eine Argumentation lediglich mit Grenz- bzw. Oberflächenladungen ohne die Einführung des elektrischen Feldbegriffes die Lernenden überfordern würde noch aus und wäre insbesondere aufgrund der damit einhergehenden höheren fachlichen Korrektheit eines solchen Modells spannend (vgl. Abschnitt 6.1.7).

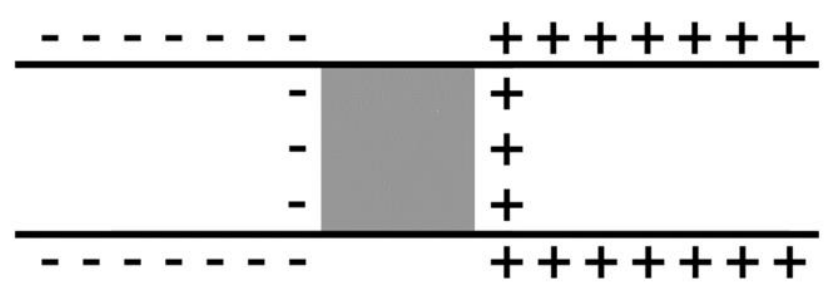

Abb. 42: Grenz- und Oberflächenladungsverteilung an einem Material mit geringer Leitfähigkeit (grau) bei einem idealen Leiter mit zu vernachlässigendem Widerstand (nach Chabay und Sherwood 2011, S. 767)

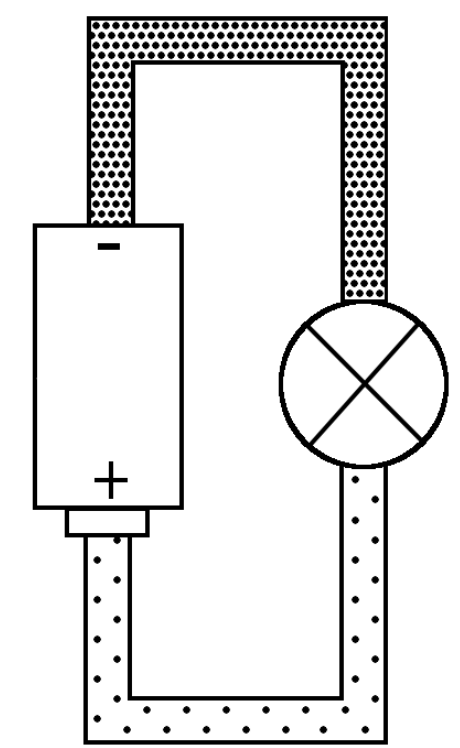

Abb. 43: Didaktische Elementarisierung der Elektronenverteilung mit Hilfe der Punktedichtedarstellung

Im didaktisch elementarisierten Elektronengasmodell wird postuliert, dass sich in Metallen wie z.B. Kupfer Elektronen in Teilchenform befinden und sich dort frei bewegen können. Im Gegensatz zu den Gasteilchen der Luft sind die Elektronen aber negativ geladen und werden daher in Materialien mit relativ hoher Leitfähigkeit im statischen Gleichgewichtszustand durch Abstoßung so weit wie möglich auseinandergetrieben, weshalb sie den ihn zur Verfügung stehenden Raum gleichmäßig ausfüllen. Dabei wird eine Komprimierbarkeit des Elektronengases angenommen. Die Batterie wird im didaktisch vereinfachten Modell als Elektronenpumpe betrachtet (vgl. Kircher 1975, S. 108), die Elektronen aus dem mit dem Pluspol verbundenen Leiter in den mit dem Minuspol verbundenen Leiter pumpt. Entsprechend des Modells sinkt damit die Elektronendichte im mit dem Pluspol verbundenen Leiterstück, während sie im mit dem Minuspol verbundenen Leiterstïck ansteigt (siehe Abb. 43). 
Im Leiter kommt es aufgrund der gegenseitigen Coulomb-Abstoßung der Elektronen zu einem von der Elektronendichte abhängigen ,elektrischen Druck“. Dieser lokale elektrische Druck wird als proportional zur Menge der dort vorhandenen Elektronen betrachtet. Im mit dem Minuspol verbundenen Leiterstück entsteht also ein hoher elektrischer Druck, wohingegen der elektrische Druck im Leiterstück, das mit dem Pluspol verbunden ist, absinkt. Durch Gleichsetzen des elektrischen Drucks mit dem elektrischen Potenzial kann die elektrische Spannung im elementarisierten Elektronengasmodell so als ,elektrischer Druckunterschied“ interpretiert werden, ohne auf den Feldbegriff zurückgreifen zu müssen, der in der Mittelstufe vermutlich eine Überforderung der Lernenden darstellen würde.

\subsubsection{Mögliche Darstellungsformen des elektrischen Potenzials ${ }^{7}$}

In Hinblick auf den Einsatz in der Schulpraxis kommt der Darstellungsform des elektrischen Potenzials im didaktisch elementarisierten Elektronengasmodell eine besondere Bedeutung zu (Gleixner 1998; Schwedes et al. 1995, S. 35), weshalb vier verschiedene Visualisierungen des elektrischen Drucks in Leitern entwickelt wurden. Eine naheliegende Visualisierung stellt die Punktedichtedarstellung dar, wie sie z.B. in ähnlicher Form schon von Kircher (1975, S. 88) vorgeschlagen wurde (vgl. Abb. 44). In dieser Darstellung befindet sich die Elektronenpumpe, d.h. die Batterie, unten und sorgt an ihren Kontakten für unterschiedliche Elektronendichten (im Beispiel von Abb. 44 für eine erhöhte Elektronendichte am Kontakt oben links und eine verminderte Elektronendichte am Kontakt oben rechts). Direkt mit den Kontakten verbunden sind zwei vergleichsweise dicke Drähte mit vernachlässigbarem Widerstand sowie zwischen diesen ein dünner Draht, der hier den elektrischen Widerstand des Stromkreises darstellt.

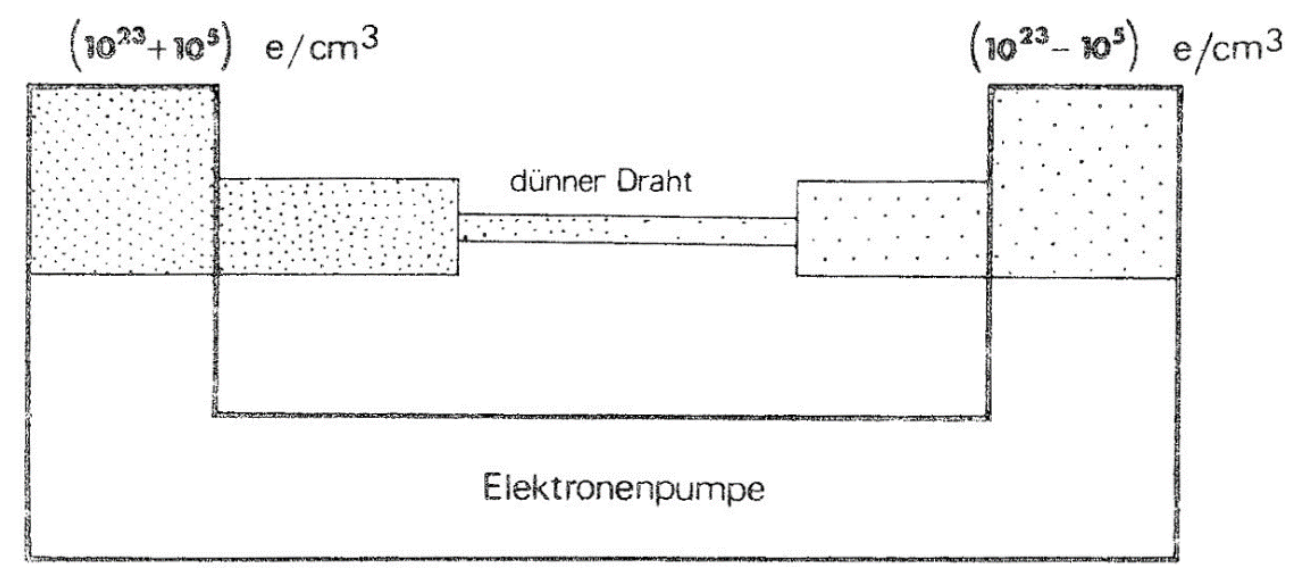

Abb. 44: Illustration der Elektronendichten in einem einfachen Stromkreis (nach Kircher 1975, S. 88). Der Widerstand entspricht dem dünnen Draht, die Zuleitung den dicken Drähten.

Die Grundidee dieser Darstellungsform ist es, die unterschiedlichen Elektronendichten im Leiter durch unterschiedliche Punktedichten darzustellen. Aufgrund der negativen Ladung der Elektronen gilt dabei: Je höher die Dichte der Punkte bzw. Elektronen und je größer damit ihre gegenseitige Abstoßung ist, desto größer ist der elektrische Druck im Leiterabschnitt. Durch die Visualisierung der unterschiedlichen Druckzustände mit Hilfe eines Teilchenbilds

\footnotetext{
${ }^{7}$ Teile dieses Abschnitts wurden bereits in Burde und Wilhelm 2015 veröffentlicht.
} 
soll der Teilchencharakter des verwendeten Modells verdeutlicht und der Zusammenhang zwischen Kompression und Druck für die Schüler sichtbarer gemacht werden.

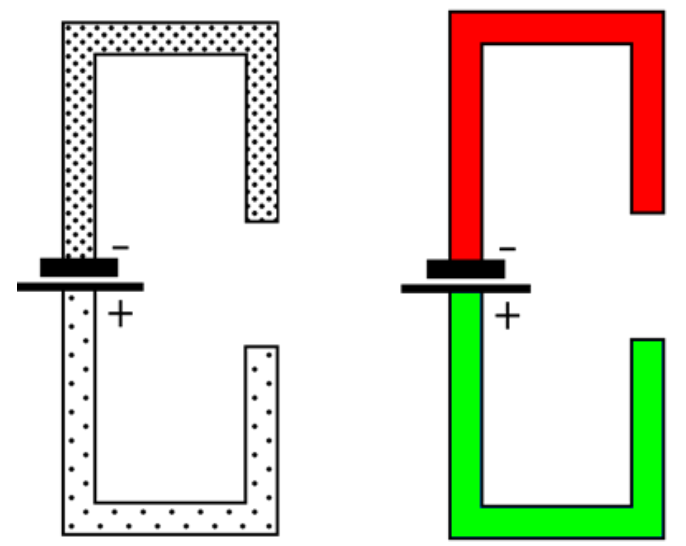

Abb. 45: Punktedichtedarstellung (links) und Farbdarstellung (rechts) des elektrischen Drucks

Eine simplifizierte Form der Visualisierung stellt die Farbdarstellung dar, wie sie bereits von Herrmann und Schmälzle (1984) vorgeschlagen wurde. Der Vorteil dieser Darstellungsform ist, dass der in den Leitern herrschende elektrische Druck direkt über die Farbe dargestellt wird und Schüler so nicht erst aus der Dichte der Elektronen auf den elektrischen Druck schließen müssen. Im Gegensatz zur häufig unsystematischen Farbwahl bei bisherigen Konzepten sollte das hier verwendete Farbschema an Konventionen angelehnt sein, die den Schülern aus dem Alltag bekannt sind. Dementsprechend fiel die Wahl zunächst auf Rot bzw. Grün für den elektrischen Über- bzw. Unterdruck, da diese beiden Signalfarben u.a. bei Druckanzeigen oder Umdrehungszählern im Auto verwendet werden. Für die Farbdarstellung in der Akzeptanzbefragung wurde daher festgelegt: Je höher der elektrische Überdruck, desto intensiver das Rot und je höher der elektrische Unterdruck, desto intensiver das Grün (vgl. Abb. 45).

Eine zur Farbdarstellung sehr ähnliche Visualisierung ist die Graustufendarstellung, wie sie in Abb. 46 zu sehen ist. Für sie gilt: Je dunkler das Grau, desto größer der elektrische Druck bzw. das Potenzial. Abstrakter ist hingegen die Visualisierung über Plus- bzw. Minuszeichen seitlich eines Leiters, wie sie z.B. im amerikanischen Lehrbuch ,Matter and Interactions" von Chabay und Sherwood (1999, S. 760) verwendet wird, in dem elektrische Stromkreise mit Hilfe von Oberflächenladungen erklärt werden. Die Idee ist hier, den Elektronenüberschuss im Leiter am Minuspol der Batterie durch entsprechende Minuszeichen links und rechts des Leiters darzustellen, während zur Visualisierung eines Elektronenmangels im Leiter entsprechend Pluszeichen verwendet werden. Da die Elektronendichte im Leiterinneren homogen ist und sich nur an der Oberfläche des Leiters ändert, ist die Plus-Minus-Darstellung physikalisch gesehen am nächsten an der Realität. Dass eine erhöhte Konzentration von Oberflächenladungen gerade an der Außenseite einer Leiterkrümmung zu finden ist (Härtel 2012b), wird hier zur Vereinfachung bewusst vernachlässigt. 

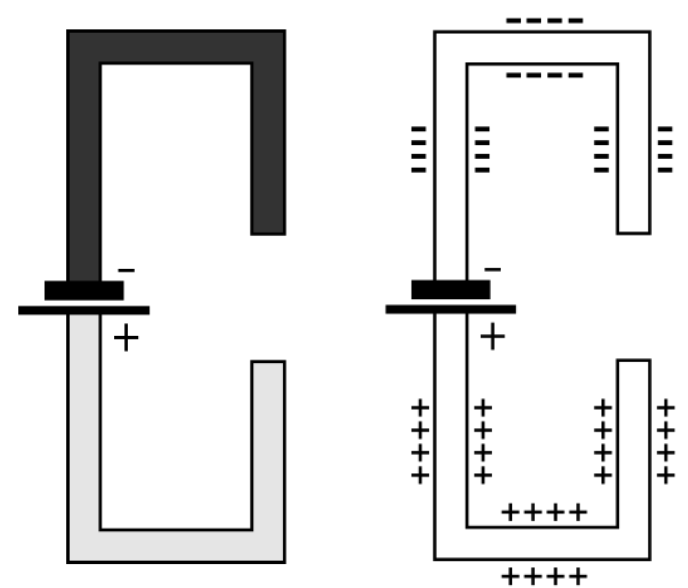

Abb. 46: Graustufendarstellung (links) und Plus-Minus-Zeichen-Darstellung (rechts) des elektrischen Drucks

Dabei ist die Anzahl der Plus- und Minuszeichen proportional zum Elektronenmangel bzw. Elektronenüberschuss und damit zum im Leiterabschnitt herrschenden elektrischen Druck bzw. Potenzial. Viele Minuszeichen stehen also für einen großen Elektronenüberschuss verglichen mit der Anzahl der positiv geladenen Atomrümpfe und damit für einen elektrischen Überdruck. Viele Pluszeichen stehen hingegen für einen großen Elektronenmangel und damit netto positiv geladenen Leiter und damit für einen elektrischen Unterdruck.

Durch die große Ähnlichkeit von Modelldarstellung und realen Schaltungen ist es denkbar, dass die Lernenden dem Elektronengasmodell einen höheren Wirklichkeitsgrad zuschreiben als anderen Modellen und Lernende mit weniger stark ausgeprägter Fähigkeit zum formalen Denken aufgrund des geringen Unterschieds zwischen Oberflächen- und Tiefenstruktur auch der Analogieschluss leichter gelingt. Insbesondere besteht die berechtigte Hoffnung, dass mit Hilfe des Elektronengasmodells und seinen mikroskopischen Erklärungen elektrischer Phänomene das von Kircher (1984, S. 58) bei Wassermodellen beschriebene „ontologische Problem" vermieden werden kann (vgl. auch Galili und Goihbarg 2005):

„Er [der Schüler] will sehen, was die Welt im Innersten zusammenhält. Er will etwas über den elektrischen Strom erfahren; das hat ihm der Lehrer und auch das Schulbuch so versprochen. Der Wasserkreislauf interessiert ihn letztlich nicht, weil ihn die Erklärungen im Wassermodell nicht zufriedenstellen: Wasser ist auf der phänomenologischen Ebene etwas ganz anderes als Elektrizität. " (Kircher, 1984, S.48).

\subsubsection{Vermittlung einer mikroskopischen Modellvorstellung}

Bereits Rhöneck (1980, 27f) erkannte, dass ,für eine Definition der Spannung über den Dichteunterschied spricht, daß damit ausgehend von der Elektrostatik durchgängig mikroskopische Modellvorstellungen benutzt werden können". Wie in den vorherigen Abschnitten dargelegt, wird hier die Auffassung vertreten, dass eine Vermittlung einer solchen mikroskopischen Modellvorstellung sinnvoll ist, weshalb die sich aus der hier verwendeten Spannungsdefinition als Dichte- bzw. Druckunterschied ergebende Chance nicht ungenutzt bleiben soll. Ausge- 
hend von den didaktischen Elementarisierungen des fachlichen Elektronengasmodells ergeben sich allerdings einige grundsätzliche Fragen dahingehend, wie diese mikroskopische Modellvorstellung genau aussehen soll. Wie in Abschnitt 5 gezeigt, unterscheiden sich die bisherigen Unterrichtskonzepte beispielsweise darin, ob sie von Elektrizität, Ladungen, Ladungsteilchen oder Elektronen in den Leitern sprechen. Da im Anfangsunterricht zur Elektrizitätslehre nahe$\mathrm{zu}$ ausschließlich metallische Leiter und hier insbesondere Kupfer benutzt werden, erscheint es gerade in einem Teilchenmodell sinnvoll, von Elektronen zu sprechen und dementsprechend auch die physikalische statt der technischen Stromrichtung zu unterrichten. Somit kann der Elektrizitätslehre eine Teilchenvorstellung zugrunde gelegt werden, die den Lernenden auch die Ladungserhaltung und somit die Konstanz der Stromstärke plausibel machen dürfte. Ein solches Vorgehen erscheint auch vor dem Hintergrund sinnvoll, dass Schüler in der Regel schon vor dem Unterricht z.B. in den Medien von Elektronen gehört haben und diese oftmals mit dem elektrischen Strom assoziieren (vgl. Härtel et al. 1981, S. 19). Der Verzicht auf dieses Vorwissen würde der epistemologischen Neugier der Schüler nicht gerecht werden, ihr Bedürfnis nach konsistenten Erklärungen nicht stillen und deshalb bestenfalls demotivierend wirken und schlimmstenfalls unangemessene eigene Modellvorstellungen bei den Schülern provozieren (Muckenfuß und Walz 1997, S. 131).

Auf der anderen Seite ist es allen bisherigen Unterrichtsmodellen auf Basis des Potenzialansatzes gemein, dass sie die technische Stromrichtung unterrichten und somit davon ausgehen, dass der Strom vom Plus- zum Minuspol fließt (Steinberg und Wainwright 1993; Gleixner 1998; Waltner et al. 2009). Der Vorteil eines solchen Vorgehens besteht darin, dass ein positives Potenzial z.B. mit einem hohen Druck und ein negatives Potenzial mit einem niedrigen Druck verglichen und sich darauf aufbauend festhalten lässt, dass der (positive) Strom der physikalischen Konvention entsprechend immer vom hohen zum niedrigen Potenzial fließt. Wird im Modell aber mit der physikalischen Stromrichtung gearbeitet, nach der die Elektronen vom Minus- zum Pluspol fließen, ist eine solche der Konvention entsprechende Argumentation nicht mehr so leicht möglich. Dies liegt daran, dass am Minuspol mit seiner hohen Elektronendichte und damit hohem elektrischen Druck ein niedriges (negatives) Potenzial vorliegt, am Pluspol mit seiner niedrigen Elektronendichte und damit niedrigen elektrischen Druck hingegen ein hohes (positives) Potenzial. Somit würde der Strom zwar von hohem zu niedrigem Druck, jedoch gleichzeitig von niedrigem zu hohem Potenzial strömen, was aus Schülersicht das Verständnis nicht unbedingt erleichtern dürfte. Eine Lösung könnte darin bestehen, im Anfangsunterricht zunächst nur den Begriff des elektrischen Drucks zu gebrauchen. Darüber hinaus stellt sich auch die grundsätzliche Frage, welcher Mehrwert in der Mittelstufe in der expliziten Einführung des Begriffs „Potenzial“ besteht, wenn die Lernenden mit der zugrundeliegenden Druckvorstellung arbeiten können.

In Hinblick auf die genaue Ausgestaltung der dem Elektronengasmodell zugrundeliegenden mikroskopischen Modellvorstellung sind zwei Ansätze denkbar. Auf der einen Seite kann das Elektronengasmodell als Nur-Elektronen-Modell konzipiert werden. In diesem Fall würden die Regeln des elektrischen Stromkreises einzig über die gegenseitige Abstoßung der 
Elektronen erklärt werden (vgl. Kircher 1975, 75ff). Auf der anderen Seite wäre aber auch eine Realisierung in Form eines Elektronen-Atomrumpf-Modells möglich, wonach ein neutraler Leiter aus gleich vielen ortsfesten Atomrümpfen und freien Elektronen besteht. Während ein Überschuss an Elektronen in diesem Modell zu einem negativ geladenen Leiterabschnitt führt, bedeutet ein Mangel an Elektronen aufgrund der dann in Überzahl vorhandenen Atomrümpfe, dass der Leiterabschnitt positiv geladen ist. Zwar entspricht das ElektronenAtomrumpf-Modell eher der fachlichen Vorstellung und kann auch elektrostatische Phänomene erklären, zur bloßen Erklärung von Potenzialunterschieden als Antrieb für den elektrischen Strom stellt es aber aufgrund der zusätzlich zu beachtenden ortsfesten, positiven Atomrümpfe eine unnötige Verkomplizierung der Zusammenhänge dar. Insbesondere ergibt sich aus der Betrachtung von Atomrümpfen kein Mehrwert hinsichtlich der Erklärungsmächtigkeit des Modells bzgl. der Regeln des Stromkreises wie z.B. den Kirchhoff'schen Gesetzen. Nichtsdestotrotz erscheint es aus mindestens zwei Gründen im Unterricht sinnvoll zu sein, Atomrümpfe zumindest anfänglich anzusprechen, auch wenn Atomrümpfe keine Voraussetzung für das Verständnis des elektrischen Drucks bzw. Potenzials sind. So kann über das Drude-Modell mit Hilfe von Atomrümpfen eine mikroskopische Erklärung für die geringere Leitfähigkeit von Widerständen gegeben werden, indem gezeigt wird, dass die Elektronen auf ihrem Weg durch den Widerstand abhängig von Temperatur, Legierung und geometrischer Form häufig gegen die Atomrümpfe stoßen (Muckenfuß und Walz 1992, S. 137). Auch kann über eine anfängliche Thematisierung der Atomrümpfe der durch das Nur-Elektronen-Modell möglicherweise provozierten Schülervorstellung entgegengewirkt werden, dass alle Leiterabschnitte grundsätzlich negativ geladen sein müssten.

\subsubsection{Didaktisch motivierte Begriffswahl zentraler Konzepte}

Vor dem Hintergrund der dem hier vorgeschlagenen Unterrichtskonzept zugrundeliegenden Druckanalogie ist auch zu überlegen, ob die im deutschsprachigen Raum üblicherweise verwendeten Fachbegriffe „Potenzial“, „Spannung“ und „Stromstärke“ sowie gängige Ausdrücke wie „Verbraucher“ oder ,Stromquelle“ aufgrund ihrer missverständlichen Semantik schon zu Beginn der Unterrichtsreihe eingeführt werden sollten. Es stellt sich insbesondere die Frage, ob es nicht sinnvoll ist, übergangsweise alternative Begriffe einzuführen, die auf den Ideen des Elektronengasmodells aufbauen und bzgl. ihrer Bedeutung im Alltag weniger missverständlich sind, da nach Muckenfuß und Walz (1997, S. 20) sonst die Gefahr besteht, dass „Lehrkräfte und Schülerinnen und Schüler [...] u. U. verschiedene Sprachen trotz gleicher Wörter [sprechen]".

Ein schönes Beispiel ist hier sicherlich der Begriff des „Verbrauchers“, der viele Schüler in ihrer Stromverbrauchsvorstellung bestärkt oder diese möglicherweise mitverursacht und deshalb durch den Begriff des „Elektrogeräts“ ersetzt werden sollte. Ähnlich sieht es mit dem Begriff der „Stromquelle“ aus, der den Lernenden suggeriert, ein Netzteil sei eine Quelle konstanten Stroms (statt konstanter Spannung) und ihnen eine Stromaussendevorstellung nahelegt. Im Unterrichtskonzept soll deshalb anfänglich nur mit Batterien gearbeitet und später korrekterweise allgemein von Spannungsquellen gesprochen werden. Gleixner (1998, 
S. 83) macht darüber hinaus noch eine Reihe weiterer interessanter Vorschläge hinsichtlich der Fachsprache, wie z.B. den Begriff der „Stromstärke“ durch „Intensität des Stroms“ (wie das Formelzeichen I) und den Begriff der „Spannung“ durch „elektrischen Unterschied“" (wie das Formelzeichen $U$ ) zu ersetzen. Im angedachten Unterrichtskonzept ist zu überlegen, diese Ideen bzgl. der Fachsprache in leicht modifizierter Form aufzugreifen, um einen stärkeren Bezug zur Druckvorstellung herzustellen. Entsprechend der Anknüpfungsstrategie von diSessa (2013) könnte insbesondere durch die Verwendung der dem Ausgangsbereich (Luftdruck) entlehnten Begriffe „elektrischer Druck“, ,elektrischer Druckunterschied“ und „Elektronenströmung" die Aktivierungspriorität geeigneter p-prims im Bereich elektrischer Stromkreise (dem Zielbereich) systematisch erhöht werden. Da in den angedachten Begriffen die angestrebten Modellvorstellungen angelegt sind, besteht so die Hoffnung, bei den Lernenden ein tieferes konzeptionelles Verständnis für einfache Stromkreise zu fördern:

- Elektrischer Druck statt elektrisches Potenzial: Hierdurch kann die in Abschnitt 6.1.5 angesprochene Vorzeichenproblematik beim Potenzial vermieden und gleichzeitig die Analogie zum Luftdruck betont werden.

- Elektrischer Druckunterschied statt elektrische Spannung: Entsprechend bietet sich der Begriff des ,elektrischen Druckunterschieds“ an, um auch hier die konzeptionelle Nähe zum Luftdruck zu betonen und den Charakter der Spannung als Differenzgröße hervorzuheben. Das Formelzeichen $U$ kann mnemotechnisch geschickt als „Unterschied des elektrischen Drucks“ eingeführt und seiner Ikonizität folgend als Erinnerungsstütze für die Anschlussbedingung von Voltmetern dienen.

- Intensität der Elektronenströmung statt elektrische Stromstärke: Der Begriff der Stromstärke ist semantisch problematisch, da die Größe leicht mit dem alltäglichen Kraftbegriff (und evtl. Druckbegriff) in Verbindung gebracht werden kann, obwohl es sich um eine rein mengenartige physikalische Größe handelt (vgl. Muckenfuß und Walz 1997, S. 32). In der Folge könnten Lernende den Begriff Stromstärke mit einer Antriebsvorstellung und daher mit der elektrischen Spannung assoziieren, was durch die vorgeschlagene Begriffswahl vermieden wird, da sie den Teilchen- und Strömungscharakter des elektrischen Stroms betont. Das Formelzeichen $I$ kann so zudem mnemotechnisch geschickt als „Intensität der Elektronenströmung“ eingeführt und auch hier seiner Ikonizität folgend als Anschlussbedingung eines Amperemeters interpretiert werden. Entsprechend der Ideen von Jung (1979) und Chi (1992) wird durch die Begriffswahl auch der ontologische Kategorienwechsel gefördert, da hierdurch der Prozess-Charakter der Stromstärke herausgestellt wird, die von den Lernenden oftmals fälschlicherweise dem Konzept „Körper“ bzw. „Substanz“ zugeordnet wird.

Die hier vorgeschlagene Sprachregelung hat auch den Vorteil, dass sie die nach Gleixner (1998, S. 58) häufig inkonsistente Verwendung von Potenzial und Spannung vermeidet. So 
wird Spannung mal als Potenzialdifferenz verstanden, mal werden aber auch die Begriffe „Spannung“ und „Potenzial“ synonym verwendet. Dies zeigt sich beispielsweise am gängigen Begriff des „Spannungsabfalls“ an einem Widerstand, wodurch den Schülern sprachlich suggeriert wird, in den einzelnen Leiterabschnitten vor und nach einem Widerstand existiere eine lokal messbare „Spannung“, obwohl hiermit eigentlich das Potenzial gemeint ist, das über einen Widerstand abfällt (oder sich implizit auf die „Masse“ bzw. „Erde“ als Referenzpunkt bezogen wird).

Im Rahmen der formativen Evaluation werden die didaktisch-motivierten und die eigentlich korrekten Fachbegriffe zunächst parallel verwendet, um herausfinden zu können, mit welchen Begriffen die Schüler weniger Lernschwierigkeiten haben. Nichtsdestotrotz steht in Hinblick auf das gesamte Unterrichtskonzept außer Frage, dass die für die Mittelstufe relevanten Fachbegriffe eingeführt werden müssen, um die Anschlussfähigkeit des Konzepts zu gewährleisten. Sollte die Verwendung der didaktischen Begriffe aber mit einer Reduktion der Lernschwierigkeiten einhergehen, wäre die Einführung der Fachbegriffe erst gegen Ende des Unterrichtskonzepts eine sinnvolle Maßnahme. Auf diese Weise könnte dann die Fachsprache aus den hier dem analogen Lernbereich entlehnten Begriffen erwachsen. Dies entspräche einer Überbrückungs- bzw. Umgehungsstrategie, da potenziell problematische Alltagsvorstellungen, die durch bestehende Fachbegriffe geweckt werden könnten, in der Anfangsphase des Unterrichts gezielt vermieden würden, um so möglichen Lernschwierigkeiten vorzubeugen (vgl. Abschnitt 3.1.6).

\subsubsection{Die Grenzen und die Anschlussfähigkeit des Elektronengasmodells}

Eine reflektierte Nutzung des Elektronengasmodells im Unterricht setzt eine Auseinandersetzung mit den fachlichen und didaktischen Grenzen des Modells sowie seiner Anschlussfähigkeit im weitergehenden Unterricht voraus. Da die grundsätzlichen fachlichen Grenzen des Elektronengasmodells u.a. bereits in Abschnitt 2.4 dargestellt sind, soll im Folgenden vor allem der Frage nachgegangen werden, wo unter didaktischen Aspekten potenzielle Schwierigkeiten in Folge der vorgenommenen Elementarisierungen zu erwarten sind und ob durch die Elementarisierungen die Anschlussfähigkeit an den weiteren Unterricht und die fachphysikalischen Modelle gefährdet ist. Bei der Nutzung von Modellen besteht grundsätzlich die Gefahr, dass sich Lehrende und Lernende nicht der Grenzen des Modells bewusst sind und in der Folge falsche Schlüsse in Hinblick auf den physikalischen Zielbereich ziehen (Duit und Glynn 1995, S. 8; Mikelskis-Seifert 2002). Sofern die Modellgrenzen allerdings im (ggf. weiterführenden) Unterricht thematisiert werden, kann den Lernenden hieran verdeutlich werden, dass Modelle prinzipiell nur Vorstellungshilfen sind, um komplexe Zusammenhänge leichter verstehbar zu machen, aber niemals mit völliger Sicherheit alle Aspekte der Realität abbilden können. Modellgrenzen stellen somit eine Chance dar, prinzipielle erkenntnistheoretische Aspekte im Unterricht zu thematisieren.

Eine klare Modellgrenze besteht in dem im Gegensatz zum Druck beliebig definierbaren Nullpunkt des elektrischen Potenzials. An diesem fundamentalen Unterschied der beiden 
Größen wird deutlich, dass das Konzept des elektrischen Drucks nur als eine erste Modellvorstellung auf dem Weg zu einem tieferen Verständnis des elektrischen Potenzials fungieren kann, ähnlich wie es in der Wissenschaftsgeschichte der Fall war (Steinberg 2008). Im Unterrichtskonzept führt diese Modellgrenze aber nicht zu Problemen, da ohnehin nur mit höheren und niedrigeren Drücken argumentiert wird und keine absoluten Werte für den elektrischen Druck bzw. das elektrische Potenzial eingeführt werden. Eine solche Einführung ist nicht notwendig, da der elektrische Strom entsprechend der Modellvorstellung einzig aus dem Bestreben des Druckausgleichs resultiert, wobei die absoluten Druck- bzw. Potenzialwerte irrelevant sind. Entsprechend ließe sich auf Basis des Elektronengasmodells auch die Thematik des beliebig definierbaren Potenzialnullpunktes leicht in den Unterricht integrieren, da im hier vorgeschlagenen Unterrichtskonzept besonderer Wert darauf gelegt wird, dass es die Druckunterschiede sind, die den elektrischen Strom verursachen und sogar darauf eingegangen wird, dass sich die an den Widerständen anliegenden Druckunterschiede und damit die Stromstärke auch bei einer Umpolung nicht ändern würden. Von hier aus ist es nur ein kleiner Schritt zu erkennen, dass die elektrischen Druckunterschiede bzw. die elektrische Spannung auch unabhängig von der Festlegung des Potenzialnullpunktes sind. Zudem können Wechselstromkreise innerhalb der Modellvorstellung leicht damit erklärt werden, dass ein Pol zwischen elektrischem Über- und Unterdruck oszilliert, womit im Gegensatz zu reinen Spannungsansätzen ohne Bezug zum elektrischen Potenzial auch der Frage nachgegangen werden kann, warum bei einer Steckdose nur von einem Pol eine Gefahr ausgeht (vgl. Herrmann und Schmälzle 1984, 480f).

Eine weitere Modellgrenze besteht darin, dass Lernende mit Wissen über das Verhalten idealer Gase auf die Idee kommen könnten, der Druck des Elektronengases im Leiter sei ähnlich eines idealen Gases abhängig von der Temperatur. Ein Kühlen des Drahtes würde demnach mit einer Absenkung des elektrischen Drucks einhergehen bzw. umgekehrt würde eine Erwärmung zu einer Erhöhung des elektrischen Drucks führen. Zudem muss die Stromstärke, im vorliegenden Unterrichtskonzept als „Intensität der Elektronenströmung“ eingeführt, im weiterführenden Unterricht dahingehend erweitert werden, dass sie fachlich gesehen nicht nur strömende Elektronen umfasst, sondern allgemein die pro Zeiteinheit durch einen Leiterquerschnitt fließende Menge elektrischer Ladung (vgl. Abschnitt 2.1).

Die im hier vorgeschlagenen Elektronengasmodell getroffene Vereinfachung, aus didaktischen Gründen nicht zwischen Leitungs- und Grenz- bzw. Oberflächenladungen zu unterscheiden und stattdessen nur die mittlere Elektronendichte in einem Leiterstück zu betrachten, stellt naturgemäß eine weitere Modellgrenze dar. Da die Anzahl der zusätzlichen Grenzund Oberflächenladungen im Vergleich zur Anzahl an Leitungselektronen im Leiter verschwindend gering ist, unterscheidet sich die mittlere Elektronendichte vor und nach einem Widerstand in Realität kaum voneinander. Um die Dichteunterschiede dennoch anschaulich darstellen zu können und den elektrischen Strom für Schüler nachvollziehbar in Analogie zu Luftdruckunterschieden als Folge von elektrischen Druckunterschieden erklären zu können, muss sich in der Punktedichtedarstellung die visualisierte Elektronendichte vor und nach ei- 
nem Widerstand merklich voneinander unterscheiden. Unter der Annahme einer konstanten Driftgeschwindigkeit der Elektronen vor und nach dem Widerstand würde das aber bedeuten, dass Elektronen entsprechend der Stromverbrauchsvorstellung im Widerstand verbraucht werden. Die Tatsache, dass die Stromstärke vor und nach dem Widerstand die gleiche ist, ließe sich im Modell nur so erklären, dass die Driftgeschwindigkeit der Elektronen in dem Maße zunimmt, wie die Elektronendichte abnimmt. Eine solche Annahme ist aber weder fachlich korrekt noch didaktisch hilfreich, weil sich Schüler mit der Differenzierung zwischen Driftgeschwindigkeit und Stromstärke ohnehin schwertun (vgl. Abschnitt 6.2.3.2). Im fertigen Unterrichtskonzept wird die Punktedichtedarstellung deshalb nur für die Überleitung von den Luftdruckbeispielen zu offenen Stromkreisen genutzt, da hier noch kein elektrischer Strom fließt und anschließend zur Farbdarstellung gewechselt, bei der direkt mit der Druck- statt der Dichtevorstellung argumentiert wird (vgl. Abschnitt 6.2.4.3). Soll der Widerspruch im Unterricht aufgelöst werden, muss erklärt werden, dass sich die zusätzlichen Ladungen nicht gleichmäßig auf das ganze Leitervolumen verteilen, sondern lediglich an der Oberfläche des Leiters sitzen und die Leitungselektronen im Leiterinneren eine homogene Dichteverteilung aufweisen.

Eine weitere wichtige Frage betrifft die Anschlussfähigkeit des hier vorgeschlagenen Elektronengasmodells in Hinblick auf den weiterführenden Unterricht. Vor dem Hintergrund, dass das elektrische Potenzial in der Oberstufe bei der Berechnung von Energien bzw. Arbeiten in einem Radialfeld einer Einzelladung bzw. im homogenen elektrischen Feld eines Plattenkondensators von großer Bedeutung ist, erscheint es sinnvoll, den Schülern bereits in der Mittelstufe eine erste qualitative Vorstellung dieser physikalischen Größe zu vermitteln. Im hier verwendeten Elektronengasmodell wird Bereichen mit einer hohen Elektronendichte ein hoher elektrischer Druck zugeschrieben, um die physikalische Stromrichtung plausibel erklären zu können. Fachlich gesehen ist es jedoch so, dass ein negativ geladener Körper über ein niedriges, d.h. negatives Potenzial und ein positiv geladener Körper über ein hohes, d.h. positives Potenzial verfügt. Spätestens in der Oberstufe, wenn der Begriff des elektrischen Potenzials explizit eingeführt wird statt nur mit einer prototypischen Vorstellung zu arbeiten, muss auf diese Vorzeichenproblematik eingegangen werden. Der Vorteil ist dann jedoch, dass die Lernenden bereits über eine qualitative Vorstellung des elektrischen Potenzials verfügen, an die in der Oberstufe angeknüpft werden kann.

Da die Vorzeichenproblematik beim elektrischen Potenzial ihren Ursprung in der Unterscheidung zwischen technischer und physikalischer Stromrichtung hat, ließe sich der scheinbare Widerspruch zwischen elektrischem Druck und elektrischem Potenzial bzgl. des Vorzeichens im Rahmen der Thematisierung der unterschiedlichen Stromrichtungen leicht aufklären. Überträgt man nämlich die Erklärung, wie es in Folge von elektrischen Druckunterschieden zum Strom kommt, auf die technische Stromrichtung, kann die konventionelle Farbkodierung von Plus- und Minuspol so interpretiert werden, dass früher am Pluspol ein „elektrischer Überdruck“ und am Minuspol ein „elektrischer Unterdruck“ von positiven Ladungsteilchen angenommen wurde. In diesem Bild entspräche dann ein „elektrischer Über- 
druck“ einem hohen (positiven) elektrischen Potenzial und ein „elektrischer Unterdruck“ einem niedrigen (negativen) elektrischen Potenzial. Indem nun die Unterschiede zur physikalischen Stromrichtung und den im Unterrichtskonzept getroffenen Annahmen bzgl. des elektrischen Drucks herausgearbeitet werden, fügt sich die hier vorgeschlagene Vorstellung des elektrischen Drucks und die gewählte Farbkodierung nahtlos in die ohnehin in der Physik übliche Unterscheidung zwischen technischer und physikalischer Stromrichtung ein. Somit ließe sich nicht nur der scheinbare Widerspruch bzgl. des Vorzeichens zwischen elektrischem Druck und elektrischem Potenzial auflösen, sondern auch die Anschlussfähigkeit an den weiteren Unterricht gewährleisten.

Hinsichtlich der typischerweise in Bezug auf das elektrische Potenzial in der Oberstufe behandelten Themen wie z.B. das Radialfeld einer Einzelladung oder das homogene Feld eines Plattenkondensators stellt sich aber dennoch die Frage, ob die hier vorgeschlagenen Visualisierungen des elektrischen Potenzials in einfachen Stromkreisen (vgl. Abschnitt 6.2.4.3) dazu geeignet sind, die mit dem Ort kontinuierliche Veränderung des elektrischen Potenzials im Raum gut darzustellen. Auch wenn eine solche kontinuierliche Veränderung des Potenzials z.B. mit kontinuierlichen Farbübergängen prinzipiell denkbar ist, erscheinen Höhenanalogien für solche Situationen die geeignetere Wahl zu sein. Sofern das Potenzial jedoch über weite Strecken konstant ist, wie dies z.B. bei einem idealen Leiter in einem Stromkreis der Fall ist, und sich nur an wenigen Stellen wie z.B. einer Glühlampe oder einem Widerstand abrupt ändert, erscheinen die hier vorgeschlagenen Visualisierungsformen u.a deswegen geeigneter zu sein, weil das Vorstellen von Höhenunterschieden in einer elektrischen Schaltung eine hohe Abstraktionsfähigkeit voraussetzt, mit der sich viele Schülerinnen und Schüler gerade im Anfangsunterricht vergleichsweise schwertun dürften (Muckenfuß und Walz 1997, S. 98).

Obwohl der Energiebegriff im Unterrichtskonzept nur angeschnitten wird, ließen sich mit Hilfe des Modells auch energetische Aspekte erklären. Der Energieumsatz an einem Widerstand $P=U I$ ist innerhalb der Modellvorstellung proportional abhängig von dem am Widerstand anliegenden elektrischen Druckunterschied $U$ (der Spannung) und der Intensität der Elektronenströmung I (der Stromstärke). Wird ein Ohm'scher Widerstand angenommen, ist entsprechend des Modells unmittelbar klar, dass eine Verdopplung des elektrischen Druckunterschieds zu einer Verdopplung der Intensität der Elektronenströmung führt. Die damit einhergehende Vervierfachung der umgesetzten Energie kann so aber nicht nur quantitativ, sondern innerhalb des Modells auch qualitativ verstanden werden. Hierzu muss nur argumentiert werden, dass pro Zeiteinheit nicht nur doppelt so viele Elektronen mit den Atomrümpfen stoBen und diese damit zu Schwingungen anregen wie vor der Verdopplung der Spannung, sondern, dass bei jedem dieser Stöße in Folge der doppelten Driftgeschwindigkeit der Elektronen nun auch der doppelte Impuls übertragen wird. Damit kann innerhalb des Elektronengasmodells also auch eine für Schüler plausible mikroskopische Erklärung für die Umwandlung von elektrischer zu thermischer Energie gegeben werden. Einschränkend ist an dieser Stelle je- 
doch anzumerken, dass fachlich gesehen nicht die Elektronen die Träger der Energie sind, sondern das elektromagnetische Feld in Form des Poynting-Vektors $\vec{S}=\vec{E} \times \vec{H}$.

Neben der Anschlussfähigkeit stellt auch die Entwicklungsfähigkeit ein wichtiges Merkmal für die didaktische Qualität eines Modells dar (vgl. Abschnitt 4.2). Hiermit ist gemeint, inwiefern sich Konzepte, Begriffe und Phänomene, die im Rahmen des hier vorgestellten Elektronengasmodells nicht thematisiert werden, durch Erweiterung der Modellvorstellungen widerspruchsfrei erklären lassen. Zu nennen ist hier beispielsweise die Einführung des elektrischen Potenzials im Raum um eine Ladung. Steinberg (2004, 140ff) erweitert hierfür das Modell des elektrischen Drucks im CASTLE-Curriculum dadurch, dass in Analogie zu einem „temperature halo“ um eine Kerzenflamme ein sogenannter ,electric pressure halo“ um eine Ladung existiere, der die Fähigkeit habe, in leitenden Materialien einen entsprechenden elektrischen Druck bzw. ein entsprechendes Potenzial zu bewirken:

„A pressure halo in empty space around charge is not a region where actual electric pressure exists - because there is no matter for pressure to exist in. Instead, a pressure halo has a property with the potential to do what electric pressure does. This property behaves exactly like actual electric pressure in any conducting body that is placed in the halo. The term "potential electric pressure" can capture the idea of a condition in space that acts like actual electric pressure in matter. For historical reasons, however, the term 'electric potential' has become standard usage among scientists. “ (Steinberg 2004, S. 149)

Seine Anschlussfähigkeit und vergleichsweise große Erklärungsmächtigkeit stellen einen klaren Mehrwert gegenüber didaktisch problematischen Modellen wie z.B. dem Rucksackmodell dar (vgl. Abschnitt 4.3). Mit seiner mikroskopischen Modellanschauung befriedigt das Elektronengasmodell zudem das Bedürfnis der Lernenden nach tiefergehenden Erklärungen am eigentlichen Lernobjekt (vgl. Kircher 1984, S. 58; Galili und Goihbarg 2005). Zu den weiteren Stärken des Modells zählt, dass auch Übergangsprozesse z.B. nach dem Ein- oder Ausschalten bis zur Einstellung eines stationären Gleichgewichtszustands diskutiert werden können, auf Oberflächenladungen eingegangen werden kann und auch Lade- und Entladevorgänge von Kondensatoren mit Hilfe des Modells erklärt werden können. All dies spricht dafür, dass Lernende mit Hilfe des Elektronengasmodells bereits in der Mittelstufe ein vergleichsweise tiefes qualitatives Verständnis der Elektrizitätslehre entwickeln können. Abschließend soll eine Bewertung des hier vorgestellten Elektronengasmodells anhand der in Abschnitt 4.2 dargelegten Kriterien an ein didaktisch gutes Modell vorgenommen werden:

1. Fachliche Relevanz: Das hier vorgestellte Elektronengasmodell hat nicht den gleichen Geltungsbereich wie ein fachliches Modell, was aber vor dem Hintergrund unproblematisch ist, dass es nur ein elementares Verständnis der wechselseitigen Beziehungen von Spannung, Stromstärke und Widerstand im Anfangsunterricht ermöglichen soll. Die im Modell vermittelten Vorstellungen sind für diesen Zweck angemessen und fachlich relevant. Das Kriterium kann deshalb als erfüllt betrachtet werden. 
2. Entwicklungsfähigkeit: Wie in diesem Abschnitt dargelegt, ist das hier vorgeschlagene Elektronengasmodell trotz seiner fachlichen und didaktischen Modellgrenzen an den weiterführenden Unterricht anschlussfähig und auch selbst entwicklungsfähig. Da die aus didaktischen Gründen vorgenommenen Elementarisierungen einer späteren fachlichen Erweiterung des Modells nicht im Wege stehen, kann dieses Kriterium als erfüllt angesehen werden.

3. Assoziabilität: Die vorgenommenen Elementarisierungen z.B. in Bezug auf die Widerstandsvorstellung (Elektronen stoßen mit Atomrümpfen vs. Streuung von Elektronenwellen an Gitterfehlern) sowie das elektrische Potenzial (als elektrischer Druck in Folge einer Ungleichverteilung von Leitungselektronen) entsprechen nicht der aktuellen fachlichen Modellvorstellung und können nur einen ersten Schritt darstellen, die Phänomene in einfachen Stromkreisen mit Hilfe einer Modellvorstellung verständlich zu machen. Das Kriterium der Assoziabilität ist deshalb nur eingeschränkt erfüllt.

\subsection{8 Übersicht über die zu evaluierende grundlegende Sachstruktur}

Vor dem Hintergrund der oben dargelegten didaktischen Überlegungen wurde die untenstehende grundlegende Sachstruktur entwickelt, die im Rahmen einer formativen Evaluation mittels Teaching Experiments, auch Akzeptanzbefragungen genannt, mit einzelnen Lernenden erprobt wurde (siehe Abschnitt 6.2). Die grundlegende Sachstruktur zeichnet sich neben einer expliziten Einführung einer mikroskopischen Modellvorstellung von Leitungsvorgängen insbesondere durch einen kurzen „Hands-on-Vorkurs“ zu Luftdruckphänomenen und der expliziten Einführung des elektrischen Potenzials vor dem Spannungs- und Stromstärkebegriff als „Elektronendruck“ aus. Bestimmte Ideen wie die Verwendung einer Spritze als MiniaturLuftpumpe, um den Lernenden Primärerfahrungen mit Luftdruckphänomenen zu ermöglichen, sowie die Argumentation mit einem elektrischen Druck in Folge einer Ladungsungleichverteilung im Stromkreis gehen dabei auf unveröffentlichte Überlegungen von $\mathrm{H}$. Wiesner aus dem Jahr 2013 zurück. Eine Übersicht über die grundlegende Sachstruktur ist nachfolgend dargestellt. Die in den Teaching Experiments genutzten Materialien können auf der Internetseite JP-Bur.de/Dissertation heruntergeladen werden.

\section{1) Elektrostatik}

- Es gibt zwei Ladungsarten: positive und negative Ladungen.

- Gleichnamige Ladungen stoßen sich ab, ungleichnamige Ladungen ziehen sich an.

- Die Anziehungs- bzw. Abstoßungskraft ist abstandsabhängig.

\section{2) Atomvorstellung}

- Metalle bestehen aus ortsfesten, positiv geladenen Atomrümpfen und freibeweglichen, negativ geladenen Elektronen.

- Ein negativ geladener Körper enthält mehr Elektronen als Atomrümpfe, ein positiv geladener Körper weniger Elektronen als Atomrümpfe und ein neutraler Körper die gleiche Anzahl an Atomrümpfen und Elektronen. 


\section{3) Der Luftdruck}

- $\quad$ Einführung des Luftdrucks und der Begriffe Überdruck, Normaldruck und Unterdruck.

- Luftströmungen als Folge von Druckunterschieden: Luft strömt immer von höherem zu niedrigerem Druck.

- Schülerexperimente, um den Zusammenhang Kompression - Luftdruck sowie Druckunterschied - Luftströmung erfahrbar zu machen.

\section{4) Das Potenzial als elektrischer Druck}

- Elektronen können sich in einem Metall so frei bewegen wie „Luftteilchen“ in einem Schlauch.

- Die Batterie sorgt für einen hohen elektrischen Druck am Minuspol und einen geringen elektrischen Druck am Pluspol.

- Der elektrische Druck eines Leiterstücks passt sich dem elektrischen Druck des jeweiligen Batteriepols, mit dem es verbunden wird, an.

- Einführung des Potenzialbegriffs als Fachbegriff für den elektrischen Druck.

\section{5) Die Spannung als elektrischer Druckunterschied}

- Einführung der elektrischen Spannung als elektrischer Druckunterschied und Ursache von Elektronenströmungen.

- Einführung eines Lämpchens als elektrischer Widerstand, der die Elektronenströmung behindert.

- Einführung des Konzepts des Stromkreises mit der Batterie als Elektronenpumpe bei gleichzeitiger Betonung, dass keine Elektronen verbraucht werden.

\section{6) Vertiefungsaufgaben}

- Kurze Einführung des Stromstärkebegriffs

- Einfluss des Widerstands: Diskussion von Spannung und Stromstärke bei Lämpchen mit unterschiedlich großen Widerständen in einem einfachen Stromkreis.

- Parallelschaltungen: Diskussion der Frage, welchen Einfluss ein bzw. zwei gleiche bzw. ungleiche parallelgeschaltete Lämpchen auf die Potenziale und die Stromstärke in den Haupt- und Parallelzweigen haben.

- Reihenschaltungen: Diskussion der Frage, welchen Einfluss zwei bzw. drei in Reihe geschaltete Lämpchen auf die Potenziale und die Stromstärke in den verschiedenen Leiterabschnitten haben. 


\subsection{Formative Evaluation der grundlegenden Konzeption}

\subsubsection{Die Methode der Teaching Experiments}

Eine zentrale Aufgabe der physikdidaktischen Forschung war und ist es, Lernschwierigkeiten und Schülervorstellungen in den verschiedenen Bereichen der Physik zu identifizieren und zu analysieren und anschließend Wege zu entwickeln, die Schülern das Verständnis der wissenschaftlichen Perspektive im Physikunterricht erleichtern. Im Sinne von Design-BasedResearch (vgl. Abschnitt 7.1) soll daher vor der Entwicklung und Evaluation eines umfassenden Unterrichtskonzepts mit entsprechenden Unterrichtsmaterialien zunächst untersucht werden, inwiefern Schüler die Grundideen der angedachten Konzeption verstehen und welche Darstellungsform des elektrischen Drucks dabei von den Schülern als am verständlichsten empfunden wird. Angestrebt wird, einen Einblick in die Lernprozesse der Schüler zu bekommen und mögliche Lernschwierigkeiten zu identifizieren, um die so gesammelten Erkenntnisse dann später in ein umfassendes Unterrichtskonzept inklusive passender Unterrichtsmaterialien auf Grundlage des in Abschnitt 6.1 dargelegten Elektronengasmodells einfließen lassen zu können.

Zur Erreichung dieser Ziele hat sich die Methode der Akzeptanzbefragung angeboten, die Mitte der 1980er Jahre in Frankfurt von Jung und Wiesner entwickelt wurde und sich als sehr effektiv zur Entwicklung erfolgreicher Unterrichtsansätze erwiesen hat (Wiesner et al. 2010). Die Akzeptanzbefragung, die heute auch unter dem Begriff „Teaching Experiments“ bekannt ist, stellt eine qualitative fachdidaktische Forschungsmethode zur gezielten Erprobung von neuen Lehrkonzeptionen dar (Jung 1992; Wiesner 1993). Ziel ist es, mit Hilfe von Lehr-Lerninterviews mit einzelnen Schülern unter möglichst kontrollierten Bedingungen einen neuen physikalischen Erklärungsansatz darauf hin zu überprüfen, inwiefern er von den Schülern akzeptiert und verstanden wird. Mit anderen Worten geht es also darum, die Reaktionen der Lernenden auf Instruktionen zu untersuchen, die auf der Basis des Wissens über Schülervorstellungen und Lernschwierigkeiten entwickelt wurden (Wiesner et al. 2010). Dabei übernimmt der Lehrende in der Regel gleichzeitig die Rolle des Forschers, der die Schülerreaktionen auf die Lernangebote erfasst und analysiert, um bereits verständliche Lernangebote zu identifizieren und potenzielle Lernhemmnisse aufzudecken, um so Ansatzpunkte zur Verbesserung des Konzepts zu finden. Um die Lernprozesse der Schüler qualitativ möglichst detailliert und umfassend analysieren zu können, beschränkt man sich dabei in der Regel auf eine geringe Anzahl an Schülern. Die Akzeptanzbefragung hat also einen explorativen Charakter. Die wesentlichen Unterschiede gegenüber dem regulären Unterricht sieht Wodzinski (1996, S. 42) vor allem in folgenden Punkten:

- Es findet keine Kommunikation zwischen den Lernenden statt.

- Die fachlichen Inhalte werden stärker komprimiert vermittelt.

- Die befragten Schüler sind konzentrierter und stärker motiviert.

- Es kann besser auf individuelle Schwierigkeiten der Lernenden eingegangen werden. 
Der Vorteil von Teaching Experiments gegenüber rein problemorientierten Befragungen der klassischen Schülervorstellungsforschung besteht u.a. darin, dass Schüler aufgrund der zuvor gegebenen Erklärungen zu weniger spontan generierten Ad-Hoc-Erklärungen neigen, die sich sonst im Laufe der Befragung oftmals erst zu elaborierten Schülervorstellungen entwickeln (Wiesner und Wodzinski 1996). In der physikdidaktischen Forschung wurden Teaching Experiments bereits in diversen Gebieten zur Untersuchung von Lernprozessen eingesetzt und führten neben grundlegenden Erkenntnissen zu Lernschwierigkeiten insbesondere zu zahlreichen erfolgreichen Unterrichtsansätzen (z.B. Wodzinski 1996; Gleixner 1998; Haagen-Schützenhöfer 2016; Wiener 2017). Im Sinne des hier verfolgten Design-Based-Research Forschungsansatzes bietet sich die Methode der Akzeptanzbefragung also als ideales Instrument an, um vor einer umfangreicheren Evaluation des Unterrichtskonzepts grundlegende Verständnisprobleme als auch geeignete p-prims für eine erfolgreiche Konzeptentwicklung im Sinne von diSessa (2013) zu identifizieren (vgl. Abschnitt 3.1.5). Die so in einem ersten formativen Zyklus aus Design und Analyse gewonnen Erkenntnisse stellen dann die Grundlage für ein Re-Design des Lernangebots und die Entwicklung eines umfassenden Unterrichtskonzepts für die Schulpraxis dar. Nach Blumör (1993) können Akzeptanzbefragungen bzw. Teaching Experiments dabei u.a. die folgenden Aspekte umfassen (vgl. Wodzinski 1996, S. 41):

1. Informationsangebot: Der Interviewer erklärt dem Lernenden in mündlicher Form ggf. unterstützt durch Grafiken und Experimente einen physikalischen Sachverhalt.

2. Frage nach der Akzeptanz: Der Lernende gibt Auskunft darüber, inwiefern die Erklärung seiner Meinung nach verständlich bzw. plausibel ist.

3. Paraphrasierung: Der Lernende gibt mit eigenen Worten die gehörte Erklärung wieder, während der Interviewer auf Auslassungen und Transformationen achtet und diese notiert.

4. Anwendung und Übung: Im letzten Schritt besteht die Aufgabe des Befragten darin, das neu Gelernte auf ein passendes Beispiel anzuwenden.

Um eine detaillierte Auswertung der Teaching Experiments zu ermöglichen, wurden alle Gespräche mit den Schülern mit Hilfe von Aufnahmegeräten aufgezeichnet und anschließend transkribiert, d.h. durch Abtippen in Schriftform übertragen. Die Transkription ist notwendig, um die Aussagen der Schüler sinnvoll analysieren und miteinander vergleichen zu können und so beispielsweise gemeinsame Lernschwierigkeiten besser identifizieren zu können. Ein Problem, das jedem Transkript inhärent ist, besteht im Spannungsfeld zwischen Detailtreue und Lesbarkeit, da ein Transkript eine Gesprächssituation naturgemäß nie völlig erfassen kann. Vor der Transkription muss also entschieden werden, wie detailgetreu und facettenreich das Gespräch in Schriftform festgehalten werden soll, um einerseits eine angemessene Rekonstruktion der Teaching Experiments zu ermöglichen, anderseits aber - nicht zuletzt aus Lesbarkeits- und Zeitgründen - genaue Details zur Aussprache wie beispielsweise 
Sprechgeschwindigkeit, Lautstärke oder gar nonverbale Aspekte bei der Transkription auszusparen.

Vor dem Hintergrund der mit den Teaching Experiments verbundenen physikdidaktischen Fragestellungen lag der Fokus der Transkription darauf, dem Leser eine spätere detailgetreue Rekonstruktion der Gesprächsinhalte zu ermöglichen, während darüberhinausgehende Aspekte wie umgangssprachliche Ausdrücke oder Dialekte im Sinne einer guten Lesbarkeit „geglättet“ wurden. Die Priorität bei der Transkription lag also auf dem, was gesagt wurde und nicht, wie es gesagt wurde. Somit ergeben sich in Anlehnung an Dresing und Pehl (2015, S. 21) folgende Transkriptionsregeln für die Audioaufnahmen:

1. Der Interviewer wurde mit dem Wort „Interviewer" gekennzeichnet und der interviewte Schüler mit dem Wort „Schüler“،. Auf das Einfügen von Zeitmarken wurde verzichtet.

2. Die Audioaufnahmen wurden wörtlich transkribiert, d.h. nicht zusammenfassend oder lautsprachlich. Ggf. genutzte Dialekte wurden möglichst wortgetreu ins Hochdeutsche übersetzt.

3. Umgangssprachliche Ausdrücke und Wortverkürzungen wurden dem Schriftdeutsch angenähert.

4. Wort- und Satzabbrüche sowie Stottern wurden in den meisten Fällen geglättet bzw. ausgelassen. Ausnahme: Es kam zu einer auffälligen Häufung solcher sprachlichen Probleme.

5. Bedeutsame Sprechpausen wurden durch drei Punkte (...) verschriftlicht.

6. Unklare Äußerungen des gerade nicht Sprechenden wie „aha, ja, ähm“, die Verständnis oder Unverständnis zum Ausdruck bringen, wurden nicht transkribiert, es sei denn, die jeweilige Äußerung stellte eine eigenständige Antwort dar.

7. Gesprächsabschnitte, die keinen Bezug zu den mit den Teaching Experiments verbundenen Fragestellungen aufweisen, wurden nicht transkribiert. In solchen Fällen wurde im Transkript in eckigen Klammern das Thema des nicht erfassten Gesprächsabschnitts kursiv vermerkt.

8. Auf die Erfassung non-verbaler Äußerungen wurde aus Zeit- und Lesbarkeitsgründen verzichtet.

Die angefertigten Transkriptionen stehen interessierten Lesern auf der Internetseite JP-Bur.de/Dissertation zur Verfügung.

\subsubsection{Beschreibung der Teaching Experiments}

Die Teaching Experiments hatten einen zeitlichen Umfang von ca. drei bis vier Schulstunden und wurden vom Autor selbst mit neun Lernenden unterschiedlicher Klassen in Einzelgesprächen im Sommer 2014 durchgeführt und anschließend transkribiert. Alle Teilnehmer der Akzeptanzbefragung waren in der sechsten Jahrgangsstufe eines Frankfurter Gymnasiums und 
hatten nach eigener Aussage sowie Auskunft der Fachlehrkräfte keine nennenswerten Vorkenntnisse zu elektrischen Stromkreisen, da in ihrem bisherigen naturwissenschaftlichen Unterricht die Elektrizitätslehre noch nicht behandelt wurde. Für die Teaching Experiments war dies eine wichtige Voraussetzung, da untersucht werden sollte, inwiefern die grundlegende Sachstruktur und die Visualisierungen des Elektronendrucks von Schülern ohne Vorkenntnisse grundsätzlich akzeptiert und verstanden werden. Ein weiteres Anliegen der Befragung war es, trotz der geringen Anzahl an befragten Schülern einen möglichst breiten Querschnitt aus dem Spektrum der Schülerschaft zu befragen. Dies wurde erreicht, indem für die Teilnahme an der Befragung eine finanzielle Vergütung angeboten wurde und so ein großer Pool an Interessenten zur Verfügung stand. Aus diesem Pool an Interessenten wurden dann durch Rücksprache mit den naturwissenschaftlichen Fachlehrern drei leistungsschwache, drei mittelmäBige und drei leistungsstarke Schüler ausgewählt. Tab. 6 zeigt, wie die Leistungsfähigkeit der einzelnen Schüler von den Fachlehrern eingeschätzt wurde, wobei leistungsstarke Schüler in den folgenden Abschnitten mit drei Sternchen, mittelmäßige Schüler mit zwei Sternchen und leistungsschwache Schüler mit einem Sternchen gekennzeichnet sind.

Tab. 6: Übersicht über die von den Lehrkräften eingeschätzte Leistungsfähigkeit der teilnehmenden Schüler

\begin{tabular}{|lll|}
\hline $\begin{array}{l}\text { eingeschätzt als } \\
\text { leistungsstark }\end{array}$ & $\begin{array}{l}\text { eingeschätzt als } \\
\text { mittelmäßig }\end{array}$ & $\begin{array}{l}\text { eingeschätzt als } \\
\text { leistungsschwach }\end{array}$ \\
\hline Schüler A *** & Schüler D ** & Schüler G* \\
\hline Schüler B *** & Schüler E ** & Schüler H* \\
\hline Schüler C $* * *$ & Schüler F $* *$ & Schüler I $*$ \\
\hline
\end{tabular}

Da mit Hilfe der Teaching Experiments einerseits die grundsätzliche Umsetzbarkeit der Unterrichtskonzeption überprüft und andererseits mögliche dabei auftretenden Lernschwierigkeiten identifiziert werden sollten, ergaben sich die folgenden drei Fragestellungen:

1. Akzeptieren und verstehen die Schüler die Grundideen hinter dem Elektronengasmodell?

2. Mit welchen Lernschwierigkeiten ist in einem weitergehenden Unterrichtskonzept auf Basis des Elektronengasmodells zu rechnen?

3. Welche der vier entwickelten Visualisierungen des elektrischen Potenzials empfinden die Schüler am verständlichsten?

Entsprechend den didaktischen Überlegungen zur grundlegenden Sachstruktur des zukünftigen Unterrichtskonzepts (vgl. Abschnitt 6.1.8) gliederte sich die Akzeptanzbefragung in sechs Einheiten, wobei das Vorgehen bei den ersten fünf Einheiten typisch für eine Akzeptanzbefragung war. Jede dieser Einheiten entsprach jeweils einem abgeschlossenen Themenblock, an den sich jeweils Fragen zur Akzeptanz (,Wie findest du diese Erklärung der Naturwissenschaftler? Ist daran irgendetwas komisch oder unverständlich?“) und eine Aufforderung zur Paraphrasierung (,Kannst du mit deinen eigenen Worten wiederholen, was die Naturwissenschaftler über Ladungen herausgefunden haben?") sowie Anwendungsaufgaben 
anschlossen. An eine kurze Erläuterung des Stromstärkebegriffs schlossen sich in der sechsten Einheit weiterführende Vertiefungsaufgaben u.a. zu Reihen- und Parallelschaltungen an. Ziel und Anspruch dieser letzten Einheit bestand nicht darin, den Schülern die gesamte elementare Elektrizitätslehre in wenigen Schulstunden zu vermitteln, sondern auch erste Hinweise darauf zu erhalten, wo mögliche Lernschwierigkeiten eines Unterrichtskonzeptes bei weitergehenden Themen zu erwarten sind.

\subsubsection{Auswertung der Teaching Experiments}

\subsubsection{Auswertung der Akzeptanz der Grundideen}

Die transkribierten Teaching Experiments stellen aufgrund ihres explorativen Charakters ein äußerst umfangreiches und komplexes Ausgangsmaterial dar. Um die erste der drei mit den Teaching Experiments verbundenen Fragestellungen (,Akzeptieren und verstehen die Schüler die Grundideen hinter dem Elektronengasmodell?“) klären zu können, wurde ein für Akzeptanzbefragungen typisches schrittweises Vorgehen gewählt (vgl. Blumör 1993). In einem ersten Schritt wurde die grundsätzliche Akzeptanz zentraler Ideen des Elektronengasmodells untersucht, bevor in einem zweiten Schritt geschaut wurde, inwiefern die Schüler in der Lage sind, die Grundideen der zuvor gehörten Erklärung eigenständig wiederzugeben. In einem dritten Schritt wurde dann die Fähigkeit der Lernenden bewertet, mit Hilfe der neu erlernten Konzepte entsprechende Anwendungsaufgaben lösen zu können.

Bei allen drei zur ersten Fragestellung „Akzeptieren und verstehen die Schüler die Grundideen hinter dem Elektronengasmodell?“ gehörenden Schritten wurden dabei jeweils zunächst gezielt diejenigen Passagen aus dem Interviewmaterial extrahiert, in denen sich die Schüler zur entsprechenden Fragestellung äußerten. Die Schülerantworten wurden dann auf Basis eines zuvor ausgearbeiteten Kodierleitfadens mit Hilfe eines ordinalskalierten Kategoriensystems (vgl. skalierende Strukturierung nach Mayring 2010, S. 92) kodiert. Um eine möglichst hohe Reliabilität dieser Kodierung zu gewährleisten, wurden alle zu einer Fragestellung gehörenden Kodierungen dann untereinander querschnittartig verglichen und zuvor getroffene Kodierungen ggf. angepasst. Für drei der neun Schüler wurden die nun querschnittsvalidierten Kodierungen dann von einer unabhängigen Person mit Hilfe des gleichen Kodierschemas noch einmal kodiert und die Interrater-Reliabilität bestimmt, die ein Maß der Zuverlässigkeit der so getroffenen Kodierungen darstellt. Mit einem Cohens-KappaKoeffizient von $\mathrm{K}=.74$ zeigte sich dabei eine gute Übereinstimmungsgüte der beiden Ratings (Fleiss und Cohen 1973). Im Folgenden soll das Vorgehen zur Analyse der Akzeptanz, Paraphrasierungen und Anwendungsaufgaben im Rahmen der ersten mit den Teaching Experiments verbundenen Fragestellung, „Akzeptieren und verstehen die Schüler die Grundideen hinter dem Elektronengasmodell?“" erklärt und die gefundenen Ergebnisse dargestellt werden.

Um die Akzeptanz der grundlegenden Ideen des Elektronengasmodells durch die Lernenden zu untersuchen, wurden die entsprechenden Textstellen im Interviewmaterial auf einer dreistufigen Skala kodiert. Dabei wurde im Kodierleitfaden zwischen vollständiger Akzep- 
tanz, eingeschränkter Akzeptanz und keiner Akzeptanz der vorgetragenen Erklärungen unterschieden, die wie folgt definiert wurden:

- Vollständige Akzeptanz: Die durch den Interviewer vorgetragene Erklärung wird von dem Schüler ohne Einschränkungen akzeptiert bzw. dieser liefert von sich aus eine sehr gute Zusammenfassung der Erklärung.

- Eingeschränkte Akzeptanz: Es wird Kritik an einzelnen Aspekten der Erklärung geäußert oder diese wird nur unter Einschränkungen akzeptiert. Dennoch kommt seitens des Schülers grundsätzliche Akzeptanz der Erklärung zum Ausdruck bzw. dieser fasst von sich aus die Erklärung zufriedenstellend zusammen.

- Keine Akzeptanz: Die Erklärung wird von dem Schüler für zu schwer erachtet bzw. nicht verstanden oder der Schüler antwortet nicht auf die Frage und liefert auch von sich aus keine sinnvolle Zusammenfassung der Erklärung.

Exemplarische Interviewpassagen zu jeder der drei Akzeptanzstufen finden sich in Abschnitt 13.1 des Anhangs. Die auf diese Weise gefundenen Ergebnisse sind in Tab. 7 zusammengefasst, wobei eine völlige Akzeptanz mit Grün bzw. dem Zahlenwert 0, eine eingeschränkte Akzeptanz mit Gelb bzw. dem Zahlenwert 0,5 und keine Akzeptanz der Erklärung durch die Schüler mit Rot bzw. dem Zahlenwert 1 kodiert wurde. Um zusätzlich Aussagen über die durchschnittliche Akzeptanz einzelner Schüler bzw. Einheiten treffen zu können, wurde auf Basis dieser Zahlenwerte ein Mittelwert zu jedem Schüler und zu jeder Einheit bestimmt. Für die Einheit „Elektrostatik“ wurde so beispielsweise ein durchschnittlicher Akzeptanzgrad von 0,2 ermittelt, wobei 0,0 dem höchsten Akzeptanzgrad und 1,0 dem niedrigsten Akzeptanzgrad entspricht. Die Anzahl der Sterne in der Schülerspalte steht für die vom Physiklehrer eingeschätzte Leistungsfähigkeit der Schüler, wobei leistungsstarke Schüler mit drei, mittelmäßige Schüler mit zwei und leistungsschwache Schüler mit einem Sternchen gekennzeichnet sind.

Tab. 7: Übersicht über den Grad der Akzeptanz der verschiedenen Erklärungen durch die Schüler

\begin{tabular}{l|rrrrr|r} 
Schüler & Elektrostatik & Atomvorst. & Luftdruck & elek. Druck & Spannung & Mittelwert \\
\hline A *** & 0 & 0 & 0 & 0 & 0 & 0,0 \\
B *** & 0,5 & 0 & 0,5 & 0,5 & 0,5 & 0,4 \\
C *** & 0 & 0 & 0 & 0 & 0 & 0,0 \\
D** & 0 & 0 & 0 & 0,5 & 0 & 0,1 \\
E** & 1 & 0 & 0 & 1 & 0,5 & 0,5 \\
F** & 0 & 0 & 0 & 0,5 & 0 & 0,1 \\
G* & 0 & 0 & 0 & 0 & 0 & 0,0 \\
H $^{*}$ & 0,5 & 0 & 0 & 0,5 & 1 & 0,4 \\
I $^{*}$ & 0 & 0 & 0 & 0 & 0 & 0,0 \\
\hline Mittelwert & 0,2 & 0,0 & 0,1 & 0,3 & 0,2 &
\end{tabular}

Die Akzeptanzbefragung zeigt, dass die Schüler mit den Grundkonzepten hinter dem Elektronengasmodell wie der Elektrostatik sowie insbesondere der Atom- und Luftdruckvor- 
stellung keine nennenswerten Schwierigkeiten zu haben scheinen und die hierzu gehörten Erklärungen weitgehend akzeptieren. Die im Zusammenhang mit der anfänglichen Einführung in die Elektrostatik geäußerte Kritik bezog sich insbesondere auf die Erklärung elektrisch neutraler Körper mit Hilfe elektrischer Ladungen. Da diese Erklärung bereits Wissen über einzelne Ladungen aus der zweiten Einheit „Atomvorstellung“ voraussetzt, ist die an dieser Stelle von den Schülern geäußerte Kritik durchaus nachvollziehbar. Volle Akzeptanz zeigte sich für die Erklärung der Atomvorstellung, bei der den Schülern eine Vorstellung von freibeweglichen, sich gegenseitig abstoßenden Elektronen und ortsfesten Atomrümpfen in Metallen vermittelt wurde. Auch die Erklärung zum Luftdruck und dass Luftströmungen immer eine Folge von Druckunterschieden sind, wurde von acht der neun Schüler vollständig akzeptiert. Als besonders hilfreich wurden hier von den Schülern die mit Spritzen als Miniatur-Luftpumpen durchgeführten Experimente zum Luftdruck bewertet, da hierbei die intuitive aber für das Konzept zentrale Vorstellung ,komprimierte Luft steht unter Druck, drückt gegen die Wände und hat das Bestreben sich auszudehnen" für die Schüler erfahrbar wurde und diese spüren konnten, dass Luft von Bereichen hohen zu Bereichen niedrigen Drucks strömt. Die Erklärung des elektrischen Drucks hat sich hingegen als problematischer erwiesen und stieß bei vier Schülern nur auf eingeschränkte Akzeptanz und wurde von einem Schüler sogar gänzlich abgelehnt. Viele der Schüler gaben an, sich durch die Vielzahl an neuen Informationen überfordert gefühlt zu haben, was vermutlich mit der verhältnismäßig geringen Anschaulichkeit der gegebenen Erklärung zusammenhängt. Anders als bei den anderen Einheiten wurden hier nämlich zunächst keinerlei Illustrationen verwendet, um eine mögliche Beeinflussung der Schüler hinsichtlich der dritten Fragestellung ,Welche der vier entwickelten Visualisierungen des elektrischen Potenzials empfinden die Schüler am verständlichsten?“" zu vermeiden. Die Erklärung der Spannung als elektrischer Druckunterschied wurde von sechs der neun Schüler völlig und von zwei Schülern eingeschränkt akzeptiert, während sie von einem Schüler abgelehnt wurde. Sofern von den Schülern Kritik an der Erklärung geäußert wurde, spiegelte sich in ihr keine grundsätzliche Ablehnung der Erklärung wider, sondern hauptsächlich ein Unbehagen mit der Vielzahl an neuen Informationen. Ein abschließender Blick auf die Gesamtakzeptanz der gegebenen Erklärungen zeigt, dass einerseits keine Einheit auf besonders große Ablehnung bei den Schülern stieß und andererseits sechs der neun Schüler mit den Erklärungen keine oder nahezu keine Schwierigkeiten hatten. Einzig Schüler B***, E** und $\mathrm{H}^{*}$ äußerten mit einem durchschnittlichen Akzeptanzgrad zwischen 0,4 und 0,5 in nennenswertem Umfang Unzufriedenheit mit den gegebenen Erklärungen. Ein Zusammenhang zwischen dem durchschnittlichen Akzeptanzgrad der Schüler und der vom Physiklehrer eingeschätzten Leistungsfähigkeit der Schüler kann dabei nicht beobachtet werden.

Nachdem die Schüler nach ihrer grundsätzlichen Akzeptanz der Erklärungen gefragt wurden, sollten sie in einem zweiten Schritt die zuvor gehörte Erklärung in eigenen Worten wiedergeben. Die entsprechenden Paraphrasierungen der Schüler wurden auf einer dreistufigen Skala kodiert, wobei entsprechend des Kodierleitfadens zwischen einer gelungenen Paraphrasierung, einer befriedigenden Paraphrasierung und einer mangelhaften Paraphrasierung unterschieden wurde. Im Abschnitt 13.2 des Anhangs findet sich der für die Kodierungen 
verwendete Erwartungshorizont sowie beispielhaft eine gelungene Paraphrasierung zu jeder Einheit. Die einzelnen Kategorien waren dabei wie folgt definiert:

- Gelungene Paraphrasierung: Die Paraphrasierung ist inhaltlich korrekt. Auch wenn dem Schüler nicht alle Fachbegriffe einfallen, fasst er doch die wesentlichen konzeptionellen Aussagen korrekt zusammen.

- Befriedigende Paraphrasierung: Die Paraphrasierung fasst die zuvor gegebene Erklärung größtenteils korrekt zusammen, allerdings wurden einzelne Aspekte inhaltlich falsch wiedergegeben.

- Mangelhafte Paraphrasierung: Die Paraphrasierung ist größtenteils falsch bzw. der Schüler ist nicht in der Lage, eigenständig eine angemessene Paraphrasierung der Erklärung zu geben.

Bei der Kodierung der Paraphrasierungen wurde dabei nur die Wiedergabe der wesentlichen Inhalte der zuvor gehörten Erklärung gewertet, nicht aber das Verständnis der Schüler von den dazugehörigen Konzepten, da die Überprüfung des Verständnisses mit Hilfe von Anwendungsaufgaben erfolgt. Die so gefundenen Bewertungen der Paraphrasierungen sind in Tab. 8 zusammengefasst, wobei eine gelungene Paraphrasierung mit Grün bzw. dem Zahlenwert 0, eine befriedigende Paraphrasierung mit Gelb bzw. dem Zahlenwert 0,5 und eine mangelhafte Paraphrasierung mit Rot bzw. dem Zahlenwert 1 kodiert wurde. Um auch hier ein Maß für die durchschnittliche Qualität der Paraphrasierungen eines Schülers bzw. einer Einheit zur Verfügung zu haben, wurde auf Basis der Zahlenwerte ein Mittelwert zu jedem Schüler bzw. zu jeder Einheit bestimmt. Für die Einheit „Spannung“ wurde so z.B. eine durchschnittliche Paraphrasierungsqualität von 0,3 ermittelt, wobei 0,0 einer sehr hohen und 1,0 einer sehr niedrigen Paraphrasierungsqualität entspricht. Die Anzahl der Sterne in der Schülerspalte steht auch hier für die vom Fachlehrer eingeschätzte Leistungsfähigkeit der Schüler, wobei leistungsstarke Schüler mit drei, mittelmäßige Schüler mit zwei und leistungsschwache Schüler mit einem Sternchen gekennzeichnet sind.

Tab. 8: Übersicht über die Bewertung der Paraphrasierungen

\begin{tabular}{l|rrrrr|r} 
Schüler & Elektrostatik & Atomvorst. & Luftdruck & elek. Druck & Spannung & Mittelwert \\
\hline A *** & 0 & 0,5 & 0 & 0 & 0 & 0,1 \\
B *** & 0 & 0 & 1 & 1 & 1 & 0,6 \\
C *** & 0 & 0 & 0 & 0 & 0 & 0,0 \\
D ** & 0 & 0,5 & 0 & 0,5 & 0 & 0,2 \\
E** & 0,5 & 0 & 0,5 & 0,5 & 0 & 0,3 \\
F** & 0 & 0 & 0 & 0 & 0 & 0,0 \\
G* & 0 & 0 & 0 & 0 & 0,5 & 0,1 \\
H* & 1 & 0,5 & 1 & 1 & 1 & 0,9 \\
I* & 0 & 0 & 0 & 0,5 & 0,5 & 0,2 \\
\hline Mittelwert & 0,2 & 0,2 & 0,3 & 0,4 & 0,3 &
\end{tabular}

Bei den Paraphrasierungen zeigt sich im Großen und Ganzen ein ähnliches Bild wie bei der Akzeptanz der Erklärungen. Im Durchschnitt gelang einem Großteil der Schüler die 
Paraphrasierung der Erklärungen verhältnismäßig gut. Einzig Schüler $\mathrm{B} * * *$ und $\mathrm{H}^{*}$ hatten große Schwierigkeiten, die zentralen Aussagen der zuvor gehörten Erklärungen in ihren eigenen Worten angemessen wiederzugeben, was insbesondere bei Schüler B***, der als leistungsstark eingeschätzt wurde, überrascht. Während Schüler B*** die anfänglichen Erklärungen zur Elektrostatik und Atomvorstellung noch angemessen paraphrasieren konnte, scheint insbesondere Schüler $\mathrm{H}^{*}$ von Anfang an mit der Aufgabe der Paraphrasierung überfordert gewesen zu sein. Überhaupt zeigte Schüler $\mathrm{H}^{*}$, der vom Physiklehrer als leistungsschwach eingeschätzt wurde, während des gesamten Interviews sehr große Unsicherheiten bzgl. der verwendeten Begriffe und Konzepte und war häufig nicht in der Lage, die physikalischen Zusammenhänge in sinnvollen Sätzen zu beschreiben. Im Durchschnitt scheinen die als leistungsschwach eingeschätzten Schüler etwas größere Probleme mit der Paraphrasierung der Erklärungen gehabt zu haben als die mittelmäßigen und leistungsstarken Schüler.

Im Folgenden werden die aufgetretenen Probleme der Schüler beim Paraphrasieren der Erklärungen zu den verschiedenen Einheiten genauer analysiert. Die Einheit „Elektrostatik“ bereitete den meisten Schülern keine großen Probleme. Auffällig war hier lediglich, dass sowohl Schüler B*** als auch Schüler $\mathrm{H}^{*}$ elektrische Ladungen mit Energie verwechselten und daher u.a. von Energieteilchen sprachen. Während Schüler E** lediglich Probleme damit hatte, die Erklärung zur Abstandsabhängigkeit der elektrostatischen Abstoßung korrekt wiederzugeben, war Schüler $\mathrm{H}^{*}$ nicht in der Lage, die zentralen Aussagen der zuvor zur „Elektrostatik“ gehörten Aussagen in eigenen Worten zusammenzufassen. Bei der Einheit „Atomvorstellung“ hatte kein Schüler besonders große Probleme, die zentralen Ideen in eigenen Worten wiederzugeben. Nichtsdestotrotz kam es bei den Paraphrasierungen zu einigen im Gespräch leicht aufklärbaren Verständnisschwierigkeiten wie beispielsweise der Gleichsetzung von Atom und Atomrumpf oder der Vorstellung, Atomrümpfe seien wie Elektronen frei beweglich und könnten daher einem metallischen Körper hinzugefügt werden, um diesen elektrostatisch positiv zu laden. Mit der Paraphrasierung der zentralen Ideen der Luftdruckeinheit hatten die meisten Schüler keine Probleme. Eine Ausnahme stellten hier wieder die Schüler B***, $\mathrm{E}^{* *}$ und $\mathrm{H}^{*}$ dar, wobei Schüler $\mathrm{E}^{* *}$ nur verhältnismäßig viele Hilfestellungen benötigte, Schüler $\mathrm{B}^{* * *}$ und $\mathrm{H}^{*}$ jedoch nicht in der Lage waren, die zentralen Aussagen der Einheit zusammenzufassen. Insbesondere konnten diese beiden Schüler nicht erklären, dass Luftströmungen immer eine Folge von Luftdruckunterschieden sind und Luft immer von Bereichen höheren Drucks zu Bereichen niedrigeren Drucks strömt. Vergleichsweise viele Unklarheiten zeigten sich bei der Paraphrasierung des elektrischen Drucks. Zwar hatten vier Schüler keinerlei Probleme mit der Paraphrasierung der zentralen Ideen, bei weiteren drei Schülern kam es jedoch zu leichten und bei den bereits bekannten Schülern $\mathrm{B}^{* * *}$ und $\mathrm{H}^{*}$ zu großen Problemen bei der Paraphrasierung der Einheit. Typischerweise hier aufgetretene Schwierigkeiten waren, dass sich die Schüler nicht mehr an den Begriff des elektrischen Potenzials erinnern konnten, ihnen nicht mehr bewusst war, dass sich die Elektronen im Leiter wie Luft in einem Schlauch verhalten oder sie schlicht verhältnismäßig viele Hilfestellungen bei ihren Erklärungen benötigten. Eine Mehrheit von fünf Schülern war in der Lage, die zuvor gehörte Erklärung zur Spannung in eigenen Worten angemessen wiederzugeben, während jeweils zwei Schüler hier- 
bei geringfügige bzw. große Probleme zeigten. Die Hauptschwierigkeit für die Schüler bestand dabei darin, mit Hilfe von elektrischen Druckunterschieden korrekt zu erklären, was die Ursache dafür ist, dass es überhaupt zu einer Elektronenströmung durch ein Lämpchen kommt. Zwar argumentierten nahezu alle Schüler mit Hilfe des elektrischen Drucks, aber einige unterschieden dabei nicht konsequent zwischen der Absolutgröße „elektrischer Druck“ und der für eine korrekte Erklärung der Ursache der Elektronenströmung nötigen Differenzgröße ,elektrischer Druckunterschied“. Vereinzelt zeigten Schüler auch die Tendenz, nur mit Hilfe der Elektronenströmung zu argumentieren, ohne dabei auf den elektrischen Druck oder elektrische Druckunterschiede einzugehen.

An die Fragen zur Akzeptanz und die Aufforderung zur Paraphrasierung schloss sich ein Abschnitt mit Anwendungsaufgaben an, der der Überprüfung des Konzeptverständnisses der Schüler diente. Auch hier wurden die Antworten der Schüler wieder auf einer dreistufigen Skala kodiert, wobei entsprechend des Kodierleitfadens zwischen einer guten, einer befriedigenden und einer mangelhaften Beantwortung der entsprechenden Aufgabe unterschieden wurde. Jede Aufgabe wurde dabei anhand der folgenden Kriterien kodiert:

- Gute Antwort: Der Schüler hat die Aufgabe ohne nennenswerte Schwierigkeiten und Hilfen lösen und seine Antworten ggf. korrekt begründen können.

- Befriedigende Antwort: Der Schüler benötigte beim Lösen der Aufgabe kleine Hilfestellungen und gab ggf. unklare Begründungen für seine Antworten.

- Mangelhafte Antwort: Der Schüler konnte die Aufgaben nicht oder nur mit - auch im Vergleich zu anderen Schülern - sehr viel Hilfe beantworten bzw. zeigte bei der Begründung große konzeptionelle Unsicherheit bzw. Fehlvorstellungen.

Bei manchen Aufgaben wurde nicht explizit nach einer Begründung der Antwort gefragt, was dazu führte, dass einige Schüler zu ihrer Antwort eine Begründung lieferten und andere nicht. Um dennoch eine Vergleichbarkeit der Bewertung der Schülerantworten sicherzustellen, wurde in solchen Fällen lediglich die Antwort an sich gewertet und nicht die von den Schülern freiwillig gegebene Begründung. Eine Übersicht der so gefundenen Bewertungen der Schülerantworten findet sich in Tab. 9, wobei eine gute Antwort mit Grün bzw. dem Zahlenwert 0, eine befriedigende Antwort mit Gelb bzw. dem Zahlenwert 0,5 und eine mangelhafte Antwort mit Rot bzw. dem Zahlenwert 1 kodiert wurde. Um auch hier Aussagen über die durchschnittliche Qualität der Antworten zu einzelnen Aufgaben bzw. Schülern zu ermöglichen, wurde auf Basis der Zahlenwerte zu jeder Aufgabe und zu jedem Schüler ein Mittelwert bestimmt. Zusätzlich wurde auf Grundlage aller zu einer Einheit gehörenden Aufgabenmittelwerte ein Schwierigkeitsindex (in Tab. 9 abgekürzt als „Sw_Index“) durch Mittelwertbildung berechnet, um so die Fähigkeit der Schüler, die Aufgaben der unterschiedlichen Einheiten lösen zu können, besser miteinander vergleichen zu können. Für die Einheit „Atomvorstellung" wurde so beispielsweise aus den Aufgabenmittelwerten 0,11 sowie 0,00 und 0,17 ein Schwierigkeitsindex von 0,09 ermittelt. 
Tab. 9: Übersicht über die Bewertungen der Aufgabenlösefähigkeit der Schüler

\begin{tabular}{|c|c|c|c|c|c|c|c|c|c|c|c|c|c|c|c|c|c|c|c|c|c|c|c|c|c|}
\hline \multirow[b]{2}{*}{ Schüler } & \multirow{2}{*}{\begin{tabular}{|l} 
ES \\
A1
\end{tabular}} & \multicolumn{3}{|c|}{ Atomvorst. } & \multicolumn{10}{|c|}{ Luftdruck } & \multicolumn{2}{|c|}{ el. Druck } & \multicolumn{8}{|c|}{ Spannung } & \multirow[b]{2}{*}{ Mittelwert } \\
\hline & & A2 & A3 & A4 & A5 & A6 & A7 & A8 & A9 & A10 & A11 & A12 & A13 & A14 & A15 & A16 & A17 & A18 & A19 & A20 & A21 & A22 & A23 & A24 & \\
\hline $\mathrm{A}^{* * *}$ & 0 & 0 & 0 & 0,5 & 0 & 0 & 0 & 0 & 0 & 0 & 0 & 0 & 0,5 & 0,5 & 0 & 0 & 0 & 1 & 0 & 0 & 0 & 0 & 0,5 & 0 & 0,13 \\
\hline $\mathrm{B}^{* * *}$ & 0 & 0 & 0 & 0 & 0 & 0 & 0,5 & 0 & 0 & 0 & 0 & 0 & 0,5 & 0 & 0,5 & 0 & 0,5 & 0,5 & $5 \quad 0,5$ & 0 & 0 & 0 & 0 & 0 & 0,13 \\
\hline $\mathrm{C} * * *$ & 0 & 0 & 0 & 0,5 & 0 & 0 & 1 & 0 & 0 & 0 & 0 & 0 & 0 & 0 & 0 & 0 & 0 & 1 & 0 & 0 & 0 & 0,5 & 0,5 & 0 & 0,15 \\
\hline $\mathrm{D}^{* *}$ & 0 & 0 & 0 & 0 & 0 & 0 & 0 & 0 & 0 & 0 & 0 & 0 & 0 & 0 & 0,5 & 0 & 0 & 0 & 0 & 0 & 0 & 0 & 0 & 0 & 0,02 \\
\hline $\mathrm{E}^{* *}$ & 0 & 0 & 0 & 0 & 0 & 0 & 0 & 0 & 0 & 0 & 0 & 0 & 1 & 0,5 & 0 & 0 & 0,5 & 0 & 0,5 & 0 & 0 & 0 & 0,5 & 0 & 0,13 \\
\hline $\mathrm{F}^{* *}$ & 0 & 0 & 0 & 0 & 0 & 0 & 0 & 0 & 0 & 0 & 0 & 0 & 0 & 0 & 0 & 0 & 0 & 0 & 0 & 0 & 0 & 0 & 0 & 0 & 0,00 \\
\hline G * & 0 & 0 & 0 & 0 & 0 & 0 & 0 & 0 & 0 & 0 & 0 & 0 & 0,5 & 0 & 0,5 & 0 & 0 & 0 & 0 & 0,5 & 0 & 0 & 0 & 0 & 0,06 \\
\hline $\mathbf{H}^{*}$ & 1 & 1 & 0 & 0,5 & 0 & 0 & 1 & 1 & 0 & 0 & 0 & 1 & 0,5 & 0 & 0,5 & 1 & 1 & 1 & 0 & 1 & 0 & 0 & 1 & 1 & 0,52 \\
\hline I* & 0 & 0 & 0 & 0 & 0 & 0 & 0 & 0 & 0 & 0 & 0 & 0 & 0 & 0 & 0 & 0 & 0,5 & 0 & 0,5 & 0,5 & 0 & 0 & 0,5 & 0 & 0,08 \\
\hline Mittelwert & 0,11 & 0,11 & 0,00 & 0,17 & 0,00 & 0,00 & 0,28 & 0,11 & 0,00 & 0,00 & 0,00 & 0,11 & 0,33 & 0,11 & 0,22 & 0,11 & 0,28 & 0,39 & 0,17 & 0,22 & 0,00 & 0,06 & 0,33 & 0,11 & \\
\hline Sw_Index & 0,11 & & 0,09 & & & & & & & 09 & & & & & 0,1 & 17 & & & & 0,1 & 19 & & & & \\
\hline
\end{tabular}

Vergleicht man wie gut die Schüler im Mittel die Aufgaben lösen konnten, zeigt sich, dass nur Schüler H* schwerwiegende Probleme beim Lösen der Aufgaben hatte, während alle anderen Schüler im Mittel sehr gut mit den gestellten Aufgaben zurechtkamen. Ein Zusammenhang zwischen der durchschnittlichen Aufgabenlösefähigkeit der Schüler und der vom Physiklehrer eingeschätzten Leistungsfähigkeit der Schüler ist nicht zu erkennen. Auch wenn Schüler $\mathrm{H}^{*}$ einige Aufgaben durchaus gut lösen konnte, bestätigt sich in diesem Abschnitt das schon zuvor sich abzeichnende Bild, wonach er mit den Anforderungen des Teaching Experiments im Großen und Ganzen insbesondere im Vergleich zu seinen Mitschülern überfordert war. Die Ergebnisse bzgl. Schüler $\mathrm{H}^{*}$ decken sich also mit den Befunden der ersten beiden Abschnitte, überraschen hingegen bzgl. Schüler B***, der mit dem Lösen der Aufgaben keine größeren Probleme zu haben schien als die anderen Schüler, obwohl er sich sowohl bei der Akzeptanz als auch den Paraphrasierungen vergleichsweise schwergetan hat.

Ein Blick auf die in Tab. 9 in fett dargestellten Schwierigkeitsindizes zeigt, dass die Schüler die Aufgaben zu allen Einheiten insgesamt gut lösen konnten. Insbesondere mit den für das Elektronengasmodell grundlegenden Einheiten „Elektrostatik“, „Atomvorstellung“ und „Luftdruck“ scheinen die Schüler mit einem Schwierigkeitsindex von etwa 0,10 nahezu keine Probleme zu haben, während sie durch die Aufgaben zum ,elektrischen Druck“ und der „Spannung“ mit einem Schwierigkeitsindex von fast 0,20 schon mehr gefordert zu sein scheinen. Mit Ausnahme von Schüler $H^{*}$ bereiten die Aufgaben zur elektrostatischen Kraftwirkung den Schülern keinerlei Probleme. Auch haben sie mit Ausnahme von Schüler H* nahezu keine Schwierigkeiten mit Hilfe von Atomrümpfen und Elektronen zu erklären, warum metallische Körper elektrostatisch geladen sind. Erfreulich ist insbesondere, dass es ihnen keinerlei Schwierigkeiten bereitet, Luftströmungen auf Luftdruckunterschiede zurückzuführen. Allen Schülern ist beispielsweise bewusst, dass der Luftdruck in einem aufgepumpten Reifen größer als in der Umgebung ist und dass die Luft daher bei Öffnung des Ventils aus dem Reifen strömt. Auch andersherum wissen alle Schüler, dass der Luftdruck in einer evakuierten Dose geringer ist als in der Umgebungsluft. Auch hier können alle Schüler erfolgreich vorhersagen, dass die Luft von Normaldruck in die evakuierte Dose strömen würde. Bis auf Schüler $\mathrm{H}^{*}$ gelingt allen Schülern die Übertragung ihres intuitiven Luftdruckverständnisses auf den im Modell in Stromkreisen herrschenden elektrischen Druck. Ihnen ist also bewusst, dass sich Elektronen im Leiter wie „Luftteilchen“ in einem Schlauch verhalten und dass Elektronen wie 
Luft solange von Leiterabschnitten hohen Drucks zu Leiterabschnitten niedrigen Drucks strömen, bis kein elektrischer Druckunterschied mehr existiert. Ferner gelingt es zwei Dritteln der Schüler von sich aus mit Hilfe der Spannung zu erklären, warum Elektronen in einem Stromkreis durch ein Lämpchen strömen und dass der Elektronenstrom mit größer werdender Spannung ansteigen muss. Die dem Elektronengasmodell zugrundeliegenden Konzepte werden also von acht der neun Schüler vollständig verstanden und sechs der neun Schüler besitzen bereits nach verhältnismäßig kurzer Zeit ein grundlegendes Verständnis der elektrischen Spannung als elektrischem Druckunterschied und Antrieb der Elektronenströmung.

Nichtsdestotrotz gab es in den Teaching Experiments eine Reihe von Aufgaben, bei denen sich bei einigen Schülern nennenswerte Verständnisschwierigkeiten zeigten. Im Folgenden soll daher für alle Aufgaben mit einem Mittelwert der Aufgabenlösefähigkeit von höher als 0,15 analysiert werden, wo genau die Schwierigkeiten der Schüler lagen. Bei Aufgabe 4 sollten die Schüler anhand einer selbsterstellten Skizze erklären, wie man sich mit Atomrümpfen und Elektronen erklären kann, dass ein Metall positiv geladen ist. Drei Schüler dachten hier zunächst, dass man dem Metall einfach zusätzliche positiv geladene Atomrümpfe hinzufügen könne, konnten ihren Denkfehler dann aber nach einer kurzen Rückfrage selbstständig korrigieren. Bei Aufgabe 7 wurde gefragt, wann Luft aufhört aus dem Ventil eines aufgepumpten Fahrradreifens zu strömen. Zwei Schüler dachten, dass die Luft erst dann aufhört aus dem Reifen zu strömen, wenn sich in dem Reifen keinerlei Luft mehr befindet. Der mit einigen Aspekten des Konzepts überforderte Schüler $\mathrm{H}^{*}$ argumentierte zunächst mit einer „,begrenzten Luftmenge“ im Reifen, ohne dies genauer ausführen zu können und glaubte nach Rückfragen dann ebenfalls, dass die Luftströmung erst dann aufhöre, wenn sich im Reifen keine Luft mehr befände. Auch bei Aufgabe 13 zeigten sich bei vier Schülern leichte und bei einem Schüler große Verständnisschwierigkeiten. Auf die Frage, warum z.B. eine Cola-Dose beim Öffnen zischt, wurde von einigen Schülern ohne Bezug zum Luftdruck geantwortet und spekuliert, dass die Cola die Luft frisst oder dass das Geräusch etwas mit dem Zuckergehalt der Cola zu tun haben müsse. Andere versuchten zwar mit dem Luftdruck bzw. Luftdruckunterschieden zu argumentieren, brauchten hierbei jedoch vergleichsweise viele Tipps und Hilfestellungen. Bei Frage 15 sollten die Schüler anhand einer von vier möglichen Darstellungen erläutern, wie sich der von einer Batterie erzeugte Druckunterschied vom jeweils linken zum rechten Bild verändert hat. Die sich bei dieser Aufgabe anfänglich ergebenden Unklarheiten waren nicht Ausdruck von grundlegenden konzeptionellen Schwierigkeiten seitens der Schüler, sondern waren schlicht dem Umstand geschuldet, dass die dargebotenen Visualisierungen des Potenzials für die Schüler neu und ungewohnt waren und ließen sich daher bei allen Schülern leicht beheben. Bei Aufgabe 17 sollten die Schüler mit Hilfe des Modells erläutern, wie es in einem Stromkreis zu einer Elektronenströmung durch ein Lämpchen kommt. Die größten Probleme hatte hier wieder Schüler H*, der mit dem Spannungskonzept als elektrischem Druckunterschied wenig anfangen konnte. Die anderen Schüler argumentierten zunächst nur mit dem Druck oder der Elektronenströmung, konnten nach einer kurzen Rückfrage die Elektronenströmung aber mit Druckunterschieden erklären. Drei Schüler hatten mit Aufgabe 18, bei der sie den Zusammenhang zwischen einer größeren Spannung und einer größeren Elekt- 
ronenströmung erkennen und erklären sollten, nennenswerte und ein Schüler leichte Schwierigkeiten. Statt über elektrische Druckunterschiede zu argumentieren, erklärte u.a. Schüler $\mathrm{H}^{*}$ diesen Sachverhalt rein mit den Pumpeigenschaften der Batterie. Ein anderer Schüler griff zwar bei seiner Begründung auf Druckunterschiede zurück, vermutete jedoch eine inverse Beziehung, wonach eine größere Spannung zu einer kleineren Elektronenströmung führen müsse. Aufgabe 19 fragte danach, ob man eine Spannung an einem Punkt messen kann und bereitete den Schülern nur geringe Schwierigkeiten. Lediglich drei Schüler benötigten eine kleine Hilfestellung, um korrekt erklären zu können, dass Spannung immer einen Unterschied des Drucks beschreibt und man eine Spannung daher nur zwischen zwei Punkten messen könne. Im Kontext von Aufgabe 20, die danach fragte, was an der Formulierung ,die Batterie ist leer" missverständlich sei, vertraten drei Schüler zumindest anfänglich die bekannte „Batterie-als-Elektronenfass"-Vorstellung, wonach eine leere Batterie keine Elektronen mehr enthalte. Nur Schüler $\mathrm{H}^{*}$ hatte mit dieser Aufgabe größere Verständnisprobleme. Bei Aufgabe 23 sollten die Schüler erklären, warum mit einem Kondensator in Reihe geschaltete Lämpchen während des Lade- und Entladevorgangs leuchten. Wie auch bei ähnlichen Aufgaben zuvor, benötigten vier Schüler eine kurze Erinnerung hierbei mit Druckunterschieden zu argumentieren, während Schüler $\mathrm{H}^{*}$ mit der Aufgabenstellung überfordert schien, da er das Konzept des elektrischen Druckunterschieds als Antrieb der Elektronenströmung nicht verstanden hatte.

\subsubsection{Auswertung der aufgetretenen Lernschwierigkeiten}

In Hinblick auf die Entwicklung eines Unterrichtskonzepts auf Basis des Elektronengasmodells sind auch die in den Teaching Experiments aufgetretenen Schülervorstellungen und Lernschwierigkeiten von besonderem Interesse. Das Ziel der zweiten mit den Teaching Experiments verbundenen Fragestellung war es daher zu klären, mit welchen Lernschwierigkeiten in einem weitergehenden Unterrichtskonzept auf Basis des Elektronengasmodells zu rechnen ist. $\mathrm{Zu}$ diesem Zweck wurden die Schüler nach der eigentlichen Akzeptanzbefragung mit verhältnismäßig anspruchsvollen Fragestellungen zu einer Reihe für sie neuer Konzepte wie z.B. Parallel- und Reihenschaltungen konfrontiert. Hierbei wurde dann geschaut, zu welchen Problemen und Schülervorstellungen es bei der Bearbeitung der Aufgaben gekommen ist und welche Verständnisschwierigkeiten sich in den Gesprächen mit den Schülern insgesamt gezeigt haben. Während sich die bisherige Auswertung also auf konkrete Akzeptanz-, Paraphrasierungs- oder Anwendungsaufgaben bezog, geht es in diesem Abschnitt darum, einen Überblick über die typischerweise auftretenden Schülervorstellungen und Lernschwierigkeiten zu bekommen, auch wenn diese von den Schülern teilweise im Laufe der Akzeptanzbefragungen überwunden werden konnten.

Aufgrund des explorativen Charakters dieser Fragestellung mussten die Kategorien, nach denen das Interviewmaterial kodiert werden sollte, zunächst einmal induktiv gefunden werden. Zu diesem Zweck wurden die Interviews Schüler für Schüler durchgearbeitet, um auf diesem Weg den individuellen Lernverlauf sowie besondere Lernhindernisse der einzelnen Schüler besser analysieren zu können. In Anlehnung an die Methode der induktiven Kategori- 
enbildung (Mayring 2010, S. 83) wurden dabei zunächst alle aufgetretenen Lernschwierigkeiten der Schüler im gesamten Interviewmaterial identifiziert und die entsprechenden Textstellen markiert. In einem zweiten Schritt wurden dann die bei den einzelnen Schülern gefundenen Lernschwierigkeiten querschnittartig miteinander verglichen und auf Basis der mehrfach vorkommenden Lernschwierigkeiten entsprechende Kategorien gebildet (z.B. „Verwechslung von elektrischen Ladungen mit Energie(teilchen)"). In einem letzten Schritt wurde dann durch einen querschnittartigen Vergleich des Interviewmaterials der Ausprägungsgrad der jeweils auftretenden Lernschwierigkeiten auf Basis einer dreistufigen Skala bestimmt, wobei hinsichtlich der Lernschwierigkeiten zwischen ,tritt nicht auf“, „tritt leicht auf (= implizit bzw. einmal)“ und „tritt verstärkt auf (= explizit bzw. mehrfach)“ unterschieden wurde. Um ein $\mathrm{Ma}$ für die Häufigkeit einer Schülervorstellung berechnen zu können, wurden den einzelnen Feldern in Tab. 10 neben Farben auch wieder Zahlenwerte bzgl. des Ausprägungsgrades der aufgetretenen Schülervorstellung zugeordnet, wobei das Nicht-Auftreten einer Schülervorstellung mit Grün bzw. dem Zahlenwert 0, das leichte Auftreten einer Schülervorstellung mit Gelb bzw. dem Zahlenwert 0,5 und das explizite bzw. mehrfache Auftreten einer Schülervorstellung mit Rot bzw. dem Zahlenwert 1 kodiert wurde. Um zusätzlich ein Maß für den durchschnittlichen Ausprägungsgrad einer Schülervorstellung zur Verfügung zu haben und diese so besser miteinander vergleichen zu können, wurde auf Basis der Zahlenwerte ein Mittelwert zu jeder Schülervorstellung bestimmt. Zur Schülervorstellung „E-Teil“ wurde so z.B. ein Mittelwert bzw. durchschnittlicher Ausprägungsgrad von 0,67 bestimmt, wobei 0,0 einem sehr niedrigen und 1,0 einem sehr hohen Ausprägungsgrad der Schülervorstellung entspricht.

Tab. 10: Übersicht über die Ausprägungsgrade der aufgetretenen Schülervorstellungen

\begin{tabular}{|c|c|c|c|c|c|c|c|c|c|c|c|c|c|c|c|c|c|c|c|}
\hline Schüler & E-Teil & R-frei & Dr-ab & Sp-lok & Str-aus E & E-Stau & Dr-LA & Inv-W & Ba-kStQ & gDU-gS & gD-gS & sv & LA & Fließ-Dr & D-vs-DU & S=StröG & SA & Übm-Str & D-vs-S \\
\hline$A^{* * *}$ & 0,5 & 1 & 0 & 0 & 0 & 1 & 1 & 0,5 & 1 & 0 & 1 & 0 & 1 & 1 & 0,5 & 1 & 0,5 & 1 & 0,5 \\
\hline $\mathrm{B}^{* * *}$ & 1 & 0 & 1 & 0,5 & 0 & 1 & 1 & 1 & 0 & 1 & & & 0,5 & 0 & 1 & & & 1 & \\
\hline $\mathrm{C}^{* * *}$ & 1 & 1 & 0 & 0 & 1 & 1 & 0 & 1 & 0 & 0 & 0 & 0,5 & 0 & 0 & 0,5 & 0,5 & 1 & 0,5 & 0 \\
\hline $\mathrm{D}^{* *}$ & 1 & 0 & 0 & 0 & 0 & 1 & 0 & 0 & 0 & 0 & 0 & 0,5 & 0 & 0,5 & 1 & 1 & 1 & 1 & 0 \\
\hline $\mathrm{E}^{* *}$ & 1 & 0 & 1 & 0,5 & 0 & & 1 & 0 & 1 & 1 & 1 & 0 & 0 & 1 & 0,5 & 1 & 0 & 1 & 0,5 \\
\hline $\mathrm{F}^{* *}$ & 0 & 0 & 1 & 0 & 0 & 1 & 1 & 0 & 1 & 1 & 1 & 1 & 0,5 & 1 & 0 & 0,5 & 0,5 & 0,5 & 0 \\
\hline $\mathrm{G}^{*}$ & 0 & 0 & 1 & 0 & 1 & 0 & 0 & 1 & 1 & 1 & 1 & 0 & 0 & 0 & 0 & 0,5 & 1 & 0 & 0,5 \\
\hline $\mathbf{H}^{*}$ & 1 & 1 & 0 & 0,5 & 1 & 1 & & 1 & & 1 & & 1 & & 0 & 0,5 & 0,5 & & 1 & 0 \\
\hline I* $^{*}$ & 0,5 & 0 & 1 & 1 & 0 & 1 & 0 & 1 & 1 & 1 & 1 & 1 & 0 & 0,5 & 0 & 0,5 & 0 & 0,5 & 0,5 \\
\hline Mittelwert & 0,67 & 0,33 & 0,56 & 0,28 & 0,33 & 0,88 & 0,50 & 0,61 & 0,63 & 0,67 & 0,71 & 0,50 & 0,25 & 0,44 & 0,44 & 0,69 & 0,57 & 0,72 & 0,25 \\
\hline
\end{tabular}

Wurde ein Thema bzw. Unterthema mit einem Schüler nicht besprochen und konnte im Interviewmaterial daher keine Aussage zu einer hierzu auftretenden Schülervorstellung gefunden werden, so wurde das entsprechende Feld grau markiert und bei der Berechnung der Mittelwerte nicht berücksichtigt. Eine Beschreibung und Kategorisierung sowie Zuordnung der aufgetretenen Schülervorstellungen zu den in Tab. 10 verwendeten Codes findet sich in Tab. 11. 
Tab. 11: Codes der aufgetretenen Schülervorstellungen mit Beschreibung, Kategorisierung und Ausprägungsgrad ( $M W=$ Mittelwert)

\begin{tabular}{|c|c|c|c|}
\hline Code & Beschreibung der Schülervorstellung & Kategorie & MW \\
\hline E-Teil & $\begin{array}{l}\text { Verwechslung von elektrischen Ladungen mit Ener- } \\
\text { gie(teilchen) }\end{array}$ & Atomvorstellung & 0,67 \\
\hline R-frei & $\begin{array}{l}\text { Es wird angenommen, Atomrümpfe seien in Metallen } \\
\text { frei beweglich }\end{array}$ & Atomvorstellung & 0,33 \\
\hline Dr-ab & $\begin{array}{l}\text { Der elektrische Druck baut sich bei Anschluss eines Lei- } \\
\text { ters in den Batteriepolen ab, so wie auch der Luftdruck } \\
\text { in der Spritze bei Ventilöffnung nachlässt }\end{array}$ & Batteriekonzept & 0,56 \\
\hline Sp-lok & Spannung als lokale Größe statt Differenzgröße & $\begin{array}{l}\text { bereits bekannte } \\
\text { Schülervorstellung }\end{array}$ & 0,28 \\
\hline Str-aus & $\begin{array}{l}\text { Batterie als Elektronenfass statt Elektronenpumpe } \\
\text { (Stromaussendevorstellung) }\end{array}$ & $\begin{array}{l}\text { bereits bekannte } \\
\text { Schülervorstellung }\end{array}$ & 0,33 \\
\hline E-Stau & $\begin{array}{l}\text { Ein größerer Widerstand führt zu einem größeren Elek- } \\
\text { tronenrückstau und damit einem größeren Druck- } \\
\text { unterschied in den mit der Batterie verbundenen Leitern }\end{array}$ & Batteriekonzept & 0,88 \\
\hline Dr-LA & $\begin{array}{l}\text { Der elektrische Druck in den mit der Batterie verbunde- } \\
\text { nen Leiterstücken ist abhängig von den Leiterabmessun- } \\
\text { gen }\end{array}$ & Batteriekonzept & 0,50 \\
\hline Inv-W & $\begin{array}{l}\text { Ein Lämpchen mit einem größeren Widerstand braucht } \\
\text { eine größere Stromstärke (inverse Widerstands- } \\
\text { vorstellung) }\end{array}$ & $\begin{array}{l}\text { Widerstands- } \\
\text { vorstellung }\end{array}$ & 0,61 \\
\hline B-kStQ & $\begin{array}{l}\text { Gleiche Batterie = gleiche Stromstärke durch das Lämp- } \\
\text { chen (unabhängig vom Widerstand) }\end{array}$ & $\begin{array}{l}\text { Widerstands- } \\
\text { vorstellung }\end{array}$ & 0,63 \\
\hline gDU-gS & $\begin{array}{l}\text { Gleicher Druckunterschied = gleiche Stromstärke durch } \\
\text { das Lämpchen (unabhängig vom Widerstand) }\end{array}$ & $\begin{array}{l}\text { Widerstands- } \\
\text { vorstellung }\end{array}$ & 0,67 \\
\hline gD-gS & $\begin{array}{l}\text { Parallelschaltung: Wo ein gleicher elektrischer Druck } \\
\text { herrscht, ist auch die Stromstärke gleich (Übergenerali- } \\
\text { sierung) }\end{array}$ & Stromvorstellung & 0,71 \\
\hline SV & $\begin{array}{l}\text { Stromverbrauchsvorstellung (= Elektronen bleiben teil- } \\
\text { weise in Lämpchen stecken) }\end{array}$ & $\begin{array}{l}\text { bereits bekannte } \\
\text { Schülervorstellung }\end{array}$ & 0,50 \\
\hline LA & $\begin{array}{l}\text { Strom teilt sich an Knoten zu gleichen Teilen auf } \\
\text { (lokale Argumentation) }\end{array}$ & $\begin{array}{l}\text { bereits bekannte } \\
\text { Schülervorstellung }\end{array}$ & 0,25 \\
\hline Fließ-Dr & $\begin{array}{l}\text { Es wird von fließendem Druck statt strömenden Elektro- } \\
\text { nen gesprochen }\end{array}$ & Stromvorstellung & 0,44 \\
\hline D-vs-DU & $\begin{array}{l}\text { Begrifflich wird nicht zwischen Druck und Druckunter- } \\
\text { schied unterschieden (auch wenn dies ggf. konzeptionell } \\
\text { der Fall ist) }\end{array}$ & Druckvorstellung & 0,44 \\
\hline S=StröG & $\begin{array}{l}\text { Verwechslung oder Gleichsetzung von Stromstärke mit } \\
\text { Strömungsgeschwindigkeit der Elektronen }\end{array}$ & Stromvorstellung & 0,69 \\
\hline $\mathbf{S A}$ & Sequentielles Denken bei Reihenschaltungen & $\begin{array}{l}\text { bereits bekannte } \\
\text { Schülervorstellung }\end{array}$ & 0,57 \\
\hline Übm-Str & $\begin{array}{l}\text { Tendenz mit Hilfe von Strömungen zu argumentieren, } \\
\text { statt Strömungen über Druckunterschiede zu erklären } \\
\text { (übermächtiger Strombegriff) }\end{array}$ & $\begin{array}{l}\text { bereits bekannte } \\
\text { Schülervorstellung }\end{array}$ & 0,72 \\
\hline D-vs-S & $\begin{array}{l}\text { Mangelnde Differenzierung zwischen elektrischem } \\
\text { Druck und elektrischem Strom }\end{array}$ & Druckvorstellung & 0,25 \\
\hline
\end{tabular}


Im Verlauf der Teaching Experiments traten eine Reihe bekannter und neuer Schülervorstellungen und Lernschwierigkeiten auf, die einerseits wertvolle Erkenntnisse in Hinblick auf die Entwicklung eines vollwertigen Unterrichtskonzepts liefern, andererseits aber vor dem Hintergrund des mit drei Schulstunden knapp bemessenen Zeitrahmens und der für die Schüler vielen neuen Inhalte ohne Übungs- und Vertiefungsphasen nicht überraschend sind. Wichtig ist an dieser Stelle, dass die hier vorgestellten Verständnisschwierigkeiten nicht die bei den Schülern nach den Teaching Experiments noch anzutreffenden Schülervorstellungen widerspiegeln, sondern eine Dokumentation der wichtigsten in den Akzeptanzbefragungen aufgetretenen Lernschwierigkeiten darstellen, auch wenn diese im Verlauf der Befragung von den Schülern erfolgreich überwunden werden konnten. Die folgende Analyse der während der Akzeptanzbefragungen aufgetretenen Schülervorstellungen bezieht sich hauptsächlich auf diejenigen Schüler, bei denen die entsprechende Schülervorstellung mehrfach bzw. explizit vorkam (in Tab. 10 in Rot dargestellt). Sofern die jeweilige Schülervorstellung jedoch auch in vereinzelter bzw. impliziter Form bei mehreren Schülern beobachtet werden konnte, wird dies explizit ausgewiesen.

Wie der in Tab. 11 vorgenommenen Kategorisierung der aufgetretenen Schülervorstellungen zu entnehmen ist, konnten im Verlauf der Teaching Experiments sieben bereits bekannte Schülervorstellungen dokumentiert werden (vgl. Abschnitt 3.2). Hierzu zählen die inverse Widerstandsvorstellung, die sequentielle und lokale Argumentation, die Stromverbrauchs- und Stromaussendevorstellung sowie ein übermächtiger Strombegriff und die Tendenz, die Spannung als lokale Größe zu sehen. Die größte Herausforderung stellte für die Schüler in diesem Kontext der Übergang vom ,übermächtigen Strombegriff“ (Rhöneck 1986, S. 13) hin zur systematischen Analyse von Stromkreisen mit Hilfe von elektrischen Druckunterschieden dar (Code: Übm-Str). So zeigten alle Schüler zumindest einmal während der Akzeptanzbefragung die Tendenz, Stromkreise ausschließlich aus Sicht der Elektronenströmung zu analysieren, ohne dabei von sich aus auf die Spannung als Ursache der Elektronenströmung einzugehen. Ebenfalls vergleichsweise häufig hatten die Schüler Probleme damit, sich des Systemcharakters von Reihenschaltungen bewusst zu werden. Stattdessen vertraten drei Schüler wiederholt und zwei Schüler vereinzelt die bekannte sequentielle Argumentation und analysierten u.a. Reihenschaltungen aus Sicht eines Elektronenstroms, der die in Reihe geschalteten Lämpchen schrittweise durchläuft (Code: SA). In Folge argumentierten die Schüler z.B. bei Reihenschaltungen, dass die Lämpchen nacheinander angehen müssten oder dass sich Widerstandsänderungen im ,hinteren“ Teil des Stromkreises nicht auf die Stromstärke im „,vorderen“ Teil auswirken würden. Häufig wurde in diesem Zusammenhang zumindest auch implizit argumentiert, dass die Batterie dabei mit einem Fass Öl vergleichbar sei, in dem Elektronen gespeichert sind, die dann ausgehend vom Minuspol in den Stromkreis strömen, was der bekannten Stromaussendevorstellung entspricht (Code: Str-aus). Auch vertraten drei Schüler die bekannte und weit verbreitete Stromverbrauchsvorstellung, wonach Elektronen auf ihrem Weg durch einen Widerstand bzw. Lämpchen verbraucht oder an dessen Atomrümpfen hängenblieben (Code: SV). Bei weiteren zwei Schülern trat die Stromverbrauchsvorstellung vereinzelt auf. Die Schülervorstellung, dass Spannung eine lokale, d.h. auf einen Lei- 
terabschnitt bezogene Größe wie beispielsweise das elektrische Potenzial ist, wurde in den Teaching Experiments von nur einem Schüler wiederholt und drei Schülern vereinzelt vertreten, was vermutlich auf die im Konzept starke Betonung der Spannung als Differenzgröße zurückzuführen ist (Code: Sp-lok). Erfreulich ist ferner, dass auch die lokale Argumentation, wonach sich der Strom an Knotenpunkten bei Parallelschaltungen zu gleichen Teilen aufteilen müsse, ebenfalls nur von einem Schüler wiederholt und von zwei Schülern vereinzelt vertreten wurde (Code: LA).

Eine Reihe von Verständnisschwierigkeiten und Schülervorstellungen gab es im Zusammenhang mit der Aufgabe einer Batterie im Stromkreis. Entgegen der bei den meisten Schülern zumindest verbal kommunizierten Einsicht, dass eine Batterie eine Quelle konstanter Spannung ist, war es mit großen Schwierigkeiten verbunden, den Schülern bewusst zu machen, welche Implikationen dies für Stromkreise hat. Insgesamt konnten so in der Kategorie „Batteriekonzept“ drei Schülervorstellungen dokumentiert werden, wobei die am häufigsten aufgetretene Schülervorstellung vermutlich u.a. auf die Verwendung des ungeschickten Wortbildes des „Elektronenstaus“ in der Akzeptanzbefragung zurückzuführen ist. Wie in Abb. 47 gezeigt, glaubten sieben Schüler möglicherweise in Folge dieses Wortbildes, dass sich in einem einfachen Stromkreis bestehend aus Batterie, Leitern und Lämpchen der elektrische Druckunterschied am Lämpchen vergrößern müsse, wenn ein Lämpchen mit einem größeren Widerstand verwendet wird (Code: E-Stau).
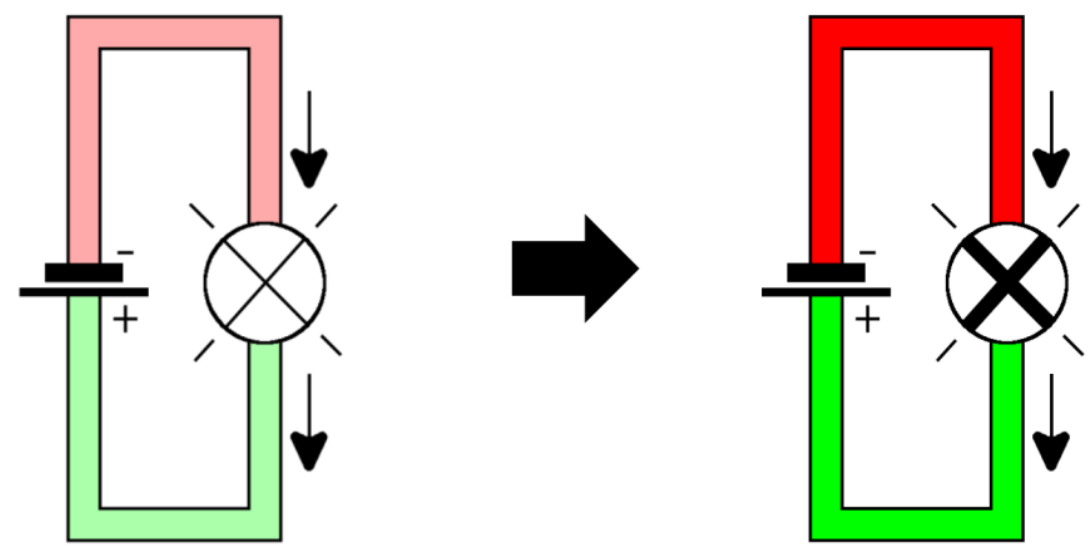

Abb. 47: Visualisierung der Schülervorstellung, wonach der elektrische Druckunterschied bei Vergrößerung des Widerstands ansteigen müsste.

Gut denkbar ist hier, dass der Begriff des „Elektronenstaus“ bei den Schülern zu einer Assoziation mit Verkehrsstaus geführt hat, bei denen eine Verengung der Unfallstelle durchaus zu einem größeren Rückstau führt. Eine Batterie ist aber keine Konstantstromquelle, verschiebt also nicht eine feste Anzahl Elektronen pro Zeiteinheit vom Plus- zum Minuspol, die sich am Widerstand immer weiter anstauen und somit dort den elektrischen Druck vergrößern, sondern stellt eine Konstantspannungsquelle dar, weshalb die Größe des Widerstands des Lämpchens in Abb. 47 keinerlei Einfluss auf die am Lämpchen anliegende Spannung hat. Interessanterweise führte die Punktedichtedarstellung bei Schüler $\mathrm{F}^{* *}$ zu einer komplementären Schülervorstellung. Er argumentierte, dass die Stromstärke bei einer geringeren Spannung 
am Lämpchen größer sein müsse, weil sich dann im mit dem Pluspol der Batterie verbundenen Leiter mehr Elektronen befänden.

Eine aus physikdidaktischer Perspektive weitere interessante neue Schülervorstellung betrifft den Zusammenhang zwischen elektrischem Druck und Volumen der Leiterstuicke, die direkt mit den Polen einer Batterie verbunden sind. So führte die bereits beschriebene Schülervorstellung von einer Batterie als Konstantstrom- statt Konstantspannungsquelle im Elektronengasmodell dazu, dass vier Schüler anfänglich einen direkten Zusammenhang zwischen Leitervolumen und dem im Leiter herrschenden Potenzial sahen (Code: Dr-LA). In der Vorstellung der Schüler steht den Elektronen bei einem größeren Leiter mehr Platz zur Verfügung, weshalb der „elektrische Druck“ bzw. das elektrische Potenzial in diesem absinken müsse.
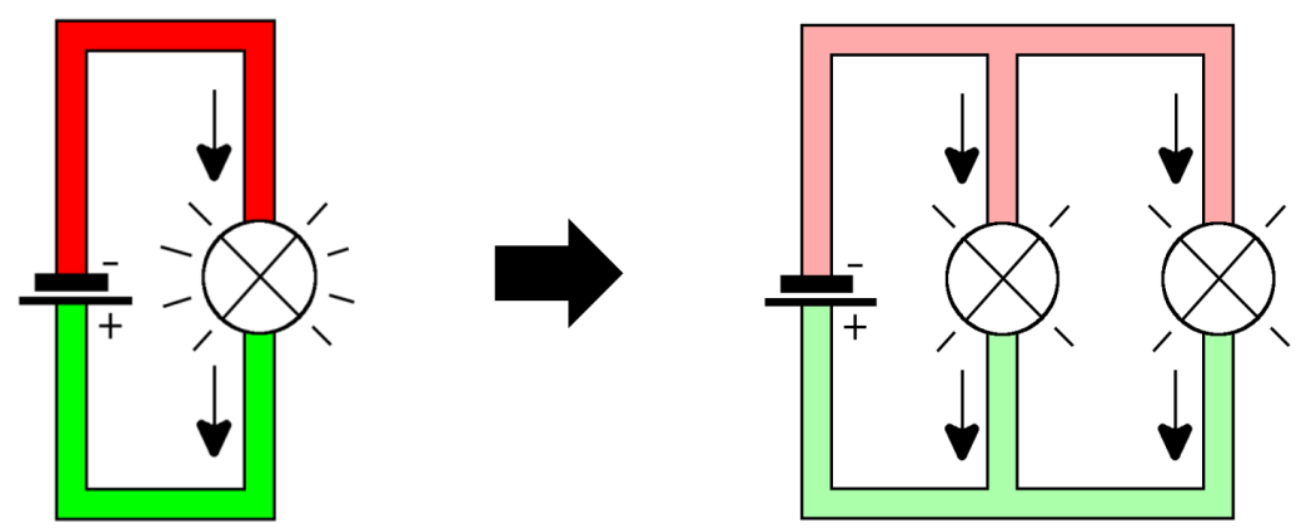

Abb. 48: Visualisierung der Schülervorstellung, wonach der elektrische Druckunterschied bei Vergrößerung des Leitervolumens absinken müsste

Konkret zeigte sich diese Schülervorstellung, wenn neben ein bisheriges Lämpchen ein weiteres, gleichartiges Lämpchen parallelgeschaltet wird, wie in Abb. 48 illustriert ist. Vor dem Hintergrund der zu Beginn eingeführten Luftdruckanalogie ist das Auftreten dieser Vorstellung nachvollziehbar: Eine Vergrößerung des ursprünglichen (oberen) Rohrvolumens durch Anschluss weiterer Rohre würde hier tatsächlich zu einem Druckabfall führen, den die Pumpe erst wieder ausgleichen müsste.

Eine möglicherweise auf die vorangegangenen Schülerexperimente zum Luftdruck in Spritzen als Miniatur-Luftpumpen zurückzuführende Schülervorstellung betrifft den elektrischen Druck an den Polen der Batterie. Wird ein elektrischer Leiter z.B. an den Minuspol der Batterie angeschlossen, so meinten fünf Schüler, dass dann durch die ausströmenden Elektronen der elektrische Druck im Minuspol abnehmen müsse (Code: Dr-ab). Wie bereits angedeutet, wird diese Vorstellung möglicherweise durch die Spritzenexperimente, wo der Luftdruck in der Spritze nach Öffnung des Ventils - anders als bei einem z.B. an Tankstellen anzutreffenden Luftdruckgerät - auch absinkt, begünstigt.

Wenige Probleme hatten die Schüler damit, mit Hilfe von Atomrümpfen und Elektronen eine grundlegende atomare Vorstellung davon $\mathrm{zu}$ entwickeln, warum ein metallischer Körper elektrisch geladen ist. Eine typische Schwierigkeit war hier, dass fünf Schüler wieder- 
holt elektrische Ladungen begrifflich mit positiver oder negativer Energie bzw. Energieteilchen verwechselten, während weitere zwei Schüler hiermit vereinzelt Probleme hatten (Code: E-Teil). Eine andere Schwierigkeit betraf Atomrümpfe, die von manchen Schülern für ebenso beweglich gehalten wurden wie Elektronen (Code: R-frei). Auf die Frage, wie ein elektrisch neutraler Körper elektrisch positiv geladen werden kann, antworteten drei Schüler daher, dass man ihm einfach positiv geladene Atomrümpfe hinzufügen müsse, auch wenn sich dieser Denkfehler in der Regel leicht korrigieren ließ.

Unerwarteterweise schienen die Schüler relativ große Probleme damit zu haben, eine angemessene Vorstellung davon zu entwickeln, wie die Stromstärke vom elektrischen Widerstand beeinflusst wird. Für eine Reihe von Schülern hatte der elektrische Widerstand keinerlei Einfluss auf die Stromstärke. Stattdessen äußerten sechs Schüler die Überzeugung, dass ein gewisser elektrischer Druckunterschied immer zu einer bestimmten Stromstärke führe - ganz unabhängig vom Widerstand des Lämpchens (Code: gDU-gS). Ähnlich hierzu vertraten fünf Schüler die Schülervorstellung, dass die Stromstärke durch ein Lämpchen einzig von der verwendeten Batterie abhinge und nicht durch den Widerstand des Lämpchens beeinflusst würde (Code: B-kStQ). In einer wieder anderen Variante vertraten fünf Schüler die in der physikdidaktischen Forschung bereits gut dokumentierte inverse Widerstandsvorstellung, wonach ein Lämpchen mit einem größeren Widerstand auch eine größere Stromstärke benötige (Code: Inv-W). Einige Schüler erklärten hierzu, dass sie unter der Größe eines Widerstands dessen geometrische Größe verstünden und ein größerer Widerstand mit mehr Strom versorgt werden müsste. Sieht man von der Länge als einer Dimension der geometrischen Größe eines Widerstands ab und betrachtet stattdessen lediglich den Querschnitt des Widerstands als dessen geometrische Größe, ist die Vorstellung dieser Schüler nah an der physikalischen Sichtweise.

Im Zusammenhang mit dem elektrischen Strom kam es zu drei nennenswerten Schülervorstellungen. An erster Stelle ist hier die Schwierigkeit der Schüler zu nennen, bei einer Parallelschaltung zwischen elektrischem Druck und der Stromstärke zu differenzieren. So war den Schülern zwar bewusst, dass der elektrische Druck in Haupt- und Nebenzweigen einer Parallelschaltung gleich groß ist, fünf der neun Schüler schlossen daraus aber fälschlicherweise, dass dann in Haupt- und Nebenzweigen auch die Stromstärke gleich groß sein müsse (Code: gD-gS). Mehr als die Hälfte der Schüler schloss also fälschlicherweise direkt vom elektrischen Druck auf die Stromstärke im jeweiligen Leiter und hatte somit Schwierigkeiten zu verstehen, dass die Stromstärke in den Ästen einer Parallelschaltung kleiner als im Hautzweig ist. Als besonders problematisch hat sich auch die in der Punktedichtedarstellung nötige Unterscheidung zwischen Stromstärke und Strömungsgeschwindigkeit der Elektronen erwiesen, die keinem der interviewten Schüler unmittelbar einleuchtete und von drei Schülern überhaupt nicht verstanden wurde. In Folge gelang es diesen Schülern nicht, konzeptionell zwischen „Stromstärke“ und „Strömungsgeschwindigkeit der Elektronen“" zu unterscheiden (Code: $\mathrm{S}=$ StröG). Dies ist insbesondere in Hinblick auf die Punktedichtedarstellung problematisch, da sich hier vor und nach einem Widerstand aufgrund der unterschiedlich großen Elektronendichten die Strömungsgeschwindigkeiten unterscheiden, die Stromstärke jedoch 
konstant bleibt. Ferner sprachen drei Schüler bei der Analyse von Stromkreisen wiederholt nicht von strömenden Elektronen, sondern von fließendem Druck und argumentierten, dass hoher Druck zu niedrigem Druck strömen würde (Code: Fließ-Dr). Weitere zwei Schüler hatten hiermit vereinzelt Schwierigkeiten. Auf Nachfrage zeigte sich dann allerdings, dass es sich hierbei weniger um ein konzeptionelles als vielmehr um ein begriffliches Problem handelte, da den Schülern bewusst war, dass eigentlich Elektronen strömen.

Die direkt im Zusammenhang mit der elektrischen Druckvorstellung aufgetretenen Verständnisschwierigkeiten sind überschaubar. Auch wenn den Schülern der Unterschied zwischen der Absolutgröße „elektrischer Druck“ und der Differenzgröße „elektrischer Druckunterschied" konzeptionell bewusst war, bestand eine begriffliche Schwierigkeit für zwei Schüler trotzdem darin, sprachlich bei der Analyse von Stromkreisen konsequent zwischen Druck und Druckunterschied zu differenzieren (Code: D-vs-DU). Weitere vier Schüler hatten hiermit zumindest vereinzelt Schwierigkeiten. Ferner hat insbesondere die Verwendung der Begriffe „Potenzial“ und „Spannung“ statt „elektrischer Druck“ und „elektrischer Druckunterschied“ bei manchem Schüler für unnötige Verständnisschwierigkeiten gesorgt, auch wenn diese begrifflichen Schwierigkeiten möglicherweise primär auf eine zu geringe Übungszeit zurückzuführen sind. Der Grund könnte darin liegen, dass die physikalischen Fachbegriffe einen zusätzlichen Abstraktionsgrad darstellen, da sie nicht zu einer direkten Assoziation mit dem zuvor an Luftdruckbeispielen erworbenen intuitiven Druckverständnis führen (vgl. Abschnitt 6.1.6). Vereinzelt konnte außerdem bei vier Schülern beobachtet werden, dass der Begriff der „Stärke“ in ,Stromstärke“ die Schüler dazu veranlasste, den Stromstärkebegriff mit „,Kraft“ oder „Druck“ in Verbindung zu bringen und somit konzeptionell in der Nähe des Druckkonzepts zu verorten. Werden Strom und elektrischer Druck aber konzeptionell nicht mehr getrennt, ist es nur noch ein kleiner Schritt zur bekannten „Stromspannung", die sich auch in den Teaching Experiments - wenn auch selten - in der Verwendung von missverständlichen Begriffen wie „Überstrom“, „Unterstrom“ oder „Stromdruck“ zeigte (Code: D-vs-S).

\subsubsection{Auswertung der Visualisierungen des Potenzials ${ }^{8}$}

In Hinblick auf das zu entwickelnde Unterrichtskonzept war die letzte mit den Teaching Experiments verbundene Fragestellung daher, welche der vier entwickelten Visualisierungsformen des elektrischen Potenzials die Schüler als am verständlichsten empfinden. Hierzu wurden ihnen die verschiedenen Darstellungsformen des elektrischen Drucks erstmals in Einheit 4 ,Das Potenzial als elektrischer Druck“ am Beispiel eines offenen Stromkreises vorgelegt. Bei den anfänglichen Erklärungen zum Luftdruck und dem Potenzial als elektrischem Druck wurde also komplett auf Visualisierungen verzichtet, um eine Beeinflussung der Schüler bzgl. ihrer Darstellungspräferenzen zu vermeiden. Nachdem im Gespräch mit den Schülern geklärt wurde, was in den unterschiedlichen Darstellungen zu sehen ist und wie diese physikalisch gesehen zu interpretieren sind, wurden die Schüler gebeten, die Visualisierungen nach ihrer

\footnotetext{
${ }^{8}$ Teile dieses Abschnitts wurden bereits in Burde und Wilhelm 2015 bzw. Burde et al. 2015 veröffentlicht. 
Verständlichkeit zu ordnen und ihre Reihenfolge nach Möglichkeit zu begründen. Im weiteren Verlauf der Teaching Experiments hatten die Schüler dann auch Gelegenheit, selbstständig Potenziale mit Hilfe einer Visualisierung ihrer Wahl in Stromkreise einzuzeichnen. Dabei ergab sich ein klares Bild, wonach die Schüler die Punktedichtedarstellung gefolgt von der Farbdarstellung für am verständlichsten empfanden. Wie in Abb. 49 unter dem Punkt „Platz I“ zu sehen, sagten sechs der neun Schüler, die Punktedichtedarstellung sei am verständlichsten und drei Schüler waren der Ansicht, die Farbdarstellung sei am verständlichsten, wohingegen die Graustufendarstellung oder die Plus-Minus-Darstellung von keinem der Schüler favorisiert wurde.

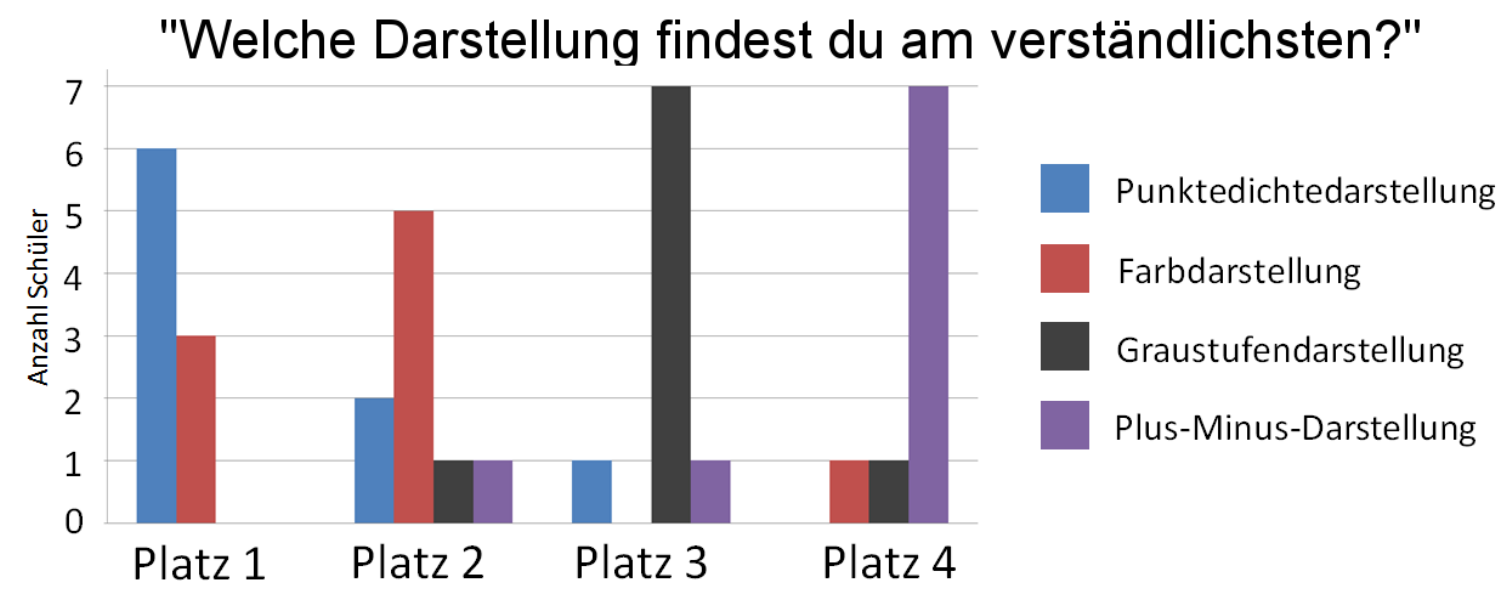

Abb. 49: Antworten der Schüler auf die Frage, welche Darstellungsform des elektrischen Drucks sie am verständlichsten finden

Auf dem zweiten Platz des Verständlichkeitsrankings sah eine Mehrheit von fünf Schülern die Farbdarstellung, während lediglich zwei Schüler die Punktedichtedarstellung und jeweils ein Schüler die Graustufen- bzw. Plus-Minus-Darstellung auf dem zweiten Platz sahen. Auf dem dritten Platz der Verständlichkeit wurde von den Schülern relativ eindeutig die Graustufendarstellung gesehen, die hier von sieben Schülern verortet wurde. Jeweils ein Schüler sah hier jedoch die Punktedichtedarstellung bzw. die Plus-Minus-Darstellung. Als eindeutig am wenigsten verständlich wurde von sieben Schülern die Plus-Minus-Darstellung gesehen, während jeweils ein Schüler die Farb- bzw. Graustufendarstellung am unverständlichsten empfand. Zusammenfassend lässt sich somit sagen, dass die Punktedichte- und Farbdarstellung des elektrischen Drucks von den Schülern in den Teaching Experiments favorisiert und als am verständlichsten empfunden wurden.

Die bei der Befragung gezeigte Darstellungspräferenz zeigte sich mit einer interessanten Ausnahme auch beim eigenständigen Lösen von Aufgaben. Entgegen den Erwartungen nutzte eine Mehrheit von fünf Schülern beim Lösen von Aufgaben die Farbdarstellung und nicht die Punktedichtedarstellung, die lediglich von drei Schülern genutzt wurde, obwohl sechs der neun Schüler in der Befragung angaben, die Punktedichtedarstellung zu favorisieren. Ein weiterer Schüler nutzte die Plus-Minus-Darstellung. 


\section{Welche Darstellung nutzten die Schüler selbst?}

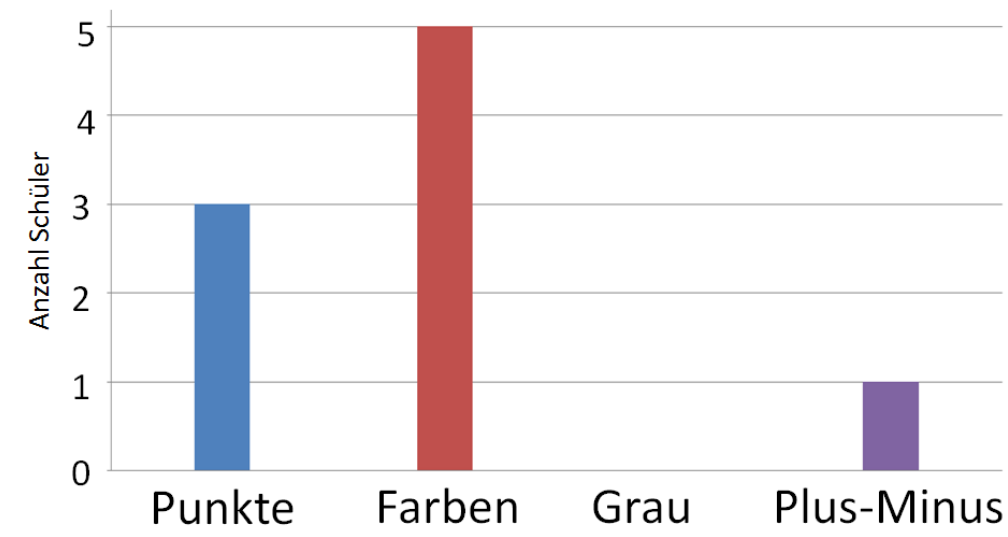

Abb. 50: Übersicht über die von den Schülern beim eigenständigen Lösen von Aufgaben genutzten Visualisierungsformen des elektrischen Drucks

Es liegt die Vermutung nahe, dass die Entscheidung der Schüler mehrheitlich die Farb- statt die Punktedichtedarstellung zu nutzen, maßgeblich durch praktische Erwägungen beeinflusst wurde, da sich das Einfärben eines Stromkreises mit Farbstiften deutlich unkomplizierter gestaltet als das Einzeichnen unzähliger Punkte per Hand. Der hierzu nötige Wechsel zwischen den unterschiedlichen Darstellungsformen bereitete den Schülern keinerlei Probleme.

\subsubsection{Zusammenfassung und Bewertung der Befunde}

Die bisherige Auswertung der Teaching Experiments hatte eine detaillierte Analyse von Akzeptanz, Paraphrasierung, Aufgabenverständnis und Schülervorstellungen zum Ziel. Im Folgenden sollen nun die wichtigsten Erkenntnisse der Akzeptanzbefragung zu einem Gesamtbild zusammengeführt und eine Bewertung vorgenommen werden.

\subsubsection{Zusammenfassung und Bewertung der Akzeptanz der Grundideen?}

Die Akzeptanzbefragung zeigt insgesamt, dass die Grundideen des angedachten Unterrichtsansatzes nach dem Elektronengasmodell von den Schülern akzeptiert und grundsätzlich verstanden werden. Besonders erfreulich ist, dass die Schüler mit den Grundkonzepten hinter dem Elektronengasmodell keine nennenswerten Schwierigkeiten zu haben scheinen. Die anfängliche Einführung in die Elektrostatik wird von den Schülern akzeptiert und bereitet ihnen mit Ausnahme eines Schülers keine grundsätzlichen Probleme. Auch die Atomvorstellung von freibeweglichen, sich gegenseitig abstoßenden Elektronen und ortsfesten Atomrümpfen in Metallen wird von allen Schülern akzeptiert und ist mit keinen nennenswerten Schwierigkeiten verbunden. Insbesondere ist positiv hervorzuheben, dass die Schüler die Erklärungen zum Thema Luftdruck und Luftströmungen für verständlich halten und es ihnen leichtfällt, an ihre Alltagserfahrungen mit Luftdruck anzuknüpfen und durch unterstützende Schülerexperimente mit Spritzen als Miniatur-Luftpumpen ein intuitives, aber für das Unterrichtskonzept

\footnotetext{
${ }^{9}$ Teile dieses Abschnitts wurden bereits in Burde und Wilhelm 2015 veröffentlicht.
} 
völlig ausreichendes Luftdruckverständnis im Sinne von „komprimierte Luft steht unter Druck, drückt gegen die Wände und hat das Bestreben sich auszudehnen" zu entwickeln. Im Anschluss gelingt es allen Schülern sich darüber bewusst zu werden, dass Luft stets von Bereichen hohen Drucks zu Bereichen niedrigen Drucks strömt, womit sie eine Ankervorstellung für das hier angestrebte Spannungskonzept besitzen.

In Anbetracht der höheren Komplexität der Kapitel „elektrischer Druck“ und „Spannung" wenig überraschend sinkt die grundsätzliche Akzeptanz und Aufgabenlösefähigkeit der Schüler im Vergleich zu den vorherigen drei Basiseinheiten etwas ab, befindet sich aber weiterhin auf einem sehr zufriedenstellenden Niveau. Vielversprechend ist insbesondere, dass den Schülern der Transfer des Luftdruckkonzepts auf den im Elektronengasmodell in elektrischen Stromkreisen herrschenden elektrischen Druck ohne Weiteres gelingt. Direkt nach der Einführung der Spannung als elektrischem Druckunterschied können etwa zwei Drittel der Schüler von sich aus mit Hilfe der Spannung erklären, warum Elektronen in einem Stromkreis durch ein Lämpchen strömen und dass dieser Elektronenstrom mit größer werdender Spannung ansteigen muss, d.h. die große Mehrheit der Schüler entwickelt bereits nach verhältnismäßig kurzer Zeit ein grundlegendes Verständnis der elektrischen Spannung als elektrischem Druckunterschied und Antrieb der Elektronenströmung. Vor dem Hintergrund, dass viele Schüler bei traditionellen Ansätzen auch nach der Sekundarstufe I kein angemessenes Spannungskonzept besitzen, sondern vielmehr glauben, die Spannung sei die Stärke bzw. Kraft des Stroms (Rhöneck und Völker 1984), ist dies ein sehr positives und ermutigendes Ergebnis. Problematisch ist hingegen, dass viele Schüler trotz ihres konzeptionellen Spannungsverständnisses von sich aus dazu tendieren, elektrische Stromkreise ausschließlich aus Sicht der Elektronenströmung zu analysieren. Obwohl den Schülern auf Nachfrage in der Regel bewusst ist, dass Strömungen eine Folge von elektrischen Druckunterschieden sind, tendieren sie teilweise bei der eigenständigen Analyse von Stromkreisen dazu, ohne elektrisches Potenzial oder elektrische Spannung zu argumentieren.

\subsubsection{Zusammenfassung und Bewertung der Lernschwierigkeiten}

Im zweiten Teil der Akzeptanzbefragung wurden die Schüler in kurzer Zeit mit verhältnismäBig anspruchsvollen Fragestellungen zu einer Reihe für sie neuer Konzepte wie beispielsweise Reihen- und Parallelschaltungen konfrontiert. Ziel war es Hinweise darauf zu erhalten, wo mögliche Lernschwierigkeiten eines weitergehenden Unterrichtskonzeptes zu erwarten sind. Es zeigt sich, dass es insbesondere beim Batterie- und Widerstandskonzept zu Verständnisschwierigkeiten kommt und die Schüler bei der Analyse von Stromkreisen teilweise mit bekannten Schülervorstellungen wie der Stromaussende- und Stromverbrauchsvorstellung argumentieren.

Die Batterie wird von vielen Schülern nicht als Konstantspannungs- sondern als Konstantstromquelle gesehen. Die daraus resultierenden Lernschwierigkeiten sind vielfältig und reichen von der Überzeugung, das Potenzial in den mit der Batterie verbundenen Leiterstücken sei abhängig von deren Abmessungen (größeres Volumen = geringerer Druck) oder den 
im Stromkreis verwendeten Widerständen (größerer Widerstand = größerer Druck) bis hin zur Schülervorstellung, der elektrische Druck an den Batteriepolen müsse bei Anschluss eines Leiterstuicks absinken bzw. ansteigen. In Anbetracht der Vielzahl der in Verbindung mit der Batterie aufgetretenen Verständnisschwierigkeiten scheint in den Teaching Experiments nicht hinreichend auf die Aufgabe von Batterien in Stromkreisen eingegangen worden zu sein.

Auch der Einfluss des elektrischen Widerstands auf die Stromstärke scheint einigen Schülern Verständnisschwierigkeiten zu bereiten. Dies zeigt sich u.a. daran, dass einige Schüler glauben, dass der Widerstand keinen Einfluss auf die Stromstärke habe oder sie sogar die inverse Widerstandsvorstellung vertreten, wonach ein größerer Widerstand zu einer höheren Stromstärke führt. Alternativ wurde von Schülern auch argumentiert, dass Elektronen auf ihrem Weg durch einen Widerstand an den Atomrümpfen hängenblieben bzw. dort verbraucht würden, was der bekannten Stromverbrauchsvorstellung entspricht.

Bei der Analyse von Parallelschaltungen zeigt sich, dass es den Schülern teilweise Probleme bereitet, die Elektronenströmung konsequent als eine Folge von Druckunterschieden zu sehen und zu erkennen, dass sich die Stromstärke in den Hauptzweigen aus der Summe der Stromstärken in den Nebenzweigen ergibt. Stattdessen analysieren einige Schüler die Parallelschaltung aus Sicht des Stroms und glauben, dieser müsse sich an Verzweigungspunkten zu gleichen Teilen aufteilen, was der lokalen Argumentation entspricht. Bei anderen Schülern kommt es zu einer Übergeneralisierung, wonach in Haupt- und Nebenzweigen die Stromstärke gleich groß sein müsse, weil dort auch der gleiche elektrische Druck herrscht.

Insbesondere bei der Analyse von Reihenschaltungen treten diverse bekannte Schülervorstellungen auf. So tendieren drei Schüler des Öfteren und zwei Schüler vereinzelt dazu, Reihenschaltungen aus Sicht der Elektronenströmung zu analysieren ohne dabei auf die eigentlich entscheidenden elektrischen Druckunterschiede einzugehen (,übermächtiger Strombegriff"). Diese Schüler sind bei der Analyse von Reihenschaltungen geneigt, die Stromaussendevorstellung zu vertreten, wonach die Elektronenströmung die Batterie am Minuspol verlässt und dann Lämpchen für Lämpchen die Reihenschaltung durchläuft (sequentielle Argumentation). Vor diesem Hintergrund ist es wenig verwunderlich, dass manche Schüler Schwierigkeiten haben, den Systemcharakter von Stromkreisen zu verstehen.

Die Verwendung der Fachbegriffe „Potenzial“ und „Spannung“ hat sich bei einigen Schülern als lernhinderlich erwiesen. Um das konzeptionelle Verständnis von Potenzial und Spannung nicht unnötig zu erschweren, bietet es sich insbesondere am Anfang einer Unterrichtseinheit an, alternative Begriffe wie „elektrischer Druck“ und „elektrischer Druckunterschied" zu nutzen. Der Vorteil eines solchen Vorgehens besteht darin, dass die Assoziation mit der Luftdruckanalogie gestärkt wird und die Schüler sich zunächst auf das Konzeptverständnis konzentrieren können statt mit wenig aussagekräftigen und teils missverständlichen Fachbegriffen zu operieren (vgl. Abschnitt 6.1.6). Eine sinnvolle Alternative zum Stromstärkebegriff stellt der Begriff der „Intensität der Elektronenströmung“ dar, da so der Teilchenund Strömungscharakter betont und eine Assoziation mit „Kraft“ bzw. „Druck“ vermieden 
wird. Die Punktedichtedarstellung hat sich zur Diskussion der Konstanz der Stromstärke vor und nach einem Widerstand als ungeeignet erwiesen, da die Schüler die beiden Konzepte „Stromstärke“ und „Strömungsgeschwindigkeit“" konzeptionell nur schwer trennen können.

\subsubsection{Zusammenfassung und Bewertung der Visualisierungen des Potenzials}

In den Teaching Experiments hat sich klar gezeigt, dass die Punktedichte- und Farbdarstellung des elektrischen Drucks von den Schülern akzeptiert und als am verständlichsten empfunden werden. Von den neun befragten Schülern favorisieren sechs Schüler die Punktedichtedarstellung und drei Schüler die Farbdarstellung, aber keiner die Graustufendarstellung oder die Plus-Minus-Darstellung. Sollen Schüler aber mit Hilfe einer Darstellungsform ihrer Wahl eigenständig Potenziale in Stromkreisen einzeichnen, entscheidet sich eine Mehrheit von fünf Schülern für die Farbdarstellung und lediglich drei Schüler für die Punktedichtedarstellung. Lediglich ein Schüler nutzt die Plus-Minus-Darstellung.

Die hohe Akzeptanz und Verständlichkeit der Punktedichtedarstellung in Kombination mit dem von den Schülern gezeigten problemlosen Wechsel der Visualisierungsform hin zur Farbdarstellung spricht dafür, beiden Darstellungen einen Platz im Unterrichtskonzept einzuräumen, so gleichzeitig ein Bewusstsein für den Modellcharakter der Darstellungen des elektrischen Drucks zu schaffen und damit auch die Modellkompetenz der Schüler zu fördern. Eine alleinige Verwendung der Punktedichtedarstellung ist nicht ratsam, da dann einige bedeutende Schwierigkeiten zu erwarten sind. So eignet sich die Punktedichtedarstellung einerseits unter praktischen Gesichtspunkten kaum für den alleinigen Einsatz im Unterricht, da das Einzeichnen verschiedener Punktedichten für die Schüler mit einem unverhältnismäßig hohen Zeitaufwand verbunden ist. Andererseits führt die Verwendung der Punktedichtedarstellung aber auch zu Erklärungs- und Verständnisschwierigkeiten in Hinblick auf die Konstanz der Stromstärke vor und nach einem Widerstand, da durch die unterschiedlichen Elektronendichten in statischen Bildern die Stromverbrauchsvorstellung ggf. ungewollt unterstützt wird. Die Konstanz der Stromstärke vor und nach dem Widerstand trotz unterschiedlicher Elektronendichten ließe sich zwar mit unterschiedlichen Strömungsgeschwindigkeiten erklären, jedoch zeigte sich in den Teaching Experiments, dass die Schüler Probleme haben, konzeptionell zwischen Stromstärke und Strömungsgeschwindigkeit zu unterscheiden. Hinzu kommt, dass eine solche Erklärung fachlich gesehen problematisch wäre (vgl. Abschnitt 6.1.7). Vor diesem Hintergrund erscheint es sinnvoll, den elektrischen Druck analog zum Luftdruck zunächst mit Hilfe der Punktedichtedarstellung an offenen Schaltungen einzuführen und anschließend bei der Diskussion von geschlossenen Stromkreisen mit fließenden Elektronenströmungen zur Farbdarstellung zu wechseln.

Das Konzept wurde zudem frühzeitig in einem Lehrergesprächskreis präsentiert und diskutiert. Viele Lehrkräfte äußerten hier die Befürchtung, die Farbdarstellung würde der typischen farblichen Kodierung von magnetischen Nord- und Südpolen zu sehr ähneln, was bei den Schülern zu Fehlvorstellungen und Verwechslungen zwischen Elektrizitätslehre und Magnetismus führen könnte. Um mögliche Lernschwierigkeiten in Verbindung mit dem 
Thema Magnetismus zu vermeiden und eine höhere Akzeptanz des Modells bei den Lehrern zu erreichen, erschien es daher sinnvoll, die Farbdarstellung leicht zu modifizieren. Wichtig war dabei, dass dem Farbschema auch weiterhin eine den Schülern aus dem Alltag bekannte und leicht zu erschließende Systematik zugrunde liegt, weshalb die Wahl auf Rot für einen hohen elektrischen Druck und Blau für niedrigen elektrischen Druck fiel. Der Vorteil ist, dass dieses Farbschema den meisten Schülern bereits aus ihrem Alltag vertraut sein dürfte, da entsprechend der Konvention z.B. bei Wetterberichten, Wärmebildkameras und Wasserhähnen hohe Temperaturen üblicherweise in Rot und niedrige Temperaturen üblicherweise in Blau dargestellt werden. Wie in Abb. 51 dargestellt, gilt für die modifizierte Farbdarstellung dementsprechend: Je höher der elektrische Überdruck, desto intensiver das Rot und je höher der elektrische Unterdruck, desto intensiver das Blau.
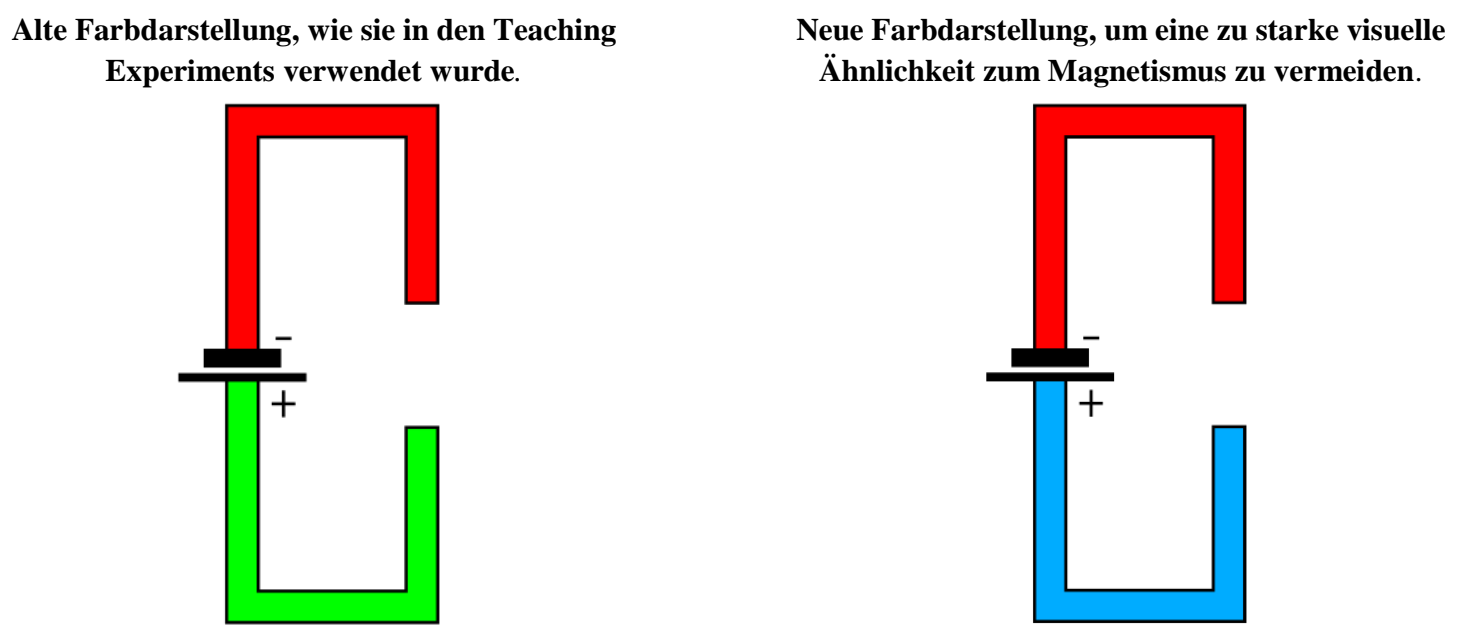

Abb. 51: Gegenüberstellung der ursprünglichen und der modifizierten Farbdarstellung des elektrischen Drucks

Ein potenzieller Nachteil der neuen Farbwahl liegt darin, dass üblicherweise z.B. an Spannungswürfeln Pluspole rot und Minuspole blau eingefärbt werden, was gegenteilig zu den im Modell verwendeten Farben ist. Inwiefern dies für die Schüler ein Problem darstellt, ist Gegenstand der unterrichtspraktischen Evaluation des Unterrichtskonzepts (vgl. Abschnitt 7.6).

\subsubsection{Konsequenzen in Hinblick auf das Unterrichtskonzept}

\subsubsection{Inhaltliche und strukturelle Änderungen am Konzept}

Wie die Akzeptanzbefragung gezeigt hat, haben die Schüler mit der Hinführung zu den Grundkonzepten hinter dem Elektronengasmodell wie der Elektrostatik und der Atom- und Luftdruckvorstellung nur minimale Schwierigkeiten. Während die ersten drei Einheiten daher nur Detailverbesserungen bedürfen, zeigen die im zweiten Teil der Akzeptanzbefragung aufgetretenen Verständnisschwierigkeiten, dass insbesondere das Widerstandskonzept und die Aufgabe einer Batterie im Stromkreis in einem Unterrichtskonzept stärker thematisiert werden müssen. Für das Unterrichtskonzept ergeben sich daher einige strukturelle Änderungen, die in Tab. 12 der bisherigen Struktur der Teaching Experiments gegenübergestellt sind und im Folgenden ausführlicher dargelegt werden. 
Tab. 12: Gegenüberstellung der Struktur der Teaching Experiments und der des neuen Unterrichtskonzepts

Struktur der Teaching Experiments

1. Elektrostatik

2. Atomvorstellung

3. Der Luftdruck

4. Das Potenzial als elektrischer Druck

5. Die Spannung als Druckunterschied

6. Die Stromstärke und Vertiefungsaufgaben

a. Einfluss von R auf I

b. Parallelschaltungen

c. Reihenschaltungen

\section{Struktur des neuen Unterrichtskonzepts}

1. Elektrostatik und Atomvorstellung

2. Luftströmungen durch Druckunterschiede

3. Batterie, elektrischer Druck und Spannung

4. Der elektrische Strom und Widerstand

5. Die Parallelschaltung

6. Der Kondensator

7. Die Reihenschaltung

8. Messung und Berechnung von Stromstärke, Spannung und Widerstand

Die inhaltliche Struktur des neuen Unterrichtskonzepts ist an die bisher in den Teaching Experiments genutzte Struktur angelehnt. Insbesondere kann im neuen Unterrichtskonzept der bewährte Einstieg über die Elektrostatik und Atomvorstellung sowie die Einheit zum Luftdruck größtenteils übernommen werden. Da in der Farbdarstellung ein Elektronenüberschuss bzw. elektrischer Überdruck mit der Farbe Rot und ein Elektronenmangel bzw. elektrischer Unterdruck mit der Farbe Blau dargestellt wird, erscheint es im Gegensatz zu den Teaching Experiments sinnvoll, bei der Einführung der Atomvorstellung auch Elektronen bereits rot und Atomrümpfe blau darzustellen. Ferner sollte in Folge des Feedbacks der Schüler die Frage danach, wie ein elektrisch geladener Körper neutralisiert werden kann, nun erst nach der Einführung der Atomvorstellung thematisiert werden.

Vor dem Hintergrund der mit dem Widerstandskonzept aufgetretenen Probleme sollte in der Luftdruckeinheit jedoch anders als in der bisherigen Konzeption bereits eine erste Widerstandsvorstellung bei den Schülern etabliert werden. Schüler sollten die wichtige Erfahrung machen, dass ein Stück Stoff eine Luftströmung behindert bzw. hemmt und somit einen Widerstand für sie darstellt. Da die Schülerexperimente mit den Spritzen als MiniaturLuftpumpen von den Schülern als sehr hilfreich empfunden wurden, ist es sinnvoll, diese auch wieder im geplanten Unterrichtskonzept zu verwenden.

In der dritten Einheit sollten dann das Potenzial und die Spannung gemeinsam an offenen Stromkreisen eingeführt werden, um zunächst den konzeptionellen Unterschied zwischen diesen beiden Größen klar herausarbeiten zu können, ohne gleichzeitig auf Stromstärke und Widerstand eingehen zu müssen. Zur Visualisierung des Elektronenüberschusses bzw. des Elektronenmangels bietet es sich an, zunächst wieder auf die bereits in der Luftdruckeinheit verwendete Punktedichtedarstellung zurückzugreifen, um die Parallelen zwischen Luftdruck und elektrischem Druck zu verdeutlichen. Anschließend sollte dann aber ein Wechsel zur Farbdarstellung stattfinden, da sich die Farbdarstellung einerseits als praktikabler erwiesen hat und andererseits damit die für Schüler schwierige aber in der Punktedichtedarstellung nötige Unterscheidung zwischen Stromstärke und Strömungsgeschwindigkeit im späteren 
Unterrichtsverlauf entfällt. Ferner sollte in der dritten Einheit die Aufgabe einer Batterie vertieft thematisiert werden und herausgearbeitet werden, dass eine Batterie den elektrischen Druck in den mit ihren Polen verbundenen Leiterstücken u.a. unabhängig von deren Länge immer konstant hält.

Unterrichtsgegenstand der vierten Einheit sind der elektrische Strom und der elektrische Widerstand. Da sich der elektrische Widerstand in den Teaching Experiments als schwieriges Konzept erwiesen hat, ist es ratsam, den elektrischen Widerstand zunächst qualitativ in Anlehnung an das in der Luftdruckeinheit erworbene Widerstandskonzept einzuführen. Hierzu sollte zunächst rein phänomenologisch argumentiert werden, dass ein elektrischer Widerstand die Elektronenströmung in einem Stromkreis genauso behindert, wie ein Stück Stoff eine Luftströmung behindert. Diese intuitive Analogie sollte dann später um ein mikroskopisches Widerstandsmodell auf Basis der anfänglich eingeführten Atomrümpfe und Elektronen ergänzt werden, um den Schülern einerseits ein tieferes Verständnis von elektrischen Widerständen zu ermöglichen und andererseits der Stromverbrauchsvorstellung entgegenzuwirken. Die Darstellung der elektrischen Stromstärke mit Hilfe unterschiedlich dicker Strompfeile hat sich in den Teaching Experiments als nicht optimal erwiesen, da es von den Schülern vorgezogen wurde mit konkreten Zahlenwerten zu arbeiten. Aus diesem Grund sollte in der vierten Einheit nun nicht nur eine konzeptionelle Vorstellung des elektrischen Stroms vermittelt, sondern auch das Ampere als quantitatives Maß für die Intensität der Elektronenströmung eingeführt und in Kombination mit einfachen Strompfeilen verwendet werden. Nachdem nun die Grundgrößen Spannung, Widerstand und Stromstärke bekannt sind, sollte die wechselseitige Beziehung der verschiedenen Größen anhand eines Schaubilds weiter gefestigt werden (vgl. Abb. 57 auf S.165). Das Ziel sollte hierbei sein, den Schülern qualitativ bewusst zu machen, dass der elektrische Druckunterschied die Stromstärke bewirkt und der elektrische Widerstand die Stromstärke lediglich beeinflusst.

Aufbauend auf den Erfahrungen im weiterführenden Teil der Akzeptanzbefragung u.a. zu Parallel- und Reihenschaltungen bietet es sich als nächstes an, Parallelschaltungen im Unterricht zu behandeln, da sich mit ihnen eine Reihe für das Unterrichtskonzept grundlegender Konzepte einüben und vertiefen lassen. Neben der Erkenntnis, dass die Batterie eine Quelle konstanter Spannung und nicht konstanten Stroms ist, zählt hierzu insbesondere, dass Stromkreise immer ausgehend von an den einzelnen Lämpchen anliegenden elektrischen Druckunterschieden zu analysieren sind. Ferner kann auch der Schülervorstellung begegnet werden, dass der elektrische Strom in Leitern gleichen elektrischen Drucks gleich groß sein muss.

Um die für die Analyse von Reihenschaltungen nötige dynamische Modellvorstellung vorzubereiten, sollten anschließend mit Hilfe von Anfangs-, Übergangs- und Endzuständen die Lade- und Entladevorgänge von Kondensatoren besprochen werden. Auf Grundlage der dynamischen Modellvorstellungen können im Anschluss dann Reihenschaltungen mit gleichen und ungleichen Widerständen diskutiert werden, wobei zu überlegen ist, den Schülern als alternative Erklärung zusätzlich auch die Fahrradkettenanalogie anzubieten. Dies würde 
den Schülern eine weitere Perspektive auf Reihenschaltungen ermöglichen und ein Anlass für eine Reflexion der Analogienutzung im Unterricht darstellen. Außerdem kann mit Hilfe der Fahrradkettenanalogie den Schülern noch einmal mit einem anderen Modell der Systemcharakter von Stromkreisen bewusstgemacht und die Stromverbrauchsvorstellung entkräftet werden. Nachdem die Anschlussbedingungen von Volt- und Amperemetern in Stromkreisen eingeübt wurden, kann dann der Widerstandswert $R$ als Maß dafür eingeführt werden, welcher elektrische Druckunterschied an einem Widerstand nötig ist, um eine Elektronenströmung von $1 \mathrm{~A} \mathrm{zu}$ verursachen. Da in dem angestrebten Unterrichtskonzept das qualitative Verständnis im Mittelpunkt steht, sollte eine Quantifizierung der physikalischen Größen sowie eine Thematisierung des Ohm'schen Gesetzes erst gegen Ende der Unterrichtsreihe stattfinden.

Um den dokumentierten Verständnisschwierigkeiten im Unterrichtskonzept vorzubeugen, sollten Schüler und Lehrer ferner mit Hilfe von Info-Boxen im Lehr- und Lernmaterial auf typische Lernschwierigkeiten und Schülervorstellungen aufmerksam gemacht werden. Neben diesen strukturellen und inhaltlichen Änderungen erscheint es insbesondere vor dem Hintergrund der mit den Begriffen „Potenzial“, „Spannung“ und „Stromstärke“ aufgetretenen Problemen sinnvoll, hier vorübergehend gewisse begriffliche Modifikationen vorzunehmen (vgl. Abschnitt 6.1.6). Die Verwendung des Begriffs ,elektrischer Druck“ statt „Potenzial“ hat beispielsweise den Vorteil, dass so einerseits die Vorzeichenproblematik beim Potenzial vermieden wird und andererseits die Analogie zum Luftdruck betont wird. Dementsprechend sollte im Unterrichtskonzept der Begriff des ,elektrischen Druckunterschieds“ zumindest vorübergehend synonym für die ,elektrische Spannung“ genutzt werden, um sowohl die Analogie zum Luftdruck als auch den Charakter der Differenzgröße der Spannung hervorzuheben.

\subsubsection{Die aufgetretenen Schülervorstellungen und ihre Folgen}

Der oben dargelegten Strukturierung des neuen Unterrichtskonzepts entsprechend soll im Folgenden für jede der aufgetretenen Schülervorstellungen dargelegt werden, welche konkreten Konsequenzen jeweils für das Unterrichtskonzept gezogen wurden. Eine Darstellung der Konsequenzen für Einheit 8 ,Messung und Berechnung von Stromstärke, Spannung und Widerstand" entfällt, da die Inhalte dieser Einheit nicht Teil der Teaching Experiments waren. In Tab. 13 sind noch einmal alle aufgetretenen Schülervorstellungen mit den dazugehörigen Codes aufgelistet, da der folgenden Abschnitt auf diesen aufbaut.

Tab. 13: Übersicht über die aufgetretenen Schülervorstellungen mit dazugehörigen Codes

\begin{tabular}{|l|l|}
\hline Code & Beschreibung der Schülervorstellung \\
\hline E-Teil & Verwechslung von elektrischen Ladungen mit Energie(teilchen) \\
\hline R-frei & Es wird angenommen, Atomrümpfe seien in Metallen frei beweglich \\
\hline Dr-ab & $\begin{array}{l}\text { Der elektrische Druck baut sich bei Anschluss eines Leiters in den Batteriepolen ab, so } \\
\text { wie auch der Luftdruck in der Spritze bei Ventilöffnung nachlässt }\end{array}$ \\
\hline Sp-lok & Spannung als lokale Größe statt Differenzgröße \\
\hline Str-aus & Batterie als Elektronenfass statt Elektronenpumpe (Stromaussendevorstellung) \\
\hline E-Stau & $\begin{array}{l}\text { Ein größerer Widerstand führt zu einem größeren Elektronenrïckstau und damit einem } \\
\text { größeren Druckunterschied in den mit der Batterie verbundenen Leitern }\end{array}$ \\
\hline
\end{tabular}




\begin{tabular}{|c|c|}
\hline Dr-LA & $\begin{array}{l}\text { Der elektrische Druck in den mit der Batterie verbundenen Leiterstücken ist abhängig } \\
\text { von den Leiterabmessungen }\end{array}$ \\
\hline Inv-W & $\begin{array}{l}\text { Ein Lämpchen mit einem größeren Widerstand braucht eine größere Stromstärke (in- } \\
\text { verse Widerstandsvorstellung) }\end{array}$ \\
\hline B-kStQ & $\begin{array}{l}\text { Gleiche Batterie = gleiche Stromstärke durch das Lämpchen (unabhängig vom Wider- } \\
\text { stand) }\end{array}$ \\
\hline gDU-gS & $\begin{array}{l}\text { Gleicher Druckunterschied = gleiche Stromstärke durch das Lämpchen (unabhängig } \\
\text { vom Widerstand) }\end{array}$ \\
\hline gD-gS & $\begin{array}{l}\text { Parallelschaltung: Wo ein gleicher elektrischer Druck herrscht, ist auch die Stromstärke } \\
\text { gleich (Übergeneralisierung) }\end{array}$ \\
\hline SV & Stromverbrauchsvorstellung (= Elektronen bleiben teilweise in Lämpchen stecken) \\
\hline LA & Strom teilt sich an Knoten zu gleichen Teilen auf (lokale Argumentation) \\
\hline Fließ-Dr & Es wird von fließendem Druck statt strömenden Elektronen gesprochen \\
\hline D-vs-DU & $\begin{array}{l}\text { Begrifflich wird nicht zwischen Druck und Druckunterschied unterschieden (auch wenn } \\
\text { dies ggf. konzeptionell der Fall ist) }\end{array}$ \\
\hline $\mathbf{S}=\mathbf{S t r o ̈ G}$ & $\begin{array}{l}\text { Verwechslung oder Gleichsetzung von Stromstärke mit Strömungsgeschwindigkeit der } \\
\text { Elektronen }\end{array}$ \\
\hline SA & Sequentielles Denken bei Reihenschaltungen \\
\hline Übm-Str & $\begin{array}{l}\text { Tendenz mit Hilfe von Strömungen zu argumentieren, statt Strömungen über Druckun- } \\
\text { terschiede zu erklären (übermächtiger Strombegriff) }\end{array}$ \\
\hline D-vs-S & Mangelnde Differenzierung zwischen elektrischem Druck und elektrischem Strom \\
\hline
\end{tabular}

\section{1) Elektrostatik und Atomvorstellung}

Schülervorstellung Konsequenzen für das Unterrichtskonzept

E-Teil Die Lehrer sollten dafür sensibilisiert werden, dass manche Schüler dazu tendieren, positive bzw. negative Ladungen mit positiver bzw. negativer Energie oder Energieteilchen zu verwechseln.

R-frei Mit Hilfe von Info-Boxen im Lernmaterial sollten die Schüler darauf hingewiesen werden, dass Atomrümpfe nicht beweglich sind. Ferner sollten hierzu auch entsprechende Übungsaufgaben angeboten werden.

\section{1) Luftströmungen durch Druckunterschiede}

\section{Schülervorstellung Konsequenzen für das Unterrichtskonzept}

Inv-W +

gDU-gS
Mit Hilfe von Schülerexperimenten sollte bereits bei der Thematisierung des Luftdrucks bei den Schülern eine Ankervorstellung für den elektrischen Widerstand etabliert werden. Die Schüler könnten hierzu Luft durch unterschiedlich dicke Stoffe blasen und so erfahren, dass eine (Luft-)Strömung umso mehr behindert bzw. gehemmt wird, desto dicker der Stoff bzw. je größer der Widerstand ist. Anschließend sollte eine Regel zur Bestimmung der Intensität der Luftströmung unter Berücksichtigung der Hemmung durch den Stoffwiderstand aufgestellt werden. Zusätzlich sollten Übungsaufgaben bearbeitet werden, bei denen der Einfluss des Stoffwiderstands auf die Intensität der Luftströmung thematisiert wird. 
D=DU Die Lehrer sollten explizit darauf hingewiesen werden, bei der Besprechung von Luftströmungen konsequent zwischen Druck und Druckunterschied zu differenzieren und dies auch von den Schülern zu erwarten. Darüber hinaus sollten entsprechende Übungsaufgaben von den Schülern bearbeitet werden, um ihnen den wichtigen konzeptionellen Unterschied zwischen den beiden Größen bewusst zu machen.

Übm-Str

Bereits in der Luftdruckeinheit sollte stärker auf den Druckunterschied als Ursache für Strömungen eingegangen werden, z.B. mit Hilfe diverser Übungsaufgaben. Die Lehrer und Schüler sollten außerdem mit Hilfe von Info-Boxen explizit auf die Bedeutung des Druckunterschieds hingewiesen werden.

\section{2) Batterie, elektrischer Druck und Spannung}

\section{Schülervorstellung Konsequenzen für das Unterrichtskonzept}

Sp-lok +

$\mathrm{D}=\mathrm{DU}$

Dr-ab

Dr-LA
Die elektrische Spannung sollte als elektrischer Druckunterschied eingeführt werden, um den Differenzcharakter der Größe stärker zu betonen. Außerdem sollte den Schülern mit Hilfe von diversen Übungsaufgaben der Unterschied zwischen elektrischem Druck und elektrischem Druckunterschied deutlicher bewusstgemacht werden. Statt von „Potenzial“ und „Spannung“ sollte zumindest anfänglich besser von ,elektrischem Druck“ und „elektrischem Druckunterschied“ gesprochen werden. Ferner sollten Lehrer und Schüler explizit auf diese Problematik hingewiesen werden.

Es sollte klar auf den wichtigen Unterschied zu den Schülerexperimenten mit Spritzen hingewiesen werden und explizit thematisiert werden, dass der elektrische Druck der Batteriepole auch bei Anschluss eines Leiters konstant bleibt.

Mit Hilfe von entsprechenden Hinweisen und Übungsaufgaben sollte den Schülern bewusstgemacht werden, dass der elektrische Druck in den mit den Batteriepolen verbundenen Leiterstücken unabhängig von deren Abmessungen ist.

\section{3) Der elektrische Strom und Widerstand}

\section{Schülervorstellung Konsequenzen für das Unterrichtskonzept}

Str-aus

Der Stromaussendevorstellung sollte u.a. dadurch begegnet werden, dass einerseits die Lehrer für diese Schülervorstellung sensibilisiert werden und andererseits den Schülern mit Hilfe von Info-Boxen und passenden Übungsaufgaben bewusstgemacht wird, dass die Batterie nicht mit einem Fass Öl sondern mit einer Pumpe zu vergleichen ist. Ferner sollten die Lehrer darauf hingewiesen werden, die Elektronenströmung nicht 
E-Stau

Inv-W

Ba-KStQ

SV

Fließ-Dr

$\mathrm{S}=\mathrm{StröG}$ immer schrittweise ausgehend vom Minuspol aus zu analysieren, da dies die Stromaussendevorstellung noch zusätzlich fördern kann.

Bei der Diskussion von Stromkreisen mit Hilfe von elektrischen Drücken sollte der Begriff des „Elektronenrückstaus“ am Lämpchen vermieden werden und stattdessen betont werden, dass die Batterie den Druck in den mit ihren Polen verbundenen Leitern auch dann konstant hält, wenn Elektronen strömen.

Das Verständnis für das elektrische Widerstandskonzept sollte dadurch gefördert werden, dass den Schülern immer wieder die Analogie zwischen der Behinderung der Luftströmung durch ein Stück Stoff und der Behinderung der Elektronenströmung durch einen elektrischen Widerstand vor Augen geführt wird. Durch Hinweise und Sensibilisierung der Lehrkräfte sollte ferner versucht werden, dem Missverständnis vorzubeugen, dass ein großer Widerstand ein geometrisch gesehen großes Bauteil darstellt. Zu diesem Zweck sollte später auch der Begriff des Widerstandswerts $R$ eingeführt werden, um sprachlich sauber zwischen dem Widerstandsbauteil und dem Wert des Widerstands differenzieren zu können.

Es sollte stärker betont werden, dass eine Batterie eine Konstantspannungs- und keine Konstantstromquelle darstellt und die Stromstärke vom elektrischen Druckunterschied und dem verwendeten Widerstand abhängt. Hierzu sollte u.a. die wechselseitige Beziehung von $U, R$ und $I$ in einem Schaubild dargestellt und besprochen werden.

Durch verschiedene Maßnahmen sollte stärker betont werden, dass Elektronen in Lämpchen oder Widerständen nicht hängen bleiben bzw. verbraucht werden. Hierzu kann u.a. ein Vergleich zu einer Luftpfeife bemüht werden, bei der durch das Pfeifgeräusch ebenso wenig „Luftteilchen“ verbraucht werden wie Elektronen in einem Lämpchen. Ferner bietet es sich mit Hilfe der Fahrradkettenanalogie an, nicht nur der Stromverbrauchsvorstellung entgegenzuwirken, sondern den Schülern auch den Systemcharakter von Stromkreisen bewusster zu machen. Entscheidend ist aber vor allem, den Schülern auf Basis der anfangs eingeführten Atomvorstellung ein anschauliches Widerstandsmodell zu vermitteln, um ihnen eine plausible Erklärung der Vorgänge auf mikroskopischer Ebene zu ermöglichen.

Die Lehrer sollten mit Hilfe einer Info-Box in den Lehrermaterialien dafür sensibilisiert werden, bei Schülern auf eine korrekte Ausdrucksweise zu achten.

Durch die Nutzung der Farb- statt der Punktedichtedarstellung bei geschlossenen Stromkreisen entfällt die Notwendigkeit der für Schüler schwierigen Unterscheidung zwischen Stromstärke und Strömungsge- 
schwindigkeit. Ein weiterer Vorteil der Farbdarstellung besteht darin, dass Schüler das Potenzial in Stromkreisen deutlich unkomplizierter einzeichnen können als mit der Punktedarstellung.

Die zusätzliche Nutzung der Fahrradkettenanalogie ermöglicht es, den Systemcharakter von Stromkreisen zu verdeutlichen und so dem sequentiellen Denken der Schüler entgegenzuwirken. Die Lehrer sollten dafür sensibilisiert werden, die Elektronenströmung möglichst nicht schrittweise ausgehend vom Minuspol aus zu analysieren, da dies ungewollt bei den Schülern das sequentielle Denken fördern kann.

\section{4) Die Parallelschaltung}

\section{$\underline{\text { Schülervorstellung Konsequenzen für das Unterrichtskonzept }}$}

$\mathrm{gD}-\mathrm{gS}+$

$\mathrm{D}=\mathrm{DU}$

$\mathrm{Ba}-\mathrm{KStQ}+$

$\mathrm{LA}+$

Übm-Str
Die Analyse von Parallelschaltungen stellt für die Schüler eine ausgezeichnete Lerngelegenheit dar, konzeptionell zwischen dem elektrischen Druck und dem elektrischen Strom sowie elektrischem Druck und elektrischem Druckunterschied differenzieren zu lernen. Eine Diskussion von Parallelschaltungen bietet sich daher nach der Einführung von Widerstand, Stromstärke und Spannung an dieser Stelle an.

Die Diskussion von Parallelschaltungen ermöglicht es ferner, den Schülern bewusst zu machen, dass Stromkreise immer ausgehend von den an Lämpchen anliegenden Druckunterschieden und den sich daraus ergebenden Elektronenströmungen $\mathrm{zu}$ analysieren sind. Auf diese Weise kann sowohl der Batterie als Konstantstromquellenvorstellung, der lokalen Argumentation als auch der Tendenz der Schüler entgegengewirkt werden, Stromkreise aus Sicht der Elektronenströmung zu analysieren. Es bietet sich ferner an, das zur systematischen Analyse von Parallelschaltungen nötige Vorgehen auch schriftlich zu fixieren und mit Hilfe passender Übungsaufgaben zu vertiefen.

\section{5) Der Kondensator}

\section{Schülervorstellung Konsequenzen für das Unterrichtskonzept}

Sp-lok +

Die Analyse der Vorgänge beim Laden bzw. Entladen eines Kondensa$\mathrm{D}=\mathrm{DU}$ tors mit Hilfe der elektrischen Druckvorstellung stellt für die Schüler eine weitere Lerngelegenheit dar, zwischen elektrischem Druck und elektrischem Druckunterschied differenzieren zu lernen und so die Spannung als Differenzgröße zu begreifen. Die Analyse der Lade- bzw. Entladevorgänge eines Kondensators mit Anfangs-, Übergangs- und Endzuständen ermöglicht es den Schülern im Besonderen eine dynamische Modellvorstellung zu entwickeln, was ihr Verständnis von Reihenschaltungen erleichtern dürfte. 
Kapitel 6: Entwicklung des Unterrichtskonzepts

\section{6) Die Reihenschaltung}

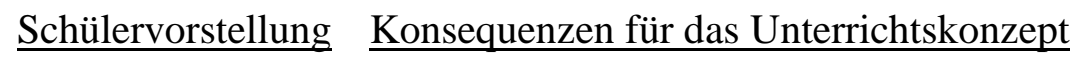

E-Stau Im Unterricht sollte wiederholt betont werden, dass der elektrische Druck in den Leiterstücken, die direkt mit der Batterie verbunden sind, von der Batterie konstant gehalten wird. Hierzu sollten entsprechende Übungen und Hinweise in die Lehrmaterialien aufgenommen und der Begriff des „Elektronenstaus“ in diesem Zusammenhang gemieden werden.

$\mathrm{SV}+\mathrm{SA} \quad$ Um der Stromverbrauchsvorstellung entgegenzuwirken und den Systemcharakter von Stromkreisen zu unterstreichen, bietet es sich an, neben der Analyse von Reihenschaltungen mit Hilfe der elektrischen Druckvorstellung an dieser Stelle auch auf die Fahrradkettenanalogie zurückzugreifen.

\subsection{Die Sachstruktur des fertigen Unterrichtskonzepts ${ }^{10}$}

Wie der vorangegangene Abschnitt gezeigt hat, konnten mit Hilfe der Teaching Experiments sowohl eine Reihe erfolgsversprechender als auch potenziell problematischer Aspekte der angedachten Konzeption identifiziert werden. Im Sinne von Design-Based-Research wurde auf Basis der so gewonnenen Erkenntnisse ein Unterrichtskonzept inklusive passender Unterrichtsmaterialien wie Overheadfolien, Übungsaufgaben und Zusammenfassungen für den praktischen Einsatz im realen Physikunterricht entwickelt. Um den Lehrkräften darüber hinaus eine Vorstellung davon zu geben, wie das Konzept im Unterricht konkret umgesetzt werden soll, wurde auch ein Lehrerleitfaden mit vielen didaktischen Hinweisen und Tipps zur Unterrichtsgestaltung erstellt. Ein solches Vorgehen ist typisch für Design-Based-Research, da für diesen Ansatz die spätere Implementation der Forschungsergebnisse in der Praxis und die damit einhergehende Überwindung des Practice-Research-Gaps ein wesentliches Anliegen darstellt. Mit der umfassenden Bereitstellung von Unterrichtsmaterialien wird zudem angestrebt, eine gewisse Vergleichbarkeit des Unterrichts in den verschiedenen Treatmentklassen bzgl. der Umsetzung wesentlicher didaktischer Ideen zu gewährleisten, um verallgemeinerbare Aussagen über die Lernwirksamkeit des Unterrichtskonzepts als Gesamtpaket in der Schulpraxis treffen zu können (vgl. Abschnitt 7.1). Die Sachstruktur und die damit verbundenen didaktischen Ideen des Unterrichtskonzepts sollen im Folgenden exemplarisch dargelegt werden. Das komplette Unterrichtskonzept inklusive aller ausgearbeiteten Unterrichtsmaterialien, die im Rahmen dieser Studie den Lehrkräften zur Verfügung gestellt wurden, können auf der Internetseite JP-Bur.de/Dissertation heruntergeladen werden.

\footnotetext{
${ }^{10}$ Teile dieses Abschnitts wurden bereits in Burde und Wilhelm 2017a veröffentlicht.
} 


\subsubsection{Elektrostatik und Atomvorstellung}

Sofern die Schüler aus ihrem bisherigen Unterricht keine Kenntnisse zur Elektrostatik mitbringen, werden zunächst die elektrostatische Kraftwirkung und ihre Abstandsabhängigkeit an einfachen Beispielen thematisiert. Mit Blick auf die für das Unterrichtskonzept zentrale Idee des Elektronengases sowie eine spätere mikroskopische Modellvorstellung des elektrischen Widerstands wird anschließend der atomare Aufbau von Metallen am Beispiel von Kupfer mit Hilfe von Atomrümpfen und Elektronen besprochen (siehe Abb. 52).

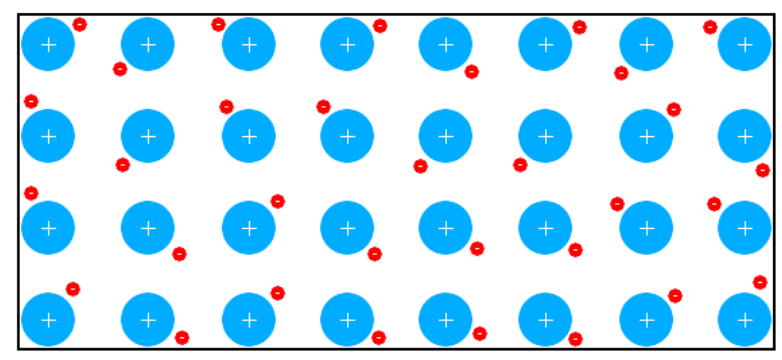

Abb. 52: Modellvorstellung des atomaren Aufbaus von Kupfer mit Atomrümpfen (blau) und Elektronen (rot)

\subsubsection{Luftströmungen durch Druckunterschiede}

Als nächstes wird an die intuitive Luftdruckvorstellung der Schüler im Sinne von „komprimierte Luft steht unter Druck, drückt gegen die Wände und hat das Bestreben sich auszudehnen" angeknüpft, um an bekannten Alltagsobjekten wie aufgeblasenen Fahrradreifen und in Schülerexperimenten zu klären, dass Luft immer von Bereichen höheren Drucks zu Bereichen niedrigeren Drucks strömt. Ergebnis sollte die Erkenntnis sein, dass Luftströmungen immer eine Folge von Druckunterschieden sind und dass zwischen den beiden Größen Druck und Druckunterschied konzeptionell unterschieden werden muss. Die explizite Thematisierung von Luftdruckphänomenen geschieht vor dem Hintergrund, dass Lernende konzeptionell Schwierigkeiten haben, zwischen Druck- und Druckdifferenz zu unterscheiden.

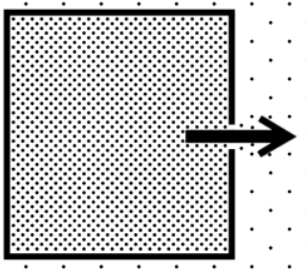

Kein (Stoff-)Widerstand, Große (Luft-)Strömung

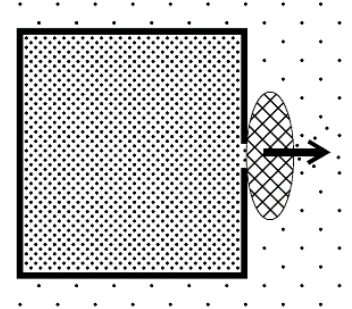

Kleiner (Stoff-)Widerstand, Mittlere (Luft-)Strömung

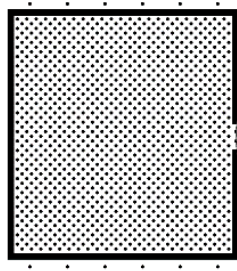

Großer (Stoff-)Widerstand,

Kleine (Luft-)Strömung

Abb. 53: Intensität der Luftströmung in Abhängigkeit vom Druckunterschied und dem (Stoff-)Widerstand

Abgeschlossen wird die Einheit mit einer Hinführung zur Widerstandsvorstellung, indem die Schüler ein Stück Stoff (z.B. ein Schal, Kragen oder Ärmel) nehmen und Luft durch dieses blasen. Je dicker das Stück Stoff dabei gefaltet wird, desto stärker ist die Hemmung bzw. Behinderung der Luftströmung (siehe Abb. 53). Die Hemmung bzw. Behinderung der Luftströmung durch den Stoff wird dann als Widerstand bezeichnet. Dieser Vorkurs zu Luftdruckphänomenen nimmt bewusst keinen großen Teil des Unterrichtskonzepts ein, da nicht 
der analoge, sondern der primäre Lernbereich im Fokus des Unterrichts stehen sollten (vgl. Abschnitt 4.1.2) und weitergehende Analogversuche aufwendig und aufgrund der fehlenden Sichtbarkeit von Luft auch wenig anschaulich wären.

\subsubsection{Batterie, Potenzial und Spannung}

In dieser Einheit wird die Vorstellung vom Luftdruck auf den in Leitern herrschenden elektrischen Druck übertragen. Die Grundidee ist dabei, dass sich in Metallen Elektronen in Teilchenform befinden und sich dort frei bewegen können. Da die Elektronen negativ geladen sind, werden sie durch Abstoßung so weit wie möglich auseinandergetrieben, weshalb sie den ihnen zur Verfügung stehenden Raum im gesamten Leiter gleichmäßig ausfüllen. Im Leiter kommt es aufgrund der gegenseitigen Coulomb-Abstoßung der Elektronen zu einem von der Elektronendichte abhängigen elektrischen Druck (für eine fachliche Auseinandersetzung sei auf Abschnitt 2.3 und für eine didaktische Auseinandersetzung auf Abschnitt 6.1.3 verwiesen). Hierzu wird angenommen, dass am Minuspol einer Batterie ein Elektronenüberschuss besteht und am Pluspol ein Elektronenmangel. Am Minuspol und dem mit ihm verbundenen Leiterstück herrscht also ein elektrischer Überdruck, während am Pluspol und dem mit ihm verbundenen Leiterstiuck ein elektrischer Unterdruck herrscht.

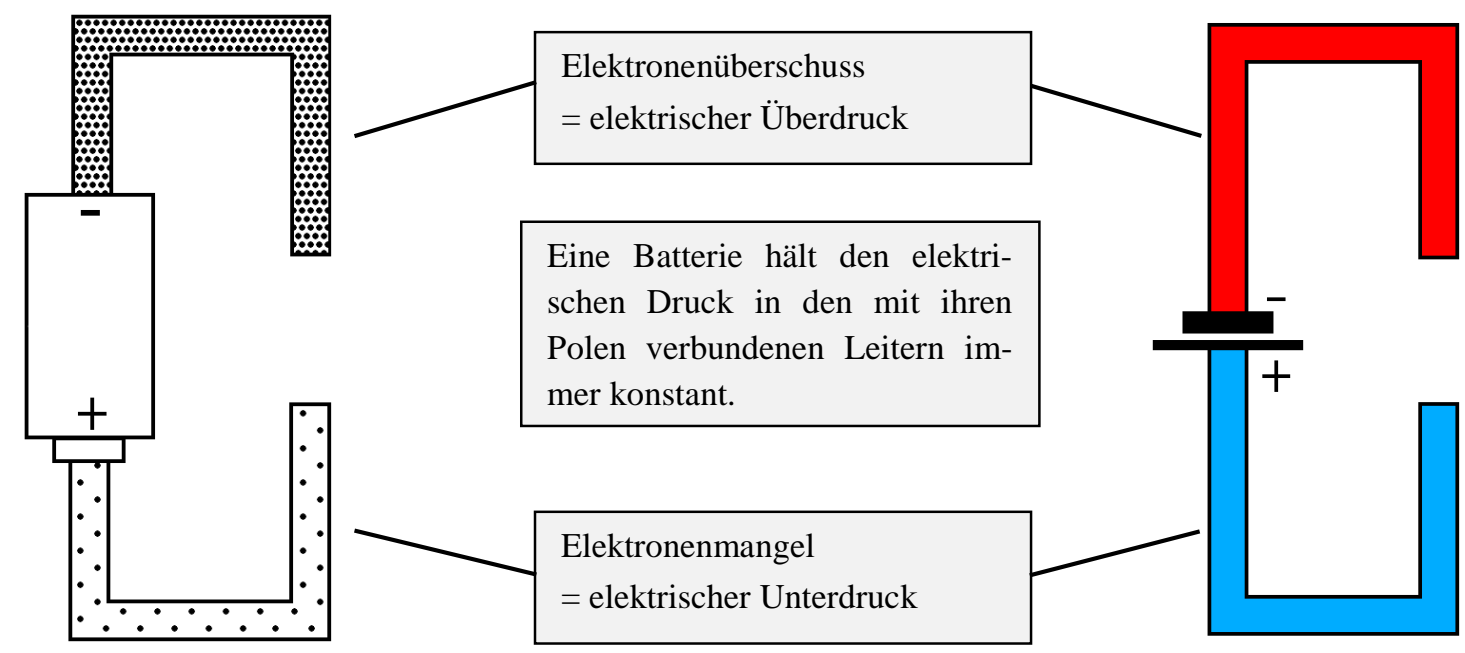

Abb. 54: Punktedichtedarstellung (links) und Farbdarstellung (rechts) des elektrischen Drucks

Um die konzeptionelle Nähe von Luftdruck und elektrischem Druck auch visuell zu unterstreichen und den Lernenden somit den Übergang hin zur Betrachtung der Vorgänge in einfachen elektrischen Stromkreisen auf Basis ihres intuitiven Luftdruckverständnisses zu erleichtern, wird die von Luftdruckphänomenen bereits bekannte Punktedichtedarstellung zunächst auf offene Stromkreise übertragen. Da es aus didaktischen Gründen geschickter ist, den elektrischen Über- und Unterdruck nicht mit Punkten, sondern mit Farben zu visualisieren, findet jedoch zügig ein Wechsel zur Farbdarstellung statt (siehe Abb. 54). So lässt sich der elektrische Druck mit Hilfe von Farbstiften von den Lernenden nicht nur deutlich unkomplizierter in Schaltkreisen einzeichnen, sondern es entfällt auch eine potenziell schwierige, aber in der Punktedichtedarstellung später nötige Unterscheidung zwischen Stromstärke und Strömungsgeschwindigkeit der Elektronen vor und nach Widerständen (vgl. Abschnitt 6.2.4.3). Im Gegensatz zur häufig unsystematischen Farbwahl bei bisherigen Konzepten, liegt dem hier 
verwendeten Farbschema eine Systematik zugrunde, die den Schülern aus ihrem Alltag vertraut sein sollte. Ein hoher elektrischer Druck wird daher in Rot und ein niedriger elektrischer Druck in Blau dargestellt, da entsprechend der Konvention z.B. auch bei Wetterberichten, Wärmebildkameras und Wasserhähnen hohe Temperaturen meist in Rot und niedrige Temperaturen meist in Blau dargestellt werden.

Um das konzeptionelle Verständnis des Potenzial- und Spannungsbegriffs nicht durch die Begriffswahl unnötig zu erschweren, wird das Potenzial zunächst als ,elektrischer Druck“ und die Spannung als „elektrischer Druckunterschied“ eingeführt. Der Vorteil dieser Begriffe liegt darin, dass die konzeptionelle Nähe zur Luftdruckeinheit betont wird und die Schüler nicht mit wenig aussagekräftigen und teils semantisch missverständlichen Fachbegriffen operieren müssen. Um Schülervorstellungen wie z.B. die konzeptionelle Gleichsetzung von Spannung und Strom zu vermeiden, wird der Begriff der „Intensität der Elektronenströmung“ statt der „Stromstärke“ verwendet, da so der Teilchen- und Strömungscharakter dieser mengenartigen Größe betont wird (vgl. Abschnitt 6.1.6). Nach der Einführung von elektrischem Druck und elektrischem Druckunterschied wird anschließend an verschiedenen offenen Schaltungen und Alltagsbeispielen (Batterie, Steckdose und Hochspannungsleitung) der Unterschied zwischen diesen beiden Größen konzeptionell weiter ausdifferenziert. Die Thematisierung der elektrischen Spannung an offenen Schaltungen geschieht insbesondere vor dem Hintergrund, dass Lernende oftmals nur ungenügend zwischen dem Spannungs- und Strombegriff differenzieren können. Indem hier Beispiele betrachtet werden, bei denen eine Spannung auch unabhängig vom elektrischen Strom existiert, soll ein eigenständiges Spannungskonzept gefördert werden. Dabei wird auch immer wieder betont, dass der elektrische Druck in den Leitern ausschließlich von der Spannungsquelle bestimmt wird und nicht von der Länge oder Abmessung der Leiter abhängt.

Da keine absoluten Werte für das elektrische Potenzial eingeführt werden, wird auf eine explizite Diskussion der Erdung eines Leiterabschnittes im Rahmen der Elementarisierung bewusst verzichtet. Darüber hinaus werden im Rahmen der Elementarisierung in Stromkreisen nur ideale Leiter und Batterien betrachtet, da sich gezeigt hat, dass dies den Lernenden das Verständnis erleichtert (Gleixner 1998, S. 232). In Bezug auf die Batterie hat dies zur Folge, dass die von ihr erzeugte elektrische Spannung im Stromkreis - entgegen einer realen Batterie mit einem Innenwiderstand - unabhängig von der Stromstärke als konstant angenommen wird. Aus der reinen Betrachtung idealer Leiter ergibt sich, dass das elektrische Potenzial innerhalb eines Leiterabschnitts konstant ist, obwohl dies bei einem realen Leiter mit geringem Widerstand nicht der Fall wäre.

\subsubsection{Der elektrische Strom und Widerstand}

Nachdem die Vorstellung vom elektrischen Druck am Beispiel offener Schaltungen ohne Stromfluss etabliert wurde, werden die elektrischen Druckunterschiede analog zur vorherigen Betrachtung von Luftdruckunterschieden nun als Ursache für Elektronenströmungen in Stromkreisen eingeführt. Hierzu wird an einem einfachen Stromkreis bestehend aus Batterie 
und Lämpchen besprochen, dass in Folge des am Lämpchen anliegenden elektrischen Druckunterschieds Elektronen durch das Lämpchen strömen und dieses zum Leuchten bringen. Die Höhe des elektrischen Drucks wird dabei mit Hilfe unterschiedlicher Farbintensitäten visualisiert. Hierbei gilt, dass der elektrische Druck umso höher ist, je intensiver das Rot ist, bzw. umso niedriger ist, desto intensiver das Blau ist (siehe Abb. 55). Da sich Lernende tendenziell an Oberflächenmerkmalen orientieren, sollte bei Experimenten darauf geachtet werden, dass Schaltplan und reale Schaltung nicht nur eine große topologische Ähnlichkeit aufweisen, sondern, dass auch die Farbe der verwendeten Kabel zur im Modell verwendeten Farbkodierung passt. Auf diese Weise wird angestrebt, die kognitive Belastung der Lernenden zu reduzieren und ihnen das Erkennen von Zusammenhängen zu erleichtern, da sich so das sonst nicht direkt beobachtbare elektrische Potenzial auch in der realen Schaltung erkennen lässt.
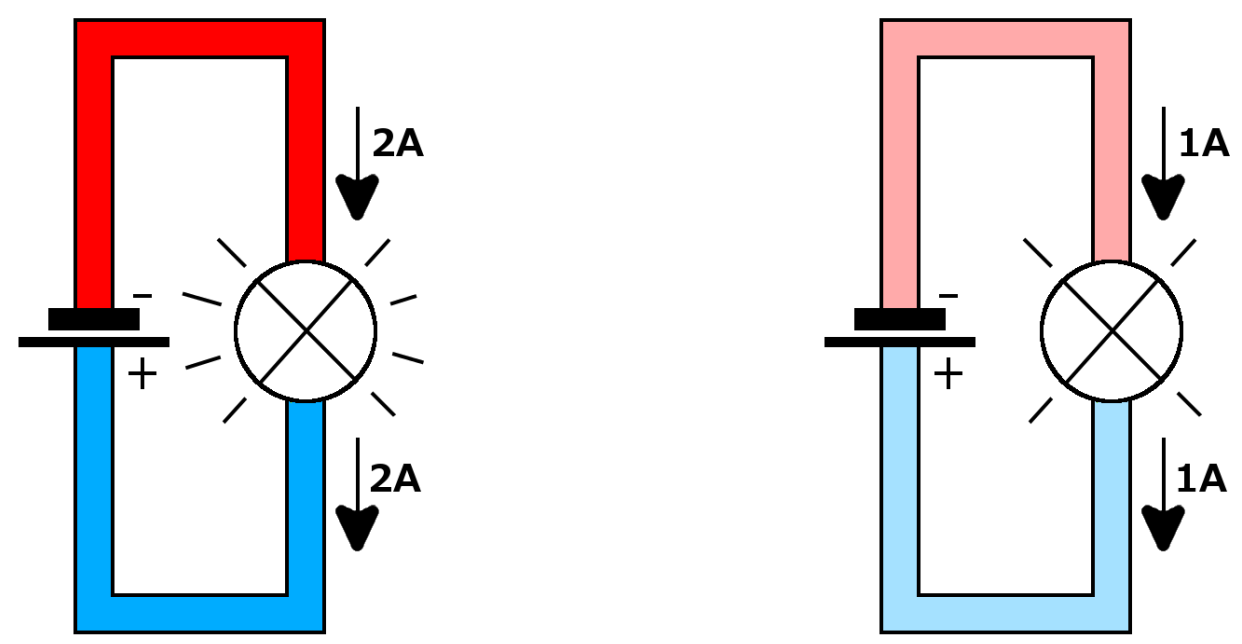

Abb. 55: Umso größer die Spannung (elektrischer Druckunterschied), desto größer die Stromstärke (Intensität der Elektronenströmung)
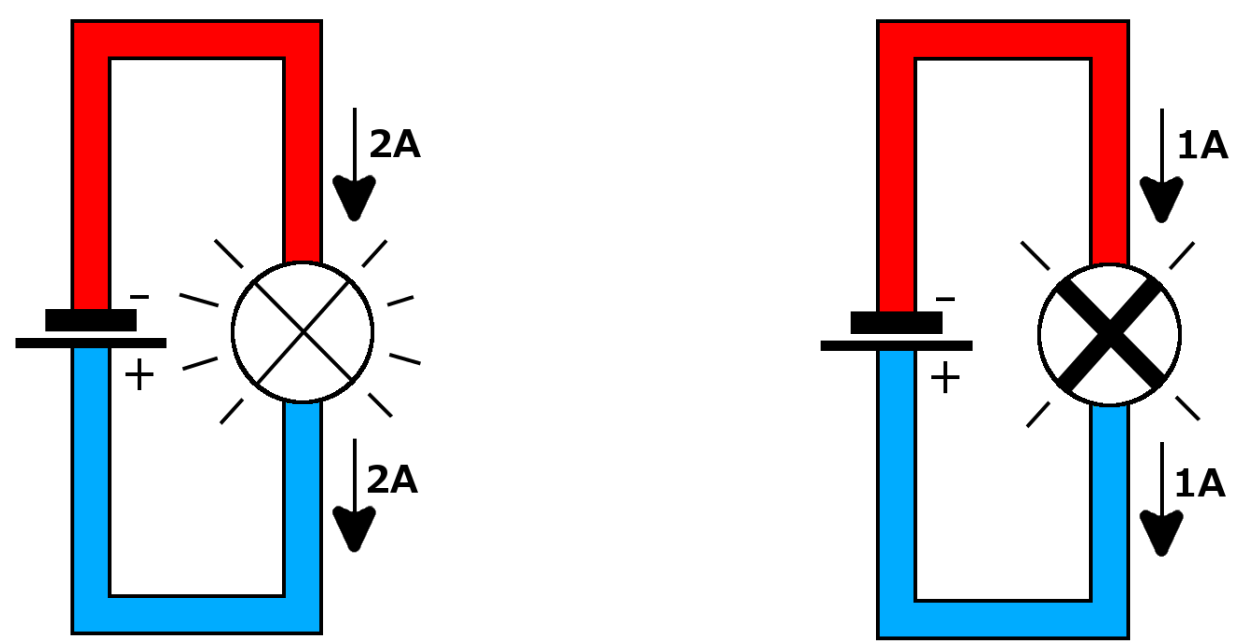

Abb. 56: Umso größer der elektrische Widerstand, desto kleiner die Stromstärke. Das dickere Kreuz steht hier für ein Lämpchen mit doppelt so großem Widerstand. 
In Anlehnung an das in der Luftdruckeinheit erworbene Widerstandskonzept wird den Schülern anschließend eine qualitative Vorstellung des elektrischen Widerstands vermittelt und sein Einfluss auf die Elektronenströmung diskutiert. Dabei wird zunächst rein phänomenologisch argumentiert, dass ein elektrischer Widerstand die Elektronenströmung genauso behindert, wie ein Stück Stoff eine Luftströmung behindert. Im Schaltbild wird die Höhe des elektrischen Widerstandswertes bei Lämpchen über die Dicke des Kreuzes und die Leuchtstärke der Lämpchen über die Anzahl der symbolischen Lichtstrahlen dargestellt (siehe Abb. 56).

Für jedes Elektron, das vom elektrischen Überdruck durch das Lämpchen zum elektrischen Unterdruck strömt, pumpt die Batterie ein anderes Elektron vom Unterdruck in den Bereich des Überdrucks, weshalb der am Lämpchen anliegende Druckunterschied konstant bleibt und nicht abnimmt. Der Einfluss der Spannung auf die Stromstärke sowie vom Widerstand auf die Stromstärke wird halb-quantitativ über Je-desto-Beziehungen erarbeitet. Dabei ist das Ziel, bei den Schülern ein qualitatives Verständnis der Wirkungszusammenhänge im Stromkreis zu erreichen, wonach die Spannung die Elektronenströmung kausal bewirkt und der Widerstand diese lediglich beeinflusst (siehe Abb. 57).

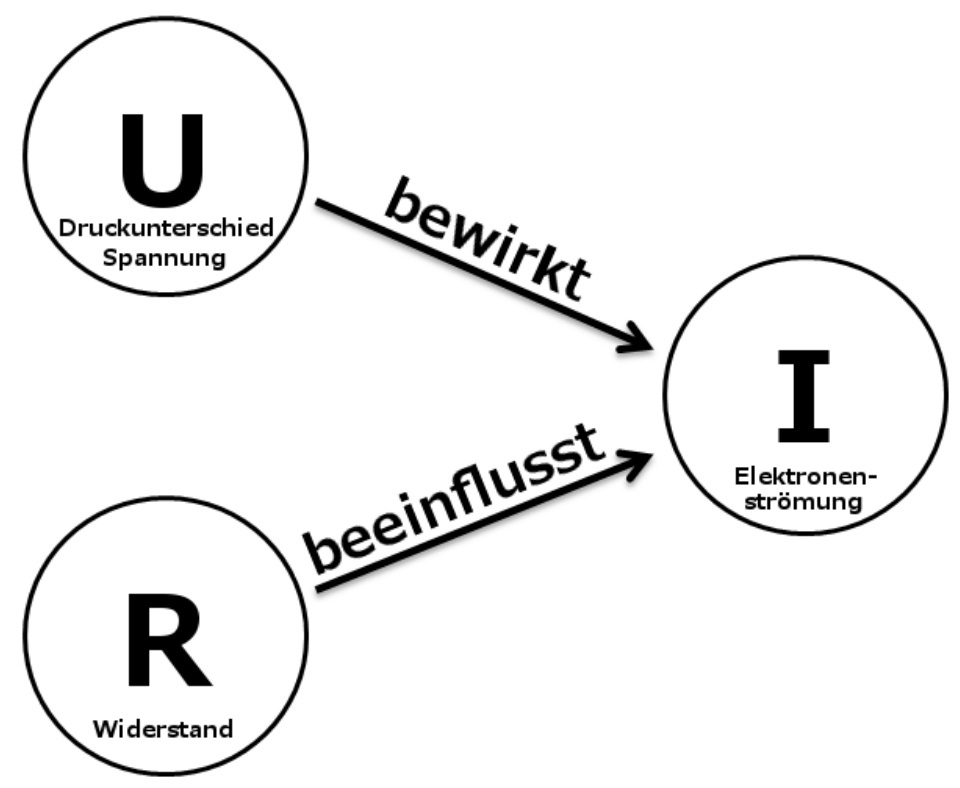

Abb. 57: Qualitativer Wirkungszusammenhang zwischen $U, R$ und I

Die bisher rein qualitative Vorstellung des elektrischen Widerstands wird anschlieBend um ein mikroskopisches Widerstandsmodell auf Basis der zu Beginn eingeführten Atomvorstellung ergänzt. Ziel hierbei ist es, den Schülern in Anlehnung an das Drude-Modell ein besseres Verständnis diverser elektrischer Leitungsvorgänge zu ermöglichen. Ideale Leiter werden beispielsweise damit erklärt, dass die Atomrümpfe in einem solchen Material sehr gleichmäßig angeordnet sind und die Elektronen daher nahezu ungestört strömen können (siehe Abb. 58). 

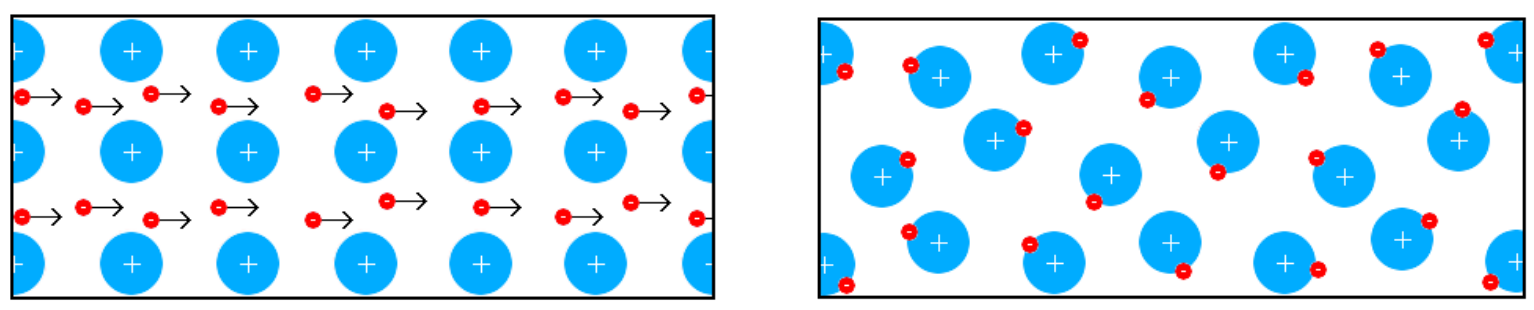

Abb. 58: Ein einfaches mikroskopisches Modell eines idealen Leiters (links) und eines Isolators (rechts)
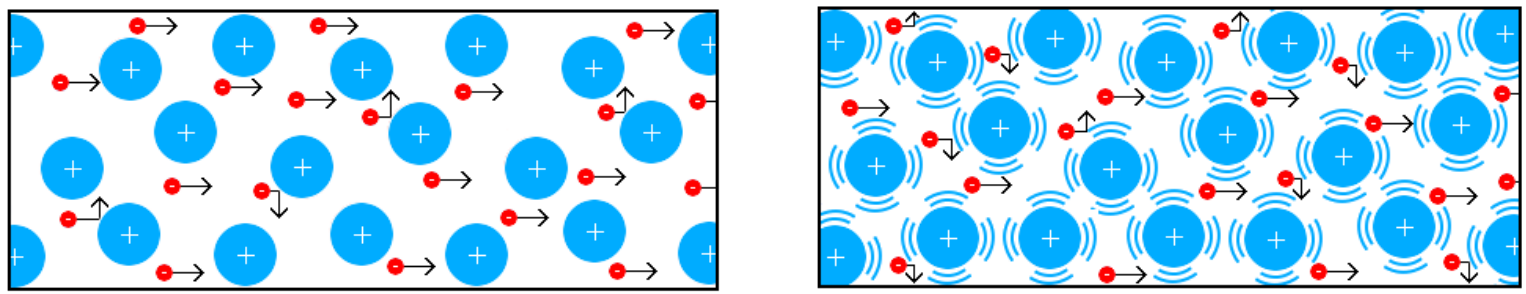

Abb. 59: Ein einfaches mikroskopisches Modell der Materialabhängigkeit (links) und Temperaturabhängigkeit (rechts) des elektrischen Widerstands

Isolatoren können mit Hilfe des Modells damit erklärt werden, dass die Elektronen aufgrund der Materialeigenschaften fest an den Atomrümpfen hängen und es daher nicht zu einer Elektronenströmung kommen kann. Der nicht zu vernachlässigende elektrische Widerstand einiger Materialien kann im Modell damit erklärt werden, dass es in Folge der ungleichmäßigen Anordnung der Atomrümpfe häufig zu Kollisionen zwischen diesen und den strömenden Elektronen kommt (siehe Abb. 59), weshalb die Elektronenströmung insgesamt verlangsamt wird. In ähnlicher Weise kann den Schülern mit dem Modell auch der Einfluss der Temperatur, des Leiterquerschnitts und der Leiterlänge auf den elektrischen Widerstand vermittelt werden.

\subsubsection{Die Parallelschaltung}

Zum Einstieg in das Thema „Parallelschaltungen“ wird zunächst ein einfacherer Stromkreis bestehend aus einer Batterie und einem Lämpchen betrachtet (Schaltung 1). Anschließend wird ein zweites identisches Lämpchen parallel zum ersten geschaltet (Schaltung 2). Nun muss herausgearbeitet werden, dass eine (ideale) Batterie die elektrischen Drücke in den mit ihren Polen direkt verbundenen Leiterstücken konstant hält - unabhängig von deren Länge oder der Anzahl der parallelgeschalteten Lämpchen. Wie in Abb. 60 zu sehen, lässt sich nun mit Hilfe der Farbdarstellung leicht zeigen, dass an beiden Lämpchen der gleiche elektrische Druckunterschied wie zuvor anliegt. Da es sich um identische Lämpchen handelt, muss die Intensität der Elektronenströmung daher durch jede der beiden Lämpchen in Schaltung 2 genauso groß sein wie durch das Lämpchen in Schaltung 1. Insgesamt hat sich die Intensität der Elektronenströmung also im Vergleich zu Schaltung 1 in den direkt mit den Polen der Batterie verbundenen Leiterstiucken verdoppelt, weshalb die Batterie entsprechend mehr Elektronen pro Zeit vom Plus- zum Minuspol „pumpen“ muss und deshalb schneller „erschöpft“ ist. 
Parallelschaltungen eignen sich im Unterrichtskonzept auf Basis des Elektronengasmodells hervorragend zur weiteren Festigung und konzeptionellen Ausdifferenzierung der grundlegenden Größen „elektrischer Druck“, ,elektrischer Druckunterschied“ und „Intensität der Elektronenströmung“. Insbesondere lernen Schüler Stromkreise immer nur ausgehend von elektrischen Druckunterschieden und nicht aus Sicht der Elektronenströmung zu analysieren. Indem Leiterabschnitte gleichen Potenzials betrachtet werden, kann zudem für die Schüler einsichtig gezeigt werden, dass zwischen zwei Punkten eines stromführenden Leitungsdrahts nicht zwangsläufig eine elektrische Spannung anliegen muss, womit eine Differenzierung des Strom- und Spannungskonzepts angestrebt wird.
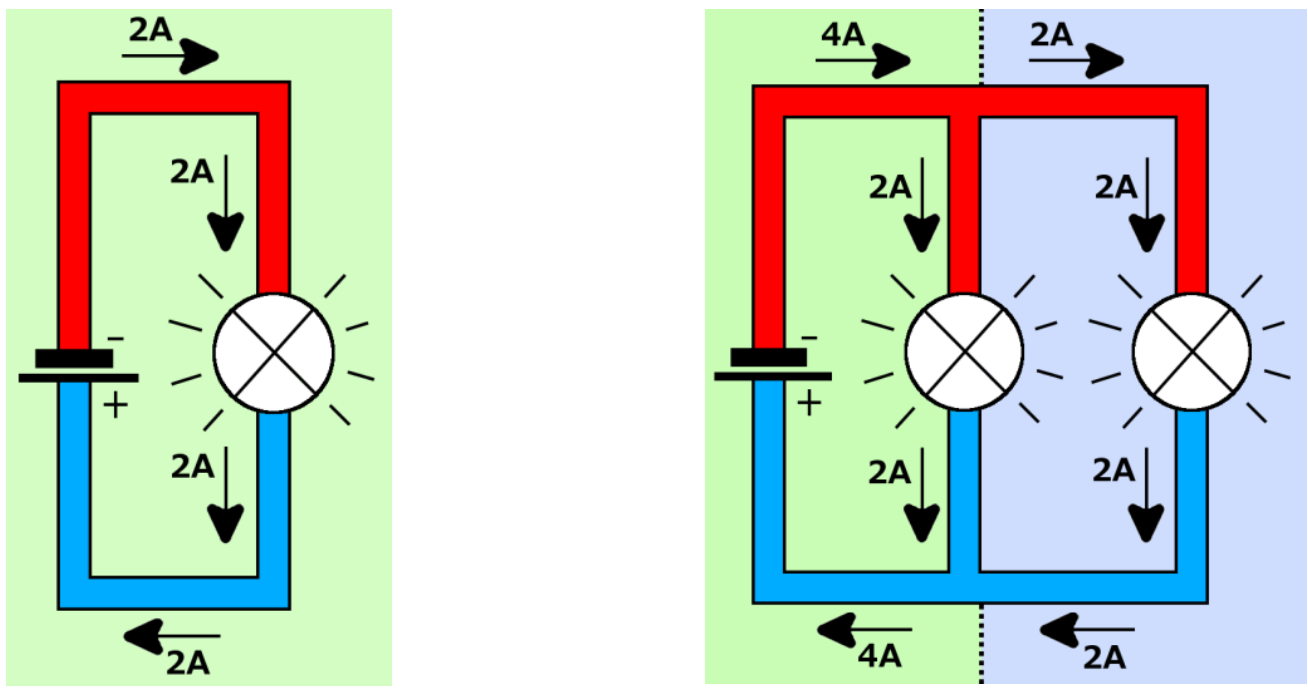

Abb. 60: Einfacher Stromkreis mit einem Lämpchen (Schaltung 1, links); Parallelschaltung eines weiteren identischen Lämpchens (Schaltung 2, rechts)

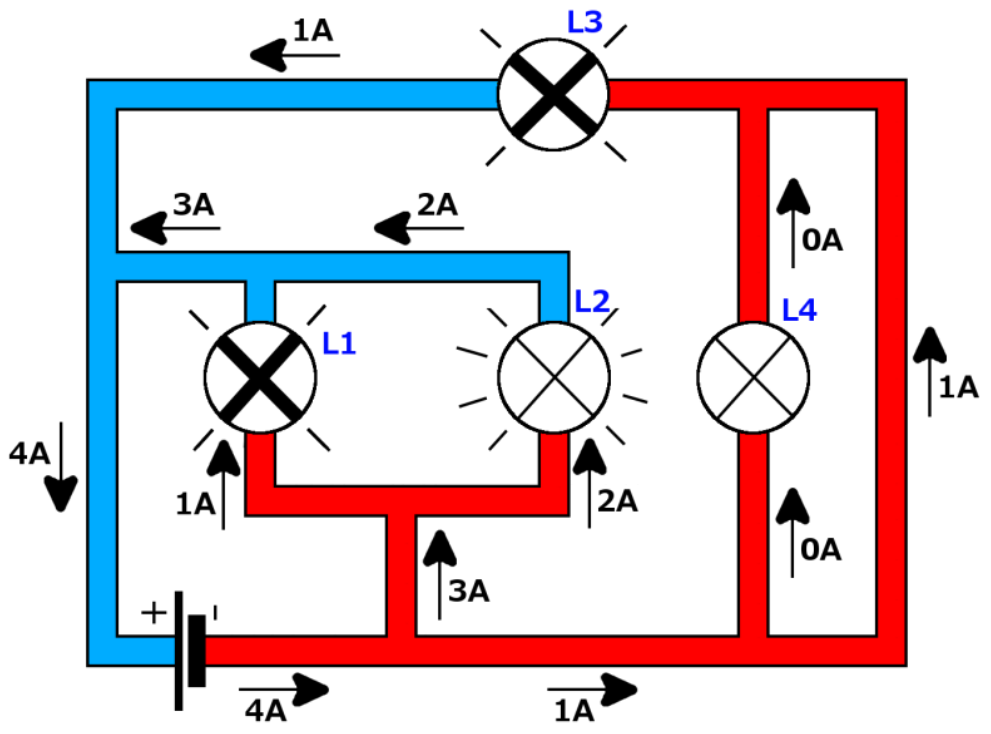

Abb. 61: Das farbliche Einzeichnen der elektrischen Drücke ermöglicht den Schülern auch bei verhältnismäßig komplexen Schaltungen die leichte Identifikation parallelgeschalteter Lämpchen.

Darüber hinaus wird das Konzept der Batterie als Quelle konstanter Spannung statt konstanten Stroms weiter vertieft und eine einfache Regel zur Identifikation von Parallelschaltungen formuliert: „Zwei Lämpchen sind dann parallelgeschaltet, wenn an ihren Seiten 
jeweils die gleichen Farben (= elektrischen Drücke) anliegen". Wie in Abb. $61 \mathrm{zu}$ sehen, lässt sich mit Hilfe dieser Regel auch bei verhältnismäßig komplexen Parallelschaltungen alleine durch das Einzeichnen der elektrischen Drücke mit Hilfe der Farbdarstellung leicht ein Überblick über die parallelgeschalteten Lämpchen gewinnen.

\subsubsection{Der Kondensator}

Um ihnen das Verständnis von Reihenschaltungen zu erleichtern, werden die Schüler am Beispiel von Lade- und Entladevorgängen von Kondensatoren zunächst an eine dynamische Modellvorstellung herangeführt. Dabei sollen sie mit Hilfe von Anfangs-, Übergangs- und Endzuständen schrittweise nachvollziehen können, wie sich die elektrischen Drücke in den einzelnen Leiterabschnitten einstellen, bis der Endzustand erreicht ist.

Im Anfangszustand herrscht dabei in allen Leiterabschnitten ein elektrischer Normaldruck (gelb), da noch keine Batterie angeschlossen wurde und sich daher in Abschnitten A, B, $\mathrm{C}$ und D noch normal-viele Elektronen befinden (siehe Abb. 62 links). Unmittelbar nach Anschluss der Batterie stellt sich in Abschnitt A ein elektrischer Überdruck und in Abschnitt C ein elektrischer Unterdruck ein, während in Abschnitten B und D weiterhin ein elektrischer Normaldruck herrscht, da zu diesem Zeitpunkt gedacht noch keine Elektronen durch die Lämpchen geströmt sind (Übergangszustand, siehe Abb. 62 Mitte). In Folge des nun an den Lämpchen anliegenden elektrischen Druckunterschieds kommt es solange zu einer Elektronenströmung durch die Lämpchen, bis sich die elektrischen Drücke jeweils angeglichen haben und nicht mehr verändern (Endzustand, siehe Abb. 62 rechts).
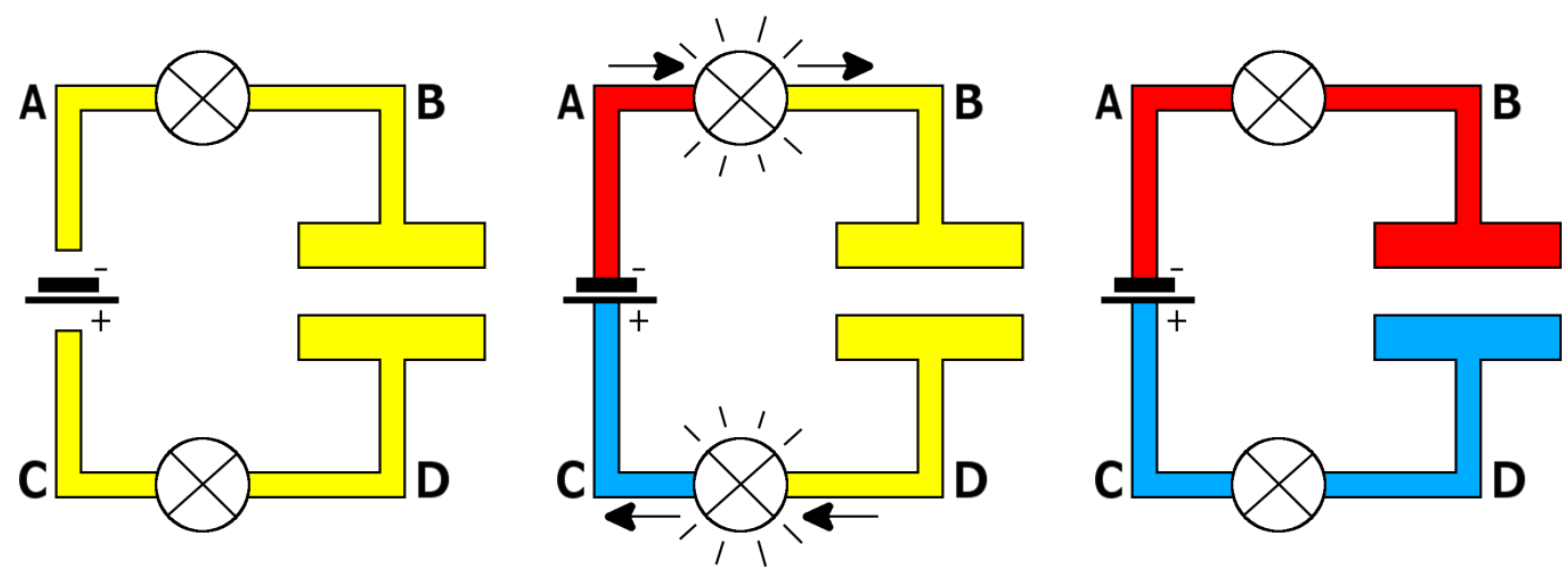

Abb. 62: Hinführung zur dynamischen Modellvorstellung am Beispiel des Ladevorgangs eines Kondensators Links: Anfangszustand / Mitte: Übergangszustand / Rechts: Endzustand

Experimentell können die unterschiedlichen Zustände an einem Kondensator mit einer Kapazität von $25.000 \mu \mathrm{F}$ in Kombination mit zwei Lämpchen von $6 \mathrm{~V}$ und 0,05 A und einer $12 \mathrm{~V}$-Gleichspannungsquelle demonstriert und diskutiert werden, da sich eine große Zeitkonstante $\tau=R \cdot C=6 s$ ergibt. Hierbei kann mit Hilfe von elektrischen Druckunterschieden analysiert werden, warum die Lämpchen während des Übergangszustands zunächst hell und dann immer schwächer leuchten. Anschließend sollte zur Entkräftung der Stromaussendevorstellung ebenfalls auf die Frage eingegangen werden, warum beim Ladevorgang auch das untere Lämpchen anfängt zu leuchten. 


\subsubsection{Die Reihenschaltung}

Während man sich in der einfachen Elektrizitätslehre in der Regel lediglich für den stationären Gleichgewichtszustand interessiert, also den Zustand, in dem sich Stromstärke und Spannung zeitlich nicht mehr ändern, werden Reihenschaltungen im Unterrichtskonzept auf Basis des Elektronengasmodells mit Hilfe der bei den Kondensatoren bereits eingeführten dynamischen Modellvorstellung analysiert. Die Grundidee ist dabei, dass sich die elektrischen Drücke und Elektronenströmungen in Reihenschaltungen zwar äußerst schnell einstellen, der stationäre Gleichgewichtszustand (hier Endzustand genannt) jedoch nur schrittweise über sogenannte Übergangszustände erreicht wird.

Bei einer Reihenschaltung von zwei unterschiedlichen Lämpchen herrscht im Anfangszustand, d.h. so lange der Stromkreis noch nicht mit der Batterie verbunden ist, in allen Abschnitten des Stromkreises ein elektrischer Normaldruck (gelb) (siehe Abb. 63 links). Sobald der Stromkreis dann mit den Polen der Batterie verbunden wird, stellen sich zunächst ein elektrischer Überdruck (rot) im Leiterstück bis zum oberen Lämpchen und ein elektrischer Unterdruck (blau) im Leiterstück bis zum unteren Lämpchen ein (siehe Abb. 63 Mitte). Im mittleren Leiterabschnitt zwischen den beiden Lämpchen herrscht noch ein elektrischer Normaldruck (gelb), weil gedacht noch keine Elektronen durch die Lämpchen geströmt sind (Übergangszustand). Da nun an beiden Lämpchen die gleichen elektrischen Druckunterschiede anliegen, der Widerstand am oberen Lämpchen aber doppelt so groß ist wie am unteren, strömen weniger Elektronen von oben in den mittleren Leiterabschnitt hinein als Elektronen durch das untere Lämpchen mit dem geringeren Widerstand herausströmen. In Folge sinkt der elektrische Druck im mittleren Leiterabschnitt so lange (gelb $\rightarrow$ hellblau), bis der elektrische Druckunterschied über dem oberen Lämpchen so groß ist, dass die Intensität der Elektronenströmung durch beide Lämpchen exakt gleich groß ist. Da sich jetzt sowohl die elektrischen Drücke als auch die Elektronenströmungen nicht mehr ändern, ist der Endzustand erreicht (siehe Abb. 63 rechts).

Mit Hilfe dieser Argumentation ist leicht nachzuvollziehen, warum sich in Reihenschaltungen an größeren Widerständen auch größere Druckunterschiede (= Spannungen) einstellen müssen. Allgemein kann die abnehmende Intensität der Elektronenströmung bei einer zunehmenden Anzahl von Widerständen im Elektronengasmodell damit erklärt werden, dass sich der von der Batterie erzeugte elektrische Druckunterschied bei Reihenschaltungen auf immer mehr Widerstände aufteilen muss. 

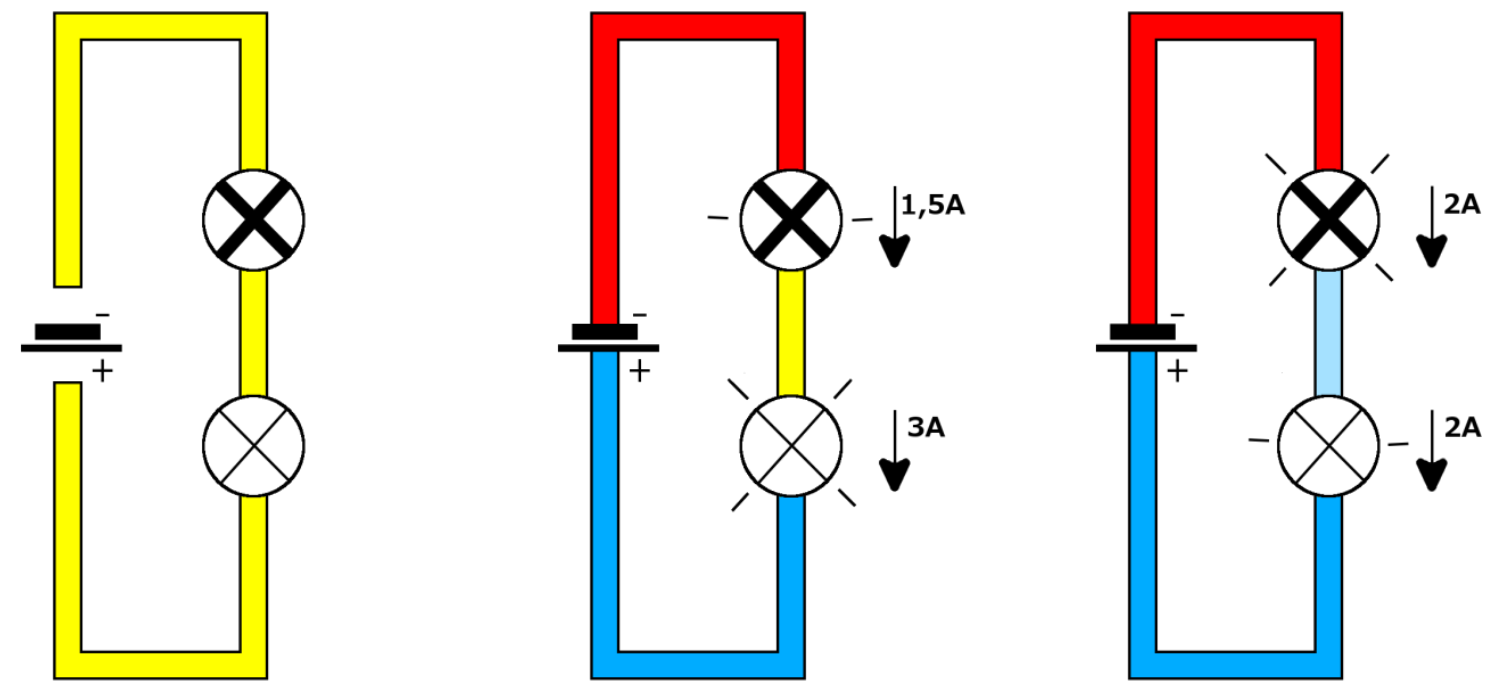

Abb. 63: Analyse einer Reihenschaltung mit Hilfe der dynamischen Modellvorstellung Links: Anfangszustand / Mitte: Übergangszustand / Rechts: Endzustand

Neben der Argumentation mit elektrischen Druckunterschieden wird an dieser Stelle zusätzlich die schon zuvor im Zusammenhang mit der elektrischen Energieübertragung eingeführte Fahrradkettenanalogie bemüht, um den Schülern eine weitere Perspektive auf Reihenschaltungen zu ermöglichen. Über diesen zusätzlichen Zugang zum elektrischen Stromkreis kann nicht nur dessen Systemcharakter betont, die Stromverbrauchsvorstellung entkräftet und dem sequentiellen Denken entgegengewirkt werden, sondern auch der Einfluss mehrerer in Reihe geschalteter Widerstände auf die Gesamtstromstärke insbesondere für diejenigen Schüler plausible erklärt werden, die Schwierigkeiten mit der dynamischen Modellvorstellung haben. Hierzu wird ein Widerstand mit einem Bremsklotz verglichen und argumentiert, dass die Fahrradkette sich umso langsamer dreht, desto mehr bzw. desto stärker die Bremsklötze an der Kette reiben.

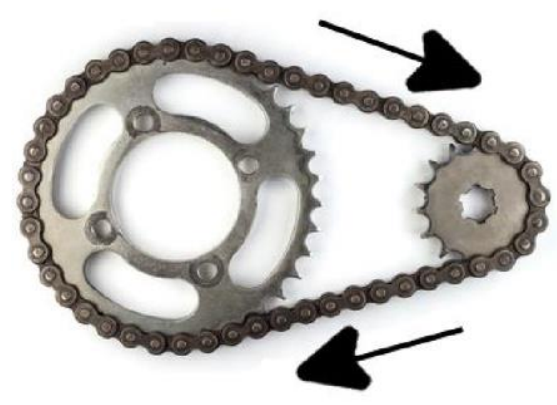

Fahrradkette

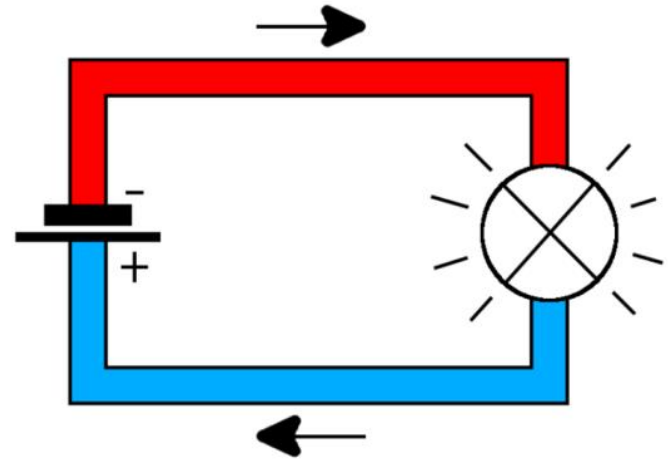

Elektronenkreislauf

Abb. 64: Fahrradkettenanalogie als zusätzliche Vorstellungshilfe insbesondere bzgl. der elektrischen Energieübertragung, des Systemcharakters von Stromkreisen und des Einflusses mehrerer Widerstände auf die Gesamtstromstärke (Quelle Fahrradkette: (C) enterphoto / Fotolia.com)

Das zusätzliche Einbeziehen des Fahrradkettenmodells bietet darüber hinaus noch die Chance im Unterricht den Stellenwert von Modellen in der Physik anzusprechen und insbesondere zu verdeutlichen, dass Modelle lediglich menschengemachte Vorstellungshilfen sind, um bestimmte Phänomene besser verstehen zu können. 


\subsubsection{Messung und Berechnung von Stromstärke, Spannung und Widerstand}

Nachdem die Schüler nun über ein fundiertes konzeptionelles Verständnis der grundlegenden Größen Spannung, Stromstärke und Widerstand verfügen, wird im nächsten Schritt die Anschlussbedingung von Volt- und Amperemetern erarbeitet, um auch quantitative Messungen vornehmen zu können, wobei von idealen Messinstrumenten ausgegangen wird. Auch wenn ab diesem Kapitel die sonst in der Physik übliche Schaltbilddarstellung mit einzelnen Leiterbahnen verwendet wird, erscheint eine dreidimensionale Darstellung von Messgeräten in Schaltplänen sinnvoll, um sie visuell klar vom restlichen Stromkreis abzugrenzen (siehe Abb. 65). Die Kabel des Voltmeters werden dabei aus dem gleichen Grund nicht entsprechend der Farbkodierung eingefärbt und deuten zusätzlich mit Hilfe von Pfeilen an, zwischen welchen Punkten des zu untersuchenden Stromkreises die Spannung gemessen wird. Die Tatsache, dass sowohl Spannung als auch Stromstärke experimentell mit ein und demselben Instrument gemessen werden, kann bei manchen Schülern unnötigerweise die Schülervorstellung verstärken, dass es sich bei Spannung und Stromstärke auch um die gleiche physikalische Größe handelt (Härtel et al. 1981, S. 45). Um dieser Schülervorstellung nicht unnötig Vorschub zu leisten, wird den Lehrkräften im Unterrichtskonzept vorgeschlagen, die Stromstärke statt mit einem Multimeter mit einer Stromzange zu messen, was darüber hinaus noch den Vorteil hat, dass der Stromkreis für die Messung nicht unterbrochen werden muss (Girwidz 1995). Alternativ wäre auch denkbar, für die Strommessung konsequent analoge Zeigerinstrumente und für die Spannungsmessung konsequent digitale Voltmeter einzusetzen, um den Schülern die Differenzierung von Strom- und Spannungsmessung zu erleichtern (Muckenfuß und Walz 1997, S. 107).

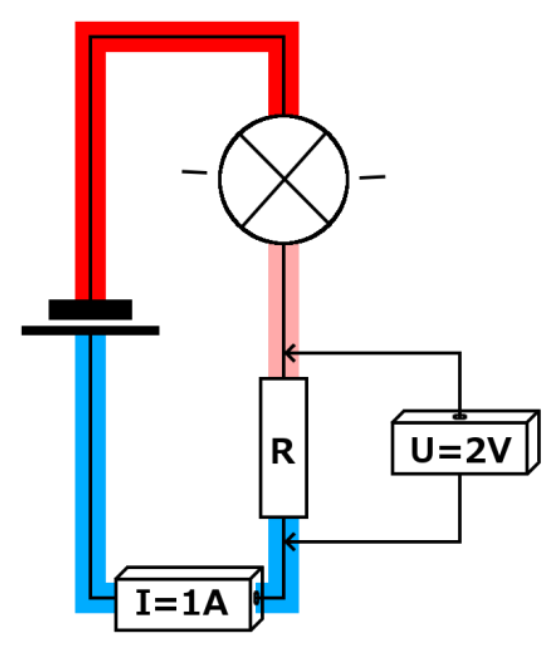

Abb. 65: Darstellung der Anschlussbedingungen von Volt- und Amperemetern in einem einfachen Stromkreis

Um ferner die Anschlussfähigkeit der vermittelten Konzepte zu gewährleisten, werden in diesem Teil des Unterrichtskonzepts vermehrt die physikalisch korrekten Fachtermini „Stromstärke“ und „Spannung“ statt „Intensität der Elektronenströmung“ und „elektrischer Druckunterschied“" genutzt. Entsprechend der Widerstandsdefinition $R:=U / I$ kommt es dann auch zu einer Quantifizierung des elektrischen Widerstands, wobei der Widerstands- 
wert $R$ interpretiert wird als Maß für den elektrischen Druckunterschied, der nötig ist, um eine Elektronenströmung von $1 \mathrm{~A}$ durch den Widerstand zu verursachen (vgl. Muckenfuß und Walz 1997, 22ff). Bei den meisten Widerständen steigt dieser Widerstandswert mit zunehmender Stromstärke an. Im Unterrichtskonzept wird der Sonderstatus des Ohm'schen Gesetzes, d.h. die Konstanz des Widerstandswertes bei manchen Materialien, deshalb gegen Ende explizit thematisiert.

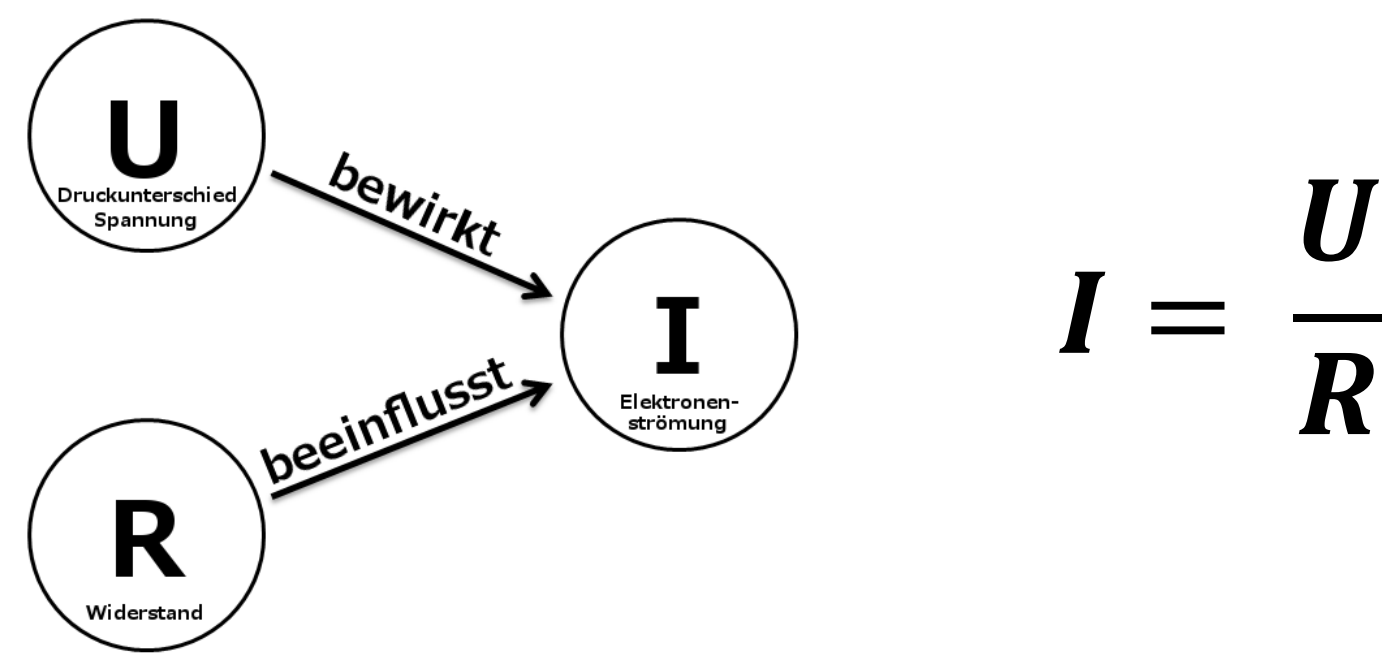

Abb. 66: Übergang von einem qualitativen (links) zu einem quantitativen (rechts) Zusammenhang der grundlegenden physikalischen Größen I, $U$ und $R$

In einem letzten Schritt wird dann der den Schülern bereits bekannte qualitative Wirkungszusammenhang, wonach die Spannung die Elektronenströmung kausal bewirkt und der Widerstand diese lediglich beeinflusst, wieder aufgegriffen und in die quantifizierte Form $I=\frac{U}{R}$ überführt, was vor dem Hintergrund der Physik als quantitative Wissenschaft einen wesentlichen Schritt darstellt (siehe Abb. 66). Da in der Elektrizitätslehre nach Gleixner (1998, S. 233) den Schülerinnen und Schülern , [...] weniger das Verständnis für die Unterschiedlichkeit der Begriffe als die Fülle der Fakten und Begriffe zu schaffen [macht]“, schließt die Unterrichtsreihe mit Übungen zur Festigung und Vertiefung der abgedeckten Themen ab. 


\section{C) Summative Evaluation}





\section{Summative Evaluation des Unterrichtskonzepts}

\subsection{Die Studie als Design-Based-Research-Projekt}

Trotz ihrer jahrzehntelangen Geschichte und zunehmenden Professionalisierung ist der Einfluss fachdidaktischer Forschung auf die Unterrichtspraxis oftmals gering (Bereiter 2002; Reinmann 2005). Der Grund für diesen Research-Practice-Gap ist nicht auf die Qualität der fachdidaktischen Forschung an sich zurückzuführen, die methodisch gesehen inzwischen ein sehr hohes Niveau erreicht hat (Wilhelm und Hopf 2014). Vielmehr wird der Grund für die Diskrepanz zwischen fachdidaktischer Forschung und Schulpraxis darin gesehen, dass die traditionellen Ansätze der Experimental- und Evaluationsforschung ungeeignet seien, nachhaltige Innovationen in der Unterrichtspraxis zu bewirken (Reinmann 2005). Mit diesem Befund ist allerdings explizit keine Herabwürdigung dieser Forschungsansätze intendiert. Im Gegenteil wird hier die Position von Wiesner et al. (2010, S. 1) geteilt, dass die verschiedenen Forschungsansätze nicht nur wesentliche Bestandteile der Physikdidaktik darstellen, sondern, dass die Stärke physikdidaktischer Forschung gerade in der Synthese der Erkenntnisse der verschiedenen Ansätze zu suchen ist.

Ein Kritikpunkt der didaktischen Experimentalforschung liegt in der angestrebten Variablenkontrolle, wie sie beispielsweise auch in der pädagogischen Psychologie üblich ist. Der Grund hierfür liegt darin, dass der Versuch, alle Stellschrauben kontrollieren und potenzielle Störfaktoren eliminieren zu können, oftmals zu Forschungsdesigns führt, die mit der Unterrichtsrealität nur noch wenig gemein haben. Hinzu kommt, dass bereits seit den 1950er Jahren bekannt ist, dass die Veränderung einzelner Treatment-Variablen nur einen sehr geringen Einfluss auf den Lernerfolg hat, weil der reale Unterricht im Gegensatz zu einem Laborsetting eine äußerst komplexe Situation mit einer Vielzahl an unterschiedlichen, subjektabhängigen und zudem wechselwirkenden Variablen darstellt (Cronbach 1957; Bereiter 2002, S. 324). Hier stellt sich die grundsätzliche Frage, ob das Ganze nicht vielleicht mehr ist als die Summe seiner Teile und ob beim Versuch, die unzähligen Einflussfaktoren und ihre komplexen Interaktionen miteinander im Einzelnen verstehen zu wollen, nicht der reale Physikunterricht an sich aus den Augen verloren wird (Wiesner et al. 2010, S. 2). Von Lehrkräften werden die Ergebnisse didaktischer Laborstudien oftmals als banal wahrgenommen und selbst interessante und relevante Forschungsergebnisse finden aufgrund der noch zu leistenden Implementationsarbeit oftmals nicht den Weg in die Schulpraxis (Wilhelm und Hopf 2014).

Darüber hinaus wird aber die eher praxisnahe Evaluationsforschung dafür kritisiert, keine wesentlichen Veränderungen in der Unterrichtsrealität anzustoßen. Bereiter (2002, S. 324) illustriert die Problematik der reinen Evaluationsforschung daran, dass diese bzgl. früher Prototypen des Automobils vermutlich zu dem Schluss gekommen wäre, die Pferdekutsche sei das verlässlichere Fortbewegungsmittel, womit dessen langfristiges Innovationspotenzial unerkannt geblieben wäre. Überhaupt sei ein solcher Forschungsansatz ungeeignet, selber 
neue Wege zu innovativen Verbesserungen aufzuzeigen, was nach Reinmann (2005, S. 67) insofern problematisch ist, als dass Innovationen in der Bildung u.a. deshalb nahezu ausschließlich aus Politik und Wirtschaft kommen, die aber nicht nur einer besseren Bildung verschrieben seien, sondern im Gegensatz zur didaktischen Forschung auch bildungsferne Interessen verfolgten. Es stellt sich deshalb die Frage, ob die Aufgabe fachdidaktischer Forschung ähnlich zu den Ingenieurswissenschaften nicht auch darin bestehen sollte, selbst Forschung zum „Zwecke der Innovation“ zu betreiben (Reinmann 2005, S. 53).

Ein Forschungsansatz mit einem solchen Anspruch stellt Design-Based-Research (DBR) dar (Design-Based Research Collective 2003; Hopf und Wiesner 2007). In Anlehnung an die wissenschaftstheoretischen Überlegungen von Stokes (1997) schlagen Wilhelm et al. (2012) vor, Design-Based-Research als nutzenorientierte Grundlagenforschung zu klassifizieren, da diese Forschungsrichtung sowohl eine theoretische Erkenntnisgewinnung als auch einen praktischen Nutzen anstrebe. Eine einfache Charakterisierung von DBR als anwendungsbezogene Forschung oder Grundlagenforschung wäre hingegen unzureichend, wie am Beispiel von Louis Pasteur deutlich wird, der als historischer Vertreter der nutzenorientierten Grundlagenforschung gesehen wird. Ausgehend von dem praktischen Problem, dass Bier und Wein teilweise bei der Herstellung verderben, machte Pasteur die bedeutende Entdeckung, dass manche Bakterien zum Leben keinen Sauerstoff benötigen. Diese aus einem praktischen Problem heraus motivierte Forschung hatte also nicht nur großen praktischen Nutzen, sondern führte auch zu einem bedeutenden theoretischen Erkenntnisgewinn.

Auf die Didaktik übertragen, besteht das Ziel von Design-Based-Research darin, für ein konkretes Problem aus der Unterrichtspraxis theoriegeleitet eine Lösung zu finden, diese im realen Unterricht zu evaluieren und dabei gleichzeitig den Stand der Wissenschaft voranzubringen, indem zum jeweiligen Kontext eine Theorie des Lernens entwickelt wird. Diesem in der deutschsprachigen Literatur auch als ,fachdidaktische Entwicklungsforschung“ bezeichneten Ansatz (Prediger und Link 2012) liegt die Überzeugung zugrunde, dass Theorie und Praxis keine getrennten Sphären sind, sondern dass die Praxis vielmehr den Nukleus der Theorieentwicklung darstellt (Reinmann 2005, S. 66). Dementsprechend zielt Design-BasedResearch nicht nur darauf ab, wie die klassische Evaluationsforschung z.B. neue Unterrichtskonzepte, Methoden oder Medien auf ihre Lernwirksamkeit hin zu überprüfen, sondern auf Basis der in der Praxis gemachten Erfahrungen ein theoretisches Verständnis davon zu entwickeln, wie Lernen in dem jeweiligen spezifischen Kontext funktioniert. Eine wesentliche Motivation aller didaktischen Forschungsbemühungen im Rahmen von DBR besteht aber allen voran darin, nachhaltige Innovationen in der Unterrichtspraxis zu bewirken. Wichtig dabei ist, dass mit Innovationen hier nicht die alleinige Existenz neuer Unterrichtsmaterialien oder didaktischer Erkenntnisse gemeint ist, sondern, dass diese auch ihren Weg in die Praxis finden und dort von den Betroffenen als signifikante Verbesserung wahrgenommen werden müssen (Reinmann 2005, 53f). Am bereits erläuterten Theorie-Praxis-Problem in der Didaktik zeigt sich allerdings, dass dies keinesfalls eine Selbstverständlichkeit darstellt. Indem DesignBased-Research also bereits bei der Forschungskonzeption die spätere Implementation der 
Forschungserkenntnisse in der Praxis im Blick hat und gleichzeitig den Anspruch hat, eine kontextspezifische Theorie des Lernens zum jeweiligen Forschungsgegenstand zu entwickeln, versucht dieser Ansatz die oft beklagte Kluft zwischen Forschung und Praxis zu überwinden.

Charakteristisch für fachdidaktische Entwicklungsforschung bzw. Design-BasedResearch ist im Allgemeinen weniger die verwendete Methodik als die beschriebene Zielsetzung, das Lehren und Lernen durch nachhaltige Innovationen in der Unterrichtspraxis voranzubringen (Jahn 2014). Wie einleitend erläutert, reicht es hierzu in der Regel nicht, nur kleine Stellschrauben zu verändern, weil DBR davon ausgeht, ,[...] dass es vermutlich keine solchen isolierbaren Einzelfaktoren gibt" und sich ,,[...], funktionierende' Lernumgebungen in einem komplexen Wechselspiel verschiedener Einflussfaktoren entwickeln" (Wilhelm und Hopf 2014, S. 33). Vielmehr müssen auf Basis fundierter Fachkompetenz und der breiten Kenntnis fachdidaktischer Zugänge und Lerntheorien neue Designs wie z.B. Unterrichtsansätze theoriegeleitet entwickelt und anschließend überprüft werden, was eine enge Zusammenarbeit mit Praktikern unerlässlich macht. Da didaktische Interventionen bei Design-Based-Research typischerweise einen holistischen Charakter haben, also z.B. ein ganzes Unterrichtskonzept inklusive neuer Sachstruktur, Visualisierungen und Experimenten umfassen können, und zudem keine verallgemeinerbare Ursache-Wirkungs-Beziehungen aufklären wollen, hat dieser Forschungsansatz keinen Anspruch auf vollständige Variablenkontrolle. Wie Jahn (2014, S. 10) in diesem Zusammenhang erklärt, besteht das Ziel von DBR vielmehr darin aufzudecken, was die Faktoren sind, die den Erfolg- oder Misserfolg der jeweiligen Intervention begünstigen. Nichtsdestotrotz strebt Design-Based-Research eine hohe externe Validität, d.h. Verallgemeinerbarkeit der Studienergebnisse innerhalb des jeweiligen Kontextes an, um gewährleisten zu können, dass die gefundenen Ergebnisse auf die Praxis übertragbar sind. Damit ähnelt der Ansatz von DBR dem Vorgehen eines Arztes, der auf Basis des aktuellen medizinischen und pharmazeutischen Wissens dem Patienten zur Heilung einer Krankheit ein Medikament verschreibt, dessen Wirksamkeit zwar wissenschaftlich belegt ist, aber dessen genaue physiologischen und pharmazeutischen Wechselwirkungen noch unbekannt sind (Wiesner et al. 2010, 2f). An diesem Beispiel wird aber auch deutlich: In dem Maße, wie die pharmazeutische Grundlagenforschung zur Verbesserung von Medikamenten beiträgt, kann Design-BasedResearch von den Erkenntnissen didaktischer Grundlagenforschung profitieren. Umgekehrt können aber auch Design-Based-Research-Projekte Fragen aufwerfen, die Anstöße für die didaktische Grundlagenforschung darstellen (Wilhelm und Hopf 2014, S. 32).

Zur Evaluation wird in der Regel ein Methodenpluralismus eingesetzt, wobei einer größeren empirischen Erhebung im Feld in der physikdidaktischen Forschung manchmal eine qualitative Befragung einzelner Schülerinnen und Schüler in Form von Teaching Experiments vorausgeht. Diese unter kontrollierten Bedingungen durchgeführten Befragungen dienen der Identifikation von potenziellen Verständnisschwierigkeiten und haben in der Regel einen formativen Charakter, d.h. die hier gemachten Erfahrungen können direkt in eine Modifikation des Designs münden (vgl. Abschnitt 6.2). Je nach Bedarf kann der Prototyp des Designs in weiteren Iterationen überarbeitet werden, bis die Lernwirksamkeit des Designs anschließend 
in einer empirischen Evaluation in der unkontrollierten Unterrichtspraxis überprüft wird (vgl. Abschnitt 7.4 und 7.5). Neben einer rein quantitativen Erhebung der Lernförderlichkeit des neuen Designs verfolgt DBR aber auch an diesem Punkt oftmals einen multiperspektivischen Ansatz, indem auch qualitative Daten zur Analyse des Designs herangezogen werden (,Triangulation“). Durch die qualitative Befragung der unterrichtenden Lehrkräfte kann so beispielsweise in Erfahrung gebracht werden, welche Aspekte des Designs besonders erfolgreich bzw. problematisch sind, was insbesondere hinsichtlich eines weiteren Entwicklungszyklus sehr hilfreich sein kann (vgl. Abschnitt 7.6). Indem DBR-Forscher im Rahmen der Evaluation gleichzeitig als Ankläger und Anwalt des eigenen Designs auftreten, versuchen sie systematisch potenzielle Stolperstellen für Lernende zu identifizieren und das Design schrittweise zu verbessern. Neben dem Anspruch, auch zur didaktischen Grundlagenforschung beizutragen, ist es insbesondere dieser für die DBR-Forschung typische zyklische Entwicklungsprozess, der sich über mehrere Jahre oder Jahrzehnte erstrecken kann, der Design-Based-Research von den eingangs kritisierten reinen Evaluationsstudien unterscheidet (Wilhelm und Hopf 2014).

Nach Reinmann (2005, S. 67) kommt bei Design-Based-Research als Forschungsansatz ,[...] trotz allen praktischen Problemlösewillens die Frage der Wissenschaftlichkeit nicht zu kurz; leitende wissenschaftliche Prinzipien werden von diesem Ansatz erfüllt"،, da DBR u.a. "[...] explizit auch ein theoretisches Erkenntnisinteresse“ verfolge und zudem ,[...] anschlussfähig an traditionelle Richtungen der Lehr-Lernforschung" sei. Durch aufeinanderfolgende Zyklen von fachdidaktischer Entwicklungsarbeit, formativer Erprobung im kleinen Rahmen, empirischer Evaluation mit großen Stichproben im realen Unterricht und Re-Design auf Basis der gewonnenen Erkenntnisse entstehen so Forschungsergebnisse, die sowohl einen evidenzbasierten Beitrag zur Verbesserung des Physikunterrichts als auch des Forschungsstands der Physikdidaktik liefern.

\subsection{Forschungsfragen und Ablauf der Studie}

Das bisher auf Grundlage von didaktischen Überlegungen und den Erkenntnissen der Teaching Experiments entwickelte Unterrichtskonzept wird nun entsprechend des Forschungsansatzes von Design-Based-Research (vgl. Abschnitt 7.1) einer summativen Evaluation in der Praxis unterzogen. Hierzu wird ein Methodenpluralismus eingesetzt, da durch einen multiperspektivischen Zugang ein umfassenderes Bild der Lernwirksamkeit des Unterrichtskonzepts erlangt werden kann und seine Stärken bzw. Schwächen aufgedeckt werden können. Im Sinne von Design-Based-Research ermöglichen die so gewonnenen Erkenntnisse das Unterrichtskonzept in einem zukünftigen Zyklus des Entwicklungsprozesses weiter zu verbessern (vgl. Abschnitt 7.1). Konkret besteht das Ziel der Evaluation des Unterrichtskonzepts einerseits darin, mit quantitativen Methoden zu untersuchen, ob das neue Unterrichtskonzept auf Basis des Elektronengasmodells verglichen mit dem traditionellen Physikunterricht zu einem höheren Lernerfolg der Schülerinnen und Schüler führt. Andererseits soll mit Hilfe einer qualitativen Befragung auch festgestellt werden, wie das Unterrichtskonzept von den Lehrkräften auf Basis ihrer praktischen Unterrichtserfahrung eingeschätzt wird. Somit erge- 
ben sich für die vorliegende DBR-Studie die folgenden konkreten Forschungsfragen, wobei die ersten drei quantitativer und die letzte qualitativer Natur sind:

1. Führt das neue Unterrichtskonzept zu einem höheren Lernzuwachs als der traditionelle Physikunterricht?

2. Zeigt sich eine Abhängigkeit der Lernwirksamkeit des neuen Unterrichtskonzepts von dem Geschlecht der Lernenden, den unterrichtenden Lehrkräften, dem Vortestergebnis, der Jahrgangsstufe oder der Anzahl an Unterrichtsstunden in der Elektrizitätslehre?

3. Unterscheidet sich die Auftretenswahrscheinlichkeit von typischen Schülervorstellungen zwischen dem traditionellen Physikunterricht und dem neuen Unterrichtskonzept?

4. Wie wird das neue Unterrichtskonzept von Lehrkräften aus der Praxis beurteilt?

Um die Brücke zwischen Forschung und Praxis zu schlagen und die Unterrichtsmaterialien im Feld evaluieren zu können, war eine enge Kooperation mit Lehrkräften nötig. Aus diesem Grund wurde das Forschungsvorhaben in einem vom Institut für Didaktik der Physik gepflegten Netzwerk von Physiklehrkräften per E-Mail und in Fortbildungsveranstaltungen beworben. Allen Lehrkräften, die nach dem neuen Unterrichtskonzept unterrichten wollten, wurde angeboten, zu einem nachmittäglichen Informationstreffen in die Universität zu kommen, um dort die Grundideen des Unterrichtskonzepts persönlich vorgestellt zu bekommen und mögliche Unklarheiten klären zu können. Dabei hatte das Informationstreffen bewusst nicht den Charakter einer verpflichtenden Schulung, da das Ziel der Studie in einer möglichst praxisnahen Evaluation der Lernwirksamkeit bestand und bei einer potenziell breiteren zukünftigen Anwendung des Konzepts auch keine flächendeckenden Lehrerfortbildungen angeboten werden können. Statt einer Schulung bekamen die Lehrkräfte einen Lehrerleitfaden zur Verfügung gestellt, der neben einer didaktischen Begründung insbesondere

- allgemeine didaktische Hinweise zum Unterrichtskonzept wie z.B. zur Gliederung der Sachstruktur und zur verwendeten Terminologie enthält,

- Informationen zu den speziell für das Unterrichtskonzept benötigten Materialien gibt,

- detaillierte Erläuterungen zu jedem Unterabschnitt der Unterrichtseinheit wie z.B. den Lernzielen, dem erwarteten zeitlichen Umfang, den benötigten Materialien und den jeweils zu besprechenden Themen beinhaltet,

- die Lehrkräfte auf Basis der Erkenntnisse der Teaching Experiments für zu erwartende Schülervorstellungen und Lernschwierigkeiten sensibilisiert.

Darüber hinaus haben die Lehrkräfte eine ganze Reihe an Unterrichtsmaterialien wie Overheadfolien, Zusammenfassungen und ein Übungsheft aber auch einen Klassensatz Einmalspritzen als Miniatur-Luftpumpen kostenfrei zur Verfügung gestellt bekommen, damit die Schülerinnen und Schüler Primärerfahrungen zum Luftdruck aufbauen können. Begleitend zum Übungsheft erhielten die Lehrerinnen und Lehrer ferner eine Musterlösung, um potenzielle Missverständnisse zu vermeiden. Mit Hilfe der so den Lehrkräften zur Verfügung gestell- 
ten standardisierten Materialien sollte eine größere Vergleichbarkeit des Unterrichts in den verschiedenen Klassen erzielt werden. Neben der auf diese Weise vorgezeichneten Sachstruktur wurden den Lehrkräften aber keine Vorgaben bzgl. der genauen unterrichtlichen Gestaltung z.B. hinsichtlich der Methoden oder zeitlichen Einteilung einzelner Unterrichtsstunden gemacht (vgl. Abschnitt 6.1.2). Ebenfalls in dem Paket enthalten waren der in dieser Studie verwendete Fragebogen auf Basis von Urban-Woldron und Hopf (2012) (vgl. Anhang 13.3) inklusive einer standardisierten Anweisung, wie dieser bearbeitet werden sollte, um die Objektivität der Erhebung zu erhöhen (vgl. Anhang 13.4). Die Unterrichtsmaterialien, die im Rahmen dieser Studie den Lehrkräften zur Verfügung gestellt wurden, können auf der Internetseite JP-Bur.de/Dissertation heruntergeladen werden.

Die eigentliche Datenerhebung für die empirische Studie zur Evaluation des Lernfortschritts fand zwischen 2013 und 2016 statt. Bei der Datenerhebung wurde zwischen zwei Gruppen unterschieden. Auf der einen Seite wurde der Lernfortschritt von Klassen erhoben, die nach dem neuen Unterrichtskonzept unterrichtet wurden (Treatmentgruppe). Auf der anderen Seite wurde auch der Lernfortschritt von Klassen erhoben, die von ihren Lehrern traditionell unterrichtet wurden (Kontrollgruppe). Der mitgeschickte Fragebogen wurde von den Schülern in beiden Gruppen vor und nach der Unterrichtseinheit zur Elektrizitätslehre als Paper-and-Pencil-Test während der Unterrichtszeit bearbeitet. Wie in Tab. 14 dargestellt, unterschied sich der Erhebungszeitraum in beiden Gruppen. Während die Datenerhebung in der Kontrollgruppe in den Schuljahren 2013/14, 2014/15 und 2015/16 stattfand, stand für die Treatmentgruppe lediglich das Schuljahr 2015/16 zur Verfügung. Die unterschiedlichen Erhebungszeiträume sind der Tatsache geschuldet, dass zunächst die Grundideen des neuen Unterrichtskonzepts entwickelt und in Teaching Experiments evaluiert werden mussten (vgl. Abschnitt 6). Auf diesen Erfahrungen aufbauend wurde anschließend dann das vollständige Unterrichtskonzept inklusive entsprechender Unterrichtsmaterialien ausgearbeitet. Eine Evaluation des fertigen Unterrichtskonzepts konnte deshalb erst im Schuljahr 2015/16 stattfinden.

Tab. 14: Darstellung des zeitlichen Verlaufs der Datenerhebung

\begin{tabular}{|l|l|l|l|}
\hline & \multicolumn{3}{|c|}{ Schuljahr } \\
\hline & $2013 / 14$ & $2014 / 15$ & $2015 / 16$ \\
\hline Kontrollgruppe & Daten-Erhebung & Daten-Erhebung & Daten-Erhebung \\
\hline Treatmentgruppe & - & - & Daten-Erhebung \\
\hline
\end{tabular}




\subsection{Konzeption der empirischen Studie zum Lernerfolg}

\subsubsection{Studiendesign}

Die vorliegende summative Evaluation des Unterrichtskonzepts hat den Charakter einer empirischen Evaluationsstudie. Bortz und Döring (2006, S. 114) führen aus, dass zu Evaluationsstudien prinzipiell verschiedene Studiendesigns denkbar sind, wobei die Wahl des Studiendesigns die Validität, d.h. die Gültigkeit der Studienergebnisse maßgeblich beeinflusst. Sie weisen darauf hin, dass in Bezug auf die Validität einer Studie zwischen der internen und der externen Validität unterschieden werden muss, wobei es innerhalb eines Studiendesigns in der Regel nicht gelingt, interne und externe Validität in gleichem Maße zu gewährleisten. Nach Bortz und Döring (2006, S. 53) ist die interne Validität einer Studie gegeben, „wenn ihre Ergebnisse kausal eindeutig interpretierbar sind“, wohingegen die externe Validität einer Studie dann gegeben ist, ,wenn ihre Ergebnisse über die besonderen Bedingungen der Untersuchungssituation und über die untersuchten Personen hinausgehend generalisierbar sind“.

In Bezug auf die Untersuchungssettings muss einerseits zwischen experimentellen und quasi-experimentellen Studien und andererseits zwischen Labor- und Feldstudien unterschieden werden. Eine Feldstudie führt verglichen mit einer Laborstudie zu einer hohen externen und einer geringen internen Validität. Experimentelle Untersuchungen zeichnen sich durch die randomisierte Zuordnung der Studienteilnehmer auf die Treatment- und Kontrollgruppe aus, da dann davon ausgegangen werden kann, dass es in Folge des statistischen Fehlerausgleichs zu einer Neutralisierung personengebundener Störvariablen (wie z.B. Motivation oder kognitiver Fähigkeiten) kommt (Bortz und Döring 2006, S. 54). Häufig ist eine solche Randomisierung in der Praxis aber nicht umsetzbar, weil natürliche Gruppen wie z.B. Schulklassen untersucht werden sollen. Hier besteht die Gefahr, dass sich die personengebundenen Störvariablen nicht mehr ausgleichen und sich in der Folge die Probanden der verschiedenen Gruppen z.B. in Bezug auf ihre Motivation systematisch unterscheiden. Die in einer Untersuchung gefundenen Unterschiede bezüglich der abhängigen Variablen lassen sich dann im Gegensatz zu einem experimentellen Studiendesign nicht mehr eindeutig auf Unterschiede in der unabhängigen Variablen zurückführen, weshalb eine quasi-experimentelle Studie verglichen mit einer experimentellen Studie über eine geringere interne Validität verfügt (Bortz und Döring 2006, S. 54).

Tab. 15: Interne und externe Validität bei verschiedenen Studiendesigns (nach Bortz und Döring 2006, S. 58)

\begin{tabular}{|l|l|l|}
\hline \multirow{2}{*}{ Feld } & Experimentell & Quasi-experimentell \\
\cline { 2 - 3 } & Interne Validität: hoch & Interne Validität: niedrig \\
\hline \multirow{2}{*}{ Labor } & Externe Validität: hoch & Externe Validität: hoch \\
\cline { 2 - 3 } & Interne Validität: hoch & Interne Validität: niedrig \\
\cline { 2 - 3 } & Externe Validität: niedrig & Externe Validität: niedrig \\
\hline
\end{tabular}


Die Unterscheidung zwischen Labor- und Feldstudien bezieht sich auf die Frage, ob die Untersuchung in der natürlichen Umgebung der Studienteilnehmer, dem sogenannten Feld, stattfindet oder in einem wissenschaftlichen Labor. Die mit einer Laborstudie zur Erhöhung der internen Validität angestrebte Kontrolle untersuchungsbedingter Störvariablen (z.B. visuelle Reize oder soziale Interaktionen) geht dabei allerdings zu Lasten ihrer externen Validität, da eine Generalisierbarkeit so gefundener Erkenntnisse auf natürliche Situationen oftmals nur bedingt möglich ist (Bortz und Döring 2006, S. 57). Auch wenn eine experimentelle Feldstudie aus theoretischer Sicht sowohl über eine sehr hohe interne und externe Validität verfügt, ist nur schwer vorstellbar, wie sie sich in einem für die Physikdidaktik relevanten Kontext praktisch umsetzen ließe. Bei der vorliegenden summativen Untersuchung handelt es sich um eine quasi-experimentelle Feldstudie, da die Lernförderlichkeit des Unterrichtskonzepts im realen Unterricht evaluiert werden sollte. Der quasi-experimentelle Charakter der Studie resultiert aus der Tatsache, dass eine zufällige Zuordnung sowohl der Lehrkräfte als auch der Schülerinnen und Schüler auf Treatment- bzw. Kontrollgruppe aus organisatorischen und pragmatischen Gründen nicht umsetzbar war. Die Zusammensetzung der Gesamtstichprobe aus natürlichen Gruppen muss aufgrund der damit einhergehenden Besonderheiten in der Datenanalyse berücksichtigt werden, weshalb in der Auswertung u.a. auf Mehrebenenanalysen zurückgegriffen wird.

Zur möglichst eindeutigen Evaluation der Lernwirksamkeit des Unterrichtskonzepts reicht es nicht, lediglich eine große Anzahl an Schülern nach dem Konzept zu unterrichten und auf Basis eines nach dem Unterricht durchgeführten Nachtests (in Folge auch „Posttest“ genannt) auf die Lernwirksamkeit des Konzepts zu schließen. Ein solcher EingruppenUntersuchungsplan ohne Vortest führt zu uneindeutigen Ergebnissen, da völlig offenbleibt, inwiefern der beobachtete Lernerfolg auf das „Treatment“, d.h. das neue Unterrichtskonzept zurückzuführen ist. Aufgrund des fehlenden Vortests (auch „Pretest“ genannt) wäre darüber hinaus nicht sichergestellt, dass die Schüler überhaupt etwas durch den Unterricht dazugelernt haben (Bortz und Döring 2006, S. 524). Bei quasi-experimentellen Untersuchungen ist die Nutzung von Vortests auch deshalb absolut essenziell, weil nur so potenzielle stichprobenspezifische Unterschiede zu Beginn der Studie identifiziert werden können (Bortz und Döring 2006, S. 552). Doch selbst wenn ein solcher Vortest im Rahmen eines Eingruppen-PretestPosttest-Design durchgeführt würde, könnte der beobachtete Lernerfolg mit einer Fülle alternativer Einflussfaktoren (sogenannte „Confounder" bzw. „konfundierende Variablen“) wie z.B. Reifungsprozessen erklärt werden, weshalb die interne Validität einer solchen Eingruppen-Untersuchung in hohem Maße gefährdet wäre (Bortz und Döring 2006, S. 558). Eine deutliche Verbesserung der internen Validität einer Studie im Pretest-Posttest-Design lässt sich nach Bortz und Döring (2006, S. 559) bei quasi-experimentellen Untersuchungen mit Vortest durch das Hinzufügen einer Kontrollgruppe erzielen, da potenzielle Confounder dann auf Kontroll- und Treatmentgruppe in gleicher Weise wirken und somit kontrolliert werden. In Bezug auf die für diese Studie relevante quasi-experimentelle Überprüfung von durch den Unterricht erzielten Veränderungen gilt dann, dass ,die einfachen Differenzen zwischen den Messungen verschiedener Messzeitpunkte sinnvolle, unverzerrte Schätzungen für »wahre" 
Veränderungen darstellen“ (Bortz und Döring 2006, S. 557) und dass es sich zur Ermittlung des Nettoeffekts des Treatments anbietet, die Differenz der Veränderung in Treatment- und Kontrollgruppe zu bilden. Empirisch wurde der Lernerfolg der Schülerinnen und Schüler in der vorliegenden Arbeit deshalb mit Hilfe einer quasi-experimentellen Feldstudie im PretestPosttest-Treatment-Kontrollgruppen-Design evaluiert.

Interessierte Lehrkräfte hatten in dieser Studie die Wahl, ob sie ihre Klassen konventionell unterrichten möchten und somit Teil der Kontrollgruppe sind oder ob sie ihre Klassen nach dem neuen Unterrichtskonzept unterrichten möchten und damit Teil der Treatmentgruppe sind. Der Verzicht auf eine randomisierte Zuordnung der Lehrkräfte zu Kontroll- und Treatmentgruppe hatte zwei Gründe. Auf der einen Seite war so gewährleistet, dass keine Lehrkraft ein Konzept unterrichten musste, von dem sie sich überfordert fühlte oder von dessen Grundideen sie nicht überzeugt war. Auf der anderen Seite wurde die Studie bereits seit dem Schuljahr 2013/14 - und damit zwei Jahre vor Fertigstellung des neuen Unterrichtskonzepts beworben und Daten in der Kontrollgruppe erhoben, da auf diesem Wege mehr Lehrkräfte und Klassen in die Studie eingebunden werden konnten. Somit bestand während zwei der drei Jahre der Studie nur die Möglichkeit zur Teilnahme an der Kontrollgruppe, was den Lehrkräften auch klar kommuniziert wurde.

Im letzten Studienjahr, d.h. dem Schuljahr 2015/16, wäre dann zwar eine randomisierte Zuordnung interessierter Lehrkräfte zu Kontroll- und Treatmentgruppe möglich gewesen, dies wäre aber aufgrund des großen Interesses der Lehrkräfte an dem neuen Konzept mit einer deutlichen Verringerung des Umfangs der Treatmentgruppe einhergegangen. Um eine möglichst große externe Validität der Studie insbesondere in Bezug auf die Ergebnisse der Treatmentgruppe zu erreichen, erschien es deshalb angemessener, auf eine randomisierte Zuordnung im letzten Studienjahr zu Gunsten einer größeren und damit repräsentativeren Treatmentgruppe zu verzichten. Insbesondere wurde auf diese Weise auch versucht, durch eine höhere Anzahl an Lehrkräften in der Treatmentgruppe die hohe Bedeutung der individuellen Lehrerkompetenz auf den Lernerfolg der Schüler zu kompensieren (Hattie 2013). Aus testtheoretischer Perspektive geht die damit erzielte höhere externe Validität der Studie zu Lasten ihrer internen Validität, da nicht ausgeschlossen werden kann, dass etwaige sich in der Studie ergebende Unterschiede zwischen den beiden Gruppen nicht auf systematische Unterschiede zwischen den sie unterrichtenden Lehrkräften z.B. in Bezug auf deren Motivation, Lehrkompetenz oder Erfahrung zurückzuführen sind (Bortz und Döring 2006, S. 57). Auch mögliche Selbstselektionseffekte der Lehrkräfte sind durch diese Vorgehensweise nicht auszuschließen. Wie allerdings bereits erläutert, liegt das Dilemma der Evaluationsforschung grundsätzlich darin, dass eine hohe interne und externe Validität einer Untersuchung in der Regel nur schwer gleichzeitig erzielt werden können.

Eine weitere, die Validität der Studie betreffende Frage ist, wie genau der Unterricht in Treatment- und Kontrollgruppe aussah. Da es aufgrund der Vielzahl an teilnehmenden Lehrkräften im Rahmen dieser Studie nicht möglich war, den Unterricht zu hospitieren oder 
gar zu videografieren, wurden die Lehrkräfte der Treatmentgruppe stattdessen gebeten, ein Unterrichtstagebuch zu führen. In diesem wurde von den Lehrkräften zu jeder Unterrichtsstunde u.a. das Thema und das Datum sowie die verwendeten Unterrichtsmaterialien protokolliert, so dass sich die grobe Struktur des Unterrichts und die Gesamtzahl der gehaltenen Unterrichtsstunden nachvollziehen ließ (vgl. Anhang 13.5). Inwiefern die teilnehmenden Lehrerinnen und Lehrer aber wirklich alle Ideen des Unterrichtskonzepts wie z.B. die leicht veränderte Terminologie zu Stromstärke, Spannung und Potenzial in ihrem eigenen Unterricht umgesetzt haben, lässt sich über diesen Weg nicht feststellen. Auch wurde bewusst nur die Sachstruktur des Unterrichts vorgegeben und den einzelnen Lehrkräften bei der (methodischen) Gestaltung der einzelnen Stunden weitgehend freie Hand gelassen, um das Konzept entsprechend einer Design-Based-Research-Studie in einem realistischen Rahmen evaluieren zu können und eine potenzielle Übertragbarkeit der Ergebnisse im Falle einer späteren umfangreicheren Implementation in der Schulpraxis zu gewährleisten (vgl. Abschnitt 6.1.2). In der Kontrollgruppe wurden die teilnehmenden Lehrkräfte nach Beendigung der Einheit zur Elektrizitätslehre zusätzlich gebeten, einen kurzen Fragebogen zu ihrem gehaltenen Unterricht auszufüllen (vgl. Anhang 13.6).

\subsubsection{Stichprobe ${ }^{11}$}

Die zwischen November 2013 und November 2016 im Frankfurter Raum durchgeführte Studie basiert auf den Daten von 790 Schülerinnen und Schülern, die in 36 Klassen von 23 verschiedenen Lehrerinnen und Lehrern unterrichtet wurden. Fehlten einzelne Schüler oder Schülerinnen bei der Durchführung des Pre- oder Posttests, wurden diese in der Auswertung nicht berücksichtigt. Alle Angaben beziehen sich deshalb auf die effektiv in dieser Studie berücksichtigten Schülerinnen und Schüler. Die Kontrollgruppe (KG) umfasste 17 Gymnasialschulklassen bzw. 357 Schülerinnen und Schüler, die von 11 Lehrkräften über durchschnittlich 23,5 Schulstunden $(S D=11,9)$ unterrichtet wurden. Die etwas größere Treatmentgruppe (TG) umfasste 19 Gymnasialschulklassen bzw. 433 Schülerinnen und Schüler, die von 14 Lehrkräften über durchschnittlich 24,3 Schulstunden $(S D=9,8)$ unterrichtet wurden. Bei der Auswertung wurde eine Klasse der Treatmentgruppe nicht berücksichtigt, da die unterrichtende Lehrkraft Schwierigkeiten mit der deutschen Sprache hatte und nach Aussage einer Kollegin darüber hinaus kein Interesse an der ordnungsgemäßen Durchführung des Unterrichtskonzepts zeigte. Wie in Abschnitt 7.3.1 erläutert, wurden die Lehrkräfte nicht randomisiert auf Kontroll- und Treatmentgruppe aufgeteilt, sondern hatten die Wahl, ob sie traditionell oder nach dem neuen Unterrichtskonzept unterrichten wollten.

\footnotetext{
${ }^{11}$ Teile dieses Abschnitts wurden bereits in Burde und Wilhelm (2017b) veröffentlicht.
} 
Tab. 16: Verteilung der Gesamtstichprobe auf Kontroll- und Treatmentgruppe

\begin{tabular}{|l|l|l|}
\hline & Kontrollgruppe & Treatmentgruppe \\
\hline Schülerinnen und Schüler & 357 & 433 \\
\hline Klassen (gymn.) & 17 & 19 \\
\hline Lehrkräfte & 11 & 14 \\
\hline Jahrgangsstufe & $29 \%$ Stufe 7 & $21 \%$ Stufe 7 \\
& $71 \%$ Stufe 8 & $79 \%$ Stufe 8 \\
\hline Schulstunden (SD) & $23,5(11,9)$ & $24,3(9,8)$ \\
\hline
\end{tabular}

Die unterrichteten Klassen verteilten sich in etwa gleichmäßig auf Jahrgangsstufe 7 (KG: 29\% bzw. TG: 21\%) und Jahrgangsstufe 8 (KG: 71\% bzw. TG: 79\%), wobei das Thema „Elektrizitätslehre" bei allen teilnehmenden Klassen zum ersten Mal im Unterricht behandelt wurde. Die beiden Gruppen sind also in Hinblick auf die Gruppengröße, die Jahrgangsstufe und die unterrichtete Stundenzahl miteinander vergleichbar. Die Verteilung der Gesamtstichprobe auf Kontroll- und Treatmentgruppe ist zur besseren Übersicht auch noch einmal in Tab. 16 dargestellt.

Wie bereits angedeutet, wurden die Lehrkräfte der Kontrollgruppe gebeten, einen kurzen Fragebogen zu ihrem gehaltenen Unterricht auszufüllen, in dem u.a. auch nach den verwendeten Modellen und den unterrichteten Konzepten gefragt wurde. Dabei zeigte sich, dass die drei am häufigsten im Unterricht genutzten Modelle das geschlossene Wasserkreislaufmodell (6 Klassen), das offene Wasserkreislaufmodell (4 Klassen) und das Fahrradkettenmodell (3 Klassen) waren. Während in allen Klassen die elektrische Stromstärke sowie Reihenund Parallelschaltungen Unterrichtsgegenstand waren, wurde nach Auskunft der Lehrkräfte jedoch nur in 14 Klassen die elektrische Spannung, in 12 Klassen der elektrische Widerstand und in 6 Klassen das elektrische Potenzial behandelt. Weitergehende Informationen wurden zur Kontrollgruppe im Rahmen dieser Studie bewusst nicht erhoben, da das Ziel nicht darin bestand zu untersuchen, was ,konventionellen“ Unterricht auszeichnet oder zu klären, inwieweit der Unterricht in der Kontrollgruppe „konventionell“ war. Stattdessen wurde wie bereits erläutert über eine hohe Teilnehmerzahl sowohl in der Kontroll- als auch Treatmentgruppe versucht, eine möglichst hohe Repräsentativität und damit externe Validität zu gewährleisten.

\subsubsection{Testinstrument}

Zur Erhebung des Verständnisses grundlegender Konzepte in der Elektrizitätslehre existieren eine Reihe von unterschiedlichen Methoden und Instrumenten. Neben der direkten Befragung von Schülern, die zwar zeitaufwändig ist, jedoch eine detaillierte Analyse ihrer Vorstellungen erlaubt und deshalb im Rahmen der Teaching Experiments genutzt wurde (vgl. Abschnitt 6.2), bieten sich zur Erhebung der Schülervorstellungen einer großen Anzahl von Probanden vor allem Multiple-Choice-Tests an. Historisch gesehen am bedeutsamsten und sicherlich am meisten zitiert ist der klassische Rhöneck-Test (Rhöneck 1986). Auch wenn dieser Test einen bedeutenden Beitrag zur Forschung über Schülervorstellungen geleistet hat, ist er aus heutiger 
Sicht aufgrund diverser testtheoretischer Mängel nicht für große empirische Untersuchungen des Schülerverständnisses geeignet (Zitzelsberger und Rabe 2011). Der in den USA entwickelte DIRECT-Test („Determining and Interpreting Resistive Electric Circuit Concepts Test”) stellt hingegen ein psychometrisch ausgereiftes diagnostisches Testinstrument dar, erlaubt aufgrund seiner einstufigen Struktur allerdings nicht das eindeutige Identifizieren von Schülervorstellungen (Engelhardt und Beichner 2004). Der dreistufige SECDT-Test von Peşman und Eryılmaz (2010) erlaubt zwar prinzipiell das Erfassen von Schülervorstellungen und sogar der Antwortsicherheit der Lernenden, weist aber für die empirische fachdidaktische Forschung eine zu niedrige Reliabilität auf.

Das konzeptionelle Verständnis von einfachen elektrischen Stromkreisen wurde in dieser Arbeit deshalb auf Basis des psychometrisch ausgereiften zweistufigen MultipleChoice-Tests von Urban-Woldron und Hopf (2012) erhoben, der 22 Items primär zu Stromstärke und Widerstand umfasst. Nach Urban-Woldron (2013, S. 211) ist das Testinstrument geeignet, ,[...] anstelle von Interviews für die Evaluation von Elektrizitätslehre-Unterricht in der Schulpraxis [...]“ genutzt zu werden und ist "[...] gleichzeitig aber psychometrisch so ausgereift [...], dass es auch für die empirische fachdidaktische Forschung verwendbar ist". Insbesondere erlaube das Testinstrument ,[...] einen fairen Vergleich verschiedener Zugänge zum Elektrizitätslehre-Unterricht, wo ja verschiedenste Ansätze miteinander konkurrieren“ (Urban-Woldron und Hopf 2012, S. 224), weshalb es für den Einsatz in dieser Studie geradezu prädestiniert erscheint.

Ein weiterer großer Vorteil des Tests besteht insbesondere darin, dass er die Diagnose von gut dokumentierten Schülervorstellungen erlaubt. Gerade bei der Evaluation eines neuen Unterrichtskonzepts ist es nämlich nicht nur von großem Interesse zu wissen, ob die Schüler mehr oder weniger Items korrekt beantworten können, sondern vor allem auch, im Detail zu untersuchen, wo weiterhin besondere Lernschwierigkeiten liegen und bestimmte Konzepte noch nicht angemessen verstanden wurden. Einen solchen detaillierten Blick auf die Schülervorstellungen ermöglicht der Test insbesondere aufgrund seiner zweistufigen Struktur, die gegenüber einstufigen Testinstrumenten wie dem DIRECT oder Rhöneck-Test eine große Weiterentwicklung darstellt. Im Gegensatz zu einem einstufigen Item müssen die Schüler bei einem zweistufigen Item eine Frage nicht nur beantworten, sondern diese in einer zweiten Stufe auch begründen. Die Items des Multiple-Choice-Tests enthalten also sowohl Distraktoren $\mathrm{zu}$ den Antworten (erste Stufe) als auch Distraktoren zu den möglichen Erklärungen (zweite Stufe), weil aus einer richtigen Antwort auf der ersten Stufe nicht zwangsläufig auf ein angemessenes konzeptionelles Verständnis geschlossen werden kann. Auf jeder Stufe gibt es dabei nur einen korrekten Attraktor. Der Vorteil einer solchen Zweistufigkeit liegt darin, dass auf diese Weise nicht nur typische Schülervorstellungen auf rasche und effiziente Weise erhoben werden können, sondern so auch die Identifikation von falsch-positiven (d.h. richtige Antworten trotz einer falschen Begründung bzw. Vorstellung) und falsch-negativen Antworten (d.h. falsche Antworten trotz einer korrekten Vorstellung) ermöglicht wird. Somit verbindet das hier verwendete Testinstrument die Vorteile eines quantitativen, psychometrisch vali- 
dierten Multiple-Choice-Tests mit den sonst nur durch zeitaufwendige, qualitative Interviews erzielbaren Einblicken in die Vorstellungen der Schüler. Nach Urban-Woldron und Hopf (2012, S. 207) lassen sich mit dem Test folgende Schülervorstellungen in der elementaren Elektrizitätslehre erfassen, wobei diese inhaltlich in Abschnitt 3.2 erläutert sind und ihre genaue Erhebung mit Hilfe des Tests in Abschnitt 7.3.4 erklärt wird:

1. Stromverbrauchsvorstellung (SV)

2. Batterie als konstante Stromquelle (BS)

3. Inverse Widerstandsvorstellung (IR)

4. Stromstärke ist unabhängig von R (UR)

5. Stromverbrauch ist proportional zu R (PR)

6. Lokales Denken (LD)

7. Sequentielle Argumentation (SA)

8. Erfassen von Parallelschaltungen (PS)

Da der hier eingesetzte Test ohne Bezug zum neuen Unterrichtskonzept entwickelt wurde, liegt der Schwerpunkt der 22 Items vor allem auf dem elektrischen Strom- und Widerstandskonzept, nicht aber auf der elektrischen Spannung, die zusammen mit dem elektrischen Potenzial die Grundlage des zu evaluierenden Unterrichtskonzepts darstellt. Um das Spannungsverständnis der Schüler dennoch beurteilen zu können, wurde der Test von UrbanWoldron und Hopf (2012) um vier weitere Items zum Spannungskonzept ergänzt. Die dabei verwendeten Spannungsitems lehnen sich an Aufgabe 8 des Rhöneck-Tests an und stellen keine Multiple-Choice-Items dar. Vielmehr müssen hier die Werte für die zwischen verschiedenen Punkten einer Schaltung bestehenden Spannungen eingetragen werden. Aufgrund der Tatsache, dass das verwendete Testinstrument fast ausschließlich Konzepte abprüft, die schwerpunktmäßig im traditionellen Unterricht thematisiert werden, gleichzeitig aber das für das neue Unterrichtskonzept zentrale Spannungskonzept nur peripher erhebt, kann davon ausgegangen werden, dass das neue Unterrichtskonzept durch den Test nicht systematisch bevorzugt wird. Da jedes Item dichotom kodiert wird $(0=$ falsche Antwort bzw. $1=$ richtige Antwort), die insgesamt acht zweistufigen Items nur dann als korrekt gezählt werden, wenn sowohl die gegebene Antwort als auch die Begründung korrekt sind, und die Spannungsitems bzw. Item 16 zur Stromstärke nur dann korrekt gewertet werden, wenn alle anzugebenden Spannungs- bzw. Stromstärkewerte stimmen, liegt der maximal erreichbare Summenscore im eingesetzten Test bei 26. Der Fragebogen wurde als Paper-and-Pencil-Test von den Schülern während der Unterrichtszeit bearbeitet.

Auf Grundlage der vier hinzugefügten Items (31a, 31b, 32a, 32b) wurde zudem versucht, Kategorien in Bezug auf die elektrische Spannung zu identifizieren, mit denen die Lernenden potenziell Schwierigkeiten haben könnten. Dies sollte eine erste Einschätzung darüber ermöglichen, inwiefern die Lernenden über ein angemessenes Spannungskonzept verfügen. Konkret wurden die folgenden drei Kategorien erhoben: 
1. Spannung als Eigenschaft des elektrischen Stroms (UI)

2. Konzeptionelle Schwierigkeiten mit der elektrischen Spannung (KS)

3. Mindestens eins von vier Spannungsitems korrekt

\subsubsection{Erhebung der Schülervorstellungen}

Die Schülervorstellungen wurden größtenteils auf Basis des Kodiermanuals von UrbanWoldron und Hopf (2012, S. 219) erhoben. Dieses gibt an, welche Schülervorstellung hinter welcher Antwortkombination steht, wie an Hand des im Test verwendeten Items 3 kurz erläutert werden soll. Wie in Abb. 67 zu sehen ist, wird in Item 3 danach gefragt, wie ein größerer Widerstand in einem einfachen geschlossenen Stromkreis die Stromstärke beeinflusst. Entsprechend der Zweistufigkeit des Items müssen die Schüler nicht nur eine Antwort auswählen, sondern in einer zweiten Stufe auch eine Begründung für ihre Antwort geben. Zweistufige Items wie beispielsweise Item 3 werden nur als korrekt kodiert, wenn sowohl die Antwort auf der ersten, als auch die Begründung auf der zweiten Stufe korrekt ist.

\begin{tabular}{|c|c|c|}
\hline Item 3 & \multicolumn{2}{|c|}{$\begin{array}{l}\text { Der Widerstand } \mathbf{R}_{1} \text { im Stromkreis (Abb. rechts oben) ist klein. Er wird } \\
\text { durch einen größeren Widerstand } \mathbf{R}_{2} \text { ersetzt (Abb. rechts unten). }\end{array}$} \\
\hline a) & $\begin{array}{l}\text { Was geschieht mit der Stromstärke im Strom- } \\
\text { kreis? }\end{array}$ & \\
\hline 1) & Sie wird größer. & \\
\hline 2) & Sie wird kleiner, aber nicht Null. & \\
\hline 3) & Sie bleibt gleich. & \\
\hline 4) & Es fließt kein Strom mehr & (kleiner Widerstand) \\
\hline b) & Wie erklärst du deine Entscheidung? & \\
\hline 1) & $\begin{array}{l}\text { Die Batterie ist nicht stark genug, um überhaupt } \\
\text { Strom durch den größeren Widerstand zu trei- } \\
\text { ben. }\end{array}$ & \\
\hline 2) & $\begin{array}{l}\text { Die Batterie kann nicht einen so großen Strom } \\
\text { wie vorher durch den größeren Widerstand trei- } \\
\text { ben. }\end{array}$ & $\begin{array}{c}\mathbf{R}_{\mathbf{2}} \\
\text { (großer Widerstand) }\end{array}$ \\
\hline 3) & $\begin{array}{l}\text { Ein größerer Widerstand braucht mehr Strom als } \\
\text { ein kleinerer Widerstand. }\end{array}$ & \\
\hline 4) & $\begin{array}{l}\text { Es ist dieselbe Batterie; daher bleibt auch die } \\
\text { Stromstärke gleich. }\end{array}$ & \\
\hline
\end{tabular}

Im vorliegenden Beispiel wäre die Antwortkombination a2b2 korrekt. Die Kombination von Antwort und Begründung erlaubt aber nicht nur die Unterscheidung zwischen falschen und korrekten Antworten, sondern ermöglicht insbesondere direkte Rückschlüsse auf spezifische Schülervorstellungen und deren Häufigkeit. Urban-Woldron und Hopf (2012, S. 208) 
führen am Beispiel von Item 3 exemplarisch aus, hinter welcher Antwortkombination welche Schülervorstellung steht:

Tab. 17: Abbildung der verschiedenen Schülervorstellungen durch Antwortkombinationen für Item 3 (entnommen aus Urban-Woldron und Hopf 2012, S. 208)

\begin{tabular}{|l|l|l|}
\hline $\begin{array}{l}\text { Antwort- } \\
\text { kombination }\end{array}$ & Beschreibung & $\begin{array}{l}\text { Schüler- } \\
\text { vorstellung }\end{array}$ \\
\hline a2b2 & Korrekte Lösung & -- \\
\hline a1b3 & $\begin{array}{l}\text { Lernende, die im Teil a) die Antwort 1 und im Teil b) die } \\
\text { Antwort 3 auswählen, sind der Meinung, dass die Strom- } \\
\text { stärke größer wird, da ein größerer Widerstand mehr Strom } \\
\text { braucht als ein kleinerer Widerstand. Sie haben also eine } \\
\text { inverse Widerstandsvorstellung. }\end{array}$ & $\begin{array}{l}\text { IR } \\
\text { derstands- } \\
\text { vorstellung) }\end{array}$ \\
\hline a3b4 & $\begin{array}{l}\text { Lernende, die im Teil a) die Antwort 3 und im Teil b) die } \\
\text { Antwort 4 auswählen, sind der Meinung, dass die Batterie } \\
\text { immer gleich viel Strom liefert und die Stromstärke auch } \\
\text { unbeeinflusst vom Widerstand immer gleich bleibt. Sie } \\
\text { sehen eine Batterie als eine Quelle für einen konstanten } \\
\text { Strom. }\end{array}$ & $\begin{array}{l}\text { Batterie als } \\
\text { konstante } \\
\text { Stromquelle) }\end{array}$ \\
\hline $\begin{array}{l}\text { Lernende, die auf Stufe a) die Antwort 2 und auf Stufe b) } \\
\text { die Antwort 3 auswählen, geben zwar die richtige Antwort } \\
\text { in Bezug auf die Verringerung der Stromstärke, erklären } \\
\text { ihre Antwort aber damit, dass ein größerer Widerstand } \\
\text { mehr Strom verbraucht. Nach Hestenes und Halloun (1995) } \\
\text { wird diese Antwort als falsch-positiv bezeichnet. Wird da- } \\
\text { her nur auf der ersten Stufe getestet, überschätzt man die } \\
\text { Anzahl der richtigen Lösungen. }\end{array}$ & $\begin{array}{l}\text { PR } \\
\text { (Strom- } \\
\text { verbrauch ist } \\
\text { zuroportional }\end{array}$ \\
\hline
\end{tabular}

Im Folgenden soll nun für alle Schülervorstellungen dargelegt werden, auf welchen Antwortkombinationen im verwendeten Multiple-Choice-Test sie erhoben wurden. Wie Tab. $18 \mathrm{zu}$ entnehmen ist, wurden die Schülervorstellung LD, SA und PS teilweise auch auf Basis mehrerer Einzelitems statt gestufter Items ermittelt. Da im Unterrichtskonzept die physikalische Stromrichtung gelehrt, im Test in Bezug auf die Stromverbrauchsvorstellung aber von der technischen Stromrichtung ausgegangen wird, wurden die bisherigen Antwortkombinationen um passende Kombinationen erweitert (SV1 = 1 für a2b5; SV2 = 1 für a3b2; SV3 = 1 für a1b2; SV4 = 1 für a2b2). Auf diese Weise kann die Stromverbrauchsvorstellung also sowohl für Lernende festgestellt werden, die von der physikalischen als auch der technischen Stromrichtung ausgehen. Da sich das Kodiermanual auf eine vorherige Version des Tests mit 23 Items bezieht, in der vorliegenden Arbeit aber eine Nachfolgeversion mit nur noch 22 Items eingesetzt wird, wurden in Tab. 18 Bezüge zu dem nicht mehr vorkommenden Item 11 entfernt. 
Kapitel 7: Summative Evaluation des Unterrichtskonzepts

Tab. 18: Übersicht über die Antwortkombinationen und die daraus abgeleiteten Schülervorstellungen (angelehnt an Urban-Woldron und Hopf 2012, S. 219)

\begin{tabular}{|c|c|}
\hline Schïlervorstellung / Konstrukt & $\begin{array}{l}\text { Antwortkombinationen bzw. } \\
\text { beobachtete Variablen }\end{array}$ \\
\hline Stromverbrauchsvorstellung (SV) & $\begin{array}{l}\mathrm{SV} 1=1 \text { für } \mathrm{a} 1 \mathrm{~b} 2 \text { oder } \mathrm{a} 2 \mathrm{~b} 5 \text { bei Item } 21 \\
\mathrm{SV} 2=1 \text { für } \mathrm{a} 1 \mathrm{~b} 2 \text { oder } \mathrm{a} 3 \mathrm{~b} 2 \text { bei Item } 4 \\
\mathrm{SV} 3=1 \text { für } \mathrm{a} 3 \mathrm{~b} 2 \text { oder } \mathrm{a} 1 \mathrm{~b} 2 \text { bei Item } 28 \\
\mathrm{SV} 4=1 \text { für } \mathrm{a} 1 \mathrm{~b} 2 \text { oder } \mathrm{a} 2 \mathrm{~b} 2 \text { bei Item } 22\end{array}$ \\
\hline Batterie als konstante Stromquelle (BS) & $\begin{array}{l}\mathrm{BS} 1=1 \text { für } \mathrm{a} 3 \mathrm{~b} 4 \text { bei Item } 3 \\
\mathrm{BS} 2=1 \text { für } \mathrm{a} 2 \mathrm{~b} 2 \mathrm{c} 2 \text { bei Item } 27 \\
\mathrm{BS} 3=1 \text { für } \mathrm{a} 2 \mathrm{~b} 2 \text { bei Item } 23 \\
\mathrm{BS} 4=1 \text { für } \mathrm{a} 2 \mathrm{~b} 2 \text { bei Item } 6\end{array}$ \\
\hline Stromstärke ist unabhängig von R (UR) & $\begin{array}{l}\text { UR } 1=1 \text { für } \text { b2 bei Item } 29 \text { und } \mathrm{b} 2 \text { bei Item } 25 \\
\text { UR2 }=1 \text { für } \mathrm{a} 2 \text { bei Item } 10 \text { und } \mathrm{a} 2 \text { bei Item } 15 \\
\text { UR } 3=1 \text { für } \mathrm{a} 2 \text { bei Item } 10 \text { und } \mathrm{b} 2 \text { bei Item } 25 \\
\mathrm{UR} 4=1 \text { für a } 2 \text { bei Item } 15 \text { und b2 bei Item } 29\end{array}$ \\
\hline Inverse Widerstandsvorstellung (IR) & $\begin{array}{l}\text { IR1 }=1 \text { für } \mathrm{a} 1 \mathrm{~b} 3 \text { bei Item } 3 \\
\text { IR2 }=1 \text { für } \mathrm{a} 1 \mathrm{~b} 1 \text { bei Item } 23 \\
\text { IR3 }=1 \text { für } \mathrm{a} 1 \mathrm{~b} 4 \text { bei Item } 6\end{array}$ \\
\hline $\begin{array}{l}\text { Stromverbrauch ist proportional zum } \\
\text { Widerstand (PR) }\end{array}$ & $\begin{array}{l}\text { PR1 }=1 \text { für } \mathrm{a} 3 \mathrm{~b} 1 \text { bei Item } 23 \\
\text { PR2 }=1 \text { für } \mathrm{a} 3 \mathrm{~b} 4 \text { bei Item } 6 \\
\text { PR3 }=1 \text { für } \mathrm{a} 2 \mathrm{~b} 3 \text { bei Item } 3\end{array}$ \\
\hline Lokales Denken (LD) & $\begin{array}{l}\mathrm{LD} 1=1 \text { für } \mathrm{I} 1=0,3 \mathrm{~A}, \mathrm{I} 2=0,3 \mathrm{~A} \\
\text { und } \mathrm{I} 3=0,6 \mathrm{~A} \text { bei Item } 16 \\
\mathrm{LD} 2=1 \text { für a } 2 \text { bei Item } 7 \\
\mathrm{LD} 3=1 \text { für a3 bei Item } 24\end{array}$ \\
\hline Sequentielle Argumentation (SA) & 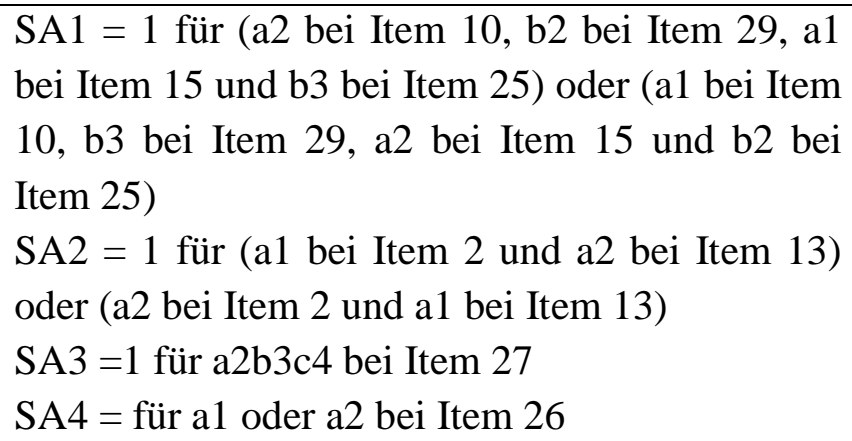 \\
\hline Erfassen von Parallelschaltungen (PS) & $\begin{array}{l}\text { PS } 1=1 \text { für a } 2 \text { oder a } 3 \text { oder a } 4 \text { bei Item } 9 \\
\text { PS } 2=1 \text { für a } 2 \text { bei Item } 20 \\
\text { PS } 3=1 \text { für a } 1 \text { oder a } 2 \text { oder a } 3 \text { oder a } 5 \text { bei } \\
\text { Item } 14 \\
\text { PS } 4=1 \text { für a } 2 \text { oder a } 3 \text { oder a } 4 \text { bei Item } 30\end{array}$ \\
\hline
\end{tabular}

Eine Schülervorstellung wird entsprechend dieses Kodiermanuals bereits dann als vorhanden angenommen, wenn ein Schüler bei einem einzigen von mehreren die jeweilige Schülervorstellung abprüfenden Items eine entsprechende Antwortkombination ankreuzt. Damit also beispielsweise die Lernschwierigkeit „Erfassen von Parallelschaltungen“ diagnos- 
tiziert wird, reicht es, wenn bei Item 20 nur das Feld a2 angekreuzt wurde. Mit anderen Worten wird also die den Schülervorstellungen zugrundeliegende Subskala dichotom kodiert, d.h. eine Schülervorstellung kann nur vorliegen (Wert 1) oder nicht vorliegen (Wert 0).

Die vier zusätzlich hinzugefügten Items 31a, 31b, 32a, 32b (siehe Anhang 13.3) dienen der Identifikation von Schülervorstellungen und Schwierigkeiten in Bezug auf die elektrische Spannung, wobei die folgenden drei Kategorien unterschieden werden:

1. Spannung als Eigenschaft des elektrischen Stroms (UI): Diese Schülervorstellung wird dann für gegeben angenommen, wenn bei einem offenen Stromkreis von den Schülern für alle gefragten Spannungswerte der Wert null angegeben wird, da in diesem Fall davon auszugehen ist, dass für sie Spannung immer nur zusammen mit elektrischem Strom auftreten kann. Diese Schülervorstellung wird bewusst nur auf Basis des Items 32b zum offenen Stromkreis erhoben, weil bei den anderen Items eine Überlagerung mit der Stromverbrauchsvorstellung nicht ausgeschlossen werden kann. Es gilt: $\mathrm{UI}=1$ für $\mathrm{b} 1=0 \mathrm{~V}$ und $\mathrm{b} 2=0 \mathrm{~V}$ und $\mathrm{b} 3=0 \mathrm{~V}$ bei Item 32 .

2. Konzeptionelle Schwierigkeiten mit der elektrischen Spannung (KS): Diese etwas allgemeinere Lernschwierigkeit wurde angenommen, wenn die Schüler zwischen zwei Punkten auf einem ununterbrochenen Leiterstück einen Spannungswert angeben, der größer ist als Null. In diesem Fall ist davon auszugehen, dass die Schüler nicht klar zwischen dem Absolutwert „Potenzial“, der einem Leiterabschnitt lokal zugeordnet werden kann, und der Differenzgröße „Spannung“, die sich auf den Potenzialunterschied zwischen zwei Punkten bezieht, unterscheiden können. Hierbei handelt es sich allerdings um eine weniger trennscharfe Kategorie, da Schüler, die die Spannung für eine Eigenschaft des Stroms halten, auch konzeptionelle Schwierigkeiten mit der Spannung haben und bei den entsprechenden Items vermutlich einen Spannungswert größer Null annehmen würden. Es gilt: KS $=1$ für a1 $>0$ oder a3 $>0$ oder b1 $>0$ bei Item 31 oder $\mathrm{a} 1>0$ bei Item 32 .

3. Mindestens eins von vier Spannungsitems korrekt: Dieser dritte Punkt ist keine Lernschwierigkeit im engeren Sinne, sondern stellt lediglich einen groben Indikator dar, inwiefern die Lernenden zumindest über ein rudimentäres Spannungskonzept verfügen. Wie oben bereits erläutert, wird hier ein Spannungsitem nur dann als korrekt gewertet, wenn jeweils alle drei pro Item anzugebenden Spannungswerte korrekt eingetragen werden.

\subsubsection{Auswertemethodik}

Im Folgenden werden die Grundideen einiger für diese Arbeit grundlegenden statistischen Verfahren und Kenngrößen beschrieben. Außerdem werden die Voraussetzungen erläutert, unter denen diese statistischen Verfahren angewendet werden dürfen. Da die Mehrebenenanalyse im Gegensatz zu $t$-Tests und Varianzanalysen in der fachdidaktischen Forschung ein ver- 
gleichsweise neues und komplexes Analysewerkzeug darstellt, wird dieses Verfahren in Abschnitt 7.5.1 ausführlicher beschrieben. Für eine mathematische Darstellung der angewendeten statistischen Methoden sei an dieser Stelle auf die entsprechende Fachliteratur verwiesen. Alle Berechnungen wurden in dieser Studie mit Hilfe von IBM SPSS Statistics 24 oder Microsoft Excel 2016 durchgeführt.

\subsubsection{Signifikanz und Effektstärke}

In dieser Arbeit wird neben der Signifikanz der gefundenen Ergebnisse nach Möglichkeit auch deren Effektstärke berichtet, damit der Leser die Bedeutung der gefundenen Effekte besser beurteilen kann. Die Signifikanz ermöglicht eine Einschätzung davon, ob ein gefundener Mittelwertsunterschied zufälligen oder systematischen Charakter hat. Für alle Signifikanzangaben soll dabei folgende Konvention gelten: signifikante Ergebnisse $(.01 \leq p<.05)$ werden mit einem Stern , ${ }^{*}$, , hoch signifikante Ergebnisse $(.001 \leq p<.01)$ werden mit zwei Sternen „**“ und höchst signifikante Ergebnisse $(p<.001)$ werden mit drei Sternen ,***“ gekennzeichnet. Bei großen Stichproben werden jedoch auch bei kleinen Mittelwertsunterschieden schnell signifikante Ergebnisse erzielt. Um die praktische Bedeutung eines gefundenen Effekts besser beurteilen zu können, wird deshalb zusätzlich die Effektstärke berichtet, die unabhängig von der Stichprobengröße ist. Als Maß für die Effektstärke wird hier Cohens $d$ verwendet, das den Abstand zweier Normalverteilungen in Einheiten ihrer gemeinsamen Standardabweichung angibt. Auf die gemeinsame Standardabweichung wird deshalb zurückgegriffen, weil sich die Stichproben in dieser Studie bzgl. ihres Umfangs und ihrer Varianz unterscheiden.

$$
d=\frac{\mu_{A}-\mu_{B}}{S D_{\text {pooled }}}
$$

mit

$$
S D_{\text {pooled }}=\sqrt{\frac{\left(N_{A}-1\right) \cdot \sigma_{A}^{2}+\left(N_{B}-1\right) \cdot \sigma_{B}^{2}}{\left(N_{A}-1\right)+\left(N_{B}-1\right)}} \quad \begin{aligned}
& N_{A}: \text { Stichprobengröße A } \\
& N_{B}: \text { Stichprobengröße B } \\
& \sigma_{A}^{2}: \text { Varianz von Stichprobe A } \\
& \sigma_{B}^{2}: \text { Varianz von Stichprobe B }
\end{aligned}
$$

$\mu_{B}:$ Mittelwert von Stichprobe B

$S D_{\text {pooled }}$ : gemeinsame Standardabweichung der beiden Stichproben

Cohens $d$ als Effektstärke lässt sich nicht nur leicht interpretieren, sondern ermöglicht auch einen Vergleich der Ergebnisse der verschiedenen in dieser Arbeit genutzten statistischen Verfahren. Nach Cohen (1988) kann dabei als Faustregel angenommen werden, dass ab einem Wert von 0.2 ein kleiner Effekt, ab einem Wert von 0.5 ein mittlerer Effekt und ab einem Wert von 0.8 ein großer Effekt vorliegt.

\subsubsection{Signifikanzniveaus, Konfidenzintervalle und t-Tests}

Mit Hilfe des $t$-Tests, einem parametrischen Testverfahren der Inferenzstatistik, kann geprüft werden, ob aus der Mittelwertdifferenz zweier Stichproben darauf geschlossen werden kann, dass sich die den Stichproben zugrundeliegenden Populationen systematisch unterscheiden. 
Dabei wird bei einem $t$-Test die Nullhypothese $\mathrm{H}_{0}$ geprüft, dass der beobachtete Unterschied zwischen den beiden Stichproben zufällig zustande gekommen ist und beide Stichproben der gleichen Grundgesamtheit (Population) entstammen. Allerdings liefert der $t$-Test nur einen Wahrscheinlichkeitswert, der angibt, wie wahrscheinlich es ist, die Nullhypothese $\mathrm{H}_{0}$ fälschlicherweise abzulehnen, obwohl sie in Wirklichkeit zutrifft (den sogenannten $\alpha$-Fehler oder Fehler 1. Art zu begehen). Eine auch bei anderen Teststatistiken sehr häufig verwendete Entscheidungsgrenze für eine solche fälschliche Ablehnung der Nullhypothese liegt bei 5\%, d.h. wenn die mittels $t$-Test gefundene Irrtumswahrscheinlichkeit unter diesem Schwellenwert bzw. Signifikanzniveau liegt, wird davon ausgegangen, dass sich die den Stichproben zugrundeliegenden Populationen signifikant voneinander unterscheiden.

Sofern möglich und sinnvoll, werden in dieser Arbeit zudem in Diagrammen die Konfidenzintervalle des Mittelwerts angegeben. Bei den gewählten Konfidenzintervallen handelt es sich um 95\%-Konfidenzintervalle. Ein solches 95\%-Konfidenzintervall gibt die Spanne von Werten an, innerhalb derer sich bei $95 \%$ aller Stichproben der wahre Populationsmittelwert befindet (Field 2011, S. 48).

In der vorliegenden Arbeit werden sowohl $t$-Tests für unabhängige Stichproben als auch $t$-Tests für abhängige Stichproben eingesetzt. Werden die gleichen Personen mit Hilfe des gleichen Testinstruments zu zwei Messzeitpunkten untersucht, so muss der $t$-Test für abhängige Stichproben (bzw. gepaarter $t$-Test oder $t$-Test für verbundene Stichproben) verwendet werden, weil die Messungen nicht unabhängig voneinander sind. Geprüft wird beim $t$-Test für abhängige Stichproben dann, ob sich die mittlere Differenz aller Messwertpaare von Null unterscheidet oder nicht. Nach Bühner und Ziegler (2009, 243f) lauten die Voraussetzungen für einen solchen gepaarten bzw. abhängigen $t$-Test dabei wie folgt:

1. Zu jedem einzelnen Zeitpunkt müssen die Messwerte der Probanden unabhängig voneinander sein.

2. Die Messwerte müssen eine Intervallskalierung aufweisen.

3. Die Differenz aller Messwertpaare (von Messzeitpunkt 1 und Messzeitpunkt 2) müssen in beiden Populationen normalverteilt sein.

Ein $t$-Test für unabhängige Stichproben hingegen wird verwendet, um zu überprüfen, ob sich die Populationsmittelwerte zweier unabhängiger Stichproben voneinander unterscheiden. Es wird also untersucht, ob sich aufgrund eines Unterschieds in der unabhängigen Variablen (z.B. Gruppenzugehörigkeit) ein Unterschied in der abhängigen Variablen (z.B. PretestErgebnis) ergibt. Die Voraussetzungen für einen unabhängigen $t$-Test sind nach Bühner und Ziegler (2009, S. 256):

1. Die Messwerte von unterschiedlichen Personen müssen voneinander unabhängig sein.

2. Die Messwerte müssen eine Intervallskalierung aufweisen. 
3. Die abhängige Variable muss in beiden Populationen (und damit auch in beiden Stichproben) normal verteilt sein.

4. Die Varianzen der abhängigen Variablen müssen in beiden Populationen gleich sein (Varianzhomogenität).

Nach Rasch et al. (2010, 59f) reagiert der $t$-Test aber robust gegenüber Verletzungen seiner Voraussetzungen, solange die Stichproben in ihrem Umfang in etwa miteinander vergleichbar sind, weshalb das Prüfen dieser Voraussetzungen in der Forschungspraxis eher unüblich ist. Da insbesondere die Verletzung der Normalverteilungsannahme bei größeren Stichproben unproblematisch sei, empfehlen Bühner und Ziegler (2009, 256f) die Normalverteilung lediglich deskriptiv mittels Histogrammen zu prüfen und weisen darauf hin, dass selbst bei einer Verletzung der Normalverteilung der $t$-Test in der Regel non-parametrischen Tests wie dem Mann-Whitney-U-Test vorgezogen werden sollte. In dieser Arbeit wird deshalb auf eine quantitative Überprüfung der Normalverteilungsannahme z.B. mittels Kolmogorov-Smirnov-Test verzichtet, zumal dieser Test aufgrund des großen Stichprobenumfangs bereits bei kleinen Abweichungen von der Normalverteilung signifikant werden würde (Field 2011, S. 144). Die Verletzung der Varianzhomogenitätsannahme ist insofern nicht problematisch, als dass in solchen Fällen auf das korrigierte Ergebnis eines $t$-Tests für heterogene Varianzen (Welch-Test) zurückgegriffen werden kann. Auch wenn in der vorliegenden Arbeit vor der Durchführung des jeweiligen statistischen Verfahrens stets eine Prüfung der Voraussetzungen stattfand, wird diese Prüfung in expliziter Form nur bei zentralen Ergebnissen dargelegt.

\subsubsection{Die Varianzanalyse (ANOVA)}

Werden mehr als zwei Gruppen miteinander verglichen, sollte auf einen paarweisen Vergleich der verschiedenen Gruppen untereinander mittels $t$-Test verzichtet werden, da dies eine Alpha-Fehler-Inflation zur Folge hätte. Der Grund für die Alpha-Fehler-Inflation ist darin begründet, dass z.B. bei drei Gruppen drei $t$-Tests durchgeführt werden müssten. Wird für jeden $t$-Test ein Signifikanzniveau von 5\%, d.h. eine Sicherheit von 95\%, keinen Alpha-Fehler zu begehen, angenommen, so sinkt diese Sicherheit bei drei $t$-Tests auf $85,7 \%$ ab $(0,95 \times$ x $0,95 \mathrm{x}$ $0,95=0,857)$. Damit liegt die Wahrscheinlichkeit mindestens einen Alpha-Fehler zu begehen bei drei $t$-Tests bereits bei 14,3\% (Field 2011, S. 348). Ein geeigneteres Mittel, um zu überprüfen, ob sich mehr als zwei Gruppen hinsichtlich eines Merkmals signifikant unterscheiden, stellt deshalb eine Varianzanalyse dar, die oft auch als ANOVA für Analysis $\underline{\text { Of }} \underline{\text { Variance }}$ bezeichnet wird. Wie der Name bereits andeutet, wird bei Varianzanalysen die Varianz der abhängigen Variablen untersucht, indem ihre Gesamtvarianz zerlegt wird in „Varianz zwischen den Gruppen“ und „Varianz innerhalb der Gruppen“. Die hier verwendete kategoriale unabhängige Variable „Gruppe“ wird bei Varianzanalysen als Faktor bezeichnet, wobei der Faktor „Gruppe“ verschiedene Ausprägungen haben kann, die als Faktorstufen bezeichnet werden (z.B. die verschiedenen Terzile im Vortest). Das Ziel der Varianzanalyse besteht dann darin, die Varianz zwischen den Gruppen ins Verhältnis zur Varianz innerhalb der Gruppen zu setzen. Dieses Verhältnis gibt der $F$-Wert an, der wie folgt definiert ist: 
$F=\frac{M S_{\text {zwischen }}}{M S_{\text {innerhalb }}}$

$M S_{\text {zwischen }}$ : Varianz zwischen den Gruppen

$M S_{\text {innerhalb }}$ : Varianz innerhalb der Gruppen

Dabei steht $M S$ für ,Mean Squares“, d.h. im Falle von $M S_{z w i s c h e n}$ für die mittlere Quadratsumme der Abweichungen der Gruppenmittelwerte vom Gesamtmittelwert und im Falle von $M S_{\text {innerhalb }}$ für die mittlere Quadratsumme der Abweichungen der Einzelwerte von den Gruppenmittelwerten (Rasch et al. 2014, 9ff). Wird der F-Wert statistisch signifikant, bedeutet dies, dass sich die abhängige Variable signifikant zwischen den verschiedenen Gruppen unterscheidet. Unklar ist dann aber noch, um welche Gruppen es sich dabei genau handelt. Dies kann mit Hilfe sogenannter Post-Hoc-Tests herausgefunden werden, mit denen die verschiedenen Gruppen paarweise miteinander verglichen werden. Für große Stichproben, die aber in ihrem Umfang nicht übereinstimmen und ggf. auch ungleiche Varianzen aufweisen, wird von Field (2011, 375f) der Games-Howell Post-Hoc-Test empfohlen, weshalb dieser in der vorliegenden Arbeit verwendet wird.

Nach Rasch et al. (2010, 30f) müssen für die Durchführung von Varianzanalysen die folgenden Voraussetzungen erfüllt sein:

1. Die Intervallskalierung der abhängigen Variable muss gegeben sein.

2. In allen Bedingungen sind die Messwerte voneinander unabhängig.

3. In der Population muss das untersuchte Merkmal normal verteilt sein.

4. Es muss eine Varianzhomogenität bzgl. der Populationen der untersuchten Gruppen vorliegen.

Dabei weisen Rasch et al. (2010, S. 31) darauf hin, dass eine Varianzanalyse ähnlich einem $t$ Test gegenüber der Verletzung der dritten und vierten Voraussetzung verhältnismäßig robust ist, solange die Stichprobenumfänge in beiden Gruppen sich nicht zu sehr unterscheiden. Werden die gleichen Personen mit Hilfe des gleichen Testinstruments zu verschiedenen Messzeitpunkten untersucht, so ist die zweite Voraussetzung verletzt, da die Messungen miteinander korrelieren. In einem solchen Fall ist dann die Anwendung einer Varianzanalyse mit Messwiederholung notwendig, die zusätzlich die Voraussetzung der ,Sphärizität“ an die Daten stellt (Rasch et al. 2010, 71f). Darunter versteht man die Homogenität aller Varianzen der Differenzen zweier Faktorstufen, die in der vorliegenden Studie jedoch immer gegeben ist, da ausschließlich mit Faktoren gearbeitet wird, die lediglich zwei Faktorstufen haben (den InnerSubjekt-Faktor der Messwiederholung mit Vor- und Nachtest).

\subsubsection{Die Kovarianzanalyse (ANCOVA)}

Die abhängige Variable, in der vorliegenden Studie das Posttest-Ergebnis, ist aber oftmals nicht ausschließlich von der unabhängigen Variable, hier der Gruppenzugehörigkeit, abhängig, sondern kann auch von anderen Faktoren wie z.B. der kognitiven Fähigkeit, der Motivation oder dem Pretest-Ergebnis beeinflusst werden. Mit Hilfe einer Kovarianzanalyse, oftmals auch als ANCOVA (스alysis of Covariance) bezeichnet, lässt sich der Einfluss solcher intervallskalierten Störvariablen bzw. Kovariaten statistisch kontrollieren. Dabei stellt die Kovari- 
anzanalyse eine Erweiterung der einfachen Varianzanalyse dar, weil diese mit einer linearen Regressionsanalyse verbunden wird. Dies geschieht vor dem Hintergrund, dass bei einer normalen Varianzanalyse die „Varianz innerhalb der Gruppen“, also die nicht durch das Modell erklärte Varianz, infolge des Einflusses der Störvariablen verhältnismäßig groß ausfallen kann (vgl. ANOVA). Damit läge aber auch ein nach unten verzerrter $F$-Wert vor, in dessen Folge der Einfluss der unabhängigen Variablen auf die abhängige Variable statistisch unterschätzt werden würde. Indem nun bei einer Kovarianzanalyse zusätzliche Kovariaten erhoben und in das Modell integriert werden, kann ein Teil dieser bisher unerklärten Varianz innerhalb der Gruppen reduziert werden, womit der Effekt der unabhängigen Variablen auf die abhängige Variable genauer erfasst werden kann. Dieses Ziel wird erreicht, indem zunächst eine Regressionsanalyse mit der Kovariate als Prädiktor für die abhängige Variable durchgeführt wird, wodurch die Varianz der abhängigen Variablen, die auf die Kovariate zurückgeht, entfernt bzw. herauspartialisiert wird. Im Anschluss wird dann eine reguläre Varianzanalyse bzgl. der in der Regressionsanalyse unerklärt gebliebenen Residualvarianz durchgeführt. Da diese in der Varianzanalyse untersuchte Residualvarianz um den Einfluss der Kovariate(n) bereinigt ist, erlaubt eine Kovarianzanalyse eine genauere Bestimmung des Einflusses der unabhängigen Variablen auf die abhängige Variable.

Da eine Kovarianzanalyse auf einer normalen Varianzanalyse aufbaut, gelten für sie grundsätzlich die gleichen Voraussetzungen. Zusätzlich werden an eine Kovarianzanalyse jedoch die folgenden beiden Anforderungen gestellt (Field 2011, 397ff):

1. Es müssen homogene Regressionskoeffizienten vorliegen. Begründung: In den verschiedenen Gruppen müssen die Regressionsgeraden der Kovariaten eine vergleichbare Steigung aufweisen, da sich sonst der Einfluss der Kovariaten auf die abhängige Variable in den verschiedenen Gruppen unterscheiden würde.

2. Die Kovariate muss unabhängig vom Gruppeneffekt sein. Begründung: Besteht eine Abhängigkeit der Kovariate vom Gruppeneffekt, sind Kovariate und Gruppeneffekt bzgl. der abhängigen Variablen konfundiert, d.h. die Kovariate würde nicht nur bisher ungeklärte Residualvarianz aufklären, sondern auch einen Teil der Varianz, die eigentlich auf den Gruppeneffekt zurückgeht.

\subsubsection{Die Mehrebenenanalyse $\mathrm{e}^{12}$}

Die bisher beschriebenen und in der didaktischen Forschung oftmals genutzten statistischen Verfahren des $t$-Tests, der Varianzanalyse und der Kovarianzanalyse gehen davon aus, dass die einzelnen Messungen statistisch gesehen unabhängig voneinander sind. Diese geforderte statistische Unabhängigkeit ist aber bei fachdidaktischer Feldforschung oftmals nicht gegeben, da die Schüler im realen Unterricht gemeinsam in Klassen lernen und sich somit beispielsweise bzgl. ihres Lernerfolges in Folge bestimmter gemeinsamer Einflüsse (wie z.B. dem gemeinsamen Unterricht, dem gleichen Lernklima und anderen sozialen Interaktionen

\footnotetext{
${ }^{12}$ Teile dieses Abschnitts wurden bereits in Burde und Wilhelm (2017b) veröffentlicht.
} 
innerhalb einer Klasse) überzufällig ähnlich sind (Kuhn 2014, 297f). In der Terminologie der Mehrebenenanalyse sind die Schüler in Schulklassen „geschachtelt“. Diese starke Abhängigkeit des erzielten Lernerfolgs von der Klassenzugehörigkeit zeigt sich in der vorliegenden Studie beispielsweise bei der Auswertung des in den verschiedenen Klassen erzielten Lernzuwachses in Abschnitt 7.4.7. Wie in Abb. 68 dargestellt, liegt der Untersuchung also eine hierarchische Datenstruktur zugrunde, bei der die Lernenden (Ebene 1) in Schulklassen (Ebene 2) geschachtelt sind.

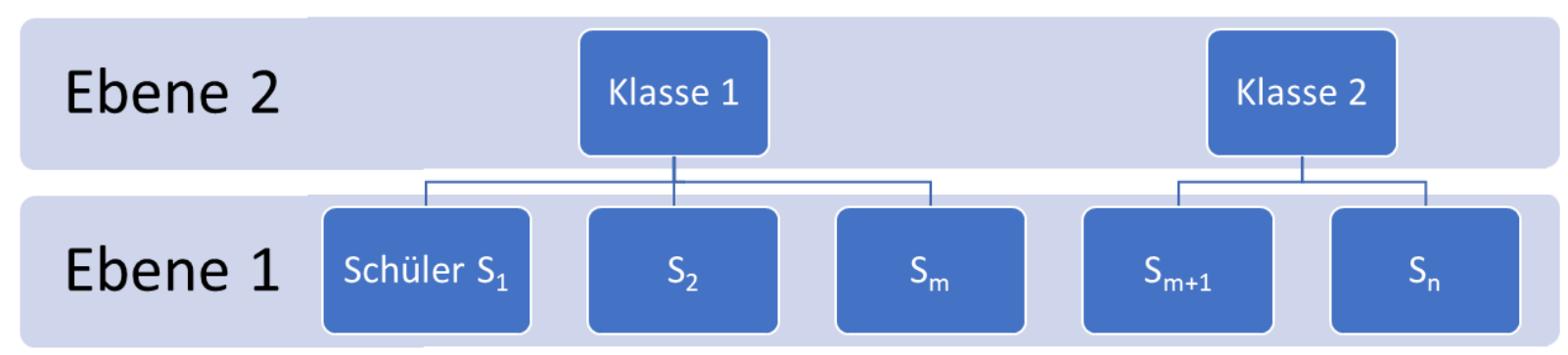

Abb. 68: Schematische Darstellung der Zwei-Ebenen-Struktur des Hierarchisch Linearen Modells (nach Kuhn 2010, S. 100)

Der grundsätzliche Vorteil der Berücksichtigung einer solchen hierarchischen Datenstruktur durch ein Hierarchisch Lineares Modell (im Englischen als MLA für Multi-LevelAnalysis bezeichnet) bzw. eine Mehrebenenanalyse besteht darin, dass zwischen Prädiktoren (d.h. unabhängigen Variablen) auf den einzelnen Ebenen unterschieden werden kann. Während kognitive Fähigkeit, Motivation, Geschlecht oder individuelles Pretest-Ergebnis potenzielle Prädiktoren auf Schülerebene darstellen, können Zugehörigkeit zur Kontroll- oder Treatmentgruppe, Stundenumfang, Schulart oder mittleres Klassen-Pretest-Ergebnis als Prädiktoren auf Klassenebene angesehen werden. Mit Hilfe einer Mehrebenenanalyse ist es im Gegensatz zu einer Kovarianzanalyse (ANCOVA) nicht nur möglich, Interaktionseffekte zwischen den verschiedenen Ebenen nachzuweisen, sondern auch konkret zu untersuchen, welche Merkmale auf höherer Ebene bei welchen Schülern zu einem höheren Lernzuwachs führen. Eine Mehrebenenanalyse stellt also ein anspruchsvolleres statistisches Verfahren dar, das die Klärung komplexer inhaltlicher Fragen erlaubt, die mit konventionellen varianzanalytischen Methoden wie z.B. einer ANCOVA nicht zu beantworten wären (Kuhn 2014, 309f; Kreft und Leeuw 1998, S. 4).

Eine für die statistische Interpretation der Daten bedeutende Konsequenz einer solchen hierarchischen Struktur besteht aber vor allem darin, dass ein zusätzlicher Schüler einer bestimmten Klasse aufgrund seiner Ähnlichkeit zu seinen Mitschülern keinen vollständig neuen Informationsbeitrag von $100 \%$ zur statistischen Schätzung liefert, sondern nur einen verminderten Informationsbeitrag von beispielsweise 80\% (Twisk 2006, S. 33; Kreft und Leeuw 1998, S. 9). Je ähnlicher sich die Schüler innerhalb einer Klasse sind, je höher also die Korrelation zwischen ihnen ist, desto geringer fällt der zusätzliche Informationsbeitrag eines weiteren Schülers innerhalb einer solchen Klasse aus (Twisk 2006, 14f). Um wie viel der Informationsbeitrag vermindert ist, hängt von der sogenannten Intraklassenkorrelation (,Intraclass Correlation") ab. Da der Standardfehler vom Stichprobenumfang abhängt, der effektive 
Stichprobenumfang mit steigender Intraklassenkorrelation in Folge des verminderten Informationsbeitrags aber abnimmt, bedeutet eine höhere Intraklassenkorrelation auch immer eine höhere statistische Unsicherheit bzgl. des gefundenen Effekts. Wird die hierarchische Datenstrukturierung wie bei $t$-Tests oder Varianzanalysen vernachlässigt, besteht deshalb die Gefahr einer Alpha-Fehler-Inflation, in deren Folge gewisse Effekte für statistisch signifikant gehalten werden könnten, obwohl sie es tatsächlich nicht sind (Kreft und Leeuw 1998, 9f). Die adäquate Berücksichtigung der Mehrebenenstruktur der Daten ermöglicht also nicht nur die Berücksichtigung von Prädiktoren auf verschiedenen Ebenen und ggf. die Untersuchung von möglichen Cross-Level-Interaktionen, sondern geht im Gegensatz zu konventionellen Varianz- und Regressionsanalysen insbesondere mit einer genaueren Berechnung der entsprechenden Effekte und einer adäquateren Schätzung ihrer Unsicherheit einher (Twisk 2006, 30ff). Eine detaillierte Beschreibung dieses statistischen Verfahrens und seiner Kennwerte findet sich in Abschnitt 7.5.1 dieser Arbeit.

\subsection{Ergebnisse der empirischen Studie zum Lernerfolg}

\subsubsection{Generelle Aspekte zum Testinstrument}

\subsubsection{Der Bearbeitungsgrad des Tests}

Zunächst soll untersucht werden, wie hoch der Bearbeitungsgrad des Tests in Kontroll- und Treatmentgruppe im Vor- und Nachtest jeweils ist. Um auch mehrstufige, aber nur teilweise bearbeitete Items in der Berechnung angemessen berücksichtigen zu können, wird hierzu der Anteil der bearbeiteten Subitems an der Gesamtzahl der Subitems $(N=45)$ betrachtet. Damit ergeben sich die in Abb. 69 für die Pre- und Posttests dargestellten Werte.

\section{Bearbeitungsgrad des Tests}

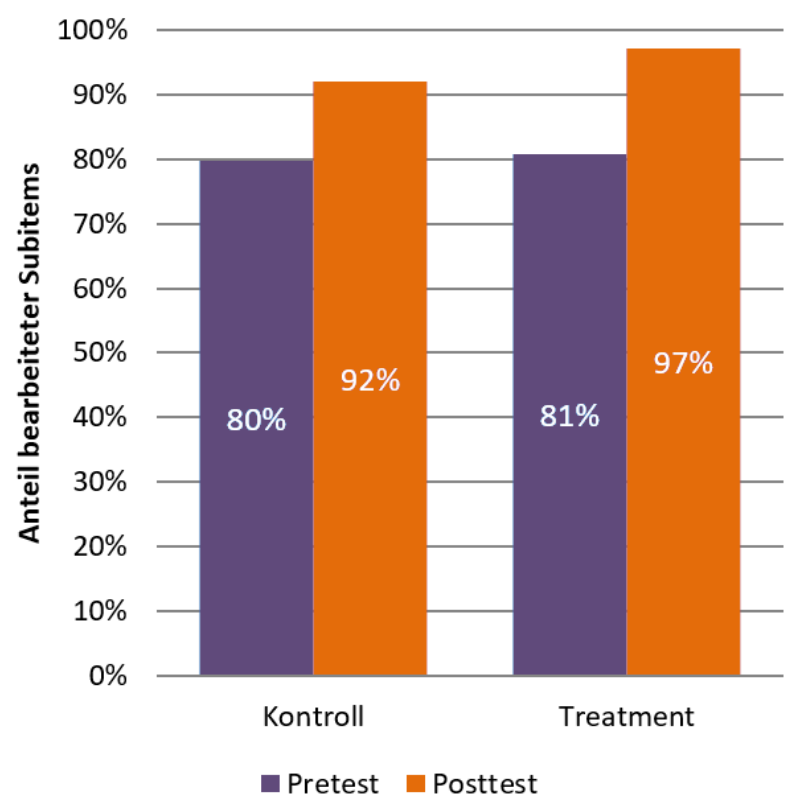

Abb. 69: Dargestellt ist der Anteil der in Kontroll- und Treatmentgruppe in Pre- und Posttest bearbeiteten Subitems. 
Ein zweiseitiger $t$-Test für unabhängige Stichproben zeigt, dass sich die beiden Gruppen bzgl. des Anteils der bearbeiteten Subitems im Pretest nicht unterscheiden $(t(88)=-0.33$, $p=.743$ ), wohingegen die Treatmentgruppe mit 97\% einen signifikant höheren Bearbeitungsanteil im Posttest aufweist als die Kontrollgruppe mit 92\% $(t(53,58)=-4.30, p<.001)$. In beiden Gruppen liegt zudem der Bearbeitungsgrad im Posttest signifikant höher als im Pretest.

Eine genauere Analyse des Bearbeitungsanteils der einzelnen Items in Abb. 70 zeigt, dass in der Kontrollgruppe insbesondere Item 16, bei dem die Stromstärke in einer Parallelschaltung bestimmt werden musste, sowie die selbstentwickelten Items zur elektrischen Spannung (Item 31a, 31b, 32a und 32b) im Posttest seltener bearbeitet wurden als in der Treatmentgruppe. Bei allen Items, die in der Kontrollgruppe deutlich seltener bearbeitet wurden als in der Treatmentgruppe, handelt es sich um Items, bei denen die Lernenden selber Zahlenwerte für die Stromstärke oder die elektrische Spannung eintragen mussten, d.h. keine MultipleChoice-Items. Wie an Hand der gelben Markierung von mehrstufigen Items in Abb. $70 \mathrm{zu}$ erkennen ist, besteht aber kein grundsätzlicher Unterschied im Bearbeitungsgrad zwischen ein- und mehrstufigen Items.

\section{Bearbeitungsanteil der einzelnen Items im Posttest}

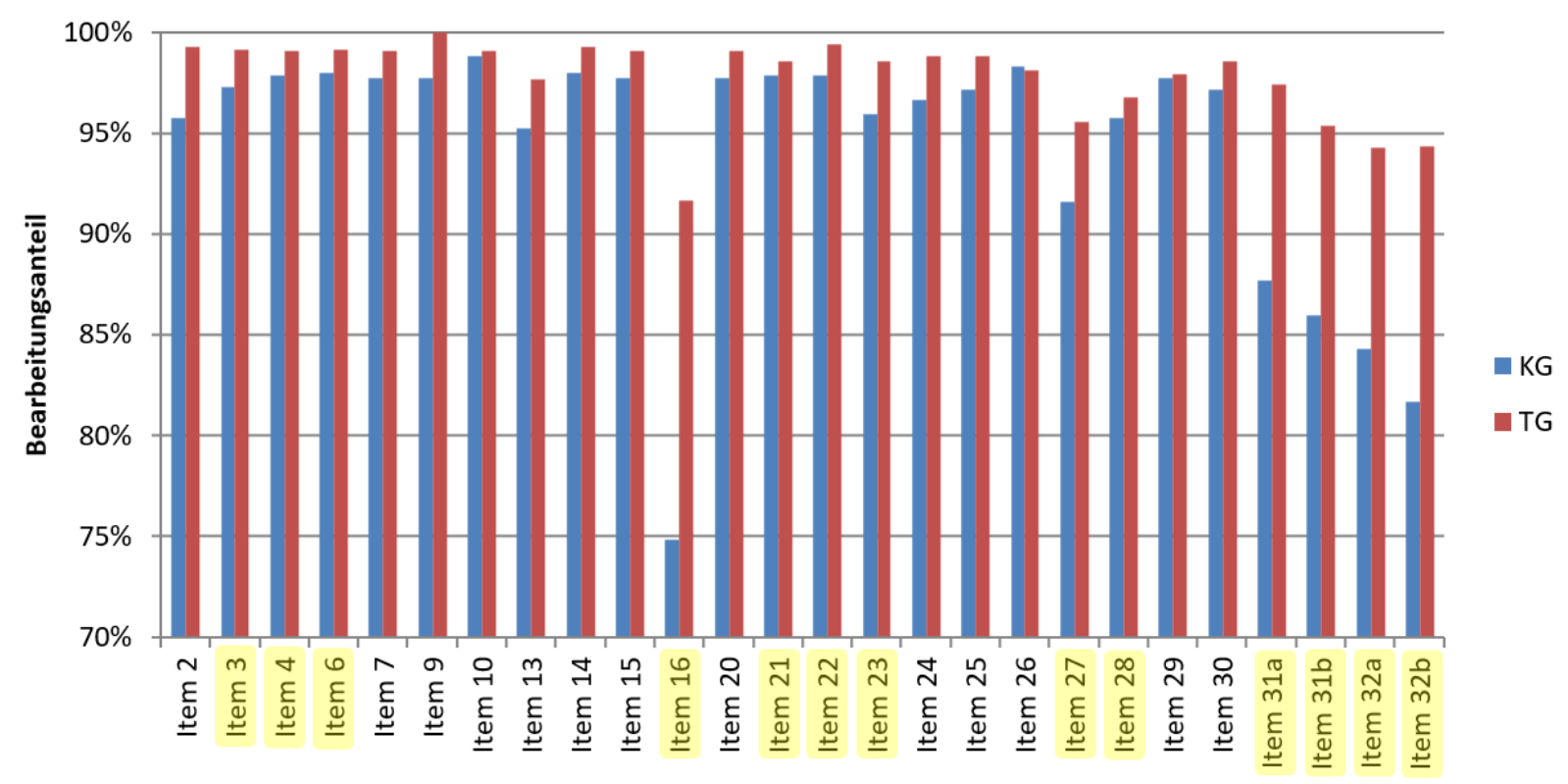

Abb. 70: Vergleich des Bearbeitungsgrads der einzelnen Items im Posttest in Kontroll- und Treatmentgruppe (mehrstufige Items sind gelb hinterlegt)

Die Berechnung des Bearbeitungsgrads eines mehrstufigen Items fand hier über die Mittelung des Bearbeitungsgrads von dessen Subitems statt. Ist ein zweistufiges Item beispielsweise auf der ersten Stufe (Antwort) aber nicht auf der zweiten Stufe (Begründung) bearbeitet worden, würde dieses Item als insgesamt zu 50\% bearbeitet kodiert werden.

\subsubsection{Anteile korrekt gelöster Items im Vortest}

Wie bereits in Abschnitt 7.3.3 erklärt, wurden alle Items dichotom kodiert $(0=$ inkorrekt; 1 = korrekt). Mehrstufige Items wurden nur dann als korrekt kodiert, wenn sie auf allen Stu- 
fen korrekt beantwortet wurden (z.B. korrekte Antwort und korrekte Begründung) bzw. wenn alle gefragten Stromstärke- bzw. Spannungswerte korrekt angegeben wurden. Betrachtet man den Anteil korrekt gelöster Items im Vortest, so zeigen sich große Unterschiede zwischen den einzelnen Items, wobei es nicht so ist, dass mehrstufige Items grundsätzlich seltener korrekt gelöst wurden als einstufige Items. Allerdings wird aus Abb. 71 deutlich, dass insbesondere die Items zur Spannung von nahezu keinem Lernenden im Vortest korrekt gelöst wurden. Vor dem Hintergrund, dass diese Items das eigenständige Einsetzen von Spannungswerten verlangen und die Lernenden das Thema Elektrizitätslehre zum Testzeitpunkt noch nicht behandelt hatten, ist dieser Befund aber wenig überraschend.

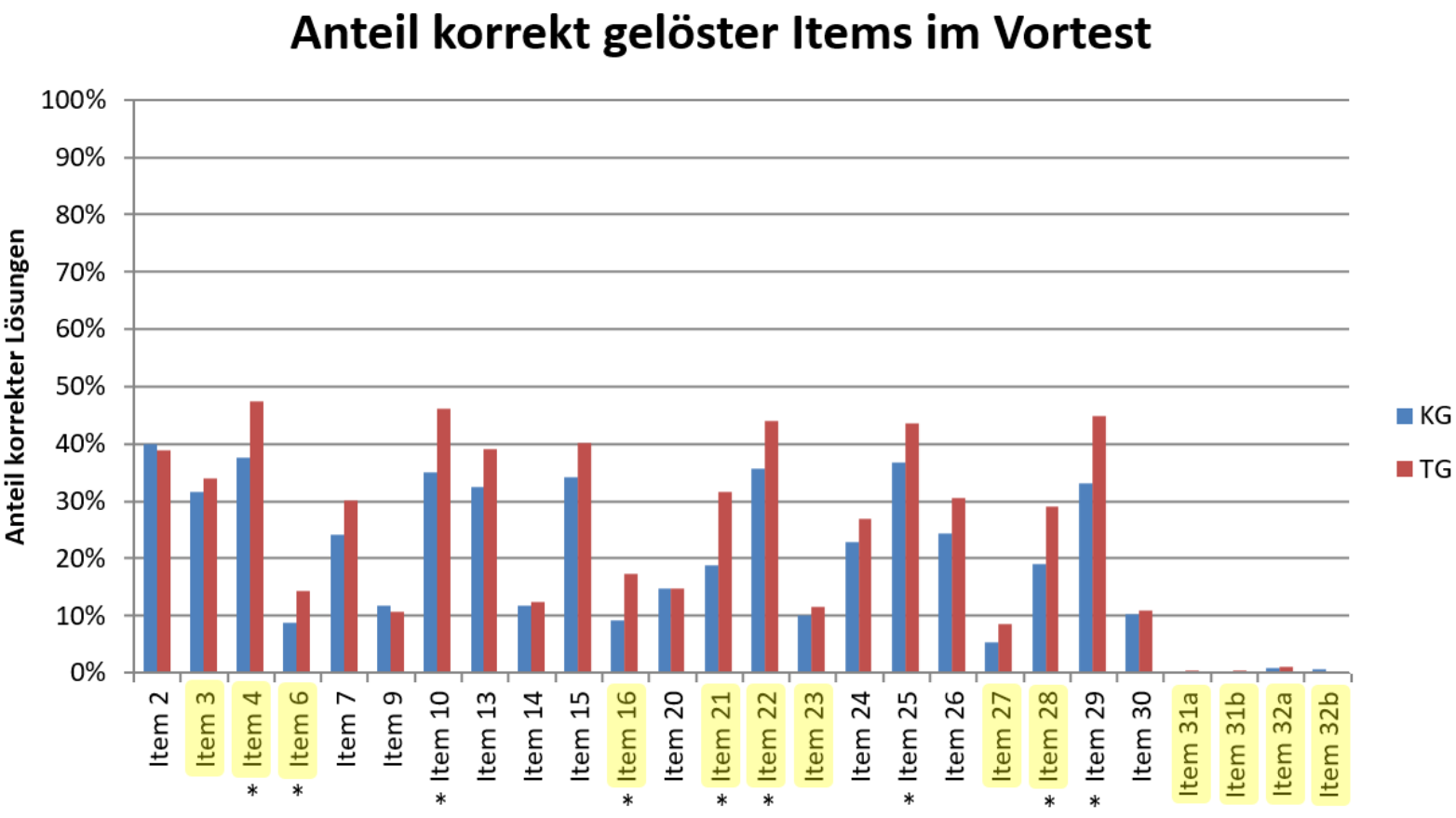

Abb. 71: Vergleich des Anteils korrekt gelöster Items im Vortest zwischen Kontroll- und Treatmentgruppe; mehrstufige Items sind gelb hinterlegt; signifikante Unterschiede $(p<.05)$ sind mit einem Stern gekennzeichnet

Mit Hilfe eines Chi-Quadrat-Tests wurde ferner untersucht, ob sich der Anteil korrekt gelöster Items im Vortest zwischen Kontroll- und Treatmentgruppe signifikant voneinander unterscheidet. Ist dies der Fall, so ist das entsprechende Item in Abb. 71 mit einem Stern gekennzeichnet. Wie man dieser Abbildung entnehmen kann, sind die Unterschiede zwischen den beiden Gruppen bei einem Großteil der Items statistisch gesehen nicht signifikant. Nichtsdestotrotz weist die Treatmentgruppe bei einem Drittel der 26 Items bereits einen signifikant höheren Lösungsanteil im Vortest auf als die Kontrollgruppe.

Die konkrete Itemschwierigkeit, die bei dichotom kodierten Items dem relativen Anteil der Probanden entspricht, die das jeweilige Item korrekt beantworten konnten, ist für den Vortest in Tab. 19 differenziert für die Kontroll- und Treatmentgruppe aufgeführt. Zusätzlich sind auch die Unterschiede bzgl. des Anteils korrekt gelöster Items zwischen den beiden Gruppen sowie deren statistische Signifikanz angegeben. 
Tab. 19: Vergleich des Anteils korrekt gelöster Items im Vortest zwischen Kontroll- und Treatmentgruppe (Lösungsanteile unter 20\% sind farblich sowie Signifikanzwerte unter 5\% fett hervorgehoben.)

\begin{tabular}{|c|c|c|c|c|}
\hline & KG & TG & Unterschied & Signifikanz \\
\hline Item 2 & $40 \%$ & $39 \%$ & $1 \%$ & $\mathrm{p}=.719$ \\
\hline Item 3 & $32 \%$ & $34 \%$ & $2 \%$ & $\mathrm{p}=.494$ \\
\hline Item 4 & $38 \%$ & $47 \%$ & $10 \%$ & $p=.006$ \\
\hline Item 6 & $9 \%$ & $14 \%$ & $6 \%$ & $p=.014$ \\
\hline Item 7 & $24 \%$ & $30 \%$ & $6 \%$ & $\mathrm{p}=.063$ \\
\hline Item 9 & $12 \%$ & $11 \%$ & $1 \%$ & $p=.612$ \\
\hline Item 10 & $35 \%$ & $46 \%$ & $11 \%$ & $p=.001$ \\
\hline Item 13 & $32 \%$ & $39 \%$ & $7 \%$ & $\mathrm{p}=.057$ \\
\hline Item 14 & $12 \%$ & $12 \%$ & $1 \%$ & $\mathrm{p}=.762$ \\
\hline Item 15 & $34 \%$ & $40 \%$ & $6 \%$ & $\mathrm{p}=.082$ \\
\hline Item 16 & $9 \%$ & $17 \%$ & $8 \%$ & $p=.001$ \\
\hline Item 20 & $15 \%$ & $15 \%$ & $0 \%$ & $\mathrm{p}=.979$ \\
\hline Item 21 & $19 \%$ & $32 \%$ & $13 \%$ & $\mathrm{p}<.001$ \\
\hline Item 22 & $36 \%$ & $44 \%$ & $9 \%$ & $p=.015$ \\
\hline Item 23 & $10 \%$ & $12 \%$ & $1 \%$ & $\mathrm{p}=.511$ \\
\hline Item 24 & $23 \%$ & $27 \%$ & $4 \%$ & $\mathrm{p}=.192$ \\
\hline Item 25 & $37 \%$ & $44 \%$ & $7 \%$ & $p=.048$ \\
\hline Item 26 & $24 \%$ & $30 \%$ & $6 \%$ & $\mathrm{p}=.056$ \\
\hline Item 27 & $5 \%$ & $9 \%$ & $3 \%$ & $\mathrm{p}=.079$ \\
\hline Item 28 & $19 \%$ & $29 \%$ & $10 \%$ & $p=.001$ \\
\hline Item 29 & $33 \%$ & $45 \%$ & $12 \%$ & $p=.001$ \\
\hline Item 30 & $10 \%$ & $11 \%$ & $0 \%$ & $p=.824$ \\
\hline Item 31a & $0 \%$ & $0 \%$ & $0 \%$ & $p=.199$ \\
\hline Item $31 \mathrm{~b}$ & $0 \%$ & $0 \%$ & $0 \%$ & $\mathrm{p}=.199$ \\
\hline Item $32 \mathrm{a}$ & $1 \%$ & $1 \%$ & $0 \%$ & $p=.660$ \\
\hline Item $32 b$ & $1 \%$ & $0 \%$ & $0 \%$ & $\mathrm{p}=.454$ \\
\hline Mittelwert & $20 \%$ & $24 \%$ & $5 \%$ & \\
\hline
\end{tabular}

Da zum Zeitpunkt der Testdurchführung das Thema Elektrizitätslehre im Unterricht noch nicht behandelt wurde und deshalb davon auszugehen ist, dass der Großteil der Schüler hier über kein signifikantes Vorwissen verfügte, lag der Anteil der korrekt beantworteten Items im Vortest erwartungsgemäß bei insgesamt lediglich $20 \%$ in der Kontrollgruppe und $24 \%$ in der Treatmentgruppe (vgl. Tab. 19). Damit wäre der Vortest aufgrund der Bodeneffekte bei vielen Items (vgl. farblich hinterlegte Zellen in Tab. 19) insgesamt zu schwer gewesen, um das Vorwissen der Lernenden differenziert erheben zu können (Bortz und Döring 2006, S. 182). Vor dem Hintergrund, dass der Vortest in der vorliegenden Studie aber nicht zu diesem Zweck eingesetzt wurde, sondern, um das bei einzelnen Schülern z.B. in Folge bestimmter Hobbys oder einer Klassenwiederholung möglicherweise in signifikantem Umfang vorhandene Vorwissen in dem Bereich kontrollieren zu können, indem lediglich der durch den Unterricht erzielte Lernfortschritt betrachtet wird, sind die hier ermittelten niedrigen Lösungsanteile im Vortest unproblematisch. 


\subsubsection{Anteile korrekt gelöster Items im Nachtest}

Betrachtet man den Anteil der korrekt gelösten Items im Nachtest, so zeigen sich nicht nur große Unterschiede zwischen den einzelnen Items, sondern auch zwischen der Kontroll- und der Treatmentgruppe insgesamt (siehe Abb. 72). Ein Chi-Quadrat-Test ergibt, dass die Unterschiede zwischen den beiden Gruppen bzgl. des Anteils korrekter Lösungen im Nachtest mit Ausnahme von Item 21 bei allen Items statistisch signifikant sind. Während die Schüler der Treatmentgruppe also bei nahezu allen Items einen höheren Anteil korrekter Lösungen aufweisen als Schüler der Kontrollgruppe, ist der Vorsprung der Treatmentgruppe bei den selbstentwickelten Spannungsitems besonders eklatant. Keines dieser offenen Items, bei denen die korrekten Spannungswerte selbstständig eingetragen werden mussten, wurde von mehr als $10 \%$ der Schüler der Kontrollgruppe richtig gelöst. In der Treatmentgruppe lag der Anteil der Schüler mit korrekter Lösung hingegen bei allen Spannungsitems etwa um einen Faktor drei höher. Wie Abb. 72 zu entnehmen ist, lässt sich aber auch hier nicht grundsätzlich sagen, dass zweistufige Items seltener korrekt gelöst wurden als einstufige Items.

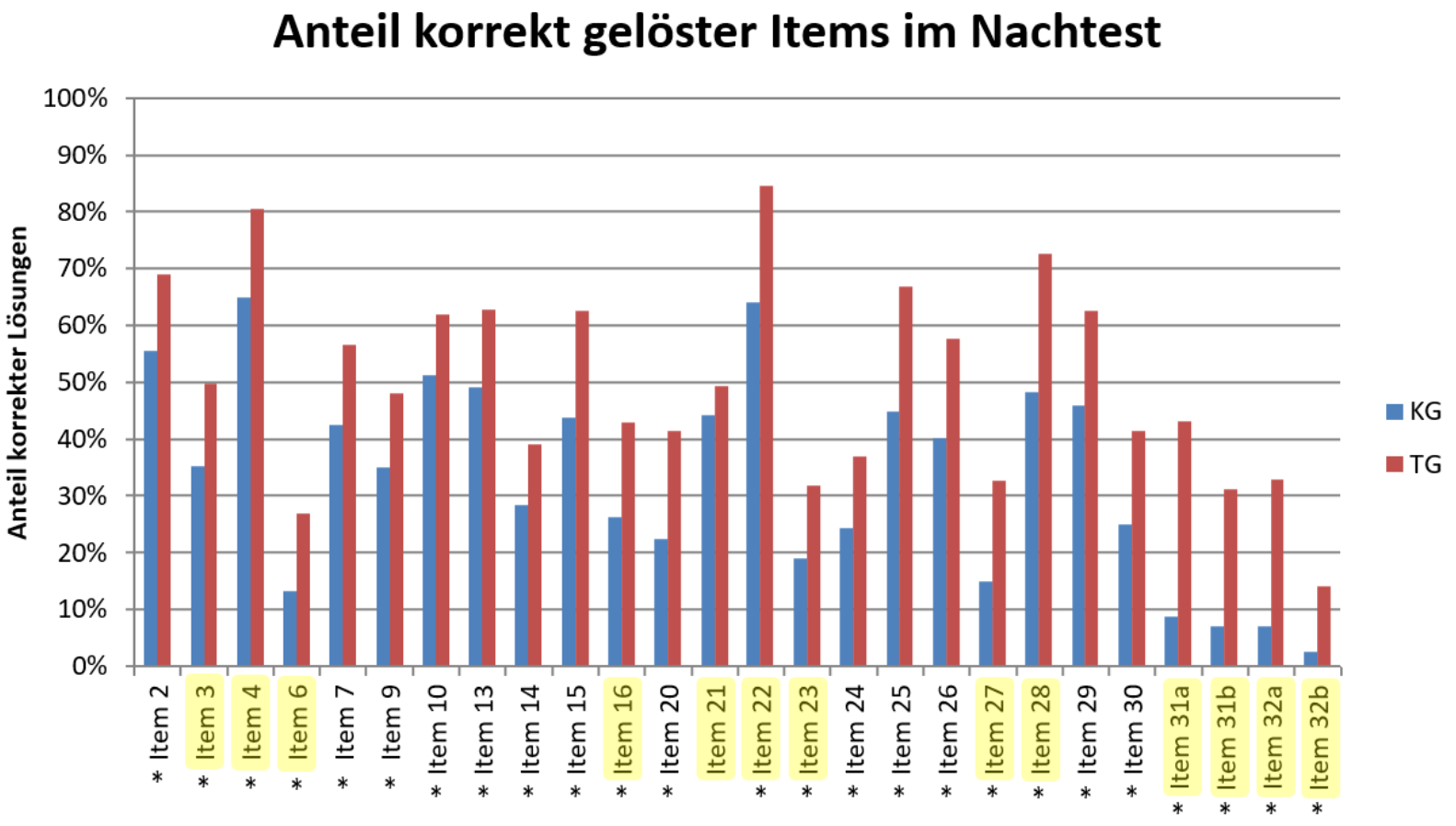

Abb. 72: Vergleich des Anteils korrekt gelöster Items im Nachtest zwischen Kontroll- und Treatmentgruppe; mehrstufige Items sind gelb hinterlegt; signifikante Unterschiede $(p<.05)$ sind mit einem Stern gekennzeichnet

Differenziert für Kontroll- und Treatmentgruppe finden sich in Tab. 20 für den Nachtest die genauen Itemschwierigkeiten, die hier dem relativen Anteil der Lernenden entsprechen, die das jeweilige Item korrekt beantworten konnten. Zusätzlich sind auch hier für jedes Item der Unterschied bzgl. des Anteils korrekter Lösungen zwischen den beiden Gruppen sowie der entsprechende Signifikanzwert angegeben, der mit Hilfe eines Chi-Quadrat-Tests ermittelt wurde. 
Tab. 20: Vergleich des Anteils korrekt gelöster Items im Nachtest zwischen Kontroll- und Treatmentgruppe (Lösungsanteile unter 20\% sind farblich sowie Signifikanzwerte unter 5\% fett hervorgehoben.)

\begin{tabular}{|c|c|c|c|c|}
\hline & KG & TG & Unterschied & Signifikanz \\
\hline Item 2 & $55 \%$ & $69 \%$ & $14 \%$ & $p<.001$ \\
\hline Item 3 & $35 \%$ & $50 \%$ & $14 \%$ & $\mathrm{p}<.001$ \\
\hline Item 4 & $65 \%$ & $81 \%$ & $16 \%$ & $\mathrm{p}<.001$ \\
\hline Item 6 & $13 \%$ & $27 \%$ & $14 \%$ & $p<.001$ \\
\hline Item 7 & $43 \%$ & $57 \%$ & $14 \%$ & $p<.001$ \\
\hline Item 9 & $35 \%$ & $48 \%$ & $13 \%$ & $p<.001$ \\
\hline Item 10 & $51 \%$ & $62 \%$ & $11 \%$ & $p=.003$ \\
\hline Item 13 & $49 \%$ & $63 \%$ & $14 \%$ & $p<.001$ \\
\hline Item 14 & $28 \%$ & $39 \%$ & $11 \%$ & $\mathrm{p}=.002$ \\
\hline Item 15 & $44 \%$ & $63 \%$ & $19 \%$ & $p<.001$ \\
\hline Item 16 & $26 \%$ & $43 \%$ & $17 \%$ & $p<.001$ \\
\hline Item 20 & $22 \%$ & $41 \%$ & $19 \%$ & $\mathrm{p}<.001$ \\
\hline Item 21 & $44 \%$ & $49 \%$ & $5 \%$ & $\mathrm{p}=.148$ \\
\hline Item 22 & $64 \%$ & $85 \%$ & $20 \%$ & $p<.001$ \\
\hline Item 23 & $19 \%$ & $32 \%$ & $13 \%$ & $\mathrm{p}<.001$ \\
\hline Item 24 & $24 \%$ & $37 \%$ & $13 \%$ & $\mathrm{p}<.001$ \\
\hline Item 25 & $45 \%$ & $67 \%$ & $22 \%$ & $p<.001$ \\
\hline Item 26 & $40 \%$ & $58 \%$ & $18 \%$ & $\mathrm{p}<.001$ \\
\hline Item 27 & $15 \%$ & $33 \%$ & $18 \%$ & $\mathrm{p}<.001$ \\
\hline Item 28 & $48 \%$ & $73 \%$ & $24 \%$ & $p<.001$ \\
\hline Item 29 & $46 \%$ & $63 \%$ & $17 \%$ & $\mathrm{p}<.001$ \\
\hline Item 30 & $25 \%$ & $41 \%$ & $16 \%$ & $\mathrm{p}<.001$ \\
\hline Item $31 \mathrm{a}$ & $9 \%$ & $43 \%$ & $35 \%$ & $\mathrm{p}<.001$ \\
\hline Item $31 \mathrm{~b}$ & $7 \%$ & $31 \%$ & $24 \%$ & $\mathrm{p}<.001$ \\
\hline Item $32 \mathrm{a}$ & $7 \%$ & $33 \%$ & $26 \%$ & $\mathrm{p}<.001$ \\
\hline Item $32 \mathrm{~b}$ & $3 \%$ & $14 \%$ & $12 \%$ & $p<.001$ \\
\hline Mittelwert & $33 \%$ & $50 \%$ & $17 \%$ & \\
\hline
\end{tabular}

Insgesamt lag der Anteil der korrekt beantworteten Items im Nachtest in der Kontrollgruppe bei $33 \%$ und in der Treatmentgruppe bei $50 \%$ (vgl. Tab. 20), weshalb davon ausgegangen werden kann, dass eine angemessene Itemschwierigkeit vorliegt und der Test in seiner Gesamtheit die Leistungsfähigkeit der Probanden im Nachtest gut erfassen kann. Nichtsdestotrotz scheinen insbesondere die selbstentwickelten Spannungsitems einen zu hohen Schwierigkeitsgrad zu besitzen, um Leistungsunterschiede im unteren Bereich angemessen unterscheiden zu können. Während also keine Hinweise auf Deckeneffekte vorliegen, können Bodeneffekte insbesondere bei den Spannungsitems nicht ausgeschlossen werden (Bortz und Döring 2006, S. 182).

\subsubsection{Einfluss der Zweistufigkeit auf die Itemschwierigkeit}

Abschließend soll an Hand der rein zweistufigen Multiple-Choice-Items 3, 4, 6, 21, 22, 23 und 28 untersucht werden, wie sich die Zweistufigkeit auf die Itemschwierigkeit auswirkt und ob aus einer korrekten Antwort auf der ersten Stufe darauf geschlossen werden kann, dass die 
Lernenden auch die dahinterstehenden Konzepte verstehen. Andere mehrstufige Items wie z.B. Item 16, 27, 31a, 31b, 32a und 32b stellen keine Multiple-Choice-Items dar bzw. weisen nicht ein rein zweistufiges Format von Antwort und Begründung auf, weshalb sie hier nicht näher betrachtet werden.

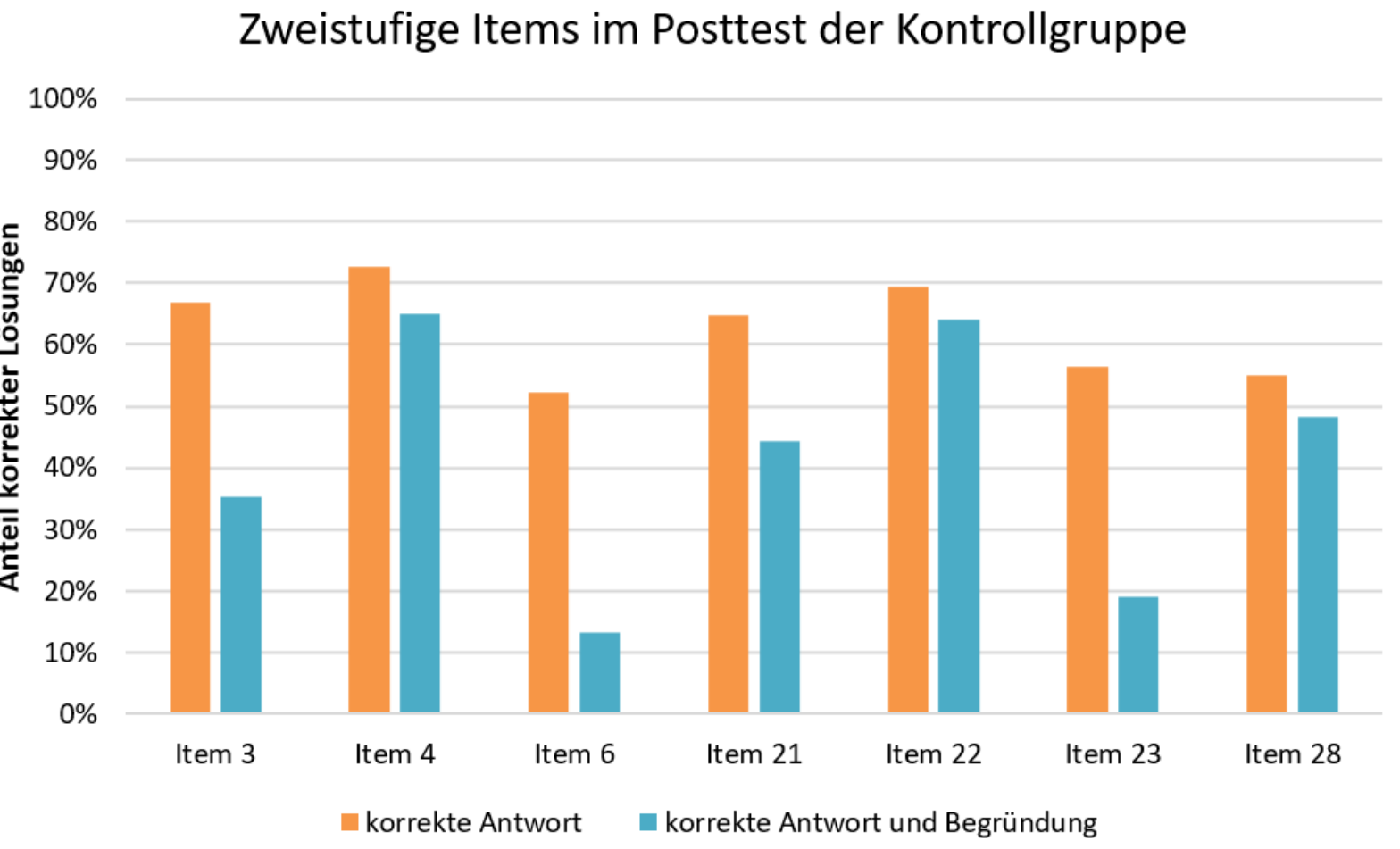

Abb. 73: Abhängigkeit der Itemschwierigkeit von der Zweistufigkeit der Items im Posttest der Kontrollgruppe

Wie anhand von Abb. 73 in Bezug auf das Posttest-Ergebnis der Kontrollgruppe zu erkennen ist, liegt insbesondere bei Item 3, Item 6 und Item 23 (jeweils zum Einfluss eines höheren Widerstands auf die Stromstärke) sowie Item 21 (zur Stromverbrauchsvorstellung) ein verhältnismäßig großer Unterschied zwischen dem relativen Anteil der Schüler vor, die eine korrekte Antwort geben können, und denen, die zusätzlich ihre richtige Antwort auch korrekt begründen können. Bei diesen Items hängt die Itemschwierigkeit also stark von der Zweistufigkeit ab, was darauf hindeutet, dass vielen dieser korrekten Antworten fachlich gesehen falsche Schülervorstellungen zugrunde liegen. Hier zeigt sich, dass ein zweistufiger Test, wie er in der vorliegenden Untersuchung eingesetzt wird, das Verständnis der Lernenden dank des Ausschlusses von falsch-positiven Antworten (d.h. richtigen Antworten mit falscher Begründung) deutlich besser erheben kann, als es mit einem rein einstufigen Test möglich wäre.

Wie aus Abb. $74 \mathrm{zu}$ entnehmen ist, ergibt sich in der Treatmentgruppe ein ähnliches Bild, wobei sowohl der Anteil der korrekten Antworten als auch der Anteil der korrekten Antworten mit korrekter Begründung bei allen Items tendenziell höher ausfällt als in der Kontrollgruppe. Dies ist insbesondere bei Item 6 der Fall, das sich mit dem Einfluss von ein bzw. zwei in Reihe geschalteten Motoren auf die Stromstärke im Stromkreis befasst. Während in der Kontrollgruppe nur etwa ein Viertel derer, die dieses Item richtig lösten, auch eine richti- 
ge Begründung geben konnten, sind es in der Treatmentgruppe immerhin 40\%. Über alle sieben hier diskutierten zweistufigen Items gemittelt, geben in der Kontrollgruppe ca. $41 \%$ der Lernenden sowohl eine korrekte Antwort als auch eine korrekte Begründung, während es in der Treatmentgruppe 56\% sind.

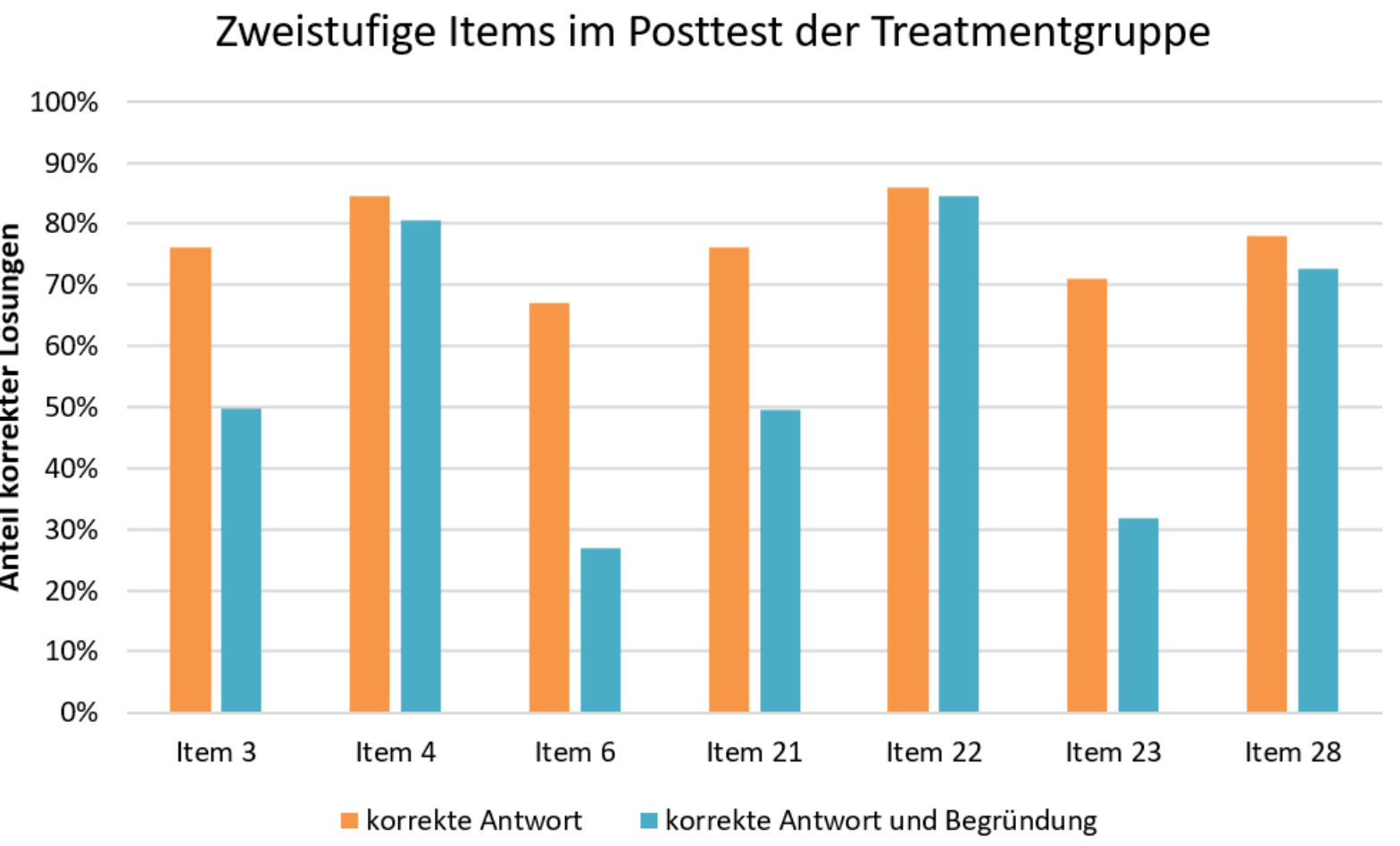

Abb. 74: Abhängigkeit der Itemschwierigkeit von der Zweistufigkeit der Items im Posttest der Treatmentgruppe

\subsubsection{Reliabilitätsanalyse des gesamten Testinstruments}

Um die interne Konsistenz des eingesetzten Testinstruments zu bestimmen, wurde Cronbachs Alpha auf Basis der Posttest-Daten der Treatmentgruppe für den gesamten Test berechnet. Dieser verfügt mit einem Cronbachs Alpha von $\alpha=.85$ über eine gute interne Konsistenz (Bortz und Döring 2006, S. 725), die trotz der hinzugefügten vier Spannungsitems mit der Reliabilität $\alpha=.84$ des ursprünglichen Testinstruments von Urban-Woldron und Hopf (2012, S. 215) vergleichbar ist. Zudem wurde für die verschiedenen Items des Tests die Trennschärfe berechnet, die ein Maß dafür ist, wie stark jedes Item mit allen anderen Items des Tests korreliert. Die Trennschärfe gibt also an, wie gut ein einzelnes Item das Gesamtergebnis des Testinstruments vorhersagt und stellt deshalb ein Gütekriterium für einzelne Items dar. In der Literatur werden Trennschärfekoeffizienten ab .30 als ausreichend beurteilt, weshalb Items mit darunterliegenden Werten in Tab. 21 farblich hervorgehoben sind (Bortz und Döring 2006, 219f). 
Kapitel 7: Summative Evaluation des Unterrichtskonzepts

Tab. 21: Übersicht über die Trennschärfe der einzelnen Items des Testinstruments. Trennschärfen unter .30 sind farblich hervorgehoben.

\begin{tabular}{|l|r|}
\hline & Trennschärfe \\
\hline Item 2 & .40 \\
\hline Item 3 & .33 \\
\hline Item 4 & .30 \\
\hline Item 6 & .35 \\
\hline Item 7 & .22 \\
\hline Item 9 & .46 \\
\hline Item 10 & .31 \\
\hline Item 13 & .44 \\
\hline Item 14 & .50 \\
\hline Item 15 & .38 \\
\hline Item 16 & .14 \\
\hline Item 20 & .43 \\
\hline Item 21 & .23 \\
\hline Item 22 & .39 \\
\hline Item 23 & .39 \\
\hline Item 24 & .20 \\
\hline Item 25 & .36 \\
\hline Item 26 & .41 \\
\hline Item 27 & .55 \\
\hline Item 28 & .41 \\
\hline Item 29 & .42 \\
\hline Item 30 & .46 \\
\hline Item 31a & .54 \\
\hline Item 31b & .56 \\
\hline Item 32a & .53 \\
\hline Item 32b & .40 \\
\hline
\end{tabular}

Aus Sicht der Trennschärfenanalyse erweisen sich Item 7, 16, 21 und 24 als problematische Items, wobei Item 7 nach der Stromstärke in einer vergleichsweise schwierigen gemischten Schaltung mit Schalter fragt und Item 16 das einzige Item des ursprünglichen Tests ist, bei dem die Zahlenwerte für die Stromstärke in einer Parallelschaltung eigenständig eingetragen werden müssen. Bei Item 21 und 24 wird nach der Helligkeit von Glühlämpchen in verschiedenen Stromkreisen gefragt.

\subsubsection{Reliabilitätsanalyse der Subskalen}

In einem nächsten Schritt wurde Cronbachs Alpha zusätzlich für die den verschiedenen Schülervorstellungen zugrundeliegenden Subskalen sowie für die dem ursprünglichen Test hinzugefügten Items zur Spannung berechnet. Es zeigt sich einerseits, dass die hinzugefügten Spannungsitems mit einem Cronbachs Alpha von $\alpha=.84$ über eine gute interne Konsistenz verfügen und somit davon ausgegangen werden kann, dass sie das gleiche Konstrukt messen. Auch die selbst erstellten Kategorien zur elektrischen Spannung „Konzeptionelle Schwierig- 
keiten mit der elektrischen Spannung“ und „Spannung als Eigenschaft des Stroms“ besitzen mit Reliabilitätskoeffizienten von $\alpha=.83$ bzw. $\alpha=.74$ eine vergleichsweise hohe interne Konsistenz. Wie aus Tab. 22 hervorgeht, weisen jedoch alle Subskalen des ursprünglichen Tests von Urban-Woldron und Hopf (2012) mit Ausnahme der Subskala „Erfassen von Parallelschaltungen“" mit einem Cronbachs Alpha von unter $\alpha=.80$ eine verhältnismäßig niedrige Reliabilität auf (Bortz und Döring 2006, S. 199). Insbesondere die Schülervorstellungen „Stromverbrauch ist proportional zum Widerstand“ und „Lokales Denken“ verfügen mit Reliabilitätskoeffizienten von $\alpha=.17$ bzw. $\alpha=.13$ über eine so niedrige interne Konsistenz, dass hier eine klassische Auswertung, bei der aus den verschiedenen Items der jeweiligen Subskala ein Mittelwert berechnet wird, nicht sinnvoll wäre.

Eine Erhöhung dieser Werte von Cronbachs Alpha durch Streichung von unpassenden Variablen (wie z.B. LD1 oder PR3, vgl. Abschnitt 7.3.4) ist keine Option, weil einerseits Testentwicklung und Anwendung des Tests nicht an der gleichen Stichprobe durchgeführt werden dürfen und weil andererseits die Testentwicklung ohnehin nicht Gegenstand dieser Arbeit ist. Insbesondere vor dem Hintergrund der hohen Kontextabhängigkeit von Schülervorstellungen (vgl. Abschnitt 3.1.4) ist es zudem leicht einsichtig, dass der Kontext eines Items einer Subskala einen großen Einfluss auf das Antwortverhalten der Lernenden haben kann. Obwohl die Items einer Subskala aus Expertensicht das gleiche Konstrukt, d.h. die gleiche Schülervorstellung messen, kann sich aufgrund der hohen Kontextabhängigkeit von Schülervorstellungen das Antwortverhalten der Lernenden zwischen den verschiedenen Items einer Subskala stark unterscheiden. Die extrem niedrigen Werte für Cronbachs Alpha der beiden Subskalen „Stromverbrauch ist proportional zum Widerstand“ und „Lokales Denken“" von $\alpha=.17$ bzw. $\alpha=.13$ können also darauf zurückzuführen sein, dass das Auftreten dieser beiden Schülervorstellungen stärker vom jeweiligen Kontext der Items abhängt als bei den anderen Subskalen.

Tab. 22: Übersicht über die Werte von Cronbachs Alpha der verschiedenen Subskalen. Werte unter .50 sind farblich hervorgehoben (vgl. Abschnitt 7.4.8.2).

\begin{tabular}{|l|r|r|}
\hline Subskala & Items $n$ & Cronbachs $\alpha$ \\
\hline Stromverbrauchsvorstellung (SV) & 4 & .57 \\
\hline Batterie als konstante Stromquelle (BS) & 4 & .61 \\
\hline Stromstärke ist unabhängig von R (UR) & 4 & .77 \\
\hline Inverse Widerstandsvorstellung (IR) & 3 & .66 \\
\hline $\begin{array}{l}\text { Stromverbrauch ist proportional zum Wi- } \\
\text { derstand (PR) }\end{array}$ & 3 & .17 \\
\hline Lokales Denken (LD) & 3 & .13 \\
\hline Sequentielle Argumentation (SA) & 4 & .53 \\
\hline Erfassen von Parallelschaltungen (PS) & 4 & .83 \\
\hline Hinzugefügte Items zur Spannung & 4 & .84 \\
\hline $\begin{array}{l}\text { Konzeptionelle Schwierigkeiten mit der } \\
\text { elektrischen Spannung (KS) }\end{array}$ & 4 & .83 \\
\hline Spannung als Eigenschaft des Stroms (UI) & & .74 \\
\hline
\end{tabular}


Vor dem Hintergrund der teilweise niedrigen internen Konsistenz der hier verwendeten Subskalen sei jedoch angemerkt, dass entsprechend des Kodiermanuals von UrbanWoldron und Hopf (2012) eine Schülervorstellung bereits dann für vorhanden angenommen wird, wenn sie bei nur einem der Items der jeweiligen Subskala auftritt (vgl. Abschnitt 7.3.4). Wenn also ein Schüler in einem der drei bzw. vier zur jeweiligen Subskala gehörenden Items die Schülervorstellung vertritt, so wird hier die gesamte Subskala mit dem Wert 1 kodiert und davon ausgegangen, dass dieser Schüler die jeweilige Schülervorstellung besitzt. Bei dieser dichotomen Kodierung der Subskalen, die in der vorliegenden Arbeit Anwendung findet (vgl. Abschnitt 7.4.8.1 und 7.5.4), wird also lediglich untersucht, ob ein Schüler eine bestimmte Schülervorstellung in dem Test überhaupt vertritt und mindestens einmal zeigt und nicht, wie stark diese Schülervorstellung bei ihm ausgeprägt ist. Würde man stattdessen aus den verschiedenen Items der jeweiligen Subskala einen Mittelwert berechnen, so wäre dieser Wert hingegen ein Maß dafür, wie stark eine bestimmte Schülervorstellung von einem Schüler vertreten wird. Während bei dieser Auswertung auf Basis der Mittelwerte der Subskalen ein hohes Cronbachs Alpha, d.h. eine gute interne Konsistenz der jeweiligen Subskala, notwendig ist, um sicherzustellen, dass das gleiche Konstrukt gemessen wird und das Ergebnis, d.h. den Ausprägungsgrad der jeweiligen Schülervorstellung, sinnvoll interpretieren zu können, ist die interne Konsistenz der Subskala nicht in gleichem Maße von Bedeutung, wenn lediglich erhoben wird, ob eine Schülervorstellung grundsätzlich einmal auftaucht oder nicht. Da in dieser Arbeit mit Ausnahme von Abschnitt 7.4.8.2 jedoch ausschließlich letzteres untersucht wird, sind die teils geringen Reliabilitätskoeffizienten der Subskalen als vergleichsweise unproblematisch zu beurteilen.

\subsubsection{Wright-Map des Testinstruments}

Auf Basis der Daten des Posttests von Kontroll- und Treatmentgruppe wurde zudem mit Hilfe des Rasch-Modells jeweils eine Wright-Map des Testinstruments berechnet, um beurteilen zu können, ob das eingesetzte Testinstrument für die jeweils untersuchte Schülergruppe eine angemessene Schwierigkeit besaß. Da bei einer Wright-Map die Personenfähigkeiten und die Itemschwierigkeiten einander gegenübergestellt werden, können darüber hinaus noch Aussagen darüber getroffen werden, welche Items für die Schüler eine zu hohe oder zu niedrige Schwierigkeit besitzen und ob Items ggf. aufgrund des gleichen Schwierigkeitsgrades redundant sind. Eine Wright-Map gliedert sich in zwei Hälften, wobei sich auf der linken Seite die Personen entsprechend ihren Fähigkeiten befinden und auf der rechten Seite die Items entsprechend ihrer Schwierigkeit. Dabei befinden sich in der linken Hälfte Personen mit der höchsten Fähigkeit oben bzw. mit der niedrigsten Fähigkeit unten in der Wright-Map, während sich in der rechten Hälfte die Items mit der höchsten Schwierigkeit oben bzw. mit der niedrigsten Schwierigkeit unten in der Wright-Map befinden. Sind Personen in der WrightMap auf der gleichen Höhe wie ein Item aufgetragen, so bedeutet dies, dass diese Personen dieses Item mit einer Wahrscheinlichkeit von 50\% lösen können. Alle Aufgaben darunter werden von diesen Personen mit einer höheren Wahrscheinlichkeit, alle Aufgaben darüber werden von diesen Personen mit einer geringeren Wahrscheinlichkeit gelöst. 


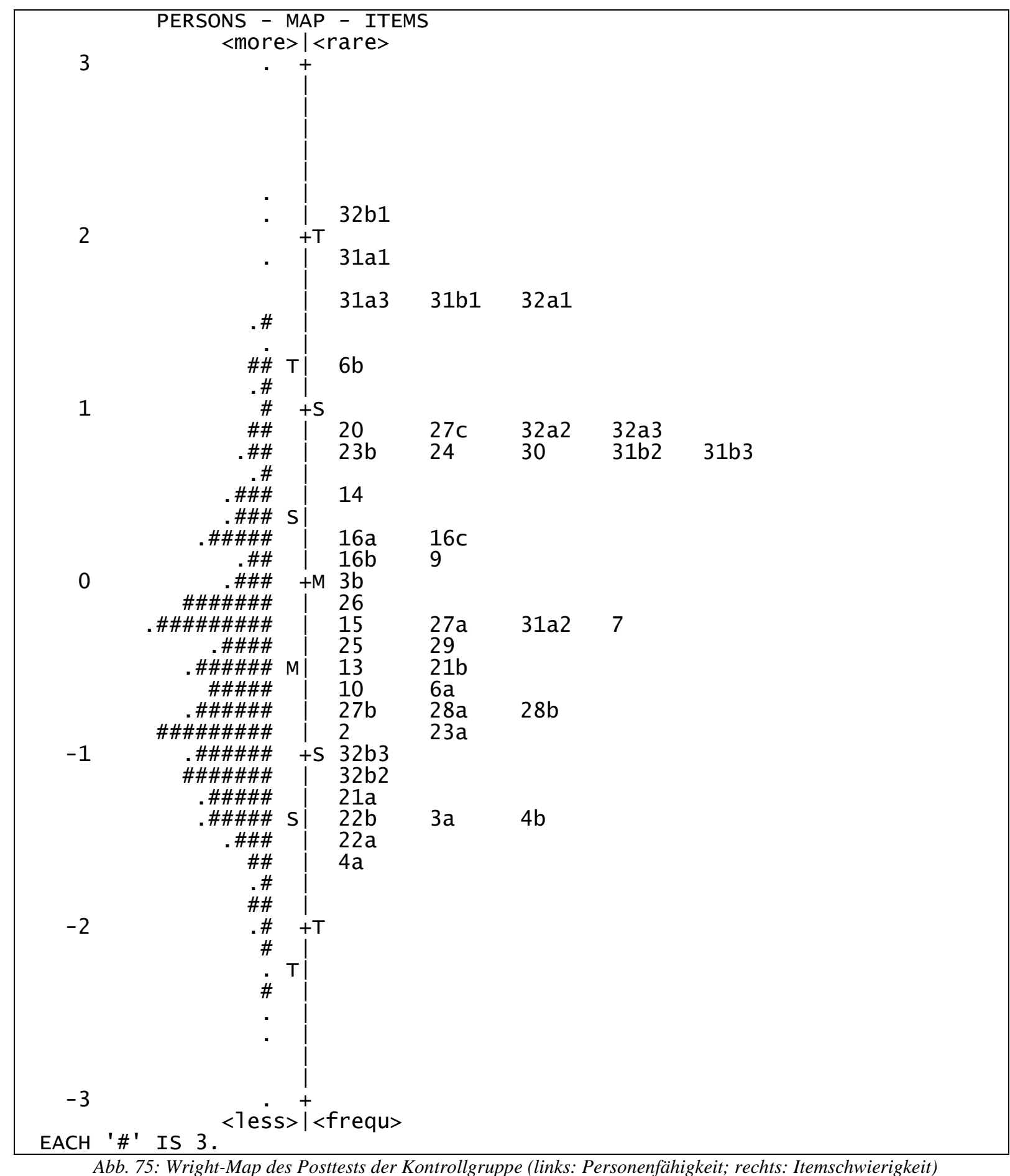

Wie an Hand der in Abb. 75 dargestellten Verteilung der Itemschwierigkeiten zu erkennen ist, besitzt das Testinstrument für die Kontrollgruppe insgesamt einen etwas zu hohen Schwierigkeitsgrad. Insbesondere die Items zur elektrischen Spannung 32b1, 31a1, 31a3, 31b1 und 32a1 weisen für diese Gruppe eine zu hohe Itemschwierigkeit auf. Demgegenüber existieren im Test aber zu wenige leichte Items, um die Leistungsfähigkeit von Schülern mit niedrigen Fähigkeiten möglichst differenziert erheben zu können. Nichtsdestotrotz decken die Items des Tests einen großen Fähigkeitsbereich der Schülerinnen und Schüler ab, weshalb das Testinstrument zur Erhebung der Schülerfähigkeiten in der Kontrollgruppe im Großen und Ganzen geeignet ist. 
Kapitel 7: Summative Evaluation des Unterrichtskonzepts

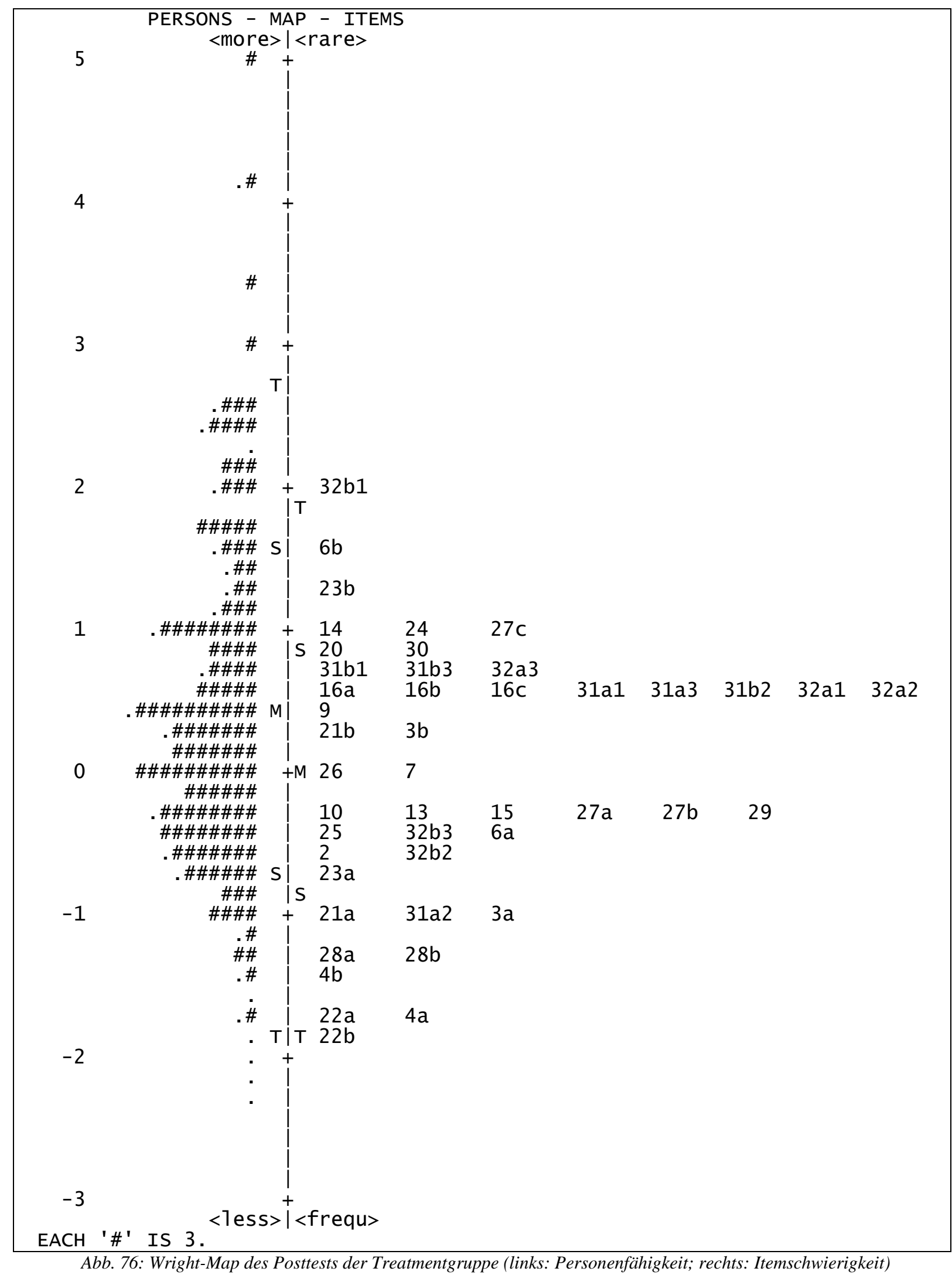

Anhand der in Abb. 76 dargestellten Verteilung der Itemschwierigkeiten in der Wright-Map ist zu erkennen, dass das Testinstrument für die Treatmentgruppe hingegen insgesamt einen etwas zu niedrigen Schwierigkeitsgrad besaß, da keine Items existieren, um die Leistungsfähigkeit der sehr guten Schüler angemessen erheben zu können. Demgegenüber 
existiert eine ausreichende Anzahl an verhältnismäßig leichten Items, um die Leistungsfähigkeit von weniger guten Schülern differenziert messen zu können. Im direkten Vergleich zur Kontrollgruppe zeigt sich auch, dass die Itemschwierigkeit im vorliegenden Fall von der Stichprobe abhängt, da beispielsweise die Spannungsitems in der Treatmentgruppe tendenziell lediglich eine mittlere Itemschwierigkeit aufweisen, in der Kontrollgruppe hingegen tendenziell eine sehr hohe. Nichtsdestotrotz lässt sich aber auch hier feststellen, dass das Testinstrument im Großen und Ganzen angemessen zur Erhebung der Schülerfähigkeiten in der Treatmentgruppe ist.

\subsubsection{Die Pre- und Posttest-Ergebnisse}

\subsubsection{Vergleich der erzielten Gesamtpunktzahlen im Nachtest}

Um die Lernförderlichkeit des neuen Unterrichtskonzepts mit traditionellen Ansätzen vergleichen zu können, werden in einem ersten Schritt zunächst die von den Schülern beider Gruppen erreichten Punktzahlen im Posttest miteinander verglichen. Es geht also um einen ersten Eindruck davon, wie die Lernenden aus beiden Gruppen nach dem Elektrizitätslehreunterricht im verwendeten Test abschneiden. In dem in Abb. 77 dargestellten Histogramm ist deshalb für jede mögliche im Test zu erzielende Punktzahl der Anteil der Schüler der jeweiligen Gruppe aufgetragen, der diese Punktzahl im Posttest erreicht hat. Da der Test aus 26 Items besteht, die jeweils dichotom kodiert wurden, liegt die maximal zu erreichende Punktzahl bei 26 (vgl. Abschnitt 7.3.3).

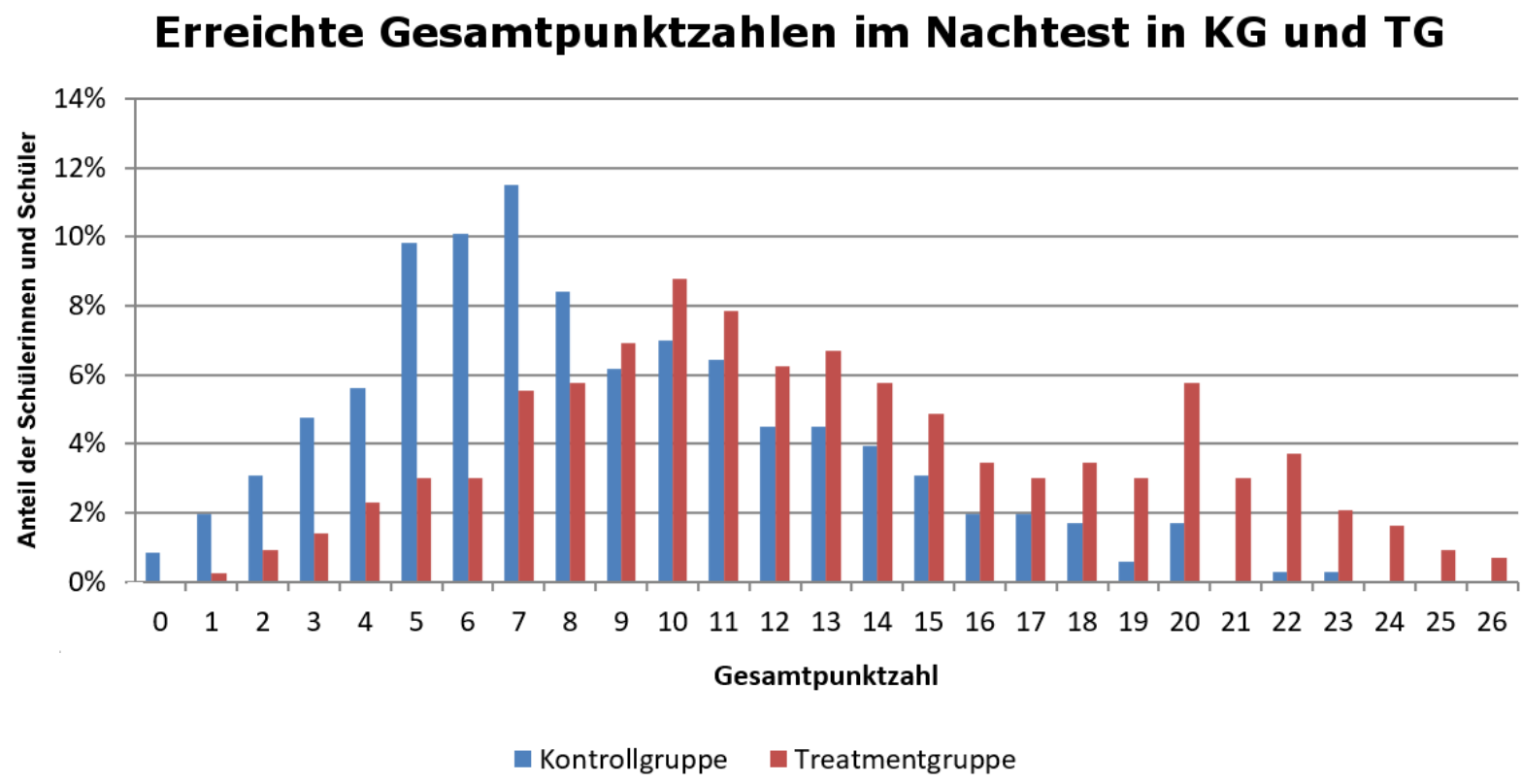

Abb. 77: Erreichte Punktezahlen von Kontroll- und Treatmentgruppe im Posttest

Wie man dem Histogramm entnehmen kann, erzielen die Schüler der Treatmentgruppe tendenziell höhere Gesamtpunktzahlen als die Schüler der Kontrollgruppe. Insbesondere fällt auf, dass ein Summenscore von mehr als 20 Punkten nahezu ausschließlich von Schülern der Treatmentgruppe erzielt wird, wohingegen Schüler der Kontrollgruppe deutlich häufiger als Schüler der Treatmentgruppe einen verhältnismäßig geringen Summenscore von acht oder 
weniger Punkten erreichen. Summenscores von neun und mehr Punkten werden hingegen mehrheitlich von Schülern der Treatmentgruppe erzielt.

Ein Vergleich der Posttest-Ergebnisse mittels Boxplots bestätigt den ersten Eindruck, dass die Schüler der Treatmentgruppe im Test nach dem Elektrizitätslehreunterricht besser abschneiden als die Schüler der Kontrollgruppe. Insbesondere zeigt sich, dass der Median für Schüler der Kontrollgruppe lediglich acht Punkte beträgt, wohingegen er bei Schülern der Treatmentgruppe mit vier Punkten mehr bei zwölf Punkten liegt.

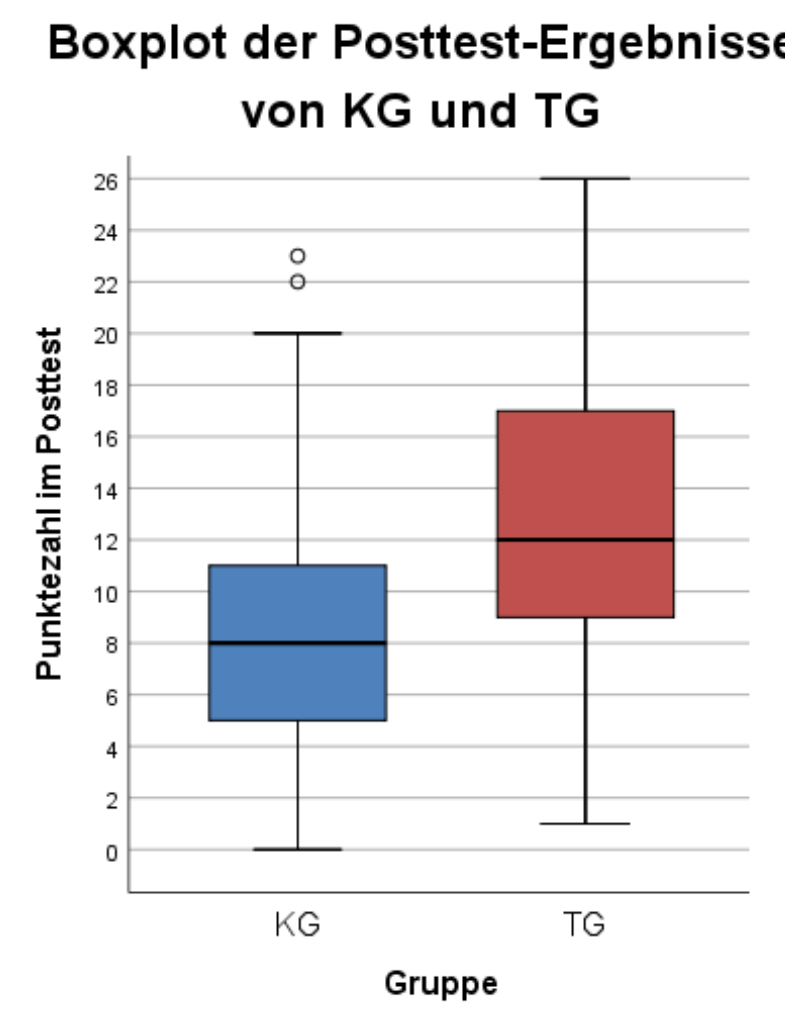

Abb. 78: Boxplot der Posttest-Ergebnisse von Kontroll- und Treatmentgruppe

\subsubsection{Mittelwertsvergleich der Posttests mittels t-Test}

Nun soll der Frage nachgegangen werden, ob die Schüler der Treatmentgruppe im Durchschnitt ein signifikant höheres Posttest-Ergebnis erzielt haben als die Schüler der Kontrollgruppe. Um dies mittels eines einseitigen $t$-Tests für unabhängige Stichproben prüfen zu können, müssen einige statistische Bedingungen erfüllt sein.

Prüfung der Voraussetzungen

1) Intervallskalierung: Die vorliegenden Daten liegen intervallskaliert vor.

2) Normalverteilung: Wie anhand der beiden untenstehenden Histogramme zu sehen ist, kann im vorliegenden Fall trotz einer geringen Schiefe der Verteilung von einer ausreichenden Normalverteilung der Posttest-Ergebnisse in Kontroll- und Treatmentgruppe ausgegangen werden. 


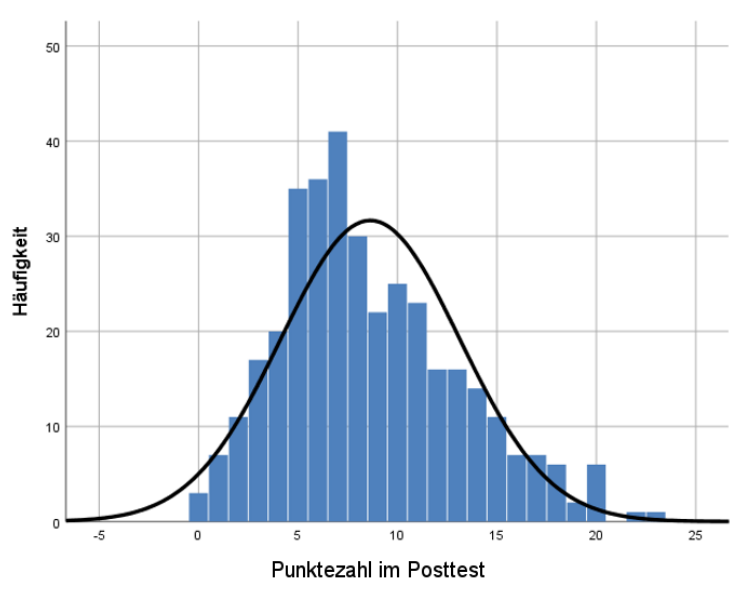

Abb. 79: Histogramm zur Prüfung der Normalverteilung der Posttest-Ergebnisse der Kontrollgruppe

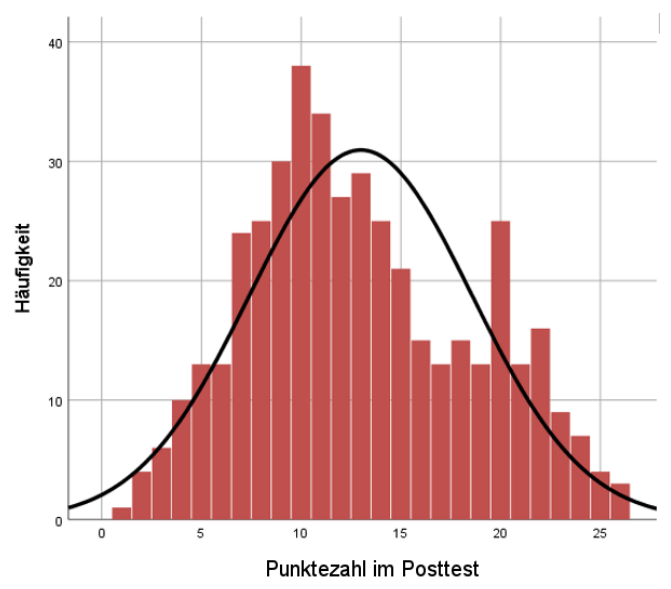

Abb. 80: Histogramm zur Prüfung der Normalverteilung der Posttest-Ergebnisse der Treatmentgruppe

3) Varianzhomogenität: Für die Anwendung eines unabhängigen $t$-Tests müssen ferner die Varianzen der beiden Stichproben miteinander vergleichbar sein. Die Varianzhomogenität wird mittels des Levene-Tests überprüft. In den vorliegenden Stichproben ist keine Varianzgleichheit gegeben $(F(1,788)=21.35, p<.001)$, weshalb die im Folgenden angegebenen Werte auf dem Welch-Test basieren, der gegenüber einer Verletzung der Varianzhomogenität als robust gilt.

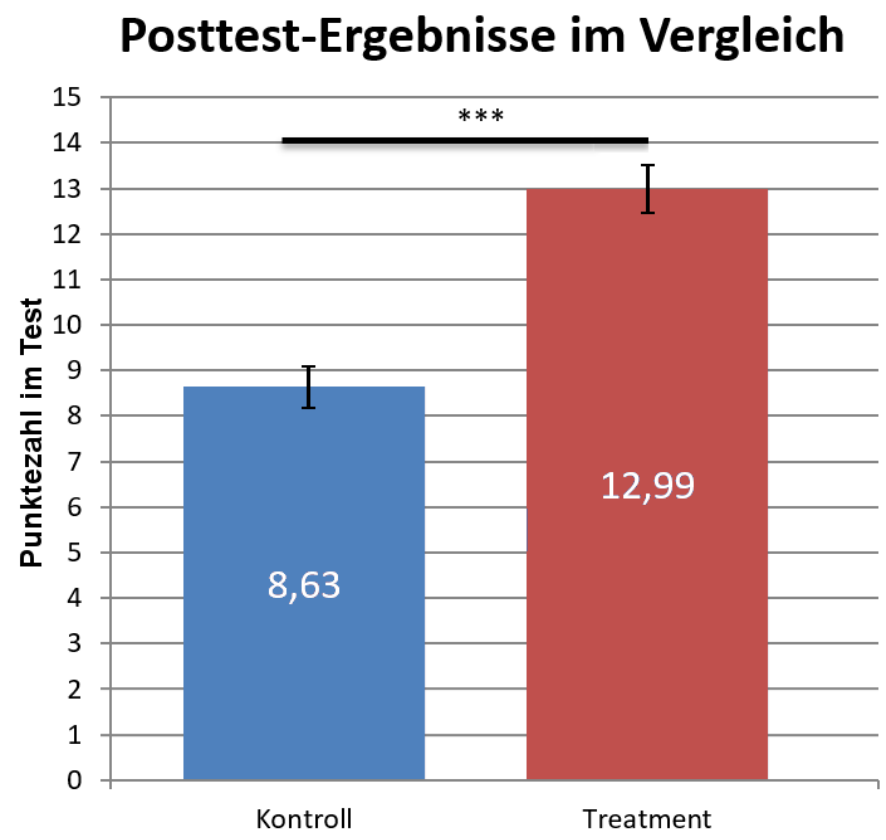

Abb. 81: Arithmetisches Mittel des Posttests für Kontroll-und Treatmentgruppe mit 95\%-Konfidenzintervallen (Signifikanzniveaus: $* * * p<.001 ; * * p<.01 ; * p<.05$ )

Betrachtet man die in Kontroll- und Treatmentgruppe nach dem Elektrizitätslehreunterricht durchschnittlich erzielten Punkte, so zeigt sich, dass die Schüler der Treatmentgruppe im Schnitt mehr Items korrekt beantworten konnten $(M=12.99, S E=0.25)$ als die Schüler der Kontrollgruppe $(M=8.63, S E=0.27)$. Im Mittel erreichten die Schüler der Treatmentgruppe damit 4.36 Punkte mehr als die Schüler der Kontrollgruppe. Dieser Unterschied ist nach dem Welch-Test höchst signifikant $(t(787.60)=12.14, p<.001)$ und entspricht einer 
großen Effektstärke von $d=.85$. Auch aus testtheoretischer Sicht ist das Abschneiden der Schüler im mittleren Punktebereich positiv zu beurteilen, da dann für den Nachtest davon ausgegangen werden kann, dass insgesamt keine Boden- oder Deckeneffekte aufgetreten sind (vgl. Abschnitt 7.4.1.3).

\subsubsection{Vergleich der Pretest-Ergebnisse}

Im folgenden Abschnitt soll untersucht werden, ob die beiden Gruppen hinsichtlich des Pretest-Ergebnisses vergleichbar sind. Hierzu wird auf das statistische Instrument des zweiseitigen $t$-Tests für unabhängige Stichproben zurückgegriffen. Geprüft wird, ob sich die beiden Gruppen bzgl. ihres arithmetischen Mittelwerts signifikant voneinander unterscheiden. Um dies mittels eines unabhängigen $t$-Tests prüfen zu können, müssen einige Voraussetzungen erfüllt sein.

\section{Prüfung der Voraussetzungen}

1) Intervallskalierung: Die vorliegenden Daten liegen intervallskaliert vor.

2) Normalverteilung: Wie anhand der beiden untenstehenden Histogramme zu sehen ist, kann im vorliegenden Fall trotz einer geringen Schiefe der Verteilung von einer ausreichenden Normalverteilung der Posttest-Ergebnisse in Kontroll- und Treatmentgruppe ausgegangen werden.

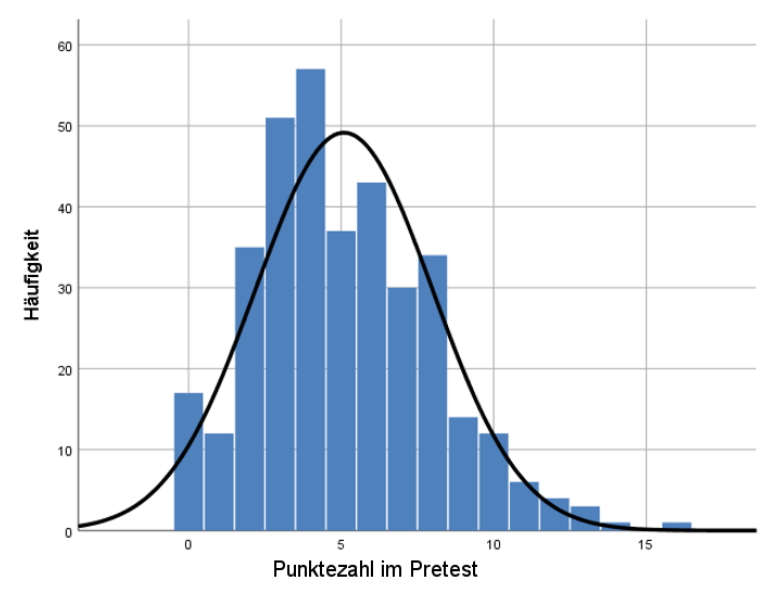

Abb. 82: Histogramm zur Prüfung der Normalverteilung der Pretest-Ergebnisse der Kontrollgruppe

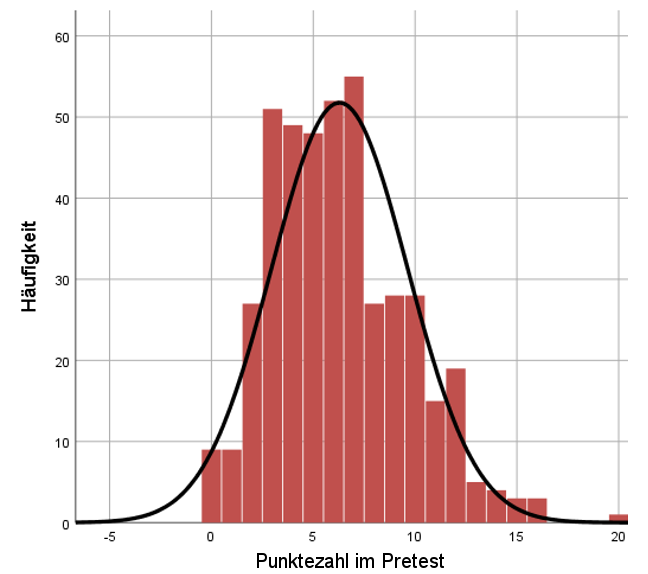

Abb. 83: Histogramm zur Prüfung der Normalverteilung der Pretest-Ergebnisse der Treatmentgruppe

3) Varianzhomogenität: Die Varianzhomogenität wird mit Hilfe des Levene-Tests überprüft. Im vorliegenden Fall sind die Varianzen der beiden Stichproben nicht gleich $(F(1,788)=6.31, p=.012)$, weshalb die im Folgenden angegebenen Werte auf dem Welch-Test basieren, der gegenüber einer Verletzung der Varianzhomogenität als robust gilt.

Betrachtet man die durchschnittlich erzielten Punkte in Kontroll- und Treatmentgruppe im Pretest, so ergibt sich folgendes Bild: 


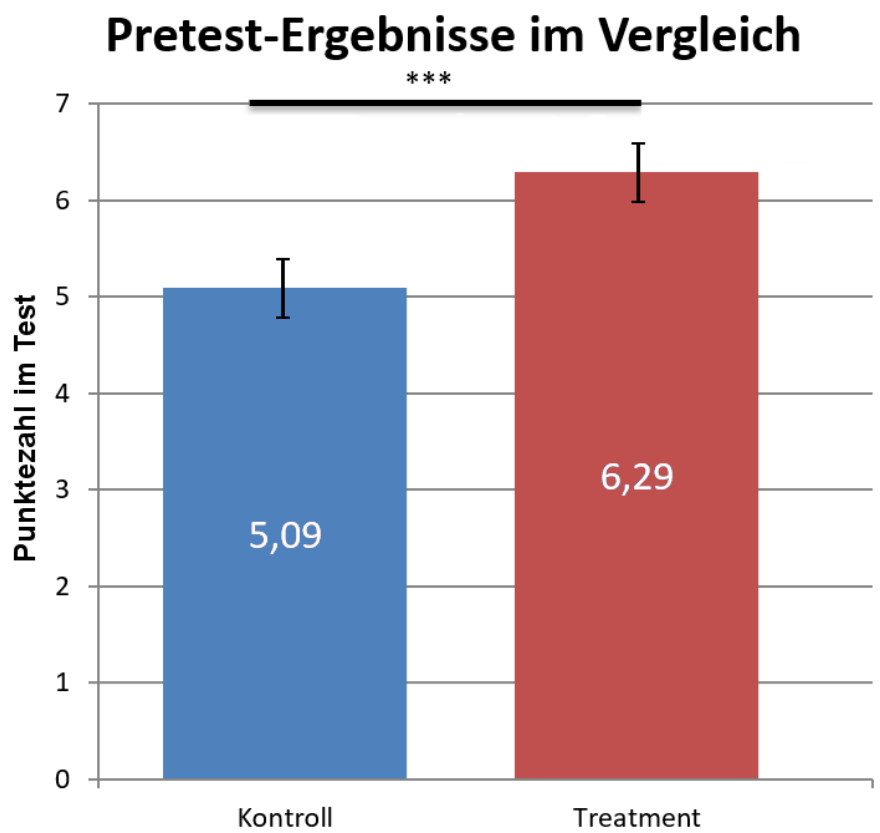

Abb. 84: Arithmetisches Mittel des Pretests von Kontroll- und Treatmentgruppe mit 95\%-Konfidenzintervallen (Signifikanzniveaus: $* * * p<.001 ; * * p<.01 ; * p<.05$ )

Es zeigt sich, dass die Schüler der Treatmentgruppe im Mittel bereits vor dem Elektrizitätslehreunterricht mehr Items korrekt beantworten konnten $(M=6.29, S E=0.15)$ als die Schüler der Kontrollgruppe $(M=5.09, S E=0.17)$. Die Schüler der Kontrollgruppe hatten im arithmetischen Mittel also ein um 1.20 Punkte schlechteres Pretest-Ergebnis als die Schüler der Treatmentgruppe. Dieser Unterschied ist nach dem Welch-Test höchst signifikant $(t(785.86)=5.41, p<.001)$. Da sich Kontroll- und Treatmentgruppe also bereits bzgl. ihrer Pretest-Ergebnisse signifikant voneinander unterscheiden, würde eine alleinige Betrachtung der Posttest-Ergebnisse zur Beurteilung der Effektivität des neuen Unterrichtskonzepts mit einer sehr geringen internen Validität einhergehen und wäre daher wenig überzeugend (Bortz und Döring 2006, S. 547). Für quasi-experimentelle Studien im Pretest-Posttest-Design empfehlen Bortz und Döring (2006, S. 554) deshalb die Bildung einer Differenzvariablen als Veränderungsindikator. Als Maß für die Effektivität des jeweiligen Unterrichts soll deshalb im Folgenden für Kontroll- und Treatmentgruppe der durch den jeweiligen Unterricht erzielte absolute Lernzuwachs analysiert werden, indem jeweils die Differenz von Post- und Pretest gebildet wird.

\subsubsection{Analyse des absoluten Lernzuwachses}

\subsubsection{Der absolute Lernzuwachs in der Kontrollgruppe}

In diesem Abschnitt wird für die Kontrollgruppe geprüft, ob das arithmetische Mittel des absoluten Lernzuwachses im Posttest signifikant höher liegt als das arithmetische Mittel des absoluten Lernzuwachses im Pretest. Dahinter steht die Frage, ob die Schüler, die traditionell unterrichtet worden sind, im Mittel einen absoluten Lernzuwachs erzielt haben, der signifikant größer ist als Null. Da in Pre- und Posttest die gleichen Schüler befragt wurden, muss dies mit Hilfe eines einseitigen $t$-Tests für abhängige Stichproben überprüft werden. Hierzu soll zu- 
nächst geschaut werden, ob die Voraussetzungen für die Durchführung eines abhängigen $t$ Tests erfüllt sind.

\section{Prüfung der Voraussetzungen}

1) Intervallskalierung: Die vorliegenden Daten liegen intervallskaliert vor.

2) Normalverteilung: Die Voraussetzung eines $t$-Tests für abhängige Stichproben ist im Gegensatz zu einem $t$-Test für unabhängige Stichproben nicht die Normalverteilung jeder einzelnen Stichprobe, sondern die Normalverteilung der Differenzwerte von Preund Posttest von allen Versuchspersonen (Rasch et al. 2010, S. 89). Wie das Histogramm in Abb. 85 zeigt, sind die Messwertdifferenzen der Messwertpaare in der Kontrollgruppe annährend normalverteilt.

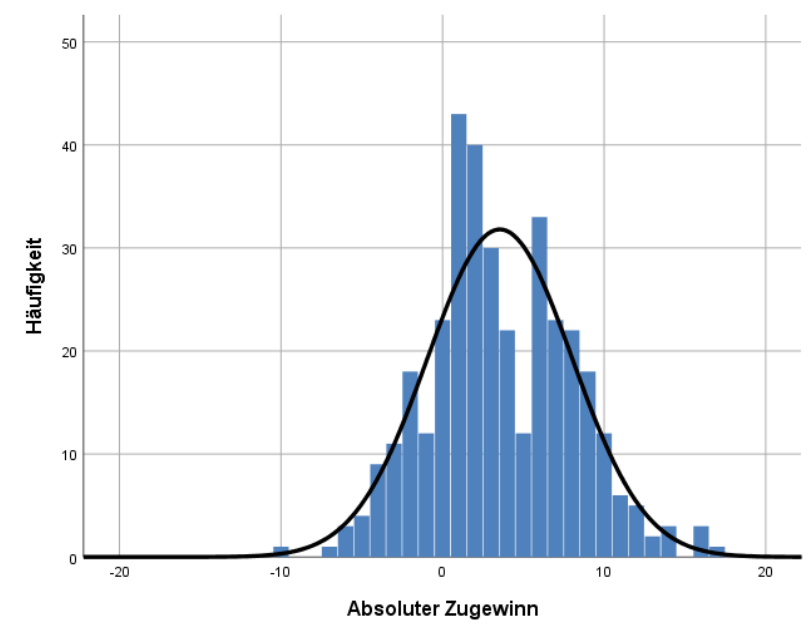

Abb. 85: Histogramm zur Prüfung der Normalverteilung der Messwertdifferenzen (absoluter Zugewinn) in der Kontrollgruppe

Der durchschnittliche absolute Lernzuwachs der Schüler in der Kontrollgruppe beträgt 3.54 Punkte $(S E=0.24)$. Dieser absolute Lernzuwachs ist entsprechend eines einseitigen $t$ Tests für abhängige Stichproben höchst signifikant $(t(356)=14.95, p<.001)$. Die Leistungen der Schüler der Kontrollgruppe in Pre- und Posttest unterscheiden sich damit signifikant voneinander, d.h. in der Kontrollgruppe fand ein Lernzuwachs statt.

\subsubsection{Der absolute Lernzuwachs in der Treatmentgruppe}

Analog zu dem vorherigen Abschnitt soll nun für die Treatmentgruppe mittels eines einseitigen $t$-Tests für abhängige Stichproben geprüft werden, ob ihr arithmetisches Mittel im Posttest signifikant höher liegt als ihr arithmetisches Mittel im Pretest. 


\section{Prüfung der Voraussetzungen}

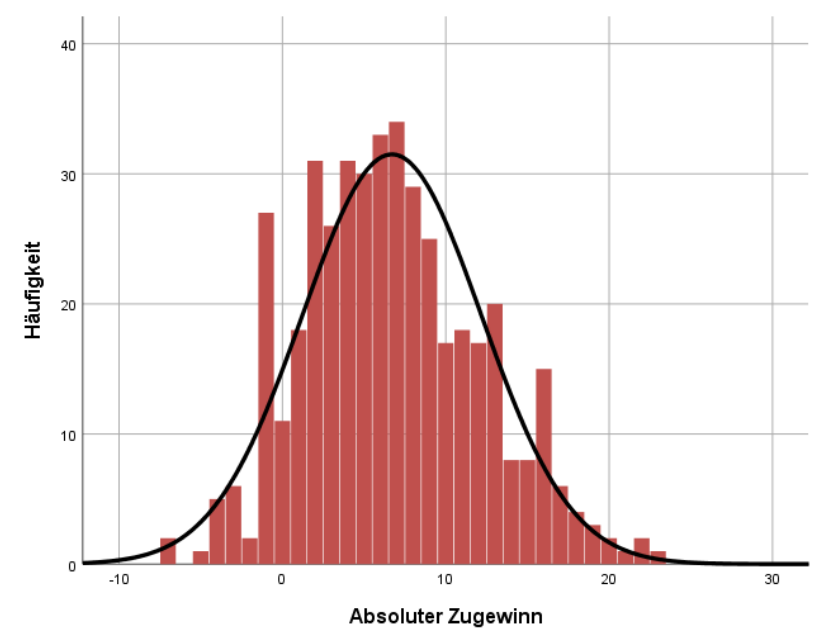

Abb. 86: Histogramm zur Prüfung der Normalverteilung der Messwertdifferenzen (absoluter Zugewinn) in der Treatmentgruppe

1) Intervallskalierung: Die vorliegenden Daten liegen intervallskaliert vor.

2) Normalverteilung: Wie das Histogramm in Abb. 86 zeigt, kann man bzgl. der Messwertdifferenzen der Messwertpaare in der Treatmentgruppe von einer annähernden Normalverteilung ausgehen.

Der durchschnittliche absolute Lernzuwachs der Schüler in der Treatmentgruppe beträgt 6.70 Punkte $(S E=0.26)$. Dieser absolute Lernzuwachs ist entsprechend eines einseitigen $t$-Tests für abhängige Stichproben höchst signifikant $(t(432)=25.41, p<.001)$. Die Leistungen der Schüler der Treatmentgruppe in Pre- und Posttest unterscheiden sich damit signifikant voneinander, d.h. auch in der Treatmentgruppe fand ein Lernzuwachs statt.

\subsubsection{Der Nettoeffekt als Maß der Lerneffektivität}

Sowohl in der Kontroll- als auch in der Treatmentgruppe hat sich gezeigt, dass die Schüler im Posttest signifikant besser abschneiden als im Pretest. Im Folgenden soll nun der jeweils erzielte absolute Lernzuwachs, definiert als Differenz des arithmetischen Mittelwerts von Postund Pretest-Ergebnis, miteinander verglichen werden, um den signifikanten Unterschieden im Pretest-Ergebnis Rechnung $\mathrm{zu}$ tragen. Zur Ermittlung des Nettoeffekts des Treatments gegenüber dem klassischen Unterricht wird von Bortz und Döring (2006, S. 559) vorgeschlagen $\mathrm{zu}$ untersuchen, wie sich der absolute Lernzuwachs in Treatment- und Kontrollgruppe unterscheidet und hierzu entsprechend die Differenz der Lernzuwächse zu bilden. Die so ermittelte Differenzvariable kann dabei als ein erstes Maß für die höhere Lerneffektivität des einen gegenüber dem anderen Unterricht gesehen werden (,Nettoeffekt des Treatments"). 
Kapitel 7: Summative Evaluation des Unterrichtskonzepts

Tab. 23: Ermittlung des Nettoeffekts des Treatments nach Bortz und Döring (2006, S. 559)

\begin{tabular}{|l|l|l|l|}
\hline & Pretest & Posttest & Zugewinn \\
\hline Kontrollgruppe & $\mathrm{KG}_{1}$ & $\mathrm{KG}_{2}$ & $\mathrm{KG}=\mathrm{KG}_{2}-\mathrm{KG}_{1}$ \\
\hline Treatmentgruppe & $\mathrm{TG}_{1}$ & $\mathrm{TG}_{2}$ & $\mathrm{TG}=\mathrm{TG}_{2}-\mathrm{TG}_{1}$ \\
\hline \multicolumn{2}{|l}{} & Nettoeffekt: TG - KG \\
\cline { 2 - 4 }
\end{tabular}

Um zu überprüfen, ob der so ermittelte Nettoeffekt des Treatments statistisch gesehen signifikant ist, wird von Bortz und Döring (2006, S. 560) für dieses Studiendesign eine zweifaktorielle Varianzanalyse mit Messwiederholung empfohlen. Kommt es bei dieser Varianzanalyse zu einer signifikanten Interaktion zwischen Gruppenfaktor und Messwiederholungsfaktor, liegt ein statistisch signifikanter Nettoeffekt des Treatments vor. Im Folgenden soll daher mit Hilfe einer univariaten zweifaktoriellen Varianzanalyse mit Messwiederholung (gemischtes Design) geprüft werden, ob sich die absoluten Lernfortschritte in Kontroll- und Treatmentgruppe signifikant voneinander unterscheiden. Der Name der Analysemethode leitet sich nach Bühner und Ziegler (2009, 478f) davon ab, dass die Varianzanalyse eine abhängige Variable untersucht (univariat), jedoch zwei Faktoren beinhaltet (zweifaktoriell), nämlich den der „Gruppe“ und den des „Messzeitpunkts“, der wiederholt gemessen wird. Somit wird ein „,between-subjects-design“ und ein „,within-subject-design“ gemischt (gemischtes Design).

Prüfung der Voraussetzungen nach Rasch et al. (2010, S. 71):

1) Intervallskalierung der abhängigen Variablen: Der absolute Lernzuwachs wird in beiden Gruppen intervallskaliert gemessen. Die Voraussetzung ist deshalb erfüllt.

2) Normalverteilung der Messwerte in allen Teilstichproben: Wie in Abschnitten 7.4.1 und 0 gezeigt, kann sowohl in den Pretests der KG und TG als auch in den Posttests der KG und TG von einer Normalverteilung der Messwerte ausgegangen werden. Zudem sind Varianzanalysen weitgehend robust gegenüber der Verletzung der Normalverteilungsannahme (Rasch et al. 2010, S. 31). Es kann deshalb davon ausgegangen werden, dass diese Voraussetzung erfüllt ist.

3) Varianzhomogenität: Die Prüfung der Varianzhomogenität zwischen den Stufen des Zwischen-Subjekt-Faktors (hier: Treatment, d.h. KG und TG) innerhalb jeder Stufe des Inner-Subjekt-Faktors (hier: Messzeitpunkt, d.h. Pre- bzw. Posttest) erfolgte ebenfalls bereits in den Abschnitten 7.4.1 und 0. Der Levene-Test zeigt in beiden Fällen, dass keine Varianzhomogenität gegeben ist. Zwar sind Varianzanalysen prinzipiell auch relativ robust gegenüber einer Verletzung der Varianzhomogenitätsannahme, allerdings weist Field (2011, S. 360) darauf hin, dass dies insbesondere bei unbalancierten Designs (ungleiche Stichprobenumfänge) nicht zutrifft und zu einer Verzerrung der Ergebnisse führen kann. Im vorliegenden Fall weist die Treatmentgruppe als größere Stichprobe auch die größere Varianz verglichen mit der kleineren Kontrollgruppe auf. Dies führt nach Field (2011, S. 360) zu einer zu konservativen Schätzung des FVerhältnisses, weshalb möglicherweise signifikante Unterschiede tendenziell übersehen werden. Sofern mit Hilfe der Varianzanalyse also ein signifikanter Unterschied in 
Bezug auf den in Kontroll- und Treatmentgruppe erzielten absoluten Lernzuwachs gefunden wird, wäre das gefundene Signifikanzniveau tendenziell sogar zu konservativ und die Verletzung der Varianzhomogenitätsannahme unproblematisch.

4) Sphärizität: Die Voraussetzung ist erfüllt, da der Inner-Subjekt-Faktor nur zwei Stufen (d.h. zwei Messzeitpunkte) hat und die Sphärizität, also die Homogenität aller Varianzen der Differenzen zweier Faktorstufen, somit automatisch gegeben ist (Rasch et al. 2010, S. 72).

Die univariate zweifaktorielle Varianzanalyse mit Messwiederholung ergibt, dass sich Kontroll- und Treatmentgruppe bzgl. des durchschnittlich erzielten absoluten Lernzuwachses höchst signifikant unterscheiden, da ein höchst signifikanter Interaktionseffekt zwischen Gruppen- und Messwiederholungsfaktor vorliegt $\left(F(1,788)=76.21, p<.001, \eta^{2}=.088\right)$. Der vorliegende Interaktionseffekt ist dabei so zu verstehen, dass sich der über die beiden Messzeitpunkte stattfindende Lernzuwachs in beiden Gruppen signifikant voneinander unterscheidet. Wie Abb. $87 \mathrm{zu}$ entnehmen ist, fällt dabei der durchschnittliche absolute Lernzuwachs in der Treatmentgruppe höher aus als in der Kontrollgruppe.

\section{Pre- und Posttest-Ergebnis von KG und TG}

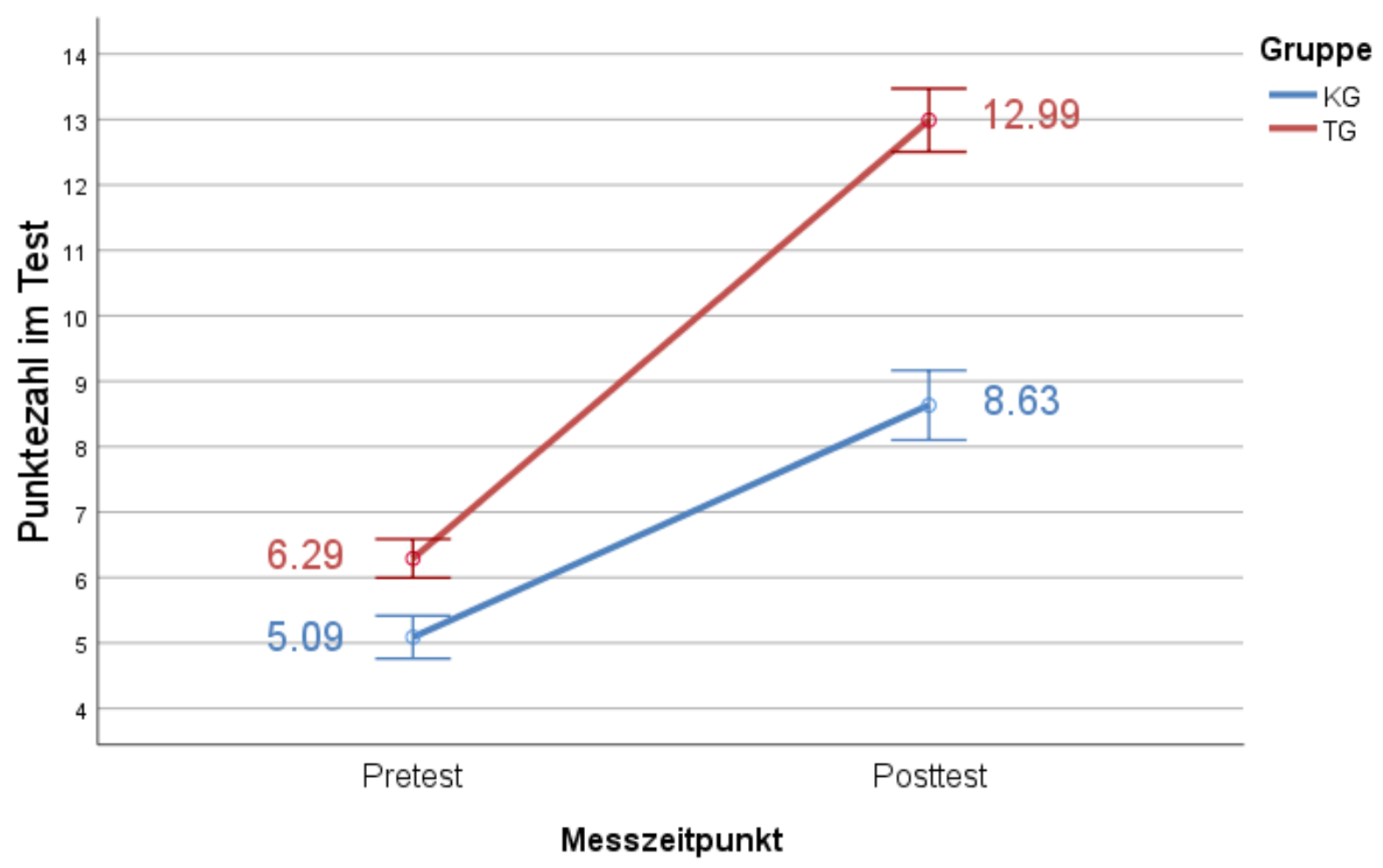

Abb. 87: Interaktionseffekt von Gruppen- und Messwiederholungsfaktor mit 95\%-Konfidenzintervallen

Die hier durchgeführte Varianzanalyse zeigt also, dass der in Abschnitt 7.4.3.2 berechnete durchschnittliche absolute Lernzuwachs der Schüler der Treatmentgruppe mit 6.70 Punkten $(S E=0.26)$ signifikant höher ist als der in Abschnitt 7.4.3.1 berechnete durchschnittliche absolute Lernzuwachs der Schüler in der Kontrollgruppe, bei der er lediglich 3.54 Punkte $(S E=0.24)$ beträgt. Der von Bortz und Döring (2006, S. 560) vorgeschlagene, zu berech- 
nende Nettoeffekt des neuen Unterrichtskonzepts als Differenz des Lernzuwachses in Kontroll- und Treatmentgruppe beträgt somit 3.16 Punkte $(S E=0.50)$. Die im Rahmen der Varianzanalyse für diesen Unterschied angegebene Effektstärke von $\eta^{2}=.088$ gibt dabei den Anteil der durch den Gruppeneffekt aufgeklärten Varianz auf Stichprobenebene an. In dieser Studie können also 8,8\% der Variabilität des absoluten Lernzuwachses alleine mit der Gruppenzugehörigkeit der Schüler erklärt werden. Nach Cohen (1988, S. 276) lässt sich dieses Effektstärkemaß mittels des untenstehenden Zusammenhangs in ein Cohens $d$ überführen.

$$
d=2 \cdot \sqrt{\frac{\eta^{2}}{1-\eta^{2}}}
$$

Somit ergibt sich für die Effektstärke ausgedrückt als Cohens $d$ ein Wert von $d=.62$, was einem mittleren Effekt entspricht. Da aus Abb. 87 zum Interaktionseffekt von Gruppenund Messwiederholungsfaktor die Werte der absoluten Lernzuwächse in Kontroll- und Treatmentgruppe nicht direkt hervorgehen, sind die bisher gefundenen Ergebnisse in einer anderen Darstellungsform in Abb. 88 noch einmal zusammengefasst.

\section{Testergebnisse im Vergleich}

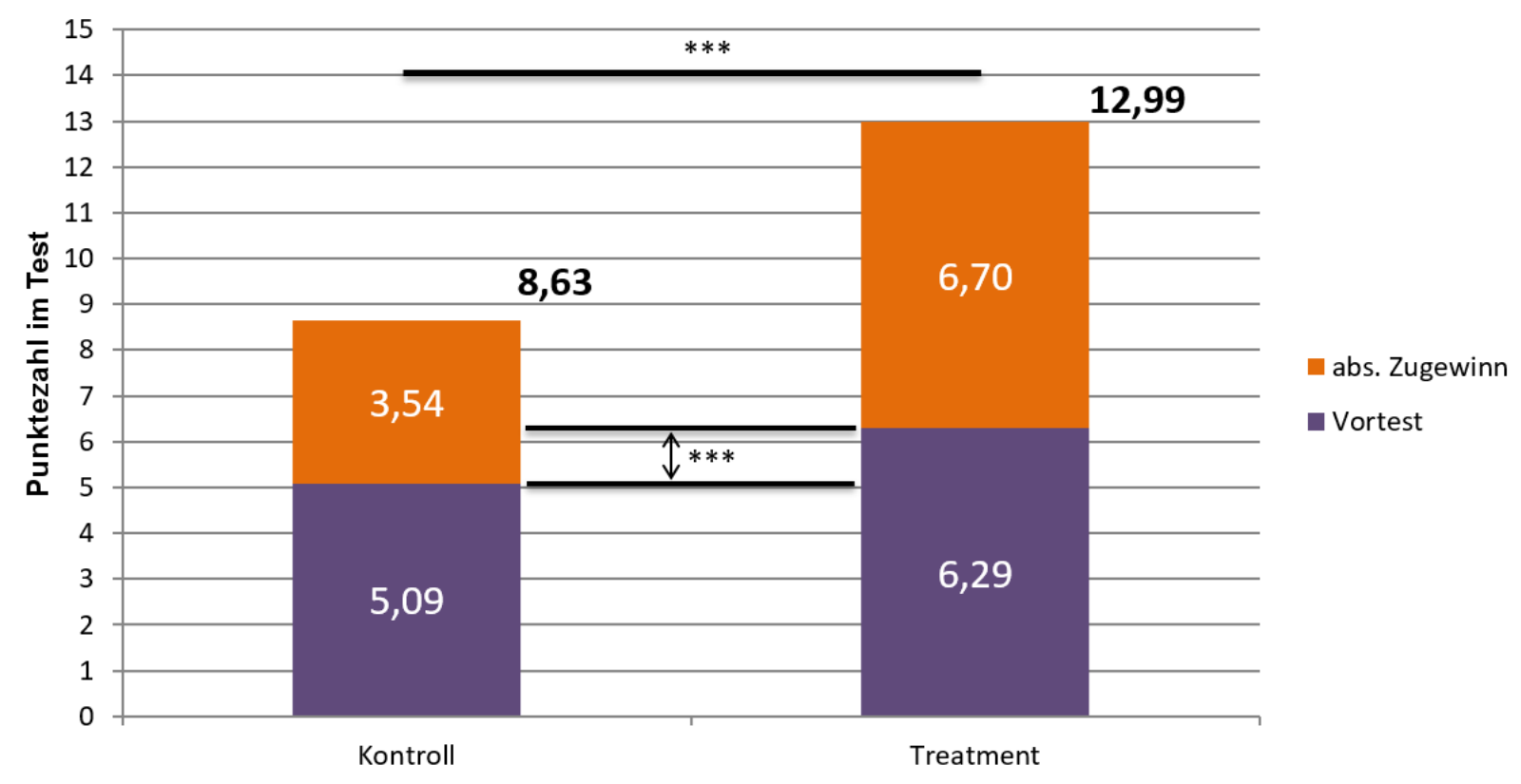

Abb. 88: Darstellung der Testergebnisse von Kontroll- und Treatmentgruppe im Vergleich (Signifikanzniveaus: $* * * p<.001 ; * * p<.01 ; * p<.05$ )

In Abb. 89 ist der in Kontroll- und Treatmentgruppe erzielte absolute Lernzuwachs zusätzlich als Boxplot aufgetragen. Während der Median des absoluten Lernzuwachses in der Kontrollgruppe bei lediglich 3 Punkten liegt, fällt er in der Treatmentgruppe mit 6 Punkten exakt doppelt so hoch aus. Der Boxplot zeigt zudem, dass die Varianz des absoluten Zugewinns in der Treatmentgruppe leicht höher ausfällt als in der Kontrollgruppe. 


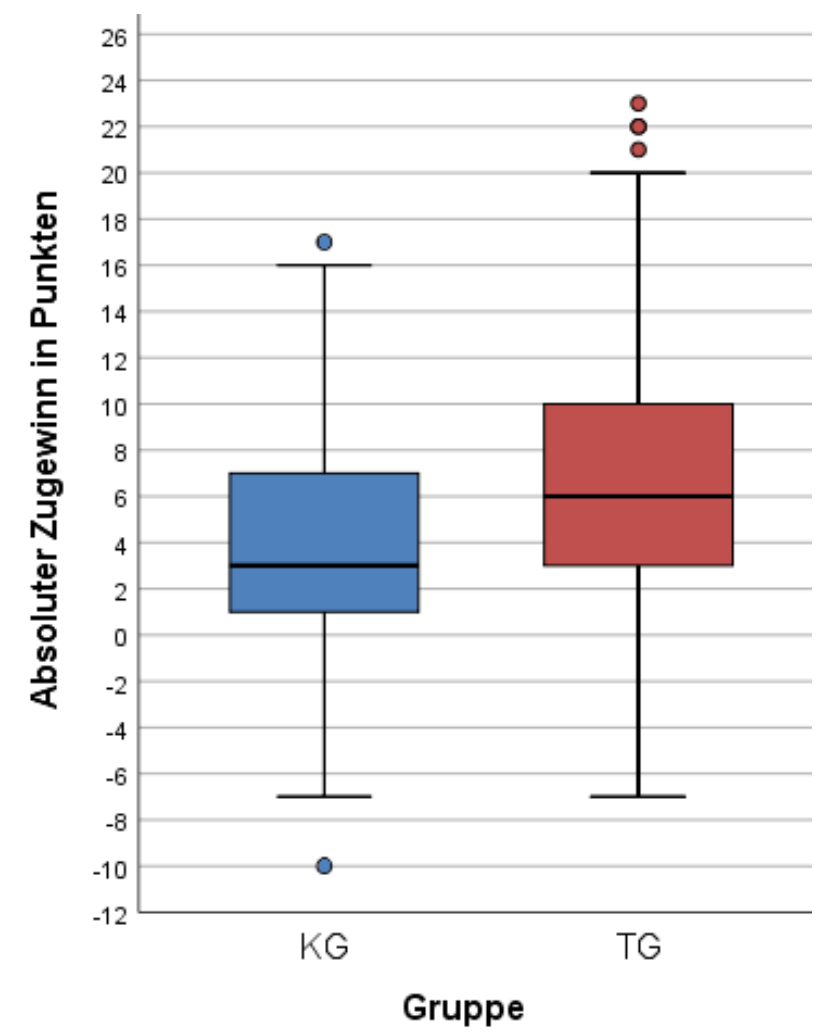

Abb. 89: Boxplot des in Kontroll- und Treatmentgruppe erzielten absoluten Lernzuwachses

Nach Bortz und Döring (2006, S. 560) ist allerdings davon auszugehen, dass sich die im Vortest gezeigte Diskrepanz zwischen Kontroll- und Treatmentgruppe im Nachtest alleine aufgrund von Regressionseffekten verkleinert. Dies hätte im vorliegenden Fall zur Folge, dass der Nettoeffekt des Treatments tendenziell unterschätzt wird. Unabhängig von der Diskrepanz im Vortest ist die alleinige Betrachtung des Zugewinns nach Todman und Dugard (1995, S. 182) nicht ausreichend, um den Einfluss des Pretest-Ergebnisses zu kontrollieren, da der absolute Zugewinn mit den Pretest-Ergebnissen korreliert. Eine Korrelationsanalyse zeigt für den vorliegenden Datensatz, dass der absolute Zugewinn negativ mit dem Pretest-Ergebnis korreliert ist $(r(790)=-.22, p<.001)$. Dies bedeutet also, dass der absolute Zugewinn tendenziell umso geringer ausfällt, desto höher das Pretest-Ergebnis ist. Vor dem Hintergrund, dass im Test maximal 26 Punkte erzielt werden können und Schüler mit hohen Pretest-Ergebnissen prinzipiell weniger Punkte hinzugewinnen können als Schüler mit niedrigen PretestErgebnissen, ist dieser Befund wenig überraschend.

\subsubsection{Unterschiedlicher Lernzuwachs in der TG zwischen Mädchen und Jungen}

Nachdem der durchschnittlich in der Treatmentgruppe von allen Lernenden erzielte absolute Lernzuwachs ermittelt wurde, wird nun untersucht, ob sich dieser zwischen den Mädchen $(N=225)$ und den Jungen $(N=208)$ signifikant unterscheidet. Hierzu wird analog zum vorangegangenen Abschnitt 7.4.3.3 auf eine univariate zweifaktorielle Varianzanalyse mit Messwiederholung (gemischtes Design) zurückgegriffen, wobei der Innersubjektfaktor wieder der Vor- bzw. Nachtest, der Zwischensubjektfaktor hier jedoch das Geschlecht der Lernenden innerhalb der Treatmentgruppe ist. 
Prüfung der Voraussetzungen nach Rasch et al. (2010, S. 71):

1) Intervallskalierung der abhängigen Variablen: Der absolute Lernzuwachs wird in beiden Gruppen intervallskaliert gemessen. Die Voraussetzung ist deshalb erfüllt.

2) Normalverteilung der Messwerte in allen Teilstichproben: Wie anhand der untenstehenden Histogramme zu erkennen ist, kann im vorliegenden Fall trotz einer geringen Schiefe der Verteilungen von einer ausreichenden Normalverteilung der Daten ausgegangen werden.

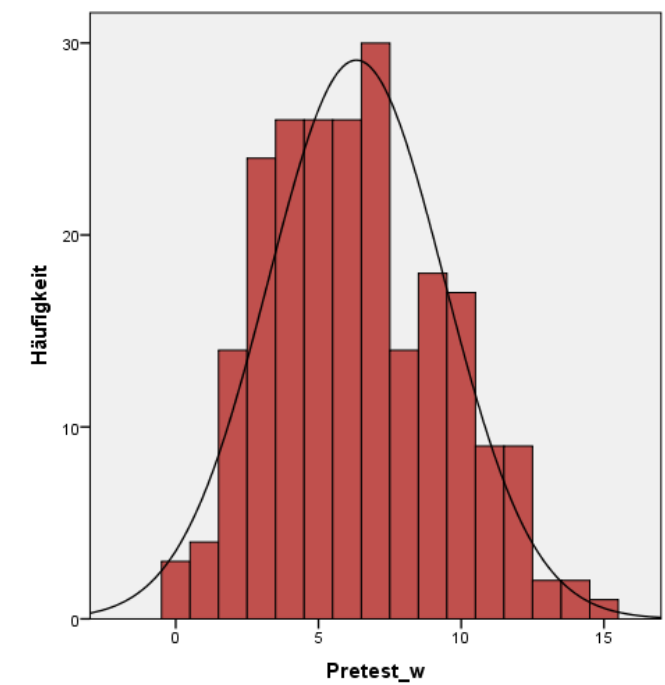

Abb. 90: Histogramm zur Prüfung der Normalverteilung der Pretest-Ergebnisse der Mädchen $(N=225)$ in der Treatmentgruppe

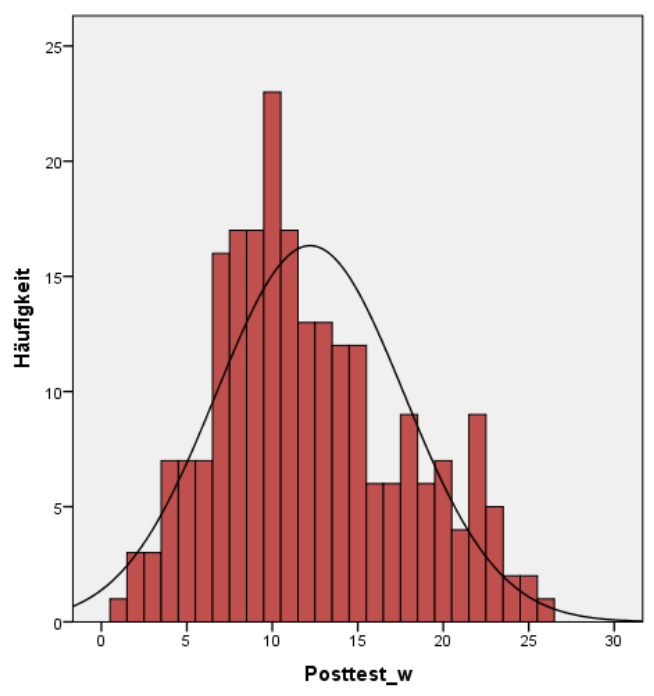

Abb. 92: Histogramm zur Prüfung der Normalverteilung der Posttest-Ergebnisse der Mädchen $(N=225)$ in der Treatmentgruppe

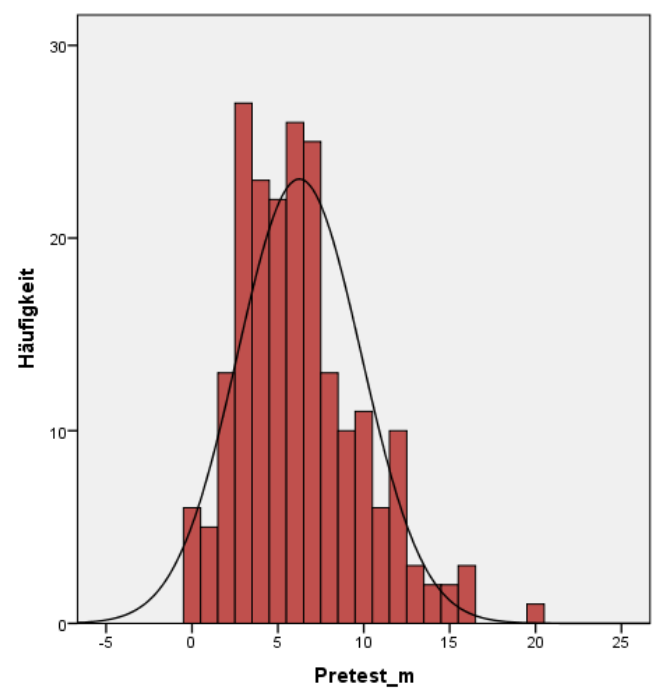

Abb. 91: Histogramm zur Prüfung der Normalverteilung der Pretest-Ergebnisse der Jungen $(N=208)$ in der Treatmentgruppe

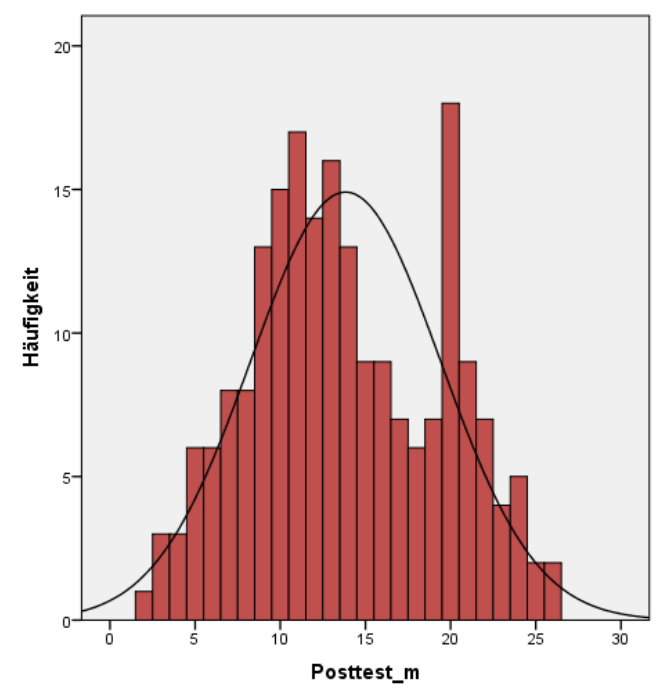

Abb. 93: Histogramm zur Prüfung der Normalverteilung der Posttest-Ergebnisse der Jungen $(N=208)$ in der Treatmentgruppe 
3) Varianzhomogenität: Die Prüfung der Varianzhomogenität zwischen den Stufen des Zwischen-Subjekt-Faktors (hier: Geschlecht, d.h. männlich oder weiblich) innerhalb jeder Stufe des Inner-Subjekt-Faktors (hier: Messzeitpunkt, d.h. Pre- bzw. Posttest) erfolgt mittels des Levene-Tests. Es zeigt sich, dass sowohl im Vortest $(F(1,431)=2.18, p=.141)$ als auch im Nachtest $(F(1,431)=0.27, p=.602)$ zwischen Mädchen und Jungen Varianzgleichheit bzgl. der Testergebnisse vorliegt, weshalb diese Bedingung erfüllt ist.

4) Sphärizität: Die Voraussetzung ist erfüllt, da der Inner-Subjekt-Faktor nur zwei Stufen (d.h. zwei Messzeitpunkte) hat und die Sphärizität, also die Homogenität aller Varianzen der Differenzen zweier Faktorstufen, somit automatisch gegeben ist (Rasch et al. 2010, S. 72).

Ein zweiseitiger $t$-Test für unabhängige Stichproben zeigt zunächst, dass sich Jungen mit 6.25 Punkten $(S E=0.25)$ und Mädchen mit 6.33 Punkten $(S E=0.21)$ in ihren PretestErgebnissen nicht signifikant voneinander unterscheiden $(t(431)=-0.25, p=.806)$. Die univariate zweifaktorielle Varianzanalyse ergibt jedoch, dass das Unterrichtskonzept bei den Jungen mit 7.58 Punkten $(S E=0.38)$ verglichen mit lediglich 5.88 Punkten $(S E=0.36)$ bei den Mädchen zu einem signifikant höheren absoluten Lernzuwachs führt $(F(1,431)=10.64$, $\left.p<.001, \eta^{2}=.024\right)$. Die Effektstärke entspricht einem Cohens $d$ von $d=.31$, was einen kleinen Effekt darstellt. Die Ergebnisse sind in Abb. 94 zusammengefasst dargestellt.

\section{Lernentwicklung in der Treatmentgruppe nach Geschlecht}

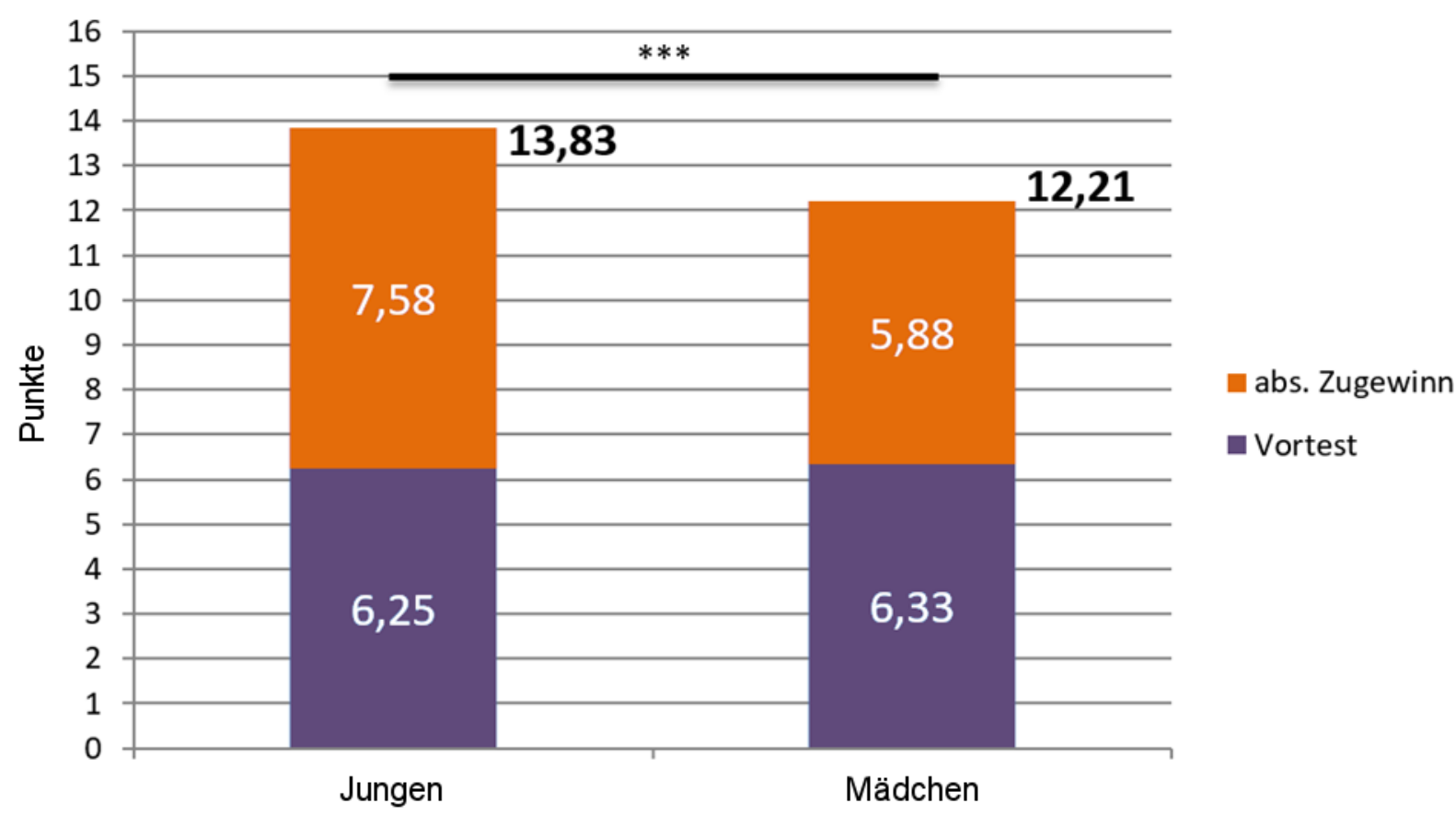

Abb. 94: Vergleich der Pre- und Posttest-Ergebnisse sowie des absoluten Lernzuwachses von Jungen und Mädchen in der Treatmentgruppe. Das angegebene Signifikanzniveau $* * * p<.001$ bezieht sich auf den erzielten abs. Zugewinn. 
An dieser Stelle wäre es interessant zu untersuchen, ob in der Kontrollgruppe auch ein signifikanter Unterschied bzgl. des absoluten Lernzugewinns zwischen Mädchen und Jungen besteht und falls ja, wie hoch dieser ausfällt. Es könnte nämlich trotz der gefundenen Diskrepanz zwischen Mädchen und Jungen in der Treatmentgruppe sein, dass die Unterschiede hinsichtlich des erzielten absoluten Lernzugewinns in der Kontrollgruppe noch größer ausgefallen sind als in der Treatmentgruppe. Ein solcher Vergleich ist jedoch leider nicht möglich, da in der Kontrollgruppe das Geschlecht der Lernenden nicht erhoben wurde.

\subsubsection{Absoluter Lernzuwachs nach Terzilen}

Nun könnte man einwenden, dass der höhere absolute Lernzuwachs in der Treatmentgruppe auf das höhere Vorwissen der Schüler zurückzuführen ist („Matthäus-Effekt $\left.{ }^{\circ}\right)$. Andersherum könnte man auch vermuten, dass der absolute Lernzuwachs der Schüler der Treatmentgruppe aufgrund ihres höheren durchschnittlichen Pretest-Ergebnisses prinzipiell geringer ausfallen müsste, weil im Test maximal 26 Punkte erzielt werden können. Aus diesem Grund soll nun untersucht werden, wie der absolut erzielte Lernzuwachs vom Pretest-Ergebnis der Schüler abhängt. Hierzu wurden die Schüler der Kontroll- und Treatmentgruppe in Abhängigkeit ihres Vortestergebnisses zunächst in die Terzile „,unteres Drittel“, „,mittleres Drittel“ und „oberes Drittel“ eingeteilt und für jedes dieser Terzile dann der durch den Unterricht erzielte durchschnittliche absolute Lernzugewinn berechnet. Die Unterschiede zwischen den jeweiligen Terzilen der Kontroll- und Treatmentgruppe wurden dann mittels eines einseitigen $t$-Tests für unabhängige Stichproben verglichen und die Unterschiede zwischen den Terzilen innerhalb der Kontroll- bzw. Treatmentgruppe mittels einer Varianzanalyse untersucht.

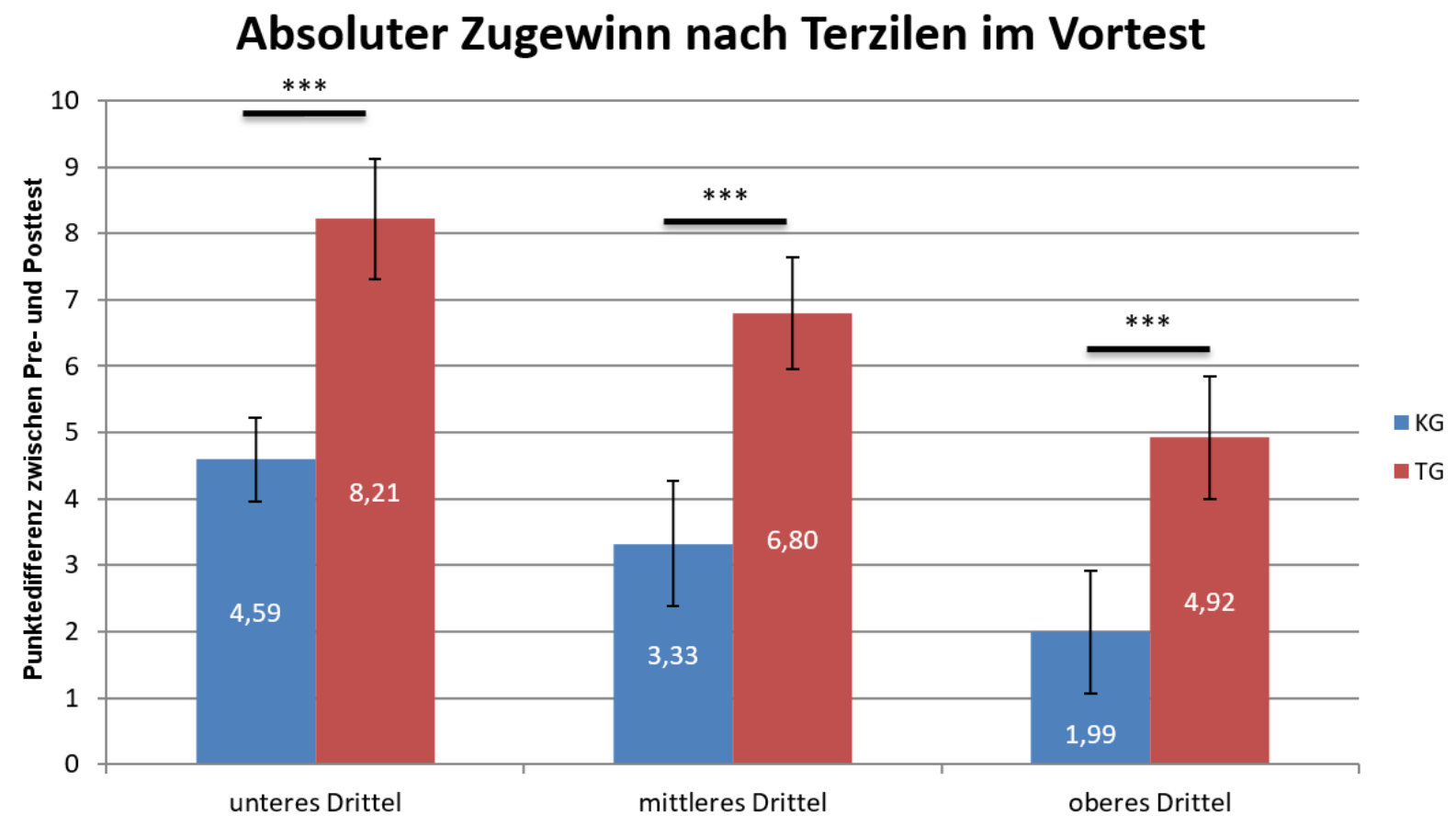

Abb. 95: Absoluter Zugewinn nach Terzilen im Vortest mit 95\%-Konfidenzintervallen (Signifikanzniveaus: $* * * p<.001 ; * * p<.01 ; * p<.05$ ) 
Wie Abb. 95 zu entnehmen ist, erzielen die Schüler der Treatmentgruppe unabhängig von ihrem Vortestergebnis in jedem Terzil einen höchst signifikant größeren absoluten Zugewinn als die Schüler der Kontrollgruppe. Konkret erzielt das untere Drittel in der Treatmentgruppe einen absoluten Lernzuwachs von 8.21 Punkten ( $S E=0.45)$, wohingegen der absolute Lernzuwachs in der Kontrollgruppe hier mit 4.59 Punkten $(S E=0.32)$ höchst signifikant geringer ausfällt $(t(265.61)=6.58, p<.001)$. Das mittlere Drittel in der Treatmentgruppe erzielt einen absoluten Lernzuwachs von 6.80 Punkten $(S E=0.42)$, während dieser mit 3.33 Punkten $(S E=0.47)$ in der Kontrollgruppe ebenfalls höchst signifikant geringer ausfällt $(t(192.01)=5.48, p<.001)$. Der absolute Lernzuwachs des oberen Drittels in der Treatmentgruppe liegt nur bei 4.92 Punkten $(S E=0.46)$ und beträgt in der Kontrollgruppe nur 1.99 Punkte $(S E=0.46)$, was auch einen höchst signifikanten Unterschied zwischen den beiden Gruppen darstellt $(t(232.45)=4.48, p<.001)$. An der Entwicklung dieser Zahlen ist auch deutlich zu erkennen, dass der absolute Lernzuwachs sowohl in der Kontroll- als auch in der Treatmentgruppe tendenziell umso geringer ausfällt, desto besser die Schüler im Vortest abgeschnitten haben. Dieses Ergebnis entspricht dem Befund aus Abschnitt 7.4.3.3, wonach der absolute Zugewinn negativ mit dem Pretest-Ergebnis korreliert ist $(r(790)=-.22, p<.001)$.

Eine univariate einfaktorielle Varianzanalyse bestätigt, dass sich der absolut erzielte Lernzuwachs in der Kontrollgruppe in den verschiedenen Terzilen höchst signifikant voneinander unterscheidet $(F(2,354)=11.81, p<.001)$. Ein anschließender Games-Howell PostHoc-Test zeigt, dass sich in der Kontrollgruppe allerdings nur das obere vom unteren Drittel in Bezug auf den erzielten absoluten Lernzuwachs signifikant unterscheidet $(p<.001)$, während der jeweilige Unterschied zum mittleren Drittel statistisch nicht signifikant ist. Auch für die Treatmentgruppe bestätigt eine univariate einfaktorielle Varianzanalyse, dass sich der absolut erzielte Lernzuwachs in den unterschiedlichen Terzilen höchst signifikant voneinander unterscheidet $(F(2,430)=13.23, p<.001)$. Eine genauere Analyse mit Hilfe eines GamesHowell Post-Hoc-Tests ergibt, dass ein statistisch signifikanter Unterschied bzgl. des erzielten Lernzuwachses in der Treatmentgruppe sowohl zwischen dem unteren und oberen Drittel $(p<.001)$ als auch zwischen dem mittleren und oberen Drittel $(p=.008)$ besteht, sich der erzielte absolute Lernzuwachs im unteren und mittleren Drittel jedoch nicht signifikant voneinander unterscheidet. Wie bereits angedeutet, ist dieses Ergebnis wenig verwunderlich, da der potenziell erzielbare absolute Lernzuwachs aufgrund der Maximalpunktzahl des Tests von 26 Punkten prinzipiell umso geringer ist, je mehr Punkte bereits im Vortest erzielt wurden. Damit ist aber auch die These des „Matthäus-Effekts“ widerlegt, wonach die Schüler der Treatmentgruppe nur deshalb einen höheren Zugewinn erzielt hätten, weil sie im Vortest signifikant besser abgeschnitten haben und somit vermutlich auch über ein höheres Vorwissen verfügten.

\subsubsection{Analyse des relativen Lernzuwachses}

Da Schüler mit einer hohen Punktzahl im Vortest prinzipiell keinen so hohen absoluten Lernzuwachs mehr erzielen können wie Schüler mit einer niedrigen Punktzahl, wird im Folgenden der durch den Unterricht erzielte relative Zugewinn betrachtet. Der relative Zugewinn gibt dabei an, wie groß der absolute Lernzugewinn in Relation zum für den Schüler überhaupt 
erzielbaren Zugewinn ausfällt (relativer Zugewinn = absoluter Zugewinn dividiert durch den möglichen Zugewinn). Der durchschnittliche relative Zugewinn in der Treatmentgruppe ist mit 34\% $(S E=1 \%)$ mehr als doppelt so hoch wie in der Kontrollgruppe, wo er lediglich $16 \%$ $(S E=1 \%)$ beträgt. Ein einseitiger $t$-Test für unabhängige Stichproben zeigt, dass dieser Unterschied zwischen den beiden Gruppen höchst signifikant ist $(t(787.73)=9.70, p<.001)$.

Relativer Zugewinn

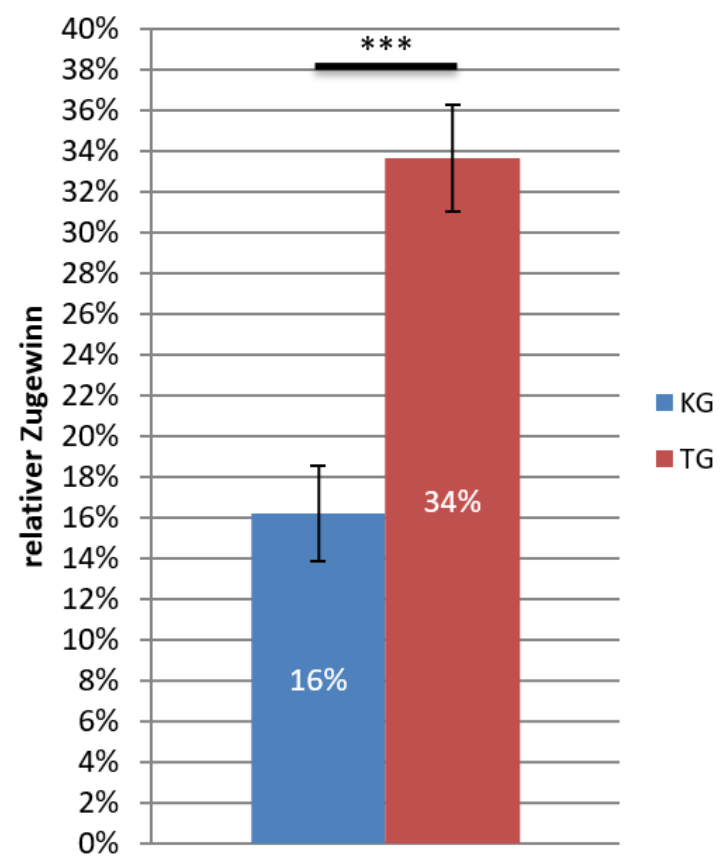

Abb. 96: Durchschnittlicher relativer Zugewinn der Kontroll- und Treatmentgruppe mit 95\%-Konfidenzintervallen (Signifikanzniveaus: $* * * p<.001 ; * * p<.01 ; * p<.05$ )

Auch hier besteht eine interessante Fragestellung darin, wie der im Unterricht erzielte relative Lernzuwachs vom Pretest-Ergebnis der Schüler abhängt. Hierzu wurden die Schüler der Kontroll- und Treatmentgruppe wieder in Abhängigkeit ihres Vortestergebnisses in die Terzile „unteres Drittel“, „mittleres Drittel“ und „oberes Drittel“ eingeteilt und die Unterschiede mittels eines einseitigen $t$-Tests für unabhängige Stichproben bzw. einer Varianzanalyse verglichen.

Wie aus Abb. 97 hervorgeht, erzielen Schüler, die nach dem neuen Unterrichtskonzept unterrichtet wurden, unabhängig vom Vortestergebnis einen deutlich höheren relativen Zugewinn als ihre traditionell unterrichteten Mitschüler. Konkret erzielt das untere Drittel der Schüler in der Treatmentgruppe einen relativen Lernzuwachs von 35\% (SE $=2 \%)$ gegenüber $19 \%(S E=1 \%)$ in der Kontrollgruppe, was einen höchst signifikanten Unterschied darstellt $(t(265.75)=6.72, p<.001)$. Das mittlere Drittel der Schüler in der Treatmentgruppe erzielt mit 34\% (SE $=2 \%)$ einen mehr als doppelt so hohen relativen Lernzuwachs wie die Schüler der Kontrollgruppe, die hier lediglich einen relativen Lernfortschritt von $16 \%(S E=2 \%) \mathrm{zu}$ verzeichnen haben, was ebenfalls einem höchst signifikanten Unterschied entspricht $(t(195.60)=5.69, p<.001)$. Der relative Lernzuwachs des oberen Drittels in der Treatment- 
gruppe liegt bei 31\% (SE =3\%) und entspricht damit fast dem Dreifachen des hier in der Kontrollgruppe erzielten relativen Lernzuwachses von 11\% $(S E=3 \%)$. Auch dies stellt einen höchst signifikanten Unterschied dar $(t(233.94)=5.00, p<.001)$.

\section{Relativer Zugewinn nach Terzilen im Vortest}

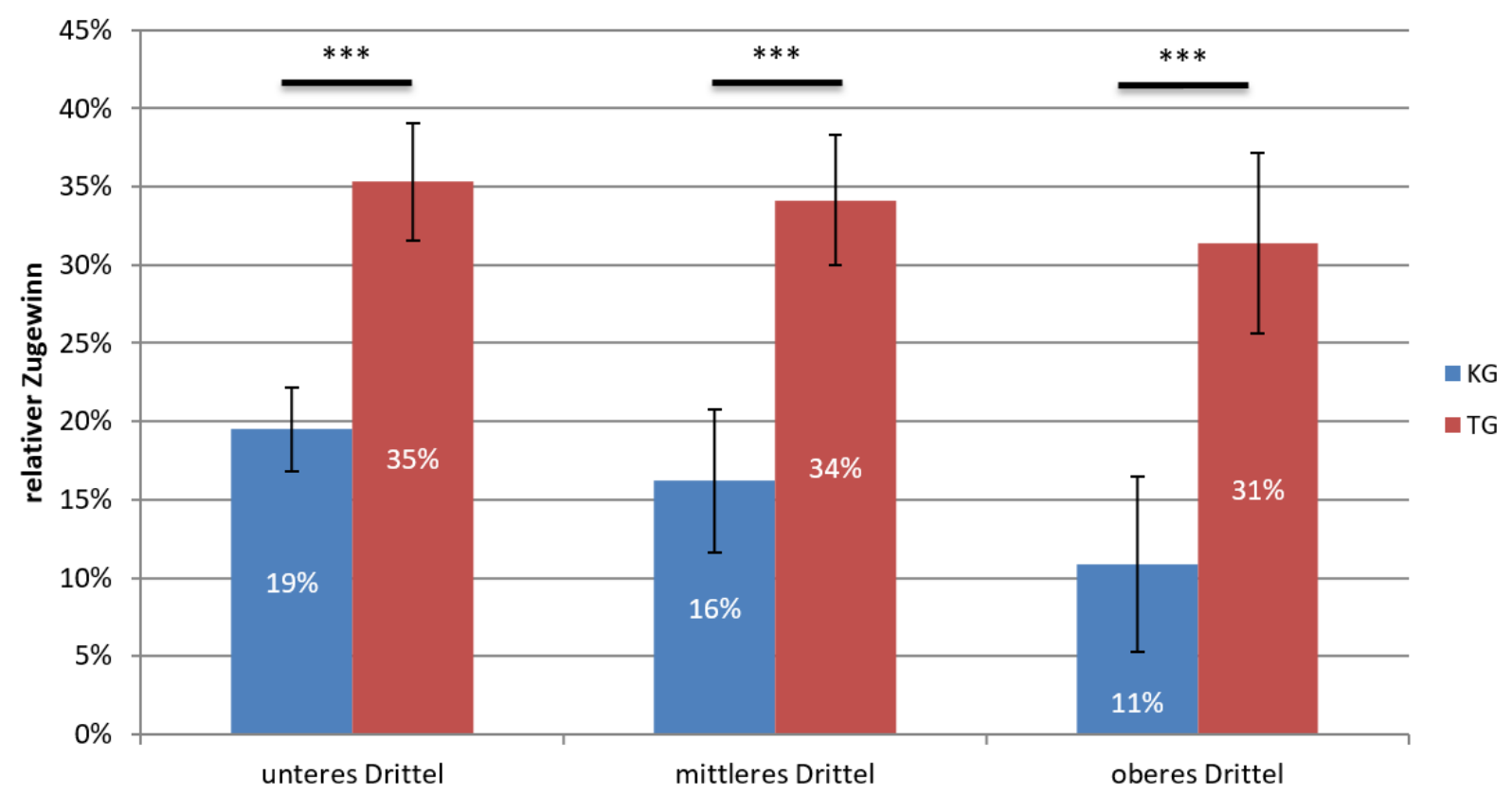

Abb. 97: Relativer Zugewinn nach Terzilen im Vortest mit 95\%-Konfidenzintervallen (Signifikanzniveaus: $* * * p<.001 ; * * p<.01 ; * p<.05$ )

Ein Vergleich des von den jeweiligen Terzilen einer Gruppe erreichten relativen Lernzuwachses mit Hilfe einer univariaten einfaktoriellen Varianzanalyse ergibt, dass sich die einzelnen Terzile der Treatmentgruppe nicht signifikant voneinander unterscheiden $(F(2,430)=.71, p=.490)$, wohingegen sich in der Kontrollgruppe signifikante Unterschiede offenbaren $(F(2,354)=4.84, p=.008)$. Eine genauere Analyse mit Hilfe eines GamesHowell Post-Hoc-Tests zeigt, dass das untere Drittel einen signifikant höheren relativen Lernzuwachs in der Kontrollgruppe erzielt als das obere Drittel $(p=.020)$, während der jeweilige Unterschied zum mittleren Drittel statistisch nicht signifikant ist.

Unabhängig von ihrem Vortestergebnis können die Schüler der Treatmentgruppe also nach dem Unterricht mehr als ein Drittel der Items korrekt beantworten, die sie vor dem Unterricht noch nicht korrekt beantworten konnten. Der relative Lernzuwachs ist also für alle Schüler in der Treatmentgruppe statistisch gesehen gleich hoch. In der Kontrollgruppe hingegen ist der relative Lernzuwachs insgesamt nicht nur halb so groß wie in der Treatmentgruppe, sondern hängt auch noch signifikant von dem Vortestergebnis ab. Je besser die Schüler im Vortest sind, desto geringer fällt ihr relativer Lernzuwachs in der Kontrollgruppe aus. Auch wenn alle Schüler unabhängig von ihrem Vortestergebnis deutlich vom neuen Unterrichtskonzept profitieren, trifft dies im Vergleich zum traditionellen Unterricht insbesondere für Schüler mit einem guten Vortestergebnis zu. Konkret zeigt das obere Drittel der Schüler der Kontrollgruppe einen nur etwa halb so großen relativen Lernzuwachs wie das untere Drittel. 
Auf einem ohnehin niedrigen Niveau bzgl. des erreichten relativen Lernzuwachses scheint der traditionelle Unterricht also insbesondere bei Schülern mit einem guten Vortestergebnis kaum dazu beizutragen, dass diese noch etwas dazulernen. Anzumerken ist hier auch, dass der sogenannte ,Matthäus-Effekt" nicht zu beobachten ist: Ein gutes Abschneiden im Vortest führt auch bei Betrachtung des relativen Zugewinns in Kontroll- und Treatmentgruppe nicht zu einem höheren Lernzuwachs. Zusätzlich zur beobachteten negativen Korrelation zwischen Pretest-Ergebnis und absolutem Zugewinn (vgl. Abschnitt 7.4.3.3) ist dies ein weiteres klares Indiz, dass der auch absolut höhere Lernzuwachs in der Treatmentgruppe nicht mit dessen im Vergleich zur Kontrollgruppe signifikant höheren Pretest-Ergebnis zu erklären ist.

\subsubsection{Kontrolle der Pretest-Unterschiede}

\subsubsection{Kontrolle der Pretest-Differenzen mittels Parallelisierung}

Neben der Betrachtung des relativen Zugewinns besteht eine weitere Möglichkeit zur Kontrolle der bereits vor der Intervention vorhandenen Pretest-Unterschieden zwischen den beiden untersuchten Gruppen in deren Parallelisierung oder der Bildung von sogenannten „,matched samples“. Bei der Parallelisierung wird eine annährend gleiche Verteilung der Störvariablen in beiden Gruppen angestrebt, so dass diese z.B. in Bezug auf das Pretest-Ergebnis übereinstimmen, indem aus den jeweiligen Stichproben gezielt entsprechende Schüler entfernt werden. Hierdurch sollen Unterschiede in Bezug auf das Pretest-Ergebnis als Ursache für unterschiedliche Lernzuwächse (weitgehend) ausgeschlossen werden. In der Folge können sich bei der Parallelisierung die beiden Gruppen bzgl. ihres Stichprobenumfangs unterscheiden. Das Matching stellt in gewisser Hinsicht eine Unterkategorie der Parallelisierung dar, weil hier bzgl. einer Störvariablen wie dem Pretest-Ergebnis identische Paare aus beiden Gruppen gebildet werden. In der Folge haben beide Stichproben dann den exakt gleichen Stichprobenumfang, Mittelwert und Streuung bzgl. der Störvariablen, weshalb man von einem „matched sample“ spricht. Durch die Anwendung dieser beiden Vorgehen wird allerdings die Datenstruktur der Stichprobe zwangsläufig verändert, worunter die externe Validität leidet (Bortz und Döring 2006, S. 527). In der vorliegenden Arbeit kamen drei Verfahren zum Einsatz:

1. Matching auf Basis gleicher Pretest-Werte durch Entfernung von Schülern mit extremen Lernfortschritten: Bei diesem Verfahren wird angestrebt, in Kontrollund Treatmentgruppe für jeden Pretest-Wert die gleiche Anzahl an Schülern zu haben (,matching“). Dies wird erreicht, indem aus jeder Gruppe von PretestWerten jeweils diejenigen Schüler der größeren Gruppe entfernt werden, die den größten bzw. kleinsten Lernfortschritt bei dem jeweiligen Pretest-Ergebnis erzielt haben. Auf diese Weise werden also Extremfälle ausgeschlossen und es wird sichergestellt, dass die Anzahl der Schüler in beiden Gruppen identisch ist. Durch das Matching reduziert sich die Größe der Gesamtstichprobe im vorliegenden Fall von 790 Schülern auf 646 Schüler im „matched sample“, die sich zu gleichen Teilen auf Kontroll- und Treatmentgruppe aufteilen. 
2. Matching auf Basis gleicher Pretest-Werte durch Entfernung von Schülern mit mittleren Lernfortschritten: Auch bei diesem Verfahren wird angestrebt, in Kontroll- und Treatmentgruppe für jeden Pretest-Wert die gleiche Anzahl an Schülern zu haben (,matching“). Dies wird jedoch erreicht, indem aus jeder Gruppe von Pretest-Werten jeweils diejenigen Schüler der größeren Gruppe entfernt werden, die sich bezogen auf den Lernfortschritt in der Mitte (Median) der Verteilung innerhalb einer Pretestwert-Gruppe befinden. Dieses Vorgehen führt also im Gegensatz zum in Punkt 1 beschriebenen Verfahren tendenziell zur Betrachtung von Schülern mit extrem hohen bzw. niedrigen Lernfortschritten innerhalb einer Pretestwert-Gruppe, führt aber auch zur Bildung von Gruppen mit der gleichen Anzahl an Schülern. Durch das Matching reduziert sich die Größe der Gesamtstichprobe im vorliegenden Fall von 790 Schülern auf 646 Schüler im „,matched sample“, die sich zu gleichen Teilen auf Kontrollund Treatmentgruppe aufteilen.

3. Parallelisierung auf Klassenebene: Hier wird Schritt für Schritt aus jeder Klasse der Kontrollgruppe derjenige Schüler mit dem schlechtesten Vortestergebnis und aus jeder Klasse der Treatmentgruppe derjenige Schüler mit dem besten Vortestergebnis entfernt, bis die beiden Gruppen in Bezug auf das durchschnittliche Vortestergebnis übereinstimmen. Der Vorteil der Parallelisierung gegenüber den beiden Matching-Verfahren besteht insbesondere darin, dass aus keiner Klasse überproportional viele Schüler entfernt werden und so die grundlegende Struktur der Stichprobe soweit wie möglich erhalten bleibt. Auch werden insgesamt weniger Schüler aus der Stichprobe entfernt. Durch die Parallelisierung reduziert sich die Größe der Gesamtstichprobe im vorliegenden Fall von 790 Schülern auf 697 Schüler, wovon sich 311 in der Kontrollgruppe und 386 in der Treatmentgruppe befinden.

Im Folgenden sind für die drei oben beschriebenen Verfahren die arithmetischen Mittel für Pre- und Posttest von Kontroll- und Treatmentgruppe inklusive ihres Standardfehlers $(S E)$ angegeben, da sich so prüfen lässt, ob die Mittelwerte im Rahmen ihrer statistischen Sicherheit miteinander vereinbar sind. Zusätzlich wird für jedes der Verfahren der von Bortz und Döring (2006, S. 560) vorgeschlagene Nettoeffekt des Treatments angegeben, der sich hier aufgrund identischer Pretest-Ergebnisse aus der Differenz der Posttest-Ergebnisse von Kontroll- und Treatmentgruppe berechnet. Sein Standardfehler ergibt sich entsprechend der Fehlerfortpflanzung aus der Summe der Standardfehler der Posttest-Ergebnisse von Kontrollund Treatmentgruppe. Analog zu Abschnitt 7.4.3.3 wurde auch hier die Signifikanz und die Effektstärke des Nettoeffekts des Treatments mittels einer univariaten zweifaktoriellen Varianzanalyse mit Messwiederholung (gemischtes Design) ermittelt. 
Kapitel 7: Summative Evaluation des Unterrichtskonzepts

Tab. 24: Ergebnisse der unterschiedlichen Matching- bzw. Parallelisierungsverfahren inklusive des jeweiligen Nettoeffekts des Treatments im Vergleich

\begin{tabular}{|c|c|c|c|c|c|}
\hline Verfahren & Gruppe & $\begin{array}{l}\text { Mittelwert } \\
\text { Pretest }\end{array}$ & $\begin{array}{l}S E \\
\text { Pretest }\end{array}$ & $\begin{array}{l}\text { Mittelwert } \\
\text { Posttest }\end{array}$ & $\begin{array}{l}S E \\
\text { Posttest }\end{array}$ \\
\hline \multirow{4}{*}{$\begin{array}{l}\text { 1) Matching: Gleiche Pretest- } \\
\text { Werte (ohne extreme Lern- } \\
\text { fortschritte) } \\
N=646 \text { Schüler }\end{array}$} & KG & 5.29 & 0.16 & 8.67 & 0.23 \\
\hline & TG & 5.29 & 0.16 & 12.20 & 0.26 \\
\hline & \multicolumn{3}{|c|}{ Nettoeffekt des Treatments: } & $3.53 * * *$ & 0.49 \\
\hline & \multicolumn{3}{|c|}{ Cohens $d$ bzgl. Nettoeffekt: } & .78 & \\
\hline \multirow{4}{*}{$\begin{array}{l}\text { 2) Matching: Gleiche Pretest- } \\
\text { Werte (ohne mittlere Lernfort- } \\
\text { schritte) } \\
N=646 \text { Schüler }\end{array}$} & KG & 5.29 & 0.16 & 8.75 & 0.26 \\
\hline & TG & 5.29 & 0.16 & 12.52 & 0.34 \\
\hline & \multicolumn{3}{|c|}{ Nettoeffekt des Treatments: } & $3.77 * * *$ & 0.60 \\
\hline & \multicolumn{3}{|c|}{ Cohens $d$ bzgl. Nettoeffekt: } & .70 & \\
\hline \multirow{4}{*}{$\begin{array}{l}\text { 3) Parallelisierung auf Klas- } \\
\text { senebene } \\
N=697 \text { Schüler }\end{array}$} & KG & 5.61 & 0.15 & 8.82 & 0.26 \\
\hline & TG & 5.61 & 0.14 & 12.63 & 0.28 \\
\hline & \multicolumn{3}{|c|}{ Nettoeffekt des Treatments: } & $3.81 * * *$ & 0.54 \\
\hline & \multicolumn{3}{|c|}{ Cohens $d$ bzgl. Nettoeffekt: } & .75 & \\
\hline
\end{tabular}

Anmerkung: $S E=$ Standardfehler; $* * * p<.001 ; * * p<.01 ; * p<.05$

Im Rahmen der statistischen Sicherheit ist der Nettoeffekt aller drei Verfahren mit dem auf Basis der unveränderten Stichprobe ermittelten Nettoeffekt von 3.16 Punkten $(S E=0.50)$ vereinbar. Ebenso wie der in Kontroll- und Treatmentgruppe erzielte relative Lernzuwachs deutet auch dieses Ergebnis darauf hin, dass der höhere Lernzuwachs der Treatmentgruppe nicht auf die bereits vor der Intervention vorhandenen Pretest-Differenzen zurückzuführen ist. In Abb. 98 ist der absolute Lernfortschritt der Parallelisierung auf Klassenebene dargestellt, da durch dieses Verfahren die grundlegende Struktur der ursprünglichen Stichprobe am wenigsten verändert wird. Die Entwicklung der Werte illustriert eindrucksvoll, wie die Schüler der Treatmentgruppe bei vergleichbaren Leistungen im Vortest einen deutlich höheren Lernzuwachs erzielen als ihre traditionell unterrichteten Peers in der Kontrollgruppe. 


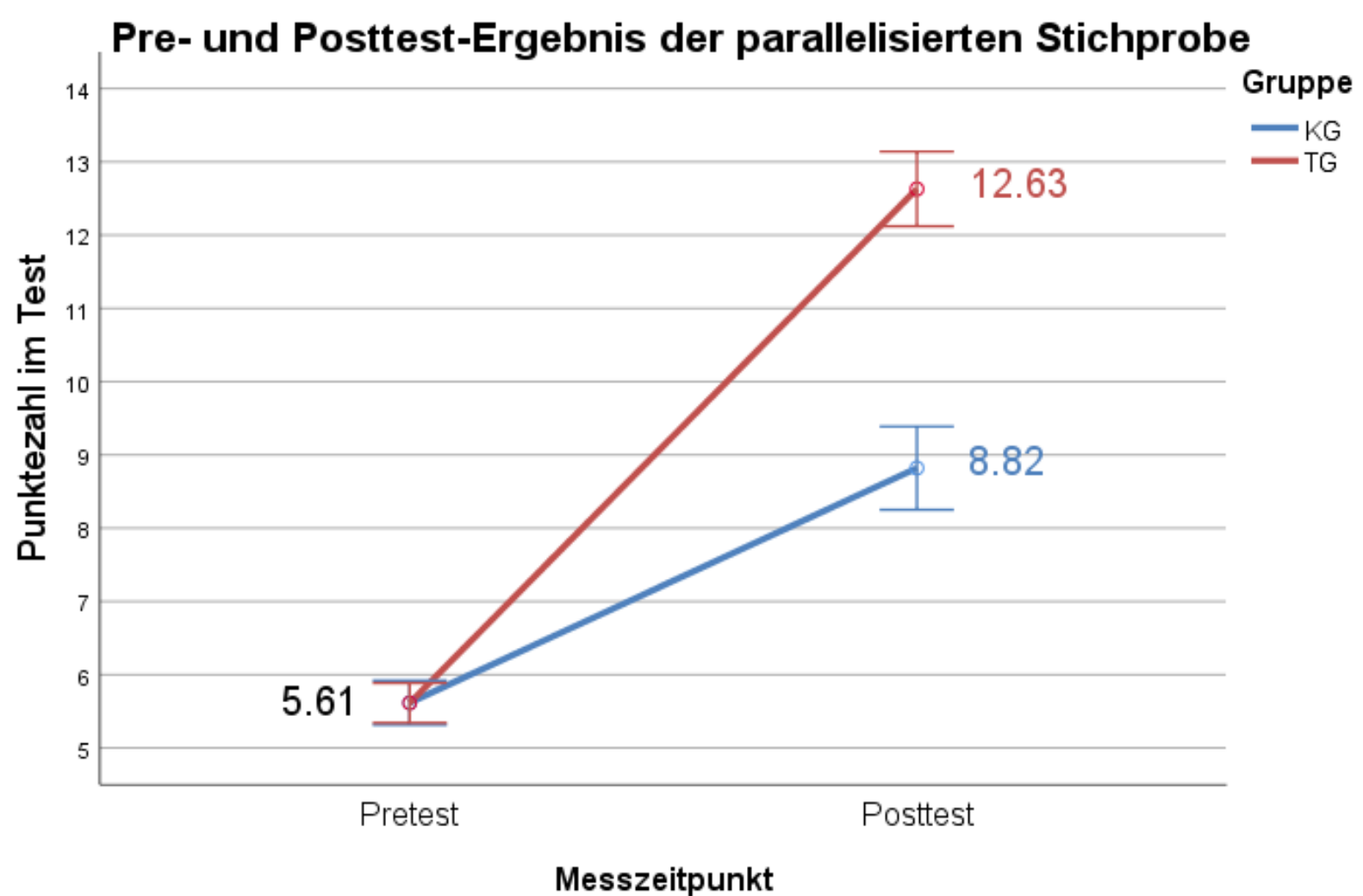

Abb. 98: Die Lernentwicklung in der parallelisierten Stichprobe mit 95\%-Konfidenzintervallen

Die Kontrolle der Pretest-Unterschiede zwischen Kontroll- und Treatmentgruppe mit Hilfe von Parallelisierung oder Matching ist jedoch insofern problematisch, als dass dieses Verfahren im vorliegenden Fall aufgrund von Regressionseffekten tendenziell zu einer Überschätzung des Nettoeffekts des Treatments führt (Bortz und Döring 2006, S. 557). Dies liegt daran, dass sich in der Treatmentgruppe aufgrund der Parallelisierung tendenziell mehr Schüler befinden, die per Zufall überdurchschnittlich schlecht im Pretest abgeschnitten haben, weil die Schüler mit gutem Pretest-Ergebnis entfernt wurden, um die durchschnittlichen PretestErgebnisse von Kontroll- und Treatmentgruppe anzugleichen. Bei wiederholter Testung ist deswegen alleine aufgrund von Regressionseffekten davon auszugehen, dass der Mittelwert der parallelisierten Treatmentgruppe ansteigt. Der durch den Unterricht erzielte Zugewinn wird aufgrund der Parallelisierung in der Treatmentgruppe in der Folge tendenziell überschätzt. Genau andersherum sieht es hingegen in der Kontrollgruppe aus, wo sich aufgrund der Parallelisierung tendenziell mehr Schüler befinden, die per Zufall überdurchschnittlich gut im Pretest abgeschnitten haben, weil hier die Schüler mit niedrigem Pretest-Ergebnis entfernt wurden. Bei wiederholter Messung ist deshalb damit zu rechnen, dass in der parallelisierten Kontrollgruppe alleine aufgrund von Regressionseffekten der Mittelwerkt absinkt. Der durch den Unterricht erzielte Zugewinn wird aufgrund der Parallelisierung in der Kontrollgruppe in der Folge tendenziell unterschätzt. Zusammengenommen ist deshalb davon auszugehen, dass der Nettoeffekt bei der Kontrolle der Pretest-Unterschiede mittels Parallelisierung oder Matching überschätzt wird. Diese regressionsbedingte Überschätzung des Nettoeffekts dürfte aber teils durch die in Abschnitt 7.4.3.3 gezeigte negative Korrelation von Pretest-Ergebnis und Zugewinn kompensiert werden. 


\subsubsection{Kontrolle der Pretest-Unterschiede mittels Kovarianzanalyse}

Ein statistisches Verfahren, das diese auf Regressionseffekte zurückgehenden Schwierigkeiten vermeidet, stellt die Kovarianzanalyse (ANCOVA) dar (vgl. Abschnitt 7.3.5.4). Der Vorteil der Kovarianzanalyse besteht insbesondere darin, dass das Pretest-Ergebnis der einzelnen Schüler als Kovariate berücksichtigt und somit kontrolliert werden kann, was eine adäquatere Schätzung des Nettoeffekts des Treatments ermöglicht. Da die Kovarianzanalyse das PretestErgebnis kontrolliert, soll im Folgenden der Nettoeffekt des Treatments über die Differenz der mittleren Posttest-Ergebnisse von Kontroll- und Treatmentgruppe ermittelt werden, wobei zunächst die Voraussetzungen dieser statistischen Methode zu prüfen sind:

Prüfung der Voraussetzungen

Zusätzlich zu den Voraussetzungen einer Varianzanalyse, die für die vorliegende Stichprobe schon in Abschnitt 7.4.3.3 geprüft wurden, werden an eine Kovarianzanalyse zwei weitere Anforderungen gestellt (Field 2011, 397ff):

1) Homogenität der Regressionssteigungen: Wie anhand der parallel verlaufenden Regressionsgeraden in der unteren Darstellung zu erkennen ist, kann von einer annähernden Homogenität der Regressionsgeraden in Kontroll- und Treatmentgruppe ausgegangen werden. Dies bedeutet, dass das Posttest-Ergebnis in beiden Gruppen in gleichem Maße vom Pretest-Ergebnis als Kovariate abhängt.

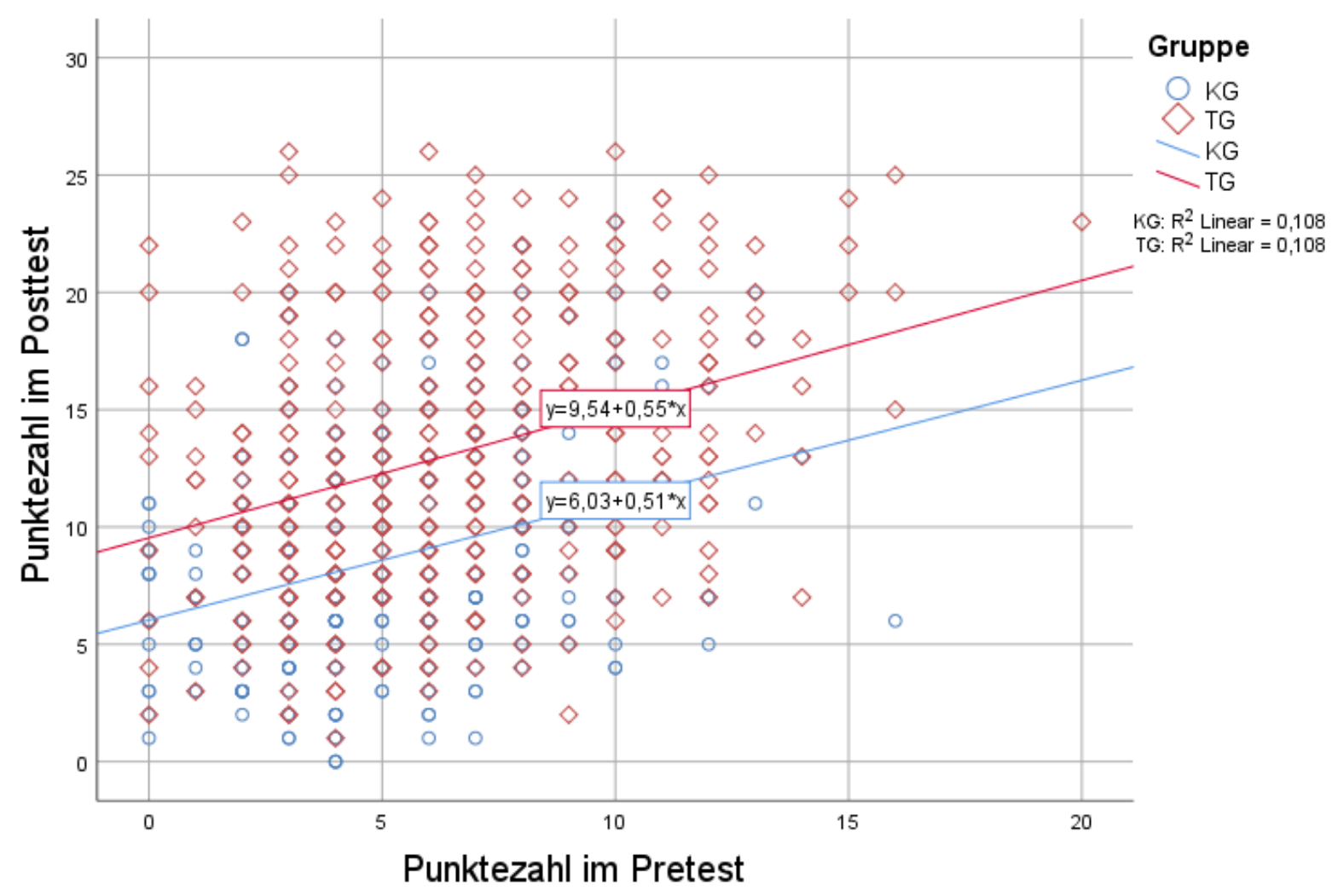

Abb. 99: Regressionsgeraden der Kontroll- und Treatmentgruppe bzgl. der Kovariate „Pretest“ und der abhängigen Variable „Posttest“" 
2) Unabhängigkeit der Kovariate vom Gruppeneffekt: Wie in Abschnitt 0 bereits gezeigt, unterscheidet sich das Pretest-Ergebnis und damit die hier verwendete Kovariate zwischen Kontroll- und Treatmentgruppe signifikant (KG: 5.09 Punkte; TG: 6.29 Punkte). Damit ist strenggenommen die Unabhängigkeit der Kovariate vom Gruppeneffekt nicht gegeben. Nach Weber (2009, S. 506) ist die Durchführung einer ANCOVA in einem solchen Fall dennoch angemessen, wenn eine ausreichende Überlappung der Kovariate, d.h. hier der Pretest-Werte beider Gruppen, vorliegt. Anders als in Fällen, die typischerweise in der Literatur kritisiert werden, bei denen nahezu keine Überlappung der Kovarianzwerte zwischen den verschiedenen Gruppen existiert und die Kovariate somit völlig von der jeweiligen Gruppe abhängt (Miller und Chapman 2001), deckt die Kovariate im vorliegenden Fall in beiden Gruppen nahezu das gleiche Spektrum an Pretest-Werten ab und ist vergleichsweise wenig von der Kontroll- oder Treatmentgruppe abhängig (vgl. Abb. 100). Weil durch die Kovariate „Pretest“ damit nur ein kleiner Teil der Varianz des Posttest-Ergebnisses aufgeklärt wird, der eigentlich auf die Gruppenzugehörigkeit zurückgeht, führt die Verletzung der Voraussetzung tendenziell zu einer Unterschätzung des Nettoeffekts des Treatments (Field 2011, 397f). Da gleichzeitig durch die Kontrolle des Pretest-Ergebnisses aber eine deutlich bessere Schätzung der Größe des Nettoeffekts infolge einer besseren Aufklärung der Residualvarianz innerhalb der Gruppen zu erwarten ist, erscheint die Durchführung der Kovarianzanalyse hier nicht nur akzeptabel, sondern sinnvoll.

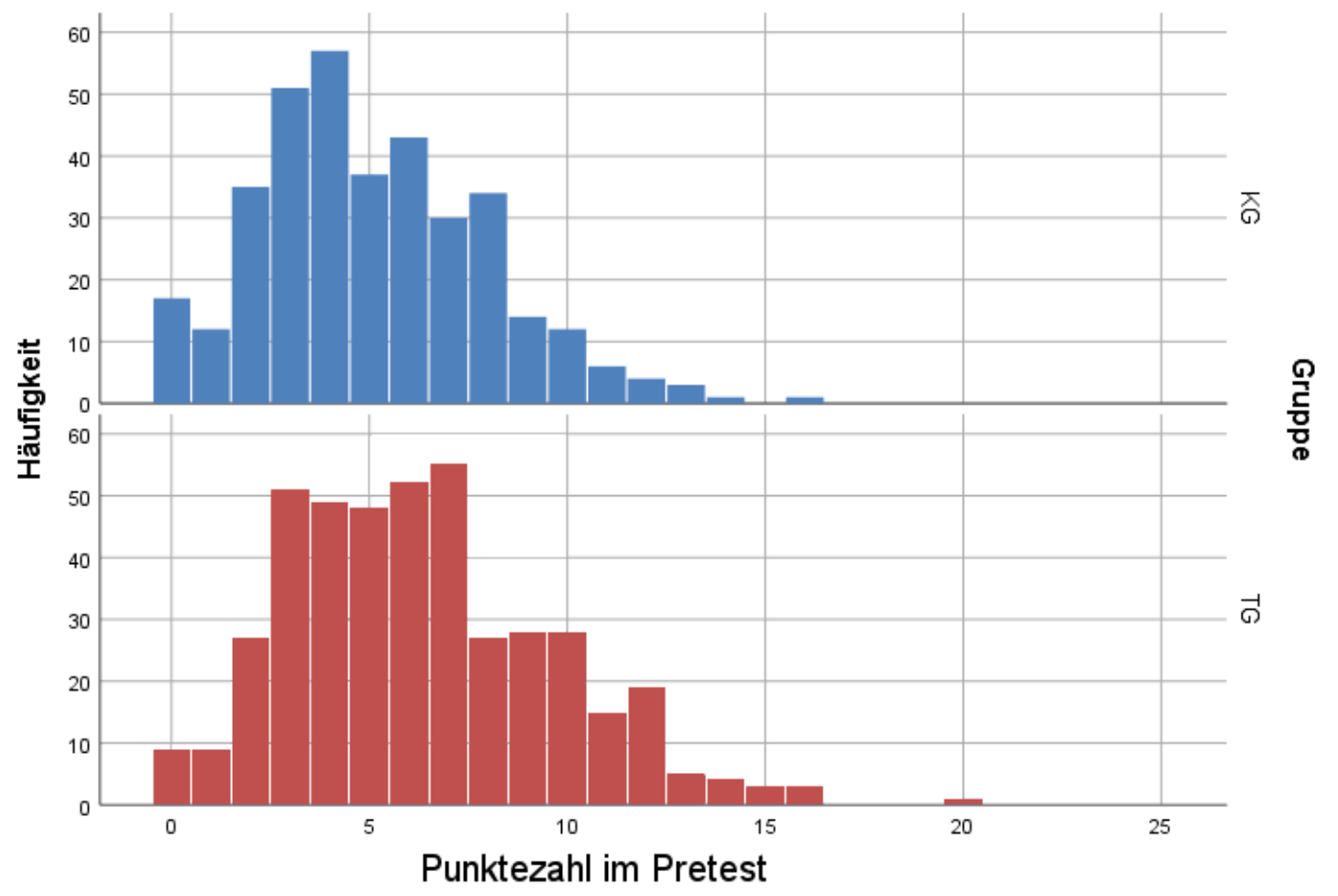

Abb. 100: Vergleich der Verteilung der Kovariate „Pretest“ in Kontroll- und Treatmentgruppe

Die Kovarianzanalyse zeigt, dass sich Kontroll- und Treatmentgruppe bzgl. des durchschnittlich erzielten Posttest-Ergebnisses unter Kontrolle des Pretest-Ergebnisses höchst signifikant unterscheiden $\left(F(1,787)=111.12, p<.001, \eta^{2}=.124\right)$. Wie in Abb. 101 dargestellt, 
ergibt sich unter der Kontrolle des Pretest-Ergebnisses für die Treatmentgruppe ein durchschnittlich erzielter Posttest-Wert von 12.70 Punkten $(S E=0.23)$, wohingegen die Schüler der Kontrollgruppe hier im Durchschnitt lediglich 8.99 Punkte $(S E=0.26)$ erreichten.

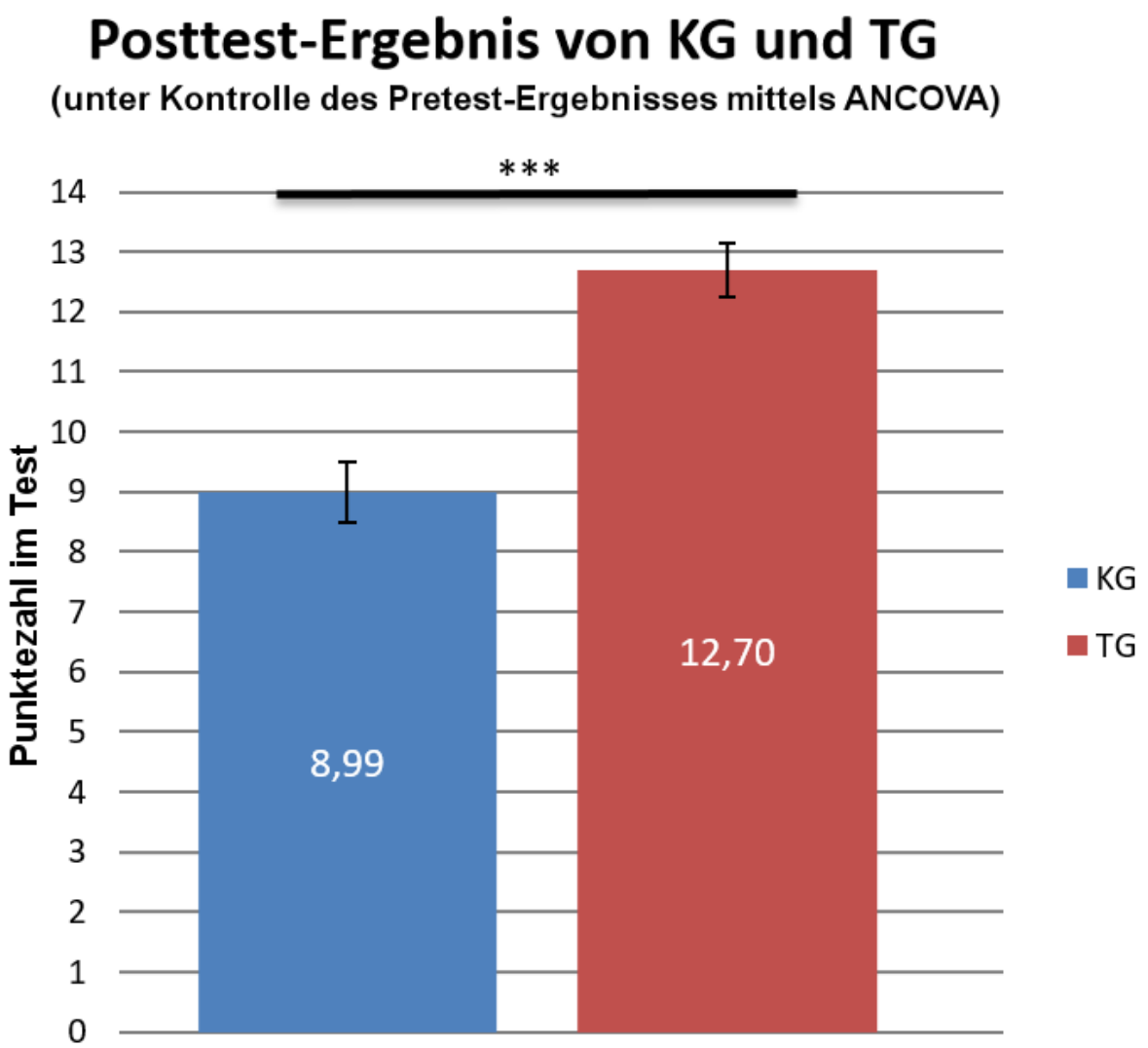

Abb. 101: Posttest-Ergebnis in Kontroll- und Treatmentgruppe unter Kontrolle des Pretest-Ergebnisses mittels ANCOVA und 95\%-Konfidenzintervallen (Signifikanzniveaus: $* * * p<.001 ; * * p<.01 ; * p<.05$ )

Für den Nettoeffekt des Treatments ergibt sich somit ein Wert von 3.71 Punkten $(S E=0.49)$, was entsprechend der in der Kovarianzanalyse für diesen Unterschied angegebenen Effektstärke von $\eta^{2}=.124$ nach Cohen (1988, S. 276) einer mittleren Effektstärke von $d=.75$ entspricht. Da die Kovariate „Pretest“ nicht völlig unabhängig von der Gruppenzugehörigkeit ist, kann davon ausgegangen werden, dass der hier ermittelte Nettoeffekt des Treatments sowie die dazugehörige Effektstärke tendenziell zu gering ausfallen, weil die Kovariate ein Teil der Varianz des Posttest-Ergebnisses aufklärt, der eigentlich auf die Gruppenzugehörigkeit zurückgeht (Field 2011, 397f).

\subsubsection{Vergleich von KG und TG bei gleichen Lehrkräften}

In der vorliegenden Studie gab es in der Kontroll- und Treatmentgruppe eine Schnittmenge von zwei Lehrkräften, die ihre Klassen einmal traditionell und einmal nach dem neuen Konzept unterrichteten. Während eine Lehrkraft mit jeweils zwei Kontroll- und zwei Treatmentklassen an der Studie teilnahm, unterrichtete eine weitere Lehrkraft jeweils eine Kontroll- und eine Treatmentklasse. Diese Untermenge von jeweils drei Klassen in Kontroll- und Treatmentgruppe ist aufgrund der hohen Bedeutung der individuellen Lehrerkompetenz auf den Lernerfolg der Schüler von besonderem Interesse (Hattie 2013), da in der restlichen Studie 
nicht auszuschließen ist, dass sich die Lehrkräfte zwischen Kontroll- und Treatmentgruppe z.B. in Bezug auf Erfahrung oder Lehrkompetenz systematisch unterscheiden. Wenn beide Gruppen hingegen von den gleichen Lehrkräften unterrichtet werden, erscheinen solche alternativen Erklärungsansätze bzgl. des unterschiedlichen Lernzuwachses in beiden Gruppen jedoch weniger plausibel, wenn auch nicht undenkbar (vgl. u.a. „Compensatory Rivalry“ und „Treatment Diffusion“ in Bortz und Döring 2006, S. 503). Die höhere interne Validität der Untersuchung dieser Teilstichprobe geht allerdings aufgrund des nun geringeren Stichprobenumfangs von insgesamt nur noch 133 Schülern (wovon sich 68 Schüler in der Kontrollgruppe und 65 Schüler in der Treatmentgruppe befinden) zu Lasten ihrer externen Validität. Die im Rahmen der Analyse dieser Teilstichprobe gefundenen Ergebnisse stellen deshalb entweder ein Indiz dafür dar, dass die bisherigen Ergebnisse möglicherweise doch auf unterschiedliche durchschnittliche Lehrerkompetenzen in den beiden Untersuchungsgruppen zurückzuführen sind, oder können als Hinweis darauf verstanden werden, dass die Lehrerkompetenzen in Kontroll- und Treatmentgruppe vergleichbar und die bisherigen Ergebnisse daher valide und verallgemeinerbar sind.

Analog zu Abschnitt 7.4.3.3 soll deshalb im Folgenden mit Hilfe einer univariaten zweifaktoriellen Varianzanalyse mit Messwiederholung (gemischtes Design) geprüft werden, ob sich die absoluten Lernfortschritte der oben beschriebenen Teilstichprobe in Kontroll- und Treatmentgruppe signifikant unterscheiden.

Prüfung der Voraussetzungen nach Rasch et al. (2010, S. 71):

1) Intervallskalierung der abhängigen Variablen: Der absolute Lernzuwachs wird in beiden Gruppen intervallskaliert gemessen. Die Voraussetzung ist deshalb erfüllt.

2) Normalverteilung der Messwerte in allen Teilstichproben: Wie den untenstehenden Histogrammen zu entnehmen ist, kann in allen Teilstichproben trotz einer gewissen Schiefe bei manchen Verteilungen eine ausreichende Normalverteilung angenommen werden. 
a. Pre- und Posttest der Kontrollgruppe

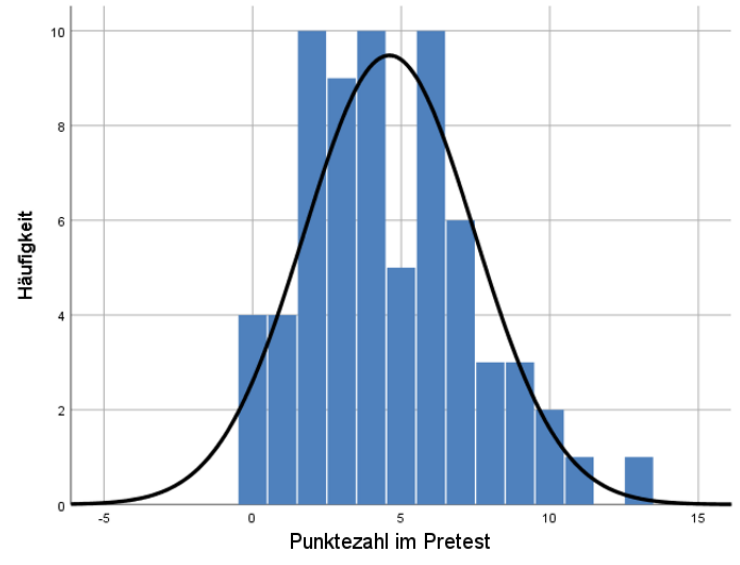

Abb. 102: Histogramm zur Prüfung der Normalverteilung der Pretest-Ergebnisse der Kontrollgruppe

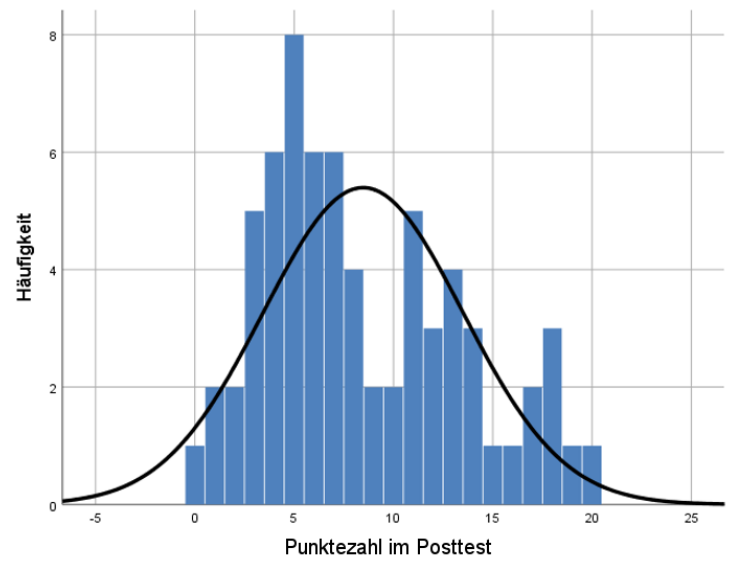

Abb. 103: Histogramm zur Prüfung der Normalverteilung der Posttest-Ergebnisse der Kontrollgruppe

\section{b. Pre- und Posttest der Treatmentgruppe}

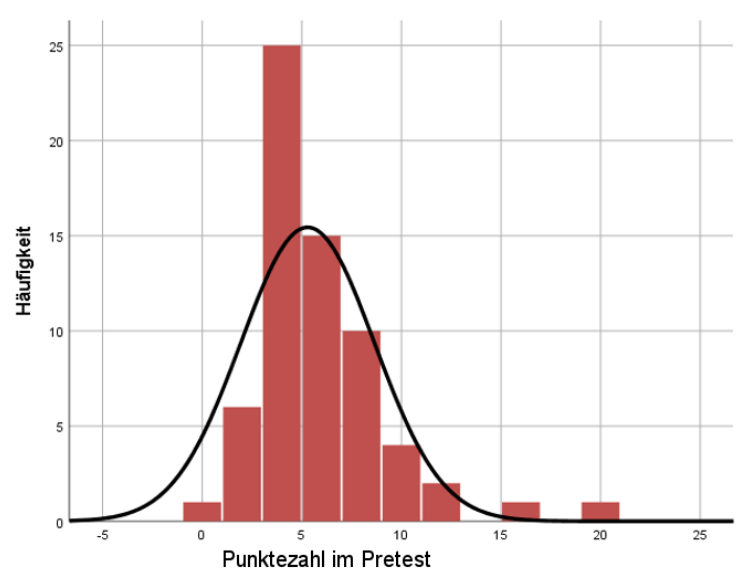

Abb. 104: Histogramm zur Prüfung der Normalverteilung der Pretest-Ergebnisse der Treatmentgruppe

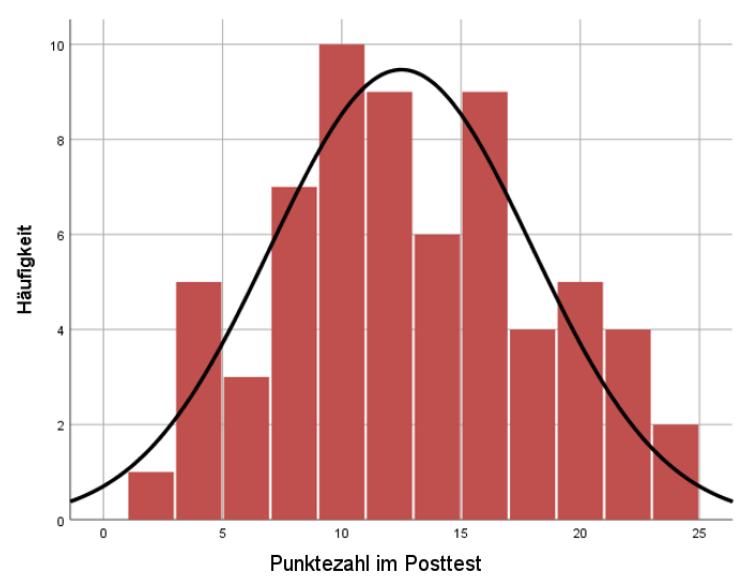

Abb. 105: Histogramm zur Prüfung der Normalverteilung der Posttest-Ergebnisse der Treatmentgruppe

3) Varianzhomogenität: Die Prüfung der Varianzhomogenität zwischen den Stufen des Zwischen-Subjekt-Faktors (hier: Treatment, d.h. KG und TG) innerhalb jeder Stufe des Inner-Subjekt-Faktors (hier: Messzeitpunkt, d.h. Pre- bzw. Posttest) erfolgt mittels eines Levene-Tests. Dieser ergibt, dass sowohl bzgl. des Pretest-Ergebnisses $(F(1,131)=.04, p=.839)$ als auch bzgl. des Posttest-Ergebnisses $(F(1,131)=.37$, $p=.545)$ zwischen Kontroll- und Treatmentgruppe Varianzhomogenität besteht und diese Bedingung deshalb erfüllt ist.

4) Sphärizität: Die Voraussetzung ist erfüllt, da der Inner-Subjekt-Faktor nur zwei Stufen (d.h. zwei Messzeitpunkte) hat und die Sphärizität, also die Homogenität aller Varianzen der Differenzen zweier Faktorstufen, somit automatisch gegeben ist (Rasch et al. 2010, S. 72). 
Die univariate zweifaktorielle Varianzanalyse mit Messwiederholung ergibt, dass sich die hier untersuchte Untermenge von Kontroll- und Treatmentgruppe bzgl. des durchschnittlich erzielten absoluten Lernzuwachses höchst signifikant unterscheiden, da ein höchst signifikanter Interaktionseffekt zwischen Gruppen- und Messwiederholungsfaktor vorliegt $\left(F(1,131)=14.88, p<.001, \eta^{2}=.102\right)$. Der vorliegende Interaktionseffekt ist dabei so zu verstehen, dass sich der über die beiden Messzeitpunkte stattfindende Lernzuwachs in beiden Gruppen signifikant voneinander unterscheidet. Dieser Umstand spiegelt sich auch in den unterschiedlichen Steigungen von Kontroll- und Treatmentgruppe in Abb. 106 wider.

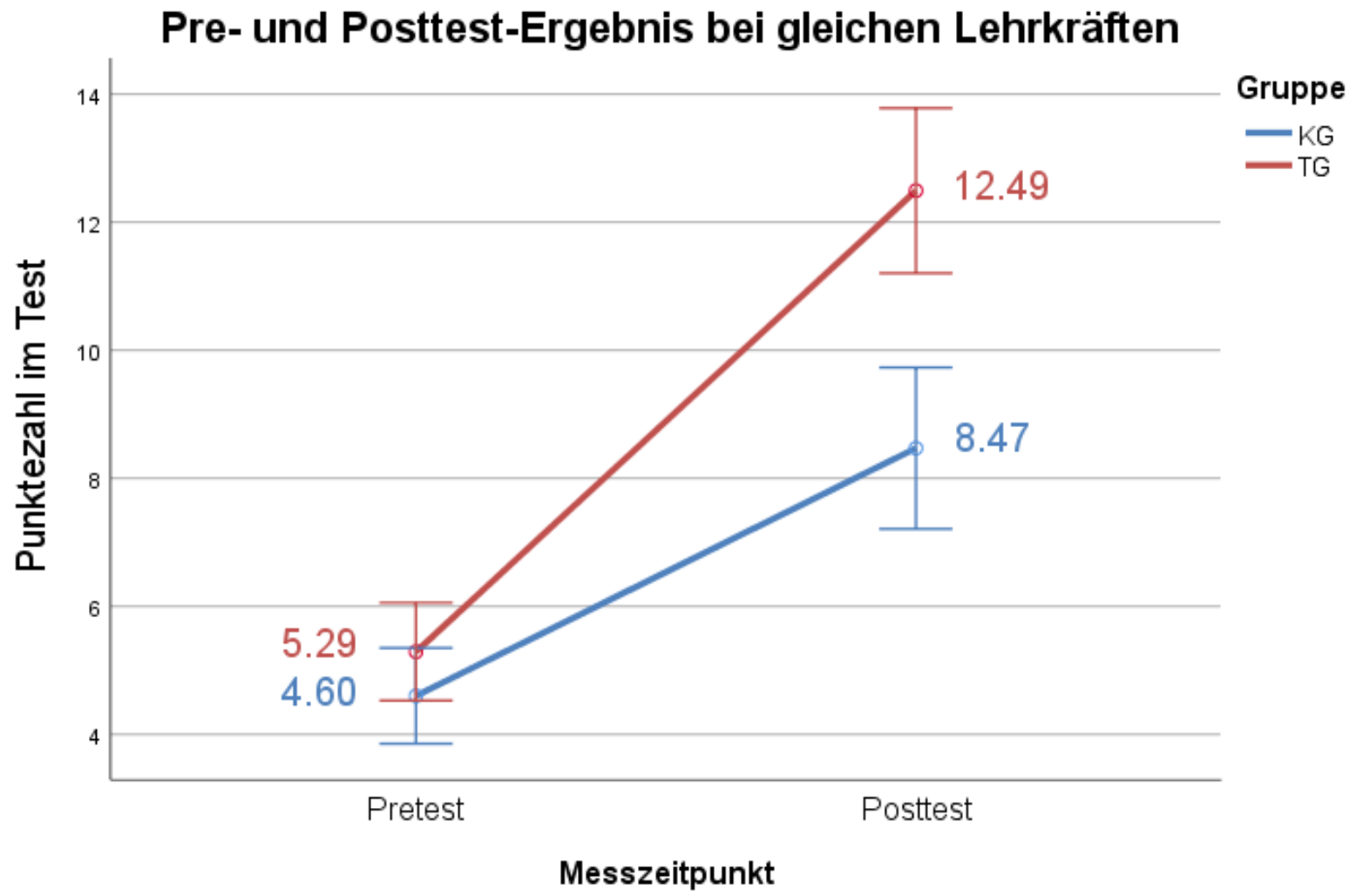

Abb. 106: Die Lernentwicklung bei gleichen Lehrkräften in Kontroll- und Treatmentgruppe mit 95\%-Konfidenzintervallen

Die hier durchgeführte Varianzanalyse zeigt, dass der absolute Lernzuwachs in der Treatmentgruppe mit 7.20 Punkten $(S E=0.68)$ höchst signifikant höher ausfällt als in der Kontrollgruppe, wo er lediglich 3.87 Punkte $(S E=0.55)$ beträgt. Im Gegensatz zur Gesamtstichprobe unterscheiden sich Kontroll- und Treatmentgruppe entsprechend eines zweiseitigen $t$-Tests für unabhängige Stichproben in der hier untersuchten Teilstichprobe zudem nicht signifikant bzgl. ihrer Leistungen im Vortest $(t(131)=1.28, p=.204)$. Der von Bortz und Döring (2006, S. 560) vorgeschlagene, zu berechnende Nettoeffekt des neuen Unterrichtskonzepts beträgt in der vorliegenden Untermenge der Gesamtstichprobe 3.33 Punkte ( $S E=1.23)$. Im Rahmen der statistischen Sicherheit ist der Nettoeffekt mit dem auf Basis der gesamten Stichprobe ermittelten Nettoeffekt von 3.16 Punkten $(S E=0.50)$ vereinbar. Die im Rahmen der Varianzanalyse für diesen Unterschied gefundene Effektstärke von $\eta^{2}=.102$ gibt dabei den Anteil der durch den Gruppeneffekt aufgeklärten Varianz auf Stichprobenebene an (Rasch et al. 2010, S. 74). In dieser Stichprobe werden also 10\% der Variabilität des absoluten Lernzu- 
wachses alleine über die Gruppenzugehörigkeit der Schüler erklärt. Nach Cohen (1988, S. 276) lässt sich dieses Effektstärkemaß im hier verwendeten Studiendesign in ein Cohens $d$ überführen, was im vorliegenden Fall einem Wert von $d=.67$ und somit einem mittleren Effekt entspricht (vgl. Abschnitt 7.4.3.3).

Die Tatsache, dass der hier gefundene Nettoeffekt bei vergleichbaren PretestErgebnissen statistisch mit dem auf Basis der gesamten Stichprobe ermittelten Nettoeffekt übereinstimmt, deutet darauf hin, dass die Gesamtstichprobe und die hier untersuchte Teilstichprobe mit identischen Lehrkräften in Kontroll- und Treatmentgruppe miteinander vergleichbar sind. Dies würde insbesondere bedeuten, dass der höhere Lernzuwachs der Treatmentgruppe nicht auf systematische Unterschiede zwischen den unterrichtenden Lehrkräften z.B. in Bezug auf Erfahrung oder Lehrkompetenz zurückzuführen ist. Da also sowohl die Analyse der Teilstichprobe mit höherer interner, aber geringerer externer Validität als auch die Analyse der Gesamtstichprobe mit geringerer interner, aber höherer externer Validität zu einem statistisch gesehen identischen Ergebnis kommen, kann davon ausgegangen werden, dass der gefundene Nettoeffekt des Treatments nicht auf Unterschiede bzgl. der Lehrerkompetenz oder anderen Selektionseffekten bei den Lehrkräften zurückzuführen ist.

\subsubsection{Der Lernzuwachs auf Klassenebene}

In der bisherigen Analyse der Daten wurden die Kontroll- und Treatmentgruppe als homogene Stichproben betrachtet und jeweils in ihrer Gesamtheit miteinander verglichen. Da es sich um eine quasi-experimentelle Feldstudie handelt, wurden die Schüler nicht völlig randomisiert auf die beiden Gruppen aufgeteilt und einheitlich unterrichtet, sondern im Klassenverbund von unterschiedlichen Lehrkräften instruiert. Im Folgenden soll daher ein genauerer Blick auf den in diesen Klassen erzielten absoluten und relativen Lernzuwachs geworfen werden, indem die individuellen Leistungen der Schüler zur durchschnittlichen Leistung der jeweiligen Klasse aggregiert werden.

\subsubsection{Vergleich des absoluten Lernzuwachses}

Abb. 107 zeigt den absoluten Zugewinn jeder einzelnen Klasse der Kontrollgruppe (blau) und der Treatmentgruppe (rot). Wie unmittelbar ersichtlich wird, fällt der mittlere absolute Zugewinn sowohl in der Kontroll- als auch in der Treatmentgruppe äußerst heterogen aus. Nichtsdestotrotz ist klar ersichtlich, dass die Klassen der Treatmentgruppe insgesamt deutlich höhere absolute Lernzuwächse zu verzeichnen haben als die Klassen der Kontrollgruppe. Während der höchste von einer Klasse im Durchschnitt erzielte absolute Zugewinn in der Kontrollgruppe bei lediglich 7.00 Punkten liegt, beträgt er in der Treatmentgruppe 12.41 Punkte. Ein bemerkenswertes Ergebnis ist ferner, dass nur eine einzige Kontrollklasse knapp den durchschnittlichen absoluten Lernzuwachs der Treatmentgruppe erreicht hat, während alle anderen Kontrollklassen darunterliegen. Eindrucksvoll ist in diesem Zusammenhang auch, dass demgegenüber 8 der 19 Klassen der Treatmentgruppe einen höheren absoluten Lernzuwachs erzielen konnten als die beste Klasse der Kontrollgruppe. Eine Klasse der Kontrollgruppe er- 
zielte sogar einen negativen absoluten Lernzuwachs; zeigte also im Posttest schlechtere Leistungen als im Pretest.

Aufgrund des innerhalb ihrer eigenen Gruppe vergleichsweise schlechten Abschneidens wurde versucht, bei den Lehrkräften der Klassen 1, 2, 3 sowie 18, 19 und 20 in Erfahrung zu bringen, welche Gründe sie für das jeweilige Ergebnis sehen. Trotz mehrfacher Rückfragen antworteten in der Kontrollgruppe nur die Lehrkräfte der Klassen 2 und 3 und in der Treatmentgruppe nur die Lehrkraft der Klasse 18. Die Lehrkraft von Klasse 2 sieht die Gründe für das schlechte Abschneiden ihrer Klasse vor allem darin, dass sie ,[...] die Klasse von einer pensionierten Lehrerin übernahm [und] die Schülerinnen und Schüler also vorher nicht kannte“ sowie der Tatsache, dass sie zusätzlich zum eigentlichen Unterricht noch mit Aufgaben in der Bildungsverwaltung beschäftigt war, was bei ihr zu ,[...] einer dauerhaften Überlastung $[. . .]^{\text {“ }}$ führte und auch die Unterrichtsvorbereitung erschwerte ${ }^{13}$. Die Lehrkraft von Klasse 3 führt den geringen Lernzuwachs darauf zurück, dass es sich schlicht um ,[...] eine besonders leistungsschwache Klasse (5 Schüler wurden nicht versetzt!)“ gehandelt habe. In der Treatmentgruppe erklärte die Lehrkraft von Klasse 18, dass in dieser Klasse der Posttest erst vier Monate nach Beendigung der Unterrichtsreihe durchgeführt wurde. Den Grund für das schlechte Abschneiden der Klasse sieht die Lehrkraft deshalb darin, dass ,[...] einigen SuS viele gelernte Dinge nicht mehr so präsent waren“" und nicht im Unterrichtskonzept selber, das ,[...] für dievSuS [sic] sehr lehrreich [war]. Die durchaus anspruchsvolle Klausur am Ende der Unterrichtsreihe ist auch gut ausgefallen"14.

\footnotetext{
${ }^{13}$ Die Antwort der Lehrkraft im Wortlaut: ,[...] die Abstimmung meines Unterrichtes in der Klasse auf deren Lernbedürfnisse war meines Erachtens in dem Schuljahr dadurch beeinträchtigt, dass 1) ich die Klasse von einer pensionierten Lehrerin übernahm, die Schülerinnen und Schüler also vorher nicht kannte; 2) ich selber das Schuljahr zuvor nur wenig und an einer anderen Schule unterrichtet hatte und gleichzeitig in die Bildungsverwaltung abgeordnet war. Die Wiederaufnahme des Unterrichts war leider nicht durch eine Entlastung für die Aufgaben in der Bildungsverwaltung begleitet. Ich befand mich also in einer dauerhaften Überlastung, die die Vorbereitung des Unterrichts erschwerte, und in einer Physiksammlung, die mir fremd geworden war. [...]“ (E-Mail erhalten am 20. Oktober 2017)

${ }^{14}$ Die Antwort der Lehrkraft im Wortlaut: „Guten Abend, ihre Unterrichtsreihe war für dievSuS sehr lehrreich. Die durchaus anspruchsvolle Klausur am Ende der Unterrichtsreihe ist auch gut ausgefallen. Das eher schwächere Abschneiden im Diagnosetest, das sie ansprechen ist jedoch vorhersehbar gewesen, weil wir die Unterrichtsreihe in Halbjahr 1 durchgeführt haben, der abschließende Diagnosetest aber leider erst über 4 Monate später bearbeitet worden ist, nachdem die SuS schon andere thematisch komplett verschiedene Unterrichtsreihen durchgegangen sind. Daher war mir bereits klar, dass einigen SuS viele gelernte Dinge nicht mehr so präsent waren.“ (E-Mail erhalten am 25. Juni 2017)
} 


\section{Absoluter Zugewinn nach Klassen}
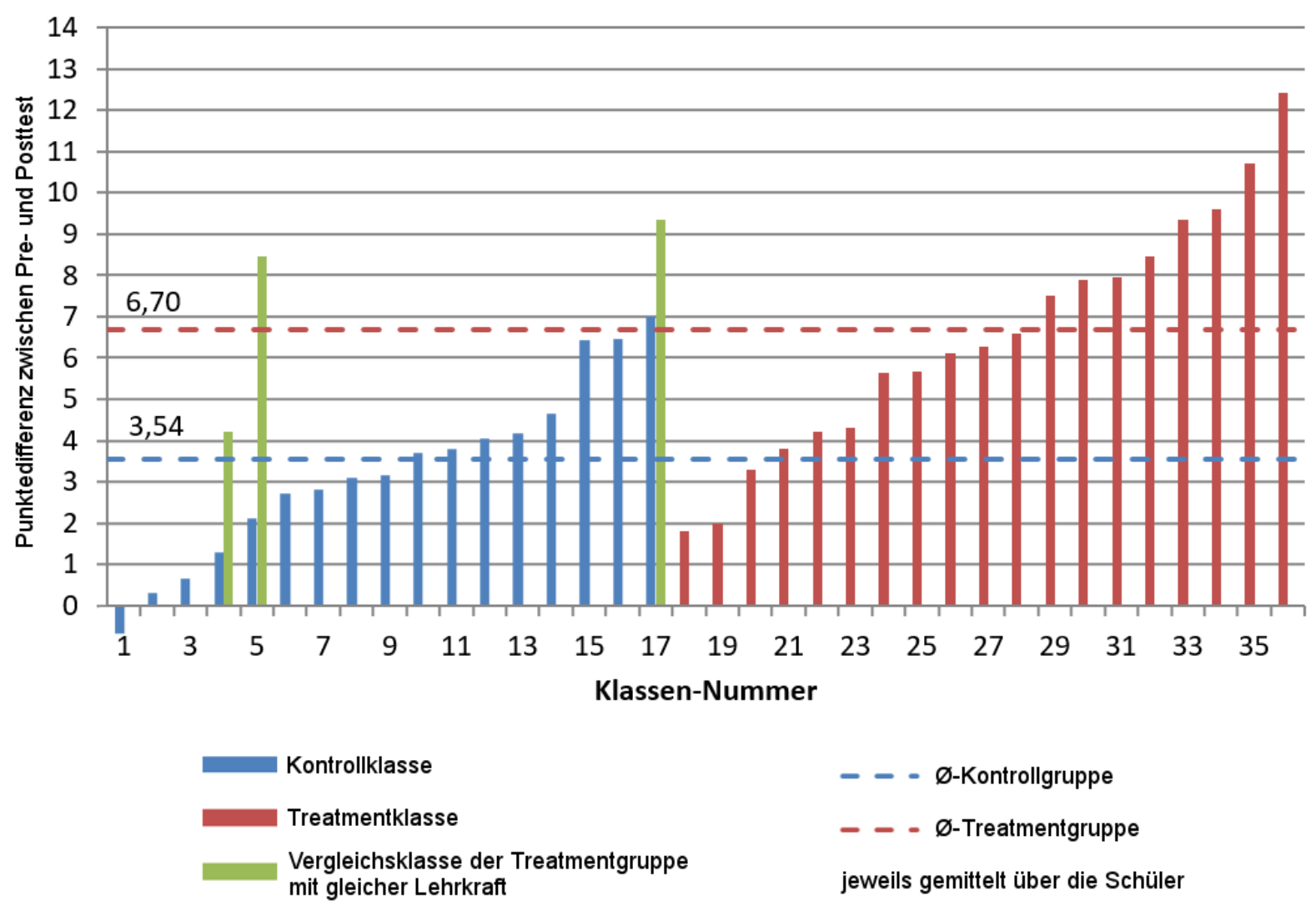

Abb. 107: Absoluter Zugewinn der Kontrollklassen (blau) und Treatmentklassen (rot). Die grünen Säulen zeigen bei drei Kontrollklassen den absoluten Lernfortschritt einer von der gleichen Lehrkraft unterrichteten Treatmentklasse. Die gestrichelten Linien geben das arithmetische Mittel des von den Schülern in KG bzw. TG erzielten absoluten Lernzuwachses an.

In der Regel wurden Kontroll- und Treatmentklassen von unterschiedlichen Lehrkräften unterrichtet, jedoch gab es zwei Lehrkräfte (Lehrkraft A: K4 und K5; Lehrkraft B: K17), die ihre Klassen einmal klassisch und einmal nach dem neuen Unterrichtskonzept unterrichteten. Wie an Hand der grünen Säulen in Abb. 107 zu sehen ist, konnten diese Lehrkräfte bei all ihren Klassen einen deutlich höheren absoluten Lernzuwachs erzielen, wenn sie diese nach dem in dieser Arbeit vorgestellten Unterrichtskonzept unterrichteten. Ein einseitiger $t$-Test für unabhängige Stichproben ergibt, dass die Unterschiede in allen drei Fällen statistisch signifikant sind $(\mathrm{K} 4: t(38.61)=2.26, p=.015 ; \mathrm{K} 5: t(35)=5.13, p<.001 ; \mathrm{K} 17: t(49)=1.76$, $p=.042)$.

Lehrkraft A teilte auf Anfrage per E-Mail ${ }^{15}$ mit, dass sie den höheren Lernzuwachs in ihren Treatmentklassen auf das neue Unterrichtskonzept und das „Druckluft-Modell“ zurück-

\footnotetext{
${ }^{15}$ Die E-Mail der Lehrkraft im Wortlaut: „[...] Aktuell habe ich 2 Klassen G9, die klassisch von anderen Kollegen unterrichtet wurden. Auch diese Klassen signalisieren mir ganz deutlich, dass sie jetzt endlich begreifen, wie die Zuteilung der Spannungen, Stromstärken und Widerstände in der Reihen- und Parallelschaltung funktioniert. Man hat den Eindruck, den Kindern fallen regelrecht die Schuppen von den Augen. :-) Mittlerweile habe ich das Konzept auch in 2 Realschulklassen (R10) getestet und stelle auch dort ein verbessertes Verständnis fest. Immerhin ist es auch schon bei den HR-Fachausbildern angekommen, denn mein LiV hat mir berichtet, dass sie das Modell auch im Seminar diskutiert haben. Das Druckluft-Modell ist meiner Meinung nach eine echte Hilfe und ich hoffe, dass auch die Verlage das Konzept letztlich in den Schulbüchern aufgreifen." (E-Mail erhalten am 11. September 2017)
} 
führt, das in ihren Augen ,eine echte Hilfe“ sei, weil ihre Schüler nun endlich begriffen, „[...] wie die Zuteilung der Spannungen, Stromstärken und Widerstände in der Reihen- und Parallelschaltung funktioniert. Man hat den Eindruck, den Kindern fallen regelrecht die Schuppen von den Augen." Die Gründe für den sehr unterschiedlichen Lernzuwachs ihrer beiden Treatmentklassen sah Lehrkraft A vor allem darin, dass die auch im Test schlechter abschneidende Klasse allgemein leistungsschwächer sei und es in dieser Klasse noch zu zusätzlichen Stundenausfällen und anderen Unterbrechungen kam $^{16}$.

Bemerkenswert ist insbesondere, dass auch Lehrkraft B mit ihrer Treatmentklasse einen signifikant höheren Lernzuwachs erzielen konnte, obwohl sie bereits mit ihrer Kontrollklasse den höchsten absoluten Lernzuwachs aller Kontrollklassen erreicht hat. Um mehr über den Unterricht dieser aus Sicht der Studie interessanten Lehrkraft in Kontroll- und Treatmentgruppe in Erfahrung zu bringen und insbesondere herauszufinden, wie sich die Lehrkraft den höheren Lernerfolg ihrer Treatmentklasse erklärt, fand am 13. März 2017 ein kurzes Interview in der Universität statt, zu dem sich Lehrkraft B freundlicherweise bereiterklärt hatte. Hier gab Lehrkraft B zu Protokoll, das Thema Elektrizitätslehre in beiden Klassen in einem Umfang von 34 Stunden unterrichtet zu haben, wobei sie in der Kontrollgruppe auf verschiedene Analogien zurückgegriffen hat, weil nach ihrer eigenen Erfahrung nicht jede Analogie von jedem Schüler in gleichem Maße angenommen wird. Während die elektrische Stromstärke in Analogie zur Wasserstromstärke oder einer Autobahn eingeführt wurde, nutzte die Lehrkraft zur Illustration des elektrischen Widerstands das Bild einer verengten Fahrbahn, ohne dies im Unterricht jedoch weiter zu vertiefen. Die elektrische Spannung wurde am Modell des offenen Wasserstromkreises erklärt, bei dem die elektrische Spannung einem Höhenunterschied entspricht. Auf die Frage, wie sich die Lehrkraft den hohen Lernerfolg ihrer Kontrollklasse erklärt, antwortete diese, dass es sich um eine sehr lernstarke Klasse mit ehrgeizigen Schülern gehandelt habe, die ein gutes naturwissenschaftliches Grundverständnis mitgebracht hätten. Insgesamt sei die Kontrollklasse nach Einschätzung der erfahrenen und engagierten Lehrkraft leistungsstärker gewesen als die Treatmentklasse, die allerdings einen noch höheren Lernerfolg erzielen konnte. Den Grund für diesen noch höheren Lernerfolg der Treatmentklasse sieht auch Lehrkraft B in dem ,gut strukturierten und logisch aufgebauten Unterrichtskonzept", das die Spannung sehr gut einführe und sehr gute Unterrichtsmaterialien, insbesondere Arbeitsblätter, enthalte.

\subsubsection{Vergleich des relativen Lernzuwachses}

Im Folgenden soll nun auch der von den einzelnen Klassen erzielte relative Lernzuwachs betrachtet werden. Dieser ist in Abb. 108 für jede einzelne Klasse der Kontrollgruppe (blau) und

\footnotetext{
${ }^{16}$ Die E-Mail der Lehrkraft im Wortlaut: „Die 8d ist generell leistungsschwächer als die 8e. Bei Vergleichsarbeiten schneidet sie in allen Fächern fast immer schlechter ab als die Vergleichslerngruppe. Auch scheint die Pubertät in der 8d heftiger zuzuschlagen (Hirnmixer...). Insofern überrascht mich das Ergebnis gar nicht. Ungünstig war auch, dass ich Stundenausfälle durch andere Termine und Unterbrechungen durch Ferien bei dieser Lerngruppe hatte, die ich bei der $8 \mathrm{e}$ nicht hatte. Ansonsten habe ich das Konzept gleichermaßen durchgearbeitet. Ich denke also, das insgesamt unterschiedliche Leistungsniveau ist die Haupt-Ursache." (E-Mail erhalten am 27. Mai 2016)
} 
der Treatmentgruppe (rot) dargestellt. Ähnlich wie der absolute Zugewinn fällt auch der mittlere relative Zugewinn sowohl in der Kontroll- als auch in der Treatmentgruppe sehr heterogen aus. Nichtsdestotrotz wird aus der Verteilung des relativen Lernzuwachses unmittelbar ersichtlich, dass die Klassen der Treatmentgruppe insgesamt deutlich höhere relative Lernzuwächse zu verzeichnen haben als die Klassen der Kontrollgruppe. Während der höchste von einer Klasse im Durchschnitt erzielte relative Zugewinn in der Kontrollgruppe bei lediglich knapp 35\% liegt, beträgt er in der Treatmentgruppe knapp über 60\%. Ein bemerkenswertes Ergebnis ist auch hier, dass nur eine einzige Kontrollklasse knapp den durchschnittlichen relativen Lernzuwachs der Treatmentgruppe erreicht hat, während alle anderen Kontrollklassen teils weit darunterliegen. Eindrucksvoll ist in diesem Zusammenhang auch, dass demgegenüber 9 der 19 Klassen der Treatmentgruppe einen höheren relativen Lernzuwachs erzielen konnten als die beste Klasse der Kontrollgruppe. Entsprechend ihres bereits negativen absoluten Lernzuwachses fällt für eine Kontrollklasse auch der durchschnittlich erzielte relative Lernzuwachs negativ aus.

\section{Relativer Zugewinn nach Klassen}

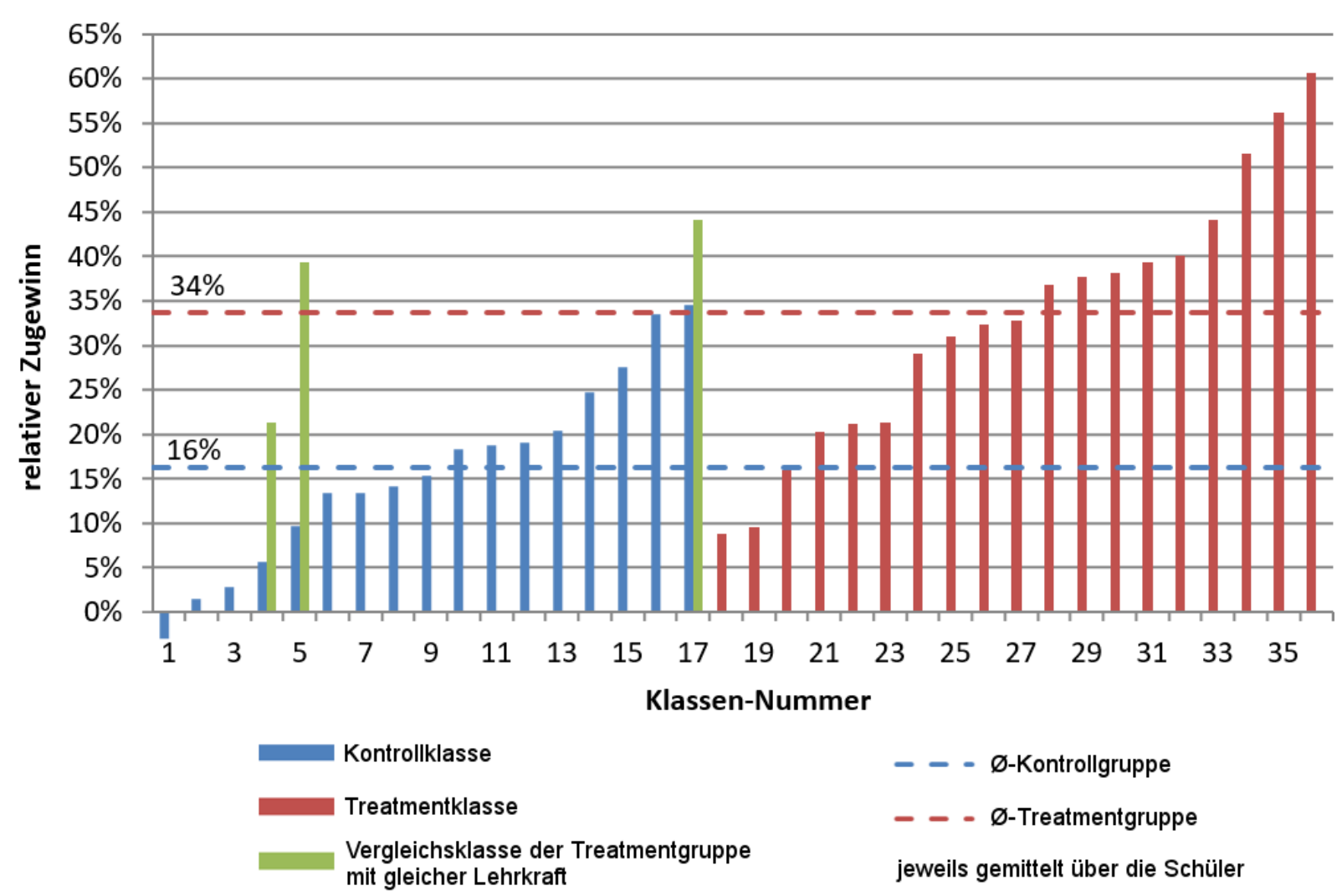

Abb. 108: Relativer Zugewinn der Kontrollklassen (blau) und Treatmentklassen (rot). Die grünen Säulen zeigen bei drei

Kontrollklassen den relativen Lernfortschritt einer von der gleichen Lehrkraft unterrichteten Treatmentklasse. Die gestrichelten Linien geben das arithmetische Mittel des von den Schülern in KG bzw. TG erzielten relativen Lernzuwachses an.

Wie bereits erwähnt, gab es zwei Lehrkräfte (Lehrkraft A: K4 und K5; Lehrkraft B: K17), die ihre Klassen einmal klassisch und einmal nach dem neuen Unterrichtskonzept auf Basis des Elektronengasmodells unterrichteten. Wie an Hand der grünen Säulen in Abb. 108 zu sehen ist, konnten diese Lehrkräfte bei all ihren Klassen auch einen deutlich höheren rela- 
tiven Lernzuwachs erzielen, wenn sie diese nach dem neuen Unterrichtskonzept auf Basis des Elektronengasmodells unterrichteten. Ein einseitiger $t$-Test für unabhängige Stichproben ergibt, dass die Unterschiede in allen drei Fällen statistisch signifikant sind (K4: $t(36.10)=2.63, p=.006$; K5: $t(35)=5.00, p<.001$; K17: $t(48)=2.02, p=.025)$. Eine Diskussion möglicher Ursachen für diese Unterschiede findet sich in Abschnitt 7.4.7.1.

\subsubsection{Darstellung des Lernzuwachses mittels Hake-Plot}

Eine weitere Möglichkeit der grafischen Darstellung der erzielten Lernzuwächse auf Klassenebene besteht in der Nutzung eines Hake-Plots, bei dem die Differenz des prozentualen Anteils korrekter Items zwischen Vor- und Nachtest einer Klasse über ihr prozentuales Vortestergebnis aufgetragen wird. Der Vorteil dieser Darstellungsform besteht u.a. darin, dass sich die unterschiedlichen Klassen von Kontroll- und Treatmentgruppe unter Berücksichtigung des Pretest-Ergebnisses hinsichtlich ihres erzielten relativen Zugewinns vergleichen lassen.

\section{Hake-Plot auf Klassenebene}

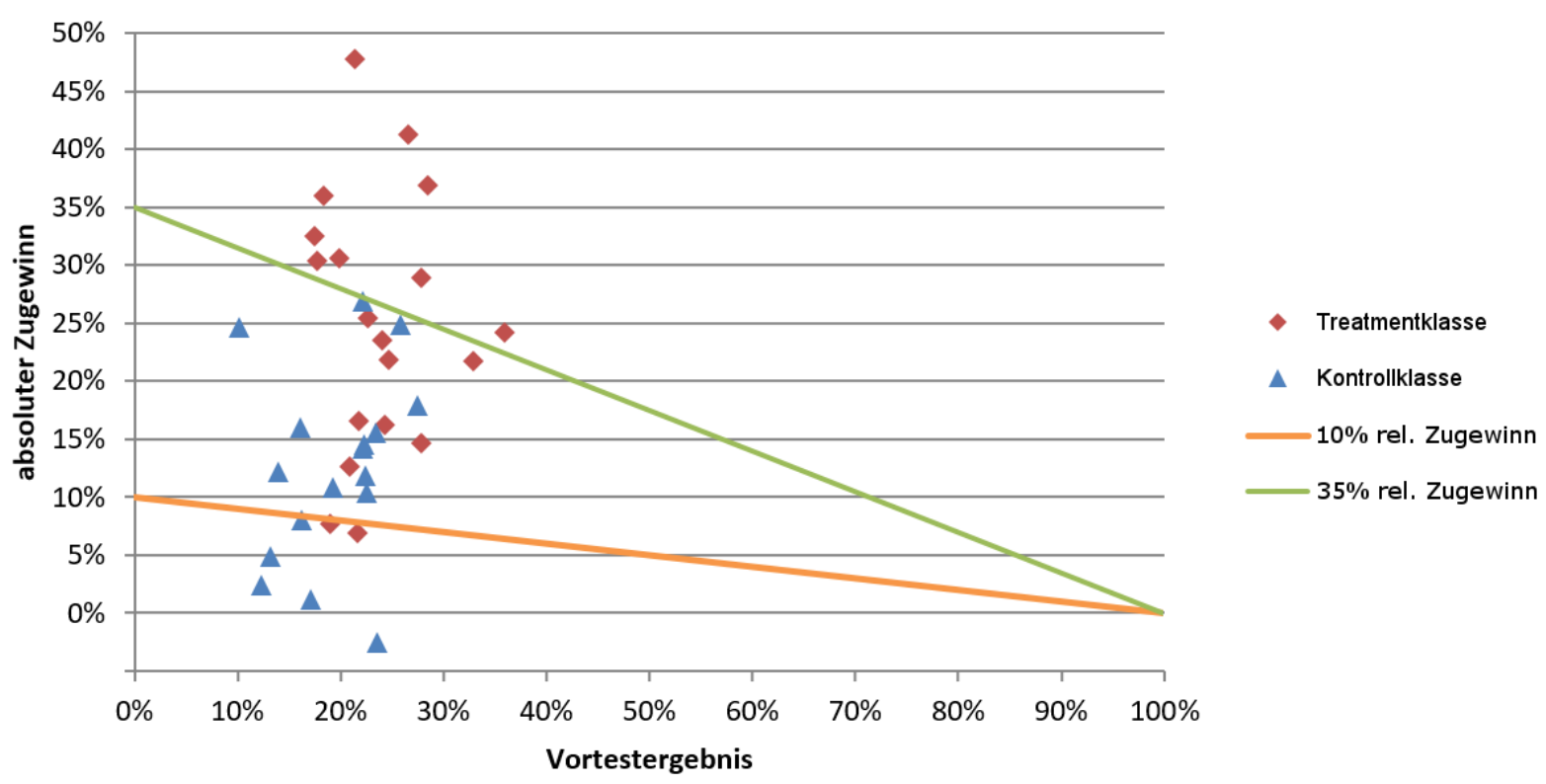

Abb. 109: Darstellung des Hake-Plots auf Klassenebene (Kontrollgruppe: blau; Treatmentgruppe: rot). Die orangefarbene Linie entspricht einem relativen Zugewinn von $10 \%$ und die grüne Linie einem relativen Zugewinn von $35 \%$.

In Abb. 109 entspricht die orangefarbene Linie einem relativen Zugewinn von 10\% und die grüne Linie einem relativen Zugewinn von 35\%. Klassen, die einen relativen Zugewinn erzielt haben, der über $35 \%$ liegt, sind im Hake-Plot deshalb oberhalb dieser grünen 35\%-Linie zu finden, während Klassen, die einen relativen Zugewinn erzielt haben, der unter $10 \%$ liegt, sich unterhalb der orangefarbenen 10\%-Linie befinden. Wie man dem Hake-Plot entnehmen kann, erzielen in dieser Studie fünf Klassen der Kontrollgruppe einen relativen Zugewinn von deutlich weniger als $10 \%$, während etwa die Hälfte der Klassen der Treatmentgruppe mit mehr als 35\% einen höheren relativen Zugewinn als die beste Klasse der Kontrollgruppe erzielt. 


\subsubsection{Analyse der Schülervorstellungen}

\subsubsection{Welcher Anteil der Lernenden zeigte welche Schülervorstellung?}

Aufgrund der Zweistufigkeit des eingesetzten Testinstruments konnten ferner auch die Schülervorstellungen der Schüler erhoben werden. Ein Vergleich der nach dem jeweiligen Unterricht noch vorhandenen Schülervorstellungen zeigt, dass in der Treatmentgruppe sieben von zehn Schülervorstellungen signifikant geringer ausgeprägt sind als in der Kontrollgruppe und bei den restlichen drei Schülervorstellungen kein signifikanter Unterschied feststellbar ist. In Hinblick auf das mit dem hier zu evaluierenden Unterrichtskonzept verbundene Ziel, den Schülern ein besseres konzeptionelles Verständnis von elektrischen Stromkreisen zu ermöglichen, ist dies grundsätzlich ein äußerst erfreuliches Ergebnis. Im Folgenden sollen zunächst die Ergebnisse zu den auf Basis des Tests von Urban-Woldron und Hopf (2012) erhobenen Schülervorstellungen vorgestellt werden, bevor anschließend die Ergebnisse der auf Basis eigener Items erhobenen Vorstellungen zur Spannung dargelegt werden (vgl. Abschnitt 7.3.4).

Tab. 25: Übersicht über die im Posttest identifizierten Schülervorstellungen (geordnet nach Signifikanzniveau). Signifikanzwerte unter $5 \%$ sind fett hervorgehoben.

\begin{tabular}{|l|l|l|l|l|l|}
\hline & \multicolumn{2}{|c|}{$\begin{array}{c}\text { Kontroll- } \\
\text { gruppe }\end{array}$} & \multicolumn{2}{c|}{$\begin{array}{c}\text { Treatment- } \\
\text { gruppe }\end{array}$} & \\
\hline Schülervorstellung & $M$ & SE & $M$ & SE & Sig. \\
\hline $\begin{array}{l}\text { Konzeptionelle Schwierigkeiten mit der } \\
\text { elektrischen Spannung (KS) }\end{array}$ & $92 \%$ & $2 \%$ & $60 \%$ & $3 \%$ & $\boldsymbol{p}<.001$ \\
\hline Stromverbrauchsvorstellung (SV) & $48 \%$ & $3 \%$ & $29 \%$ & $3 \%$ & $\boldsymbol{p}<.001$ \\
\hline Stromstärke ist unabhängig von R (UR) & $35 \%$ & $3 \%$ & $20 \%$ & $2 \%$ & $\boldsymbol{p}<.001$ \\
\hline Erfassen von Parallelschaltungen (PS) & $85 \%$ & $2 \%$ & $72 \%$ & $2 \%$ & $\boldsymbol{p}<.001$ \\
\hline Batterie als konstante Stromquelle (BS) & $39 \%$ & $4 \%$ & $25 \%$ & $3 \%$ & $\boldsymbol{p}=.001$ \\
\hline Sequentielle Argumentation (SA) & $46 \%$ & $4 \%$ & $33 \%$ & $3 \%$ & $\boldsymbol{p}=. \mathbf{0 0 7}$ \\
\hline Stromverbrauch ist proportional zu R (PR) & $52 \%$ & $3 \%$ & $43 \%$ & $3 \%$ & $\boldsymbol{p}=. \mathbf{0 2 8}$ \\
\hline Lokales Denken (LD) & $84 \%$ & $3 \%$ & $77 \%$ & $3 \%$ & $p=.111$ \\
\hline Spannung als Eigenschaft des Stroms (UI) & $60 \%$ & $4 \%$ & $54 \%$ & $3 \%$ & $p=.257$ \\
\hline Inverse Widerstandsvorstellung (IR) & $14 \%$ & $2 \%$ & $16 \%$ & $2 \%$ & $p=.462$ \\
\hline
\end{tabular}

Anmerkung: $M=$ Mittelwert; $S E=$ Standardfehler

Eine Übersicht über die genauen Werte, wie häufig welche Schülervorstellung in Kontroll- und Treatmentgruppe nach dem jeweiligen Unterricht identifiziert werden konnte, findet sich in Tab. 25. Die Signifikanzniveaus wurden dabei mittels Chi-Quadrat-Test berechnet. Eine genaue Darlegung, wie die einzelnen Schülervorstellungen erhoben wurden, findet sich in Abschnitt 7.3.4 und eine Diskussion der internen Konsistenz der den erhobenen Schülervorstellungen zugrundeliegenden Subskalen findet sich in Abschnitt 7.4.1.6. Es sei an dieser Stelle noch einmal darauf hingewiesen, dass hier entsprechend des Kodiermanuals von Urban-Woldron und Hopf (2012) eine Schülervorstellung bereits dann als vorhanden gewertet wird, wenn bei einem von mehreren der jeweiligen Subskala zugrundeliegenden Items eine 
entsprechende Antwortkombination gewählt wurde (vgl. Abschnitt 7.3.4 und 7.4.1.6). Ein Wert von $M=48 \%$ bedeutet also beispielsweise, dass $48 \%$ der Schüler mindestens einmal die entsprechende Schülervorstellung im Test vertraten.

Schüler, die nach dem neuen Unterrichtskonzept unterrichtet wurden, vertreten im Vergleich zu ihren traditionell unterrichteten Peers signifikant seltener die Schülervorstellung, dass der Strom verbraucht werden würde, die Stromstärke unabhängig vom Widerstand sei, dass die Batterie eine konstante Stromquelle und dass der Stromverbrauch proportional zum Widerstand sei. Auch haben in der Treatmentgruppe signifikant weniger Schüler Probleme mit dem Erfassen von Parallelschaltungen sowie der sequentiellen Argumentation. Keine signifikanten Unterschiede zwischen den beiden Gruppen konnten hingegen hinsichtlich des lokalen Denkens und der inversen Widerstandsvorstellung festgestellt werden. Abb. 110 gibt einen Überblick über die Häufigkeit aller erhobenen Schülervorstellungen, wobei diese entsprechend der Signifikanzniveaus der gefundenen Unterschiede zwischen Kontroll- und Treatmentgruppe geordnet sind.

\section{Schülervorstellungen nach dem Unterricht in KG und TG}

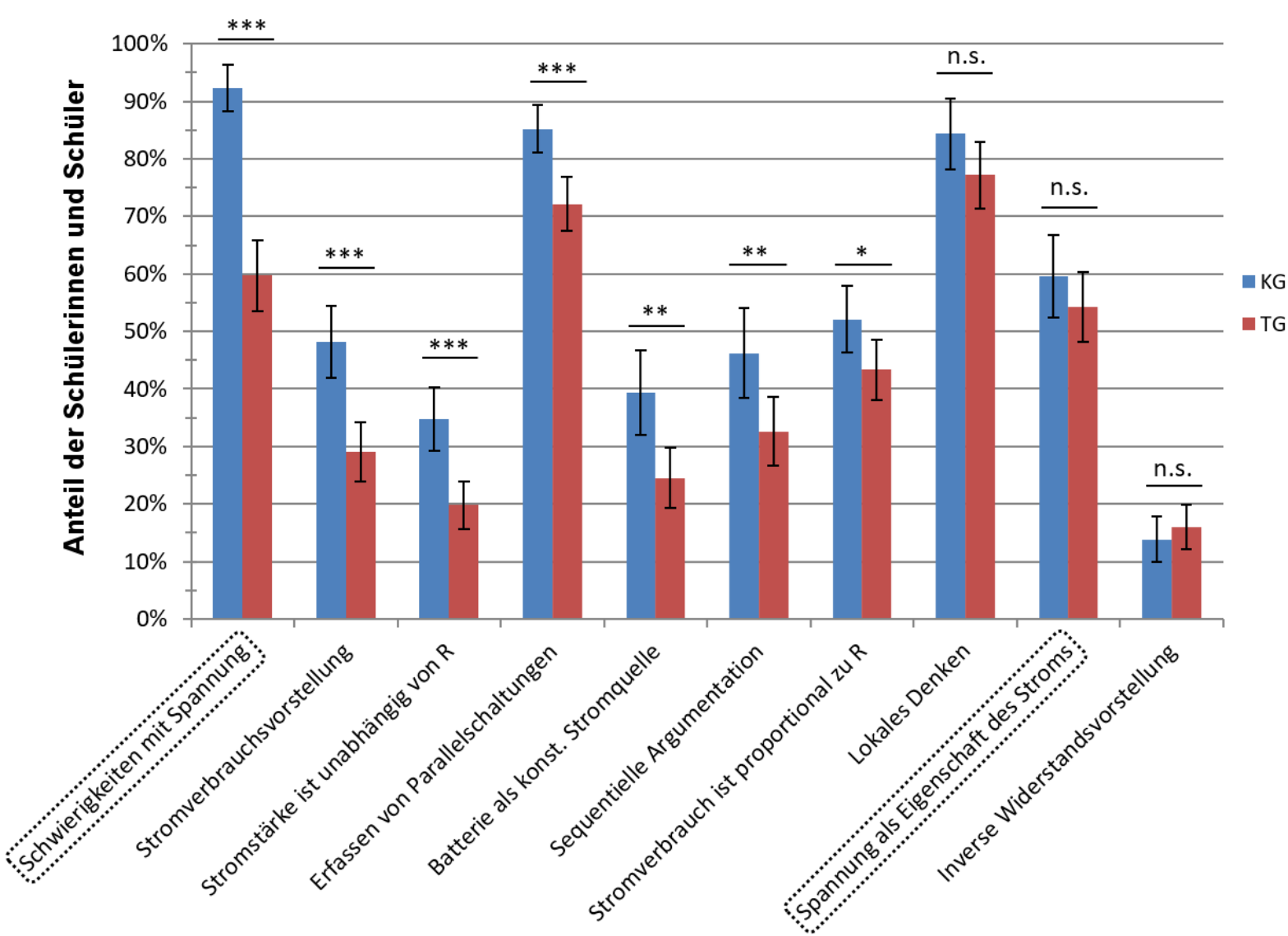

Abb. 110: Übersicht über die Schülervorstellungen im Posttest mit 95\%-Konfidenzintervallen (geordnet nach Signifikanzniveau). Die umrandeten Schülervorstellungen zur Spannung wurden auf Basis eigener Items erhoben. Es gelten die folgenden Signifikanzniveaus: $* * * p<.001 ; * * p<.01 ; * p<.05 ;$ n.s. $=$ nicht signifikant.

Erfreulicherweise scheint das neue Unterrichtskonzept im direkten Vergleich zum traditionellen Unterricht auch zu einem besseren konzeptionellen Verständnis der elektrischen 
Spannung zu führen. Dies wird einerseits daran deutlich, dass $48 \%(S E=2 \%)$ der Schüler der Treatmentgruppe mindestens eines der vier Spannungsitem vollständig korrekt lösen konnten (also alle drei gefragten Spannungswerte bei einem Item richtig angeben konnten), während dieser Wert in der Kontrollgruppe bei lediglich 12\% (SE = 2\%) liegt, was einen höchst signifikanten Unterschied darstellt $\left(\chi^{2}(1)=114.44, p<.001\right)$. Andererseits deutet auch das Ergebnis der Kategorie „Konzeptionelle Schwierigkeiten mit der elektrischen Spannung“ darauf hin, dass die Schüler der Treatmentgruppe die Spannung signifikant häufiger als Schüler der Kontrollgruppe als Differenz- und nicht Absolutgröße wahrnehmen. Nichtsdestotrotz betrachteten etwas mehr als die Hälfte der Schüler in beiden Gruppen die elektrische Spannung als Eigenschaft oder Bestandteil des elektrischen Stroms, wobei zwischen Kontroll- und Treatmentgruppe hier kein signifikanter Unterschied existiert. In beiden Gruppen wurde von den Schülern hier angenommen, dass, wenn in einem Stromkreis aufgrund eines geöffneten Schalters kein Strom fließt, auch die elektrische Spannung zwischen allen Punkten des Stromkreises null sein müsse, d.h. auch am offenen Schalter. Vor dem Hintergrund, dass auch nur etwa die Hälfte der Schüler der Treatmentgruppe überhaupt eines von vier Items zur elektrischen Spannung vollständig korrekt lösen konnten, wird damit klar, dass das neue Unterrichtskonzept zwar tendenziell zu einem besseren Verständnis der elektrischen Spannung führt, diese für die Schüler aber auch weiterhin eine nur schwer zu verstehende physikalische Größe darstellt.

\subsubsection{Der Ausprägungsgrad der Schülervorstellungen}

Statt lediglich zu untersuchen, ob eine Schülervorstellung grundsätzlich auftritt oder nicht, soll nun der Frage nachgegangen werden, wie stark die jeweiligen Schülervorstellungen bei den Lernenden ausgeprägt sind. Im Gegensatz zur sonst verwendeten dichotomen Kodierung der jeweiligen Subskalen (vgl. Abschnitt 7.3.4 und 7.4.1.6) entsprechend des Kodiermanuals von Urban-Woldron und Hopf (2012) wird hierzu aus den jeweiligen Items der Subskalen der Mittelwert berechnet. Der Mittelwert ist dann ein Maß dafür, wie stark die jeweilige Schülervorstellung ausgeprägt ist. Wird bei einer von vier der Subskala zugrundeliegenden Items beispielsweise eine entsprechende Schülervorstellung diagnostiziert, so läge der Ausprägungsgrad dieser Schülervorstellung nach dieser Auswertemethodik bei 25\%, während entsprechend der dichotomen Kodierung ein Wert von 1 angenommen würde. Der über alle Lernenden gemittelte Ausprägungsgrad einer Schülervorstellung muss dementsprechend niedriger ausfallen als der Anteil der Lernenden, die die jeweilige Schülervorstellung bei mindestens einem Item vertreten.

Im Gegensatz zur dichotomen Kodierung der Subskalen setzt diese Auswertemethodik jedoch voraus, dass die den Schülervorstellungen zugrundeliegenden Subskalen über eine angemessene interne Konsistenz verfügen. Wie in Abschnitt 7.4.1.6 gezeigt, weisen einige der Subskalen jedoch lediglich ein Cronbachs Alpha zwischen $\alpha=.50$ und $\alpha=.80$ auf, was einer verhältnismäßig niedrigen Reliabilität entspricht (Bortz und Döring 2006, S. 199). Schecker (2014, S. 5) weist allerdings darauf hin, dass es keinen objektiven Grenzwert für Cronbachs Alpha gibt, ab dem eine Skala nicht mehr sinnvoll einsetzbar ist. Insbesondere bei fachdidak- 
tischer Forschung, bei der eine Operationalisierung der oftmals komplexen Konstrukte wie z.B. Schülervorstellungen vergleichsweise schwer ist, seien niedrigere Reliabilitätskoeffizienten deshalb kein Grund, auf die entsprechende Subskala zu verzichten. Vor diesem Hintergrund und der Tatsache, dass eine konfirmatorische Faktorenanalyse von Urban-Woldron und Hopf (2012, S. 221) auf Basis eines anderen Datensatzes zu dem Ergebnis kommt, dass sich die Schülervorstellungen mit dem Testinstrument sinnvoll erheben lassen, können Reliabilitätskoeffizienten der Schülervorstellungen von $\alpha>.50$ für die folgende Analyse noch als akzeptabel betrachtet werden. Mit Reliabilitätskoeffizienten von $\alpha=.17$ bzw. $\alpha=.13$ verfügen die beiden Subskalen „Stromverbrauch ist proportional zum Widerstand“ und „Lokales Denken“ jedoch eindeutig über eine zu niedrige interne Konsistenz, um den Ausprägungsgrad dieser beiden Schülervorstellungen sinnvoll bestimmen zu können. Aus diesem Grund wird im Folgenden auf eine Auswertung des Ausprägungsgrades dieser beiden Schülervorstellungen verzichtet. Da die Kategorie „Spannung als Eigenschaft des Stroms“ zudem lediglich auf einem einzelnen Item basiert und folglich eine alternative Auswertung durch Mittelwertberechnung keinen Sinn ergibt, wird auch diese im Folgen nicht separat aufgeführt.

\section{Einfluss der Kodierung der Subskalen}

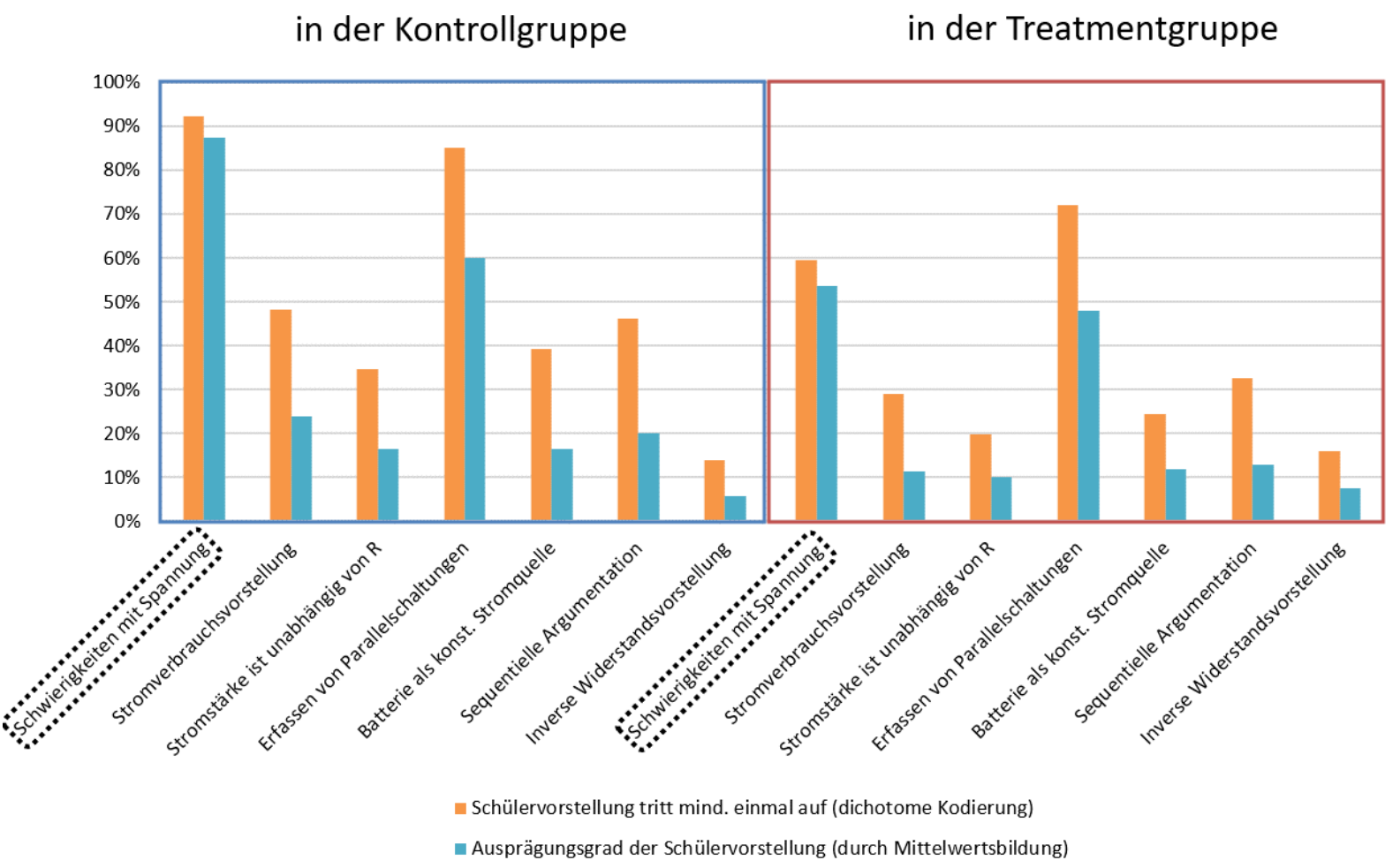

Abb. 111: Vergleich der im Posttest mittels dichotomer Kodierung der Subskalen (d.h. die Schülervorstellung tritt mindestens bei einem Item der Subskala auf) sowie durch Mittelwertbildung (d.h. des Ausprägungsgrades der Schülervorstellung) ermittelten Werte für die Kontroll- und Treatmentgruppe.

Eine Gegenüberstellung der mit Hilfe der beiden oben beschriebenen Auswerteverfahren ermittelten Werte ist für die Posttest-Ergebnisse der Kontroll- und Treatmentgruppe in Abb. 111 dargestellt. Erwartungsgemäß liegt der über alle Items einer Subskala gemittelte Ausprägungsgrad einer Schülervorstellung deutlich unter dem Anteil der Lernenden, der diese Schülervorstellung bei mindestens einem Item der jeweiligen Subskala vertritt. Eine genauere 
Auswertung zeigt, dass der Ausprägungsgrad der Schülervorstellungen durch Mittelung über die Items der Subskala sowohl in der Kontroll- als auch in der Treatmentgruppe im Durchschnitt nur halb so hoch ausfällt wie der Anteil der Lernenden, der die jeweilige Schülervorstellung bei mindestens einem Item der jeweiligen Subskala vertritt (dichotome Kodierung). Dies ist in Hinblick auf die Interpretation des vergleichsweise hohen Anteils an Lernenden, die in Abschnitten 7.4.8.1 und 7.5.4 eine bestimmte Schülervorstellung mindestens einmal im Test gezeigt haben, ein wichtiges Ergebnis. Wie Abb. $111 \mathrm{zu}$ entnehmen ist, fällt der Unterschied zwischen dichotomer Kodierung und Mittelwertbildung bei der Kategorie „konzeptionelle Schwierigkeiten mit der elektrischen Spannung“ besonders gering aus. Dies deutet darauf hin, dass die Items dieser Subskala einerseits eine geringe Kontextabhängigkeit aufweisen, andererseits mit hoher Wahrscheinlichkeit alle Spannungsitems falsch beantwortet werden, wenn nur ein Spannungsitem falsch beantwortet wird. Eine genaue Übersicht über die ermittelten Werte inklusive Angabe der entsprechenden Signifikanzniveaus findet sich in Tab. 26.

Tab. 26: Vergleich der im Posttest mittels dichotomer Kodierung der Subskalen (D) bzw. auf Basis der Mittelwerte der Subskalen (M) ermittelten Werte für die grundsätzliche Auftretenswahrscheinlichkeit bzw. den Ausprägungsgrad der verschiedenen Schülervorstellungen. Signifikanzwerte unter 5\% sind fett hervorgehoben.

\begin{tabular}{|c|c|c|c|c|c|}
\hline Schülervorstellung & CA & Kod. & KG & TG & Sig. \\
\hline \multirow{2}{*}{$\begin{array}{l}\text { Konzeptionelle Schwierigkeiten mit } \\
\text { der elektrischen Spannung (KS) }\end{array}$} & \multirow{2}{*}{.83} & $\mathrm{M}$ & $87 \%$ & $54 \%$ & $p<.001$ \\
\hline & & $\mathrm{D}$ & $92 \%$ & $60 \%$ & $p<.001$ \\
\hline \multirow{2}{*}{$\begin{array}{l}\text { Stromverbrauchsvorstellung } \\
\text { (Kürzel: SV) }\end{array}$} & \multirow{2}{*}{.57} & $\mathrm{M}$ & $24 \%$ & $11 \%$ & $p<.001$ \\
\hline & & $\mathrm{D}$ & $48 \%$ & $29 \%$ & $p<.001$ \\
\hline \multirow{2}{*}{$\begin{array}{l}\text { Stromstärke ist unabhängig von } \mathrm{R} \\
\text { (Kürzel: UR) }\end{array}$} & \multirow{2}{*}{.77} & $\mathrm{M}$ & $17 \%$ & $10 \%$ & $p=.001$ \\
\hline & & $\mathrm{D}$ & $35 \%$ & $20 \%$ & $p<.001$ \\
\hline \multirow{2}{*}{$\begin{array}{l}\text { Erfassen von Parallelschaltungen } \\
\text { (Kürzel: PS) }\end{array}$} & \multirow{2}{*}{.83} & $\mathrm{M}$ & $60 \%$ & $48 \%$ & $p<.001$ \\
\hline & & $\mathrm{D}$ & $85 \%$ & $72 \%$ & $p<.001$ \\
\hline \multirow{2}{*}{$\begin{array}{l}\text { Batterie als konstante Stromquelle } \\
\text { (Kürzel: BS) }\end{array}$} & \multirow{2}{*}{.61} & $\mathrm{M}$ & $16 \%$ & $12 \%$ & $p=.015$ \\
\hline & & $\mathrm{D}$ & $39 \%$ & $25 \%$ & $p=.001$ \\
\hline \multirow{2}{*}{$\begin{array}{l}\text { Sequentielle Argumentation } \\
\text { (Kürzel: SA) }\end{array}$} & \multirow{2}{*}{.53} & $\mathrm{M}$ & $20 \%$ & $13 \%$ & $p<.001$ \\
\hline & & $\mathrm{D}$ & $46 \%$ & $33 \%$ & $p=.007$ \\
\hline \multirow{2}{*}{$\begin{array}{l}\text { Inverse Widerstandsvorstellung } \\
\text { (Kürzel: IR) }\end{array}$} & \multirow{2}{*}{.66} & $\mathrm{M}$ & $6 \%$ & $8 \%$ & $p=.222$ \\
\hline & & $\mathrm{D}$ & $14 \%$ & $16 \%$ & $p=.462$ \\
\hline
\end{tabular}

Anmerkung: $\mathrm{CA}=$ Cronbachs Alpha; Kod. $=$ Kodierungsmethode 


\subsection{Erstellung eines Hierarchisch Linearen Modells}

\subsubsection{Beschreibung des allgemeinen Vorgehens und der Kenngrößen}

Im Folgenden sollen in Anlehnung an Kuhn (2010, 101ff) das allgemeine Vorgehen beim Arbeiten mit Mehrebenenanalysen sowie typische Kenngrößen vorgestellt werden. Wie in Abschnitt 7.3.5.5 beschrieben, wird in der vorliegenden mehrebenenanalytischen Auswertung zwischen der Individualebene der Schüler (Ebene 1) und der Kontextebene der Klassen (Ebene 2) unterschieden (,Zwei-Ebenen-Design“). Die Mehrebenenanalyse ähnelt prinzipiell einer linearen Regression, unterscheidet sich von dieser jedoch dadurch, dass für jede der beiden Ebenen eine eigene Modellgleichung formuliert wird und die Regressionsparameter variieren können. Die Regressionskonstanten bzw. Regressionskoeffizienten der jeweils tieferen Ebene werden dabei durch passende Gleichungen auf der jeweils höheren Ebene definiert. Entsprechend steigt die Anzahl der nötigen Gleichungen auf höherer Ebene mit der Anzahl der auf tieferer Ebene verwendeten Prädiktoren bzw. Regressionskoeffizienten.

Der erste Schritt einer jeden Mehrebenenanalyse besteht in der Regel darin, ein NullModell aufzustellen, mit dem untersucht wird, wie sich die Varianz der abhängigen Variablen auf die verschiedenen Ebenen des Modells verteilt. Aus diesem Grund enthält das NullModell auch noch keine Prädiktoren, die diese Varianz aufklären könnten. Für das hier verwendete Zwei-Ebenen-Design ergeben sich somit folgende zwei Gleichungen, wobei jede Gleichung eine andere Ebene repräsentiert (,hierarchisches Modell $\left.{ }^{\circ}\right)$ :

Ebene 1: Post $_{i j}=\beta_{0 j}+e_{i j} \quad$ Post $_{i j}$ : Posttest-Ergebnis eines einzelnen Schülers $i$ in Klasse $j$

$\beta_{0 j}$ : Regressionskonstante von Ebene 1 (entspricht hier dem mittleren Posttest-Ergebnis der Klasse $j$ )

$e_{i j}$ : Zufallseffekt auf Ebene 1 (hier die Differenz zwischen dem tatsächlichen Posttest-Ergebnis des Schülers $i$ in Klasse $j$ und dem durchschnittlichen PosttestErgebnis von Klasse $j$ )

Ebene 2: $\beta_{0 j}=\gamma_{00}+r_{0 j}$

$\beta_{0 j}$ : Regressionskonstante von Ebene 1

$\gamma_{00}$ : Gesamtmittelwert (entspricht hier dem mittleren Posttest-Ergebnis aller Schüler aller Klassen)

$r_{0 j}$ : Zufallseffekt auf Ebene 2 (hier die Differenz zwischen dem durchschnittlichen Posttest-Ergebnis der Klasse $j$ und dem mittleren Posttest-Ergebnis aller Schüler aller Klassen $\gamma_{00}$ )

mit $i$ : Index der Schüler (Ebene 1); $j$ : Index der Klassen (Ebene 2)

Durch Einsetzen der Definitionen der Regressionskonstanten auf Ebene 2 in die Regressionsgleichungen auf Ebene 1 lassen sich die beiden Gleichungen des hierarchischen Modells zusammenfassen zu einem sogenannten ,gemischten Modell“, das auf Basis des Intercepts $\gamma_{00}$, hier dem Gesamtmittelwert des Posttest-Ergebnisses, und den Zufallseffekten der verschiede- 
nen Ebenen, hier der Klassen bzw. Schüler, das Posttest-Ergebnis jedes einzelnen Schülers beschreibt:

Post $_{i j}=\gamma_{00}+r_{0 j}+e_{i j}$

Auf Basis der Zufallseffekte $r_{0 j}$ und $e_{i j}$ des ,Intercept-Only-Models“ werden dann die absoluten Varianzkomponenten $\operatorname{Var}\left(r_{0 j}\right)$ und $\operatorname{Var}\left(e_{i j}\right)$ ermittelt. Da sich also der Fehlerterm aus den beiden Komponenten $r_{0 j}$ und $e_{i j}$ zusammensetzt, kann zwischen der Varianz der Klassenmittelwerte um den Gesamtmittelwert $\operatorname{Var}\left(r_{0 j}\right)$ und zwischen der Varianz der individuellen Posttest-Ergebnisse der Schüler innerhalb der verschiedenen Klassen $\operatorname{Var}\left(e_{i j}\right)$ unterschieden werden. Das Ziel besteht dann darin, auf Basis dieser beiden Varianzkomponenten den Intra-

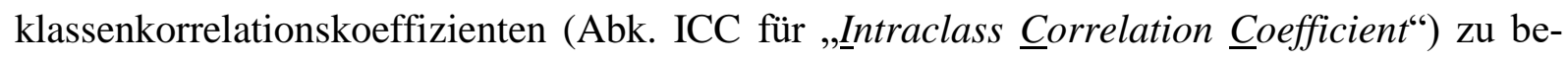
rechnen, der eine quantifizierte Größe der Intraklassenkorrelation darstellt. Der ICC ist dabei ein Maß für den Anteil der ebenenspezifischen Varianz an der Gesamtvarianz der abhängigen Variable; gibt also an, welcher Anteil der Varianz der abhängigen Variablen durch Prädiktoren auf der jeweiligen Ebene potenziell erklärbar ist. Nach Hartig und Rakoczy (2010, S. 544) sollte ab einem ICC-Wert von 10\% auf Ebene 2 (hier der Klassenebene) auf eine Mehrebenenanalyse zurückgegriffen werden, da sonst mit einer gravierenden Alpha-FehlerInflation zu rechnen ist. Der ICC wird für jede Ebene einzeln angegeben, hier also:

$$
\begin{aligned}
I C C_{\text {Schüler }}=\frac{\operatorname{Var}\left(e_{i j}\right)}{\operatorname{Var}\left(e_{i j}\right)+\operatorname{Var}\left(r_{0 j}\right)} & \operatorname{Var}\left(e_{i j}\right): \text { Varianz zwischen den Schülern } \\
I C C_{\text {Klassen }}=\frac{\operatorname{Var}\left(r_{0 j}\right)}{\operatorname{Var}\left(e_{i j}\right)+\operatorname{Var}\left(r_{0 j}\right)} &
\end{aligned}
$$

Sofern die abhängige Variable wie z.B. bei Schülervorstellungen nicht intervallskaliert vorliegt, sondern dichotom kodiert ist, lässt sich der ICC in einem Zwei-Ebenen-Design nach Twisk (2006, S. 46) wie folgt berechnen:

$$
I C C=\frac{\operatorname{Var}\left(r_{0 j}\right)}{\operatorname{Var}\left(r_{0 j}\right)+\frac{\pi^{2}}{3}} \quad \operatorname{Var}\left(r_{0 j}\right): \text { Varianz zwischen den Klassen }
$$

Typisch für die Erstellung eines Hierarchisch Linearen Modells ist die sukzessive Modellentwicklung, also das schrittweise Hinzufügen von Prädiktoren, um zu untersuchen, ob der hinzugefügte Prädiktor einen statistisch signifikanten Beitrag zur Aufklärung der bisher ungeklärten Varianz der entsprechenden Ebene liefert. Die Auswahl der Prädiktoren erfolgt hierbei zunächst theoriegeleitet auf Grundlage der Forschungsfragen, kann aber auch explorativ erfolgen. Sofern ein Prädiktor einen signifikanten Beitrag zur Aufklärung der Varianz liefert und zu einer signifikanten Modellverbesserung beiträgt, wird er in das Modell aufgenommen. Entsprechend dieser Step-Up-Strategie kann dann für jeden so gefundenen festen Effekt auch untersucht werden, ob zusätzlich signifikante Zufalls- oder Interaktionseffekte vorliegen (Raudenbush und Bryk 2002; Twisk 2006). 
Um beurteilen zu können, ob ein Modell durch Hinzufügen eines Prädiktors verbessert und damit die Modellgüte erhöht wird, greift man auf die Devianz $D$ der Modelle zurück, die sich entsprechend $D=-2 \cdot \log (L)$ berechnet, wobei $L$ für die Log-Likelihood des Modells steht. Grundsätzlich gilt bei der sukzessiven Modellentwicklung, dass die Modellgüte umso höher ist, desto kleiner die Devianz $D$ des Modells ist. Der absolute Wert der Devianz $D$ hat dabei keine Bedeutung an sich, sondern kann nur in Relation zur Devianz eines verwandten Modells interpretiert werden. Wurde das Modell M2 aus dem alten Modell M1 z.B. durch das Hinzufügen eines neuen Prädiktors (= fester Effekt) oder eines Zufallseffekts entwickelt, so ist die Differenz der Devianzen $\Delta D=D_{\mathrm{M} 1}-D_{\mathrm{M} 2}$ ein Maß für die Güte des neuen Modells M2 im Vergleich zum bisherigen Modell M1. Ob die vorliegende Modellverbesserung statistisch signifikant ist, wird mittels eines Chi-Quadrat-Tests ermittelt, wobei sich die Zahl der Freiheitsgrade aus der Differenz der Modellparameter $\mathrm{zu} d f=$ Modellparameter von MIModellparameter von M2 ergibt. Nach Twisk (2006, 13f) wird zur Prüfung der Signifikanz der Modellverbesserung ein einseitiger Chi-Quadrat-Test eingesetzt, weil nicht geprüft wird, ob ein Unterschied zwischen den Devianzen vorliegt, sondern ob die Devianz des neuen Modells im Vergleich zum alten Modell kleiner geworden ist. Um bei der Modellselektion eine Balance zwischen dem Erklärungsgehalt eines Modells und dessen Komplexität durch zusätzlich aufgenommene Parameter zu finden, wurden auf Basis der Devianz $D$ diverse korrigierte Gütemaße wie z.B. das Akaike Informationskriterium (AIC) oder das Bayessche Informationskriterium (BIC) entwickelt. Während das AIC lediglich die Anzahl der geschätzten Parameter strafend berücksichtigt, wird zur Bewertung der Modellgüte nach dem BIC auch die Stichprobengröße herangezogen. Nach Field (2011, S. 737) gilt das Bayessche Informationskriterium (BIC) als das konservativere Gütekriterium und eignet sich insbesondere für Studien mit einer geringen Anzahl an Parametern, weshalb es in der vorliegenden Untersuchung zur Anwendung kommt.

Für die Schätzung der Regressionsparameter und Varianzkomponenten wird bei Mehrebenenanalysen in der Regel entweder auf das „Maximum-Likelihood“ (ML) oder „Restricted-Maximum-Likelihood" (REML) Verfahren zurückgegriffen. Da jedes dieser iterativen Schätzverfahren seine Vor- und Nachteile hat, besteht kein Konsens darüber, welches grundsätzlich das bessere ist. Während die Stärken des REML-Verfahrens bei kleinen Stichproben und bei der Schätzung der Varianzkomponenten bzw. Zufallseffekte gesehen werden, führt die Anwendung der ML-Methode insbesondere bei unbalancierten Designs zu akkurateren Werten bzgl. der Regressionskoeffizienten eines Prädiktors (,feste Effekte ${ }^{\text {") }}$ (Raudenbush und Bryk 2002; Twisk 2006, S. 29). Ein bedeutender Nachteil der REML-Methode besteht insbesondere darin, dass es im Gegensatz zur ML-Methode nicht möglich ist, verschiedene Modelle auf Basis ihrer Modellgüte miteinander zu vergleichen (Field 2011, S. 737). Da Haupteffekte bzw. feste Effekte in der vorliegenden Studie von höherem Interesse als Zufallseffekte sind und auf die Analyse der Modellgüte nicht verzichtet werden soll, wird bei der sukzessiven Modellentwicklung auf die Maximum-Likelihood-Methode (ML) zurückgegriffen. 
Voraussetzung für die Anwendung einer Mehrebenenanalyse ist ein ausreichend groBer Stichprobenumfang insbesondere in Bezug auf die Aggregatebene (hier: Ebene 2 bzw. Klassenebene). Für die Untersuchung fester Effekte wird oftmals eine Mindestanzahl von ca. 30 Einheiten auf der Aggregatsebene empfohlen, wobei sich innerhalb jeder Level-2-Einheit mindestens zehn Level-1-Einheiten befinden sollten (Hartig und Rakoczy 2010, S. 545). Sofern darüber hinaus auch Zufalls- und Interaktionseffekte betrachtet werden, sollten noch größere Gruppengrößen vorliegen (Snijders 2005, S. 1571). Tendenziell scheint es so zu sein, dass Zufallseffekte und deren Standardfehler bei geringen Einheitenzahlen auf der Aggregatebene unterschätzt werden, während die gefundenen Effekte auf der ersten Ebene auch in diesem Fall noch solide Schätzungen darstellen (Hox 2010, S. 234). Kuhn (2010, S. 146) weist jedoch darauf hin, dass hier in der Literatur kein Konsens besteht und insbesondere neuere Befunde darauf hindeuten, dass Mehrebenenanalysen auch schon ab zehn Einheiten pro Aggregatebene eine ausreichende Robustheit aufweisen (z.B. Maas und Hox 2005).

Ein weiterer wichtiger Aspekt, insbesondere in Hinblick auf die Interpretation der Regressionskonstanten (,Intercepts“) bei Mehrebenenanalysen, betrifft die Zentrierung des Prädiktors. Wie im Folgenden dargelegt, geht es dabei um die Frage, wie der Nullpunkt der unabhängigen Variable (Prädiktor) definiert wird:

1. Keine Zentrierung: Ohne Zentrierung, d.h. bei der Beibehaltung der natürlichen Metrik der Daten, entspricht die Regressionskonstante dem Wert der abhängigen Variablen, der entsprechend des Mehrebenenmodells für den Fall vorhergesagt wird, dass alle Prädiktoren den Wert null annehmen. Wird beispielsweise der Einfluss des individuellen Pretest-Ergebnisses auf das PosttestErgebnis untersucht, gäbe die Regressionskonstante das mittlere zu erwartende Posttest-Ergebnis für Schüler mit einem Pretest-Ergebnis von null Punkten an. Möchte man Aussagen darüber treffen, wie ein durchschnittlicher Schüler im Nachtest unter bestimmten Bedingungen abgeschnitten hat, ist ein solcher Wert der Regressionskonstanten wenig hilfreich.

2. Zentrierung am Gesamtmittelwert: Beim sogenannten „Grand-MeanCentering“ wird der Prädiktor transformiert, indem von jedem Wert des bisherigen Prädiktors der Gesamtmittelwert subtrahiert wird: $\mathrm{X}_{\mathrm{GMC}}=\mathrm{X}_{\mathrm{ij}}-\overline{\mathrm{X}}$. Durch die Mittelwertzentrierung entspricht der Mittelwert $\mathrm{X}_{\mathrm{GMC}}$ nun dem Wert null. Die Regressionskonstante gibt dann den Wert der abhängigen Variablen an, den das Mehrebenenmodell für den Wert null von $\mathrm{X}_{\mathrm{GMC}}$ annehmen würde, der hier aber dem Durchschnittswert des Prädiktors entspricht. Bezogen auf unser Beispiel würde die Regressionskonstante dann dem Posttest-Ergebnis entsprechen, das von einem in Bezug auf das Pretest-Ergebnis durchschnittlichen Schüler erzielt wird.

3. Zentrierung am Gruppenmittelwert: Eine andere Form der MittelwertZentrierung besteht darin, von jedem Wert des bisherigen Prädiktors den Gruppenmittelwert (z.B. der Klasse) abzuziehen und auf diese Weise die Vari- 
anzkomponenten zwischen den einzelnen Einheiten (hier den Klassen) zu entfernen (Paccagnella 2006, S. 70). Diese Art der Zentrierung führt jedoch zu völlig anderen Interpretationen der Regressionskonstanten und eignet sich nur für sehr spezifische Fragestellungen bspw. bzgl. des Gruppenkontexts auf die Schülerleistung, da nur noch die relative Position eines Schülers innerhalb einer Klasse betrachtet wird. In Bezug auf unser Beispiel würde die Regressionskonstante dann die Leistung eines in jeder Klasse bzgl. des Pretests durchschnittlichen Schülers im Posttest entsprechen.

Neben der Signifikanz stellt vor allem die Effektstärke ein wichtiges Maß für die Beurteilung der Bedeutung eines Effektes eines Prädiktors dar. Sofern das mehrebenenanalytische Gesamtmodell nur feste Effekte umfasst, lässt sich die Effektstärke verhältnismäßig leicht berechnen, wobei zwischen ebenenspezifischen und ebenenübergreifenden Effekten unterschieden wird.

1. Ebenenspezifisch: Innerhalb einer Ebene kann die Größe eines Effekts dadurch quantifiziert werden, dass die durch den Prädiktor erzielte Varianzaufklärung ins Verhältnis gesetzt wird zur gesamten ungeklärten ebenenspezifischen Varianz des Null-Modells. Konkret erfolgt die Berechnung des durch Hinzufügen des Prädiktors anteilig aufgeklärten Varianzanteils $R^{2} P$ wie folgt:

$$
\begin{aligned}
& R^{2}{ }_{P} \text { : Anteil der durch den hinzugefügten Prä- } \\
& \text { diktor innerhalb der Ebene aufgeklärten Vari- } \\
& \text { anz } \\
& R_{P}^{2}=\frac{\operatorname{Var}_{S o P}-\operatorname{Var}_{S m P}}{\operatorname{Var}_{\text {Null }}} \quad \begin{array}{l}
\operatorname{Var}_{S o P} \text { : ungeklärte Varianz des Submodels } \\
\text { ohne Prädiktor }
\end{array} \\
& \text { Var }_{S m P} \text { : ungeklärte Varianz des Submodells } \\
& \text { mit Prädiktor } \\
& \text { Var }_{\text {Null: }} \text { ungeklärte Gesamtvarianz des Null- } \\
& \text { Modells }
\end{aligned}
$$

2. Ebenenübergreifend: Bei der Ermittlung der Größe eines festen Effekts auf höherer Ebene auf die abhängige Variable auf Ebene 1 muss nach Tymms (2004) bei der Berechnung der Effektstärke zwischen dichotomen (z.B. Gruppenzugehörigkeit) und kontinuierlichen Variablen (z.B. Pretest-Ergebnis) unterschieden werden. In beiden Fällen kann als Maß für die Effektstärke Cohens $d$ berechnet werden, wodurch eine Vergleichbarkeit zwischen den mittels Mehrebenenanalyse und Varianzanalyse berechneten Effekten gewährleistet ist. Für dichotome Prädiktoren gilt folgende Beziehung (Tymms 2004, S. 57): 
$d_{N}$ : Cohens $d$ als Effektstärke des Prädiktors

von Ebene $N$ auf die abhängige Variable auf Ebene 1

$$
d_{N}=\frac{\beta_{N}}{\sigma_{E 1}}
$$

$\beta_{N}$ : Regressionskonstante des Prädiktors eines Haupteffekts auf Ebene $N$

$\sigma_{E 1}$ : Standardabweichung der Einheiten auf Ebene 1

Für kontinuierliche Prädiktoren muss zusätzlich noch deren Standardabweichung berücksichtigt werden (Tymms 2004, S. 62):

$d_{N}$ : Cohens $d$ als Effektstärke des Prädiktors von Ebene $N$ auf die abhängige Variable auf Ebene 1

$$
\begin{array}{ll}
d_{N}=\frac{2 \cdot \beta_{N} \cdot \sigma_{P}}{\sigma_{E 1}} \quad \begin{array}{l}
\beta_{N}: \text { Regressionskoeffizient des Prädiktors } \\
\text { eines Haupteffekts auf Ebene } N
\end{array} \\
\begin{array}{l}
\sigma_{P}: \text { Standardabweichung des betrachteten } \\
\text { Prädiktors }
\end{array}
\end{array}
$$$$
\sigma_{E 1}: \text { Standardabweichung der Einheiten auf }
$$$$
\text { Ebene } 1
$$

\subsubsection{Entwicklung eines Hierarchisch Linearen Modells}

Aufgrund der vorliegenden hierarchischen Datenstruktur stellt eine Mehrebenenanalyse mit Schüler- und Klassenebene gegenüber klassischen inferenzstatistischen Verfahren grundsätzlich die adäquatere Analysemethode dar. Da der Lernerfolg der Schülerinnen und Schüler jedoch nicht nur von ihrer Klassenzugehörigkeit, sondern auch von der unterrichtenden Lehrkraft abhängt (Hattie 2013), wäre prinzipiell auch ein Drei-Ebenen-Design mit den Lehrkräften auf der dritten Ebene denkbar. Auf Lehrerebene könnten z.B. Merkmale wie PCK (pedagogical content knowledge, d.h. fachdidaktisches Wissen), CK (content knowledge, d.h. Fachwissen), Unterrichtserfahrung oder Motivation potenzielle Prädiktoren für den Lernerfolg der Schüler sein. Da diese Prädiktoren in der vorliegenden Studie jedoch nicht erhoben wurden und mit 23 Lehrkräften die Mindestanzahl von 30 Ebene-3-Einheiten unterschritten werden würde, wurde sich für ein Zwei-Ebenen-Design entschieden. Vor dem Hintergrund, dass viele Lehrkräfte in dieser Untersuchung nur eine Klasse unterrichtet haben, wäre in einem Drei-Ebenen-Design zudem eine eindeutige Unterscheidung der durch die verschiedenen Prädiktoren erzielten Varianzaufklärung zwischen Lehrer- und Klassenebene nicht möglich. Das gewählte Zwei-Ebenen-Design hingegen erfüllt mit 790 Schülern bzw. 36 Klassen alle Voraussetzungen an die Mindestzahl an Ebene-1 bzw. Ebene-2-Einheiten. Ein mehrebenenanaly- 
tischer Vergleich der Teilstichprobe mit gleichen Lehrkräften (vgl. Abschnitt 7.4.6) ist aufgrund der zu geringen Anzahl an Einheiten auf Klassenebene jedoch nicht möglich.

\subsubsection{M0: Das Null-Modell}

Mit Hilfe des Null-Modells soll zunächst untersucht werden, ob die Anwendung der Mehrebenenanalyse aufgrund einer hohen Intraklassenkorrelation im vorliegenden Datensatz überhaupt nötig ist. Hierzu wird mit Hilfe des Null-Modells, das entsprechend seines Namens keinerlei Prädiktoren enthält, die Gesamtvarianz der abhängigen Variablen (hier der PosttestErgebnisse) auf die Schüler- und Klassenebene aufgeteilt. An dem gemischten Modell kann man sehen, dass es sich hierbei um ein Random-Intercept-Modell handelt, da lediglich der Intercept bzw. die Regressionskonstante $\gamma_{00}$ (hier das mittlere Posttest-Ergebnis aller Schüler aller Klassen) sowie die beiden Zufallseffekte $r_{0 j}$ (= Abweichung des Klassenmittelwerts vom Gesamtmittelwert $\gamma_{00}$ ) und $e_{i j}$ (= Abweichung des jeweiligen individuellen PosttestErgebnisses vom Klassenmittelwert) geschätzt werden. Anschließend wird für jeden dieser Zufallseffekte die Varianz bestimmt und aus den so ermittelten Varianzanteilen der unterschiedlichen Ebenen kann dann die Intraklassenkorrelation bestimmt werden. In den folgenden Darstellungen der einzelnen Modellgleichungen wird im Sinne einer besseren Lesbarkeit auf eine Beschreibung der einzelnen Modellparameter verzichtet und hierfür stattdessen auf die ausführlichen Erklärungen am Beispiel des Null-Modells in Abschnitt 7.5.1 verwiesen.

Tab. 27: Modellgleichungen zum Modell MO

\begin{tabular}{|l|l|l|}
\hline$\overline{\bar{O}}$ & Ebene 1 & Post $_{i j}=\beta_{0 j}+e_{i j}$ \\
\cline { 2 - 3 }$\sum_{0}^{\circ}$ & Ebene 2 & $\beta_{0 j}=\gamma_{00}+r_{0 j}$ \\
\hline Gemischtes Modell & Post $_{i j}=\gamma_{00}+r_{0 j}+e_{i j}$ \\
\hline Modellgüte & BIC $=4668.84($ geschätzte Parameter $\mathrm{d} f=3)$ \\
\hline
\end{tabular}

Für den Intercept bzw. die Regressionskonstante $\gamma_{00}$ wird der Wert 10.67 ermittelt $(S E=0.61)$, d.h. die durchschnittlich erzielte Punktzahl aller 790 erhobenen Schülerinnen und Schüler im Posttest beträgt unabhängig von ihrer Gruppenzugehörigkeit ca. 11 Punkte. Der Wert des Bayesschen Informationskriteriums (BIC) beträgt für das Null-Modell 4668.84 bei drei geschätzten Parametern $\left(\mathrm{d} f_{0}=3\right)$.

Tab. 28: Schätzung der festen Parameter von Modell MO

\begin{tabular}{|l|l|l|l|l|l|l|l|}
\hline \multicolumn{5}{|l|}{} & $95 \%$-Konfidenzintervall \\
\hline Parameter & Schätzung & $S E$ & $\mathrm{~d} f$ & $t$-Wert & Sig. & Untergr. & Obergr. \\
\hline$\gamma_{00}$ & 10.67 & 0.61 & 35.94 & 17.62 & $p<.001$ & 9.44 & 11.89 \\
\hline
\end{tabular}

Anmerkung: $S E=$ Standardfehler; $\mathrm{d} f=$ Freiheitsgrade

In Tab. 29 ist die Verteilung der Varianz des Posttest-Ergebnisses (= der abhängigen Variablen) auf die Schüler- und Klassenebene dargestellt. Es zeigt sich, dass auf beiden Ebenen eine 
statistisch signifikante Varianz der Zufallseffekte vorliegt. In anderen Worten variiert das Posttest-Ergebnis statistisch gesehen nicht nur signifikant zwischen den einzelnen Schülern innerhalb der Klassen, sondern auch zwischen den verschiedenen Klassen mit ihren jeweiligen Klassenmittelwerten.

Tab. 29: Die Verteilung der ungeklärten Varianz des Posttest-Ergebnisses auf die beiden Ebenen im Modell MO

\begin{tabular}{|l|l|l|l|l|l|}
\hline Ebene & Varianz & Std.-Fehler & $\chi^{2}$-Wert & Signifikanz & ICC \\
\hline Schüler, $e_{i j}$ & 18.58 & 0.96 & 376.97 & $p<.001$ & .60 \\
\hline Klassen, $r_{0 j}$ & 12.32 & 3.11 & 15.67 & $p<.001$ & .40 \\
\hline
\end{tabular}

Wie an Hand der Intraklassenkorrelationskoeffizienten (ICC) in Tab. 29 zu erkennen ist, geht mit 60\% ein Großteil der Varianz der Posttest-Ergebnisse auf Unterschiede zwischen den einzelnen Schülern zurück, während $40 \%$ entsprechend auf Unterschiede zwischen den verschiedenen Klassen zurückgeführt werden können. Da also in der vorliegenden Stichprobe der ICC auf Klassenebene größer ist als 10\%, muss nach Hartig und Rakoczy (2010, S. 544) die Mehrebenenstruktur der Daten in der weiteren Modellentwicklung berücksichtigt werden, um eine Alpha-Fehler-Inflation zu vermeiden.

Das hier vorgestellte Null-Modell stellt dabei das erste Submodell einer sukzessiven Modellentwicklung durch das gezielte Hinzufügen von Prädiktoren dar. Jeder hinzugefügte Prädiktor, der einen statistisch signifikanten Beitrag zur Varianzaufklärung liefert, repräsentiert dabei einen festen Effekt bzw. Haupteffekt und wird im Folgenden in Anlehnung an Kuhn (2014, S. 306) mit einer eigenen Modellnummer gefolgt von einem „a“ (z.B. M1a oder M2a) gekennzeichnet. Zusätzlich sollen für jeden ins Modell aufgenommenen Haupteffekt auch noch Zufalls- und Interaktionseffekte untersucht werden, die dann alphabetisch mit Buchstaben $\mathrm{ab}, \mathrm{b}^{\circ}$ in der Modellbezeichnung kenntlich gemacht werden (z.B. M1b). Die Auswahl der hinzugefügten Prädiktoren erfolgt zunächst theoriegeleitet anhand der Forschungsfrage und im Anschluss dann explorativ auf Basis der erhobenen Kontrollvariablen. Bei der explorativen Implementation der Prädiktoren richtet sich die Reihenfolge nach dem erwarteten Einfluss des jeweiligen Prädiktors auf das Posttest-Ergebnis, wobei mit dem Prädiktor mit dem größten erwarteten Einfluss („Pretest-Ergebnis“) begonnen wird. Somit ergeben sich in dieser Untersuchung hinsichtlich der Haupteffekte folgende Modellschritte:

1. M1: Prüfung des Einflusses der Gruppenzugehörigkeit

2. M2: Prüfung des Einflusses des Pretest-Ergebnisses

3. M3: Prüfung des Einflusses der Jahrgangsstufe

4. M4: Prüfung des Einflusses der unterrichteten Stundenanzahl

5. M5: Prüfung des Einflusses des mittleren Klassenergebnisses im Pretest 


\subsubsection{M1: Prüfung des Einflusses der Gruppenzugehörigkeit}

\section{M1a: Der Haupteffekt ,Gruppe“}

Auf Basis der Forschungsfrage wird zunächst der dichotome Level-2-Prädiktor der Gruppenzugehörigkeit dem bisherigen Null-Modell hinzugefügt. Auf diese Weise wird geprüft, ob sich ein Unterschied zwischen Kontroll- und Treatmentgruppe bzgl. des mittleren PosttestErgebnisses nur aufgrund der Zugehörigkeit zu einer dieser beiden Gruppen zeigt. Das so entstehende Modell M1a modelliert also einen Haupteffekt mit den folgenden Modellgleichungen:

Tab. 30: Modellgleichungen zum Modell M1a

\begin{tabular}{|l|l|l|}
\hline$\overline{\overline{0}}$ & Ebene 1 & Post $_{i j}=\beta_{0 j}+e_{i j}$ \\
\cline { 2 - 3 }$\sum_{\bar{\delta}}^{0}$ & Ebene 2 & $\beta_{0 j}=\gamma_{00}+\gamma_{01}$ Gruppe $+r_{0 j}$ \\
\hline Gemischtes Modell & Post $_{i j}=\gamma_{00}+\gamma_{01}$ Gruppe $+r_{0 j}+e_{i j}$ \\
\hline Modellgüte & $\mathrm{BIC}=4659.27$ (geschätzte Parameter $\mathrm{d} f=4)$ \\
\hline
\end{tabular}

In diesem Modell besitzt die Gruppenzugehörigkeit einen signifikanten Einfluss auf das Nachtestergebnis $(F(1,36.05)=20.50, p<.001)$. Während Schüler der Treatmentgruppe nach diesem Modell im Durchschnitt im Posttest 12.74 Punkte $(S E=0.66)$ erzielen, führt die Zugehörigkeit zur Kontrollgruppe hier zu einem negativen Effekt von -4.38 Punkten $(S E=0.97)$ auf das Nachtestergebnis im Vergleich zur Treatmentgruppe. Der auf die Gruppenzugehörigkeit zurückzuführende Unterschied entspricht in diesem Modell einem Cohens $d$ von $d_{\text {Gr. }}=1.02$, was einen großen Effekt darstellt. Die Modellanpassung von M0 nach M1a führt zu einer statistisch bedeutsamen Verbesserung der Modellgüte nach dem Bayesschen Informationskriterium $\left(\Delta \mathrm{BIC} \mathrm{M}_{\mathrm{M} 1 \mathrm{a}}=-9.57, \mathrm{~d} f_{\mathrm{M} 0 \mathrm{M} 1 \mathrm{a}}=1, p<.001\right)$.

Tab. 31: Schätzung der festen Parameter von Modell M1a

\begin{tabular}{|l|l|l|l|l|l|l|l|}
\hline \multicolumn{9}{|c|}{} & \multicolumn{2}{l|}{$95 \%$-Konfidenzintervall } \\
\hline Parameter & Schätzung & $S E$ & $\mathrm{~d} f$ & $t$-Wert & Sig. & Untergr. & Obergr. \\
\hline$\gamma_{00}$ & 12.74 & 0.66 & 35.68 & 19.20 & $p<.001$ & 11.39 & 14.09 \\
\hline$\gamma_{01}[=\mathrm{KG}]$ & -4.38 & 0.97 & 36.05 & 4.53 & $p<.001$ & -6.35 & -2.42 \\
\hline$\gamma_{01}[=\mathrm{TG}]$ & 0 & 0 & - & - & - & - & - \\
\hline
\end{tabular}

Anmerkung: $S E=$ Standardfehler; $\mathrm{d} f=$ Freiheitsgrade

Tab. 32 kann entnommen werden, dass durch den Level-2-Prädiktor „Gruppe“ ein großer Teil der im Null-Modell ungeklärten Varianz auf Klassenebene aufgeklärt werden kann, so dass sich die unerklärte Varianz zwischen den Klassen von 12.32 auf nur noch 7.54 deutlich reduziert. Dieser Befund ist bemerkenswert, unterstreicht er doch die verhältnismäßig große Erklärungsmächtigkeit des Prädiktors „Gruppe“ in Bezug auf die im Nachtest erreichte durchschnittliche Punktzahl einer Klasse. Diese Erklärungsmächtigkeit spiegelt sich auch in 
einer ebenenspezifischen Effektstärke von $R^{2}{ }_{G r}$. $=.39$ wider, d.h. alleine durch den Level-2Prädiktor „Gruppe“ werden auf der Klassenebene 39\% der im Null-Modell ungeklärten Varianz aufgeklärt. Die Varianz der Posttest-Ergebnisse innerhalb der Klassen wird durch den Level-2-Prädiktor „Gruppe“ erwartungsgemäß nicht verringert.

Tab. 32: Die ebenenspezifische unerklärte Restvarianz im Modell MIa

\begin{tabular}{|l|l|l|l|l|l|}
\hline Ebene & Varianz & Std.-Fehler & $\chi^{2}$-Wert & Signifikanz & Ebenensp. $R^{2}$ \\
\hline Schüler, $e_{i j}$ & 18.58 & 0.96 & 377.02 & $p<.001$ & $R_{G r .}^{2}<.01$ \\
\hline Klassen, $r_{0 j}$ & 7.54 & 1.98 & 14.47 & $p<.001$ & $R_{G r .}^{2}=.39$ \\
\hline
\end{tabular}

\subsubsection{M2: Prüfung des Einflusses des Pretest-Ergebnisses}

\section{M2a: Der Haupteffekt „Pre“}

Aufbauend auf dem Modell M1a wird nun der Prädiktor „Pre“, also das individuelle PretestErgebnis der Schüler, auf der ersten Ebene des Modells im Sinne eines Haupteffekts eingebaut, um zu untersuchen, wie das Vortestergebnis das Nachtestergebnis beeinflusst und diesen Effekt zu kontrollieren. Um den Einfluss des Vortestergebnisses kontrollieren und die Regressionskonstanten sinnvoll interpretieren zu können, wird das Vortestergebnis um den Mittelwert der gesamten Stichprobe zentriert (,grand-mean-centering“). Da das Modell nun um den Effekt der unterschiedlich hohen Vortestergebnisse in den beiden Gruppen bereinigt und zentriert ist, spiegeln die Regressionskonstanten das in Kontroll- und Treatmentgruppe zu erwartende Nachtestergebnis von Schülern wider, die im Vortest die gleichen und bezogen auf die Gesamtstichprobe durchschnittlichen Leistungen zeigen. Die Modellgleichungen zu Modell M2a lauten wie folgt:

Tab. 33: Modellgleichungen zum Modell M2a

\begin{tabular}{|l|l|l|}
\hline$\overline{\bar{\rho}}$ & Ebene 1 & Post $_{i j}=\beta_{0 j}+\beta_{1 j}$ Pre $+e_{i j}$ \\
\cline { 3 - 3 }$\overline{\overline{0}}$ & Ebene 2 & $\beta_{0 j}=\gamma_{00}+\gamma_{01}$ Gruppe $+r_{0 j}$ \\
& & $\beta_{1 j}=\gamma_{10}$ \\
\hline \multicolumn{2}{|l|}{ Gemischtes Modell } & Post $_{i j}=\gamma_{00}+\gamma_{01}$ Gruppe $+\gamma_{\mathbf{1 0}}$ Pre $+r_{0 j}+e_{i j}$ \\
\hline Modellguite & BIC $=4594.97$ (geschätzte Parameter $\mathrm{d} f=5$ ) \\
\hline
\end{tabular}

In diesem Modell besitzt sowohl der bisherige Level-2-Prädiktor „Gruppe“ $(F(1,36.27)=18.91, \quad p<.001) \quad$ als auch der neue Level-1-Prädiktor „Pre“ $(F(1,779.39)=74.40, p<.001)$ einen signifikanten Einfluss auf das Nachtestergebnis. Die Modellanpassung von M1a zu M2a führt ebenfalls zu einer statistisch bedeutsamen Verbesserung der Modellgüte nach dem Bayesschen Informationskriterium $\left(\triangle \mathrm{BIC}_{\mathrm{M} 1 \mathrm{aM} 2 \mathrm{a}}=-64.30\right.$, $\mathrm{d} f_{\mathrm{M} 1 \mathrm{aM} 2 \mathrm{a}}=1, p<.001$ ), weshalb Modell M2a das neue Basismodell darstellt. In Tab. 34 sind die genauen Parameterschätzungen aufgeführt. 
Tab. 34: Schätzung der festen Parameter von Modell M2a

\begin{tabular}{|l|l|l|l|l|l|l|l|}
\hline \multicolumn{9}{|c|}{} & 95\%-Konfidenzintervall \\
\hline Parameter & Schätzung & $S E$ & $\mathrm{~d} f$ & $t$-Wert & Sig. & Untergr. & Obergr. \\
\hline$\gamma_{00}$ & 12.54 & 0.61 & 35.67 & 20.56 & $p<.001$ & 11.30 & 13.78 \\
\hline$\gamma_{01}[=\mathrm{KG}]$ & -3.88 & 0.89 & 36.27 & -4.35 & $p<.001$ & -5.68 & -2.07 \\
\hline$\gamma_{01}[=\mathrm{TG}]$ & 0 & 0 & - & - & - & - & - \\
\hline$\gamma_{10}[=$ Pre $]$ & 0.44 & 0.05 & 779.39 & 8.63 & $p<.001$ & 0.34 & 0.54 \\
\hline
\end{tabular}

Anmerkung: $S E=$ Standardfehler; $\mathrm{d} f=$ Freiheitsgrade

Die Schätzung des Regressionskoeffizienten zum Prädiktor „Pre“ von $\gamma_{10}=0.44$ $(S E=0.05)$ bedeutet, dass jeder zusätzlich im Vortest erzielte Punkt zu einem um 0.44 Punkte höheren Nachtestergebnis führt. Dieses Ergebnis bedeutet einerseits, dass das Nachtestergebnis umso höher ausfällt, je mehr Punkte im Vortest erzielt wurden. Andererseits bedeutet dieser Zusammenhang aber auch, dass der durch den Unterricht erzielte absolute Zugewinn umso geringer ausfällt, je höher das Vortestergebnis war, denn hätte das Vortestergebnis keinen Einfluss auf den absoluten Lernzuwachs, so müsste das Nachtestergebnis mit jedem zusätzlichen Punkt im Vortest ebenfalls um einen Punkt ansteigen. Der absolute Lernzuwachs, gemessen in der Anzahl der korrekt beantworteten Items, fällt also umso geringer aus, desto höher das Pretest-Ergebnis der Schüler ist. Dieses Ergebnis zeigt, dass der höhere Lernzuwachs in der Treatmentgruppe nicht mit dem „Matthäus-Effekt“, also ihrem höheren durchschnittlichen Pretest-Ergebnis, erklärt werden kann (vgl. Abschnitt 7.4.4).

Da das neue Modell M2a im Gegensatz zum bisherigen Modell M1a die Vortestunterschiede zwischen den beiden Gruppen kontrolliert, ändern sich auch die geschätzten Regressionskonstanten. Während Schüler der Treatmentgruppe im Durchschnitt ein PosttestErgebnis von 12.54 Punkten $(S E=0.61)$ erzielen, liegt dieser Wert in der Kontrollgruppe um 3.88 Punkte $(S E=0.89)$ niedriger. Aufgrund der Kontrolle der Vortestunterschiede zwischen Kontroll- und Treatmentgruppe fällt der im Nachtest modellierte Unterschied zwischen den beiden Gruppen geringer aus als in Modell M1a, wo Gruppenzugehörigkeit und PretestErgebnis, das einen höchst signifikanten positiven Effekt auf das Posttest-Ergebnis hat, noch konfundiert waren. Der auf die Gruppenzugehörigkeit zurückzuführende Unterschied entspricht in diesem Modell einem Cohens $d$ von $d_{\text {Gr. }}=.94$, was einen großen Effekt darstellt.

Tab. 35: Die ebenenspezifische unerklärte Restvarianz im Modell M2a

\begin{tabular}{|l|l|l|l|l|l|}
\hline Ebene & Varianz & Std.-Fehler & $\chi^{2}$-Wert & Signifikanz & Ebenensp. $R^{2}$ \\
\hline Schüler, $e_{i j}$ & 17.05 & 0.88 & 376.97 & $p<.001$ & $R_{\text {Pre }}^{2}=.08$ \\
\hline Klassen, $r_{0 j}$ & 6.29 & 1.67 & 14.14 & $p<.001$ & $R_{\text {Pre }}^{2}=.10$ \\
\hline
\end{tabular}

Obwohl der Level-1-Prädiktor des Vortestergebnisses „Pre“ signifikant ist, zeigt sich in Bezug auf die noch nicht aufgeklärte Varianz, dass er auf Schülerebene nur zu einer geringen Reduktion der Restvarianz beiträgt und auf dieser Ebene somit nicht viel erklärt, da die unaufgeklärte Restvarianz von $e_{i j}$ zwischen M1a und M2a nur von 18.58 auf 17.05 zurück- 
geht $\left(R_{\text {Pre }}^{2}=.08\right)$. Die Restvarianz von $r_{0 j}$ auf Klassenebene geht zwischen M1a und M2a ebenfalls nur leicht von 7.54 auf 6.29 zurück, was aber eine leicht größere Varianzaufklärung darstellt $\left(R_{\text {Pre }}^{2}=.10\right)$. Damit ist die unerklärte Varianz auf der Schüler- und Klassenebene weiterhin höchst signifikant $(p<.001)$. Eine denkbare Erklärung dafür, dass der Level-1Prädiktor „Pre“ den größten Varianzaufklärungsanteil auf der Klassen- und nicht der Schülerebene besitzt, besteht darin, dass sich die Schüler bzgl. des Pretest-Ergebnisses nicht zufällig über die verschiedenen Klassen verteilen, sondern dass z.B. Schüler mit besonders niedrigen Pretest-Ergebnissen gehäuft in bestimmten Klassen zu finden sind.

\section{M2b: Zufallseffekt auf Ebene 2 für den Level-1-Prädiktor ,Pre“}

Mit Hilfe der Erweiterung des Modells M2a um den Zufallseffekt $r_{1 j}$ wird untersucht, wie stark sich die Abhängigkeit des Nachtestergebnisses vom Vortestergebnis zwischen den verschiedenen Klassen unterscheidet, indem die Varianz dieses Zufallseffekts $r_{1 j}$ betrachtet wird.

Tab. 36: Modellgleichungen zum Modell M2b

\begin{tabular}{|c|c|c|}
\hline \multirow[b]{2}{*}{$\begin{array}{l}\overline{\overline{0}} \\
\overline{\bar{\theta}} \\
\sum_{\Sigma}^{0} \\
\dot{I}\end{array}$} & Ebene 1 & Post $_{i j}=\beta_{0 j}+\beta_{1 j}$ Pre $+e_{i j}$ \\
\hline & Ebene 2 & $\begin{array}{l}\beta_{0 j}=\gamma_{00}+\gamma_{01} \text { Gruppe }+r_{0 j} \\
\beta_{1 j}=\gamma_{10}+r_{1 j}\end{array}$ \\
\hline \multicolumn{2}{|c|}{ Gemischtes Modell } & Post $_{i j}=\gamma_{00}+\gamma_{01}$ Gruppe $+\gamma_{10}$ Pre $+\boldsymbol{r}_{1 j}$ Pre $+r_{0 j}+e_{i j}$ \\
\hline \multicolumn{2}{|c|}{ Modellgüte } & $\mathrm{BIC}=4596.90($ geschätzte Parameter $\mathrm{d} f=6)$ \\
\hline
\end{tabular}

Die Abhängigkeit des Nachtestergebnisses vom Vortestergebnis unterscheidet sich zwischen den verschiedenen Klassen nicht signifikant $\left(\operatorname{var}\left(r_{1 j}\right)=0.05, \chi^{2}(1)=2.47, p=.116\right)$. Damit bedarf die Abhängigkeit des Posttest-Ergebnisses vom Pretest-Ergebnis keiner weiteren erklärender Level-2-Prädiktoren (Klassenmerkmalen). Die Modellanpassung von M2a zu M2b führt zudem zu keiner statistisch signifikanten Verbesserung der Modellgüte nach dem Bayesschen Informationskriterium $\left(\Delta \mathrm{BIC}_{\mathrm{M} 2 \mathrm{aM} 2 \mathrm{~b}}=1.93, \mathrm{~d} f_{\mathrm{M} 2 \mathrm{aM} 2 \mathrm{~b}}=1, p=.082\right)$, weshalb das Modell M2b verworfen wird.

M2c: Interaktionseffekt von „Gruppe“ (E2) und „Pre“ (E1)

Mit Hilfe der Erweiterung des Modells M2a um den Interaktionseffekt $\gamma_{11}$ Gruppe $\cdot$ Pre wird untersucht, ob sich die Abhängigkeit des Nachtestergebnisses vom Vortestergebnis zwischen Kontroll- und Treatmentgruppe unterscheidet. 
Tab. 37: Modellgleichungen zum Modell M2c

\begin{tabular}{|c|c|c|}
\hline \multirow[b]{2}{*}{$\begin{array}{l}\overline{\bar{\theta}} \\
\bar{\delta} \\
\dot{\Sigma} \\
\bar{\Sigma}\end{array}$} & Ebene 1 & Post $_{i j}=\beta_{0 j}+\beta_{1 j}$ Pre $+e_{i j}$ \\
\hline & Ebene 2 & $\begin{array}{l}\beta_{0 j}=\gamma_{00}+\gamma_{01} \text { Gruppe }+r_{0 j} \\
\beta_{1 j}=\gamma_{10}+\gamma_{11} \text { Gruppe }\end{array}$ \\
\hline \multicolumn{2}{|c|}{ Gemischtes Modell } & Post $_{i j}=\gamma_{00}+\gamma_{01}$ Gruppe $+\gamma_{10}$ Pre $+\gamma_{11}$ Gruppe $\cdot$ Pre $+r_{0 j}+e_{i j}$ \\
\hline \multicolumn{2}{|c|}{ Modellguite } & $\mathrm{BIC}=4599.75$ (geschätzte Parameter $\mathrm{d} f=6)$ \\
\hline
\end{tabular}

In diesem Modell ist der Interaktionseffekt $\gamma_{11}$ Gruppe Pre statistisch nicht signifikant $(F(1,781.03)=1.90, p=.169)$. Der Einfluss des Vortestergebnisses auf das Nachtestergebnis unterscheidet sich also nicht bedeutsam zwischen Kontroll- und Treatmentgruppe. Die Modellanpassung von M2a zu M2c führt zudem zu einer statistisch signifikanten Verschlechterung der Modellgüte nach dem Bayesschen Informationskriterium $\left(\triangle \mathrm{BIC}_{\mathrm{M} 2 \mathrm{aM} 2 \mathrm{c}}=4.78\right.$, $\left.\mathrm{d} f_{\mathrm{M} 2 \mathrm{aM} 2 \mathrm{c}}=1, p=.014\right)$, weshalb das Modell M2c verworfen wird.

\subsubsection{M3: Prüfung des Einflusses der Jahrgangsstufe}

\section{M3a: Der Haupteffekt ,Stufe}

Aufbauend auf dem Modell M2a wird nun der dichotome Level-2-Prädiktor „Stufe“, also die Jahrgangsstufe der unterschiedlichen Klassen, auf der zweiten Ebene des Modells im Sinne eines Haupteffekts hinzugefügt. Auf diese Weise soll untersucht werden, ob das Nachtestergebnis signifikant davon abhängt, in welcher Jahrgangsstufe sich die Schüler befinden. Die Modellgleichungen zu Modell M3a lauten wie folgt:

Tab. 38: Modellgleichungen zum Modell M3a

\begin{tabular}{|c|c|c|}
\hline \multirow[b]{2}{*}{$\begin{array}{l}\overline{\overline{0}} \\
\overline{8} \\
\sum^{\circ} \\
\end{array}$} & Ebene 1 & Post $_{i j}=\beta_{0 j}+\beta_{1 j}$ Pre $+e_{i j}$ \\
\hline & Ebene 2 & $\begin{array}{l}\beta_{0 j}=\gamma_{00}+\gamma_{01} \text { Gruppe }+\gamma_{02} \text { Stufe }+r_{0 j} \\
\beta_{1 j}=\gamma_{10}\end{array}$ \\
\hline \multicolumn{2}{|c|}{ Gemischtes Modell } & Post $_{i j}=\gamma_{00}+\gamma_{01}$ Gruppe $+\gamma_{02}$ Stufe $+\gamma_{10}$ Pre $+r_{0 j}+e_{i j}$ \\
\hline \multicolumn{2}{|c|}{ Modellgüte } & $\mathrm{BIC}=4598.25($ geschätzte Parameter $\mathrm{d} f=6)$ \\
\hline
\end{tabular}

In diesem Modell ist der Haupteffekt „Stufe“ statistisch nicht signifikant $(F(1,36.40)=3.55, p=.068)$. Das durchschnittlich erzielte Posttest-Ergebnis von Schülern der siebten und achten Jahrgangsstufe unterscheidet sich also nicht bedeutsam voneinander. In anderen Worten hat die Jahrgangsstufe also keinen signifikanten Einfluss auf das PosttestErgebnis der Schüler. Zudem geht die Modellanpassung von M2a zu M3a mit einer statistisch signifikanten Verschlechterung der Modellgüte nach dem Bayesschen Informationskriterium einher $\left(\Delta \mathrm{BIC}_{\mathrm{M} 2 \mathrm{aM} 3 \mathrm{a}}=3.28, \mathrm{~d} f_{\mathrm{M} 2 \mathrm{aM} 3 \mathrm{a}}=1, p=.035\right)$, weshalb das Modell M3a verworfen wird. Da der hier getestete Haupteffekt weder einen signifikanten Einfluss auf das Posttest-Ergebnis 
hat, noch zur Verbesserung der Modellgüte beiträgt, erübrigt sich eine weitere Untersuchung dieser Modellreihe bzgl. potenzieller Zufalls- und Interaktionseffekten.

\section{$\underline{\text { M3b: Der Interaktionseffekt von ,Gruppe“ (E2) und ,Stufe“ (E2) }}$}

Nichtsdestotrotz soll einmal interessehalber untersucht werden, wie groß der Interaktionseffekt zwischen der Gruppenzugehörigkeit und der Jahrgangsstufe ausfällt, indem statt des Haupteffekts „Stufe“ der Interaktionseffekt $\gamma_{02}$ Gruppe S Stufe in die Modellgleichung von M2a eingefügt wird. Sollte dieser signifikant werden, würde sich der Einfluss der Jahrgangsstufe auf das Posttest-Ergebnis zwischen Kontroll- und Treatmentgruppe unterscheiden. Die Modellgleichungen zu Modell M3b lauten wie folgt:

Tab. 39: Modellgleichungen zum Modell M3b

\begin{tabular}{|c|c|c|}
\hline \multirow{2}{*}{$\begin{array}{l}\overline{0} \\
\frac{8}{8} \\
\sum^{2}\end{array}$} & Ebene 1 & Post $_{i j}=\beta_{0 j}+\beta_{1 j}$ Pre $+e_{i j}$ \\
\hline & Ebene 2 & $\begin{array}{l}\beta_{0 j}=\gamma_{00}+\gamma_{01} \text { Gruppe }+\gamma_{02} \text { Gruppe } \cdot \text { Stufe }+r_{0 j} \\
\beta_{1 j}=\gamma_{10}\end{array}$ \\
\hline \multicolumn{2}{|c|}{ Gemischtes Modell } & Post $_{i j}=\gamma_{00}+\gamma_{01}$ Gruppe $+\gamma_{10}$ Pre $+\gamma_{02}$ Gruppe $\cdot$ Stufe $+r_{0 j}+e_{i j}$ \\
\hline \multicolumn{2}{|c|}{ Modellguite } & $\mathrm{BIC}=4604.48$ (geschätzte Parameter $\mathrm{d} f=7)$ \\
\hline
\end{tabular}

Erwartungsgemäß ist der Interaktionseffekt $\gamma_{02}$ Gruppe $\cdot$ Stufe in diesem Modell statistisch nicht signifikant $(F(2,36.44)=2.02, p=.147)$. Das durchschnittlich erzielte PosttestErgebnis von Schülern der siebten und achten Jahrgangsstufe unterscheidet sich zwischen Kontroll- und Treatmentgruppe damit nicht bedeutsam voneinander. Die Regressionskonstante für Schüler der siebten Jahrgangsstufe in der Kontrollgruppe ist mit -2.49 Punkten $(S E=1.36)$ statistisch gesehen nicht signifikant geringer als die von Schülern aus der achten Jahrgangsstufe $(p=.075)$, weshalb es keinen bedeutsamen Unterschied innerhalb der Kontrollgruppe zwischen Schülern der siebten und achten Jahrgangsstufe hinsichtlich des erzielten Posttest-Ergebnisses gibt. Die Regressionskonstante für Schüler der siebten Jahrgangsstufe in der Treatmentgruppe ist mit -1.17 Punkten $(S E=1.42)$ statistisch gesehen ebenfalls nicht signifikant geringer als die von Schülern aus der achten Jahrgangsstufe ( $p=.414)$, weshalb es auch in der Treatmentgruppe keinen bedeutsamen Unterschied zwischen Schülern der siebten und achten Jahrgangsstufe hinsichtlich des erzielten Posttest-Ergebnisses gibt. Zudem geht die Modellanpassung von M2a zu M3b mit einer statistisch signifikanten Verschlechterung der Modellgüte nach dem Bayesschen Informationskriterium einher $\left(\Delta \mathrm{BIC}_{\mathrm{M} 2 \mathrm{aM} 3 \mathrm{~b}}=9.51\right.$, $\left.\mathrm{d} f_{\mathrm{M} 2 \mathrm{aM} 3 \mathrm{~b}}=2, p=.004\right)$, weshalb das Modell M3b verworfen wird. 


\subsubsection{M4: Prüfung des Einflusses der unterrichteten Stundenanzahl}

\section{M4a: Der Haupteffekt „Stunden“"}

Aufbauend auf dem Modell M2a wird nun der Level-2-Prädiktor „Stunden“, also die in jeder Klasse stattgefundene Anzahl an Unterrichtsstunden zur Einheit Elektrizitätslehre, auf der zweiten Ebene des Modells im Sinne eines Haupteffekts modelliert, um zu untersuchen, ob dieser Prädiktor einen signifikanten Einfluss auf das Posttest-Ergebnis hat. Die Modellgleichungen zu Modell M4a lauten wie folgt:

Tab. 40: Modellgleichungen zum Modell M4a

\begin{tabular}{|l|l|l|}
\hline \multirow{\overline{\rho}}{\overline{0}}{} & Ebene 1 & Post $_{i j}=\beta_{0 j}+\beta_{1 j}$ Pre $+e_{i j}$ \\
\cline { 3 - 3 }$\overline{0}$ & Ebene 2 & $\beta_{0 j}=\gamma_{00}+\gamma_{01}$ Gruppe $+\gamma_{02}$ Stunden $+r_{0 j}$ \\
& $\beta_{1 j}=\gamma_{10}$ \\
\hline Gemischtes Modell & Post $_{i j}=\gamma_{00}+\gamma_{01}$ Gruppe $+\gamma_{\mathbf{0 2}}$ Stunden $+\gamma_{10}$ Pre $+r_{0 j}+e_{i j}$ \\
\hline Modellguite & $\mathrm{BIC}=4599.39$ (geschätzte Parameter $\mathrm{d} f=6)$ \\
\hline
\end{tabular}

In diesem Modell ist der Haupteffekt „Stunden“ statistisch nicht signifikant $(F(1,35.81)=2.32, p=.136)$. Die Anzahl der Unterrichtsstunden hat also keinen bedeutsamen Einfluss auf die Leistungen im Nachtest. Dies könnte darauf zurückzuführen sein, dass der Test lediglich das grundlegende Konzeptverständnis der Lernenden prüft und dieses zu einem durchschnittlichen Niveau auch schon nach verhältnismäßig wenigen Stunden erreicht werden kann. Da über dieses grundlegende Konzeptverständnis hinausgehende Fähigkeiten und Kompetenzen, die möglicherweise in Klassen mit höherer Stundenanzahl verstärkt vermittelt werden, im Test nicht abgeprüft werden, lässt sich aus dem Ergebnis nicht folgern, dass die Kompetenzen und Fähigkeiten der Lernenden im Unterrichtsfach Physik durch eine höhere Anzahl an Physikstunden nicht weiter gefördert werden könnten. Zudem geht auch hier die Modellanpassung von M2a zu M4a mit einer statistisch signifikanten Verschlechterung der Modellgüte nach dem Bayesschen Informationskriterium einher $\left(\triangle \mathrm{BIC} \mathrm{M}_{\mathrm{M} 2 \mathrm{aM} 4 \mathrm{a}}=4.42\right.$, $\mathrm{d} f_{\mathrm{M} 2 \mathrm{aM} 4 \mathrm{a}}=1, p=.018$ ), weshalb das Modell M4a verworfen wird. Da der hier getestete Haupteffekt weder einen signifikanten Einfluss auf das Posttest-Ergebnis hat, noch zur Verbesserung der Modellgüte beiträgt, erübrigt sich hier ebenfalls eine weitere Untersuchung dieser Modellreihe bzgl. Zufalls- und Interaktionseffekten.

\section{M4b: Der Interaktionseffekt von „Gruppe“ (E2) und „Stunden“ (E2)}

Nichtsdestotrotz soll auch hier interessehalber untersucht werden, wie groß der Interaktionseffekt zwischen der Gruppenzugehörigkeit und der Stundenanzahl ausfällt, indem statt des Haupteffekts ,Stunden“ der Interaktionseffekt $\gamma_{02}$ Gruppe · Stunden in die Modellgleichung von M2a eingefügt wird. Sollte dieser signifikant werden, würde sich der Einfluss der unterrichteten Stunden auf das Posttest-Ergebnis zwischen Kontroll- und Treatmentgruppe unterscheiden. Die Modellgleichungen zu Modell M4b lauten wie folgt: 
Kapitel 7: Summative Evaluation des Unterrichtskonzepts

Tab. 41: Modellgleichungen zum Modell M4b

\begin{tabular}{|l|l|l|}
\hline \multirow{2}{*}{$\overline{\overline{0}}$} & Ebene 1 & Post $_{i j}=\beta_{0 j}+\beta_{1 j}$ Pre $+e_{i j}$ \\
\cline { 3 - 3 }$\overline{0}$ & Ebene 2 & $\beta_{0 j}=\gamma_{00}+\gamma_{01}$ Gruppe $+\beta_{02}$ Gruppe $\cdot$ Stunden $+r_{0 j}$ \\
& $\beta_{1 j}=\gamma_{10}$ \\
\hline & \\
\hline & \\
\hline
\end{tabular}

Erwartungsgemäß ist der Interaktionseffekt $\gamma_{02}$ Gruppe $\cdot$ Stunden in diesem Modell statistisch nicht signifikant $(F(2,35.90)=1.60, p=.216)$. Der Einfluss der unterrichteten Stunden auf das Posttest-Ergebnis der Schüler unterscheidet sich somit zwischen Kontrollund Treatmentgruppe nicht bedeutsam voneinander. Die Schätzung des Regressionskoeffizienten für Schüler der Kontrollgruppe von $\gamma_{02}=0.09(S E=0.05)$ bedeutet, dass mit jeder Physikstunde in der Kontrollgruppe das zu erwartende Posttest-Ergebnis der Schüler um 0.09 Punkte ansteigt, wobei dieser Effekt nicht signifikant ist $(p=.087)$. Für Schüler der Treatmentgruppe liegt dieser Wert bei $\gamma_{02}=0.02(S E=0.06)$, was bedeutet, dass mit jeder Physikstunde in der Treatmentgruppe das zu erwartende Posttest-Ergebnis der Schüler um 0.02 Punkte ansteigt, wobei auch dieser Effekt nicht signifikant ist $(p=.746)$. Tendenziell deutet dieses Ergebnis darauf hin, dass mehr Unterrichtsstunden in der Kontrollgruppe eine höhere Punktzahl im Nachtest zu Folge haben, während die Anzahl der Unterrichtsstunden in der Treatmentgruppe keinen Einfluss auf das Posttest-Ergebnis zu haben scheint. Hier könnte eine Erklärung darin bestehen, dass ein grundlegendes Konzeptverständnis beim neuen Unterrichtskonzept schon nach verhältnismäßig wenigen Stunden erzielt werden kann, während dies im traditionellen Unterricht erst mit zunehmenden Stundenzahlen gelingt. Die Modellanpassung von M2a zu M4b geht mit einer statistisch signifikanten Verschlechterung der Modellgüte nach dem Bayesschen Informationskriterium einher $\left(\Delta \mathrm{BIC}_{\mathrm{M} 2 \mathrm{aM} 4 \mathrm{~b}}=10.27\right.$, $\left.\mathrm{d} f_{\mathrm{M} 2 \mathrm{aM} 4 \mathrm{~b}}=2, p=.003\right)$, weshalb das Modell M4b verworfen wird.

\subsubsection{M5: Prïfung des Einflusses des mittleren Klassenergebnisses im Pretest}

\section{M5a: Der Haupteffekt ,PCM“"}

Aufbauend auf dem Modell M2a wird nun der Level-2-Prädiktor „PCM“, was für „Pre Class Mean“, also das durchschnittliche in jeder einzelnen Klasse erzielte Pretest-Ergebnis steht, auf der zweiten Ebene des Modells im Sinne eines Haupteffekts modelliert, um zu untersuchen, ob dieser Prädiktor einen signifikanten Einfluss auf das Posttest-Ergebnis der Schüler hat. Sollte dies zutreffen, wäre das individuelle Posttest-Ergebnis einzelner Schüler abhängig vom durchschnittlichen Pretest-Ergebnis ihrer Klasse. Nehmen wir beispielsweise zwei Schüler A und B mit gleichem Pretest-Ergebnis an, wobei Schüler A sich in einer Klasse mit einem niedrigem durchschnittlichen Pretest-Ergebnis befindet und Schüler B in einer Klasse mit hohem durchschnittlichen Pretest-Ergebnis. Wenn das mittlere Klassenergebnis im Pretest einen positiven Einfluss auf das individuelle Nachtestergebnis der Schüler hat, dann würde 
Schüler B trotz sonst identischer Ausgangsbedingungen alleine aufgrund seiner Klassenzugehörigkeit im Posttest besser abschneiden als Schüler A, z.B. weil ein hohes mittleres Klassenergebnis im Pretest möglicherweise einen Indikator für gute Lernbedingungen in der Klasse darstellt. Die Modellgleichungen zu Modell M5a lauten wie folgt:

Tab. 42: Modellgleichungen zum Modell M5a

\begin{tabular}{|c|c|c|}
\hline \multirow[b]{2}{*}{$\begin{array}{l}\overline{0} \\
\frac{0}{8} \\
\sum^{\circ}\end{array}$} & Ebene 1 & Post $_{i j}=\beta_{0 j}+\beta_{1 j}$ Pre $+e_{i j}$ \\
\hline & Ebene 2 & $\begin{array}{l}\beta_{0 j}=\gamma_{00}+\gamma_{01} \text { Gruppe }+\gamma_{02} \text { PCM }+r_{0 j} \\
\beta_{1 j}=\gamma_{10}\end{array}$ \\
\hline \multicolumn{2}{|c|}{ Gemischtes Modell } & Post $_{i j}=\gamma_{00}+\gamma_{01}$ Gruppe $+\gamma_{02}$ PCM $+\gamma_{10}$ Pre $+r_{0 j}+e_{i j}$ \\
\hline \multicolumn{2}{|c|}{ Modellgüte } & $\mathrm{BIC}=4597.08$ (geschätzte Parameter $\mathrm{d} f=6)$ \\
\hline
\end{tabular}

In diesem Modell wird der Haupteffekt „PCM“ statistisch signifikant $(F(1,38.11)=4.84, p=.034)$. Das durchschnittliche Pretest-Ergebnis einer Klasse hat also einen bedeutsamen Einfluss auf das Posttest-Ergebnis einzelner Schüler. Die Schätzung des Regressionskoeffizienten zum Prädiktor „PCM“ von $\gamma_{02}=0.73(S E=0.33)$ bedeutet, dass mit jedem Punkt, der in einer Klasse im Vortest durchschnittlich erzielt wurde, ein einzelner Schüler unabhängig von seinem individuellen Pretest-Ergebnis mit einem um 0.73 Punkten höheren Nachtestergebnis rechnen kann. Im Rahmen der statistischen Sicherheit hängt das individuelle Abschneiden eines Schülers im Nachtest damit in gleichem Maße von seinem individuellen Vortestergebnis $(0.44 \pm 0.05$ Punkte $)$ wie vom durchschnittlichen Vortestergebnis seiner Klasse $(0.73 \pm 0.33$ Punkte $)$ ab.

Tab. 43: Schätzung der festen Parameter von Modell M5a

\begin{tabular}{|l|l|l|l|l|l|l|l|}
\hline \multicolumn{9}{|c|}{} & $95 \%$-Konfidenzintervall \\
\hline Parameter & Schätzung & $S E$ & $\mathrm{~d} f$ & $t$-Wert & Sig. & Untergr. & Obergr. \\
\hline$\gamma_{00}$ & 8.03 & 2.13 & 37.95 & 3.77 & $p=.001$ & 3.71 & 12.34 \\
\hline$\gamma_{01}[=\mathrm{KG}]$ & -3.05 & 0.92 & 36.41 & 3.31 & $p=.002$ & -4.91 & -1.18 \\
\hline$\gamma_{01}[=\mathrm{TG}]$ & 0 & 0 & - & - & - & - & - \\
\hline$\gamma_{10}[=$ Pre $]$ & 0.42 & 0.05 & 754.32 & 8.23 & $p<.001$ & 0.32 & 0.52 \\
\hline$\gamma_{02}[=\mathrm{PCM}]$ & 0.73 & 0.33 & 38.11 & 2.20 & $p=.034$ & 0.06 & 1.40 \\
\hline
\end{tabular}

Anmerkung: $S E=$ Standardfehler; $\mathrm{d} f=$ Freiheitsgrade

Der Level-2-Prädiktor „PCM“ führt auf Schülerebene nicht zu einer ebenenspezifischen Varianzaufklärung $\left(R_{P C M}^{2}<.01\right)$, d.h. die innerhalb einer Klasse im Nachtest bestehenden Leistungsunterschiede sind unabhängig vom mittleren im Pretest erzielten Klassenergebnis. Die ungeklärte Restvarianz auf Klassenebene geht allerdings in Folge des Level-2Prädiktors „PCM“ von 6.29 leicht auf 5.48 zurück, was einer ebenenspezifischen Varianzaufklärung von $7 \%$ gegenüber dem Modell M2a entspricht $\left(R_{P C M}^{2}=.07\right)$. Vor dem Hintergrund, dass das individuelle Pretest-Ergebnis das individuelle Posttest-Ergebnis signifikant beeinflusst (Modell M2a), ist es wenig überraschend, dass auch das durchschnittliche Pretest- 
Kapitel 7: Summative Evaluation des Unterrichtskonzepts

Ergebnis einer Klasse das durchschnittliche Posttest-Ergebnis einer Klasse signifikant beeinflusst.

Tab. 44: Die ebenenspezifische unerklärte Restvarianz im Modell M5a

\begin{tabular}{|l|l|l|l|l|l|}
\hline Ebene & Varianz & Std.-Fehler & $\chi^{2}$-Wert & Signifikanz & Ebenensp. $R^{2}$ \\
\hline Schüler, $e_{i j}$ & 17.05 & 0.88 & 377.16 & $p<.001$ & $R_{P C M}^{2}<.01$ \\
\hline Klassen, $r_{0 j}$ & 5.48 & 1.47 & 13.82 & $p<.001$ & $R_{P C M}^{2}=.07$ \\
\hline
\end{tabular}

Da die Modellanpassung von M2a zu M5a allerdings nicht zu einer statistisch signifikanten Verbesserung der Modellgüte nach dem Bayesschen Informationskriterium führt $\left(\Delta \mathrm{BIC}_{\mathrm{M} 2 \mathrm{aM} 5 \mathrm{a}}=2.11, \mathrm{~d} f_{\mathrm{M} 2 \mathrm{aM} 5 \mathrm{a}}=1, p=.073\right)$, bleibt das Modell M2a weiterhin das Basismodell. Dies hat auch den Vorteil, dass eine Vergleichbarkeit der mittels Mehrebenenanalyse gefundenen Ergebnisse und hier insbesondere der Regressionskonstanten mit den z.B. mittels Kovarianzanalyse ermittelten Ergebnissen gewahrt bleibt, da in beiden Fällen nur das individuelle Pretest-Ergebnis und die Gruppenzugehörigkeit berücksichtigt werden.

\subsubsection{Zusammenfassung der Ergebnisse der Mehrebenenanalyse}

Die mehrebenenanalytische Auswertung der Daten der Studie zeigt, dass grundsätzlich 40\% der Varianz der Posttest-Ergebnisse auf Unterschiede zwischen den verschiedenen Klassen zurückgehen und 60\% auf Unterschiede zwischen den einzelnen Schülern innerhalb der Klassen. Ohne zwischen Kontroll- und Treatmentgruppe zu unterscheiden, liegt der Gesamtmittelwert aller Schüler im Nachtest bei $10.67 \pm 0.61$ Punkten. Durch die Berücksichtigung der Gruppenzugehörigkeit im Modell M1a können 39\% der ursprünglich nicht-erklärten Unterschiede im Nachtest zwischen den verschiedenen Klassen erklärt werden. Dieses Ergebnis zeigt, wie stark das mittlere Nachtestergebnis einer Klasse davon abhängt, ob sie klassisch oder nach dem neuen Unterrichtskonzept unterrichtet wurde. Betrachtet man das durchschnittlich erzielte Posttest-Ergebnis, zeigt sich in Modell M1a, dass die Schüler der Treatmentgruppe mit $12.74 \pm 0.66$ Punkten im Durchschnitt $4.38 \pm 0.97$ Punkte mehr im Nachtest erzielen als ihre traditionell unterrichteten Peers.

Indem im Modell M2a zusätzlich das Pretest-Ergebnis der einzelnen Schüler berücksichtigt wird, können $8 \%$ der Unterschiede im Nachtest zwischen den einzelnen Schülern innerhalb der Klassen und weitere $10 \%$ der Unterschiede zwischen den verschiedenen Klassen erklärt werden. Konkret zeigt sich im Modell M2a, dass für einen Lernenden das Nachtestergebnis mit jedem im Vortest erzielten Punkt um $0.44 \pm 0.05$ Punkte ansteigt. Die Kontrolle der Vortestunterschiede in Modell M2a führt dazu, dass der modellierte durchschnittliche Punktevorsprung der Treatment- gegenüber der Kontrollgruppe im Nachtestergebnis auf $3.88 \pm 0.89$ Punkte zurückgeht, wobei Schüler der Treatmentgruppe im Durchschnitt nunmehr mit $12.54 \pm 0.61$ Punkten im Nachtest rechnen können. Dieser Unterschied zwischen den beiden Gruppen entspricht einem Cohens $d$ von $d_{\text {Gr. }}=.94$, was einen großen Effekt darstellt. Modell M2b zeigt, dass sich der Einfluss des Vortestergebnisses auf das Nachtestergebnis nicht signifikant zwischen den verschiedenen Klassen unterscheidet und Modell M2c zeigt 
darüber hinaus, dass sich die Abhängigkeit des Nachtestergebnisses vom Vortestergebnis insbesondere nicht signifikant zwischen Kontroll- und Treatmentgruppe unterscheidet.

Die weitere Modellentwicklung kommt zu dem Ergebnis, dass die Jahrgangsstufe der Schüler (Modell M3) und die Anzahl der Unterrichtsstunden (Modell M4) keinen signifikanten Einfluss auf das Nachtestergebnis haben. Das mittlere Klassenergebnis im Pretest (Modell M5) hat hingegen einen signifikanten Einfluss auf das individuelle Nachtestergebnis der Schüler und erklärt 7\% der Unterschiede im Nachtest zwischen den Klassen. Auch wenn entsprechend dieses Modells jeder Punkt, der von einer Klasse im Vortest durchschnittlich erzielt wurde, dazu führt, dass das Nachtestergebnis einzelner Schüler unabhängig von ihrem individuellen Vortestergebnis um $0.73 \pm 0.33$ Punkte höher ausfällt, führt Modell M5 nicht zu einer signifikanten Verbesserung der Modellgüte nach dem Bayesschen Informationskriterium, weshalb Modell M2a das Gesamtmodell der Mehrebenenanalyse darstellt.

Tab. 45: Übersicht über die Kennzahlen der Hauptmodelle der Mehrebenenanalyse

\begin{tabular}{|c|c|c|c|c|c|}
\hline & \multicolumn{3}{|c|}{$\begin{array}{l}\text { Die Hauptmodelle der } \\
\text { Mehrebenenanalyse }\end{array}$} & \multicolumn{2}{|c|}{$\begin{array}{l}\text { Effektstärken der } \\
\text { einzelnen Prädiktoren }\end{array}$} \\
\hline & M0 & M1a & M2a & Gruppe (M1a) & Pre (M2a) \\
\hline Haupteffekte & & & & \multicolumn{2}{|c|}{ Cohens $d$} \\
\hline Intercept, $\gamma_{00}$ & $10.67 * * *$ & $12.74 * * *$ & $12.54 * * *$ & & \\
\hline Gruppe, $\gamma_{01}[=\mathrm{KG}]$ & & $-4.38 * * *$ & $-3.88 * * *$ & $d_{\text {Gr. }}=1.02$ & $d_{\mathrm{Gr} .}=.94$ \\
\hline Pre, $\gamma_{10}$ & & & $0.44 * * *$ & & \\
\hline Restvarianz & & & & \multicolumn{2}{|c|}{ Ebenenspez. $R^{2}$} \\
\hline auf Ebene $1, e_{i j}$ & $18.58 * * *$ & $18.58 * * *$ & $17.05 * * *$ & $R_{G r .}^{2}<.01$ & $R_{\text {Pre }}^{2}=.08$ \\
\hline auf Ebene $2, r_{0 j}$ & $12.32 * * *$ & $7.54 * * *$ & $6.29 * * *$ & $R_{G r .}^{2}=.39$ & $R_{\text {Pre }}^{2}=.10$ \\
\hline \multicolumn{6}{|l|}{ Modellgüte } \\
\hline $\mathrm{BIC}$ & 4668.84 & 4659.27 & 4594.97 & & \\
\hline$\Delta \mathrm{BIC}$ & & $-9.57 * * *$ & $-64.30 * * *$ & & \\
\hline
\end{tabular}

Signifikanzniveaus: $* * * p<.001 ; * * p<.01 ; * p<.05$

Um eine bessere Übersicht über die Entwicklung der Hauptmodelle der Mehrebenenanalyse zu ermöglichen, sind jeweils die Regressionsparameter und ihre statistische Signifikanz sowie die in Abschnitt 7.5.1 erklärten Effektstärken und Modellgütekennzahlen in Tab. 45 zusammengefasst. Unter Hauptmodellen werden dabei neben dem Null-Modell solche Modelle verstanden, die nach dem Bayesschen Informationskriterium zu einer signifikanten Verbesserung der Modellgüte beitragen. Während Cohens $d$ auch bei der varianzanalytischen Auswertung der Daten genutzt wurde, stellt das ebenenspezifische $R^{2}$ hier eine Besonderheit der Mehrebenenanalyse dar und ist ein Maß für die durch einen hinzugefügten Prädiktor erzielte Varianzaufklärung auf einer Ebene. Dementsprechend bedeutet beispielsweise die Angabe von $R_{\text {Pre }}^{2}=.08$ bei der Restvarianz auf Ebene 1, dass der Level-1-Prädiktors „Pre“ (das individuelle Vortestergebnis der Schüler) insgesamt 8\% der bisher in Modell M1a ungeklärten Restvarianz auf der Schülerebene aufklären kann. 


\subsubsection{Darstellung des Gesamtmodells M2a}

Das Ergebnis der oben skizzierten sukzessiven Modellentwicklung stellt das Modell M2a dar, das den dichotomen Level-2-Prädiktor „Gruppe“ (also die Zugehörigkeit zu Kontroll- oder Treatmentgruppe) und den Level-1-Prädiktor „Pre“ (also das individuelle Vortestergebnis) als Haupteffekte modelliert und gleichzeitig die unterschiedlichen Vortestergebnisse der beiden Gruppen kontrolliert. Bei gleichem Vortestergebnis erzielen Schüler, die nach dem neuen Unterrichtskonzept auf Basis des Elektronengasmodells unterrichtet worden sind, entsprechend Modell M2a im Nachtest durchschnittlich 12.54 Punkte ( $S E=0.61)$, während dieser Wert bei traditionell unterrichteten Schülern 3.88 Punkte $(S E=0.89)$ niedriger liegt.

\section{Posttest-Ergebnis von KG und TG}
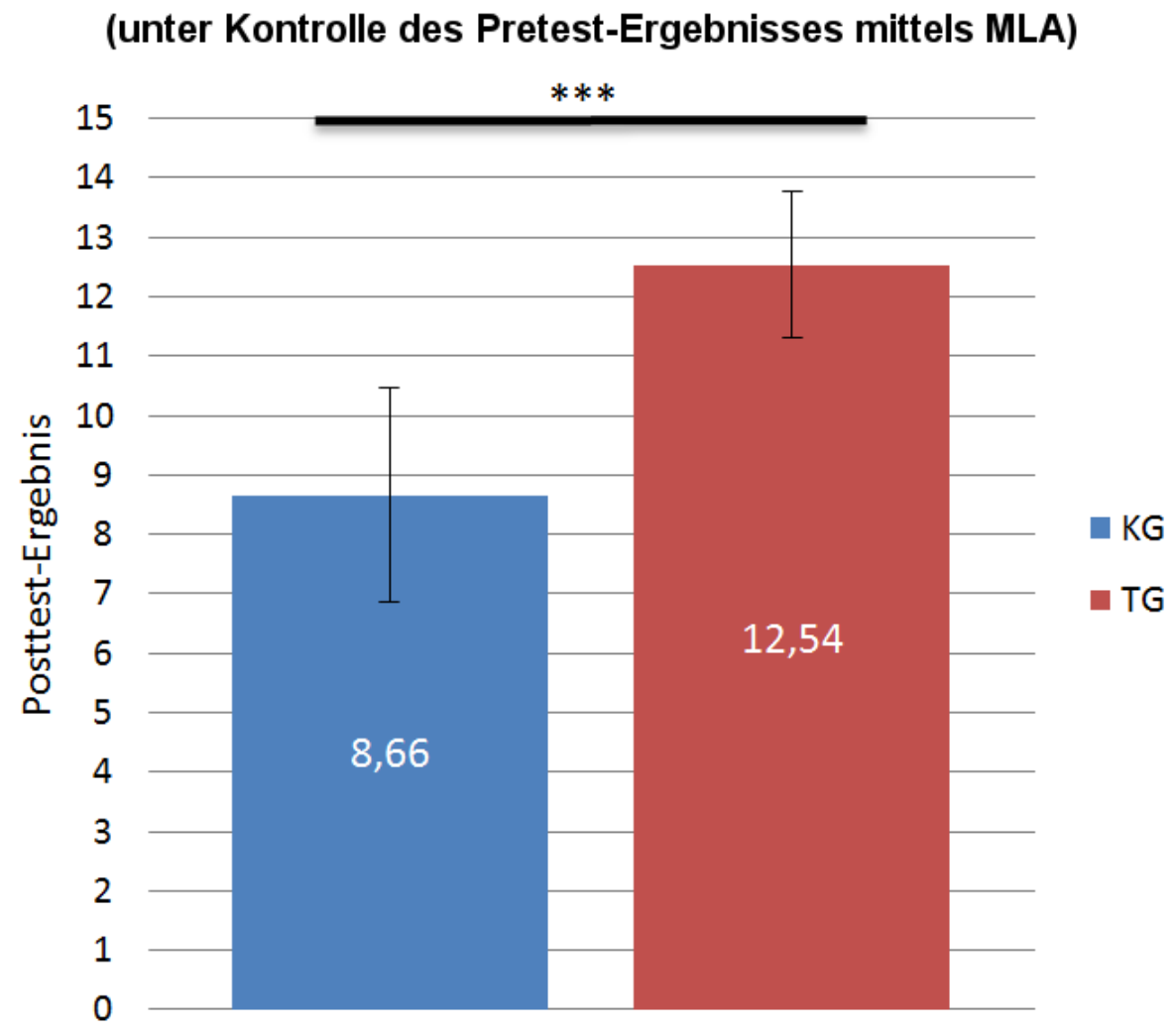

Abb. 112: Das entsprechend des Gesamtmodells M2a modellierte Posttest-Ergebnis für die KG und TG (Signifikanzniveaus: $* * * p<.001 ; * * p<.01 ; * p<.05 ;$ MLA $=$ Multilevel Analysis)

Dieser alleine auf die Gruppenzugehörigkeit zurückzuführende Unterschied ist höchst signifikant $(F(1,36.27)=18.91, p<.001)$ und entspricht einem Cohens $d$ von $d_{\text {Gr. }}=.94$, was einen großen Effekt darstellt. Interessant ist ferner der höchstsignifikante Effekt, dass jeder zusätzlich im Vortest erzielte Punkt mit einer Erhöhung des Nachtestergebnisses von 0.44 Punkten $(S E=0.05)$ einhergeht $(F(1,779.39)=74.40, p<.001)$. Auch wenn das Posttest-Ergebnis damit umso höher ausfällt, desto höher das Pretest-Ergebnis ist, zeigt dieses Ergebnis auch, dass der absolute Lernzuwachs umso geringer ausfällt, desto höher das Vortestergebnis der Schüler ist. Damit weist auch die mehrebenenanalytische Auswertung darauf hin, dass der höhere Lernzuwachs in der Treatmentgruppe nicht auf deren höheres PretestErgebnis zurückzuführen ist und damit kein „Matthäus-Effekt“" vorliegt (vgl. Abschnitt 7.4.4). 
Tab. 46: Zentrale Kenngrößen des Gesamtmodells M2a zum Posttest-Ergebnis

\begin{tabular}{|c|c|c|c|c|c|c|}
\hline & \multicolumn{6}{|c|}{ Zentrale Kenngrößen des Gesamtmodells M2a } \\
\hline Haupteffekte & $\beta$ & $S E$ & $d f$ & $\bar{t}$ & Sig. & Cohens $d$ \\
\hline Intercept, $\gamma_{00}$ & 12.54 & 0.61 & 35.67 & 20.56 & $p<.001$ & \\
\hline Gruppe, $\gamma_{01}[=\mathrm{KG}]$ & -3.88 & 0.89 & 36.27 & -4.35 & $p<.001$ & $d_{\text {Gr. }}=.94$ \\
\hline Pre, $\gamma_{10}$ & 0.44 & 0.05 & 779.39 & 8.63 & $p<.001$ & \\
\hline Restvarianz & Var & $S E$ & Anteil & $\chi^{2}$ & Sig. & Ebenensp. $R^{2}$ \\
\hline auf Ebene $1, e_{i j}$ & 17.05 & 0.88 & $73 \%$ & 376.97 & $p<.001$ & $R_{E 1}^{2}=.08$ \\
\hline auf Ebene $2, r_{0 j}$ & 6.29 & 1.67 & $27 \%$ & 14.14 & $p<.001$ & $R_{E 2}^{2}=.49$ \\
\hline \multirow{2}{*}{\multicolumn{6}{|c|}{$\begin{array}{l}\text { Anmerkung: } \beta=\text { Regressionsparameter; } S E=\text { Standardfehler; } \\
\mathrm{d} f=\text { Freiheitsgrade; } t=t \text {-Wert; Var }=\text { Varianz; Anteil }=\text { Anteil } \\
\text { der ebenensp. Varianz an der Gesamtvarianz; } \chi^{2}=\chi^{2} \text {-Wert }\end{array}$}} & Gesamtes $R^{2}$ \\
\hline & & & & & & $R_{g e s}^{2}=.24$ \\
\hline
\end{tabular}

Das Gesamtmodell M2a kann mit seinen Prädiktoren insgesamt 24\% der im NullModell vorliegenden Varianz bzgl. der Posttest-Ergebnisse aufklären $\left(R_{\text {ges }}^{2}=.24\right)$. Ein Vergleich der ebenenspezifischen Varianzaufklärung zeigt, dass mit Hilfe des Modells M2a in Hinblick auf die Nachtestergebnisse nur 8\% der Unterschiede innerhalb der Klassen, aber insgesamt 49\% der Unterschiede zwischen den Klassen aufgeklärt werden können. Von den Unterschieden zwischen den Klassen wird mit 39\% der größte Anteil durch die Zugehörigkeit zu Kontroll- oder Treatmentgruppe aufgeklärt (vgl. Abschnitt 7.5.2.2) und weitere 10\% durch das individuelle Vortestergebnis der Schüler (vgl. Abschnitt 7.5.2.3). Die mit 39\% verhältnismäßig große Varianzaufklärung durch die Zugehörigkeit zu Kontroll- oder Treatmentgruppe zeigt, wie stark das mittlere Nachtestergebnis einer Klasse davon abhängt, ob sie klassisch oder nach dem neuen Unterrichtskonzept unterrichtet wurde.

Von der im Gesamtmodell M2a noch ungeklärten Varianz der Posttest-Ergebnisse gehen $73 \%$ auf unerklärte Unterschiede innerhalb der Klassen bzw. zwischen den Schülern zurück und 27\% auf unerklärte Unterschiede zwischen den Klassen. Damit liegen nahezu drei Viertel der unerklärten Varianz auf Schülerebene vor, da durch das individuelle Vortestergebnis lediglich $8 \%$ der ohnehin großen Unterschiede zwischen den Schülern aufgeklärt werden können. Der große Anteil der unerklärten Varianz auf Schülerebene ist vor dem Hintergrund wenig überraschend, dass in der Studie außer dem individuellen Vortestergebnis keine weiteren potenziellen Level-1-Prädiktoren wie Motivation, kognitive Fähigkeit oder Lesekompetenz erhoben wurden, die im Rahmen einer Modellentwicklung Unterschiede im Nachtestergebnis hätten aufklären können. Neben der Schülerebene liegen jedoch auch auf der Klassenebene noch signifikante Unterschiede zwischen den Posttest-Ergebnissen vor, die auf Basis der erhobenen Prädiktoren mit Hilfe des Modells M2a nicht erklärt werden können. 


\subsubsection{Mehrebenenanalytischer Vergleich der Schülervorstellungen}

Bei der bisherigen Auswertung der nach dem jeweiligen Unterricht noch vorhandenen Schülervorstellungen wurde ignoriert, dass der Lernerfolg der Schüler nicht völlig unabhängig voneinander ist, sondern aufgrund der hierarchischen Datenstruktur innerhalb einer Klasse korreliert ist (vgl. Abschnitt 7.3.5.5). Da in der vorliegenden Stichprobe eine hohe Intraklassenkorrelation vorliegt, ist der effektive Stichprobenumfang geringer, was einerseits mit einer höheren statistischen Unsicherheit bzgl. der gefundenen Unterschiede zwischen Kontroll- und Treatmentgruppe in Hinblick auf die Schülervorstellungen einhergeht. Andererseits kann die Berücksichtigung der Klassenzugehörigkeit auch mit einer Veränderung der Schätzung des Anteils der in Kontroll- und Treatmentgruppe nach dem Unterricht noch vorhandenen Schülervorstellungen einhergehen, weil im Gegensatz zur traditionellen Mittelwertberechnung davon ausgegangen wird, dass nicht jeder Schüler den gleichen Informationsbeitrag zur statistischen Schätzung liefert. Die adäquate Berücksichtigung der Mehrebenenstruktur der Daten auch bei der Analyse der Schülervorstellungen ermöglicht also im Gegensatz zu konventionellen statistischen Methoden eine genauere Berechnung der zwischen Kontroll- und Treatmentgruppe vorliegenden Unterschiede bzgl. der nach dem jeweiligen Unterricht noch vorhandenen Schülervorstellungen sowie insbesondere eine adäquatere Schätzung, inwiefern diese Unterschiede statistisch signifikant sind. Da eine Schülervorstellung bei einem Schüler entweder vorliegt oder nicht vorliegt, wurde zur Auswertung auf die Methode der binären logistischen Mehrebenenanalyse zurückgegriffen. Eine allgemeine Beschreibung der Methode findet sich in Twisk (2006) und für eine mathematische Erläuterung des Verfahrens sei auf Goldstein (2011) verwiesen.

In Tab. 47 sind die Ergebnisse der unterschiedlichen Auswerteverfahren in Hinblick auf die Schülervorstellungen gegenübergestellt, wobei sich die mittels binärer logistischer Mehrebenenanalyse (MLA: Multilevel Analysis) gefundenen Werte in der oberen und die mittels klassischer Mittelwertbildung (vgl. Abschnitt 7.4.8.1) gefundenen Werte in der unteren Zeile finden. Vergleicht man die jeweiligen Signifikanzwerte miteinander, stellt man fest, dass nun in der Treatmentgruppe nicht mehr sieben von zehn Schülervorstellungen auf dem $5 \%$ Niveau signifikant geringer ausgeprägt sind als in der Kontrollgruppe, sondern nur noch fünf von zehn. Außerdem fällt das Signifikanzniveau bzgl. der gefundenen Unterschiede aufgrund der vorliegenden Intraklassenkorrelation erwartungsgemäß insgesamt geringer aus als bei der klassischen Auswertung durch Mittelwertberechnung. Es sei an dieser Stelle zudem darauf hingewiesen, dass bei beiden Auswerteverfahren entsprechend des Kodiermanuals von Urban-Woldron und Hopf (2012) eine Schülervorstellung bereits dann als vorhanden gewertet wird, wenn bei einem von mehreren der jeweiligen Subskala zugrundeliegenden Items eine entsprechende Antwortkombination gewählt wurde (vgl. Abschnitt 7.3.4 und 7.4.1.6).

Für jede der Schülervorstellungen wurde zudem der Intraklassenkorrelationskoeffizient (Abk. ICC für „Intraclass Correlation Coefficient" ${ }^{\circ}$ ) berechnet, der ein Maß für die jeweils vorliegende Intraklassenkorrelation darstellt. Je höher die Intraklassenkorrelation ist, 
desto stärker unterscheiden sich die Klassen in Bezug darauf, wie häufig die Schülervorstellung vorliegt oder nicht. Liegt beispielsweise ein ICC von .10 vor, bedeutet dies, dass $10 \%$ der Gesamtvarianz dieser Schülervorstellung auf Unterschiede zwischen den verschiedenen Klassen und 90\% auf Unterschiede zwischen den einzelnen Schülerinnen und Schülern innerhalb der Klassen zurückzuführen sind (vgl. Abschnitt 7.5.1). Ein Blick auf Tab. 47 zeigt, dass die mit Abstand höchste Intraklassenkorrelation bei ,konzeptionellen Schwierigkeiten mit der Spannung" mit einem Wert von ICC = .44 vorliegt, während der Intraklassenkorrelationskoeffizient für die restlichen Schülervorstellungen bei etwa ICC $\approx .10$ liegt. Dies deutet darauf hin, dass die Vermittlung eines angemessenen Spannungsbegriffs sehr klassenabhängig ist, also in manchen Klassen insgesamt deutlich besser gelingt als in anderen. In Kombination mit den Häufigkeiten, mit denen die jeweilige Schülervorstellung von den Schülern nach dem Unterricht vertreten wird, scheint es so, als ob in Klassen der Treatmentgruppe ein wesentlich besseres Spannungskonzept erzielt wird als in der Kontrollgruppe (vgl. Abschnitt 7.4.8).

Tab. 47: Vergleich der im Posttest durch Mittelwertberechnung (MW) und binärer logistischer Mehrebenenanalyse (MLA: Multilevel Analysis) identifizierten Schülervorstellungen (geordnet nach Signifikanzniveau)

\begin{tabular}{|c|c|c|c|c|c|}
\hline \multicolumn{2}{|l|}{ Schïlervorstellung } & \multirow{2}{*}{$\frac{\mathrm{KG}}{96 \%}$} & \multirow{2}{*}{$\frac{\mathrm{TG}}{60 \%}$} & \multirow{2}{*}{$\begin{array}{l}\text { Sig. } \\
p<.001\end{array}$} & \multirow{3}{*}{$\frac{\mathrm{ICC}}{.44}$} \\
\hline \multirow{2}{*}{$\begin{array}{l}\text { Konzeptionelle Schwierigkeiten mit } \\
\text { der elektr. Spannung (Kürzel: KS) }\end{array}$} & MLA & & & & \\
\hline & MW & $92 \%$ & $60 \%$ & $p<.001$ & \\
\hline \multirow{2}{*}{$\begin{array}{l}\text { Stromverbrauchsvorstellung } \\
\text { (Kürzel: SV) }\end{array}$} & MLA & $50 \%$ & $29 \%$ & $p=.002$ & \multirow[t]{2}{*}{.12} \\
\hline & MW & $48 \%$ & $29 \%$ & $p<.001$ & \\
\hline \multirow{2}{*}{$\begin{array}{l}\text { Stromstärke ist unabhängig von } \mathrm{R} \\
\text { (Kürzel: UR) }\end{array}$} & MLA & $35 \%$ & $20 \%$ & $p=.006$ & \multirow[t]{2}{*}{.11} \\
\hline & MW & $35 \%$ & $20 \%$ & $p<.001$ & \\
\hline \multirow{2}{*}{$\begin{array}{l}\text { Erfassen von Parallelschaltungen } \\
\text { (Kürzel: PS) }\end{array}$} & MLA & $87 \%$ & $73 \%$ & $p=.006$ & \multirow[t]{2}{*}{.13} \\
\hline & MW & $85 \%$ & $72 \%$ & $p<.001$ & \\
\hline \multirow{2}{*}{$\begin{array}{l}\text { Batterie als konstante Stromquelle } \\
\text { (Kürzel: BS) }\end{array}$} & MLA & $37 \%$ & $24 \%$ & $p=.037$ & \multirow[t]{2}{*}{.10} \\
\hline & MW & $39 \%$ & $25 \%$ & $p=.001$ & \\
\hline \multirow{2}{*}{$\begin{array}{l}\text { Sequentielle Argumentation } \\
\text { (Kürzel: SA) }\end{array}$} & MLA & $46 \%$ & $33 \%$ & $p=.067$ & \multirow[t]{2}{*}{.09} \\
\hline & MW & $46 \%$ & $33 \%$ & $p=.007$ & \\
\hline \multirow{2}{*}{$\begin{array}{l}\text { Stromverbrauch ist proportional zu R } \\
\text { (Kürzel: PR) }\end{array}$} & MLA & $52 \%$ & $43 \%$ & $p=.083$ & \multirow[t]{2}{*}{.03} \\
\hline & MW & $52 \%$ & $43 \%$ & $p=.028$ & \\
\hline \multirow{2}{*}{$\begin{array}{l}\text { Lokales Denken } \\
\text { (Kürzel: LD) }\end{array}$} & MLA & $84 \%$ & $78 \%$ & $p=.174$ & \multirow[t]{2}{*}{.03} \\
\hline & MW & $84 \%$ & $77 \%$ & $p=.111$ & \\
\hline \multirow{2}{*}{$\begin{array}{l}\text { Spannung als Eigenschaft des Stroms } \\
\text { (Kürzel: UI) }\end{array}$} & MLA & $59 \%$ & $54 \%$ & $p=.590$ & \multirow[t]{2}{*}{.16} \\
\hline & MW & $60 \%$ & $54 \%$ & $p=.257$ & \\
\hline \multirow{2}{*}{$\begin{array}{l}\text { Inverse Widerstandsvorstellung } \\
\text { (Kürzel: IR) }\end{array}$} & MLA & $14 \%$ & $15 \%$ & $p=.794$ & \multirow[t]{2}{*}{.11} \\
\hline & MW & $14 \%$ & $16 \%$ & $p=.462$ & \\
\hline
\end{tabular}

Anmerkung: $\quad$ Sig. = Signifikanzniveau; $\quad$ ICC = Intraklassenkorrelationskoeffizient; Signifikanzwerte unter $5 \%$ sind fett hervorgehoben 
Wie in Abb. 113 dargestellt, vertreten zudem signifikant weniger Schüler der Treatmentgruppe die Stromverbrauchsvorstellung als Schüler der Kontrollgruppe. Auch in Bezug auf das Erfassen von Parallelschaltungen sowie die Schülervorstellungen, die Stromstärke sei unabhängig vom Widerstand oder die Batterie sei eine konstante Stromquelle, führt das neue Unterrichtskonzept verglichen mit dem Unterricht der Kontrollgruppe zu einem signifikant besseren Verständnis bei den Lernenden.

Im Gegensatz zu den Ergebnissen durch einfache Mittelwertberechnung deutet die Analyse der Daten mit Hilfe der binären logistischen Mehrebenenanalyse aber darauf hin, dass die Unterschiede zwischen den beiden Gruppen in Bezug auf die sequentielle Argumentation sowie die Schülervorstellung, der Stromverbrauch sei proportional zum Widerstand, statistisch nicht mehr signifikant sind. Sowohl nach der klassischen durch Mittelwertbildung durchgeführten als auch der mehrebenenanalytischen Auswertung existieren hinsichtlich der Schülervorstellungen Lokales Denken, Spannung als Eigenschaft des Stroms und der Inversen Widerstandsvorstellung keine Unterschiede zwischen dem traditionellen Unterricht und dem neuen Unterrichtskonzept.

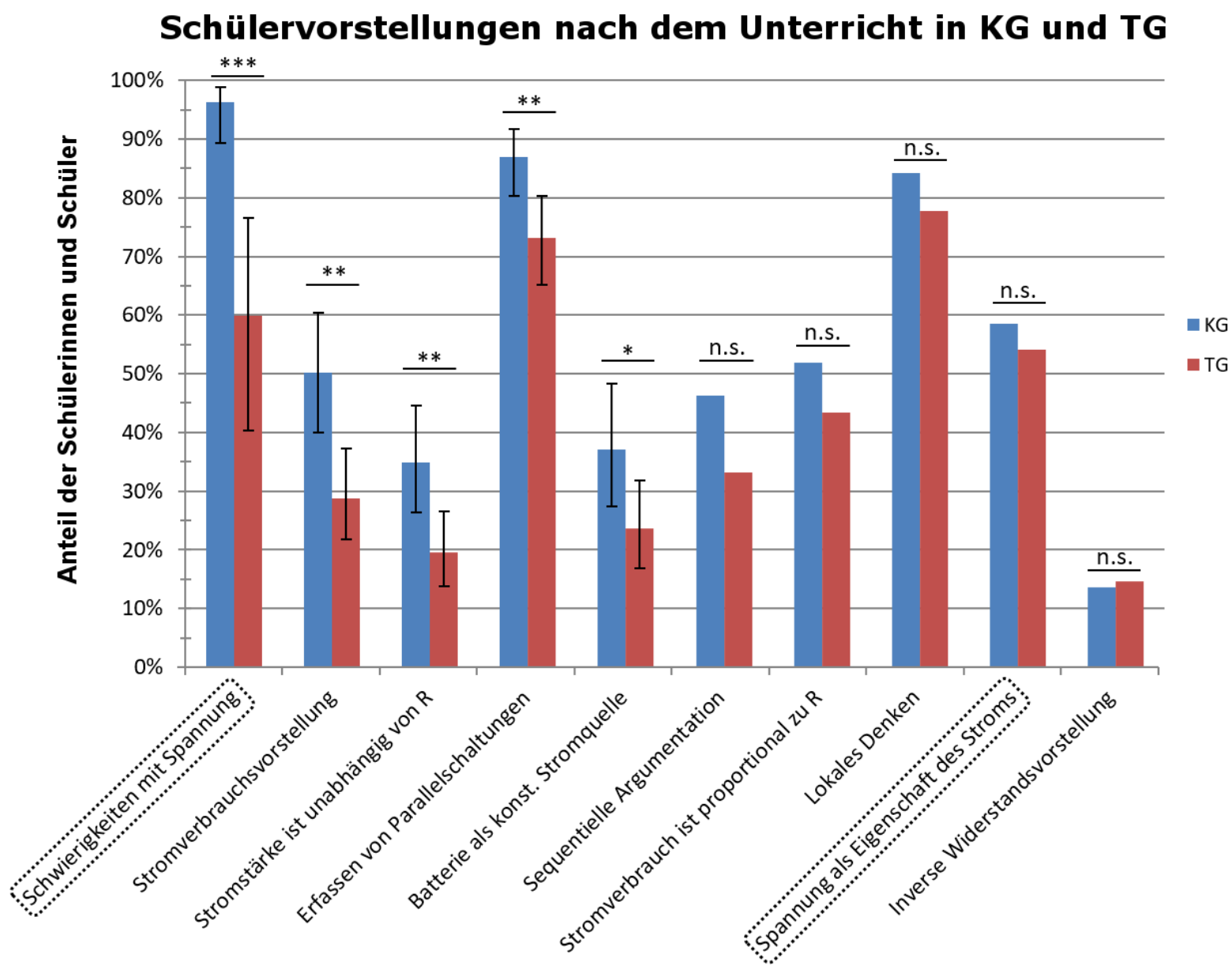

Abb. 113: Übersicht über die mittels binärer logistischer Mehrebenenanalyse ermittelten Schülervorstellungen im Posttest (geordnet nach Signifikanzniveau). Die umrandeten Schülervorstellungen zur Spannung wurden auf Basis eigener Items erhoben. Die 95\%-Konfidenzintervalle wurden von SPSS lediglich für signifikante Unterschiede angegeben. Es gelten die folgenden Signifikanzniveaus: $* * * p<.001 ; * * p<.01 ; * p<.05 ; n . s .=$ nicht signifikant. 
Trotz dieser hier beschriebenen Unterschiede bzgl. der Ergebnisse der beiden Auswerteverfahren, stimmen beide doch in Bezug auf das wesentliche Ergebnis überein: Das neue Unterrichtskonzept scheint gegenüber dem traditionellen Unterricht zu signifikant weniger Schülervorstellungen zu führen, wobei auch nach dem neuen Konzept viele Schülervorstellungen noch von einem bedeutsamen Anteil der Schüler vertreten wird. Auch wenn das neue Unterrichtskonzept also zu einem besseren Verständnis der Basiskonzepte elektrischer Stromkreise führt, scheint dieses Gebiet der Physik aber für viele Lernende auch weiterhin mit groBen Lernschwierigkeiten verbunden zu sein.

\subsection{Schulpraktische Erfahrungen der Lehrkräfte}

\subsubsection{Stichprobe und Datenerhebung}

Im Sinne des Forschungsansatzes von Design-Based-Research (vgl. Abschnitt 7.1) ist es nicht nur von Interesse zu untersuchen, ob die Schülerinnen und Schüler die Konzepte mit Hilfe des neuen Unterrichtskonzepts besser verstehen, sondern auch, wie das neue Unterrichtskonzept von den Lehrkräften auf Basis ihrer Praxiserfahrungen eingeschätzt wird. Der Grund hierfür ist, dass das neue Unterrichtskonzept nur dann in der Schulpraxis ankommen kann, wenn es bei den unterrichtenden Lehrkräften auf Akzeptanz stößt. Auch in Hinblick auf eine mögliche Überarbeitung des Unterrichtskonzepts zu einem späteren Zeitpunkt ist es wichtig, die Sicht der Lehrkräfte zu kennen, die bereits Praxiserfahrungen mit dem Unterrichtskonzept sammeln konnten.

Um ein Bild davon zu bekommen, wie die Lehrerinnen und Lehrer das Unterrichtskonzept bewerten, d.h. wo sie beispielsweise die Stärken des Unterrichtskonzepts sehen und wo tendenziell Verbesserungsbedarf, wurde ein Online-Fragebogen erstellt und per E-Mail an die Lehrkräfte verteilt. Die Entscheidung, die Befragung online durchzuführen, fiel vor allem aus organisatorischen und logistischen Gründen. Der Fragebogen hatte einen Umfang von etwa 30 Minuten, gliederte sich in verschiedene thematische Abschnitte und nutzte zwei verschiedene Frageformate (vgl. Anhang 13.7). Mit Hilfe von vorformulierten Aussagen und einer Antwortskala vom Likert-Typ wurde einerseits versucht, von den Lehrkräften eine Gesamteinschätzung zum Unterrichtskonzept zu bekommen. Dieses geschlossene Frageformat wurde gewählt, um eine Vergleichbarkeit der Antworten zu gewährleisten und eine schnelle Bearbeitung zu ermöglichen. Die andererseits nahezu ausschließlich offenen Fragen zu unterschiedlichen Aspekten des Unterrichtskonzepts sollten den Lehrkräften hingegen ermöglichen, eine differenzierte Beschreibung ihrer jeweiligen Sichtweisen und Erfahrungen zu geben. Während die geschlossenen Fragen zur Gesamteinschätzung des Unterrichtskonzepts im Online-Fragebogen zwingend zu beantworten waren, hatten viele offene Fragen einen optionalen Charakter, weil davon ausgegangen wurde, dass nicht jede Lehrkraft zu jeder Frage etwas beizutragen hat.

Insgesamt beteiligten sich 14 Lehrkräfte der Treatmentgruppe an dem Fragebogen. Für 12 dieser 14 Lehrkräfte waren Neugier und Interesse an neuen Unterrichtsansätzen das 
Hauptmotiv, an der Studie teilzunehmen. Weitere zwei Lehrkräfte gaben an, mit ihrem bisherigen Vorgehen im Unterricht unzufrieden gewesen zu sein. Während ihrer bisherigen Lehrertätigkeit hatten zwölf Lehrkräfte bereits das Modell des geschlossenen Wasserkreislaufs, fünf das Modell des offenen Wasserkreislaufs und drei bereits das Autobahn-Modell im Unterricht genutzt. Einzelne Lehrkräfte berichteten darüber hinaus, bereits das Stäbchenmodell, das Fahrradkettenmodell bzw. den starren Elektronenring, das Rucksack-, Hütchen- bzw. Bienenmodell, das Elektronengasdruckmodell bzw. kein Modell im Unterricht eingesetzt zu haben.

\subsubsection{Förderung des Konzeptverständnisses}

Wie bereits angedeutet, sollten die Lehrkräfte mit Hilfe von vorformulierten Aussagen und einer Antwortskala vom Likert-Typ (,trifft gar nicht zu“, ,trifft eher nicht zu“, ,teils-teils“,

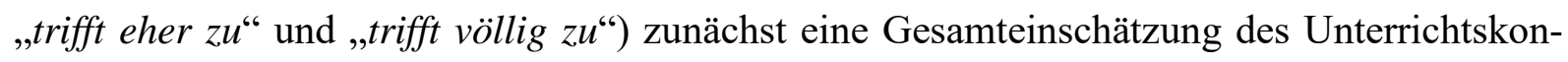
zepts vornehmen. In einem ersten Abschnitt wurden sie hier gebeten zu beurteilen, ob das Unterrichtskonzept zu einem guten konzeptionellen Verständnis von verschiedenen grundlegenden Konzepten in der Elektrizitätslehre führt, wobei zu jedem Konzept die MedianEinschätzung $M$ der Lehrkräfte ermittelt wurde. Zusätzlich wurde die ordinale Skala aufgrund der Tatsache, dass sie näherungsweise intervallskaliert ist, in eine quantitative Form übertra-

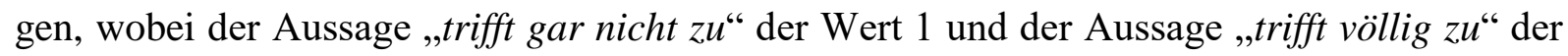
Wert 5 zugeordnet wurde. Hierdurch konnte zu jeder Aussage der Mittelwert $\mu$ als Maß für den Zustimmungsgrad der Lehrkräfte sowie die Standardabweichung $\sigma$ als Maß für die Streuung der Antworten berechnet werden, was die Vergleichbarkeit der Aussagen erleichtert. Auf eine Angabe der Spannweite der Aussagen wird verzichtet, weil sich diese bei nahezu allen Aussagen über die gesamte Skala erstreckt.

Die Einschätzungen der Lehrkräfte darüber, inwiefern das Unterrichtskonzept ein gutes konzeptionelles Verständnis verschiedener Konzepte in der Elektrizitätslehre fördert, sind in Abb. 114 zusammengefasst. Allgemein fällt auf, dass es kaum Konzepte gibt, bei denen das Unterrichtskonzept aus Sicht von mehr als einer Lehrkraft gar nicht oder eher nicht zu einem guten Verständnis bei den Schülern führt. Eine Mehrheit bescheinigt dem Unterrichtskonzept, zu einem guten konzeptionellen Verständnis der elektrischen Spannung, der Stromstärke, des Widerstands, des Potenzials und von Parallelschaltungen zu führen. Etwas unschlüssig sind sich die Lehrkräfte hingegen bzgl. der Frage, ob das Unterrichtskonzept zu einem guten konzeptionellen Verständnis von Kondensatoren und Reihenschaltungen führt. 


\section{Das Unterrichtskonzept führt zu einem guten konzeptionellen Verständnis...}

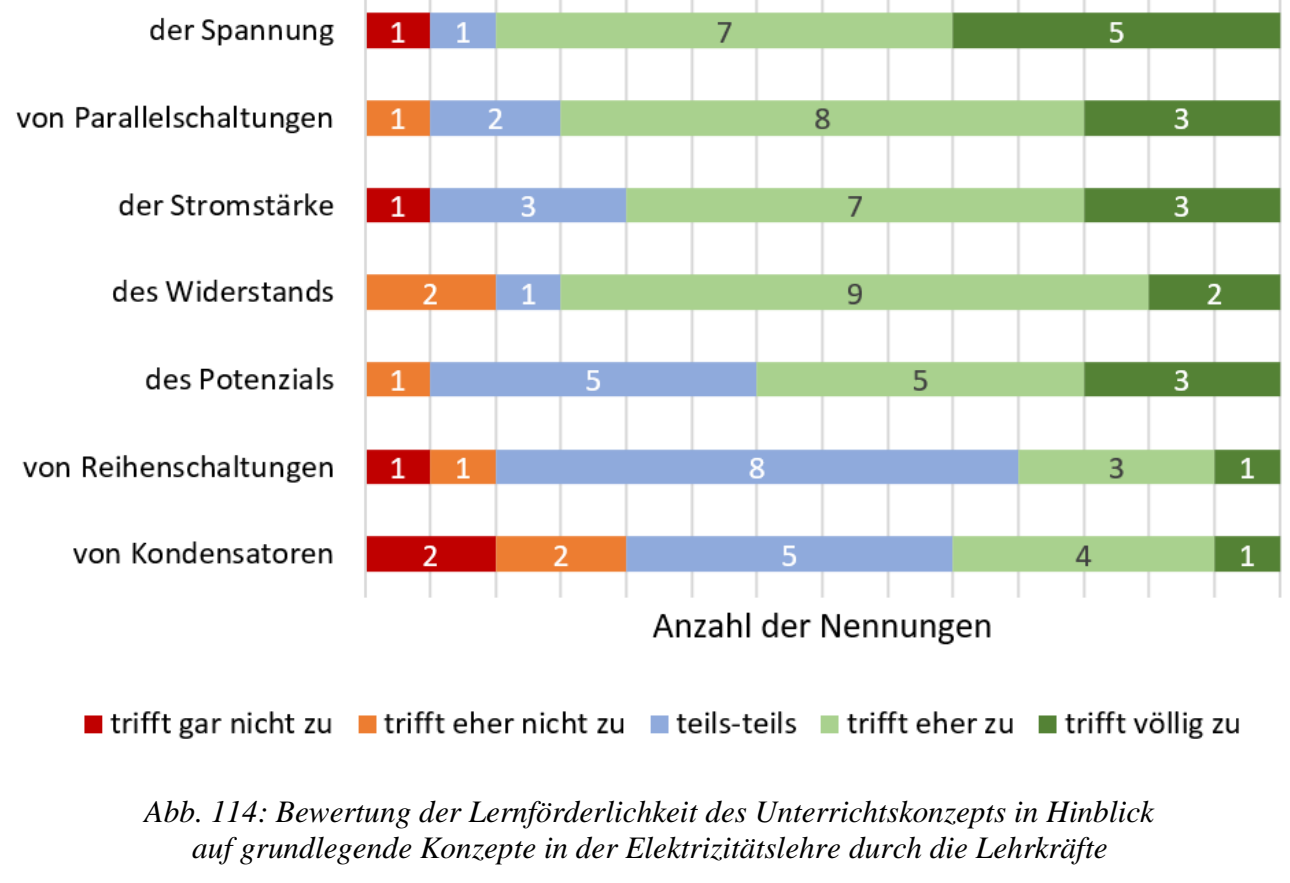

Besonders stark trägt das neue Unterrichtskonzept nach Aussage der Lehrkräfte zu einem guten konzeptionellen Verständnis der elektrischen Spannung bei $(M=4=$ trifft eher zu, $\mu=4.1, \sigma=1.1$ ). Darüber hinaus führt das neue Unterrichtskonzept aber auch zu einem vergleichbar guten konzeptionellen Verständnis von Parallelschaltungen ( $M=4=$ trifft eher zu, $\mu=3.9, \sigma=0.8)$, der Stromstärke $(M=4=$ trifft eher zu, $\mu=3.8, \sigma=1.1)$, des Widerstands $(M=4=$ trifft eher zu, $\mu=3.8, \sigma=0.9)$ und des elektrischen Potenzials $(M=4=$ trifft eher $\mathrm{zu}, \mu=3.7, \sigma=0.9)$. Etwas geringer wird hingegen die Lernförderlichkeit bzgl. eines guten konzeptionellen Verständnisses von Reihenschaltungen ( $M=3=$ teils-teils, $\mu=3.1, \sigma=0.9$ ) und Kondensatoren $(M=3=$ teils-teils, $\mu=3.0, \sigma=1.2)$ eingeschätzt.

Da das Unterrichtskonzept vor dem Hintergrund entwickelt wurde, den Lernenden ein gutes konzeptionelles Verständnis der elektrischen Spannung zu vermitteln, weil dies in der Regel im traditionellen Physikunterricht nicht gelingt (vgl. Abschnitt 6.1.1), ist es sehr erfreulich, dass die Lehrkräfte dem Unterrichtskonzept bzgl. der elektrischen Spannung die höchste Lernförderlichkeit bescheinigen. Der Umstand, dass das Unterrichtskonzept nach Ansicht einer großen Mehrheit der Lehrkräfte ebenfalls zu einem guten konzeptionellen Verständnis von Parallelschaltungen, der Stromstärke, des Widerstands und des elektrischen Potenzials führt, deutet darauf hin, dass diese vergleichsweise hohe Lernförderlichkeit der elektrischen Spannung nicht auf Kosten des übrigen Konzeptverständnisses zu gehen scheint. Die von den Lehrkräften etwas geringer bewertete Lernförderlichkeit in Bezug auf Reihenschaltungen und Kondensatoren ist wenig überraschend, da Lade- und Entladevorgänge von Kondensatoren lediglich zur Hinführung zu Reihenschaltungen dienten und bereits bei der Konzeption des Unterrichtskonzepts antizipiert wurde, dass die Analyse von Reihenschaltungen mit Hilfe von 
Übergangszuständen den Lernenden möglicherweise nicht leichtfallen würde, weshalb hierzu auch diverse offene Fragen in den Fragebogen aufgenommen wurden (vgl. Abschnitt 7.6.4.3).

\subsubsection{Didaktische Einschätzung des Unterrichtskonzepts}

In dem zweiten Abschnitt der vorformulierten Aussagen mit einer Antwortskala vom LikertTyp wurden die Lehrkräfte gebeten, eine didaktische Einschätzung des Unterrichtskonzepts vorzunehmen. Auch hier wurde zu jedem Aspekt die Median-Einschätzung $M$ der Lehrkräfte ermittelt und zusätzlich der Mittelwert $\mu$ als Maß für den Zustimmungsgrad der Lehrkräfte sowie die Standardabweichung $\sigma$ als Maß für die Streuung der Antworten berechnet (vgl. Abschnitt 7.6.2). Da sich die Spannweite der Aussagen hier nicht unterscheidet, erübrigt sich auch hier ihre Angabe.

\section{Das Unterrichtskonzept...}

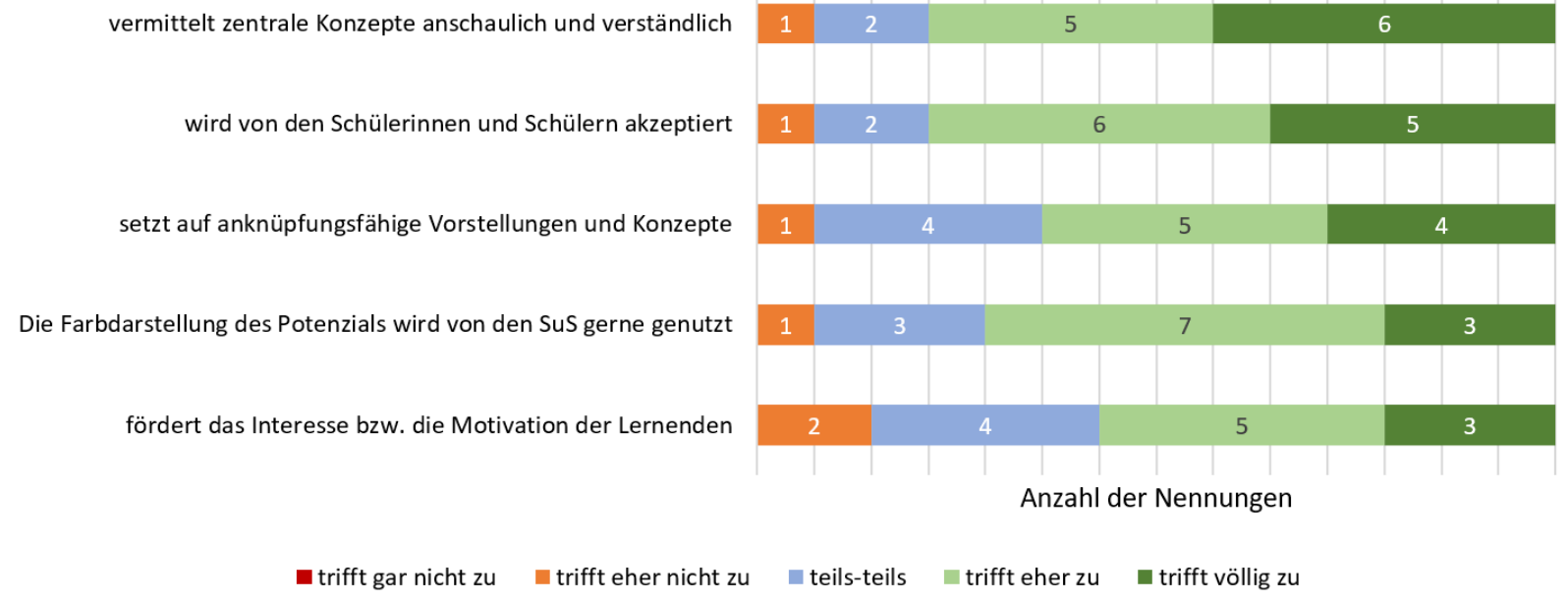

Abb. 115: Bewertung unterschiedlicher didaktischer Aspekte des Unterrichtskonzepts durch die Lehrkräfte

Wie die Lehrkräfte die unterschiedlichen didaktischen Aspekte des Unterrichtskonzepts beurteilt haben, ist in Abb. 115 dargestellt. Es zeigt sich unter den Lehrkräften eine allgemeine Zustimmung, dass das Unterrichtskonzept die abgefragten didaktischen Kriterien im Großen und Ganzen erfüllt. Keine Lehrkraft vertrat die Auffassung, dass eines der didaktischen Kriterien von dem Unterrichtskonzept überhaupt nicht erfüllt würde. Die MedianZustimmung liegt bei allen Aspekten bei $M=4=$ trifft eher zu. Betrachtet man die Mittelwerte, so zeigt sich die höchste Zustimmung bzgl. der Aussagen „Das Unterrichtskonzept vermittelt zentrale Konzepte anschaulich und verständlich“ $(\mu=4.1, \sigma=0.9)$ und „Das Unterrichtskonzept wird von den Schülerinnen und Schülern akzeptiert" $(\mu=4.1, \sigma=0.9)$. Minimal geringer fällt die Zustimmung zu den Aussagen „Das Unterrichtskonzept setzt auf anknüpfungsfähige Vorstellungen und Konzepte“ $(\mu=3.9, \sigma=0.9)$ und „Die Farbdarstellung des Potenzials wird von den SuS gerne genutzt" $(\mu=3.9, \sigma=0.9)$ aus. Die vergleichsweise geringste $\mathrm{Zu}-$ stimmung zeigen die Lehrkräfte hingegen bzgl. der Aussage „Das Unterrichtskonzept fördert das Interesse bzw. die Motivation der Lernenden" ( $\mu=3.6, \sigma=1.0)$. 


\subsubsection{Detailanalyse zu einzelnen Aspekten des Unterrichtskonzepts}

\subsubsection{Allgemeine Bewertung des gewählten Ansatzes}

Alle Lehrkräfte waren der grundsätzlichen Auffassung, dass es sich im Unterricht als fruchtbar erwiesen hat, die elementare Elektrizitätslehre mit Hilfe des elektrischen Potenzials zu erklären. Auf die Frage, ob die Schülerinnen und Schüler ein für das Unterrichtskonzept ausreichendes intuitives Luftdruckverständnis im Sinne von „,komprimierte Luft steht unter Druck, drückt gegen die Wände und hat das Bestreben sich auszudehnen“" besitzen, gaben neun Lehrkräfte an, dies sei definitiv der Fall, wohingegen vier Lehrkräfte der Meinung waren, die Schülerinnen und Schüler hätten zu Beginn der Unterrichtsreihe kaum über ein solches Luftdruckkonzept verfügt, dies habe sich aber mit Hilfe des Unterrichtskonzepts schnell aufbauen lassen („Die Schüler tuen sich sehr schwer mit dem Modell eines Druckes. Durch die Einführung dieses Konzeptes mit Hilfe der Spritzen, ließ sich dieses Konzept jedoch für die Schülerinnen und Schüler recht anschaulich einführen “; ,, Verständnis ist vorhanden bzw. kann mit dem Konzept sehr schnell hergestellt werden, auch ohne, dass Druck als phys. Größe vorher bekannt ist"). Eine Lehrkraft gab jedoch an, die Lernenden würden nicht über ein ausreichendes intuitives Luftdruckverständnis verfügen.

Bezüglich der Frage, welche Schülergruppe am meisten von dem neuen Unterrichtskonzept profitierte, gab es bei den Lehrkräften verschiedene Einschätzungen. Nach Ansicht von fünf Lehrkräften profitierten leistungsschwache Schülerinnen und Schüler am meisten von dem neuen Unterrichtskonzept, weil die Luftdruckanalogie in Kombination mit der vorgeschlagenen Farbkodierung sehr intuitiv sei. Weitere drei Lehrkräfte gaben an, dass insbesondere selbstmotivierte und fleißige Schülerinnen und Schüler ihrer Ansicht nach am meisten von dem neuen Unterrichtskonzept profitieren, weil es eine Fülle an gut ausgearbeiteten Materialien, Übungsaufgaben und Erklärungen enthält. Zwei Lehrkräfte waren hingegen der Meinung, dass allen voran leistungsstarke Schülerinnen und Schüler von dem neuen Unterrichtskonzept profitieren würden, weil sie in der Oberstufe bzgl. der Elektrizitätslehre weniger Schülervorstellungen besitzen würden. Weitere zwei Lehrkräfte vertraten die Position, dass Mädchen am meisten von dem neuen Unterrichtskonzept profitierten, weil die Verwendung der Farbkodierung und der intuitiven Luftdruckanalogie für sie einen besseren Zugang zum Thema dargestellt habe.

\subsubsection{Vor- und Nachteile des Konzeptes sowie aufgetretene Verständnisschwierigkeiten}

Die größten Vorteile des neuen Unterrichtskonzepts auf Basis des Elektronengasmodells sahen die Lehrkräfte darin, dass

- die Luftdruckanalogie in Kombination mit der vorgeschlagenen Farbkodierung zu einem besseren konzeptionellen Verständnis der Grundgrößen elektrischer Stromkreise führt (,eine Vorstellung von Spannung, Stromstärke und Widerstand fällt einem fast in den Schoß“; ,, die Schülerinnen und Schüler können sehr schnell und auf einfache 
Weise komplexe Stromkreise analysieren und korrekt bestimmen, welche Lampe leuchtet, wie sich die Spannungen verteilen und welche Lampen parallel geschaltet sind"),

- insbesondere der Unterschied zwischen Spannung und Stromstärke deutlich wird und die Spannung als Ursache des elektrischen Stroms eingeführt wird,

- das Unterrichtskonzept gutes und reichliches Übungsmaterial enthält („Die SuS waren sehr begeistert von dem liebevoll gestalteten Info-Material und fanden das Übungsheft prima“; „Hervorragend zusammengestelltes Material, tolle inhaltliche Durchdringung des Themas, gute Strukturierung"),

- die Schüler eine mikroskopische Modellvorstellung der Vorgänge in elektrischen Stromkreisen bekommen.

Während hinsichtlich der Vorteile des Unterrichtskonzepts weitgehende Einigkeit bei den Lehrkräften bestand, zeigte sich bzgl. der Nachteile bzw. Schwächen des Unterrichtskonzeptes kein klares Bild. Sieht man von Punkten ab, die nur von einzelnen Lehrkräften kritisiert wurden, bestand die Hauptkritik darin, dass

- die gewählte Farbkodierung (rot für den elektrischen Überdruck am Minuspol bzw. blau für den elektrischen Unterdruck am Pluspol) der in der Physik gängigen Konvention widerspricht, wonach der Pluspol rot und der Minuspol blau dargestellt werden,

- das Unterrichtskonzept insgesamt zu wenige Experimente vorsieht und insbesondere die Erklärungen zu den Reihenschaltungen mit Hilfe der Übergangszustände zu abstrakt bzw. theoretisch für den Anfangsunterricht seien,

- das Unterrichtskonzept mit allen Materialien in der Umsetzung teils mehr Zeit in Anspruch genommen hätte als veranschlagt.

Auch wenn die unkonventionelle Farbwahl von den Lehrkräften kritisiert wurde, gibt die große Mehrheit von 11 Lehrkräften an, dass die Schülerinnen und Schüler selber mit der im Unterrichtskonzept genutzten Farbkodierung keine Probleme hatten und diese auch nicht zu Verständnisschwierigkeiten geführt habe. Vielmehr scheint es so zu sein, dass sich manche Lehrkräfte mit der unkonventionellen Farbkodierung schwergetan haben, wie die beiden folgenden Zitate aus dem Fragebogen illustrieren: „die Schülerinnen und Schüler haben die Farbwahl so verinnerlicht, dass sie diese in der Lernkontrolle überwiegend korrekt angewendet haben, obwohl mir in der Aufgabenstellung ein Fehler (Farbvertauschung) unterlaufen ist“; „Die SuS kamen damit ganz gut zurecht. Ich musste mich konzentrieren um es nicht falsch zu machen“. Weitere drei Lehrkräfte gaben an, dass es in ihrem Unterricht in Folge der Farbkodierung zu Verständnisschwierigkeiten bei den Schülerinnen und Schülern gekommen sei.

Auf die Frage, welche Verständnisschwierigkeiten bei den Schülerinnen und Schülern in Folge des Elektronengasmodells aufgetreten sind, die im traditionellen Unterricht nicht auftraten, gaben die meisten Lehrkräfte an, keine neuen Verständnisschwierigkeiten beobachtet zu haben. Allerdings gingen mit dem Elektronengasmodell nach Aussage einzelner Lehrkräfte die folgenden neuen Verständnisschwierigkeiten einher: 
- Schülern fiel es schwer, die Erklärung des Widerstands an Hand von Luftdruckbeispielen auf Stromkreise zu übertragen.

- Die Übergangszustände und die Druckverteilung bei Reihenschaltungen erwiesen sich für manche Schüler als schwer verständlich.

- Die Art und Weise, wie die Stromstärke im Konzept eingeführt wird, ließ manche Schüler glauben, bei 1A würde genau ein Elektron pro Sekunde fließen.

- Die Argumentation mit einem Überschuss an Elektronen am Minuspol förderte bei manchen Lernenden eine sequentielle Vorstellung vom Stromkreis.

\subsubsection{Bewertung verschiedener Aspekte in Bezug auf Reihenschaltungen}

Um den Schülerinnen und Schülern eine dynamische Modellvorstellung mit Übergangszuständen zur Analyse von Reihenschaltungen zu erleichtern, wurden im Unterrichtskonzept zunächst Lade- und Entladevorgänge von Kondensatoren betrachtet, obwohl Kondensatoren in der Mittelstufe normalerweise nicht behandelt werden. Bei der Frage, ob dieses Vorgehen sinnvoll sei oder nicht, zeigten sich die Lehrkräfte gespalten. Auf der einen Seite waren vier Lehrkräfte der Ansicht, dass dieses Vorgehen prinzipiell sinnvoll sei, aber dass Kondensatoren aus Zeitgründen aus dem Unterrichtskonzept gestrichen werden sollten. Weitere zwei Lehrkräfte waren der Meinung, die Behandlung des Themas Kondensatoren sei schlicht unnötig. Auf der anderen Seite vertraten vier Lehrkräfte die Ansicht, die Analyse von Lade- und Entladevorgängen bei Kondensatoren sei wichtig für das Verständnis von Übergangszuständen bzw. Reihenschaltungen.

Eindeutig fiel hingegen das Urteil der Lehrkräfte bzgl. der Frage aus, ob die Schülerinnen und Schüler Reihenschaltungen auch alleine mit Hilfe von Übergangszuständen und ohne die Fahrradkettenanalogie analysieren könnten. Die Mehrheit von acht Lehrkräften war hier der Meinung, eine solche Analyse rein mit Hilfe von Übergangszuständen würde den Schülerinnen und Schülern sehr schwer fallen („Ich glaube, da werden viele Schüler Verständnisschwierigkeiten haben, die Fahrradkette ist eigentlich jedem Schüler bekannt und man kann damit gut das Vorhandensein der Elektronen und gleichzeitigen Strömungsbeginn erklären."), während weitere zwei Lehrkräfte angaben, leistungsstärkere Schülerinnen und Schüler kämen auch alleine mit den Übergangszuständen zurecht. Somit scheinen sich die Lehrkräfte darin einig, dass Reihenschaltungen im Anfangsunterricht von den meisten Schülerinnen und Schülern nicht alleine mit Übergangszuständen analysiert werden können.

Bei der Frage, ob die parallele Verwendung der Fahrradkettenanalogie neben der Betrachtung der Übergangszustände sinnvoll sei oder nicht, zeigten sich die Lehrkräfte gespalten. Die eine Hälfte der Lehrkräfte gab an, dass die Fahrradkettenanalogie hier eine klare Hilfe für die Schülerinnen und Schüler gewesen sei - insbesondere in Bezug auf die Addition von Widerständen und die Konstanz der Stromstärke bei Reihenschaltungen. Die andere Hälfte teilte sich einerseits auf drei Lehrkräfte auf, die der Meinung waren, die Fahrradkettenanalogie sei für ein Verständnis von Reihenschaltungen nicht notwendig und würde von den Schülerinnen und Schülern sogar abgelehnt bzw. nicht verstanden. Weitere zwei Lehrkräfte 
vertraten andererseits die Auffassung, dass im Unterrichtskonzept neben dem Elektronengasmodell keine weiteren Modelle verwendet werden sollten.

\subsubsection{Der Einsatz, des Konzepts im zukünftigen Unterricht}

Alles in allem scheinen die Lehrkräfte mit dem Unterrichtskonzept sehr zufrieden zu sein, da 12 der 14 Lehrkräfte angaben, die Elektrizitätslehre auch in Zukunft auf Grundlage des hier vorgeschlagenen Elektronengasmodells unterrichten zu wollen $(,[\ldots]$ weil ich es für sehr anschaulich und gut durchdacht halte. Zudem bietet es schwächeren Schülern die Möglichkeit einen Zugang zu diesem doch sehr anspruchsvollen Themenbereich zu erlangen"). Eine weitere Lehrkraft war sich noch nicht ganz sicher und eine Lehrkraft lehnte es ab, ihren Elektrizitätslehreunterricht in Zukunft auf dem Elektronengasmodell aufzubauen (,Falls ich sehr intelligente Schüler haben werde, könnte ich mir vorstellen, ihnen das als Differenzierung reinzugeben, aber als Unterrichtsmodell nicht mehr"). Auf die Frage, was sie am Unterrichtskonzept ändern würden, sofern sie in Zukunft wieder danach unterrichten, gaben vier Lehrkräfte an, das Konzept zeitlich straffen zu wollen, indem sie Texte kürzen und Aufgaben streichen. Drei Lehrkräfte erklärten ferner, experimentellen Phasen insbesondere zu einem früheren Zeitpunkt im Unterrichtskonzept einen größeren Raum geben zu wollen. Einzelne Lehrkräfte planten darüber hinaus, in Zukunft u.a. auf die konventionelle Farbkodierung zurückzugreifen, eine stärkere Kontextorientierung vorzunehmen und die Argumentation mit Übergangszuständen bei Reihenschaltungen lediglich als Differenzierungsmaßnahme für leistungsstärkere Schülerinnen und Schüler zu nutzen.

Entsprechend der großen persönlichen Zustimmung zu dem Unterrichtskonzept gaben 11 der 14 Lehrkräfte an, das Unterrichtskonzept auch an Kolleginnen und Kollegen weiterempfehlen zu wollen oder dies bereits getan zu haben (,Ja, weil ich die Erfahrungen damit als sehr positiv empfinde und weil ich glaube, dass ein besseres konzeptionelles Verständnis vermittelt wird als bei einem konventionellen Unterrichtsgang. “; „Das Konzept ist durchdacht und gefällt mir wesentlich besser als das Wassermodell“; „Ich habe den Eindruck, dass die SuS dieses abstrakt erscheinende Thema besser verstehen"). Bei zwei weiteren Lehrkräften war das Unterrichtskonzept den Kolleginnen bzw. Kollegen schon bekannt, z.B. weil diese auch an der Studie teilgenommen haben, und eine Lehrkraft plant nicht, das Unterrichtskonzept weiterzuempfehlen („Ich präferiere trotz meiner jetzigen Erfahrung mit dem Elektronengasmodell das Wasserkreislaufmodell. [...] Mein persönlicher Eindruck ist, dass ich mit dem Wassermodell im Unterricht mehr Physik transportieren kann als mit dem Elektronengasmodell“').

\subsubsection{Erfahrungen im Rahmen einer Pädagogischen Facharbeit}

Zentrale Ideen des hier vorgeschlagenen Elektronengasmodells wurden auch in einer Unterrichtssequenz im Rahmen einer Pädagogischen Facharbeit zur Zweiten Staatsprüfung zur Einführung des Strom- und Spannungskonzepts in der 9. Jahrgangsstufe eines Gymnasiums von Amar-Youcef (2017) evaluiert. Dabei war das Elektronengasmodell zentraler Unterrichtsgegenstand von drei Schulstunden und wurde in den darauffolgenden fünf Unterrichtsstunden 
nur bemüht, sofern es von den Lernenden gefordert wurde. Neben der grundsätzlichen Argumentation mit elektrischen Drücken, die in Analogie zu Luftdruckunterschieden zu einer Elektronenströmung in einem Stromkreis führen, wurde dabei insbesondere auf die Farbdarstellung und die diesem Unterrichtskonzept zugrundeliegende Begriffswahl zurückgegriffen. Insgesamt kommt der Verfasser zu dem Schluss, dass „,das Elektronengasmodell [...] gut geeignet [ist], um die vorgesehenen Lerninhalte zu entwickeln [...] [und sich als] sehr intuitiv und verständlich erwiesen [hat].“ (Amar-Youcef 2017, S. 27). Auch wenn die im Rahmen dieser Facharbeit dokumentierten Erfahrungen nicht repräsentativ sind, stellen sie für ein Design-Based-Research-Projekt einen weiteren interessanten Einblick in die Lernwirksamkeit zentraler Ideen des Unterrichtskonzepts in der Unterrichtspraxis dar. Aus diesem Grund sollen an dieser Stelle wesentliche Aussagen der Pädagogischen Facharbeit wiedergegeben werden:

Zur generellen Lernwirksamkeit:

- „Die SuS entwickelten ein intuitives Wissen zu den physikalischen Größen Spannung, Stromstärke und Widerstand angelehnt an den analogen Lernbereich und dessen Begrifflichkeiten und konnten dies in dem fortlaufenden Unterricht gut einbringen." (ebd., S.25)

- „Besonders die entlehnten Begrifflichkeiten (elektrischer Druck und Druckunterschied anstelle von Potential und Spannung) und Darstellungsmöglichkeiten des Potentials über eine Farbskala haben sich als einprägsam und lernfördernd erwiesen." (ebd., S.30)

\section{Zum Potenzialansatz:}

- „Die Bedeutung des Elektronengasmodells für die Herangehensweise der SuS ist insofern gegeben, als dass die überwältigende Mehrheit der SuS, wenn nicht alle, die Schaltung vom Potential her durchdacht haben." (ebd., S.25)

\section{Zur Begriffswahl:}

- „[Die verwendeten Begriffe] haben sich als sehr einprägsam und hilfreich erwiesen. Daher war es für die SuS eine große Umstellung, die korrekte Fachsprache anzuwenden [...].“ (ebd., S.27)

- „Die SuS haben im Verlauf der Einheit ihre Sprache kaum hin zur Fachsprache adaptiert, da sie anscheinend weniger einprägend ist. So wurde nach wie vor der Begriff elektrischer Druck benutzt, auch wenn die Bedeutung des Begriffs Potentials bekannt ist. Ähnlich häufig wird der Begriff Druckunterschied anstelle der Spannung benutzt.“ (ebd., S.25) 
Kapitel 7: Summative Evaluation des Unterrichtskonzepts

Zur Farbdarstellung:

- „Ebenso hat sich die Farbskala als sehr hilfreich erwiesen. Die Farbskala wurde eigenständig von den SuS von der Darstellung von Schaltkreisen innerhalb des Modells in die konventionelle übertragen." (ebd., S.27)

- „Obwohl die Farbskala über die unterschiedlichen Luftdrücke entwickelt wurde, stellte sie eine eigene Verständnisebene, ähnlich der Verwendung im Stäbchenmodell, dar und konnte ohne weiteren Umweg über den analogen Lernbereich belastet werden. Dadurch hat die Farbskala dazu beigetragen, sich allmählich von dem analogen Lernbereich zu lösen und die Grenzen des Modells zu meiden. Im Vergleich zu den Begrifflichkeiten hat sich die Farbskala als weniger beständig erwiesen. Die SuS haben gegen Ende der Unterrichtsreihe, wie beispielsweise bei der Betrachtung der Brückenschaltung, bewusst darauf verzichtet, da sie anscheinend nicht mehr als Gedankenstütze gebraucht wurde.“ (ebd., S.28)

Zur Motivation der Lernenden:

- „Ich denke, dass vor allem der einfache Zugang über das Analogiemodell und die sich daraus ergebenden intuitiven Begrifflichkeiten sowie ansprechenden Materialien und Tätigkeiten ihren Teil zur Motivation der SuS beigetragen haben. [...] Nicht nur deswegen finde ich, hat sich das Analogiemodell für die Klasse gut geeignet, sondern auch deswegen da es ziemlich gut den Vorerfahrungen und dem Interesse der SuS entsprochen hat. Es sei angemerkt, dass dies meine erste Unterrichtsreihe Elektrizitätslehre ist und ich daher keine persönliche Referenz habe.“ (ebd., S.27)

\section{$\underline{\mathrm{Zu} \text { aufgetretenen Lernschwierigkeiten: }}$}

- „Schwierigkeiten traten bei manchen SuS in der Auffassung der Unveränderlichkeit der Potentiale im geschlossenen Stromkreis und im Vergleich zum offenen Stromkreis auf. Dies war den SuS im analogen Lernbereich nur schwer vorstellbar. Ebenso kam die Frage auf, ob sich das Potential ändern würde, wenn der Leiter noch viel größer wäre.“ (ebd., S.28f) (Anmerkung: Diese Schülervorstellungen konnten bereits im Rahmen der Teaching Experiments in Abschnitt 6.2.3.2 unter den Codes „Dr-ab“ und „Dr-LA“ dokumentiert werden. Einzuschränken ist hier allerdings, dass eine reale Spannungsquelle, ganz ähnlich der ersten geäußerten Vermutung der Schüler, in einem geschlossenen Stromkreis in Folge ihres Innenwiderstands tatsächlich einen kleineren Potenzialunterschied aufweist als im offenen Stromkreis.) 


\subsection{Zentrale Ergebnisse der summativen Evaluation}

\subsubsection{Zentrale Ergebnisse zu Forschungsfrage 1}

Forschungsfrage 1 lautet:

Führt das neue Unterrichtskonzept zu einem höheren Lernzuwachs als der traditionelle Physikunterricht?

Die im Rahmen dieser Studie erhobenen Daten wurden in dieser Arbeit mit Hilfe verschiedener statistischer Methoden analysiert. Auf diese Weise sollte einerseits ein differenzierter Blick auf den Einfluss des Treatments ermöglicht werden (z.B. Analyse des absoluten und relativen Zugewinns nach Terzilen im Vortest), andererseits aber auch eine leichtere Vergleichbarkeit der Ergebnisse mit anderen fachdidaktischen Studien ermöglicht werden. Trotz der jeweiligen statistischen Limitationen sind beispielsweise die Betrachtung der Differenzvariablen „Zugewinn“, das Bilden von parallelisierten Stichproben oder die Durchführung einer Kovarianzanalyse gängige Auswerteverfahren in der fachdidaktischen Forschung.

Tab. 48: Zusammenfassung zentraler Ergebnisse von gängigen Auswertemethoden zum Nettoeffekt des Treatments

\begin{tabular}{|c|c|c|c|c|c|}
\hline Methode & Stichprobe & $M$ & $S E$ & Sig. & Cohens $d$ \\
\hline $\begin{array}{l}\text { Betrachtung der Dif- } \\
\text { ferenzvariablen ,Zu- } \\
\text { gewinn“ }\end{array}$ & $\begin{array}{l}\text { Gesamte Stichprobe } \\
N_{\text {Schüler }}=790 \\
N_{\text {Klassen }}=36\end{array}$ & 3.16 & 0.50 & $p<.001$ & $d=.62$ \\
\hline $\begin{array}{l}\text { Betrachtung der Dif- } \\
\text { ferenzvariablen ,Zu- } \\
\text { gewinn“ }\end{array}$ & $\begin{array}{l}\text { Gleiche Lehrkräfte } \\
N_{\text {Schüler }}=133 \\
N_{\text {Klassen }}=3\end{array}$ & 3.33 & 1.23 & $p<.001$ & $d=.67$ \\
\hline $\begin{array}{l}\text { Parallelisierung auf } \\
\text { Klassenebene bzgl. } \\
\text { Pretest-Ergebnis mit } \\
\text { anschließender Be- } \\
\text { trachtung der Diffe- } \\
\text { renzvariablen „Zu- } \\
\text { gewinn“ }\end{array}$ & $\begin{array}{l}\text { Untermenge der } \\
\text { Gesamtstichprobe } \\
N_{\text {Schüler }}=697 \\
N_{\text {Klassen }}=36\end{array}$ & 3.81 & 0.54 & $p<.001$ & $d=.75$ \\
\hline $\begin{array}{l}\text { Kovarianzanalyse } \\
\text { (ANCOVA) }\end{array}$ & $\begin{array}{l}\text { Gesamte Stichprobe } \\
N_{\text {Schüler }}=790 \\
N_{\text {Klassen }}=36\end{array}$ & 3.71 & 0.49 & $p<.001$ & $d=.75$ \\
\hline $\begin{array}{l}\text { Mehrebenenanalyse } \\
\text { (MLA) }\end{array}$ & $\begin{array}{l}\text { Gesamte Stichprobe } \\
N_{\text {Schüler }}=790 \\
N_{\text {Klassen }}=36\end{array}$ & 3.88 & 0.89 & $p<.001$ & $d=.94$ \\
\hline
\end{tabular}

Anmerkung: $M=$ Mittelwert; $S E=$ Standardfehler

Wie in Tab. 48 zusammengefasst, zeigen in der vorliegenden Studie jedoch alle statistischen Verfahren, dass ein höchst signifikanter Nettoeffekt des Treatments vorliegt, dass also 
das neue Unterrichtskonzept zu einem eindeutig höheren Lernzuwachs führt als der traditionelle Unterricht. Der Nettoeffekt beschreibt dabei, wie viele Punkte im eingesetzten Test die Treatmentgruppe mehr erzielen konnte als die Kontrollgruppe. Es zeigt sich, dass die mittels unterschiedlicher statistischer Methoden ermittelten Nettoeffekte im Rahmen ihrer statistischen Sicherheit miteinander übereinstimmen.

Wie in Abschnitt 7.4.3.3 diskutiert, führt die Betrachtung der Differenzvariablen „Zugewinn" auf Basis der gesamten Stichprobe zu einer Unterschätzung des Nettoeffekts, da einerseits im Posttest eine Regression zur Mitte angenommen werden muss und andererseits die Differenzvariable „Zugewinn“ negativ mit den Pretest-Ergebnissen korreliert $(r(790)=-.22$, $p<.001)$. Die gleichen Effekte dürften auch bei der Teilstichprobe mit identischen Lehrkräften tendenziell zu einer Unterschätzung des Nettoeffekts des Treatments beitragen, wobei hier der Standardfehler aufgrund der kleineren Stichprobe entsprechend größer ausfällt (siehe Abschnitt 7.4.6). Bei der Parallelisierung bzgl. der Pretest-Ergebnisse ist von einer regressionsbedingten Überschätzung des Nettoeffekts auszugehen, wobei die vorliegende negative Korrelation zwischen Pretest-Ergebnis und dem absoluten Zugewinn diesen Effekt teils kompensieren dürfte (vgl. Abschnitt 7.4.5.1). Der mit Hilfe der Kovarianzanalyse ermittelte Nettoeffekt des Treatments stellt aufgrund der adäquateren Kontrolle der Pretest-Ergebnisse eine deutlich bessere Schätzung dar als die alleinige Betrachtung des Zugewinns. Allerdings dürfte der Nettoeffekt des Treatments auch hier leicht nach unten verzerrt sein, weil die Kovariate „Pretest“ nicht völlig unabhängig von der Gruppenzugehörigkeit ist und somit einen Teil der Varianz des Posttest-Ergebnisses aufklärt, der eigentlich auf die Gruppenzugehörigkeit zurückgeht (vgl. Abschnitt 7.4.5.2).

\section{Posttest-Ergebnis von KG und TG}

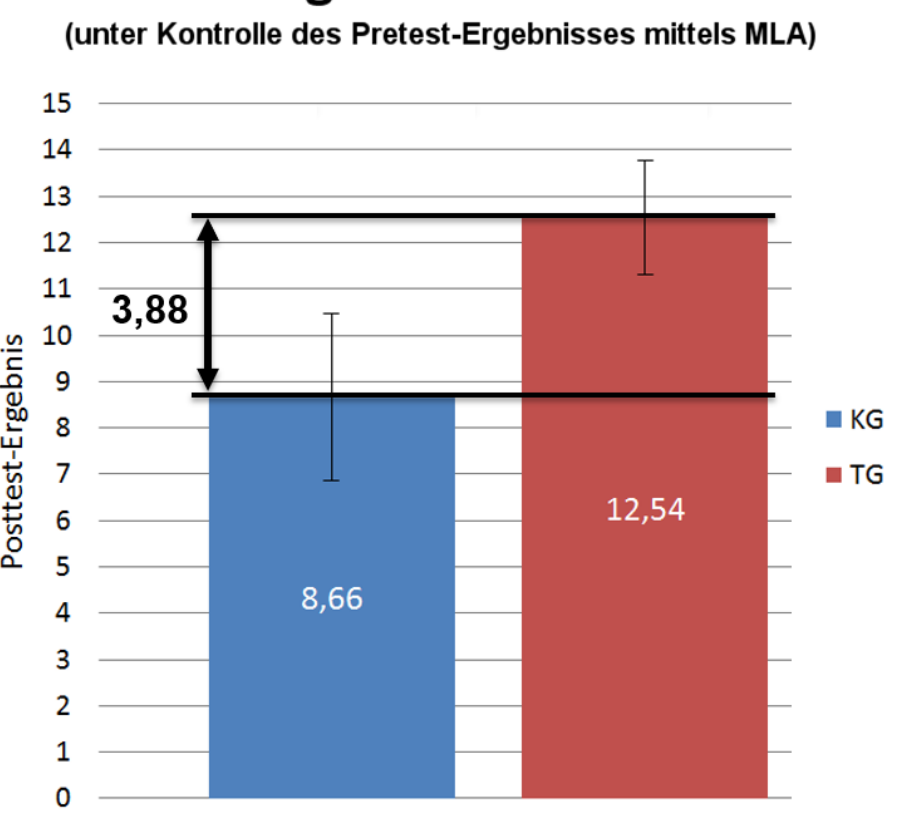

Abb. 116: Die Posttest-Ergebnisse von KG und TG mit 95\%-Konfidenzintervallen sowie der Nettoeffekt des Treatments (fett) entsprechend des Gesamtmodells der Mehrebenenanalyse (MLA = Multilevel Analysis) 
Die adäquateste Schätzung des Nettoeffekts des Treatments sowie seiner statistischen Unsicherheit stellt eindeutig das Ergebnis der Mehrebenenanalyse dar, weil hier neben einer adäquaten Kontrolle der Pretest-Ergebnisse auch die hierarchische Datenstruktur der vorliegenden Stichprobe angemessen berücksichtigt wird. Zusätzlich führt die Mehrebenenanalyse aufgrund der sukzessiven Modellentwicklung zu einem Gesamtmodell, das alle bedeutsamen Effekte und Prädiktoren beinhaltet (vgl. Abschnitt 7.5.3). Das Ergebnis der Mehrebenenanalyse zeigt, dass der höhere Lernzuwachs der Treatment- gegenüber der Kontrollgruppe mit 3.88 Punkten höchst signifikant ist $(F(1,36.27)=18.91, p<.001)$ und einem Cohens $d$ von $d_{\text {Gr. }}=.94$ entspricht, was einen großen Effekt darstellt (siehe auch Abb. 116). Es bleibt somit festzuhalten, dass das neue Unterrichtskonzept zu einem deutlich höheren Lernzuwachs führt als der traditionelle Physikunterricht. Vor dem Hintergrund, dass die Lehrkräfte zum ersten Mal nach dem Konzept unterrichteten und keine Schulung bekamen, ist dies ein bemerkenswertes Ergebnis.

\subsubsection{Zentrale Ergebnisse zu Forschungsfrage 2}

Forschungsfrage 2 lautet:

Zeigt sich eine Abhängigkeit der Lernwirksamkeit des neuen Unterrichtskonzepts von dem Geschlecht der Lernenden, den unterrichtenden Lehrkräften, dem Vortestergebnis, der Jahrgangsstufe oder der Anzahl an Unterrichtsstunden in der Elektrizitätslehre?

A) Zur Abhängigkeit von dem Geschlecht der Lernenden

\section{Lernentwicklung in der Treatmentgruppe nach Geschlecht}

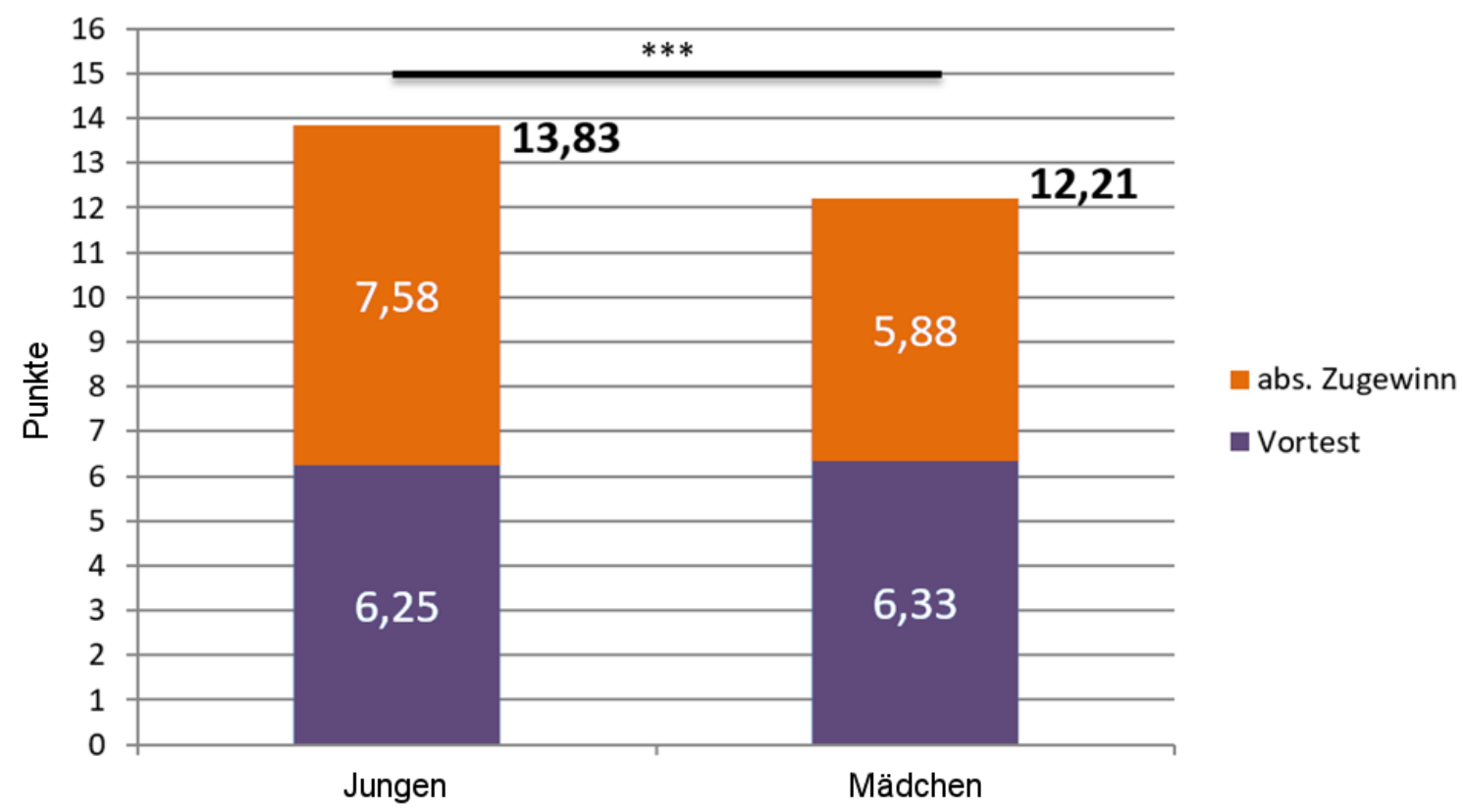

Abb. 117: Vergleich der Pre- und Posttest-Ergebnisse sowie des absoluten Lernzuwachses von Jungen und Mädchen in der Treatmentgruppe. Das angegebene Signifikanzniveau *** $p<.001$ bezieht sich aufden erzielten abs. Zugewinn. 
Wie in Abb. 117 dargestellt, fällt der absolute Lernzuwachs in der Treatmentgruppe bei den Jungen mit 7.58 Punkten $(S E=0.38)$ verglichen mit lediglich 5.88 Punkten $(S E=0.36)$ bei den Mädchen signifikant höher aus $(F(1,431)=10.64, p<.001)$, obwohl sich Jungen mit 6.25 Punkten $(S E=0.25)$ und Mädchen mit 6.33 Punkten $(S E=0.21)$ in ihren Pretest-Ergebnissen nicht signifikant voneinander unterscheiden $(t(431)=-0.25, p=.806)$. Das Unterrichtskonzept zeigt also bei den Jungen eine höhere Lernwirksamkeit als bei den Mädchen (vgl. Abschnitt 7.4.3.4).

B) Zur Abhängigkeit von den unterrichtenden Lehrkräften

Zwar wurden Kontroll- und Treatmentklassen in der Regel von unterschiedlichen Lehrkräften unterrichtet, jedoch gab es in der vorliegenden Studie eine Schnittmenge von zwei Lehrkräften, die ihre Klassen einmal traditionell und einmal nach dem neuen Unterrichtskonzept unterrichteten. Diese Untermenge von jeweils drei Klassen bzw. insgesamt 133 Schülern ist insofern von besonderem Interesse in dieser Studie, als dass hier Unterschiede bei den Lehrkräften in Bezug auf Erfahrung oder Lehrkompetenz zwischen der Kontroll- und Treatmentgruppe ausgeschlossen werden können. Vor dem Hintergrund der hohen Bedeutung der individuellen Lehrerkompetenz auf den Lernerfolg der Schüler (Hattie 2013) kann das Abschneiden der von diesen Lehrkräften unterrichteten Kontroll- und Treatmentklassen als Indiz dafür verstanden werden, ob der in der gesamten Stichprobe gefundene Nettoeffekt des Treatments auf systematische Unterschiede zwischen den jeweils unterrichtenden Lehrkräften zurückzuführen ist oder nicht.

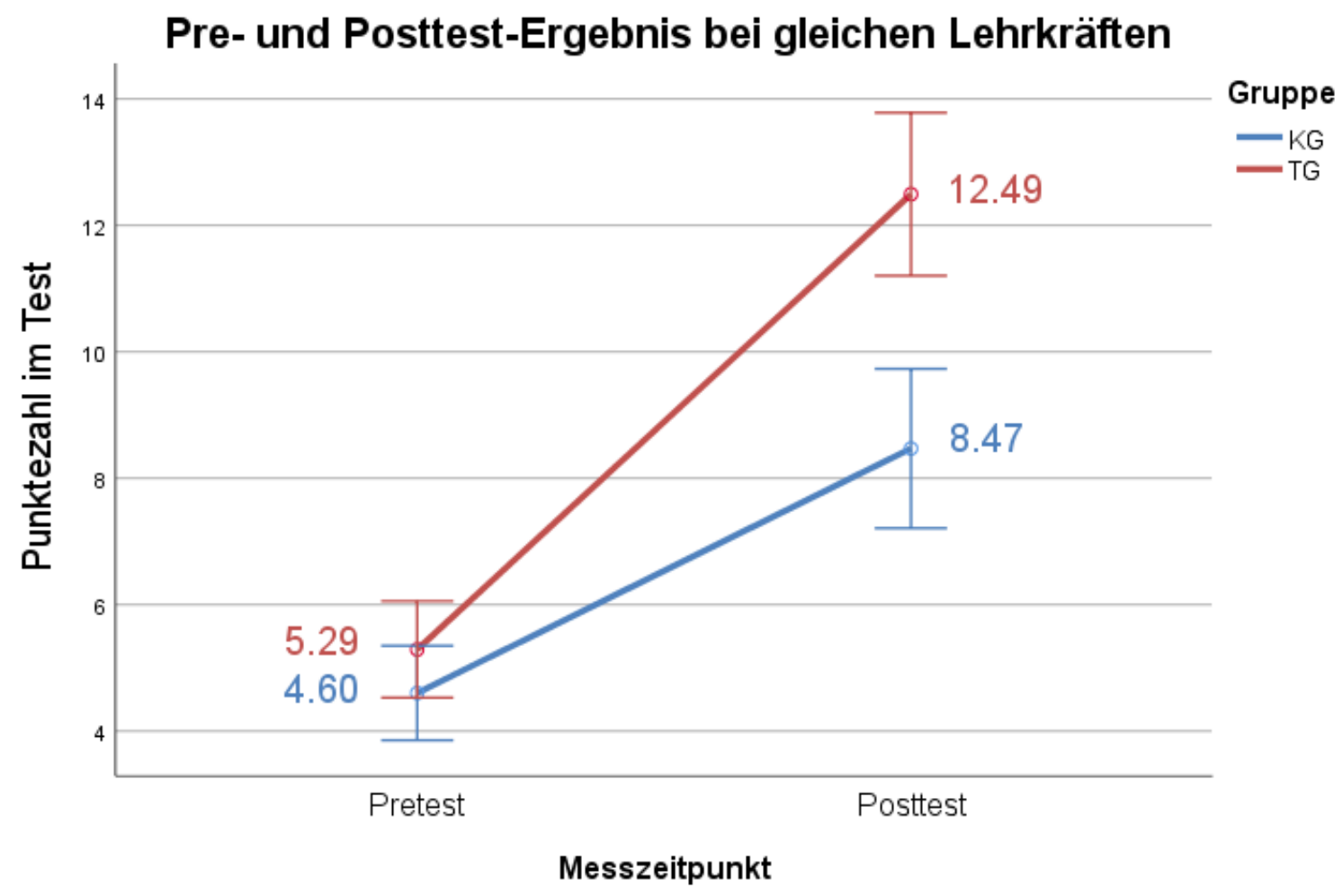

Abb. 118: Die Lernentwicklung bei gleichen Lehrkräften in Kontroll- und Treatmentgruppe mit 95\%-Konfidenzintervallen 
Wie Abb. 118 zu entnehmen ist, erzielen die gleichen Lehrkräfte bei ihren Treatmentklassen einen signifikant höheren absoluten Lernzuwachs als bei ihren Kontrollklassen $(F(1,131)=14.88, p<.001)$, wobei sich die beiden Gruppen bzgl. ihres durchschnittlichen Pretest-Ergebnisses nicht signifikant unterscheiden $(t(131)=1.28, p=.204)$. Die Tatsache, dass der hier gefundene Nettoeffekt der Teilstichprobe von $3.33 \pm 1.23$ Punkten $(p<.001$; $d=.67$ ) mit dem mit der gleichen Methode ermittelten Nettoeffekt der Gesamtstichprobe von $3.16 \pm 0.50$ Punkten $(p<.001 ; d=.62)$ vergleichbar ist, deutet darauf hin, dass der höhere Lernzuwachs der Treatmentgruppe in der Gesamtstichprobe nicht auf systematische Unterschiede oder Selektionseffekte bzgl. der unterrichtenden Lehrkräfte zurückzuführen ist (vgl. Abschnitt 7.4.6). Nichtsdestotrotz ist zu vermuten, dass die Lehrervariable maßgeblich die großen Unterschiede innerhalb der Kontroll- und Treatmentgruppe (z.B. hinsichtlich des mittleren absoluten und relativen Zugewinns auf Klassenebene) bedingt, da die Klassen in der Regel von verschiedenen Lehrkräften unterrichtet wurden. Zusammenfassend kann deshalb davon ausgegangen werden, dass der unterschiedliche Lernzuwachs von Kontroll- und Treatmentgruppe nicht auf die Lehrervariable zurückzuführen ist, die unterschiedlichen Lernzuwächse zwischen den Klassen innerhalb jeder Gruppe hingegen vermutlich schon.

\section{C) Zur Abhängigkeit von dem Vortestergebnis}

Um die Abhängigkeit der Lernförderlichkeit des neuen Unterrichtskonzepts vom Pretest-Ergebnis zu untersuchen, wurden die Schüler der Kontroll- und Treatmentgruppe in Abhängigkeit ihres Vortestergebnisses in die Terzile „unteres Drittel“, „mittleres Drittel“ und „oberes Drittel“ eingeteilt und anschließend der in diesen Terzilen erzielte relative Lernzuwachs (= Lernzugewinn) betrachtet. Der relative Zugewinn gibt dabei an, wie groß der absolute Lernzugewinn in Relation zum für den Schüler überhaupt erzielbaren Zugewinn ausfällt (relativer Zugewinn = absoluter Zugewinn dividiert durch den möglichen Zugewinn).

Während die Schüler der Treatmentgruppe unabhängig von dem jeweiligen Terzil immer einen signifikant höheren relativen Lernzuwachs als die Schüler der Kontrollgruppe erzielen konnten (siehe Abb. 119), zeigt eine univariate einfaktorielle Varianzanalyse, dass kein signifikanter Unterschied in Bezug auf den erreichten relativen Lernzuwachs innerhalb der Treatmentgruppe existiert $(F(2,430)=.71, p=.490)$. Unabhängig von ihrem PretestErgebnis erzielen also alle Schüler der Treatmentgruppe statistisch gesehen den gleichen relativen Lernzuwachs. In der Kontrollgruppe hingegen fällt der relative Lernzuwachs im Durchschnitt nicht nur halb so groß aus wie in der Treatmentgruppe, sondern hängt zudem signifikant vom Pretest-Ergebnis der Schüler ab $(F(2,354)=4.84, p=.008)$. Eine genauere Analyse mit Hilfe eines Games-Howell Post-Hoc-Tests zeigt, dass das untere Drittel der Kontrollgruppe einen signifikant höheren relativen Lernzuwachs in der Kontrollgruppe erzielt als das obere Drittel $(p=.020)$, während der jeweilige Unterschied zum mittleren Drittel statistisch nicht signifikant ist. Je höher also das Pretest-Ergebnis der Schüler in der Kontrollgruppe ist, desto geringer fällt ihr relativer Lernzuwachs aus, während in der Treatmentgruppe der relative Lernzuwachs unabhängig vom Vortestergebnis ist (vgl. Abschnitt 7.4.4). 


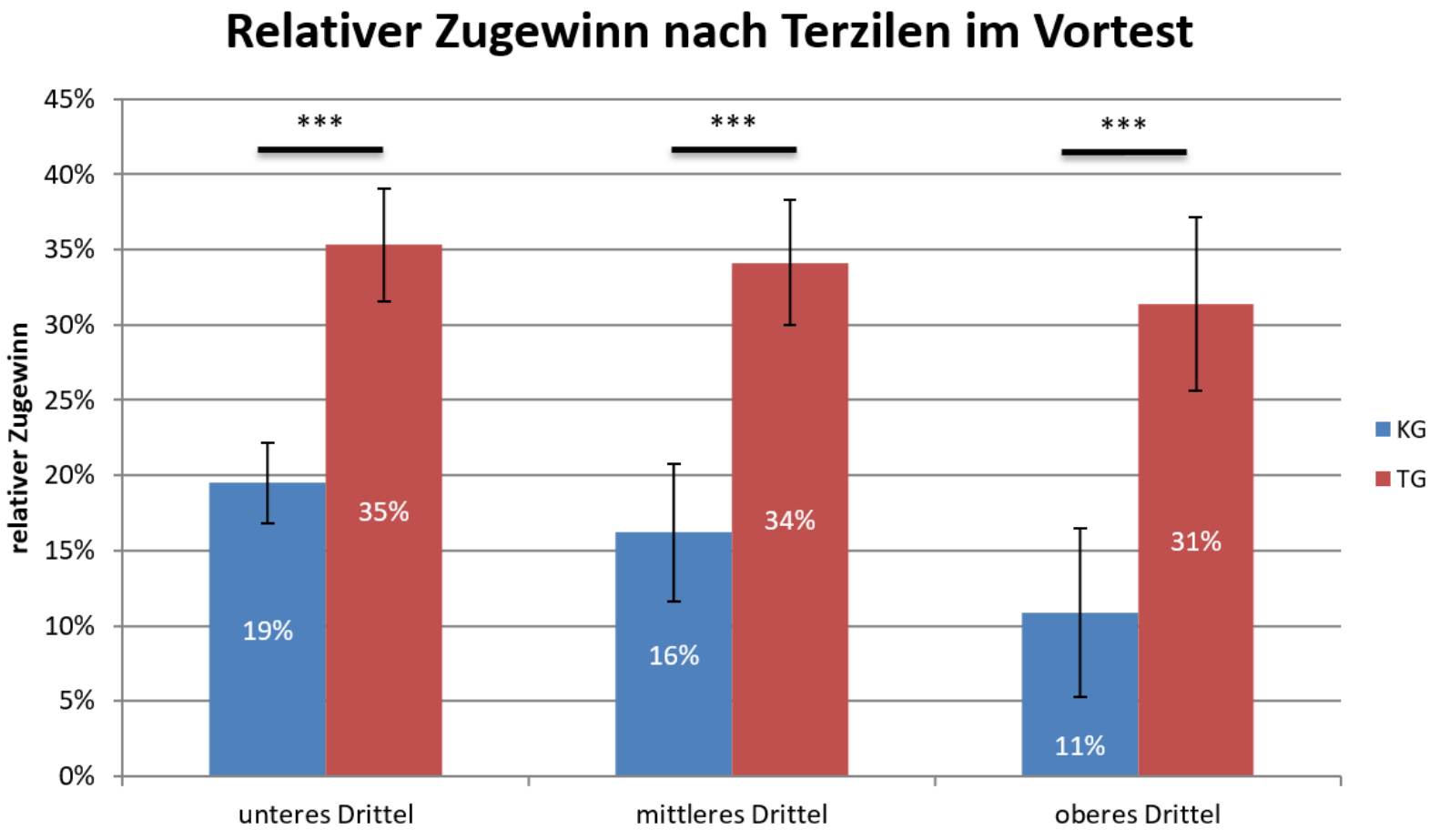

Abb. 119: Relativer Zugewinn nach Terzilen im Vortest mit 95\%-Konfidenzintervallen (Signifikanzniveaus: $* * * p<.001 ; * * p<.01 ; * p<.05$ )

Eine mehrebenenanalytische Auswertung des gesamten Datensatzes zeigt ferner, dass das Nachtestergebnis mit jedem im Vortest erzielten Punkt um $0.44 \pm 0.05$ Punkte höher ausfällt. Dabei unterscheidet sich der Einfluss des Vortestergebnisses auf das Nachtestergebnis insgesamt nicht zwischen Kontroll- und Treatmentgruppe $(F(1,781.03)=1.90, p=.169)$. Dieser Zusammenhang bedeutet auch, dass der absolute Lernzuwachs, gemessen in der Anzahl der im Posttest zusätzlich korrekt beantworteten Items, in beiden Gruppen umso geringer ausfällt, desto höher das Pretest-Ergebnis der Schüler ist. Entgegen der Annahme des „Matthäus-Effekts“ ist der höhere absolute Lernzuwachs der Treatmentgruppe daher nicht auf ihr verglichen mit der Kontrollgruppe höheres durchschnittliches Vortestergebnis zurückzuführen (vgl. Abschnitt 7.5.2.3).

D) Zur Abhängigkeit von der Jahrgangsstufe und der Anzahl an Unterrichtsstunden in der Elektrizitätslehre

Die Jahrgangsstufe hat insgesamt gesehen keinen signifikanten Einfluss auf die Posttest-Ergebnisse der Schüler $(F(1,36.40)=3.55, p=.068)$. Insbesondere zeigt sich in der Treatmentgruppe kein signifikanter Unterschied $(p=.414)$ bzgl. der Lernwirksamkeit des neuen Unterrichtskonzepts zwischen der siebten und der achten Jahrgangsstufe (vgl. Abschnitt 7.5.2.4). Ähnlich sieht es mit der Anzahl an Unterrichtsstunden in der Elektrizitätslehre aus, die weder in der gesamten Studie einen signifikanten Einfluss auf das Nachtestergebnis hat $(F(1,35.81)=2.32, p=.136)$, noch in der Treatmentgruppe das Posttest-Ergebnis signifikant beeinflusst $(p=.746)(\mathrm{vgl}$. Abschnitt 7.5.2.5). 
Somit zeigt sich insbesondere weder eine Abhängigkeit der Lernwirksamkeit des neuen Unterrichtskonzepts von der Jahrgangsstufe noch von der Anzahl der Unterrichtsstunden in der Elektrizitätslehre. Letzteres könnte darauf zurückzuführen sein, dass der Test lediglich das grundlegende Konzeptverständnis der Lernenden prüft und dieses zu einem durchschnittlichen Niveau auch schon nach verhältnismäßig wenigen Unterrichtsstunden erreicht werden kann. Über dieses grundlegende Konzeptverständnis hinausgehende Fähigkeiten und Kompetenzen wurden mit Hilfe des eingesetzten Testinstruments nicht überprüft, weshalb aus diesem Ergebnis nicht geschlossen werden kann, dass die Kompetenzen der Lernenden im Unterrichtsfach Physik durch eine höhere Anzahl an Unterrichtsstunden nicht weiter gefördert werden könnten.

\subsubsection{Zentrale Ergebnisse zu Forschungsfrage 3}

Forschungsfrage 3 lautet:

Unterscheidet sich die Auftretenswahrscheinlichkeit von typischen Schülervorstellungen zwischen dem traditionellen Physikunterricht und dem neuen Unterrichtskonzept?

Die Auswertung der nach dem Unterricht noch vorhandenen Schülervorstellungen mittels einer binären logistischen Mehrebenenanalyse zeigt, dass das neue Unterrichtskonzept entweder zu einer vergleichbaren oder signifikant geringeren Auftretenswahrscheinlichkeit von typischen Schülervorstellungen als der traditionelle Unterricht führt. Wie Abb. $120 \mathrm{zu}$ entnehmen ist, haben Schüler der Treatmentgruppe signifikant weniger konzeptionelle Schwierigkeiten mit der elektrischen Spannung und vertreten zudem signifikant weniger die Stromverbrauchsvorstellung als Schüler der Kontrollgruppe. Auch in Bezug auf das Erfassen von Parallelschaltungen sowie die Schülervorstellungen, die Stromstärke sei unabhängig vom Widerstand oder die Batterie sei eine konstante Stromquelle, führt das neue Unterrichtskonzept verglichen mit dem Unterricht der Kontrollgruppe zu einem signifikant besseren Verständnis der Lernenden. Bei den übrigen Schülervorstellungen haben sich keine statistisch signifikanten Unterschiede zwischen den beiden Gruppen gezeigt (vgl. Abschnitt 7.5.4). Es sei an dieser Stelle noch einmal darauf hingewiesen, dass hier entsprechend des Kodiermanuals von Urban-Woldron und Hopf (2012) eine Schülervorstellung bereits dann als vorhanden gewertet wird, wenn bei einem von mehreren der jeweiligen Subskala zugrundeliegenden Items eine entsprechende Antwortkombination gewählt wurde (vgl. Abschnitt 7.3.4 und 7.4.1.6). Würde hingegen der Ausprägungsgrad der Schülervorstellungen durch Mittelung über alle Items der jeweiligen Subskala betrachtet, lägen die für die verschiedenen Schülervorstellungen ermittelten Werte sowohl in der Kontroll- als auch in der Treatmentgruppe nur etwa halb so hoch (vgl. Abschnitt 7.4.8.2). 


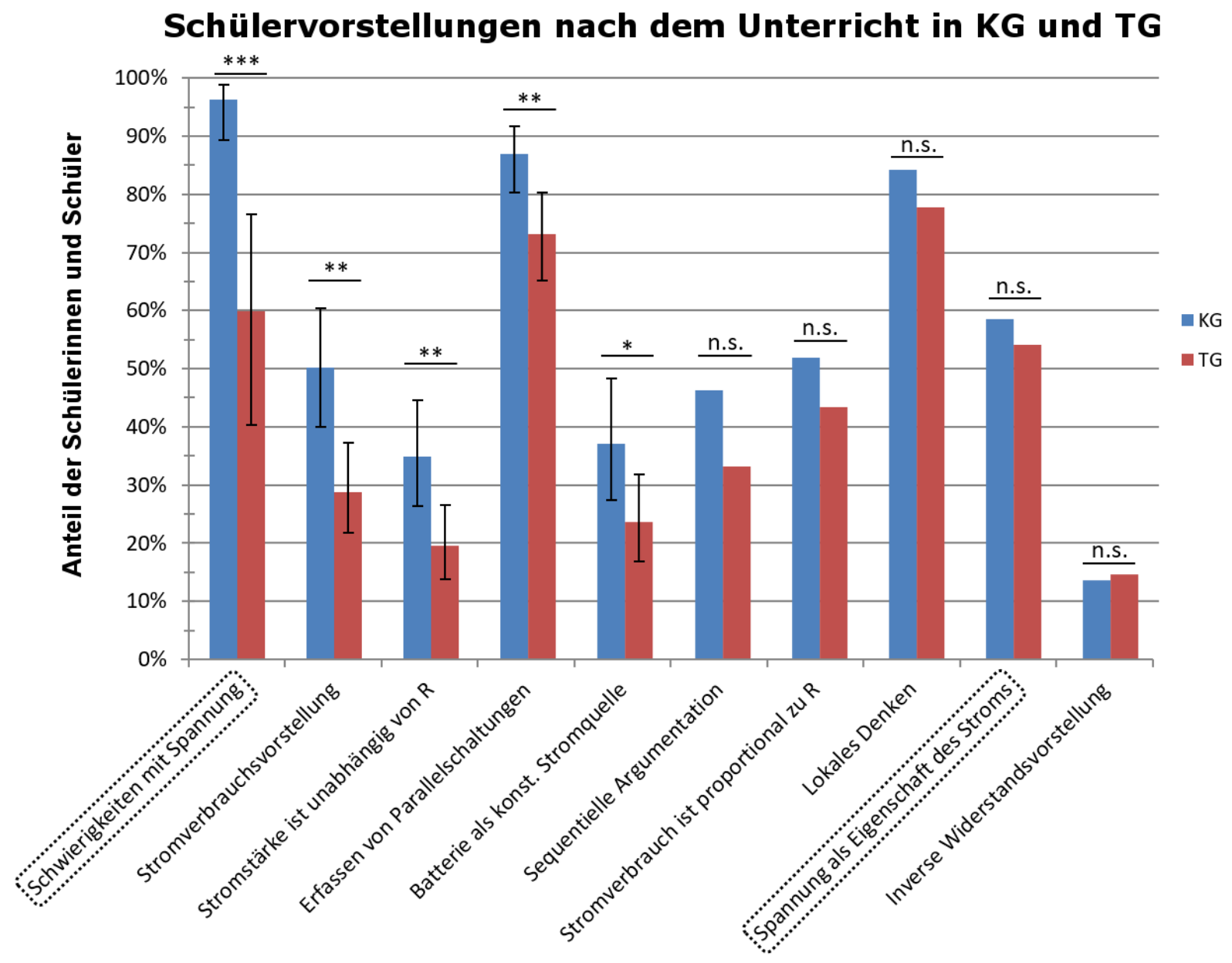

Abb. 120: Übersicht über die mittels binärer logistischer Mehrebenenanalyse ermittelten Schülervorstellungen im Posttest (geordnet nach Signifikanzniveau). Die umrandeten Schülervorstellungen zur Spannung wurden auf Basis eigener Items erhoben. Die 95\%-Konfidenzintervalle wurden von SPSS lediglich für signifikante Unterschiede angegeben. Es gelten die folgenden Signifikanzniveaus: $* * * p<.001 ; * * p<.01 ; * p<.05 ; n . s .=$ nicht signifikant.

An Hand der Kategorie „Schwierigkeiten mit Spannung“ zeigt sich zudem, dass die Vermittlung eines angemessenen Spannungsbegriffs sehr klassenabhängig ist, also in manchen Klassen insgesamt deutlich besser gelingt als in anderen (ICC $=.44$ bei ,Schwierigkeiten mit Spannung“"vs. ICC $\approx .10$ bei den restlichen Schülervorstellungen). Da 48\% (SE =2\%) der Schüler der Treatmentgruppe gegenüber lediglich $12 \%(S E=2 \%)$ der Schüler der Kontrollgruppe mindestens eines der vier Spannungsitems vollständig korrekt lösen konnten (also alle drei gefragten Spannungswerte bei einem Item richtig angeben konnten), scheint das neue Unterrichtskonzept auf Basis des Elektronengasmodells im Vergleich zum traditionellen Physikunterricht zu einem signifikant besseren Verständnis der elektrischen Spannung zu führen $\left(\chi^{2}(1)=114.44, p<.001\right)($ vgl. Abschnitt 7.4.8).

Nichtsdestotrotz bedeutet dieses Ergebnis auch, dass es mehr als der Hälfte der Schüler der Treatmentgruppe nicht gelungen ist, mindestens eines der vier Items zur elektrischen Spannung vollständig korrekt zu lösen. Damit wird deutlich, dass das neue Unterrichtskonzept im direkten Vergleich zum traditionellen Unterricht zwar zu einem deutlich besseren Verständnis der elektrischen Spannung führt, ein Großteil der Lernenden aber weiterhin große konzeptionelle Schwierigkeiten mit dieser wichtigen Größe hat. Dies gilt in unterschiedlicher 
Ausprägung auch für die anderen erhobenen Schülervorstellungen, die in der Treatmentgruppe zwar tendenziell geringer ausgeprägt sind als in der Kontrollgruppe, aber immer noch von einem hohen Anteil an Schülern vertreten werden. Auch wenn das neue Unterrichtskonzept also zu einem besseren Verständnis der Basiskonzepte elektrischer Stromkreise führt, ist die einfache Elektrizitätslehre für viele Lernende auch weiterhin mit großen Verständnisschwierigkeiten verbunden.

\subsubsection{Zentrale Ergebnisse zu Forschungsfrage 4}

Forschungsfrage 4 lautet:

Wie wird das neue Unterrichtskonzept von Lehrkräften aus der Praxis beurteilt?

Wie in Abschnitt 7.6 gezeigt, wird das Unterrichtskonzept von den Lehrkräften im Allgemeinen sehr positiv beurteilt, weshalb 12 von 14 Lehrkräften angeben, auch in Zukunft auf Grundlage des hier vorgeschlagenen Unterrichtskonzepts unterrichten zu wollen. Eine große Mehrheit von 11 der 14 Lehrkräfte gibt darüber hinaus an, das Unterrichtskonzept bereits an Kolleginnen und Kollegen weiterempfohlen zu haben oder dies zu planen. Die gleiche Anzahl an Lehrkräften stimmt ferner den Aussagen eher oder völlig zu, dass das Unterrichtskonzept zentrale Konzepte anschaulich und verständlich vermittelt und dass es von den Schülerinnen und Schülern akzeptiert wird. Lediglich 8 der 14 Lehrkräfte geben jedoch an, dass das Unterrichtskonzept das Interesse bzw. die Motivation der Lernenden eher oder eindeutig fördern würde. Der dem Unterrichtskonzept zugrundeliegende Potenzialansatz, nach dem die Spannung erst nach Einführung des elektrischen Potenzials als Potenzialdifferenz eingeführt wird, hat sich hingegen nach der Einschätzung aller Lehrkräfte als fruchtbar erwiesen. Außer einer Lehrkraft vertraten darüber hinaus alle Lehrkräfte die Ansicht, dass die Schüler ein für das Unterrichtskonzept ausreichendes intuitives Luftdruckverständnis im Sinne von „komprimierte Luft steht unter Druck, drückt gegen die Wände und hat das Bestreben sich auszudehnen“ besitzen oder dass sich dieses mit Hilfe des Unterrichtskonzepts leicht aufbauen lässt.

Danach gefragt, inwiefern das Unterrichtskonzept bei den Lernenden zu einem guten Verständnis von grundlegenden Konzepten in der Elektrizitätslehre führt, bescheinigen 12 der 14 Lehrkräfte dem neuen Unterrichtskonzept eine eher große oder eindeutig große Lernförderlichkeit in Bezug auf die elektrische Spannung. Da das Unterrichtskonzept vor dem Hintergrund entwickelt wurde, den Lernenden ein gutes konzeptionelles Verständnis der elektrischen Spannung zu vermitteln, u.a. weil dies in der Regel im traditionellen Physikunterricht nicht gelingt, ist dies ein sehr erfreuliches Ergebnis. 


\section{Das Unterrichtskonzept führt zu einem guten konzeptionellen Verständnis...}

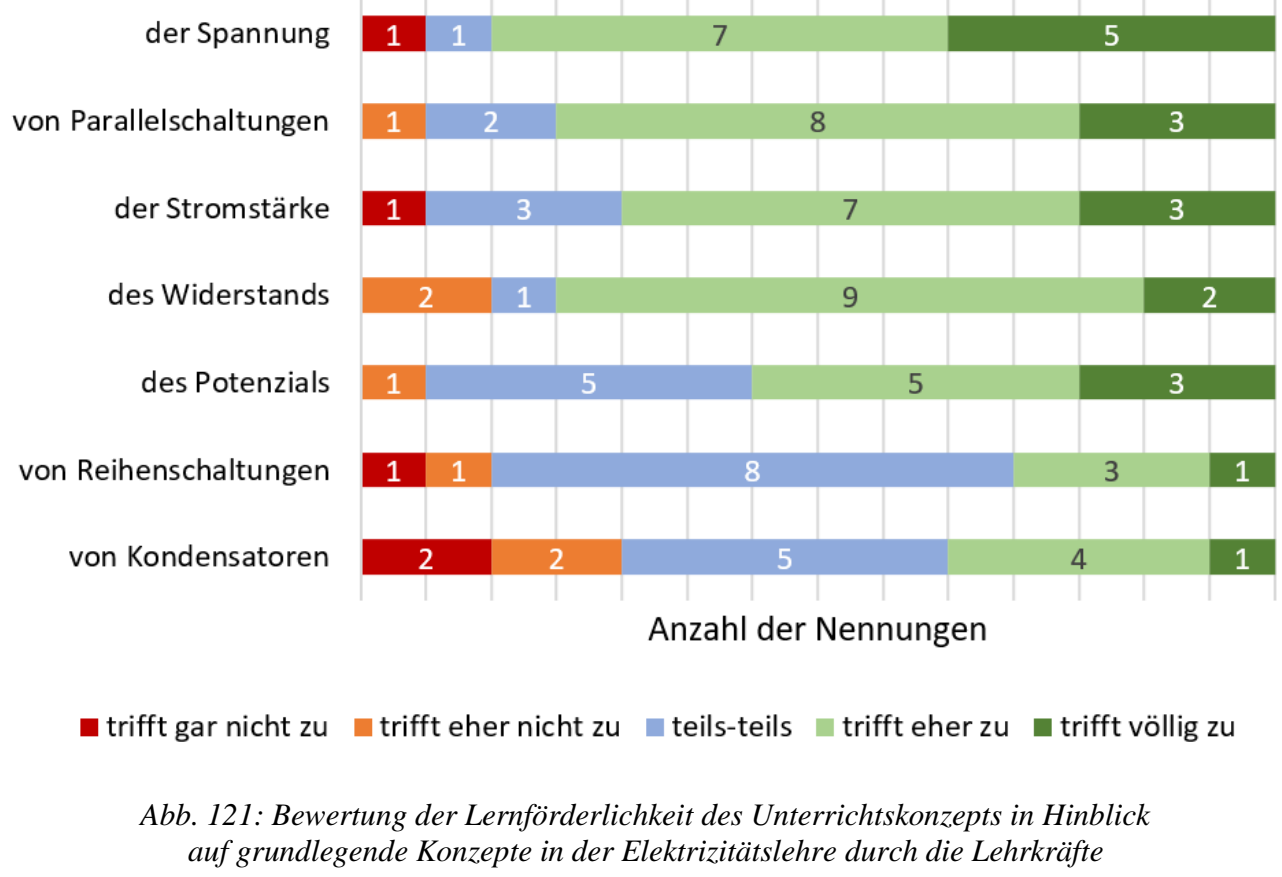

Wie in Abb. 121 dargestellt, führt das neue Unterrichtskonzept aber nach Einschätzung der Lehrkräfte auch hinsichtlich der Stromstärke, des Widerstands und von Parallelschaltungen zu einem vergleichbar guten Verständnis. Etwas geringer wird die Lernförderlichkeit bzgl. des elektrischen Potenzials eingeschätzt, wohingegen lediglich fünf bzw. vier Lehrkräfte der Aussage eher oder völlig zustimmen, das Unterrichtskonzept führe zu einem guten konzeptionellen Verständnis von Kondensatoren bzw. von Reihenschaltungen.

Weitgehende Einigkeit besteht unter den Lehrkräften dahingehend, dass die größten Vorteile des Unterrichtskonzepts darin bestehen, dass

- die Luftdruckanalogie in Kombination mit der vorgeschlagenen Farbkodierung des Potenzials zu einem besseren konzeptionellen Verständnis der Grundgrößen von elektrischen Stromkreisen führt,

- insbesondere der Unterschied zwischen Spannung und Stromstärke deutlich wird und die Spannung als Ursache des elektrischen Stroms eingeführt wird,

- das Unterrichtskonzept gutes und reichliches Übungsmaterial enthält,

- die Schüler eine mikroskopische Modellvorstellung der Vorgänge in elektrischen Stromkreisen bekommen.

Hinsichtlich der Nachteile bestand hingegen weniger Einigkeit bei den Lehrkräften. Sieht man von Punkten ab, die nur von einzelnen Lehrkräften geäußert wurden, bestand die Hauptkritik darin, dass 
- die gewählte Farbkodierung der in der Physik gängigen Konvention widerspricht, wonach der Pluspol rot und der Minuspol blau eingefärbt werden, was aber eher ein Akzeptanzproblem bei den Lehrkräften darstellt als ein Verständnisproblem bei den Lernenden,

- die Erklärungen zu den Reihenschaltungen mit Hilfe von Übergangszuständen zu abstrakt bzw. zu theoretisch für den Anfangsunterricht seien und mehr mit Experimenten gearbeitet werden sollte,

- das Unterrichtskonzept mit allen Materialien in der Umsetzung teils mehr Zeit in Anspruch genommen hätte als veranschlagt.

Insbesondere der im Unterrichtskonzept verfolgte Ansatz, Reihenschaltungen mit Hilfe von Übergangszuständen zu analysieren, wird von den Lehrkräften kritisch gesehen. Sie geben an, dass es den meisten Schülerinnen und Schülern im Anfangsunterricht nicht gelingt, Reihenschaltungen alleine mit Übergangszuständen zu analysieren und sie sich die Thematisierung von Übergangszuständen in diesem Zusammenhang höchstens für leistungsstärkere Schülerinnen und Schüler als Differenzierungsmaßnahme vorstellen könnten. Bei der Frage, ob die parallele Verwendung der Fahrradkettenanalogie neben der Betrachtung der Übergangszustände den Schülerinnen und Schülern das Verständnis von Reihenschaltungen erleichtere, zeigen sich die Lehrkräfte gespalten: Während eine Hälfte in der Fahrradkettenanalogie eine klare Hilfe für die Lernenden sieht, hält die andere Hälfte sie für unnötig oder sogar verständniserschwerend. 



\section{D) Schlussbetrachtungen}





\section{Die Design-Based-Research-Studie im Rückblick}

\subsection{Zentrale Erkenntnisse der Studie}

\subsubsection{Für die Unterrichtspraxis}

Ein wesentliches Anliegen dieser Arbeit bestand im Sinne von Design-Based-Research darin, einen didaktisch fundierten Lösungsansatz für das Problem zu entwickeln, dass es im traditionellen Elektrizitätslehreunterricht in der Regel nicht gelingt, den Lernenden ein angemessenes Verständnis der Grundbegriffe Spannung, Stromstärke und Widerstand sowie ihrer wechselseitigen Beziehung in einfachen Stromkreisen zu vermitteln. Auch wenn entsprechend des DBR-Forschungsparadigmas (vgl. Abschnitt 7.1) nicht gesagt werden kann, auf welche Faktoren die höhere Lernwirksamkeit des hier untersuchten Unterrichtskonzepts genau zurückzuführen ist, hat sich dieses in seiner Gesamtheit als sehr lernwirksam und verständnisfördernd erwiesen. Vor dem Hintergrund der Ergebnisse dieser Arbeit lässt sich deshalb in Hinblick auf die Unterrichtspraxis festhalten, dass es zusammengenommen empfehlenswert ist,

1. vor der Einführung der Stromstärke zunächst den Potenzial- und Spannungsbegriff einzuführen, um die Spannung als Primärkonzept bei den Lernenden zu verankern und so der Entstehung eines ,übermächtigen Strombegriffs“ proaktiv vorzubeugen;

2. das elektrische Potenzial in Analogie zu Luftdruckphänomenen als elektrischen Druck im Leiter einzuführen und auf diese Weise in den Alltagserfahrungen der Lernenden zu verankern;

3. das elektrische Potenzial direkt im Schaltkreis mit Hilfe einer für die Lernenden intuitiven, dem Alltag entlehnten Farbkodierung darzustellen, um die Anforderungen an das nötige Abstraktionsvermögen möglichst gering zu halten;

4. auf dem Potenzialkonzept aufbauend die elektrische Spannung als ,elektrischen Druckunterschied" und Antrieb des elektrischen Stroms einzuführen, um ein Verständnis für den Differenzcharakter der Spannung zu fördern;

5. der Entwicklung eines qualitativen Verständnisses der Grundgrößen der Elektrizitätslehre und ihrer wechselseitigen Beziehung gegenüber einer quantitativen Auseinandersetzung im Anfangsunterricht eine höhere Priorität zu geben;

6. bei der Begriffsbildung zunächst auf ein Vokabular zurückzugreifen, das Schülervorstellungen vermeidet und an die Alltagserfahrungen der Lernenden anknüpft, um daraus die Fachsprache und Fachkonzepte zu entwickeln;

7. Stromkreise immer ausgehend von „elektrischen Druckunterschieden“, d.h. den an den Widerständen anliegenden Spannungen zu analysieren und insbesondere bei Parallelschaltungen nicht mit einer Aufteilung des Stroms an Verzweigungspunkten $\mathrm{zu}$ argumentieren, da dies diverse Schülervorstellungen 
provozieren kann (u.a. Batterie als Konstantstromquelle sowie lokales und sequentielles Denken);

8. den Lernenden für die Vorgänge in elektrischen Stromkreisen auch eine mikroskopische Modellvorstellung zu geben, um ihrer epistemologischen Neugier nach tiefgehenden Erklärungen am eigentlichen Lernobjekt gerecht zu werden;

9. gezielt im Unterricht Beispiele zu thematisieren, bei denen Spannung und Stromstärke nicht gemeinsam auftreten (z.B. einen offenen Schalter), da sonst durch den Zusammenhang $U=R \cdot I$ die Schülervorstellung gestärkt wird, die Spannung sei eine Eigenschaft des elektrischen Stroms;

10. durch die parallele Verwendung des Fahrradkettenmodells zur Verdeutlichung diverser Aspekte wie des Systemcharakters von Stromkreisen und der Konstanz der Stromstärke den reflektierten Umgang mit Modellen aktiv zu fördern;

11. weiterführende Aspekte wie den Innenwiderstand einer Batterie oder eines Volt- bzw. Amperemeters, den Widerstand von Kupferleitungen und die Beliebigkeit des Potenzialnullpunkts im Anfangsunterricht zunächst auszuklammern.

Um die Implementation dieser Erkenntnisse in der Schulpraxis zu vereinfachen und dem oft beklagten Practice-Research-Gap aktiv entgegenzuwirken, wurde die Internetseite www.einfache-elehre.de ins Leben gerufen. Hier können Lehrkräfte kostenfrei aktuelle Unterrichtsmaterialien herunterladen, in denen neben den oben genannten Punkten auch zukünftige didaktische Weiterentwicklungen Berücksichtigung finden sollen.

\subsubsection{Für eine kontextspezifische Lerntheorie}

Ein wesentliches Ziel von Design-Based-Research besteht neben der Lösung eines für die Unterrichtspraxis bedeutsamen Problems auch darin, zum jeweiligen Forschungsgegenstand eine kontextspezifische Theorie des Lernens zu entwickeln und so die Physikdidaktik als Wissenschaft voranzubringen. Auf Basis der in dieser Studie gemachten Erfahrungen und den der Intervention zugrundeliegenden lerntheoretischen Annahmen sollen hier deshalb einige für die weitere physikdidaktische Forschung relevante Aspekte diskutiert werden.

Zunächst ist festzuhalten, dass in dieser Studie unter praxistauglichen Umständen alleine über die Bereitstellung von didaktisch begründeten Unterrichtsmaterialien ein verglichen mit dem traditionellen Unterricht deutlich höherer Lernerfolg bei den Schülerinnen und Schülern erzielt werden konnte. Damit hat sich nicht nur die Materialbereitstellung an sich als eine effektive Methode erwiesen, den Physikunterricht nachhaltig zu verbessern, sondern insbesondere auch der diesen Materialien zugrundeliegende Ansatz der ,instruktionsinduzierten Begriffsentwicklung“" (Wilhelm et al. 2012, S. 35). Wie in Abschnitt 3.1.5 dargelegt, besteht im wissenschaftlichen Diskurs Uneinigkeit über die Frage, wie die vorunterrichtlichen Vorstellungen der Schülerinnen und Schüler auf kognitiver Ebene organisiert sind. Während einerseits insbesondere von Vosniadou (2013) argumentiert wird, die Präkonzepte der Lernenden hätten den Charakter einer stabilen und kohärenten Theorie, wird andererseits insbeson- 
dere von diSessa (2013) die Auffassung vertreten, das Vorwissen der Lernenden hätte eher den Charakter unzusammenhängender und unstrukturierter einzelner Wissenselemente, den sogenannten ,p-prims“ bzw. ,phenomenological primitives”. Aus physikdidaktischer Perspektive ist diese Frage deshalb von großer Bedeutung, weil sich abhängig von der Art und Weise, wie das Vorwissen organisiert ist, andere zu präferierende Strategien in Hinblick darauf ergeben, wie ein Konzeptwechsel, also der Übergang von den Alltagsvorstellungen hin zur physikalischen Vorstellung, im Unterricht erzielt werden kann.

Legt man die Theorie von Vosniadou zugrunde, kann ein Konzeptwechsel nur gelingen, wenn nicht an einzelnen Wissenselementen, sondern an den grundlegenden Annahmen (der sogenannten „Framework Theory") der Lernenden angesetzt wird, da es sonst in Folge einer misslungenen Äquilibration der wissenschaftlichen Vorstellung und der eigenen Rahmentheorie zur Entstehung von Misskonzepten kommt. Vosniadou und Skopeliti (2014) gehen deshalb davon aus, dass ein Konzeptwechsel ein schwieriger und langwieriger Prozess ist, da hierzu ein ganzes Netz an tief verwurzelten konzeptionellen, epistemologischen und ontologischen Überzeugungen geändert werden müsse. Dem hier entwickelten Unterrichtskonzept liegt jedoch die maßgeblich von diSessa (2013) geprägte ,knowledge-in-pieces“ Sichtweise zugrunde, die das Vorwissen der Lernenden nicht als Hindernis, sondern vielmehr als wertvolle Ressource in Hinblick auf die Entwicklung eines wissenschaftlichen Verständnisses betrachtet. Dieses soll entsprechend der Idee der ,instruktionsinduzierten Begriffsentwicklung“ dadurch erzielt werden, dass gezielt an geeignete Wissenselemente, sogenannte , $p$ prims“", der Lernenden angeknüpft wird. Eine Aufgabe der physikdidaktischen Forschung besteht dementsprechend darin, durch eine sorgfältige Auswahl der Sachstruktur und der Kontexte eine systematische Erhöhung der Aktivierungspriorität anknüpfungsfähiger p-prims zu erreichen und auf diese Weise den Lernenden schrittweise den Aufbau eines angemessenen physikalischen Konzepts zu ermöglichen.

Die Ergebnisse der vorliegenden Studie zeigen deutlich, dass das hier entwickelte und an Schülervorstellungen orientierte Unterrichtskonzept, das systematisch versucht, an geeignete Wissenselemente der Lernenden anzuknüpfen, nicht nur eine verglichen mit dem traditionellen Unterricht deutlich höhere Lernförderlichkeit aufweist, sondern insbesondere auch zu deutlich weniger Schülervorstellungen führt. Gerade letzterer Punkt ist in Hinblick auf die noch andauernde Debatte bzgl. der Frage, wie das Vorwissen der Schülerinnen und Schüler organisiert ist und wie im Unterricht damit umgegangen werden sollte, von Interesse, da entsprechend der Rahmentheorie von Vosniadou (2008, XVII) ein solches Vorgehen mit einer Zunahme und keiner Reduktion an Schülervorstellungen hätte einhergehen müssen: „It appears that 'instruction-induced' conceptual change is more difficult to happen and is likely to lead to the formation of misconceptions and the creations of inert knowledge". Diese Position ist vor dem Hintergrund der hier erzielten Lernerfolge insbesondere in Hinblick auf die Schülervorstellungen nicht haltbar. Es zeigt sich im Gegenteil, dass die Strategie der ,instruktionsinduzierten Begriffsentwicklung “ in Kombination mit einer entsprechenden Materialbereitstel- 
lung einen für die Fachdidaktik erfolgsversprechenden und pragmatischen Weg darstellt, zu einer nachhaltigen Verbesserung des Physikunterrichts beizutragen.

\subsection{Kritische Reflexion der Studie}

In der folgenden kritischen Betrachtung sollen einerseits konzeptionelle Entscheidungen zum Studiendesign und ihre Folgen reflektiert, Limitationen der Studie aufgezeigt und auf ungeklärte Fragestellungen hingewiesen werden. Mit der Grundausrichtung als Design-BasedResearch-Projekt besteht das Ziel dieser Studie nicht darin, ähnlich der pädagogischen Psychologie oder physikdidaktischen Laboruntersuchungen durch Variablenkontrolle verallgemeinerbare Ursache-Wirkungs-Beziehungen aufzuklären. Wie in Abschnitt 7.1 dargelegt, wird vielmehr die Auffassung vertreten, dass sich nachhaltige Innovationen in der Unterrichtspraxis aufgrund der Komplexität des Unterrichtsgeschehens nur erzielen lassen, wenn Interventionen einen holistischen Charakter haben. Bezogen auf die vorliegende Arbeit bedeutet dies, dass vor dem Hintergrund des aktuellen Standes der physikdidaktischen Forschung ein ganzes Unterrichtskonzept inklusive neuer Sachstruktur, neuer Modellvorstellungen, neuer Visualisierungen und dazu passenden Unterrichtsmaterialien entwickelt wurde und engagierte Lehrkräfte nach diesem neuen Unterrichtskonzept in der unkontrollierten Schulpraxis unterrichteten. Es sei daher an dieser Stelle exemplarisch auf einige Fragen hingewiesen, deren Klärung prinzipiell von Interesse wäre, die in der vorliegenden Arbeit u.a. in Folge des gewählten Studiendesigns aber weder beantwortet werden können, noch beantwortet werden sollten:

- Welcher Anteil des in der Treatmentgruppe erzielten Lernzuwachses ist auf die verwendete Modellvorstellung, welcher auf die zugrundeliegende Sachstruktur und welcher auf die bereitgestellten Unterrichtsmaterialien zurückzuführen?

- Inwiefern war der Unterricht der verschiedenen Klassen z.B. in Hinblick auf die eingesetzten Methoden miteinander vergleichbar?

- $\mathrm{Zu}$ welchem Grad wurden die dem Unterrichtskonzept zugrundeliegenden didaktischen Ideen z.B. in Hinblick auf die vorgeschlagenen Begriffe von den Lehrkräften im Unterricht so umgesetzt wie intendiert?

In der vorliegenden Studie konnte also die im Vergleich zum traditionellen Unterricht höhere Lernwirksamkeit des Unterrichtskonzepts in der Schulpraxis nachgewiesen werden, ungeklärt bleiben aber insbesondere Fragen nach den genauen Ursachen dieser erhöhten Lernförderlichkeit.

Um genauere Aussagen über die Erfolgsbedingungen des Unterrichtskonzepts machen zu können, wäre es rückblickend sinnvoll gewesen, von den teilnehmenden Klassen bzw. Lehrkräften sowie den Schülerinnen und Schülern mehr Kovariaten zu erheben. Insbesondere hätte auch in der Kontrollgruppe das Geschlecht der Lernenden erfasst werden müssen, um überprüfen zu können, ob hier die Diskrepanz zwischen Mädchen und Jungen hinsichtlich des erzielten Lernzuwachses größer oder kleiner ausfällt als in der Treatmentgruppe. Wie die 
mehrebenenanalytische Auswertung in Abschnitt 7.5.2 gezeigt hat, bestehen die größten unerklärten Leistungsunterschiede zwischen den Lernenden selbst, die zumindest teilweise durch die Erhebung von zusätzlichen kognitiven bzw. affektiven Merkmalen hätten aufgeklärt werden können. Auf der Ebene der Lernenden hätten so auch mögliche Interaktionseffekte, sogenannte Aptitude-Treatment-Interactions (ATI), aufgedeckt werden können, wonach die Lernförderlichkeit des Unterrichtskonzepts möglicherweise maßgeblich von individuellen Merkmalen der Lernenden abhängt. In Anbetracht der Tatsache, dass der Lernzuwachs auch zwischen den Klassen äußerst heterogen ausfällt (vgl. Abschnitt 7.4.7), stellt sich auch hier die Frage, worauf diese enormen Unterschiede zurückzuführen sind. Eine Erhebung von Kovariaten wie der Unterrichtserfahrung, der Motivation und Aspekten des fachdidaktischen Wissens bei den Lehrkräften hätte hier etwas Licht ins Dunkel bringen können. Interessant wäre hier insbesondere auch die Frage, ob der Lernerfolg der Schülerinnen und Schüler z.B. von dem Wissen ihrer Lehrkräfte über Lernschwierigkeiten in der Elektrizitätslehre abhängt und falls ja, ob diese Abhängigkeit durch das vorliegende Unterrichtskonzept gemindert werden kann (vgl. Tobias 2010, S. 273). Eine Untersuchung dieser zusätzlichen Aspekte hätte allerdings den Rahmen dieser ohnehin relativ umfangreichen Arbeit vermutlich gesprengt.

Das Forschungsinteresse der vorliegenden Studie galt dem grundlegenden Konzeptverständnis der Lernenden im Anfangsunterricht zu einfachen Stromkreisen, weshalb der Lernerfolg hier mit Hilfe des Testinstruments von Urban-Woldron und Hopf (2012) ermittelt wurde. Über die Lernwirksamkeit des neuen Unterrichtskonzepts gegenüber dem traditionellen Unterricht zu darüberhinausgehenden wichtigen Fähigkeiten und Kompetenzen, die im Physikunterricht ebenfalls vermittelt werden sollten, können in dieser Studie deshalb keine Aussagen getroffen werden. Um beurteilen zu können, ob das Unterrichtskonzept auch über einen längeren Zeitraum betrachtet zu einem besseren Konzeptverständnis führt und gleichzeitig die Reliabilität der Erhebung zu erhöhen (vgl. Bortz und Döring 2006, S. 554), wäre zudem die Durchführung eines zeitverzögerten Follow-Up-Tests vorteilhaft gewesen. Abschließend sei an dieser Stelle auch noch einmal auf die in Abschnitt 7.3.1 beschriebene grundsätzliche Limitation der Studie hingewiesen, dass in Kontroll- und Treatmentgruppe bis auf wenige Ausnahmen unterschiedliche Lehrkräfte unterrichteten und daher nicht ausgeschlossen werden kann, dass die gefundenen Unterschiede auf eine Positivauswahl an Lehrkräften in der Treatmentgruppe zurückzuführen sind. Allerdings erscheint eine solche Alternativerklärung des höheren Lernerfolgs der Treatmentgruppe vor dem Hintergrund unwahrscheinlich, dass sich der Nettoeffekt des Treatments nicht wesentlich zwischen der Gesamtstichprobe und der Teilstichprobe mit identischen Lehrkräften unterscheidet (vgl. Abschnitt 7.4.6 und 7.7.2).

Nichtsdestotrotz ist es denkbar, dass die in dieser Studie ermittelte Lernwirksamkeit sowohl des traditionellen Unterrichts als auch des neuen Unterrichtskonzepts etwas höher ausfällt als im regulären Unterricht. Dies liegt einerseits daran, dass sich möglicherweise nur überdurchschnittlich engagierte Lehrkräfte überhaupt zur Studienteilnahme bereiterklärt haben (,Selbstselektionseffekt“, vgl. Bortz und Döring 2006, S. 503), andererseits aber auch da- 
ran, dass sich manche Lehrkräfte dazu entschlossen, vorzeitig aus der Studie auszusteigen. In solchen Fällen liegt die Vermutung nahe, dass tendenziell solche Klassen betroffen sind, bei denen die unterrichtenden Lehrkräfte mit den erzielten Lernerfolgen unzufrieden waren. Kommt es bei diesem in der Fachliteratur unter dem Begriff der ,experimentellen Mortalität“ bekannten Phänomen zu entsprechenden systematischen Selektionsfehlern, ist die Validität der Studienergebnisse gefährdet (Bortz und Döring 2006, S. 560). Da sich in der vorliegenden Studie die experimentelle Mortalität in Kontroll- und Treatmentgruppe mit 20\% bzw. $25 \%$ der Klassen jedoch in einer vergleichbaren Größenordnung bewegt, dürfte dieser Effekt lediglich einen Einfluss auf die externe, nicht jedoch die interne Validität der Studie haben. Mit anderen Worten ist es zwar denkbar, dass die ermittelte Lernwirksamkeit in dieser Studie in Folge der experimentellen Mortalität höher als im Regelunterricht ausfällt, da dieser Effekt jedoch in vergleichbarem Umfang in beiden Untersuchungsgruppen auftrat, ist nicht davon auszugehen, dass dieser Effekt für die ermittelte höhere Lernwirksamkeit des neuen Unterrichtskonzepts gegenüber dem klassischen Unterricht verantwortlich ist. Zudem ist es vorstellbar, dass möglicherweise gerade besonders gute Lehrkräfte für eine Teilnahme in der Treatmentgruppe nicht zur Verfügung standen, da diese aufgrund ihres bereits erfolgreichen Unterrichts keine Notwendigkeit für unterrichtliche Veränderungen sahen. In diesem Fall würde die Lernwirksamkeit des neuen Unterrichtskonzepts gegenüber dem klassischen Unterricht in dieser Studie tendenziell sogar unterschätzt werden.

\subsection{Ideen zur Weiterentwicklung und Ausblick}

\subsubsection{Verbesserungsvorschläge zum Unterrichtskonzept}

Entsprechend des Design-Based-Research-Ansatzes (vgl. Abschnitt 7.1) mit seinen Zyklen aus Design, Erprobung und Re-Design kann das hier vorgestellte Unterrichtskonzept trotz seiner bereits hohen Lernförderlichkeit nicht als ein abgeschlossenes Produkt betrachtet werden. Die Idee besteht vielmehr darin, das Unterrichtskonzept in einem weiteren Entwicklungszyklus auf Basis der in dieser Studie gesammelten Erfahrungen weiter zu optimieren und Lösungen für die noch bestehenden Schwächen und Unzulänglichkeiten zu finden. Dahinter steht die Überzeugung der DBR-Community, dass sich eine wirkliche Verbesserung der Schulpraxis nur auf evolutionärem Wege erreichen lässt und es Didaktiker braucht, die sich dem Ziel einer nachhaltigen didaktischen Innovation in ähnlichem Maße verschreiben wie die Ingenieure beim Automobil, das trotz seiner anfänglichen Unzuverlässigkeit und hohen Lautstärke durch stetige Verbesserungen zu einem großen Erfolg wurde (Bereiter 2002, 321f; Reinmann 2005, S. 56). Auf Basis der schulpraktischen Erfahrungen mit dem Unterrichtskonzept sollen deshalb im Folgenden exemplarisch einige Aspekte angesprochen werden, die im Rahmen eines weiteren Entwicklungszyklus von besonderem Interesse sind:

\section{$\underline{\text { Die gewählte Farbkodierung widerspricht der physikalischen Konvention }}$}

In der Physik wird der elektrische Pluspol üblicherweise rot und der elektrische Minuspol blau eingefärbt, während dies im Unterrichtskonzept entsprechend der gewählten Farbkodie- 
rung genau andersherum ist. Ein Anpassen der Farbkodierung an die übliche Konvention wäre leicht umzusetzen, hätte aber einen entscheidenden Nachteil: Die Farbe Blau stünde dann für einen hohen elektrischen Druck, was der Alltagserfahrung widerspricht, dass hohe Werte, z.B. bei Druck oder Temperatur, in der Regel in Rot dargestellt werden. Vor dem Hintergrund, dass die Lernenden mit der bisherigen Farbkodierung kein Problem hatten, erscheint es daher hilfreicher, den Widerspruch im Unterrichtskonzept proaktiv aufzugreifen und im Rahmen einer Diskussion von technischer vs. physikalischer Stromrichtung zu thematisieren. Überträgt man nämlich die Erklärung, wie es in Folge von elektrischen Druckunterschieden zum Strom kommt, auf die technische Stromrichtung, kann die konventionelle Farbkodierung von Plus- und Minuspol so interpretiert werden, dass früher am Pluspol ein elektrischer Überdruck und am Minuspol ein elektrischer Unterdruck von positiven Ladungsteilchen angenommen wurde (vgl. Abschnitt 6.1.7).

Eine Analyse von Reihenschaltungen mittels Übergangszuständen ist für den Anfangsunter$\underline{\text { richt zu abstrakt }}$

Viele Lehrkräfte vertraten die Ansicht, dass eine Analyse von Reihenschaltungen alleine mit Hilfe von Übergangszuständen die meisten Schülerinnen und Schüler im Anfangsunterricht überfordert (vgl. Abschnitt 7.6.4.3). Es ist deshalb zu überlegen, ob nicht zunächst ausgehend von einfachen Spannungsmessungen an einer Reihenschaltung von unterschiedlichen Widerständen erarbeitet werden sollte, dass an größeren Widerständen auch eine größere Spannung abfällt. Darauf aufbauend könnte dann gemeinsam überlegt werden, wie dies innerhalb des Elektronengasmodells zu erklären ist. Im Rahmen einer Binnendifferenzierung könnte hier angestrebt werden, dass die meisten Lernenden diese Erklärung zwar nachvollziehen, jedoch nur leistungsstärkere Schülerinnen und Schüler die Argumentation mit Übergangszuständen auch auf unbekannte Schaltungen anwenden können sollen.

$\underline{\text { Das Unterrichtskonzept benötigt zu viel Unterrichtszeit }}$

Ein weiterer Kritikpunkt einiger Lehrkräfte bestand darin, dass das Unterrichtskonzept mit allen Materialien in der Umsetzung teils mehr Zeit in Anspruch genommen hätte als veranschlagt (vgl. Abschnitt 7.6.4.2). Es wird deshalb angestrebt, das Unterrichtskonzept zu straffen, indem bestimmte Texte gekürzt und Aufgaben gestrichen werden. Sofern in einem überarbeiteten Unterrichtskonzept der Analyse von Reihenschaltungen mit Hilfe von Übergangszuständen eine geringere Bedeutung zukommt, könnte zur Erreichung dieses Ziels auch auf eine Thematisierung von Lade- und Entladevorgängen von Kondensatoren verzichtet werden.

\section{Interessensförderung durch Kontextorientierung}

Ein Vergleich, wie das Unterrichtskonzept hinsichtlich verschiedener didaktischer Aspekte von den Lehrkräften bewertet wurde (vgl. Abschnitt 7.6.3), legt den Schluss nahe, dass das Interesse und die Motivation der Lernenden durch das Unterrichtskonzept stärker gefördert werden könnte. Auch wenn dieser Punkt nicht explizit kritisiert wurde, ist in der Physikdidaktik heute unumstritten, dass eine geeignete Kontextorientierung ein wesentliches Merkmal 
guten Physikunterrichts darstellt, da die Einbettung von physikalischen Themen in Alltagskontexte es den Schülerinnen und Schülern erlaubt, die Physik als sinnvoll und für ihr Leben relevant wahrzunehmen (Labudde 2001). Insbesondere haben zahlreiche Studien zeigen können, dass sich ein an für Mädchen interessanten Kontexten orientierter Physikunterricht positiv auf das Sachinteresse und das Selbstkonzept von Mädchen auswirkt (Häußler und Hoffmann 1995; Berger 2002; Lubben et al. 2005). Unklar hingegen ist, inwiefern eine stärkere Kontextorientierung im Unterricht auch einen positiven Einfluss auf die Lernwirksamkeit des Physikunterrichts hat, wobei Stern und Schuhmacher (2004, S. 134) hier auf folgenden grundsätzlichen Zusammenhang hinweisen:

„Intelligent wird Wissen erst durch seine Anwendung in unterschiedlichen Kontexten. Benötigt werden dazu Lerngelegenheiten, in denen nicht einfach nur das auf die Bewältigung einer Anforderung zugeschnittene Wissen abgerufen werden muss, sondern in denen bereits verfügbares Wissen umstrukturiert und an die neue Anforderung angepasst werden muss. Auf diese Weise wird Wissen vielfältig vernetzt und kann bei Bedarf zunehmend schneller und flexibler aktiviert werden."

Es ist deshalb naheliegend anzunehmen, dass eine stärkere Kontextorientierung im Unterrichtskonzept zu einer besseren Vernetzung des gelernten Wissens führt, womit ,intelligentes Wissen" bei den Lernenden gefördert wird (vgl. Weinert 2000). Vor dem Hintergrund, dass Mädchen in dieser Studie einen geringeren Lernzuwachs erzielen konnten als Jungen (vgl. Abschnitt 7.4.3.4), besteht eine interessante Fragestellung darin, wie sich eine stärkere Orientierung an interessanten Kontexten auf affektive Merkmale und das Konzeptverständnis insbesondere von Mädchen auswirkt.

Im Rahmen einer internationalen Nachfolgestudie eines Konsortiums der vier Universitäten Wien, Graz, Darmstadt und Frankfurt soll das hier entwickelte Unterrichtskonzept deshalb u.a. vor dem Hintergrund der oben genannten Punkte weiterentwickelt und evaluiert werden (Wilhelm et al. 2018). In diesem Zusammenhang wird auch das Studiendesign verbessert, indem z.B. die Lehrervariable über die verschiedenen Kontroll- und Treatmentgruppen konstant gehalten wird, um ausschließen zu können, dass mögliche Unterschiede zwischen den Gruppen auf eine Positivauswahl der Lehrkräfte zurückzuführen sind. Auch wird bei dieser Studie ein zeitverzögerter Follow-Up-Test durchgeführt, um mögliche Effekte über einen längeren Zeitraum besser beurteilen zu können. Konkret sieht das Studiendesign wie folgt aus: 


\section{Darstellung des Studiendesigns der internationalen Nachfolgestudie}

1. Jahr

2. Jahr

3. Jahr

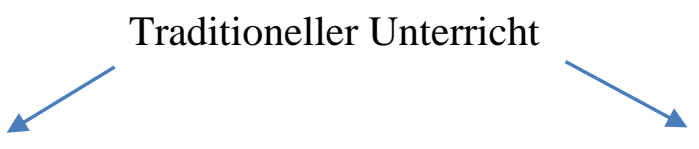

Elektronengasmodell ohne Kontextorientierung

Abb. 122: Das Studiendesign der internationalen Nachfolgestudie

Wie in der Veranschaulichung des Studiendesigns dargestellt, unterrichten die teilnehmenden Lehrkräfte im ersten Jahr die Elektrizitätslehre so, wie sie es bisher immer getan haben. Im zweiten Jahr unterrichtet die eine Hälfte der Lehrkräfte die Elektrizitätslehre weiterhin traditionell, aber ergänzt um interessante Kontexte, während die andere Hälfte der Lehrkräfte nach einer überarbeiteten Fassung des hier vorgestellten Unterrichtskonzepts auf Basis des Elektronengasmodells unterrichtet, jedoch ohne Kontextorientierung. Im dritten Jahr unterrichten dann alle Lehrkräfte nach einem kontextorientierten und überarbeiteten Unterrichtskonzept auf Basis des Elektronengasmodells.

\subsubsection{Offene Fragen}

Neben den dargelegten Ideen zur Weiterentwicklung des Unterrichtskonzepts auf Basis der in dieser Studie gemachten Erfahrungen existieren zudem noch weiterführende grundlegende Fragen, von denen einige hier abschließend kurz angesprochen werden sollen:

- Zwar konnte gezeigt werden, dass Lernende, die nach dem hier vorgeschlagenen Unterrichtskonzept auf Basis des Elektronengasmodells unterrichtet wurden, weniger klassische Schülervorstellungen vertreten als ihre traditionell unterrichteten Peers, im Rahmen der Teaching Experiments konnte aber auch gezeigt werden, dass die zugrundeliegende Vorstellung des Elektronengasmodells zu neuen Schülervorstellungen führen kann (vgl. Abschnitt 6.2.3.2). Eine interessante Frage besteht deshalb darin, wie stark diese neuen Schülervorstellungen nach einem Unterricht auf Basis des hier vorgeschlagenen Konzepts bei den Lernenden tatsächlich ausgeprägt sind.

- Dem Unterrichtskonzept liegt die Annahme zugrunde, dass eine Erklärung der elektrischen Spannung als elektrischer Druckunterschied in Analogie zu Luftdruckunterschieden den Lernenden ein besseres Verständnis der Ursache-Wirkungs-Beziehung zwischen Strom und Spannung ermöglicht (vgl. Abschnitt 6.1.2). Auch wenn in dieser Studie gezeigt werden konnte, dass dieser Ansatz sehr lernförderlich ist, stellt sich die Frage, ob die Lernenden insbesondere gegen Ende der Unterrichtseinheit die Farben im Stromkreis noch mit einer Druckvorstellung assoziieren oder ob es ihnen gelingt, sich von dem analogen Lernbereich des Luftdrucks zu lösen und ein eigenständiges 
Verständnis für den Zielbereich elektrischer Stromkreise zu entwickeln, dem eine implizite Potenzialvorstellung zugrunde liegt.

- Im Rahmen der didaktischen Elementarisierung des Elektronengasmodells wurde sich dafür entschieden, nicht zwischen Leitungs- und Oberflächenelektronen zu unterscheiden und stattdessen nur die mittlere Elektronendichte in einem Leiterstück zu betrachten (vgl. Abschnitt 6.1.3). Da die Punktedichtedarstellung nur anfänglich bei offenen Stromkreisen verwendet wurde und zur Betrachtung von strömenden Elektronen bei geschlossenen Stromkreisen die Farbdarstellung genutzt wurde, konnte die Problematik, wie die Stromstärke trotz unterschiedlicher Elektronendichten vor und nach einem Widerstand die gleiche sein kann, im Unterrichtskonzept gemieden werden. Auch wenn keine Lehrkraft davon berichtete, dass es von den Lernenden zur Konstanz der Stromstärke hier kritische Rückfragen gab, bestünde ein saubererer Weg womöglich darin, schon in der Mittelstufe zwischen Leitungs- und Oberflächenelektronen zu unterscheiden. Es könnte argumentiert werden, dass sich die zusätzlichen Elektronen nur an der Oberfläche eines Leiters ansammeln können, aufgrund ihrer negativen Ladung jedoch einen hohen elektrischen Druck bei den Elektronen im Leiterinneren bewirken. In Analogie zu Luftdruckunterschieden könnte dann wie im hier vorgeschlagenen Unterrichtskonzept erklärt werden, dass die Leitungselektronen von Bereichen mit hohem elektrischen Druck zu Bereichen mit niedrigem elektrischen Druck strömen. Dabei könnten die bewährten Visualisierungen des elektrischen Potenzials beibehalten werden, sofern bei der Punktedichtedarstellung darauf hingewiesen wird, dass sich die unterschiedlichen Elektronendichten auf die Oberflächenelektronen beziehen. Eine weitere zu klärende Frage besteht deshalb darin, inwiefern eine solche Einführung von Oberflächenelektronen von Lernenden in der Mittelstufe akzeptiert wird und womöglich zu einem besseren Verständnis elektrischer Stromkreise beitragen kann. 


\section{Zusammenfassung}

Die Elektrizitätslehre stellt für viele Schülerinnen und Schüler eine der größten Herausforderungen des Physikunterrichts der Mittelstufe dar. Trotz oftmals intensiver unterrichtlicher Bemühungen seitens der Lehrkräfte gelingt es den Lernenden oftmals nicht, ein angemessenes Verständnis einfacher elektrischer Stromkreise zu entwickeln. Der traditionelle Unterricht hat sich insbesondere in Bezug auf die Vermittlung eines angemessenen konzeptionellen Verständnisses der elektrischen Spannung als ineffektiv erwiesen. Dies ist nicht nur problematisch, weil die elektrische Spannung eine wichtige physikalische Größe im Alltag darstellt, sondern insbesondere, weil ohne sie ein grundlegendes Verständnis elektrischer Stromkreise nur schwer vorstellbar ist. Die Gründe für die Lernschwierigkeiten sind zahlreich und reichen von einer verfrühten Mathematisierung der Zusammenhänge über tief verankerte Schülervorstellungen zu diesem Thema bis hin zu seiner prinzipiellen Unanschaulichkeit sowie der Sachstruktur des traditionellen Unterrichts und die dort teilweise verwendeten ungeeigneten Modelle (vgl. Abschnitt 3.2, 4.3 und 6.1.1).

Im Sinne von Design-Based-Research bestand das Ziel dieser Arbeit deshalb darin, sich diesem für den Physikunterricht wichtigen Problem anzunehmen und theoriegeleitet auf Basis einer ausführlichen Analyse typischer Lernschwierigkeiten und bisheriger Unterrichtsansätze in der Elektrizitätslehre einen umfassenden didaktischen Lösungsansatz für die Schulpraxis zu erarbeiten (vgl. Abschnitt 3.2 und 5). Gleichzeitig sollte aber auch die Physikdidaktik als Wissenschaft vorangebracht werden, indem im Bereich der Elektrizitätslehre die kontextspezifische Lehr-Lerntheorie weiterentwickelt wird. Ausgehend von einer konstruktivistischen Grundposition (vgl. Abschnitt 3.1.2) und insbesondere der von diSessa geprägten „knowledge-in-pieces“ Perspektive auf das Lernen (vgl. Abschnitt 3.1.5) wurde bei der Entwicklung des Unterrichtskonzepts eine Anknüpfungsstrategie verfolgt. Durch die gezielte Auswahl geeigneter Kontexte und einer passenden Abfolge der Inhalte wurde versucht, an solche Vorstellungen bei den Lernenden anzuknüpfen, die einer physikalischen Begriffsentwicklung förderlich sind. Ein solches Vorgehen wird nach Wilhelm et al. (2012, S. 35) ,instruktionsinduzierte Begriffsentwicklung“"genannt.

Auf Grundlage eines didaktisch elementarisierten Elektronengasmodells und anknüpfend an die Erfolge bisheriger Potenzialansätze (u.a. von Steinberg und Wainwright 1993, Gleixner 1998 sowie Waltner et al. 2009) wurde eine erste grundlegende Sachstruktur entwickelt. Diese unterscheidet sich von vielen anderen Ansätzen u.a. dadurch, dass entsprechend den Überlegungen von Cohen et al. (1983) die elektrische Spannung als Potenzialdifferenz noch vor der Stromstärke eingeführt wird, um der Entstehung eines ,übermächtigen Strombegriffs“ entgegenzuwirken und die Ursache-Wirkungs-Beziehung zwischen der elektrischen Spannung und dem elektrischen Strom zu verdeutlichen. Hierzu wird ähnlich dem CASTLECurriculum von Steinberg und Wainwright (1993) an das intuitive Luftdruckverständnis der Lernenden z.B. mit Luftmatratzen angeknüpft und erarbeitet, dass Luftströmungen immer die Folge von Luftdruckunterschieden sind. Indem der Luftdruck mit einem in Leitern herrschen- 
den „elektrischen Druck“ verglichen wird, kann die elektrische Spannung so in Analogie zu den Luftdruckbeispielen als „elektrischer Druckunterschied“ und Antrieb des elektrischen Stroms eingeführt werden (vgl. Abschnitt 6.1.2).

Zur formativen Evaluation der grundlegenden Sachstruktur wurde auf die Mitte der 1980er Jahre in Frankfurt von Jung und Wiesner entwickelte Methode der Akzeptanzbefragung, heute auch Teaching Experiments genannt, zurückgegriffen. Diese hat sich zur Erprobung und Entwicklung von neuen Unterrichtsansätzen als äußerst effektiv erwiesen, da mit ihr durch Einzelbefragungen überprüft werden kann, welche Erklärungsansätze von Lernenden akzeptiert und verstanden werden und welche nicht (vgl. Abschnitt 6.2). Es zeigte sich, dass die wichtigsten dem Unterrichtskonzept zugrundeliegenden Ideen von den Lernenden grundsätzlich akzeptiert und verstanden werden. Insbesondere besaßen die Schüler bereits über ein für das Konzept ausreichendes intuitives Luftdruckverständnis und konnten dieses erfolgreich auf den in Stromkreisen herrschenden ,elektrischen Druck“ übertragen. Mit Hilfe der Teaching Experiments konnten aber auch eine ganze Reihe an Stolperstellen in der bisherigen Konzeption identifiziert sowie neue Schülervorstellungen aufgedeckt werden, die in Hinblick auf die Entwicklung eines Unterrichtskonzepts von großem Wert waren (vgl. Abschnitt 6.2.4 und 6.2.5).

Auf Basis der mit den Teaching Experiments gewonnenen Erkenntnissen wurde dann ein umfassendes Unterrichtskonzept inklusive passender Unterrichtsmaterialien wie Overheadfolien, Übungsaufgaben und Zusammenfassungen entwickelt (vgl. Abschnitt 6.3), das anschließend im Rahmen einer empirischen Evaluation in der Unterrichtspraxis auf seine Lernwirksamkeit hin überprüft wurde. Als Testinstrument wurde der psychometrisch ausgereifte zweistufige Multiple-Choice-Tests von Urban-Woldron und Hopf (2012) mit 22 Items hauptsächlich zum Stromstärke- und Widerstandsbegriff verwendet, der um vier Items zur elektrischen Spannung erweitert wurde und die Erhebung typischer Schülervorstellungen zu einfachen Stromkreisen erlaubt. Eine testbedingte Bevorzugung des evaluierten Unterrichtskonzepts liegt nicht vor, da der Test im Gegensatz zum Unterrichtskonzept schwerpunktmäBig nicht auf das Spannungsverständnis abzielt. Da jedes Item dichotom kodiert wurde $(0=$ falsche Antwort bzw. 1 = richtige Antwort), konnten im Test insgesamt 26 Punkte erzielt werden. Im Rahmen einer mit 790 Schülern bzw. 36 Gymnasialschulklassen durchgeführten Studie wurde dieses Unterrichtskonzept dann im Schuljahr 2015/16 im Frankfurter Raum empirisch evaluiert. Ziel der quasi-experimentellen Feldstudie auf Basis eines Pretest-PosttestTreatment-Kontrollgruppen-Designs war es, den Verständniszuwachs von traditionell unterrichteten Klassen (Kontrollgruppe) mit dem Verständniszuwachs von Klassen zu vergleichen, die nach dem neuen Unterrichtskonzept auf Grundlage des Elektronengasmodells unterrichtet wurden (Treatmentgruppe) (vgl. Abschnitt 7.3.1 und 7.3.2).

Zur Auswertung der Daten wurde auf einen Methodenpluralismus zurückgegriffen, um einerseits einen differenzierten Blick auf die Lernwirksamkeit des neuen Unterrichtskonzepts zu erhalten, andererseits aber auch eine leichtere Vergleichbarkeit der Ergebnisse mit anderen 
fachdidaktischen Studien zu ermöglichen. Eine Mehrebenenanalyse zeigt, dass der Lernzuwachs der Treatment- gegenüber der Kontrollgruppe mit 3.88 Punkten höchst signifikant ist $(F(1,36.27)=18.91, p<.001)$ und einem Cohens $d$ von $d_{\text {Gr. }}=.94$ entspricht, was einen großen Effekt darstellt (vgl. Abschnitt 7.5.3). Hinzu kommt, dass der relative Lernzuwachs der Lernenden in der Kontrollgruppe umso geringer ausfällt, desto höher ihr Pretest-Ergebnis ist, während sich in der Treatmentgruppe keine Abhängigkeit des relativen Lernzuwachses vom Vortestergebnis zeigt. Insgesamt fällt der relative Lernzuwachs in der Treatmentgruppe mit $34 \%(S E=1 \%)$ mehr als doppelt so hoch aus wie in der Kontrollgruppe, wo er lediglich $16 \%$ ( $S E=1 \%$ ) beträgt (vgl. Abschnitt 7.4.4). Betrachtet man den in den einzelnen Klassen durchschnittlich erzielten relativen Lernzuwachs, so fällt dieser in beiden Gruppen sehr heterogen aus (vgl. Abschnitt 7.4.7). Eine Mehrebenenanalyse ergibt, dass 39\% der Unterschiede zwischen den Klassen im Nachtest alleine darauf zurückzuführen sind, ob diese nach dem neuen Unterrichtskonzept unterrichtet wurden oder nicht (vgl. Abschnitt 7.5.2.2). Eine Auswertung der nach dem Unterricht noch vorhandenen Schülervorstellungen mittels einer binären logistischen Mehrebenenanalyse zeigt, dass das neue Unterrichtskonzept je nach Schülervorstellung entweder zu einer vergleichbaren oder signifikant geringeren Auftretenswahrscheinlichkeit als der traditionelle Unterricht führt. Insbesondere scheint das Unterrichtskonzept im direkten Vergleich zum traditionellen Unterricht zu einem besseren Verständnis der elektrischen Spannung zu führen, auch wenn hier einzuschränken ist, dass ein Großteil der Lernenden weiterhin große konzeptionelle Schwierigkeiten mit dieser wichtigen Größe zu haben scheint (vgl. Abschnitt 7.4.8, 7.5.4 und 7.7.3). Vor dem Hintergrund, dass die Lehrkräfte allerdings zum ersten Mal nach dem Konzept unterrichteten, sind die erzielten Lernerfolge beachtlich.

Zusätzlich zur empirischen Erhebung des Lernerfolgs der Schülerinnen und Schüler wurden die unterrichtenden Lehrkräfte zu ihren schulpraktischen Erfahrungen mit dem neuen Unterrichtskonzept befragt, um herauszufinden, welche Aspekte des Unterrichtskonzepts sich aus ihrer Sicht in der Praxis als besonders erfolgreich bzw. problematisch erwiesen haben. Ein solcher multiperspektivischer Ansatz („Triangulation“) von quantitativen und qualitativen Daten kann insbesondere in Hinblick auf eine weitere Optimierung des Konzepts von Interesse sein und ist typisch für Design-Based-Research, da für diesen Ansatz die spätere Implementation der Forschungsergebnisse in der Praxis und die damit einhergehende Überwindung des oft beklagten Practice-Research-Gaps ein wesentliches Anliegen darstellt (vgl. Abschnitt 7.1). Hier zeigte sich, dass das Unterrichtskonzept von den Lehrkräften im Allgemeinen sehr positiv beurteilt wird, da 12 von 14 Lehrkräften angaben, auch in Zukunft nach ihm unterrichten zu wollen. Eine ebenso große Anzahl an Lehrkräften bescheinigt dem Unterrichtskonzept eine eher große oder eindeutig große Lernförderlichkeit in Bezug auf die elektrische Spannung, was vor dem Hintergrund des Anliegens dieser Arbeit ein sehr erfreuliches Ergebnis darstellt. Kritisch sehen die Lehrkräfte u.a., dass die gewählte Farbkodierung des elektrischen Potenzials der in der Physik gängigen Konvention widerspricht, dass das Unterrichtskonzept teils mehr Zeit benötigte als geplant und dass der verfolgte Ansatz, Reihenschaltungen mit Hilfe von Übergangszuständen zu analysieren, für viele Lernende zu abstrakt sei. Gelobt wird insbesondere, dass die verwendete Luftdruckanalogie in Kombination mit der vorgeschlage- 
nen Farbkodierung zu einem besseren konzeptionellen Verständnis der Grundgrößen von elektrischen Stromkreisen führt und das Unterrichtskonzept gutes und reichliches Übungsmaterial enthält. Besonders hervorgehoben wird von den Lehrkräften darüber hinaus, dass im Unterrichtskonzept nicht nur der Unterschied zwischen Spannung und Stromstärke deutlich wird, sondern auch die Ursache-Wirkungs-Beziehung zwischen diesen beiden Größen (vgl. Abschnitt 7.6).

Zusammenfassend lässt sich somit sagen, dass sich der diesem Design-BasedResearch-Projekt zugrundeliegende Ansatz, ein Unterrichtskonzept theoriegeleitet auf Basis aktueller fachdidaktischer und lerntheoretischer Erkenntnisse zu entwickeln, es mit Hilfe von Teaching Experiments zu optimieren und es anschließend in der Schulpraxis unter realistischen Bedingungen zu evaluieren, im Lichte der empirisch nachgewiesenen hohen Lernwirksamkeit des so entstandenen Unterrichtskonzepts als Erfolg erwiesen hat. Es zeigt sich zudem, dass die instruktionsinduzierte Begriffsentwicklung eine effektive Methode darstellt, den Lernenden ein konzeptionelles Verständnis auch komplexer physikalischer Größen wie der elektrischen Spannung zu ermöglichen. Neben dem erzielten besseren Konzeptverständnis der elektrischen Spannung und anderer Grundgrößen der Elektrizitätslehre bei den Lernenden wird dies u.a. daran deutlich, dass die Lehrkräfte in der gewählten Sachstruktur einen Gewinn gegenüber dem herkömmlichen Elektrizitätslehreunterricht sehen. Die gravierendsten und vermutlich überzeugendsten Unterschiede zum traditionellen Vorgehen bestehen dabei darin, dass

1. vor einer mathematischen Formalisierung zunächst ein qualitatives, konzeptionelles Verständnis der Grundbegriffe Potenzial, Spannung, Stromstärke und Widerstand sowie ihrer wechselseitigen Beziehung in einfachen Stromkreisen entwickelt wird,

2. nicht die Stromstärke, sondern zunächst das elektrische Potenzial und darauf aufbauend die elektrische Spannung eingeführt werden, um der Entwicklung eines übermächtigen Strombegriffs gezielt vorzubeugen und die elektrische Spannung als Ursache des elektrischen Stroms bei den Lernenden als Primärkonzept zu verankern,

3. bei der Begriffsentwicklung auch sprachlich gezielt an das Vorverständnis der Lernenden angeknüpft wird, indem beispielsweise die elektrische Spannung in Analogie $\mathrm{zu}$ einem Luftdruckunterschied, der z.B. bei einer Luftmatratze eine Luftströmung bewirkt, als „elektrischer Druckunterschied“" und somit Antrieb des elektrischen Stroms eingeführt wird,

4. den Lernenden mit Hilfe des Elektronengasmodells bereits im Anfangsunterricht eine erste mikroskopische Modellvorstellung der Vorgänge in elektrischen Stromkreisen vermittelt wird, die ihr Bedürfnis nach tiefergehenden Erklärungen am eigentlichen Lernobjekt befriedigt und in Hinblick auf den weiterführenden Unterricht anschlussfähig ist. 
Bemerkenswert ist ferner, dass die hohe Lernwirksamkeit des Unterrichtskonzepts $(d=.94 ; p<.001)$ alleine durch die Bereitstellung von didaktisch durchdachten Unterrichtsmaterialien ohne weitere Schulung der Lehrkräfte erzielt werden konnte. Damit dürfte nicht nur eine breite und vergleichsweise einfache Implementation des Unterrichtskonzepts in der Schulpraxis möglich sein, sondern es ist davon auszugehen, dass dabei auch eine zu dieser Studie vergleichbar hohe Lernwirksamkeit erzielt werden kann. Bedenkt man, dass die Lehrkräfte der Treatmentgruppe im Gegensatz zu den Lehrkräften der Kontrollgruppe zum ersten Mal nach dem neuen Konzept unterrichteten und keine Schulung erhielten, ist davon auszugehen, dass die potenzielle Lernförderlichkeit des hier vorgestellten Unterrichtskonzepts noch höher liegt. Vor diesem Hintergrund und der Tatsache, dass das Unterrichtskonzept von den Lehrkräften akzeptiert, weiterempfohlen und als signifikante Verbesserung ihres Unterrichts wahrgenommen wird, kann das in dieser Arbeit entwickelte und evaluierte Unterrichtskonzept zu einer nachhaltigen Innovation des Elektrizitätslehreunterrichts in der Mittelstufe entsprechend des Begriffsverständnisses von Reinmann $(2005,53 f)$ beitragen. 



\section{Summary of the Doctoral Thesis ${ }^{17}$}

\subsection{Motivation}

Despite many years of science education, students often do not develop the ability to reason qualitatively about electric circuits. Instead, they tend to resort to algorithmic manipulations when analysing simple circuits, as the mathematical introduction of Ohm's law often precedes a robust conceptual understanding of its underlying physical quantities (Cohen et al. 1983). However, even when considerable time is spent on developing a qualitative understanding of electric circuits, research on students' conceptual difficulties in electricity shows that voltage and electric potential typically remain unlearned even after instruction (Duit et al. 1985). A particularly widespread alternative conception in this context is that voltage is a property or a component of an electric current, rather than an independent physical quantity that refers to a difference in electric potential (Rhöneck 1986). Not realising the importance of voltage as the cause of the flow of current, students tend to reason exclusively with current and resistance when analysing electric circuits.

Even though these two concepts may appear to be more concrete and intuitive than voltage at first sight, a robust conceptual understanding of voltage as a difference in electric potential is key to the effective analysis of electric circuits. Furthermore, the exclusive reasoning with current and resistance often contributes to the emergence of several other alternative conceptions such as sequential reasoning (Shipstone et al. 1988). Cohen et al. (1983, p. 411) have hypothesised that the electric current dominates students' thinking about electric circuits because the concept of current is introduced before potential difference in almost every introductory course on electricity. In this context, they argue that "first impressions are strong and may impede a later, more rigorous, study of electricity" and ask whether a possible remedy may lie in a curriculum that not only "[...] clearly spells out the relation of cause and effect between pd [potential difference] and current", but also introduces the concept of potential difference first. Moreover, they suggest an analogy in which the battery exerts a certain 'pressure' on the electric charges in the wire. While the latter idea was picked up in the CASTLE curriculum (Steinberg \& Wainwright 1993) in the form of an air pressure analogy, the concept of voltage was still introduced after the electric current. Nonetheless, Steinberg \& Wainwright (1993) report that students taught according to their curriculum show significantly greater achievement gains than traditionally taught students and identify a major factor for the curriculum's effectiveness in its underlying, highly intuitive, yet powerful air pressure analogy. Unfortunately, the results of a statistical study on the effectiveness of the CASTLE curriculum have never been published. What has been missing to date is the empirical evaluation of a curriculum that is not only based on the air pressure analogy, but one which also introduces the concept of potential difference before electric current.

\footnotetext{
${ }^{17}$ Parts of this section have been published in Burde \& Wilhelm (2016b) and Burde \& Wilhelm (2018).
} 


\subsection{The new curriculum}

Taking into account the results from earlier studies on typical alternative conceptions in electricity (see chapter 3) and previous teaching concepts (see chapter 5), a first draft of a new teaching concept was developed (see chapter 6.1.8). The formative evaluation of the basic ideas of the new teaching concept was conducted with the help of nine grammar school students in year 6, who had never previously had a physics lesson, using one-to-one interviews. This qualitative method, also known today as Teaching Experiments, was first developed by Wiesner and Jung (Jung 1992; Wiesner 1993) in Frankfurt in the mid-1980s and has proven to be extremely effective in probing the acceptance of new teaching ideas as well as investigating possible learning difficulties. It was found that the ideas of the new teaching concept, in particular the air pressure analogy, were widely accepted and understood by the students (see chapter 6.2.4). With the help of the Teaching Experiments, however, it was also possible to identify certain ideas and explanations in the first draft of the teaching concept that had proven to be difficult to understand. Based on the experience gained from this qualitative evaluation of the basic ideas of the new teaching concept (see chapter 6.2), a new curriculum including appropriate course materials was developed for junior high schools (see chapter 6.3).

The objective of the new curriculum is to give students a qualitative, but robust, conception of voltage, current and resistance and to help them overcome typical alternative conceptions. It constantly endeavours to provide students with intuitive explanations that have their origins in everyday physical intuitions. In contrast to the CASTLE curriculum, it focuses on potential difference as the cause of an electric current from the very beginning. For this reason, the electric potential and potential differences are introduced even before the electric current by comparing the electric potential to air pressure. Another important difference to the CASTLE curriculum lies in the fact that the curriculum described here has been adapted to German school standards and, hence, places less emphasis on hands-on experiments with capacitors. Compared to traditional introductory courses to electricity in Germany, the most significant change of the new curriculum lies in the additional, explicit introduction of the electric potential to explain voltage as a difference in electric potential. Although this may appear obvious as voltage is defined as potential difference, traditional approaches in Germany have tried to explain voltage as "energy per charge" or "the cause of current flow" without any reference to electric potential or potential differences (see chapter 4.3 and 5). Not surprisingly, most students failed to develop an adequate conceptual understanding of voltage.

By building on students' everyday physical intuitions with air pressure, the new curriculum is in accordance with the ideas of diSessa (1993), who interprets learning as the construction and reorganisation of previously only loosely connected elements of knowledge, called "p-prims", into a coherent mental structure. These p-prims ("phenomenological primitives") represent naïve understandings of the physical world and are primitive in the sense that they represent minimal abstractions from everyday experience and that they only constitute a fragmented collection of ideas. In order to facilitate effective teaching, diSessa (2013, p. 41) 
proposes to build upon appropriate prior knowledge: "Students have a richness of conceptual resources to draw on. Attend to their ideas and help them build on the best of them" (see chapter 3.1.5). The pressure concept, as it is used here, is a good example for such a p-prim, as it only refers to the everyday experience that compressed air is "under pressure" and tries to push itself out of a container (e.g. an air mattress or a bicycle tyre). In particular, the p-prim does not correspond to the refined physical scalar concept of pressure as a state variable that is difficult to understand for most young students. The curriculum then endeavours to embed this initially isolated p-prim into a cognitive structure by linking it to other p-prims such as " $a$ pressure difference leads to an air flow". By using examples of everyday objects, students experience via hands-on experiments that air always flows from areas of high pressure to areas of low pressure and that pressure differences are the cause for an air flow (see Fig. 1). In addition, students learn that several layers of fabric reduce the intensity of an air flow in order to help them develop a prototype conception of resistance in electric circuits.

The air pressure analogy is more compelling to learners than the water analogy, mainly because of the palpable compressibility of air and the fact that students gather experiences with it in everyday life (Steinberg \& Wainwright 1993, p. 355). In contrast, water is often perceived by students as an incompressible fluid and they have no experience with water pressure from everyday life. Therefore, the water analogy is not as helpful as commonly assumed because water under high pressure differs neither visibly, nor palpably, from water under low pressure (Burde \& Wilhelm 2016).
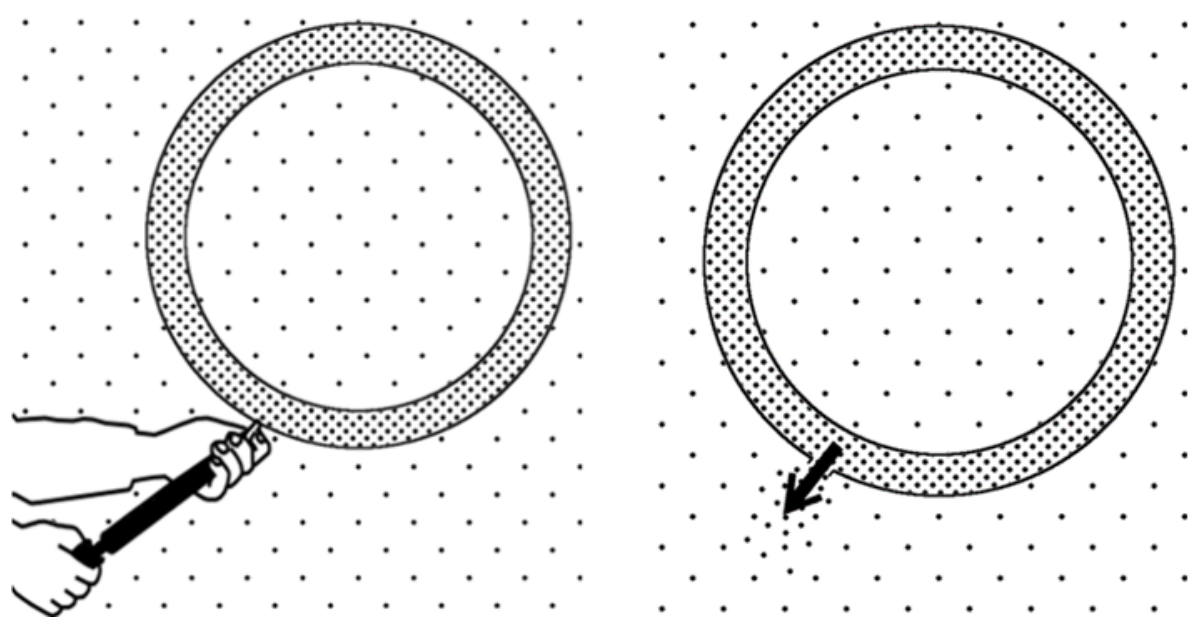

Fig. 1: Illustration of the p-prim "a pressure difference leads to an air flow" using the example of a bicycle tyre.

As a next step, the curriculum builds on these p-prims and tries to connect the familiar with the new. It is proposed that electrons are always present in every (copper) wire in the form of tiny, freely mobile particles. These electrons form a compressible electron gas that spreads evenly across a wire as the electrons are negatively charged and, therefore, repel each other. The battery's role in an electric circuit is to maintain a certain electron density in the wires directly attached to its terminals. A high electron density in the wire attached to the negative terminal corresponds to a high electric pressure, whereas, on the other hand, a low 
electron density in the wire attached to the positive terminal corresponds to a low electric pressure.

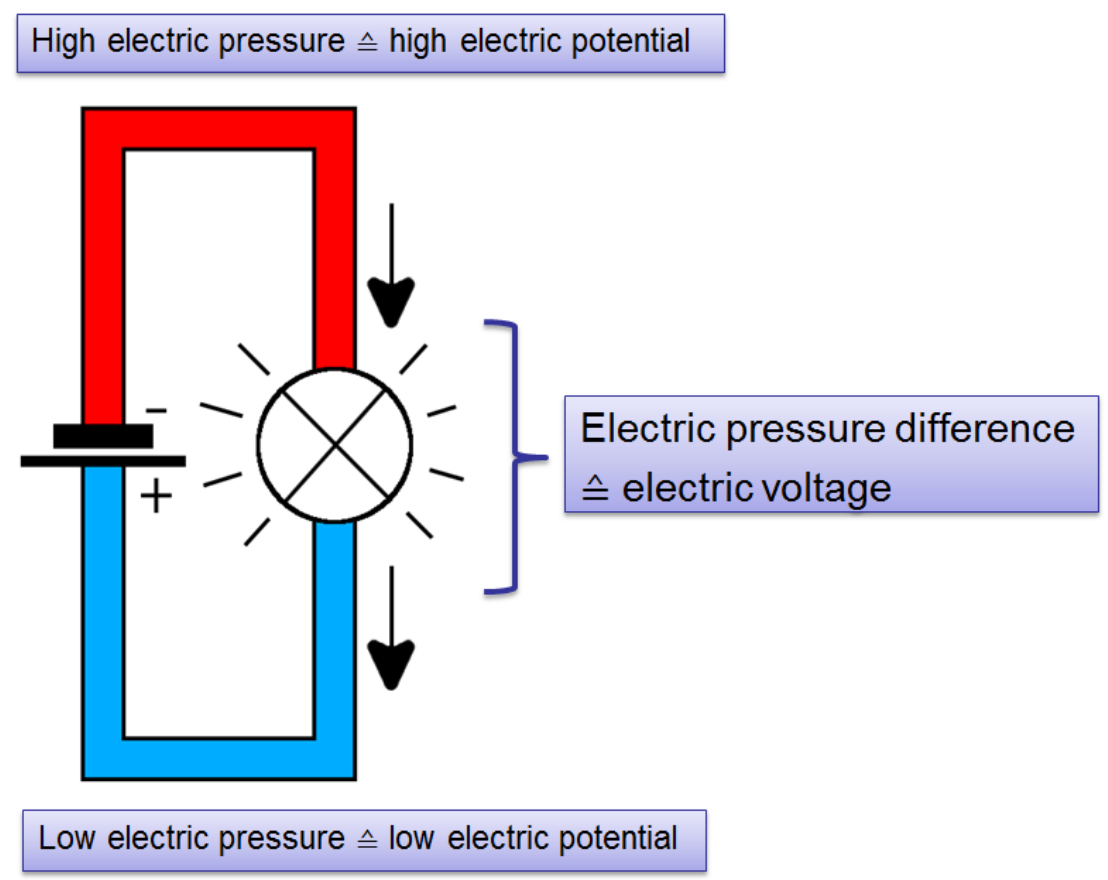

Fig. 2: Electric pressure difference (= voltage) across a light bulb in a simple electric circuit.

The key idea is that the intuitive air pressure concept from the beginning serves as a qualitative, low-abstraction prototype of the electric potential and potential difference in electric circuits. In the new curriculum, voltage as potential difference can thus be understood as an electric pressure difference across a resistor that is as much the cause for an electric current as air pressure differences are the cause for air flow. In contrast to the air pressure examples, the electric pressure in the wires of the circuit is visualised using colours. In analogy to the colours used for different temperatures on weather maps, red represents a high electric pressure and blue a low electric pressure (see Fig. 2).

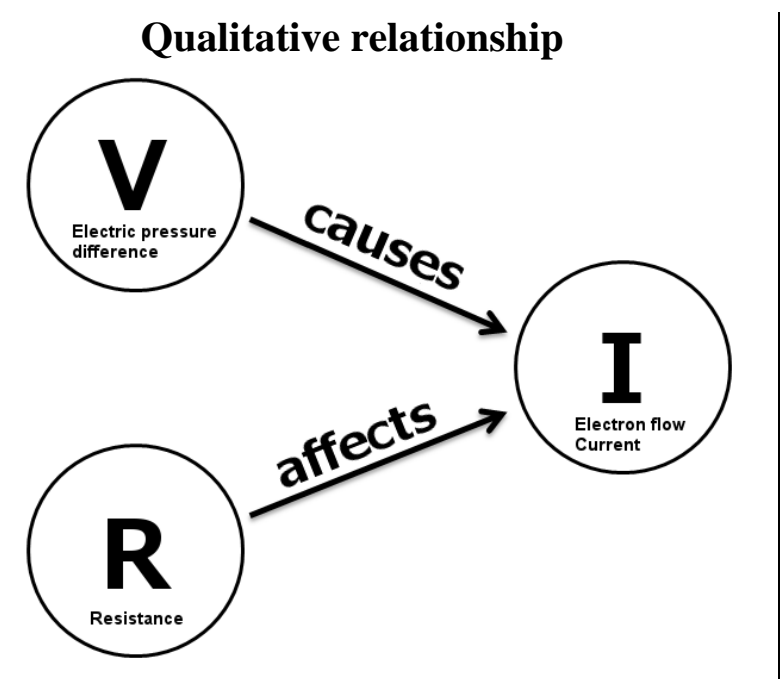

\section{Quantitative relationship}

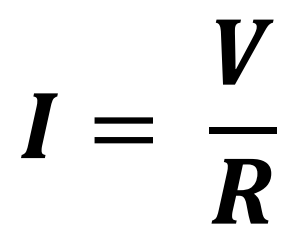

Fig. 3: Transition from a qualitative to a quantitative relationship of the physical quantities current, voltage and resistance. 
The main objective of the teaching concept is to give students a qualitative, but robust, conception of the physical quantities voltage, current and resistance. In contrast to other teaching concepts, much emphasis is initially placed on helping students develop an intuitive understanding of the electric potential as an "electric pressure" in order to then introduce voltage as an electric pressure difference that drives the electric current through resistors. Against the background of physics as a quantitative science, the qualitative understanding of the relationship between the physical quantities voltage, current and resistance is then transformed into a quantitative understanding as illustrated in Fig. 3.

From a technical perspective, it could be argued that the density of the conduction electrons is the same throughout the circuit and that these electrons do not represent a compressible fluid. Although the conduction electrons are, indeed, homogeneously distributed over the entire circuit, surface charges, that are the source of the electric field that drives the electric current in the wire, are not (see chapter 2.3). While the amount of surface charges is negligible compared to the amount of conduction electrons inside the wire, the density of surface electrons is higher nearer the negative terminal than the positive terminal. By only looking at the mean electron density in a wire and considering "electric pressure differences" instead of the electric field that drives the electric current, the electron gas model, as it is used here, represents a simplification for pedagogical reasons (see chapter 6.1.3). A more in-depth discussion of surface charges and their role in electric circuits can be found in Chabay \& Sherwood (2011).

\subsection{Sample and test instrument}

The quasi-experimental field study followed a pretest-posttest-control-group design and was based on the testing of $N=790$ students from Frankfurt/Main, Germany. The control group (CG), which was taught the traditional way by 11 teachers for an average of 23.5 lessons ( $S D=11.9$ ), consisted of 17 junior high school classes and a total of $N=357$ students. The slightly larger experimental group (EG), which was taught according to the new curriculum by 14 teachers for an average of 24.3 lessons $(S D=9.8)$, consisted of 19 junior high school classes and a total of $N=433$ students. The topic of electric circuits had not been covered before in any of the participating classes. In terms of group size and the amount of lessons taught, the CG and EG were, thus, comparable. However, for technical reasons, the participating teachers were not randomly assigned to the CG or EG, but had the choice of whether they wanted to teach traditionally or according to the new curriculum (see chapter 7.3.1). From a methodological perspective, this represents a weakness of the empirical study, because it cannot be ruled out that the differences observed between the CG and EG are in fact not attributable to the new curriculum, but to different levels of motivation, experience or aptitude on the teachers' side. However, a detailed analysis of the test results of teachers who participated in both the CG and EG, suggests that this is not the case (see chapter 7.4.6).

In both groups, the same valid and reliable two-tier diagnostic multiple-choice test was used for the pre- and posttest. The original test was constructed without reference to the new 
curriculum by an independent research group from Vienna and contained 22 items primarily on current and resistance, but not on voltage (Urban-Woldron \& Hopf 2012). Therefore, in order to be able to evaluate students' understanding of voltage, the original test was extended by another four items on the concept. Since 22 out of the 26 items primarily dealt with current and resistance, the test used here is clearly unbiased towards the new curriculum that puts a particular emphasis on potential and potential difference. The advantage of the two-tier structure of the test is that students have to justify and explain their answers. This not only allows for the identification of false-positive answers (i.e., correct answers despite an inadequate explanation), but, more importantly, for the identification of typical alternative conceptions about electric circuits. An item was only counted as correct if students gave both a correct answer and a correct explanation. The highest achievable score in the test, therefore, was 26 points (see chapter 7.3.3). A general discussion of the different statistical methods used in this study can be found in chapter 7.3.5.

\subsection{Empirical results}

As shown in Fig. 4, a first analysis of the test results shows that the mean test score before instruction was lower in the control group $(M=5.09, S E=0.15)$ than in the experimental group $(M=6.29, S E=0.16)$. An unequal variances $t$-test shows that this difference in the pretest is highly significant.

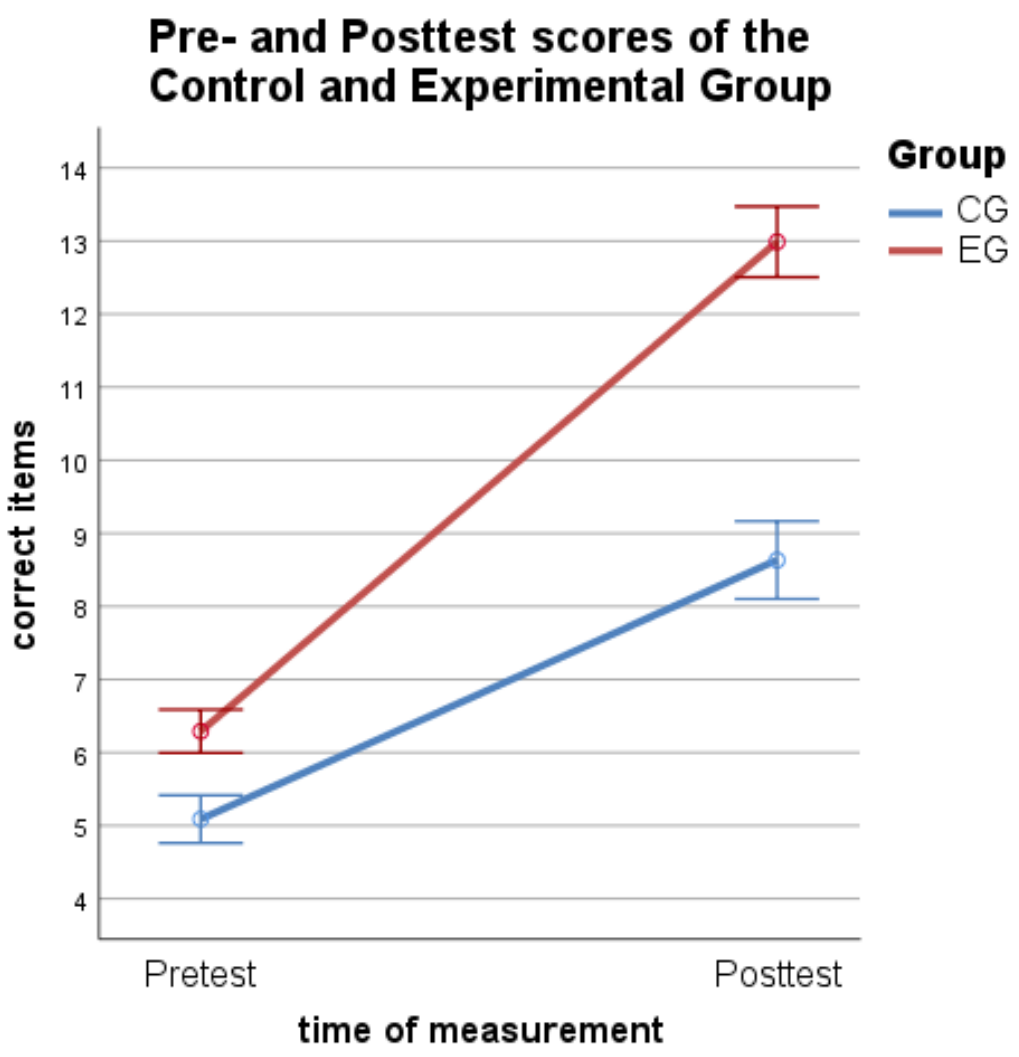

Fig. 4: Pre-and posttest (raw) scores of the control and experimental group with 95\% confidence intervals. The difference in pretest scores, as well as posttest scores, is highly significant ( $p<.001)$. 
One way to compare the net effect of instruction is to compare the difference between the pre- and posttest results of the two groups. On average, the absolute achievement gain by students who were taught according to the new curriculum $(M=6.70, S E=0.26)$ is almost twice as high as that of traditionally taught students $(M=3.54, S E=0.24)$. A mixed ANOVA with time of measurement (pretest, posttest) as a within-subjects factor and group (CG, EG) as between-subjects factor shows that the difference in absolute achievement gain between the EG and CG is highly significant and represents a medium-sized effect $d=.62$ (see chapter 7.4.3).

As the highest achievable score in the test is 26 points, a student with a high pretest result cannot achieve as high an absolute achievement gain as a student with a low pretest result (defined as the difference between the pre- and posttest). An alternative approach to compare the learning progress of students with different pretest results would be to look at the relative achievement gain, which is defined as the absolute achievement gain divided by the highest possible achievement gain. In other words, the relative achievement gain is a measure for the achieved learning progress in relation to the learning progress that could have theoretically been achieved. In the experimental group, the average relative achievement gain $(M=34 \%$, $S E=1 \%)$ is more than twice as high as in the control group $(M=16 \%, S E=1 \%)$. An unequal variances $t$-test shows that the difference between the two groups is highly significant (see chapter 7.4.4). In addition, the relative achievement gain of students in the CG is significantly dependent on their pretest result. A more detailed analysis shows that the higher the pretest result of the students in the CG, the lower is their relative achievement gain. In the EG, however, all students show the same relative achievement gain, regardless of their pretest result (see chapter 7.4.4).

The statistical methods used above are widespread in educational research, but are based on the assumption that the individual measurements are statistically independent of each other. However, this required statistical independence is often not given in educational field research, as the students are not taught independently of each other, but are grouped in different classes. As a result, their learning success depends heavily upon in which class they were taught (see chapter 7.4.7). In the terminology of Multi-Level Analyses (MLA), the students are "nested" in school classes. In order to appropriately account for the hierarchical data structure of the sample and to statistically control for the differences in pretest results between the CG and EG, a multi-level analysis was conducted. Such a multi-level analysis, also called Hierarchical Linear Model (HLM), provides the most adequate estimate of the net effect of the treatment and its statistical uncertainty and includes all significant effects and predictors due to the stepwise development of the model (see chapter 7.5.2). According to the HLM, the net effect of instruction is 3.88 points, which is a highly significant result and corresponds to a large effect of $d=.94$ (see chapter 7.5.3). It can, therefore, be assumed that the new curriculum leads to a significantly greater learning progress than traditional physics lessons. Given the fact that the teachers of the treatment group taught their classes according to the new curriculum for the first time and did not receive any training, this is a remarkable result. 
Thanks to the two-tier structure of the test instrument, it was also possible to evaluate the students' alternative conceptions after instruction by means of a binary logistic multi-level analysis (see chapter 7.5.4). In general, the new curriculum seems to lead to a better conceptual understanding as students of the EG either have a comparable, or significantly lower, probability to hold typical alternative conceptions after instruction than traditionally taught students. A comparison of the students' conceptual understanding of voltage after instruction suggests that the new curriculum leads to a better understanding of voltage than the traditional approaches. On average, more students of the EG could answer at least one of the four items on voltage correctly $(M=48 \%, S E=2 \%)$ than students of the CG $(M=12 \%, S E=2 \%)$, which represents a highly significant difference. The new curriculum particularly appears to lead to a better understanding that voltage, in contrast to current, can only be measured between two points in a circuit because voltage refers to a difference in electric potential. However, based on the answers given to an item on an open circuit, there seems to be no significant difference between the EG and the CG regarding the widespread alternative conception that voltage is seen as a property of the electric current. Here, the majority of students of both groups wrongly concluded that if the current is zero, the voltage had to be zero too across all points in the open circuit - including across the open switch.

In summary, it can be said that the new teaching concept, developed and evaluated in this study, has proven to be a promising approach to teaching electricity in junior high schools as it not only leads to achievement gains of about twice that of traditional physics classes, but also to a significantly better conceptual understanding. In particular, the new curriculum has led to a better understanding of voltage compared to traditional approaches, although the empirical results clearly show that voltage remains a difficult physical quantity for many students. 


\section{Danksagungen}

Abschließend möchte ich mich bei einigen Personen bedanken, die auf unterschiedliche Art und Weise zum Entstehen dieser Arbeit beigetragen bzw. dessen Grundlagen gelegt haben:

- An erster Stelle möchte ich mich von ganzem Herzen bei meinen Eltern Jörg und Beate Burde bedanken, die mich stets uneingeschränkt unterstützten und so erst die Grundlage für meinen bisherigen Werdegang geschaffen haben.

- Meinem Oberstufenphysiklehrer Herrn Andreas Helmbrecht möchte ich dafür danken, dass er mich mit seinem spannenden Unterricht für die Physik begeistert hat.

- Frau Prof. Dr. Rita Wodzinski möchte ich dafür danken, dass sie mein Interesse an der Physikdidaktik geweckt und mir zur richtigen Zeit die richtigen Impulse gegeben hat, dieses weiterzuverfolgen.

- Herrn Prof. Dr. Dr. Hartmut Wiesner möchte ich für seine vielen Anregungen zur Umsetzung des Elektronengasmodells in der Sekundarstufe I insbesondere zu Beginn meiner Promotionszeit danken.

- Herrn Prof. Dr. Thomas Wilhelm bin ich zutiefst dankbar, dass er trotz seiner unzähligen physikdidaktischen Engagements mir in den letzten viereinhalb Jahren stets mit Rat und Tat zur Seite stand und mich in jeder Hinsicht absolut vorbildlich betreut, unterstützt und gefördert hat. Es macht mich froh und stolz, ihn zum Doktorvater zu haben.

- Zu guter Letzt möchte ich mich bei allen Lehrkräften - und natürlich Schülerinnen und Schülern - für ihre Teilnahme an dieser Studie bedanken. Ohne ihr Engagement wäre die Arbeit in dieser Form nicht umsetzbar gewesen. 



\section{Literaturverzeichnis}

Amar-Youcef, S. (2017): Inwiefern eignet sich das Elektronengasmodell als unterstützendes Element bei der Einführung von Stromstärke und Spannung? Ein Unterrichtsversuch im Physik-Erweiterungskurs der Jahrgangsstufe 9. Pädagogische Facharbeit zur Zweiten Staatsprüfung für das Lehramt an Gymnasien. Studienseminar für Gymnasien, Offenbach.

Anderson, C. A. (2007): Belief Perseverance. In: R. F. Baumeister und K. D. Vohs (Hg.): Encyclopedia of social psychology. Thousand Oaks: SAGE, S. 109-110.

Andersson, B.; Karrqvist; C. (1979): EKNA Report No. 2, Elektriska Kretsar [Electric Circuits]. University of Gothenburg. Mölndal, Schweden.

Arnold, M.; Millar, R. (1987): Being constructive: An alternative approach to the teaching of introductory ideas in electricity. In: International Journal of Science Education 9 (5), S. $553-$ 563.

Assis, A.K.T.; Hernandes, J. A. (2013): Elektrischer Strom und Oberflächenladungen. was Wilhelm Weber schon vor mehr als 150 Jahren wußte. Montreal: Apeiron.

Ausubel, D. P. (1968): Educational psychology: A cognitive view. New York: Holt, Rinehart \& Winston.

Backhaus, U. (1987): Der Energietransport durch elektrische Ströme und elektromagnetische Felder. In: Praxis der Naturwissenschaften - Physik 36 (3), S. 30-35.

Bader, M. (2001): Vergleichende Untersuchung eines neuen Lehrganges „Einführung in die mechanische Energie und Wärmelehre“. Dissertation. Universität München, München.

Baser, M. (2006): Fostering conceptual change by cognitive conflict instruction on students' understanding of heat and temperature concepts. In: Eurasia Journal of Mathematics, Science and Technology Education 2 (2), S. 96-144.

Bereiter, C. (2002): Design Research for Sustained Innovation. In: Cognitive Studies 9 (3), S. 321-327.

Berger, R. (2002): Einfluss kontextorientierten Physikunterrichts auf Interesse und Leistung in der Sekundarstufe II. In: Zeitschrift für Didaktik der Naturwissenschaften (8), S. 119-132.

Bierwirth, R. (2014): Untersuchung von Lernproblemen zum elektrischen Potential. TU Braunschweig.

Blumör, R. (1993): Schülerverständnisse und Lernprozesse in der elementaren Optik. Essen: Westarp-Wissenschaften.

Bortz, J.; Döring, N. (2006): Forschungsmethoden und Evaluation für Human- und Sozialwissenschaftler. 4. Auflage. Berlin: Springer.

Brown, D. E. (1992): Teaching electricity with capacitors and causal models. National Association for Research in Science Teaching. Boston, MA, 1992. 
Bruner, J. S. (1986): Actual minds, possible worlds. Cambridge, MA: Harvard University Press.

Bühner, M.; Ziegler, M. (2009): Statistik für Psychologen und Sozialwissenschaftler. München: Pearson.

Burde, J.-P.; Wilhelm, T. (2015): Akzeptanzbefragung zum Elektronengasmodell. Didaktik der Physik - Frühjahrstagung Wuppertal 2015. In: PhyDid-B. Online verfügbar unter http://phydid.physik.fu-berlin.de/index.php/phydid-b/article/view/602/734.

Burde, J.-P.; Wilhelm, T. (2016a): Moment mal... (22) Hilft die Wasserkreislaufanalogie? In: Praxis der Naturwissenschaften - Physik in der Schule 65 (1), S. 46-49.

Burde, J.-P.; Wilhelm, T. (2016b): The electron gas model as an introduction to electricity in middle school science. In: J. Lavonen, K. Juuti, J. Lampiselkä, A. Uitto und K. Hahl (Hg.): Science Education Research: Engaging Learners for a Sustainable Future. Proceedings of ESERA 2015. Helsinki. European Science Education Research Association, S. 26-36.

Burde, J.-P.; Wilhelm, T. (2017a): Die Elektrizitätslehre mit dem Elektronengasmodell. In: Praxis der Naturwissenschaften - Physik in der Schule 65 (8), S. 18-24.

Burde, J.-P.; Wilhelm, T. (2017b): Ergebnisse einer empirischen Studie zum Elektronengasmodell. Didaktik der Physik - Frühjahrstagung Dresden 2017. In: PhyDid-B. Online verfügbar unter http://www.phydid.de/index.php/phydid-b/article/viewFile/760/909.

Burde, J.-P.; Wilhelm, T. (2017c): Modelle in der Elektrizitätslehre. In: Unterricht Physik 28 (157), S. 8-13.

Burde, J.-P.; Wilhelm, T. (2018): Concept and empirical evaluation of a new curriculum to teach electricity with a focus on voltage. Proceedings of the Physics Education Research Conference 2017. Cincinnati (angenommen).

Burde, J.-P.; Wilhelm, T.; Wiesner, H. (2014): Das Elektronengasmodell in der Sekundarstufe I. Didaktik der Physik - Frühjahrstagung Frankfurt 2014. In: PhyDid-B. Online verfügbar unter http://phydid.physik.fu-berlin.de/index.php/phydid-b/article/view/505/652.

Burde, J.-P.; Wilhelm, T.; Wiesner, H. (2015): Das Elektronengasmodell und Möglichkeiten seiner Visualisierung. In: S. Bernholt (Hg.): Heterogenität und Diversität - Vielfalt der Voraussetzungen im naturwissenschaftlichen Unterricht, Bd. 35. Jahrestagung in Bremen 2014. Gesellschaft für Didaktik der Chemie und Physik. Münster: Lit-Verlag, S. 438-440.

Caillot, M. (1985): Problem representations and problem-solving procedures in electricity. In: R. Duit, W. Jung und C. v. Rhöneck (Hg.): Aspects of Understanding Electricity - Proceedings of an International Workshop. IPN-Arbeitsberichte. Kiel: Schmidt \& Klaunig, S. 139151.

Carey, S. (1991): Knowledge acquistion: Enrichment or conceptual change? In: S. Carey und R. Gelman (Hg.): The epigenesis of mind. Hillsdale, NJ: Lawrence Erlbaum Associates Inc, S. 257-291. 
Chabay, R. W.; Sherwood, B. A. (1999): A unified treatment of electrostatics and circuits. Online verfügbar unter http://www.matterandinteractions.org/Content/Articles/circuit.pdf, zuletzt geprüft am 26.09.2013.

Chabay, R. W.; Sherwood, B. A. (2011): Matter \& Interactions. Hoboken N.Y.: Wiley.

Champagne, A. B.; Gunstone; R.F.; Klopfer, L. E. (1985): Instructional consequences of students' knowledge about physical phenomena. In: L.H.T West und A.L Pines (Hg.): Cognitive structure and conceptual change. New York: Academic Press, S. 61-90.

Chan, C.; Burtis, J.; Bereiter, C. (1997): Knowledge building as a mediator of conflict in conceptual change. In: Cognition and Instruction 15 (1), S. 1-40.

Chi, M.T.H. (1992): Conceptual change across ontological categories: Examples from learning and discovery in science. In: F. Giere (Hg.): Cognitive models of science: Minnesota studies in the philosophy of science. Minneapolis: University of Minnesota Press, S. 129-160.

Chinn, C. A.; Brewer, W. F. (1993): The role of anomalous data in knowledge acquistion: A theoretical framework and implications for science instruction. In: Review of Educational Research 63 (1), S. 1-49.

Clement, J. J. (1993): Using Bridging Analogies and Anchoring Intuitions to Deal with Students' Preconceptions in Physics. In: Journal of Research in Science Teaching 30 (10), S. 1241-1257.

Clement, J. J.; Steinberg, M. S. (2002): Step-Wise Evolution of Mental Models of Electric Circuits: A „Learning-Aloud“ Case Study. In: The Journal of The Learning Sciences 11 (4), S. 389-452.

Closset, J.-L. (1983): Le raisonnement séquentiel en électrocinétique. Dissertation, Université Paris VII.

Closset, J.-L. (1984): Woher stammen bestimmte „Fehler“ von Schülern und Studenten aus dem Bereich der Elektrizitätslehre? Kann man sie beheben? In: Der Physikunterricht 18 (2), S. 21-31.

Clough, E. E.; Driver, R. (1986): A study of consistency in the use of students' conceptual frameworks across different task contexts. In: Science Education 70 (4), S. 473-496.

Cohen, J. (1988): Statistical power analysis for the behavioral sciences. 2. Aufl. Hillsdale, NJ: Lawrence Erlbaum Associates.

Cohen, R.; Eylon, B.; Ganiel, M. (1983): Potential difference and current in simple electric circuits: A study of students' concepts. In: American Journal of Physics 51 (5), S. 407-412.

Cronbach, L. J. (1957): The two disciplines of scientific psychology. In: American Psychologist 12, S. 671-684.

Design-Based Research Collective (2003): Design-Based Research: An Emerging Paradigm for Educational Inquiry. In: Educational Researcher 32 (1), S. 5-8. 


\section{Literaturverzeichnis}

diSessa, A. A. (1988): Knowledge in Pieces. In: G. Forman und P. Pufall (Hg.): Constructivism in the Computer Age. Hillsdale, NJ: Lawrence Erlbaum Publishers, S. 49-70.

diSessa, A. A. (1993): Toward an Epistemology of Physics. In: Cognition and Instruction 10 (2-3), S. 105-225.

diSessa, A. A. (2013): A bird's-eye view of the "pieces" vs. "coherence" controversy (from the "pieces" side of the fence). In: S. Vosniadou (Hg.): International handbook of Research on Conceptual Change. 2. Aufl. New York: Routledge, S. 31-48.

Dresing, T.; Pehl, T. (2015): Praxisbuch Interview, Transkription \& Analyse. Anleitungen und Regelsysteme für qualitativ Forschende. 6. Auflage. Marburg. Online verfügbar unter www.audiotranskription.de/praxisbuch, zuletzt geprüft am 20.04.2016.

Dreyfus, A.; Jungwirth, E.; Eliovitch, R. (1990): Applying the "Cognitive Conflict” Strategy for Conceptual Change - Some Implications, and Problems. In: Science Education 74 (5), S. 555-569.

Driver, R.; Easley; J. (1978): Pupils and paradigms: A review of the literature related to concept development in adolescent science students. In: Studies in Science Education 5 (1), S. 61-84.

Driver, R.; Guesne, E.; Tiberghien, A. (1985): Children's Ideas in Science. Milton Keynes: Open University Press.

Dudeck, W.-G.; Schwedes, H. (1990): Der elektrische Stromkreis als System - Konzepterwerb mit Hilfe von Wasserstromkreisen in der Sek. I. In: W. Kuhn (Hg.): Didaktik der Physik / Vorträge Physikertagung Gießen. Gießen-Wieseck: Gahmig Druck, S. 456-461.

Dudeck, W.-G.; Schwedes, H. (1992): Und sie lernen doch, was Spannung ist! Fallbeispiele aus einem analogieorientierten Physikunterricht der Sek I. In: H. Behrendt (Hg.): Zur Didaktik der Physik und Chemie - Probleme und Perspektiven. Erfurt. Gesellschaft für Didaktik der Chemie und Physik, S. 253-255.

Duit, R. (1983): Strom - ein Wort sowohl der Alltagssprache als auch der Fachsprache der Physik. In: Naturwissenschaften im Unterricht. Physik/Chemie 10 (31), S. 344-347.

Duit, R. (1986): Energievorstellungen. In: Naturwissenschaften im Unterricht. Physik/Chemie 34 (13), S. 7-9.

Duit, R. (1993a): Alltagsvorstellungen berücksichtigen! In: Praxis der NaturwissenschaftenPhysik in der Schule 42 (6), S. 7-11.

Duit, R. (1993b): Schülervorstellungen - von Lerndefiziten zu neuen Unterrichtsansätzen. In: Naturwissenschaften im Unterricht - Physik 4 (16), S. 4-10.

Duit, R. (1994): An Schülervorstellungen anknüpfend Physik lehren und lernen. In: Naturwissenschaften im Unterricht - Physik 5 (22), S. 4-6. 
Duit, R. (1995a): Vorstellungen und Lernen von Physik und Chemie. Zu den Ursachen vieler Lernschwierigkeiten. In: PLUS LUCIS (2), S. 11-18.

Duit, R. (1995b): Zur Rolle der konstruktivistischen Sichtweise in der naturwissenschaftsdidaktischen Lehr- und Lernforschung. In: Zeitschrift für Pädagogik 41 (6), S. 905-923.

Duit, R. (2009a): Alltagsvorstellungen und Physik lernen. In: R. Girwidz, P. Häußler und E. Kircher (Hg.): Physikdidaktik. Theorie und Praxis. Berlin, Heidelberg: Springer, S. 605-630.

Duit, R. (2009b): Bibliography - STCSE: Students' and Teachers' Conceptions and Science Education. IPN - Leibniz-Institut für die Pädagogik der Naturwissenschaften. Kiel. Online verfügbar unter http://archiv.ipn.uni-kiel.de/stcse/, zuletzt aktualisiert am 2009, zuletzt geprüft am 10.02.2017.

Duit, R. (2009c): Elektrizitätslehre aus Schülersicht - Schülervorstellungen und Lernschwierigkeiten im Bereich der Elektrizitätslehre. Sammelband Elektrizitätslehre. In: Naturwissenschaften im Unterricht - Physik, S. 4-9.

Duit, R.; Glynn, S. (1995): Analogien - Brücken zum Verständnis. In: Naturwissenschaften im Unterricht - Physik 27 (6), S. 4-10.

Duit, R.; Jung, W.; Rhöneck, C. v. (Hg.) (1985): Aspects of Understanding Electricity - Proceedings of an International Workshop. IPN-Arbeitsberichte. Kiel: Schmidt \& Klaunig.

Duit, R.; Treagust, D.; Widodo, A. (2008): Teaching science for conceptual change: Theory and practice. In: S. Vosniadou (Hg.): International Handbook of Research on Conceptual Change. London: Routledge, S. 629-646.

Duit, R.; Treagust, D. F. (1998): Learning in science - From behaviourism towards social constructivism and beyond. In: Fraser, B. J. und K. G. Tobin (Hg.): International Handbook of Science Education. Dordrecht: Kluwer Academic, S. 3-26.

Duit, R.; Treagust, D. F. (2003): Conceptual change: a powerful framework for improving science teaching and learning. In: International Journal of Science Education 25 (6), S. 671688 .

Dupin, J.-J.; Johsua, S. (1987): Conceptions of French pupils concerning electric circuits: Structure and evolution. In: Journal of Research in Science Teaching 24 (9), S. 791-806.

Engelhardt, P. V.; Beichner, R. J. (2004): Students' understanding of direct current resistive electrical circuits. In: American Journal of Physics 72 (1), S. 98-115.

Evans, J. (1978): Teaching electricity with batteries and bulbs. In: The Physics Teacher 16 (1), S. 15-22.

Field, A. (2011): Discovering Statistics using SPSS. (and sex and drugs and rock 'n' roll). 3. Aufl. London: SAGE. 
Fleiss, J. L.; Cohen, J. (1973): The equivalence of weighted kappa and the intraclass correlation coefficient as measures of reliability. In: Educational and Psychological Measurement 33, S. 613-619.

Fredette, N. H.; Lochhead, J. (1980): Student conceptions of simple circuits. In: The Physics Teacher 18, S. 194-198.

Galili, I.; Goihbarg, E. (2005): Energy transfer in electrical circuits: A qualitative account. In: American Journal of Physics 73 (2).

Gauld, C. (1986): Models, meters and memory. In: Research in Science Education 16 (1), S. 49-54.

Gentner, D. (1989): The Mechanisms of Analogical Learning. In: S. Vosniadou und A. Ortony (Hg.): Similarity and Analogical Reasoning. Cambridge: Cambridge University Press, S. 199-244.

Gentner, D.; Gentner, D. R. (1983): Flowing Waters or Teeming Crowds: Mental Models of Electricity. In: D. Gentner und A. L. Stevens (Hg.): Mental Models. Hillsdale, NJ: Lawrence Erlbaum Associates Inc, S. 99-129.

Gerstenmaier, J.; Mandl, H. (1995): Wissenserwerb unter konstruktivistischer Perspektive. In: Zeitschrift für Pädagogik 41 (6), S. 867-888.

Giancoli, D. C. (2006): Physik. 3. aktualisierte Auflage. München: Pearson.

Girwidz, R. (1995): Die Stromzange - neue experimentelle Möglichkeiten für den Physikunterricht. In: PLUS LUCIS (1), S. 12-17. Online verfügbar unter http://pluslucis.univie.ac.at/PlusLucis/951/stromzan.pdf, zuletzt geprüft am 10.04.2014.

Glasersfeld, E. von (1993): Questions and answers about radical constructivism. In: K. G. Tobin (Hg.): The practice of constructivism in science education. Washington: AAAS Press, S. 23-38.

Gleixner, C. (1998): Einleuchtende Elektrizitätslehre mit Potenzial. Dissertation. LMU München.

Goldstein, H. (2011): Multilevel Statistical Models. 4. Aufl. Chichester: Wiley.

Gott, R. (1985): Predicting and explaining the operation of simple DC circuits. In: R. Duit, W. Jung und C. v. Rhöneck (Hg.): Aspects of Understanding Electricity - Proceedings of an International Workshop. IPN-Arbeitsberichte. Kiel: Schmidt \& Klaunig, S. 63-72.

Grob, K.; Rhöneck, C.v.; Völker, B.; Wettern, K. (1988): Die Gravitationsanalogie zur Einführung des Spannungsbegriffs. In: Naturwissenschaften im Unterricht. Physik/Chemie 36 (31), S. 14-19.

Grosslight, L.; Unger, C.; Jay, E.; Smith, C. L. (1991): Understanding models and their use in science. Conceptions of middle and high school students and experts. In: Journal of Research in Science Teaching 28 (9), S. 799-822. DOI: 10.1002/tea.3660280907. 
Haagen-Schützenhöfer, C. (2016): Lehr-und Lernprozesse im Anfangsoptikunterricht der Sekundarstufe I. Habilitationsschrift. Universität Wien, Wien. Online verfügbar unter https://online.unigraz.at/kfu_online/wbFPCompsCallBacks.cbExecuteDownload?pDocStoreNr=3644316.

Halliday, D.; Resnick, R.; Walker, J. (2001): Halliday Physik - Bachelor Edition. Weinheim: Wiley-VCH Verlag.

Harlen, W. (1999): Effective teaching of science: A review of research. Edinburgh: Scottish Council for Research in Education.

Härtel, H. (2012a): Der alles andere als einfache elektrische Stromkreis. In: Praxis der Naturwissenschaften - Physik in der Schule 61 (5), S. 17-24.

Härtel, H. (2012b): Spannung und Oberflächenladungen. Was Wilhelm Weber schon vor mehr als 150 Jahren wusste. In: Praxis der Naturwissenschaften - Physik in der Schule 61 (5), S. 25-31.

Härtel, H.; Duit, R.; Lauterbach, R.; Mie, K.; Mikelskis; H. et al. (1981): IPN Curriculum Physik - Unterrichtseinheiten für das 7. und 8. Schuljahr. Unterrichtseinheit 2: Der elektrische Stromkreis als System. Stuttgart: Klett.

Hartig, J.; Rakoczy, K. (2010): Mehrebenenanalyse - Multilevel Analysis. In: H. Holling und B. Schmitz (Hg.): Handbuch der Psychologie: Handbuch Statistik, Methoden und Evaluation. Göttingen: Hogrefe, S. 538-547.

Hartmann, S. (2004): Erklärungsvielfalt. Berlin: Logos (Studien zum Physiklernen, 37).

Hattie, J. (2013): Lernen sichtbar machen. Baltmannsweiler: Schneider Verlag Hohengehren. Häußler, P.; Bünder, W.; Duit, R.; Gräber, W.; Mayer, J. (1998): Naturwissenschaftliche Forschung: Perspektiven für die Unterrichtspraxis. Kiel: IPN.

Häußler, P.; Hoffmann, L. (1995): Physikunterricht - an den Interessen von Mädchen und Jungen orientiert. In: Unterrichtswissenschaft 23 (2), S. 107-126.

Heller, P. M.; Finley, F. N. (1992): Variable uses of alternative conceptions: A case study in current electricity. In: Journal of Research in Science Teaching 29 (3), S. 259-275.

Herdt, D. (1989): Einführung in die elementare Optik. Vergleichende Untersuchung eines neuen Lehrgangs. Essen: Westarp.

Herrmann, F. (1995): Der Karlsruher Physikkurs. In: Praxis der Naturwissenschaften - Physik in der Schule (5), S. 41-47.

Herrmann, F. (1998): Mengenartige Größen im Physikunterricht. In: Physikalische Blätter 54 (9), S. 830-833.

Herrmann, F. (2003): Der Karlsruher Physikkurs: Ein Lehrbuch für die Sekundarstufe I. Teil 2: Daten, Elektrizität, Licht. 6. durchgesehene Auflage. Köln: Aulis-Verl. Deubner. 
Herrmann, F.; Laukenmann, M.; Mingirulli, L.; Morawietz, P.; Schmälzle, P. (2014a): Der Karlsruher Physikkurs für die Sekundarstufe I. Band 1: Energie - Impuls - Entropie. Universität Karlsruhe. Karlsruhe. Online verfügbar unter http://www.physikdidaktik.unikarlsruhe.de/dl-counter/download/Sek_I_Band_1.pdf, zuletzt geprüft am 06.12.2016.

Herrmann, F.; Laukenmann, M.; Mingirulli, L.; Morawietz, P.; Schmälzle, P. (2014b): Der Karlsruher Physikkurs für die Sekundarstufe I. Band 2: Daten - Elektrizität - Licht. Universität Karlsruhe. Karlsruhe. Online verfügbar unter http://www.physikdidaktik.unikarlsruhe.de/dl-counter/download/Sek_I_Band_2.pdf, zuletzt geprüft am 05.12.2016.

Herrmann, F.; Schmälzle, P. (1984): Das elektrische Potential im Unterricht der Sekundarstufe I. In: Der mathematische und naturwissenschaftliche Unterricht 37 (8), S. 476-482.

Hesse, M. (1963): Models and Analogies in Science. London: Clowes.

Hestenes, D.; Halloun, I. (1995): Interpreting the Force Concept Inventory. In: The Physics Teacher 33, S. 502-506.

Hopf, M.; Wiesner, H. (2007): Paradigmen für physikdidaktische Forschung - ein Rück- und Ausblick: Physikdidaktik und Design-Based Research. In: S. Kolling (Hg.): Beiträge zur Experimentalphysik, Didaktik und computergestützten Physik. Festschrift zum 70. Geburtstag von Prof. Dr. Hans-Josef Pratt. Berlin: Logos, S. 37-57.

Hox, J. J. (2010): Multilevel Analysis. Techniques and Applications. Second Edition. New York: Routledge.

Jahn, D. (2014): Durch das praktische Gestalten von didaktischen Designs nützliche Erkenntnisse gewinnen: Eine Einführung in die Gestaltungsforschung. In: Wirtschaft \& Erziehung 66 (1), S. 3-15.

Jochmann, E.; Hermes, O.; Spies, P. (1914): Grundriss der Experimentalphysik und Elemente der Chemie sowie der Astronomie und mathematischen Geographie. Berlin: Winkelmann \& Söhne.

Jung, W. (1973): Fachliche Zulässigkeit aus didaktischer Sicht. Kiel: IPN Seminar II.

Jung, W. (1979): Aufsätze zur Didaktik der Physik und Wissenschaftstheorie. Frankfurt a. M.: Diesterweg.

Jung, W. (1980): Mechanik für die Sekundarstufe I. Frankfurt a. M.: Diesterweg.

Jung, W. (1981): Beiträge zur Methodik und Didaktik der Physik. Frankfurt am Main: Diesterweg.

Jung, W. (1986): Alltagsvorstellungen und das Lernen von Physik und Chemie. In: Naturwissenschaften im Unterricht. Physik/Chemie 34 (13), S. 2-6.

Jung, W. (1992): Probing acceptance: A technique for investigating learning difficulties. In: R. Duit, F. Goldberg und H. Niedderer (Hg.): Research in Physics Learning. Theoretical Is- 
sues and Empirical Studies. Proceedings of an International Workshop at the University of Bremen. Kiel: IPN, S. 278-295.

Kattmann, U. (2008): Learning biology by means of anthropomorphic conceptions? In: M. Hammann, M. Reiss, C. Boulter und S. Tunicliffe (Hg.): Biology in context: Learning and teaching for the 21st century. London: Institute of Education, S. 21-26.

Kersten, E. (1990): Systematisches Arbeiten mit Alltagskenntnissen - eine Notwendigkeit bei der Behandlung physikalischer Begriffe und Gesetze. In: Physik in der Schule 28, S. 135-142. Kircher, E. (1975): IPN Curriculum Physik für das 9. Und 10. Schuljahr. Modelle des elektrischen Stromkreises. Stuttgart: Klett Verlag.

Kircher, E. (1984): Analogmodelle für den elektrischen Stromkreis. In: Der Physikunterricht 18 (2), S. 46-61.

Kircher, E. (2015a): Elementarisierung und didaktische Rekonstruktion. In: E. Kircher, P. Häußler und R. Girwidz (Hg.): Physikdidaktik. Theorie und Praxis. 3. Aufl. Berlin: Springer, S. 107-139.

Kircher, E. (2015b): Modellbegriff und Modellbildung in der Physikdidaktik. In: E. Kircher, P. Häußler und R. Girwidz (Hg.): Physikdidaktik. Theorie und Praxis. 3. Aufl. Berlin: Springer, S. 783-807.

Kircher, E.; Duit, R. (1975): Modelle des elektrischen Stromkreises in der Sekundarstufe I das Wassermodell und das Elektronenmodell. In: Der Physikunterricht 9 (4), S. 17-42.

Kircher, E.; Girwidz, R.; Häußler, P. (2001): Physikdidaktik. Eine Einführung. 2., aktualisierte Aufl. Berlin, Heidelberg, New York, Barcelona, Hongkong, London, Mailand, Paris, Singapur, Tokio: Springer (Springer-Lehrbuch).

Kircher, E.; Rhöneck, C. v. (1975): Einige Bemerkungen zum fachdidaktischen Aspekt des Modellbegriffs. In: Der Physikunterricht 9 (4), S. 13-16.

Klahr, D.; Nigam, M. (2004): The equivalence of learning paths in early science instruction: effect of direct instruction and discovery learning. In: Psychological Science 15 (10), S. 661667.

Koller, D. (2008a): Einführung in die Elektrizitätslehre. Lehrer-Version. LMU München. München. Online verfügbar unter http://www.didaktik.physik.unimuenchen.de/archiv/inhalt_materialien/einf_elektrizitaet/konzept.zip, zuletzt geprüft am 07.12.2016.

Koller, D. (2008b): Entwurf und Erprobung eines Unterrichtskonzepts zur Einführung in die Elektrizitätslehre. Zulassungsarbeit. LMU München, zuletzt geprüft am 20.01.2014.

Koller, D.; Waltner, C.; Wiesner, H. (2008): Zur Einführung von Stromstärke und Spannung. In: Praxis der Naturwissenschaften - Physik in der Schule 57 (6), S. 6-18.

Kreft, I.; Leeuw, J. (1998): Introducing Multilevel Modeling. London: SAGE. 


\section{Literaturverzeichnis}

Kuhn, J. (2010): Authentische Aufgaben im theoretischen Rahmen von Instruktions- und Lehr-Lern-Forschung: Effektivität und Optimierung von Ankermedien für eine neue Aufgabenkultur im Physikunterricht. Wiesbaden: Vieweg.

Kuhn, J. (2014): Mehrebenenanalyse am Beispiel der Lernwirkung von Aufgaben. In: D. Krüger, I. Parchmann und H. Schecker (Hg.): Methoden in der naturwissenschaftsdidaktischen Forschung. Berlin: Springer Spektrum, S. 297-310.

Kuhn, T. S. (1962): The structure of scientific revolutions. Chicago: University of Chicago Press.

Kuhn, W. (1994): Analogien in historischer, methodologischer und didaktischer Sicht. In: Praxis der Naturwissenschaften - Physik in der Schule 43 (5), S. 2-16.

Labudde, P. (2001): Chancen für den Physikunterricht in der heutigen Zeit. Zehn Thesen zur physikalischen Bildung. In: PLUS LUCIS (2), S. 2-6.

Leisner, A. (2005): Entwicklung von Modellkompetenz im Physikunterricht. Eine Evaluationsstudie in der Sekundarstufe I. Berlin: Logos Verlag.

Licht, P.; Thijs; G.D. (1990): Method to trace coherence and persistence of preconceptions. In: International Journal of Science Education 12 (4), S. 403-416.

Limón, M.; Carretero, M. (1999): Conflicting data and conceptual change in history experts. In: W. Schnotz, S. Vosniadou und M. Carretero (Hg.): New perspectives on conceptual change. Oxford: Pergamon, S. 137-159.

Linn, M. C. (2006): The Knowledge Integration Perspective on Learning and Instruction. In: R. K. Sawyer (Hg.): Cambridge handbook of the learning sciences. Cambridge, UK: Cambrdige University Press, S. 243-264.

Lubben, F.; Bennett, J.; Hogarth, S.; Robinson, A. (2005): A systematic review of the effects of context-based and Science-Technology-Society (STS) approaches in the teaching of secondary science on boys and girls, and on lower-ability pupils. Review. University of London, London. Institute of Education.

Maas, C.J.M; Hox, J. J. (2005): Sufficient Sample Sizes for Multilevel Modeling. In: Methodology 1 (3), S. 86-92.

Maichle, U. (1982): Schülervorstellungen zu Stromstärke und Spannung. In: Naturwissenschaften im Unterricht. Physik/Chemie 30 (11), S. 383-387.

Mandl, H.; Gruber, H.; Renkl, A. (1993): Misconceptions and knowledge compartmentalization. In: G. Strube und K. F. Wender (Hg.): The cognitive psychology of knowledge. Amsterdam: Elsevier, S. 161-176.

Manthei, W. (1992): Das Analogische im Physikunterricht. Analoga, Analogien, Analogisches stärker in den Mittelpunkt des Unterrichts rücken. In: Physik in der Schule 30 (7), S. 250-256. 
Mason, L. (2001): Responses to anomalous data on controversial topics and theory change. In: Learning and Instruction 11, S. 453-483.

Mayer, R. E. (2004): Should there be a three-strike rule against pure discovery learning? The Case for Guided Methods of Instruction. In: American Psychologist 59 (1), S. 14-19.

Mayring, P. (2010): Qualitative Inhaltsanalyse: Grundlagen und Techniken. 11. vollständig überarbeitete Auflage. Weinheim und Basel: Beltz.

McCloskey, M. (1983): Naive theories of motion. In: D. Gentner und A. L. Stevens (Hg.): Mental Models. Hillsdale, NJ: Lawrence Erlbaum Associates Inc, S. 299-323.

McDermott, L. C.; Shaffer, P. S. (1992): Research as a guide for curriculum development: An example from introductory electricity. Part I: Investigation of student understanding. In: American Journal of Physics 60 (11), S. 994-1013.

McDermott, L. C.; van Zee, E. H. (1985): Identifying and Addressing Student Difficulties with Electric Circuits. In: R. Duit, W. Jung und C. v. Rhöneck (Hg.): Aspects of Understanding Electricity - Proceedings of an International Workshop. IPN-Arbeitsberichte. Kiel: Schmidt \& Klaunig, S. 39-48.

Meschede, D. (2006): Gerthsen Physik. 23. überarbeitete Auflage. Berlin: Springer.

Mikelskis-Seifert, S. (2002): Die Entwicklung von Metakonzepten zur Teilchenvorstellung bei Schülern. Untersuchung eines Unterrichts über Modelle mit Hilfe eines Systems multipler Repräsentationsebenen. Dissertation. Frei Universität Berlin.

Mikelskis-Seifert, S.; Kasper, L. (2011): Modellieren in der Physik, im Alltag und im Unterricht. In: Naturwissenschaften im Unterricht - Physik 122, S. 4-12.

Mikelskis-Seifert, S.; Leisner, A. (2003): Anbahnung eines Modellbewusstseins während einer fächerübergreifenden Projektwoche. In: CD zur Frühjahrstagung des Fachverbandes Didaktik der Physik der Deutschen Physikalischen Gesellschaft in Augsburg.

Mikelskis-Seifert, S.; Thiele, M.; Wünscher, T. (2005): Modellieren - Schlüsselfähigkeit für physikalische Forschungs- und Lernprozesse. In: PhyDid - Physik und Didaktik in Schule und Hochschule 4 (1), S. 30-46.

Miller, G. A.; Chapman, J. P. (2001): Misunderstanding Analysis of Covariance. In: Journal of Abnormal Psychology 110 (1), S. 40-48.

Minstrell, J. (1982): Explaining the ''at rest'" condition of an object. In: The Physics Teacher 20 (10), S. 10-14.

Minstrell, J. (1992): Facets of students' knowledge and relevant instruction. In: R. Duit, F.

Goldberg und H. Niedderer (Hg.): Research in physics learning: Theoretical issues and empirical studies. Kiel: Institut für die Pädagogik der Naturwissenschaften, S. 110-128.

Muckenfuß, H. (1982): Vorschläge zur Einführung der Begriffe Strom, Spannung und Widerstand. In: Naturwissenschaften im Unterricht. Physik/Chemie 30 (11), S. 399-406. 


\section{Literaturverzeichnis}

Muckenfuß, H. (1995): Lernen im sinnstiftenden Kontext. Entwurf einer zeitgemäßen Didaktik des Physikunterrichts. Berlin: Cornelsen.

Muckenfuß, H.; Walz, A. (1992): Neue Wege im Elektrikunterricht. Köln: Aulis Deubner.

Muckenfuß, H.; Walz, A. (1997): Neue Wege im Elektrikunterricht. Zweite berarbeitete Auflage. Köln: Aulis Deubner.

Müller, R. (2012): Was ist Spannung? In: Praxis der Naturwissenschaften - Physik in der Schule 61 (5), S. 8-16.

Nachtigall, D. (1992): Zum Verstehen von Physik im Unterricht. In: Physik in der Schule 30 (1), S. 10-13.

Niedderer, H.; Goldberg, F. (1995): Lernprozesse beim elektrischen Stromkreis. In: Zeitschrift für die Didaktik der Naturwissenschaften 1 (1), S. 73-86.

Niedderer, H.; Schecker, H. (1992): Towards an explicit description of cognitive systems for research in physics learning. In: R. Duit, F. Goldberg und H. Niedderer (Hg.): Research in Physics Learning - Theoretical Issues and Empirical Studies. Proceedings of an International Workshop in Bremen, S. 74-98.

Ohm, G. S. (1827): Die galvanische Kette, mathematisch bearbeitet. Berlin: Riemann.

Osborne, R. (1980): Some aspects of the students' view of the world. In: Research in Science Education 10 (1), S. 11-18.

Osborne, R. (1981): Children's ideas about electric current. In: New Zealand Science Teacher 29, S. 12-19.

Osborne, R. (1983): Towards modifying children's ideas about electric current. In: Research in Science and Technology Education 1, S. 73-82.

Paccagnella, O. (2006): Centering or not Centering in Multilevel Models? The Role of the Group Mean and the Assessment of Group Effects. In: Evaluation Review 30 (1), S. 66-85.

Perkins, D. N.; Simmons, R. (1988): Patterns of misunderstanding: An integrative model of science, math, and programming. In: Review of Educational Research 58 (3), S. 303-326.

Peşman, H.; Eryılmaz, A. (2010): Development of a Three-Tier Test to Assess Misconceptions About Simple Electric Circuits. In: The Journal of Educational Research 103 (3), S. 208-222.

Piaget, J. (1954): The Construction of Reality in the Child. New York: Basic Books.

Piaget, J. (1967): Six psychological studies. New York: Random House.

Pintrich, P. R.; Marx, R. W.; Boyle, R. A. (1993): Beyond Cold Conceptual Change: The Role of Motivational Beliefs and Classroom Contextual Factors in the Process of Conceptual Change. In: Review of Educational Research 63 (2), S. 167-199. 
Posner, G. J.; Strike, K. A.; Hewson, P. W.; Gertzog, W. A. (1982): Accommodation of a scientific conception: Toward a theory of conceptual change. In: Science Education 66 (2), S. 211-227.

Prediger, S.; Link, M. (2012): Fachdidaktische Entwicklungsforschung - Ein lernprozessfokussierendes Forschungsprogramm mit Verschränkung fachdidaktischer Arbeitsbereiche. In: H. Bayrhuber, U. Harms, B. Muszynski, B. Ralle, M. Rothgangel, L.-H. Schön et al. (Hg.): Formate Fachdidaktischer Forschung. Empirische Projekte - historische Analysen - theoretische Grundlegungen. Fachdidaktische Forschungen, Bd. 2. Münster: Waxmann, S. 29-46. Preece, P.F.W. (1984): Intuitive science: Learned or triggered? In: European Journal of Science Education 6 (1), S. 7-10.

Rasch, B.; Friese, M.; Hofmann, W.; Naumann, E. (2010): Quantitative Methoden. Einführung in die Statistik für Psychologen und Sozialwissenschaftler. 3., erweiterte Auflage. Berlin: Springer (2).

Rasch, B.; Friese, M.; Hofmann, W.; Naumann, E. (2014): Quantitative Methoden 2. Einführung in die Statistik für Psychologen und Sozialwissenschaftler. 4. Aufl. Berlin: Springer.

Raudenbush, S. W.; Bryk, A. S. (2002): Hierarchical Linear Models. Applications and Data Analysis Methods. Thousand Oaks: SAGE.

Reich, K. (2001): Konstruktivistische Ansätze in den Sozial- und Kulturwissenschaften. In: T. Hug (Hg.): Die Wissenschaft und ihr Wissen, Bd. 4. Baltmannsweiler: Schneider, S. 356-376. Reinmann, G. (2005): Innovation ohne Forschung? Ein Plädoyer für den Design-Based Research Ansatz. In: Unterrichtswissenschaft 33 (1), S. 52-69.

Renkl, A. (1996): Träges Wissen: Wenn Erlerntes nicht genutzt wird. In: Psychologische Rundschau 47 (2), S. 78-92.

Rhöneck, C. v. (1980): Schüleräußerungen zum Begriff der elektrischen Spannung beim Erklären realer Experimente. In: Der Physikunterricht 14 (4), S. 16-29.

Rhöneck, C. v. (1981a): Schüleräußerungen zum Begriff der elektrischen Spannung beim Erklären der Meßwerte am Schalter. In: Naturwissenschaften im Unterricht. Physik/Chemie 29 (6), S. 210-215.

Rhöneck, C. v. (1981b): Students' conceptions of the electric circuit before physics instruction. In: W. Jung, H. Pfundt und C. v. Rhöneck (Hg.): International Workshop on Problems concerning students' representation of physics and chemistry knowledge. PH Ludwigsburg, S. 194-213.

Rhöneck, C. v. (1986): Vorstellungen vom elektrischen Stromkreis und zu den Begriffen Strom, Spannung und Widerstand. In: Naturwissenschaften im Unterricht - Physik 34 (13), S. $10-14$.

Rhöneck, C. v. (1988): Wege zum Spannungsbegriff. In: Naturwissenschaften im Unterricht. Physik/Chemie 36 (31), S. 4-11. 
Rhöneck, C. v.; Grob, K. (1989): Schülervorstellungen im Zusammenhang mit dem elektrischen Widerstand. In: Naturwissenschaften im Unterricht. Physik/Chemie 37 (10), S. 16-20. Rhöneck, C. v.; Völker, B. (1982): Einfache Elektrizitätslehre zwischen physikalischem Anspruch und Lernschwierigkeiten. In: Naturwissenschaften im Unterricht. Physik/Chemie 30 (11), S. 406-412.

Rhöneck, C. v.; Völker, B. (1984): Vorstellungen vom Stromkreis und ihr Einfluss auf den Lernprozess. In: Der Physikunterricht 18 (2), S. 4-16.

Rhöneck, C. v.; Völker, B. (1985): Semantic Structures Describing the Electric Circuit Before and After Instruction. In: R. Duit, W. Jung und C. v. Rhöneck (Hg.): Aspects of Understanding Electricity - Proceedings of an International Workshop. IPN-Arbeitsberichte. Kiel: Schmidt \& Klaunig, S. 95-113.

Rosser, W.G.V. (1970): Magnitudes of Surface Charge Distributions Associated with Electric Current Flow. In: American Journal of Physics 38 (2), S. 265-266.

Schecker, H. (1985): Das Schülervorverständnis zur Mechanik. Eine Untersuchung in der Sekundarstufe II unter Einbeziehung historischer und wissenschaftlicher Aspekte. Dissertation. Universität Bremen, Bremen.

Schecker, H. (2014): Überprüfung der Konsistenz von Itemgruppen mit Cronbachs $\alpha$. Springer Verlag. Online verfügbar unter http://www.springer.com/cda/content/document/cda_downloaddocument/Cronbach+Alpha.pd f?SGWID=0-0-45-1426184-p175274210.

Schlichting, H. J. (1991): Zwischen common sense und physikalischer Theorie - wissenschaftstheoretische Probleme beim Physiklernen. In: Der mathematische und naturwissenschaftliche Unterricht 44 (2), S. 74-80.

Schumacher, M.; Wiesner, H. (1997): Erprobung des Potentialansatzes in der Elektrizitätslehre in Form einer Akzeptanzbefragungssequenz. In: Deutsche Physikalische Gesellschaft - Tagung 1996, S. 573-578.

Schwedes, H.; Dudeck, W.-G. (1993a): Lernen mit der Wasseranalogie. Eine Einführung in die elementare Elektrizitätslehre. In: Naturwissenschaften im Unterricht - Physik 4 (16), S. $16-23$.

Schwedes, H.; Dudeck, W.-G. (1993b): Wasserkreisel und die Doppelwassersäule - Ein Modell für verzweigte elektrische Stromkreise. In: Praxis der Naturwissenschaften - Physik in der Schule 42 (6), S. 12-17.

Schwedes, H.; Dudeck, W.-G.; Seibel, C. (1995): Elektrizitätslehre mit Wassermodellen. In: Praxis der Naturwissenschaften - Physik in der Schule 44 (2), S. 28-36.

Schwedes, H.; Schilling, P. (1983): Schülervorstellungen zu Wasserstromkreisen. In: Physica Didactica 10, S. 159-170. 
Schwedes, H.; Schilling, P. (1984): Wasser und Strom - Eine spielorientierte Unterrichtseinheit zum Begriff der elektrischen Stromstärke. In: Naturwissenschaften im Unterricht. Physik/Chemie (8), S. 263-273.

Séré, M.-G. (2004): Der gasförmige Zustand. In: R. Müller, R. Wodzinski und M. Hopf (Hg.): Schülervorstellungen zur Physik. Köln: Aulis Verlag Deubner, S. 199-214.

Shipstone, D. M. (1984): A study of children's understanding of electricity in simple D.C. Circuits. In: European Journal of Science Education 6 (2), S. 185-198.

Shipstone, D. M. (1985): On children's use of conceptual models in reasoning about current electricity. In: R. Duit, W. Jung und C. v. Rhöneck (Hg.): Aspects of Understanding Electricity - Proceedings of an International Workshop. IPN-Arbeitsberichte, Bd. 59. Kiel: Schmidt \& Klaunig, S. 73-82.

Shipstone, D. M.; Gunstone, R. F. (1985): Teaching children to discriminate between current and energy. In: R. Duit, W. Jung und C. v. Rhöneck (Hg.): Aspects of Understanding Electricity - Proceedings of an International Workshop. IPN-Arbeitsberichte. Kiel: Schmidt \& Klaunig, S. 287-297.

Shipstone, D. M.; Rhöneck, C. v.; Jung, W.; Kärrqvist, C.; Dupin, J.-J.; Johsua, S.; Licht, P. (1988): A study of secondary students' understanding of electricity in five European countries. In: International Journal of Science Education 10 (3), S. 303-316.

Snijders, T.A.B. (2005): Power and Sample Size in Multilevel Linear Models. In: B.S Everitt und D. C. Howell (Hg.): Encyclopedia of Statistics in Behavioral Science, Bd. 3. 4 Bände. Hoboken N.Y.: Wiley, S. 1570-1573.

Solomon, J. (1983): Learning about energy: how pupils think in two domains. In: European Journal of Science Education 5 (1), S. 49-59.

Späth, S. (2009): Überarbeitung und empirische Untersuchung eines Unterrichtskonzepts zur Einführung in die Elektrizitätslehre. Zulassungsarbeit. Universität München, München.

Stachowiak, H. (1973): Allgemeine Modelltheorie. Berlin: Springer.

Stachowiak, H. (1980): Der Weg zum Systematischen Neopragmatismus und das Konzept der Allgemeinen Modelltheorie. In: H. Stachowiak (Hg.): Modelle und Modelldenken im Unterricht. Bad Heilbrunn: Klinkhardt, S. 9-49.

Starauschek, E. (2001): Physikunterricht nach dem Karlsruher Physikkurs: Ergebnisse einer Evaluationsstudie. Dissertation. Berlin: Logos.

Starauschek, E. (2002): Ergebnisse einer Evaluationsstudie zum Physikunterricht nach dem Karlsruher Physikkurs. In: Zeitschrift für die Didaktik der Naturwissenschaften 8, S. 7-23. Stark, R. (2003): Conceptual Change: kognitiv oder situiert? In: Zeitschrift für Pädagogische Psychologie 14 (2), S. 133-144.

Steffe, L. P.; Gale; J. (1995): Constructivism in education. Hillsdale: Erlbaum. 
Steinberg, M. S. (2004): Electricity visualized - The CASTLE project. Student Manual. Roseville, CA: PASCO Scientific.

Steinberg, M. S. (2008): Inventing Electric Potential. In: Foundations of Science 13 (2), S. 163-175.

Steinberg, M. S.; Wainwright, C. L. (1993): Using Models to Teach Electricity - The CASTLE Project. In: The Physics Teacher 31 (6), S. 353-357.

Stern, E.; Schuhmacher, R. (2004): Lernziel: Intelligentes Wissen. In: Universitas - Orientierung in der Wissensgesellschaft (2), S. 121-134.

Stokes, D. E. (1997): Pasteur's Quadrant: Basic Science and Technological Innovation. Washington/DC: Brookings Institution Press.

Stork, E.; Wiesner, H. (1981): Schülervorstellungen zur Elektrizitätslehre und Sachunterricht. In: Sachunterricht und Mathematik in der Primarstufe 9, S. 218-230.

Strike, K. A.; Posner, G. J. (1992): A revisionist theory of conceptual change. In: R. A. Duschl und R. J. Hamilton (Hg.): Philosophy of science, cognitive science, and educational theory and practice. Albany: New York University Press, S. 147-176.

Tenney, Y. J.; Gentner, D. (1985): What makes analogies accessible: Experiments on the water-flow analogy for electricity. In: R. Duit, W. Jung und C. v. Rhöneck (Hg.): Aspects of Understanding Electricity - Proceedings of an International Workshop. IPN-Arbeitsberichte, Bd. 59. Kiel: Schmidt \& Klaunig, S. 311-318.

Tiberghien, A. (1980): Modes and conditions of learning - an example: The learning of some aspects of the concept of heat. In: Archenhold, W. F., Driver, A, A. Orton und C. WoodRobinson (Hg.): Cognitive development research in science and mathematics. Proceedings of an international seminar held in the Centre for Studies in Science Education. Leeds: University of Leeds, S. 288-309.

Tiberghien, A.; Delacôte, G. (1976): Manipulations et représentations de circuits électriques simples chez les enfants de 7 à 12 ans. In: Revue Française de Pédagogie 34, S. 32-44.

Tiemann, A. (1993): Analogie. Analyse einer grundlegenden Denkweise in der Physik. Thun: Deutsch (Reihe Physik, 11).

Tipler, P. A.; Llewellyn, R. A. (2003): Moderne Physik. München: Oldenbourg.

Tipler, P. A.; Mosca, G. (2006): Physik. Für Wissenschaftler und Ingenieure. Zweite deutsche Auflage. München: Elsevier, Spektrum.

Tobias, V. (2010): Newtonsche Mechanik im Anfangsunterricht. Die Wirksamkeit einer Einführung über die zweidimensionale Dynamik auf das Lehren und Lernen. Dissertation. LMU München, München.

Todman, J.; Dugard, P. (1995): Analysis of Pre-test-Post-test Control Group Designs in Educational Research. In: Educational Psychology 15 (2), S. 181-198. 
Twisk, J.W.R. (2006): Applied Multilevel Analysis. A Practical Guide. Cambridge: Cambridge University Press.

Tymms, P. (2004): Effect sizes in multilevel models. In: I. Schagen und K. Elliot (Hg.): But what does it mean? The use of effect sizes in educational research. Rotherham: B\&B Press, S. 55-66.

Urban-Woldron, H. (2013): Das Verständnis in der Elektrizitätslehre überprüfen. In: S. Bernholt (Hg.): Inquiry-based learning - Forschendes Lernen, Bd. 33. Jahrestagung in Hannover 2012. Gesellschaft für Didaktik der Chemie und Physik. Münster: Lit-Verlag, S. 209-211.

Urban-Woldron, H.; Hopf, M. (2012): Entwicklung eines Testinstruments zum Verständnis in der Elektrizitätslehre. In: Zeitschrift für die Didaktik der Naturwissenschaften 18, S. 201-227. Online verfügbar unter http://archiv.ipn.uni-kiel.de/zfdn/pdf/18_Urbahn.pdf.

Vollmer, G. (1998): Evolutionäre Erkenntnistheorie. Angeborene Erkenntnisstrukturen im Kontext von Biologie, Psychologie, Linguistik, Philosophie und Wissenschaftstheorie.

Stuttgart: Hirzel Verlag.

Vosniadou, S. (1994): Capturing and Modeling the Process of Conceptual Change. In: Learning and Instruction 4, S. 45-69.

Vosniadou, S. (1999): Conceptual change research: State of the art and future directions. In: W. Schnotz, S. Vosniadou und M. Carretero (Hg.): New perspectives on conceptual change. Oxford: Pergamon, S. 3-14.

Vosniadou, S. (Hg.) (2008): International Handbook of Research on Conceptual Change. London: Routledge.

Vosniadou, S. (Hg.) (2013): International handbook of Research on Conceptual Change. 2. Aufl. New York: Routledge.

Vosniadou, S.; Brewer, W. F. (1992): Mental models of the earth: A Study of conceptual change in childhood. In: Cognitive Psychology 24 (4), S. 535-585.

Vosniadou, S.; Skopeliti, I. (2014): Conceptual Change from the Framework Theory Side of the Fence. In: Science \& Education 23 (7), S. 1427-1445.

Vygotsky, L. S. (1978): Mind in society. The Development of Higher Psychological Processes. Cambridge, MA: Harvard University Press.

Waltner, C.; Späth; S., Koller; D.; Wiesner, H. (2009): Einführung von Stromstärke und Spannung - Ein Unterrichtskonzept und Ergebnisse einer Vergleichsstudie. In: D. Höttecke (Hg.): Entwicklung naturwissenschaftlichen Denkens zwischen Phänomenen und Systematik. Jahrestagung der GDCP in Dresden 2009, Bd. 30. Gesellschaft für Didaktik der Chemie und Physik. Münster: Lit-Verlag, S. 182-184.

Walz, A. (1985): Fields that accompany currents. In: R. Duit, W. Jung und C. v. Rhöneck (Hg.): Aspects of Understanding Electricity - Proceedings of an International Workshop. IPN-Arbeitsberichte. Kiel: Schmidt \& Klaunig, S. 403-412. 
Weber, E. (2009): Quantifying Student Learning: How to Analyze Assessment Data. In: Bulletin of the Ecological Society of America 90 (4), S. 501-511.

Weerda, J. (1982): Untersuchungen zum Modellbegriff in der Chemie: eine empirische Untersuchung bei Schülern. Frankfurt a. M.: Verlag Peter Lang.

Weinert, F. E. (1998): Lehrerkompetenz als Schlüssel der inneren Schulreform. In: schulreport (2), S. 24-27.

Weinert, F. E. (2000): Lehren und Lernen für die Zukunft - Ansprüche an das Lernen in der Schule. Vortragsveranstaltungen mit Prof. Dr. Franz E. Weinert, Max-Planck-Institut für psychologische Forschung, gehalten am 29. März 2000 im Pädagogischen Zentrum in Bad Kreuznach. Online verfügbar unter http://www2.ibw.uniheidelberg.de/ gerstner/WeinertLehren\&Lernen.pdf.

Whitehead, A. N. (1929): The Aims of Education and Other Essays. New York: The Free Press.

Widodo, A.; Duit, R. (2004): Konstruktivistische Sichtweisen vom Lehren und Lernen und die Praxis des Physikunterrichts. In: Zeitschrift für Didaktik der Naturwissenschaften 10, S. 233-255.

Wiener, G. (2017): Elementary particle physics in early physics education. Dissertation. Universität Wien, Wien. Fakultät für Physik.

Wiesner, H. (1986): Schülervorstellungen und Lernschwierigkeiten im Bereich der Optik. In: Naturwissenschaften im Unterricht. Physik/Chemie 34 (13), S. 25-29.

Wiesner, H. (1993): Verbesserung des Lernerfolgs durch Untersuchungen von Lernschwierigkeiten im Physikunterricht. Habilitation. Universität Frankfurt, Frankfurt a. M.

Wiesner, H. (1994a): Ein neuer Optikkurs für die Sekundarstufe I, der sich an Lernschwierigkeiten und Schülervorstellungen orientiert. In: Naturwissenschaften im Unterricht - Physik (22), S. 7-15.

Wiesner, H. (1994b): Verbesserung des Lernerfolgs im Unterricht über Mechanik: Schülervorstellungen, Lernschwierigkeiten und fachdidaktische Folgerungen. In: Physik in der Schule, S. 122-126.

Wiesner, H. (2008): Physikunterricht - an Schülervorstellungen orientiert. In: Praxis der Naturwissenschaften - Physik in der Schule 57 (6), S. 4-5.

Wiesner, H.; Jung, W.; Kiowski, I.; Weber, E. (1982): Zur Einführung von Stromstärke und Spannung. In: Naturwissenschaften im Unterricht. Physik/Chemie 30, S. 388-393.

Wiesner, H.; Tobias, V.; Waltner, C.; Hopf, M.; Wilhelm, T.; Sen, A. (2010): Dynamik in den Mechanikunterricht. Didaktik der Physik - Beiträge zur DPG-Frühjahrstagung. In: PhyDid-B. 
Wiesner, H.; Wodzinski, R. (1996): Akzeptanzbefragungen als Methode zur Untersuchung von Lernschwierigkeiten und Lernverläufen. In: R. Duit und C. v. Rhöneck (Hg.): Lernen in den Naturwissenschaften. Kiel: IPN, S. 250-274.

Wilhelm, T. (2005): Konzeption und Evaluation eines Kinematik/Dynamik-Lehrgangs zur Veränderung von Schülervorstellungen mit Hilfe dynamisch ikonischer Repräsentationen und graphischer Modellbildung. Berlin: Logos (46).

Wilhelm, T. (2015): Moment mal... (18): Elektronen als Energieträger? In: Praxis der Naturwissenschaften - Physik in der Schule 64 (6), S. 47-49.

Wilhelm, T.; Burde, J.-P.; Spatz, V.; Haagen-Schützenhöfer, C.; Hopf, M. (2018): Elektronengasmodell und Kontextorientierung - ein binationales Projekt. In: C. Maurer (Hg.): Qualitätsvoller Chemie- und Physikunterricht - normative und empirische Dimensionen, Bd. 38. Jahrestagung in Regensburg 2017. Gesellschaft für Didaktik der Chemie und Physik. Münster: Lit-Verlag.

Wilhelm, T.; Hopf, M. (2014): Design-Forschung. In: D. Krüger, I. Parchmann und H. Schecker (Hg.): Methoden in der naturwissenschaftsdidaktischen Forschung. Berlin: Springer Spektrum, S. 31-42.

Wilhelm, T.; Tobias, V.; Waltner, C.; Hopf, M.; Wiesner, H. (2012): Design-Based Research am Beispiel der zweidimensional-dynamischen Mechanik. In: S. Bernholt (Hg.): Konzepte fachdidaktischer Strukturierung für den Unterricht, Bd. 32. Jahrestagung der GDCP in Oldenburg 2011. Gesellschaft für Didaktik der Chemie und Physik. Münster: Lit-Verlag, S. 31-47.

Wilkinson, D. J. (1972): A Study of the development of Flow with Reference to the Introduction of Electric Current in the early years of the Secondary School. M.A. Research Exercise. Leeds.

Willer, J. (1992): Analogie. Zur Geschichte und Bedeutung des Begriffs. In: Physik in der Schule 30 (7), S. 247-250.

winlab.de (2018): Der handgetriebene Generator "DynaMot". Online verfügbar unter https://www.winlab.de/media/image/5c/38/ba/54850_xxl_600x600.png, zuletzt geprüft am 22.01.2018.

Wodzinski, R. (1996): Untersuchungen von Lernprozessen beim Lernen Newtonscher Dynamik im Anfangsunterricht. Münster: Lit Verlag.

Wodzinski, R. (2004): Schülervorstellungen und Lernschwierigkeiten. In: R. Müller, R.

Wodzinski und M. Hopf (Hg.): Schülervorstellungen zur Physik. Köln: Aulis Verlag Deubner, S. 23-36.

Wodzinski, R. (2013): Lernhilfe oder Lernhindernis? Modelle von Leitungsvorgängen in Stromkreisen unter der Lupe. In: Unterricht Physik 23 (133), S. 12-16. 


\section{Literaturverzeichnis}

Zembylas, M. (2005): Three Perspectives on Linking the Cognitive and the Emotional in Science Learning: Conceptual Change, Socio-Constructivism And Poststructuralism. In: Studies in Science Education 41 (1), S. 91-115.

Zitzelsberger, S.; Rabe, T. (2011): Eine Analyse des Rhöneck-Tests. In: D. Höttecke (Hg.): Naturwissenschaftliche Bildung als Beitrag zur Gestaltung partizipativer Demokratie. Jahrestagung der GDCP in Potsdam 2010. Gesellschaft für Didaktik der Chemie und Physik. Münster: Lit-Verlag, S. 217-219. 


\section{Anhang}

13.1 BEISPIELE FÜR DIE AKZEPTANZSTUFEN ....................344

13.2 BEISPIELE FÜR PARAPHRASIERUNGEN ........................345

13.3 DAS EINGESETZTE TESTINSTRUMENT ...........................349

13.4 DAS BEGLEITSCHREIBEN ZUM TEST ..............................357

13.5 DAS UNTERRICHTSTAGEBUCH DER TG .......................358

13.6 FRAGEBOGEN ZUM UNTERRICHT IN DER KG.............359

13.7 FRAGEBOGEN „PRAXISERFAHRUNGEN““......................360 


\subsection{Beispiele für die Akzeptanzstufen}

\section{1) Vollständige Akzeptanz}

Interviewer Wie findest du diese Erklärung der Naturwissenschaftler? Ist da irgendwas komisch dran, irgendwas unverständlich?

Schüler Also man kann es eigentlich wunderbar verstehen, wenn es eben mehr Elektronen gibt, dann ist der Körper negativ geladen, und bei Rümpfen positiv. Das ist nicht schwierig.

\section{2) Eingeschränkte Akzeptanz}

Interviewer Wie findest du diese Erklärung der Naturwissenschaftler? Ist da irgendwas komisch dran, irgendwas unverständlich?

Schüler Ich find da sind, es ist halt, es ist zu viel auf einmal irgendwie und es gibt ein paar Worte die ich nicht so richtig, das sind halt viele Fachwörter drinnen, also würd ich jetzt mal sagen.

Interviewer Zum Beispiel?

Schüler Hmm, elektrisches Potenzial, Pluspol, Minuspol, Elektronendruck, verbundenen Leiter Leiterstück und äh, und angeschlossene Leiterstücke.

Interviewer Also Leiterstücke, hm, okay. Irgendwas positiv an der Erklärung? Irgendwas was du gut fandest?

Schüler Ja, sie war halt zwar sehr lang, aber es war halt diesmal detailliert, also man konnte halt, hm, man konnte es halt verstehen, also da wurde viel erklärt. Ich fande halt nur es war zu viel auf einmal. Aber trotzdem es war ganz okay, dass das so detailliert ist. Aber beim zweiten Mal lesen mindestens weiß man das mindestens ja dann.

\section{3) Keine Akzeptanz}

Interviewer Wie findest du diese Erklärung der Naturwissenschaftler? Ist da irgendwas komisch dran, irgendwas unverständlich?

Schüler Hmmm naja also das ist schon schwer, würde ich sagen, weil es ist nicht so leicht.

Interviewer Okay, und was ist da nicht so leicht, was ist schwer?

Schüler Hier also, hier, also ich frage mal kurz ob ich es richtig verstanden habe: Wenn ein Körper neutral ist, also genauso viele positive wie negative, also ich muss mir das nochmal kurz durchlesen, ich habe das nicht so ganz verstanden... 


\subsection{Beispiele für Paraphrasierungen}

\section{Erwartungshorizont der Paraphrasierungen}

1. Elektrostatik

a. Es gibt zwei Ladungsarten: positive und negative Ladungen.

b. Gleichnamige Ladungen stoßen sich ab, ungleichnamige Ladungen ziehen sich an.

c. Die Anziehungs- bzw. Abstoßungskraft hängt vom Abstand der Körper ab.

2. Atomvorstellung

a. Atome bestehen aus Atomrümpfen und Elektronen

b. Atomrümpfe sind positiv und ortsfest, Elektronen negativ und freibeweglich

c. Ggf. gleiche Anzahl an Atomrümpfen und Elektronen bei neutralen Körpern

3. Luftdruckvorstellung

a. Luft strömt von höherem zu niedrigerem Druck.

b. Es strömen „Luftteilchen“, nicht Druck

4. Elektrischer Druck

a. Elektronen sind im Leiter mit „Luftteilchen“ vergleichbar.

b. Die Batterie sorgt für einen hohen elektrischen Druck am Minuspol und einen geringen elektrischen Druck am Pluspol.

c. Potenzial als Fachbegriff für elektrischen Druck

5. Spannung

a. Spannung als Druckunterschied: Überdruck vor Lämpchen, Unterdruck nach Lämpchen

b. Das Lämpchen stellt einen Widerstand für die Elektronen dar.

c. Die Batterie fungiert als Elektronenpumpe, die für den Druckunterschied sorgt.

Beispiele für gelungene Paraphrasierungen

\section{1) Elektrostatik}

Interviewer Okay. Kannst du mit deinen eigenen Worten wiederholen, was die Naturwissenschaftler über Ladungen herausgefunden haben?

Schüler Also positive und positive Ladungen, die stoßen sich ab, genauso wie negative Ladungen. Aber wenn jetzt zwei ungleiche Ladungen haben, z.B. positiv und negativ dann ziehen sie sich halt an.

Interviewer Super

Schüler Und je näher sie zueinanderkommen, also näher positiv z.B. an positiv ist, umso stärker ist die Abstoßungskraft und es ist auch so bei negativ und negativer Ladung. Wenn sie jetzt aber weiter weg sind, dann ist die Abstoßungskraft nicht so stark.

Interviewer Super. Genau. Und was zeichnet jetzt so einen elektrisch neutralen Körper aus? 
Schüler Also der Körper, der hat halt genauso viele positive Ladungen wie negative Ladungen und ja.

\section{2) Atomvorstellung}

Interviewer Ja kannst du mit deinen eigenen Worten wiederholen, wie sich die Naturwissenschaftler das mit den Atomen und Ladungen erklären?

Schüler Die erklären sich halt, dass es Atomrümpfe gibt, also das sind positiv geladene Teilchen und die können sich halt nicht bewegen und dann gibt es noch Elektronen, das sind halt negativ geladene Teilchen und die können sich bewegen und wenn halt genauso viele Elektronen wie Atomrümpfe in einem Gegenstand sind, dann ist der elektrisch neutral und wenn es jetzt aber mehr Elektronen gibt, dann ist er negativ geladen und wenn es aber weniger Elektronen gibt, dann ist er positiv geladen.

\section{3) Luftdruck}

Interviewer Kannst du mit deinen eigenen Worten wiederholen, wie sich die Naturwissenschaftler erklären, dass es zu Luftströmungen kommt, z.B. hier bei der Spritze?

Schüler Das liegt halt daran, dass die Luft immer gleichverteilt sein möchte und wenn jetzt z.B. in dieser Spritze halt der Luftdruck größer ist, da versucht die Luft es halt auszugleichen und strömt dann halt raus aus der Spritze und wenn der Luftdruck halt niedriger ist, dann versucht sie es wieder auszugleichen und strömt dann halt in die Spritze.

Interviewer Genau und wenn man so eine ganz allgemeine Regel formulieren sollte, wie Luft strömt, wie würde die dann lauten?

Schüler $\quad$ Ähhh ja, dass es immer gleich ist

Interviewer Das ist das Ziel, aber von wo nach wo strömt die Luft immer, wenn du das mit dem Druck beschreibst?

Schüler In das Objekt entweder mit weniger oder mit mehr Druck

Interviewer Strömt die Luft dahin wo mehr Druck ist?

Schüler Ne ne ne. Nur wo weniger Druck ist.

Interviewer Genau. Also die Luft strömt immer von Bereichen höheren Drucks zu niedrigeren Drucks. Wie du vorhin gesagt hast, sie hört dann auf zu strömen, wenn die Drücke sich angeglichen haben. Ja und strömt die Luft oder strömt der Druck?

Schüler Die Luft strömt.

Interviewer Genau, super. 


\section{4) Elektrostatik}

Interviewer Womit ist denn das Verhalten der Elektronen in so einem Leiter vergleichbar?

Schüler Mit der Luft, denke ich.

Interviewer Ja genau, die Elektronen verhalten sich im Leiter wie Luft. Ja und wie wir wissen, ist ein Pol der Batterie positiv und der andere negativ geladen. An welchem Pol gibt es mehr Elektronen als im normalen Draht, d.h. wo ist der Druck höher?

Schüler Am Minuspol?

Interviewer Genau, am Minuspol. Ja was passiert jetzt, wenn ich ein Leiterstück an so ein Pol anschließe?

Schüler Das halt die Elektronen in das Leiterstück strömen...

Interviewer Genau, und warum tun die das?

Schüler Glaube ich, um auszugleichen auch

Interviewer Genau, weil der Druck im Leiterstück...?

Schüler Niedriger ist

Interviewer Als am Minuspol, genau. Das Leiterstück hat ja einfach einen normalen Druck. An welchem Pol gibt es weniger Elektronen, ist der Druck geringer?

Schüler Am Pluspol

Interviewer Genau und was passiert, wenn ich jetzt ein Leiterstück an den Pluspol anschließe?

Schüler Ich glaube, dann ... weiß ich nicht, kann ich jetzt nicht so genau sagen.

Interviewer Das können wir uns überlegen. Wir haben ja gesagt, der elektrische Druck am Pluspol ist geringer als normal. Und wie ist der Druck im normalen Leiterstück?

Schüler Ausgeglichen

Interviewer Ausgeglichen, genau. Sind einfach normal-viele Elektronen drinnen. Wenn ich jetzt so einen Leiter habe mit Normaldruck an den Pluspol, wo ein Unterdruck herrscht, was passiert dann?

Schüler Dann strömen glaube ich vielleicht von dem Normaldruck Elektronen in den Pluspol, um das wieder auszugleichen, oder?

Interviewer Genau, also wir haben ja gelernt, dass Luft und auch Elektronen immer dahin strömen, wo der Druck niedriger ist. Und da der Pluspol einen sehr niedrigen Druck hat, einen Unterdruck hat und der geringer ist als der Normaldruck in dem Leiterstück, strömen die Elektronen dann dahin. Das erklärt auch deine Frage, wie man das hinbekommt, dass z.B. weniger Elektronen in so einem Leiterstück drinnen sind.

Schüler Also wie Luft dann...?

Interviewer Wie Luft, genau

Schüler Ahhhh 
Interviewer Ja und wie lautet das Fachwort für den elektrischen Druck?

Schüler Potenzial

Interviewer Genau, super, das Potenzial.

\section{5) Spannung}

Interviewer Kannst du die Erklärung mit deinen eigenen Worten wiederholen?

Schüler Also da ist einmal hier der positive, also die positiv geladenen Leiterstücke und die negativ geladenen und die finden sich dann an einem Punkt zusammen, wo das Lämpchen eingesetzt wird und da ist eben dann die Spannung, also der Druckunterschied, und deswegen leuchtete das dann, da fließen ja dann auch die Elektronen durch

Interviewer Das heißt was macht erstmal die Batterie?

Schüler Also die Batterie sorgt dafür, dass es einen gewissen Druckunterschied gibt, dass es nicht überall einen neutralen Druck gibt.

Interviewer Okay, genau, super. D.h. was für einen Druck haben wir jetzt hier oben im oberen Leiterabschnitt, der mit dem Minuspol verbunden ist?

Schüler Da haben wir einen höheren Druck und einen negativen

Interviewer Okay, das wäre negativ geladen.

Schüler Und da ist es jetzt positiv geladen, einen niedrigeren Druck

Interviewer Man könnte auch sagen einen Unterdruck da unten.

Schüler Da oben Oberdruck - Überdruck und Unterdruck.

Interviewer Okay, genau. Und die Spannung war jetzt nochmal was?

Schüler Die Spannung war ein Maß für den Druckunterschied, ja. 


\subsection{Das eingesetzte Testinstrument}

nach Urban-Woldron und Hopf (2012)
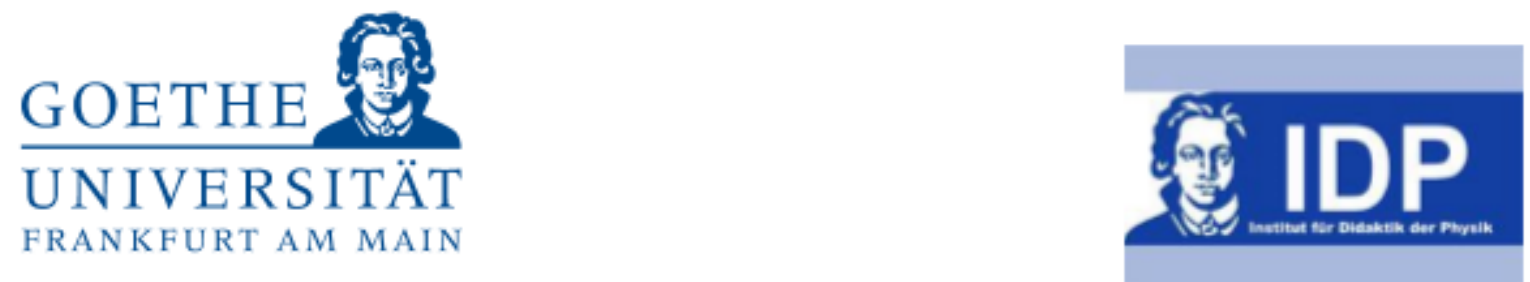

\section{Liebe Schülerinnen und Schüler,}

zunächst einmal herzlichen Dank für eure Teilnahme an diesem Forschungsprojekt des Instituts für Didaktik der Physik der Goethe-Universität Frankfurt zur Verbesserung des Physikunterrichts. Damit die Bearbeitung des Forschungsfragebogens anonym erfolgen kann und wir deine Ergebnisse von einer ersten Durchführung mit den Ergebnissen einer zweiten Durchführung nach Behandlung des Themas vergleichen können, trage bitte in das Feld "Kennung" unten die ersten zwei Buchstaben des Vornamens deines Vaters und gefolgt von den ersten zwei Buchstaben des Vornamens deiner Mutter ein (siehe Beispiel).

\section{Beispiel:}

Vorname des Vaters: Peter

Vorname der Mutter: $\underline{\text { Julia }}$

Kennung: \begin{tabular}{l|l|l|l}
\hline $\mathbf{P}$ & $\mathbf{E}$ & $\mathbf{J}$ & $\mathbf{U}$ \\
\hline
\end{tabular}

Bitte trage nun deine Daten in die Felder unten ein:

\begin{tabular}{|l|l|}
\hline Name deiner Schule & \\
\hline Heutiges Datum & \\
\hline Dein Geschlecht & $\square$ männlich \\
\hline $\begin{array}{l}\text { Deine Kennung } \\
\text { (in Großbuchstaben) }\end{array}$ & \begin{tabular}{l} 
(Reihenfolge: Vater, Mutter) \\
\hline
\end{tabular} \\
\hline
\end{tabular}

Es ist äußerst wichtig, dass du dir bei der Bearbeitung des Fragebogens Mühe gibst und die Fragen gewissenhaft beantwortest. Ich bedanke mich herzlich und wünsche viel Erfolg beim Bearbeiten des Forschungsfragebogens!

Jan-Philipp Burde

Institut für Didaktik der Physik,

Goethe-Universität Frankfurt am Main

Hildegard Urban-Woldron 
Anhang

\section{Forschungsfragebogen „Elektrizitätslehre“}

Bei den in diesem Forschungsfragebogen vorkommenden Batterien handelt es sich ausschließlich um ideale Batterien. Sie besitzen eine konstante Spannung und keinen Innenwiderstand.

Ebenso sind verbindende Leitungen widerstandslas.

Die Lampen besitzen nicht wie die meisten tatsāchlichen Lampen einen vom Strom abhängigen Widerstand. Falls nicht anders angegeben, sind alle Lampen, alle Batterien und alle Widerstände in einem Beispiel gleich.

Es werden die bekannten Symbole verwendet:

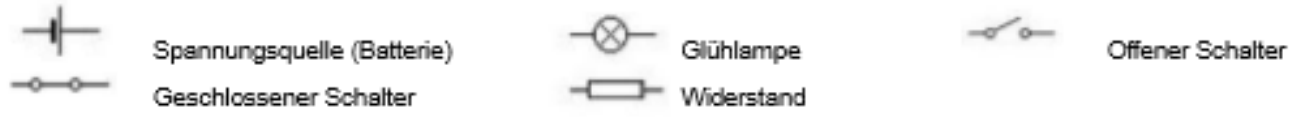

\begin{tabular}{|c|c|c|c|}
\hline \multirow[t]{2}{*}{ Item 2} & \multicolumn{3}{|c|}{$\begin{array}{l}\text { In einem Stromkreis mit einer Glühbirne und den Widerständen } R_{1}=10 \Omega \text { und } R_{2}=10 \Omega \text { beträgt die Stromstärke I = } \\
0,4 \mathrm{~A} \text {. Der Widerstand } R_{1} \text { wird nun durch den Widerstand } R_{3}=20 \Omega \text { ersetzt. }\end{array}$} \\
\hline & Welchen Einfluss hat das auf die Stromstärke durch die Glühbirne? & $1=0,4 \mathrm{~A}$ & $R_{1}=10 \Omega$ \\
\hline$\square$ & Die Stromstärke ist nun kleiner als 0,4 A. & & \\
\hline$\square$ & Die Stromstärke ist genau so groß wie vorher. & & \\
\hline$\square$ & Die Stromstärke ist jetzt größer als 0,4 A. & & $R_{2}=10 \Omega$ \\
\hline
\end{tabular}

\begin{tabular}{|c|l|}
\hline Item 3 & $\begin{array}{l}\text { Der Widerstand } \mathrm{R}_{1} \text { im Stromkreis (Abb. rechts oben) ist klein. Er wird durch einen } \\
\text { größeren Widerstand } \mathrm{R}_{2} \text { ersetzt (Abb. rechts unten). } \\
\text { Was geschieht mit der Stromstärke im Stromkreis? }\end{array}$ \\
\hline$\square$ & Sie wird größer. \\
\hline$\square$ & Sie wird kleiner, aber nicht Null. \\
\hline$\square$ & Sie bleibt gleich. \\
\hline$\square$ & Es fließt kein Strom mehr. \\
\hline b) & Wie erklärst du deine Entscheidung? \\
\hline$\square$ & $\begin{array}{l}\text { Die Batterie ist nicht stark genug. um überhaupt Strom durch den größeren } \\
\text { Widerstand zu treiben. }\end{array}$ \\
\hline$\square$ & $\begin{array}{l}\text { Die Batterie kann nicht einen so großen Strom wie vorher durch den größeren } \\
\text { Widerstand treiben. }\end{array}$ \\
\hline$\square$ & Ein größerer Widerstand braucht mehr Strom als ein kleinerer Widerstand. \\
\hline$\square$ & Es ist cieselbe Batterie; daher bleibt auch die Stromstärke gleich. \\
\hline
\end{tabular}

\begin{tabular}{|c|l|}
\hline Item 4 & Im Stromkreis rechts ist eine Batterie mit einem Motor verbunden. \\
\hline a) & $\begin{array}{l}\text { Was kannst du über die Anzeigen der beiden Amperemeter } \\
\text { aussagen? }\end{array}$ \\
\hline$\square$ & A1 zeigt eine höhere Stromstãrke an. \\
\hline$\square$ & Beide Amperemeter zeigen die gleiche Stromstärke an. \\
\hline$\square$ & A2 zcigt eine höhere Stromstãrke an. \\
\hline b) & Wie erklärst du deine Entscheidung? \\
\hline$\square$ & Im gesamten Stromkreis ist die Stromstärke gleich. \\
\hline$\square$ & Ein Teil des Stroms vird vom Motor verbraucht. \\
\hline$\square$ & Der gesamte Strom wird vom Motor verbraucht. \\
\hline
\end{tabular}




\begin{tabular}{|c|l|}
\hline Item 6 & $\begin{array}{l}\text { Ein Stromkreis besteht aus einer Batterie und einem Motor. Der Motor läuft (Abb. rechts } \\
\text { oben). Dann wird ein zweiter gleicher Motor hinzugefügt (Abb. rechts unten). }\end{array}$ \\
\hline a) & Welchen Einfluss hat das auf die Stromstärke im Stromkreis? \\
\hline$\square$ & Sie wird größer. \\
\hline$\square$ & Sie bleibt gleich. \\
\hline$\square$ & Sie wird kleiner, aber nicht Null. \\
\hline$\square$ & Es fließstkein Strom mehr. \\
\hline b) & Wie erklärst du deine Entscheidung? \\
\hline$\square$ & Die Batterie ist nicht stark genug, überhaupt Strom durch zwei Motoren anzutreiben. \\
\hline$\square$ & Es ist dieselbe Batterie; daher liefert sie denselben Strom. \\
\hline$\square$ & Die Batterie kann nicht so viel Strom wie varher durch beide Motoren treiben. \\
\hline$\square$ & Zwei Motoren brauchen mehr Strom als einer. \\
\hline$\square$ & Der Strom teilt sich auf beide Motoren auf; so wird er halbiert. \\
\hline
\end{tabular}

\begin{tabular}{|c|l|}
\hline Item 7 & $\begin{array}{l}\text { Im Stromkreis rechts siehst du vier identische Lampen, die mit einer Batterie } \\
\text { verbunden sind. Der Schalter } S \text { ist, wie dargestellt, anfänglich geschlossen. }\end{array}$ \\
& $\begin{array}{l}\text { Was geschieht mit dem Strom durch Lampe } \mathrm{L}_{2} \text {, wenn der Schalter } \mathrm{S} \\
\text { geöffnet wird? }\end{array}$ \\
\hline$\square$ & Die Stromstärke durch $\mathrm{L}_{2}$ wird größer. \\
\hline$\square$ & Die Stromstärke durch $\mathrm{L}_{2}$ bleibt gleich. \\
\hline$\square$ & Die Stromstärke durch $\mathrm{L}_{2}$ wird kleiner. \\
\hline$\square$ & Mit den gegebenen Informationen ist die Frage nicht zu beantworten. \\
\hline
\end{tabular}

\begin{tabular}{|c|l|l|}
\hline Item 9 & $\begin{array}{l}\text { In welcher Schaltung oder in welchen Schaltungen (siehe Abbildungen } \\
\text { rechts) sind die Widerstände } \mathrm{R}_{1} \text { und } \mathrm{R}_{2} \text { parallel zur Batterie geschaltet? }\end{array}$ \\
\hline$\square$ & Stromkreise 1, 2 und 3 \\
\hline$\square$ & Stromkreis 2 \\
\hline$\square$ & Stromkreis 1 \\
\hline$\square$ & Stromkreise 1 und 2 & \\
\hline$\square$ & Stromkreise 2 und 3 & \\
\hline
\end{tabular}

\begin{tabular}{|c|l|}
\hline Item 10 & $\begin{array}{l}\text { Im Stromkreis rechts unten sind zwei Widerstände und eine Glühlampe an eine Batterie angeschlossen. } \\
\text { Was passiert mit der Helligkeit der Lampe, wenn } R_{2} \text { gleich bleibt und } R_{1} \text { verkleinert wird? }\end{array}$ \\
\hline$\square$ & Die Lampe leuchtet heller. \\
\hline$\square$ & Die Lampe leuchtet gleich hell. \\
\hline$\square$ & Die Lampe leuchtet weniger hell. \\
\hline
\end{tabular}

\begin{tabular}{|c|c|c|c|}
\hline Item 13 & $\begin{array}{l}\text { In einem Stromkreis mit einer Glühbime und den Widerständen } \\
R_{1}=10 \Omega \text { und } R_{2}=10 \Omega \text { beträgt die Stromstärke } \\
\text { I = 0,4 A. Widerstand } R_{2} \text { wird nun durch den Widerstand } R_{3}=20 \Omega \text { ersetzt. }\end{array}$ & & \\
\hline & Welchen Einfluss hat das auf die Stromstärke durch die Glühbime? & \multicolumn{2}{|c|}{$1=0.4 \mathrm{~A} \quad \mathrm{R}_{1}=10 \Omega$} \\
\hline$\square$ & Die Stromstärke ist nun kleiner als 0,4 A. & \multirow{2}{*}{\multicolumn{2}{|c|}{$\frac{1}{5}$}} \\
\hline$\square$ & Die Stromstärke ist genau so groß vie vorher. & & \\
\hline$\square$ & Die Stromstärke ist jetzt größer als 0,4 A. & \multicolumn{2}{|r|}{$R_{2}=10 \Omega$} \\
\hline
\end{tabular}




\begin{tabular}{|c|l|}
\hline Item $\mathbf{2 1}$ & $\begin{array}{l}\text { Betrachte den Stromkreis auf der rechten Seite. } \\
\text { a) Wie hell werden die Glühbirnen leuchten? }\end{array}$ \\
\hline$\square$ & Beide Glühbimen leuchten. L1 leuchtet heller als L2. \\
\hline$\square$ & Beide Glühbirnen leuchten. L2 leuchtet heller als L1. \\
\hline$\square$ & Beide Glühbirnen leuchten gleich hell. \\
\hline$\square$ & L1 leuchtet. L2 leuchtet nicht. \\
\hline$\square$ & L2 leuchtet. L1 leuchtet nicht. \\
\hline b) & Wie erklärst du deine Entscheidung? \\
\hline$\square$ & L1 verbraucht den gesamten Strom. Es ist daher kein Strom mehr für L2 übrig. \\
\hline$\square$ & L1 verbraucht einen Teil des Stroms. Es ist daher nur mehr weniger Strom für L2 übrig. \\
\hline$\square$ & Der elektrische Strom ist überall im Stromkreis gleich. \\
\hline$\square$ & Der Strom wird gleichmäßig auf beide Glühbirnen aufgeteilt. \\
\hline$\square$ & L2 ist näher bei der Batterie. Daher bekommt sie mehr Strom. \\
\hline
\end{tabular}

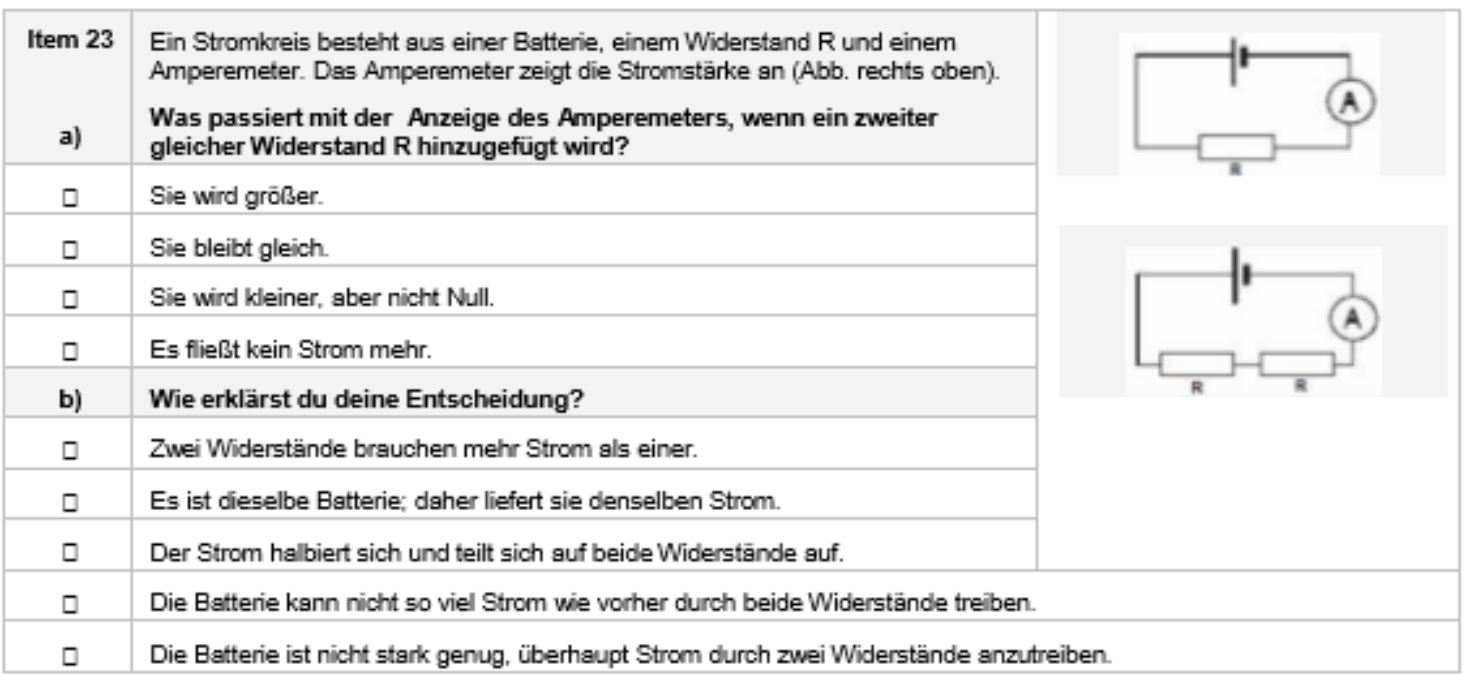

\begin{tabular}{|c|l|}
\hline \multirow{2}{*}{ Item 24} & Vergleiche die Helligkeiten der Lampen $\mathrm{L} 1, \mathrm{~L} 2$ und $\mathrm{L} 3$ in den beiden Stromkreisen (Abb. unten) \\
& Welche Lampe oder welche Lampen leuchten am hellsten? \\
\hline$\square$ & Lampe $\mathrm{L}_{1}$ leuchtet am hellsten. \\
\hline$\square$ & Lampe $\mathrm{L}_{2}$ leuchtet am hellsten. \\
\hline$\square$ & Lampe $\mathrm{L}_{3}$ leuchtet am hellsten. \\
\hline$\square$ & Die Lampen $\mathrm{L}_{1}$ und $\mathrm{L}_{2}$ leuchten am hellsten. \\
\hline$\square$ & Die Lampen $\mathrm{L}_{1}$ und $\mathrm{L}_{3}$ leuchten am hellsten.
\end{tabular}

\begin{tabular}{|c|l|}
\hline Item $\mathbf{2 5}$ & $\begin{array}{l}\text { Im Stromkreis rechts unten sind zwei Widerstände und eine Glühlampe an eine Batterie angeschlossen. } \\
\text { Wie ändert sich die Helligkeit der Lampe, wenn } \mathbf{R}_{1} \text { gleich bleibt und } \mathbf{R}_{2} \text { vergrößert wird? }\end{array}$ \\
\hline$\square$ & Die Lampe leuchtet heller. \\
\hline$\square$ & Die Lampe leuchtet gleich hell. \\
\hline$\square$ & Die Lampe leuchtet weniger hell. \\
\hline
\end{tabular}


Anhang

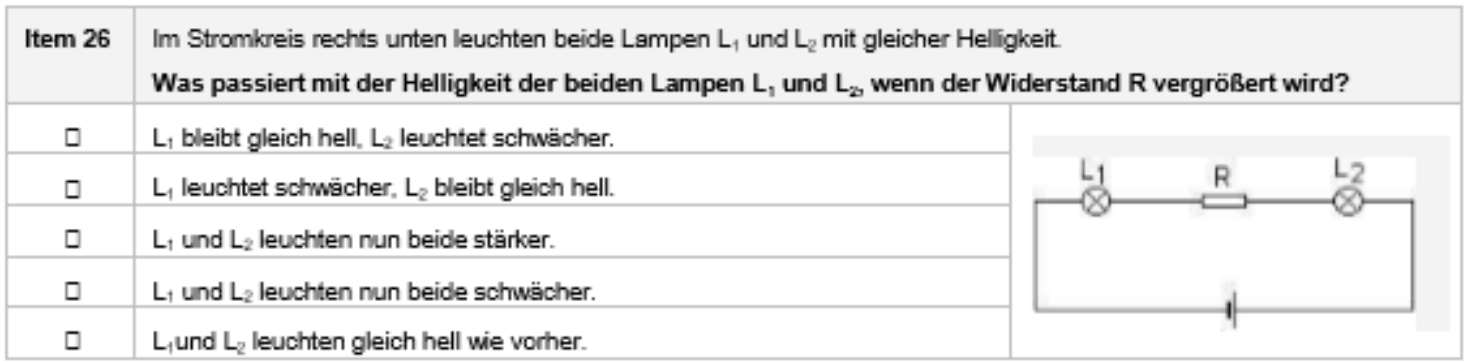

\begin{tabular}{|c|c|c|c|}
\hline \multirow{3}{*}{$\begin{array}{c}\text { Item } 27 \\
\text { a) }\end{array}$} & \multirow{2}{*}{\multicolumn{3}{|c|}{$\begin{array}{l}\text { Der Stromkreis rechts besteht aus zwei Amperemetern und einem regelbaren } \\
\text { Widerstand. } \\
\text { Beide Amperemeter zeigen die Stromstärke an. } \\
\text { Nun wird der Widerstand R vergrößert. }\end{array}$}} \\
\hline & & & \\
\hline & $\begin{array}{l}\text { Wie verändert sich dadurch die Anzeige von } \\
\text { Amperemeter } A_{t} \text { ? }\end{array}$ & b) & $\begin{array}{l}\text { Wie verändert sich die Anzeige von } \\
\text { Amperemeter } A_{\text {Q }} \text { ? }\end{array}$ \\
\hline$n$ & Sie wird größer. & $n$ & Sie wird größer. \\
\hline$\square$ & Sie bleibt gleich. & $\square$ & Sie bleibt gleich. \\
\hline$\square$ & Sie wird kleiner. & $\square$ & Sie wird kleiner. \\
\hline c) & \multicolumn{3}{|l|}{ Wie erklärst du deine Entscheidung? } \\
\hline$\square$ & \multicolumn{3}{|c|}{ Ein größerer Widerstand braucht mehr Strom als ein kleinerer Widerstand. } \\
\hline$\square$ & \multicolumn{3}{|c|}{ Es ist dieselbe Batterie; daher liefert sie denselben Strom. } \\
\hline$\square$ & \multicolumn{3}{|c|}{ Eine Vergrößerung des Widerstands führt zu einer Verringerung der Stromstärke überall im Stromkreis. } \\
\hline$\square$ & \multicolumn{3}{|c|}{$\begin{array}{l}\text { Eine Vergrößerung des Widerstands führt zu einer Verringerung der Stromstärke nach dem Widerstand. Sie } \\
\text { beeinflusst daher den Strom vor dem Widerstand nicht. }\end{array}$} \\
\hline$\square$ & \multicolumn{3}{|c|}{$\begin{array}{l}\text { Eine Vergrößerung des Widerstands führt zu einer Verringerung der Stromstärke nach dem Widerstand. Daher wird } \\
\text { der Strom vor dem Widerstand größer. }\end{array}$} \\
\hline
\end{tabular}

\begin{tabular}{|c|c|c|}
\hline Item 28 & $\begin{array}{l}\text { Im Stromkreis rechts leuchtet die Glūhbirne und das Amperemeter } A_{1} \text { zeigt eine Stromstärke } \\
\text { von } 0.2 \mathrm{~A} \text { an. } \\
\text { Nun wird ein zweites Amperemeter } A_{2} \text { auf der anderen Seite des Stromkreises eingebaut } \\
\text { (siehe Abb. rechts unten). } \\
\text { Was zeigt das Amperemeter } A_{2} \text { an? }\end{array}$ & 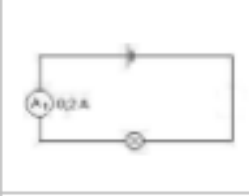 \\
\hline$\square$ & Mehr als $0,2 \mathrm{~A}$. & \\
\hline$\square$ & Genau $0.2 \mathrm{~A}$. & \\
\hline$\square$ & Weniger als $0,2 \mathrm{~A}$, aber nicht $0 \mathrm{~A}$. & \\
\hline$\square$ & OA. & \\
\hline b) & Wie erklärst du deine Entscheidung? & \\
\hline$\square$ & Im gesamten Stromkreis ist die Stromstärke gleich. & \\
\hline$\square$ & Ein Teil des Stroms vird von der Glühbime verbraucht. & \\
\hline$\square$ & Der gesamte Strom wird von der Glūhbirne verbraucht. & \\
\hline
\end{tabular}

\begin{tabular}{|c|l|}
\hline Item 29 & $\begin{array}{l}\text { Im Stromkreis rechts unten sind zvei Widerstände und eine Glühlampe an eine Batterie angeschlossen. } \\
\text { Was passiert mit der Helligkeit der Lampe, wenn } R_{2} \text { gleich bleibt und } R_{1} \text { vergrößert wird? }\end{array}$ \\
\hline$\square$ & Die Lampe leuchtet heller. \\
\hline$\square$ & Die Lampe leuchtet gleich hell. \\
\hline$\square$ & Die Lampe leuchtet weniger hell. \\
\hline
\end{tabular}




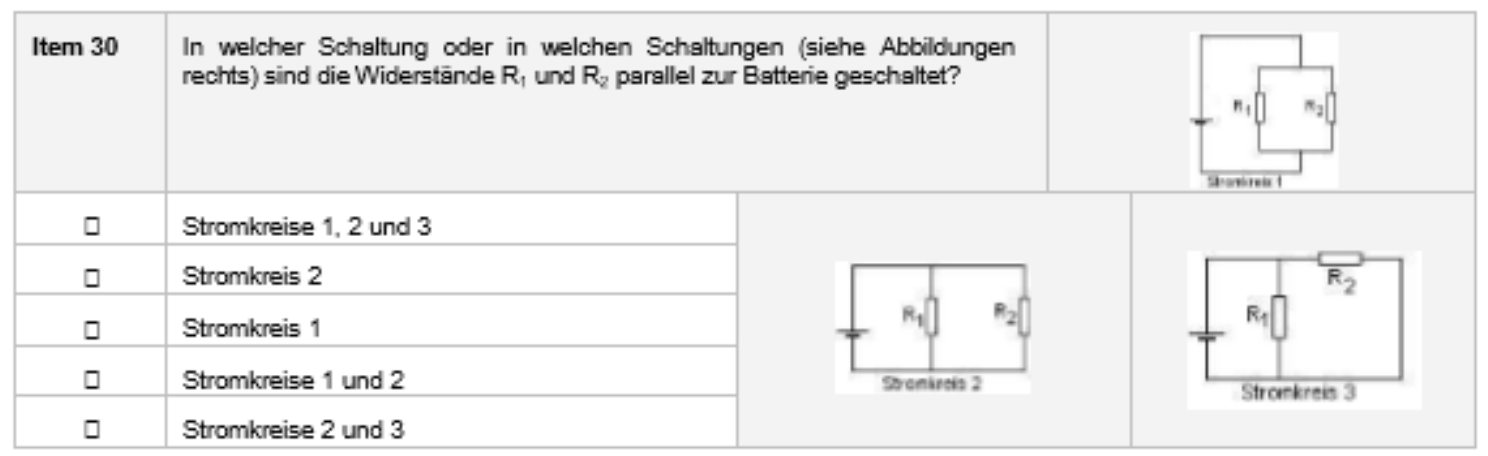

Item 31 Schaue dir die folgende Schaltung an:

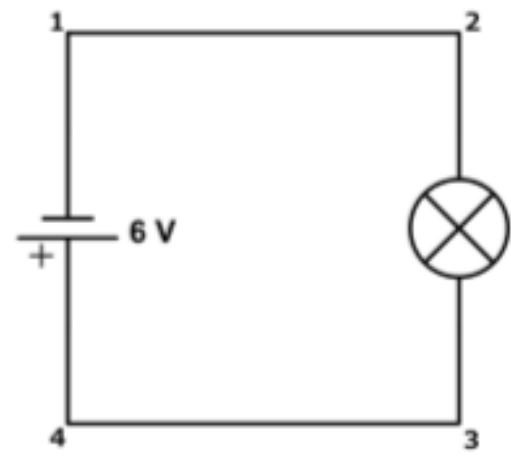

Wie groß ist in diesem Stromkreis die Spannung zwischen den Punkten:

1 und 2 und $3:$

Zwischen den Punkten 3 und 4 wird nun ein zweites Lämpchen der gleichen Sorte eingebaut:

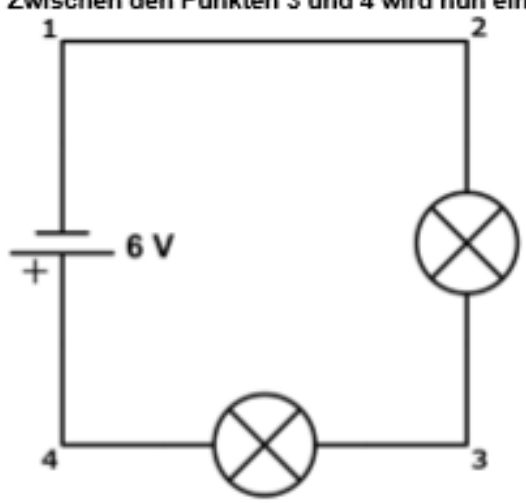

Wie groß ist in dem Stromkreis mit zwei Lämpchen die Spannung zwischen den Punkten:

1 und 2 :

2 und $3:$

3 und 4: 
Anhang

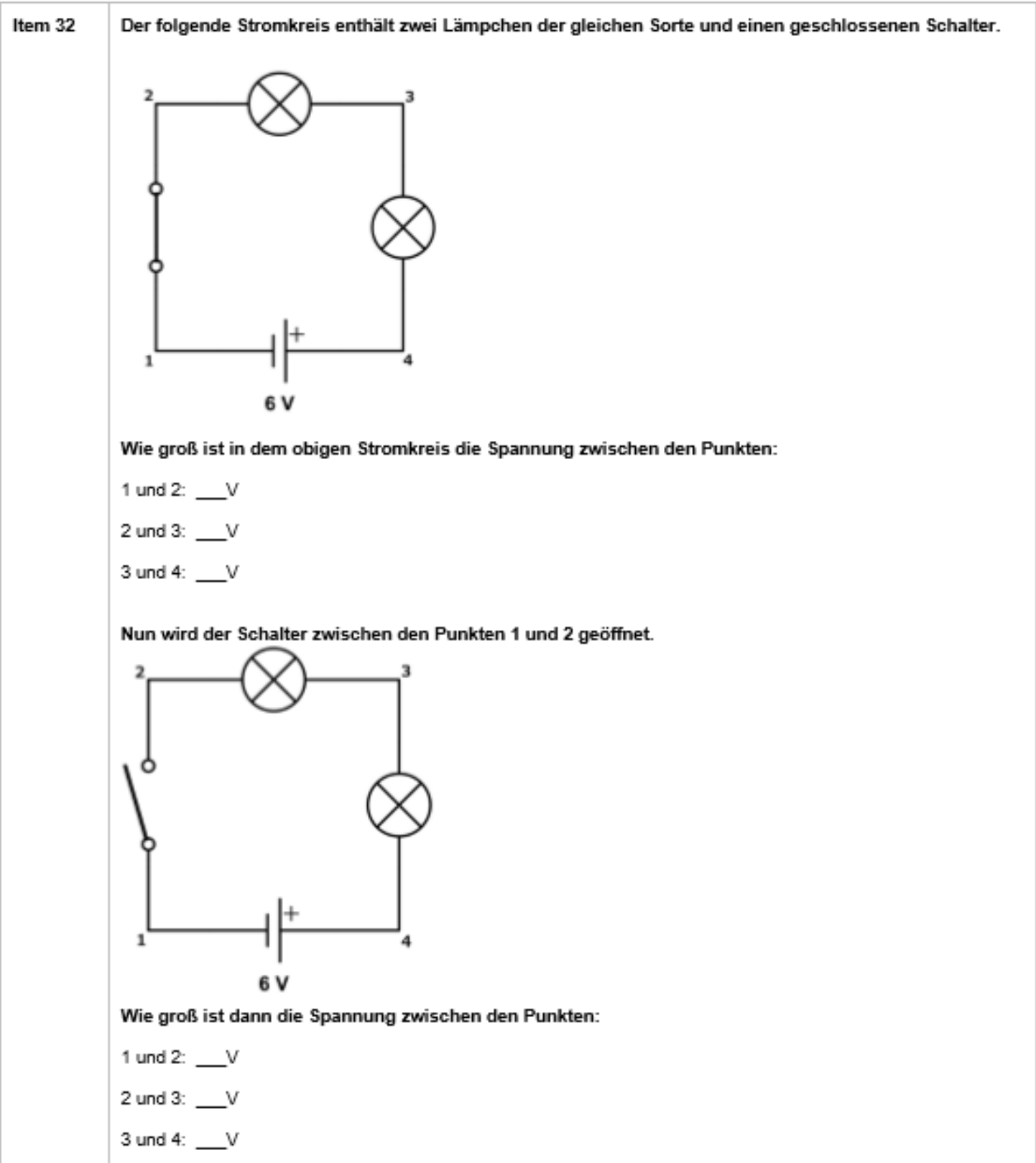

ENDE DES FRAGEBOGENS

Hildegard Urban-Woldron 


\subsection{Das Begleitschreiben zum Test}

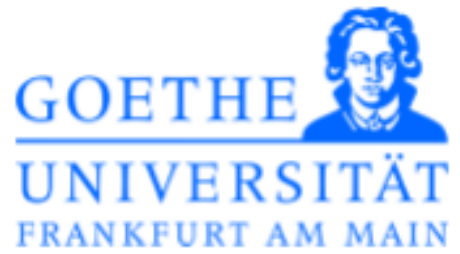

Jan-Philine Burde, IDP, 60438 Frankfurt am Main

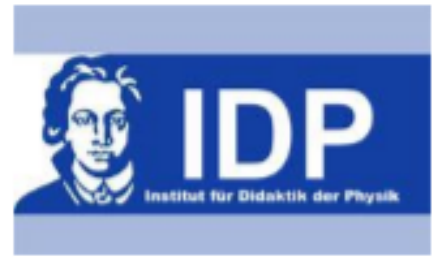

Jan-Philipp Burde

Institut für Didaktik der Physik

Goethe-Universitāt Frankfurt am Main

Max-von-Laue-Str. 1

60438 Frankfurt am Main

Tel.: (089) $798-46820$

E-Mail: burde@physik.uni-frankfurt.de

Frankfurt, den 14.08.2015

\section{Studie zur E-Lehre}

Liebe Kolleginnen und Kollegen!

Sie haben sich bereit erklärt, von Ihren Schülern vor und nach dem Unterricht zu einfachen Stromkreisen einen Test bearbeiten zu lassen. Zunächst einmal ein großes Dankeschön für diese Unterstützung unserer physikdidaktischen Forschung. Der vorliegende Verständnistest zur E-Lehre wurde von Frau Prof. Dr. Urban-Woldron von der Universität Wien zur gezielten Überprüfung des Verständnisses der Schülerinnen und Schüler im Gebiet der Elektrizitätslehre entwickelt. Bei der Durchführung des Tests würden wir Sie bitten, folgende Punkte zu beachten:

- Um den Lernfortschritt der Schülerinnen und Schüler messen zu können, soll der E-Lehre-Test von den Schülern zweimal bearbeitet werden: Einmal vor und einmal nach dem E-LehreUnterricht.

- Statt ihres Namens, sollen die Schülerinnen und Schüler eine Kennung auf die Tests schreiben, die sich aus dem Vornamen ihrer Väter und Mütter ergibt (siehe Test). Die Kennung ermöglicht es uns, die Tests anonym auszuwerten und dennoch die Vor- mit den Nachtests zu vergleichen.

- Geben Sie den Schülern nach Möglichkeit so viel Zeit zur Bearbeitung des Tests, wie diese benötigen.

- Achten Sie darauf, dass der Test von den Schülern einzeln bearbeitet wird und es somit nicht zu „Teamwork" kommt.

- Lassen Sie sich von der etwas eigenartigen Nummerierung der Test-Items nicht verwirren. Die teilweise falsche Nummerierung kommt daher, dass manche Items von Frau Urban-Woldron im Nachhinein wieder gestrichen oder verschoben wurden.

- Zu jeder Frage gibt es nur eine korrekte Antwort.

Wir würden uns freuen, wenn Sie die bearbeiteten Tests mit dem bereits frankierten Rückumschlag zurück an das Institut für Didaktik der Physik schicken würden.

Herzlichen Dank für Ihre Unterstützung, 
Anhang

13.5 Das Unterrichtstagebuch der TG

Unterrichtstagebuch

\begin{tabular}{l|l}
\hline Heutiges Datum & \\
\hline Thema der Stunde & \\
& \\
\hline
\end{tabular}

\begin{tabular}{|l|c|}
$\begin{array}{c}\text { verwendete Materialien } \\
\text { (bitte unbedingt angeben) }\end{array}$ & $\begin{array}{c}\text { aufgetretene Probleme / Kommentar } \\
\text { (optional) }\end{array}$ \\
Overheadfolien: z.B. 3.1, 3.2 und 3.3 & \\
Arbeitsblätter: z.B. 3.1 und 3.2 & \\
\hline Sonstiges: z.B. durchgefunrte Experimente & \\
\hline
\end{tabular}

weitere Anmerkungen (optional) 


\subsection{Fragebogen zum Unterricht in der KG}
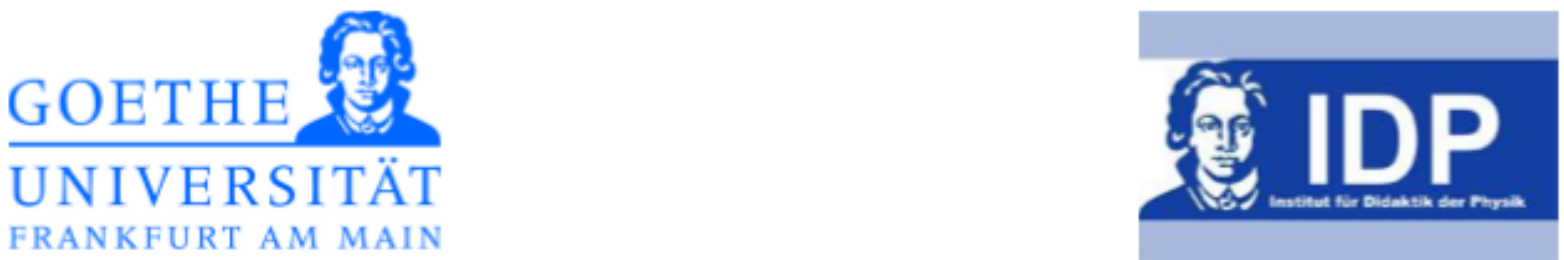

\section{Ergänzende Fragen zum E-Lehre-Unterricht}

Liebe Kolleginnen und Kollegen!

Zunächst einmal ganz herzlichen Dank für die Rücksendung der Fragebögen des Vortests. Anbei finden Sie die Fragebögen zur Erhebung des Lernstands nach dem E-Lehre-Unterricht. Um die Ergebnisse besser interpretieren zu können, würden wir uns sehr freuen, wenn wir von Ihnen ein paar grundlegende Informationen zu dem von Ihnen gehaltenen E-Lehre-Unterricht bekommen könnten.

1. Der Unterricht fand in Jahrgangsstufe statt (Schulform:

2. Das Thema Elektrizitätslehre wurde über insgesamt Schulstunden unterrichtet.

3. Im Unterricht wurde hauptsächlich die folgende Analogie bzw. das folgende Modell verwendet (z.B geschlossener ebener Wasserkreislauf, Höhenanalogie mit offenem Wasserkreislauf, Hūtchenmodell, Stäbchenmodell, Fahrradkettenmodell, Elektronengasdruckmodell, 0.a.):

4. Welche der untenstehenden Konzepte wurden im Unterricht explizit thematisiert?

\begin{tabular}{|l|l|l|}
\hline Konzept & Unterrichtsgegenstand? & Thema über wie viele Stunden? \\
& Ja: Nein: & \\
\hline Stromstärke & & \\
\hline Widerstand & & \\
\hline Potenzial & & \\
\hline Spannung & & \\
\hline Reihenschaltungen & & \\
\hline Parallelschaltungen & & \\
\hline
\end{tabular}

Wir bedanken uns noch einmal herzlich für Ihre Unterstützung und verbleiben mit freundlichen Grüßen, 


\subsection{Fragebogen „Praxiserfahrungen“}

Bei den mit einem Stern (*) markierten Fragen ist eine Antwort im Online-Formular zwingend erforderlich gewesen.

\section{Allgemeine Fragen zum Einstieg}

1. Warum haben Sie sich dazu entschieden, an der Studie teilzunehmen?

2. Im Elektrizitätslehreunterricht werden häufig Modelle und Analogien eingesetzt. Welche Modelle bzw. Analogien haben Sie bisher zur Einführung in die E-Lehre benutzt? (Mehrfachnennung möglich)
a. Ebener geschlossener Wasserkreislauf
b. Offenes Wasserhöhenmodell
c. Rucksackmodell
d. Stäbchenmodell
e. Fahrradkettenmodell
f. Elektronengasmodell
g. Ich nutze keine Modelle bzw. Analogien
h. Sonstiges (bitte angeben):

\section{Gesamteinschätzung des Unterrichtskonzepts}

3. *Wie würden Sie das Unterrichtskonzept in Bezug auf die folgenden Aspekte bewerten? (jeweils zu beantworten mittels einer 5-wertigen Likert-Skala:

1: trifft gar nicht zu; 2: trifft eher nicht zu; 3: teils-teils; 4: trifft eher zu; 5: trifft völlig zu)

Das Unterrichtskonzept

a. ...erreicht bei den Schülerinnen und Schülern ein gutes konzeptionelles Verständnis des Potenzials (elektrischen Drucks)

b. ...erreicht bei den Schülerinnen und Schülern ein gutes konzeptionelles Verständnis der Spannung (elektrischer Druckunterschied)

c. ...erreicht bei den Schülerinnen und Schülern ein gutes konzeptionelles Verständnis der Stromstärke

d. ...erreicht bei den Schülerinnen und Schülern ein gutes konzeptionelles Verständnis des Widerstands

e. ...erreicht bei den Schülerinnen und Schülern ein gutes konzeptionelles Verständnis von Kondensatoren

f. ...erreicht bei den Schülerinnen und Schülern ein gutes konzeptionelles Verständnis von Parallelschaltungen

g. ...erreicht bei den Schülerinnen und Schülern ein gutes konzeptionelles Verständnis von Reihenschaltungen 
4. *Wie würden Sie das Unterrichtskonzept in Bezug auf die folgenden Aspekte bewerten? (jeweils zu beantworten mittels einer 5-wertigen Likert-Skala:

1: trifft gar nicht zu; 2: trifft eher nicht zu; 3: teils-teils; 4: trifft eher zu; 5: trifft völlig zu)

Das Unterrichtskonzept

h. ...wird von den Schülerinnen und Schüler akzeptiert.

i. ...vermittelt zentrale Konzepte anschaulich und verständlich.

j. ...setzt auf anknüpfungsfähige Vorstellungen und Konzepte.

k. ...fördert das Interesse bzw. die Motivation der Lernenden.

1. ...setzt mit der Farbdarstellung auf eine Visualisierung des Potenzials, die von den Schülerinnen und Schülern gerne genutzt wird.

\section{Allgemeine Bewertung des gewählten Ansatzes}

5. Hat es sich im Unterricht als fruchtbar erwiesen, die elementare Elektrizitätslehre mit Hilfe des elektrischen Potenzials zu erklären (im vorliegenden Unterrichtskonzept als „elektrischer Druck“ bezeichnet)?

6. *Für das Unterrichtskonzept ist es unerlässlich, dass die Schülerinnen und Schüler ein intuitives Luftdruckverständnis im Sinne von „komprimierte Luft steht unter Druck, drückt gegen die Wände und hat das Bestreben sich auszudehnen“ besitzen. Beschreiben Sie kurz auf Grundlage Ihrer Erfahrungen mit dem Unterrichtskonzept, inwiefern die Schülerinnen und Schüler ein solches Luftdruckverständnis besitzen.

7. Profitieren aus Ihrer Perspektive bestimmte Schülergruppen besonders vom neuen Unterrichtskonzept (z.B. Mädchen/Jungen oder leistungsstarke bzw. schwache Schüler)?

\section{Vor- und Nachteile des Konzepts sowie aufgetretene Verständnisschwierigkeiten}

8. *Worin liegen Ihrer Meinung nach die größten Stärken des Unterrichtskonzepts und warum?

9. *Was sind Ihrer Meinung nach die größten Schwächen des Unterrichtskonzepts und warum?

10. Kam es in Folge der Farbwahl für das Potenzial (rot = elektr. Überdruck; blau = elektr. Unterdruck) zu Verständnisschwierigkeiten bei den Schülerinnen und Schülern (z.B. weil Rot an Spannungswürfeln klassischerweise für den Pluspol und Blau für den Minuspol steht)? Falls ja, beschreiben Sie diese bitte kurz.

11. Beschreiben Sie auf Grundlage Ihrer Erfahrungen mit dem Elektronengasmodell die größten aufgetretenen Verständnisschwierigkeiten auf Seiten der Schülerinnen und Schüler (die in Ihrem bisherigen Unterricht nicht auftraten). 


\section{Bewertung verschiedener Aspekte in Bezug auf Reihenschaltungen}

12. Im Unterrichtskonzept wird auch der Lade- und Entladevorgang eines Kondensators analysiert. Erklären Sie kurz, inwiefern Sie dieses Vorgehen für sinnvoll erachten oder nicht.

13. *Wie schätzen Sie die Fähigkeit der Schülerinnen und Schüler ein, Reihenschaltungen alleine mit Hilfe von Übergangszuständen zu analysieren (d.h. ohne Verwendung der Fahrradkettenanalogie)?

14. *Beschreiben Sie kurz, inwiefern Sie die parallele Verwendung der Fahrradkettenanalogie zur Erklärung von Reihenschaltungen für sinnvoll erachten oder nicht.

\section{Der Einsatz des Konzepts im zukünftigen Unterricht}

15. *Werden Sie die E-Lehre in Zukunft wieder nach dem Elektronengasmodell unterrichten?

16. Was würden Sie am Unterrichtskonzept ändern, wenn Sie noch einmal danach unterrichten würden?

17. *Haben bzw. werden Sie das Unterrichtskonzept an Ihre Kollegen weiterempfehlen? Warum? 

Bisher erschienene Bände der Reihe „Studien zum Physik- und Chemielernen“

ISSN 1614-8967（vormals Studien zum Physiklernen ISSN 1435-5280)

1 Helmut Fischler, Jochen Peuckert (Hrsg.): Concept Mapping in fachdidaktischen Forschungsprojekten der Physik und Chemie

ISBN 978-3-89722-256-4 40.50 EUR

2 Anja Schoster: Bedeutungsentwicklungsprozesse beim Lösen algorithmischer Physikaufgaben. Eine Fallstudie zu Lernprozessen von Schülern im Physiknachhilfeunterricht während der Bearbeitung algorithmischer Physikaufgaben

ISBN 978-3-89722-045-4 40.50 EUR

3 Claudia von Aufschnaiter: Bedeutungsentwicklungen, Interaktionen und situatives Erleben beim Bearbeiten physikalischer Aufgaben

ISBN 978-3-89722-143-7 40.50 EUR

4 Susanne Haeberlen: Lernprozesse im Unterricht mit Wasserstromkreisen. Eine Fallstudie in der Sekundarstufe I

ISBN 978-3-89722-172-7 40.50 EUR

5 Kerstin Haller: Über den Zusammenhang von Handlungen und Zielen. Eine empirische Untersuchung zu Lernprozessen im physikalischen Praktikum

ISBN 978-3-89722-242-7 40.50 EUR

6 Michaela Horstendahl: Motivationale Orientierungen im Physikunterricht ISBN 978-3-89722-227-4 50.00 EUR

7 Stefan Deylitz: Lernergebnisse in der Quanten-Atomphysik. Evaluation des Bremer Unterrichtskonzepts

ISBN 978-3-89722-291-5 40.50 EUR

8 Lorenz Hucke: Handlungsregulation und Wissenserwerb in traditionellen und computergestützten Experimenten des physikalischen Praktikums

ISBN 978-3-89722-316-5 50.00 EUR

9 Heike Theyßen: Ein Physikpraktikum für Studierende der Medizin. Darstellung der Entwicklung und Evaluation eines adressatenspezifischen Praktikums nach dem Modell der Didaktischen Rekonstruktion

ISBN 978-3-89722-334-9 40.50 EUR

10 Annette Schick: Der Einfluß von Interesse und anderen selbstbezogenen Kognitionen auf Handlungen im Physikunterricht. Fallstudien zu Interessenhandlungen im Physikunterricht

ISBN 978-3-89722-380-6 40.50 EUR

11 Roland Berger: Moderne bildgebende Verfahren der medizinischen Diagnostik. Ein Weg zu interessanterem Physikunterricht

ISBN 978-3-89722-445-2 40.50 EUR 
12 Johannes Werner: Vom Licht zum Atom. Ein Unterrichtskonzept zur Quantenphysik unter Nutzung des Zeigermodells

ISBN 978-3-89722-471-1 40.50 EUR

13 Florian Sander: Verbindung von Theorie und Experiment im physikalischen Praktikum. Eine empirische Untersuchung zum handlungsbezogenen Vorverständnis und dem Einsatz grafikorientierter Modellbildung im Praktikum

ISBN 978-3-89722-482-7 40.50 EUR

14 Jörn Gerdes: Der Begriff der physikalischen Kompetenz. Zur Validierung eines Konstruktes

ISBN 978-3-89722-510-7 40.50 EUR

15 Malte Meyer-Arndt: Interaktionen im Physikpraktikum zwischen Studierenden und Betreuern. Feldstudie zu Bedeutungsentwicklungsprozessen im physikalischen Praktikum

ISBN 978-3-89722-541-1 40.50 EUR

16 Dietmar Höttecke: Die Natur der Naturwissenschaften historisch verstehen. Fachdidaktische und wissenschaftshistorische Untersuchungen

ISBN 978-3-89722-607-4 40.50 EUR

17 Gil Gabriel Mavanga: Entwicklung und Evaluation eines experimentell- und phänomenorientierten Optikcurriculums. Untersuchung zu Schülervorstellungen in der Sekundarstufe I in Mosambik und Deutschland

ISBN 978-3-89722-721-7 40.50 EUR

18 Meike Ute Zastrow: Interaktive Experimentieranleitungen. Entwicklung und Evaluation eines Konzeptes zur Vorbereitung auf das Experimentieren mit Messgeräten im Physikalischen Praktikum

ISBN 978-3-89722-802-3 40.50 EUR

19 Gunnar Friege: Wissen und Problemlösen. Eine empirische Untersuchung des wissenszentrierten Problemlösens im Gebiet der Elektrizitätslehre auf der Grundlage des Experten-Novizen-Vergleichs

ISBN 978-3-89722-809-2 40.50 EUR

20 Erich Starauschek: Physikunterricht nach dem Karlsruher Physikkurs. Ergebnisse einer Evaluationsstudie

ISBN 978-3-89722-823-8 40.50 EUR

21 Roland Paatz: Charakteristika analogiebasierten Denkens. Vergleich von Lernprozessen in Basis- und Zielbereich

ISBN 978-3-89722-944-0 40.50 EUR

22 Silke Mikelskis-Seifert: Die Entwicklung von Metakonzepten zur Teilchenvorstellung bei Schülern. Untersuchung eines Unterrichts über Modelle mithilfe eines Systems multipler Repräsentationsebenen

ISBN 978-3-8325-0013-9 40.50 EUR

23 Brunhild Landwehr: Distanzen von Lehrkräften und Studierenden des Sachunterrichts zur Physik. Eine qualitativ-empirische Studie zu den Ursachen

ISBN 978-3-8325-0044-3 40.50 EUR 
24 Lydia Murmann: Physiklernen zu Licht, Schatten und Sehen. Eine phänomenografische Untersuchung in der Primarstufe

ISBN 978-3-8325-0060-3 40.50 EUR

25 Thorsten Bell: Strukturprinzipien der Selbstregulation. Komplexe Systeme, Elementarisierungen und Lernprozessstudien für den Unterricht der Sekundarstufe II

ISBN 978-3-8325-0134-1 40.50 EUR

26 Rainer Müller: Quantenphysik in der Schule

ISBN 978-3-8325-0186-0 40.50 EUR

27 Jutta Roth: Bedeutungsentwicklungsprozesse von Physikerinnen und Physikern in den Dimensionen Komplexität, Zeit und Inhalt

ISBN 978-3-8325-0183-9 40.50 EUR

28 Andreas Saniter: Spezifika der Verhaltensmuster fortgeschrittener Studierender der Physik

ISBN 978-3-8325-0292-8 40.50 EUR

29 Thomas Weber: Kumulatives Lernen im Physikunterricht. Eine vergleichende Untersuchung in Unterrichtsgängen zur geometrischen Optik

ISBN 978-3-8325-0316-1 40.50 EUR

30 Markus Rehm: Über die Chancen und Grenzen moralischer Erziehung im naturwissenschaftlichen Unterricht

ISBN 978-3-8325-0368-0 40.50 EUR

31 Marion Budde: Lernwirkungen in der Quanten-Atom-Physik. Fallstudien über Resonanzen zwischen Lernangeboten und SchülerInnen-Vorstellungen

ISBN 978-3-8325-0483-0 40.50 EUR

32 Thomas Reyer: Oberflächenmerkmale und Tiefenstrukturen im Unterricht. Exemplarische Analysen im Physikunterricht der gymnasialen Sekundarstufe

ISBN 978-3-8325-0488-5 40.50 EUR

33 Christoph Thomas Müller: Subjektive Theorien und handlungsleitende Kognitionen von Lehrern als Determinanten schulischer Lehr-Lern-Prozesse im Physikunterricht ISBN 978-3-8325-0543-1 40.50 EUR

34 Gabriela Jonas-Ahrend: Physiklehrervorstellungen zum Experiment im Physikunterricht

ISBN 978-3-8325-0576-9 40.50 EUR

35 Dimitrios Stavrou: Das Zusammenspiel von Zufall und Gesetzmäßigkeiten in der nichtlinearen Dynamik. Didaktische Analyse und Lernprozesse

ISBN 978-3-8325-0609-4 40.50 EUR

36 Katrin Engeln: Schülerlabors: authentische, aktivierende Lernumgebungen als Möglichkeit, Interesse an Naturwissenschaften und Technik zu wecken ISBN 978-3-8325-0689-6 40.50 EUR

37 Susann Hartmann: Erklärungsvielfalt

ISBN 978-3-8325-0730-5 40.50 EUR 
Knut Neumann: Didaktische Rekonstruktion eines physikalischen Praktikums für Physiker

ISBN 978-3-8325-0762-6 40.50 EUR

39 Michael Späth: Kontextbedingungen für Physikunterricht an der Hauptschule. Möglichkeiten und Ansatzpunkte für einen fachübergreifenden, handlungsorientierten und berufsorientierten Unterricht

ISBN 978-3-8325-0827-2 40.50 EUR

40 Jörg Hirsch: Interesse, Handlungen und situatives Erleben von Schülerinnen und Schülern beim Bearbeiten physikalischer Aufgaben

ISBN 978-3-8325-0875-3 40.50 EUR

41 Monika Hüther: Evaluation einer hypermedialen Lernumgebung zum Thema Gasgesetze. Eine Studie im Rahmen des Physikpraktikums für Studierende der Medizin ISBN 978-3-8325-0911-8 40.50 EUR

42 Maike Tesch: Das Experiment im Physikunterricht. Didaktische Konzepte und Ergebnisse einer Videostudie

ISBN 978-3-8325-0975-0 40.50 EUR

43 Nina Nicolai: Skriptgeleitete Eltern-Kind-Interaktion bei Chemiehausaufgaben. Eine Evaluationsstudie im Themenbereich Säure-Base

ISBN 978-3-8325-1013-8 40.50 EUR

44 Antje Leisner: Entwicklung von Modellkompetenz im Physikunterricht ISBN 978-3-8325-1020-6 40.50 EUR

45 Stefan Rumann: Evaluation einer Interventionsstudie zur Säure-Base-Thematik ISBN 978-3-8325-1027-5 40.50 EUR

46 Thomas Wilhelm: Konzeption und Evaluation eines Kinematik/Dynamik-Lehrgangs zur Veränderung von Schülervorstellungen mit Hilfe dynamisch ikonischer Repräsentationen und graphischer Modellbildung - mit CD-ROM

ISBN 978-3-8325-1046-6 45.50 EUR

47 Andrea Maier-Richter: Computerunterstütztes Lernen mit Lösungsbeispielen in der Chemie. Eine Evaluationsstudie im Themenbereich Löslichkeit ISBN 978-3-8325-1046-6 40.50 EUR

48 Jochen Peuckert: Stabilität und Ausprägung kognitiver Strukturen zum Atombegriff ISBN 978-3-8325-1104-3 40.50 EUR

49 Maik Walpuski: Optimierung von experimenteller Kleingruppenarbeit durch Strukturierungshilfen und Feedback

ISBN 978-3-8325-1184-5 40.50 EUR

50 Helmut Fischler, Christiane S. Reiners (Hrsg.): Die Teilchenstruktur der Materie im Physik- und Chemieunterricht

ISBN 978-3-8325-1225-5 34.90 EUR

51 Claudia Eysel: Interdisziplinäres Lehren und Lernen in der Lehrerbildung. Eine empirische Studie zum Kompetenzerwerb in einer komplexen Lernumgebung

ISBN 978-3-8325-1238-5 40.50 EUR 
52 Johannes Günther: Lehrerfortbildung über die Natur der Naturwissenschaften.

Studien über das Wissenschaftsverständnis von Grundschullehrkräften

ISBN 978-3-8325-1287-3 40.50 EUR

53 Christoph Neugebauer: Lernen mit Simulationen und der Einfluss auf das Problemlösen in der Physik

ISBN 978-3-8325-1300-9 40.50 EUR

54 Andreas Schnirch: Gendergerechte Interessen- und Motivationsförderung im Kontext naturwissenschaftlicher Grundbildung. Konzeption, Entwicklung und Evaluation einer multimedial unterstützten Lernumgebung

ISBN 978-3-8325-1334-4 40.50 EUR

55 Hilde Köster: Freies Explorieren und Experimentieren. Eine Untersuchung zur selbstbestimmten Gewinnung von Erfahrungen mit physikalischen Phänomenen im Sachunterricht

ISBN 978-3-8325-1348-1 40.50 EUR

56 Eva Heran-Dörr: Entwicklung und Evaluation einer Lehrerfortbildung zur Förderung der physikdidaktischen Kompetenz von Sachunterrichtslehrkräften ISBN 978-3-8325-1377-1 40.50 EUR

57 Agnes Szabone Varnai: Unterstützung des Problemlösens in Physik durch den Einsatz von Simulationen und die Vorgabe eines strukturierten Kooperationsformats ISBN 978-3-8325-1403-7 40.50 EUR

58 Johannes Rethfeld: Aufgabenbasierte Lernprozesse in selbstorganisationsoffenem Unterricht der Sekundarstufe I zum Themengebiet ELEKTROSTATIK. Eine Feldstudie in vier 10. Klassen zu einer kartenbasierten Lernumgebung mit Aufgaben aus der Elektrostatik

ISBN 978-3-8325-1416-7 40.50 EUR

59 Christian Henke: Experimentell-naturwissenschaftliche Arbeitsweisen in der Oberstufe. Untersuchung am Beispiel des HIGHSEA-Projekts in Bremerhaven ISBN 978-3-8325-1515-7 40.50 EUR

60 Lutz Kasper: Diskursiv-narrative Elemente für den Physikunterricht. Entwicklung und Evaluation einer multimedialen Lernumgebung zum Erdmagnetismus ISBN 978-3-8325-1537-9 40.50 EUR

61 Thorid Rabe: Textgestaltung und Aufforderung zu Selbsterklärungen beim Physiklernen mit Multimedia ISBN 978-3-8325-1539-3 40.50 EUR

62 Ina Glemnitz: Vertikale Vernetzung im Chemieunterricht. Ein Vergleich von traditionellem Unterricht mit Unterricht nach Chemie im Kontext

ISBN 978-3-8325-1628-4 40.50 EUR

63 Erik Einhaus: Schülerkompetenzen im Bereich Wärmelehre. Entwicklung eines Testinstruments zur Überprüfung und Weiterentwicklung eines normativen Modells fachbezogener Kompetenzen

ISBN 978-3-8325-1630-7 40.50 EUR 
64 Jasmin Neuroth: Concept Mapping als Lernstrategie. Eine Interventionsstudie zum Chemielernen aus Texten

ISBN 978-3-8325-1659-8 40.50 EUR

65 Hans Gerd Hegeler-Burkhart: Zur Kommunikation von Hauptschülerinnen und Hauptschülern in einem handlungsorientierten und fächerübergreifenden Unterricht mit physikalischen und technischen Inhalten

ISBN 978-3-8325-1667-3 40.50 EUR

66 Karsten Rincke: Sprachentwicklung und Fachlernen im Mechanikunterricht. Sprache und Kommunikation bei der Einführung in den Kraftbegriff

ISBN 978-3-8325-1699-4 40.50 EUR

67 Nina Strehle: Das Ion im Chemieunterricht. Alternative Schülervorstellungen und curriculare Konsequenzen

ISBN 978-3-8325-1710-6 40.50 EUR

68 Martin Hopf: Problemorientierte Schülerexperimente

ISBN 978-3-8325-1711-3 40.50 EUR

69 Anne Beerenwinkel: Fostering conceptual change in chemistry classes using expository texts

ISBN 978-3-8325-1721-2 40.50 EUR

70 Roland Berger: Das Gruppenpuzzle im Physikunterricht der Sekundarstufe II. Eine empirische Untersuchung auf der Grundlage der Selbstbestimmungstheorie der Motivation

ISBN 978-3-8325-1732-8 40.50 EUR

71 Giuseppe Colicchia: Physikunterricht im Kontext von Medizin und Biologie. Entwicklung und Erprobung von Unterrichtseinheiten ISBN 978-3-8325-1746-5 40.50 EUR

72 Sandra Winheller: Geschlechtsspezifische Auswirkungen der Lehrer-Schüler-Interaktion im Chemieanfangsunterricht

ISBN 978-3-8325-1757-1 40.50 EUR

73 Isabel Wahser: Training von naturwissenschaftlichen Arbeitsweisen zur Unterstützung experimenteller Kleingruppenarbeit im Fach Chemie

ISBN 978-3-8325-1815-8 40.50 EUR

74 Claus Brell: Lernmedien und Lernerfolg - reale und virtuelle Materialien im Physikunterricht. Empirische Untersuchungen in achten Klassen an Gymnasien (Laborstudie) zum Computereinsatz mit Simulation und IBE

ISBN 978-3-8325-1829-5 40.50 EUR

75 Rainer Wackermann: Überprüfung der Wirksamkeit eines Basismodell-Trainings für Physiklehrer

ISBN 978-3-8325-1882-0 40.50 EUR

76 Oliver Tepner: Effektivität von Aufgaben im Chemieunterricht der Sekundarstufe I ISBN 978-3-8325-1919-3 40.50 EUR 
77 Claudia Geyer: Museums- und Science-Center-Besuche im naturwissenschaftlichen Unterricht aus einer motivationalen Perspektive. Die Sicht von Lehrkräften und Schülerinnen und Schülern

ISBN 978-3-8325-1922-3 40.50 EUR

78 Tobias Leonhard: Professionalisierung in der Lehrerbildung. Eine explorative Studie zur Entwicklung professioneller Kompetenzen in der Lehrererstausbildung ISBN 978-3-8325-1924-7 40.50 EUR

79 Alexander Kauertz: Schwierigkeitserzeugende Merkmale physikalischer Leistungstestaufgaben

ISBN 978-3-8325-1925-4 40.50 EUR

80 Regina Hübinger: Schüler auf Weltreise. Entwicklung und Evaluation von Lehr-/ Lernmaterialien zur Förderung experimentell-naturwissenschaftlicher Kompetenzen für die Jahrgangsstufen 5 und 6

ISBN 978-3-8325-1932-2 40.50 EUR

81 Christine Waltner: Physik lernen im Deutschen Museum

ISBN 978-3-8325-1933-9 40.50 EUR

82 Torsten Fischer: Handlungsmuster von Physiklehrkräften beim Einsatz neuer Medien. Fallstudien zur Unterrichtspraxis

ISBN 978-3-8325-1948-3 42.00 EUR

83 Corinna Kieren: Chemiehausaufgaben in der Sekundarstufe I des Gymnasiums. Fragebogenerhebung zur gegenwärtigen Praxis und Entwicklung eines optimierten Hausaufgabendesigns im Themenbereich Säure-Base

978-3-8325-1975-9 37.00 EUR

84 Marco Thiele: Modelle der Thermohalinen Zirkulation im Unterricht. Eine empirische Studie zur Förderung des Modellverständnisses

ISBN 978-3-8325-1982-7 40.50 EUR

85 Bernd Zinn: Physik lernen, um Physik zu lehren. Eine Möglichkeit für interessanteren Physikunterricht

ISBN 978-3-8325-1995-7 39.50 EUR

86 Esther Klaes: Außerschulische Lernorte im naturwissenschaftlichen Unterricht. Die Perspektive der Lehrkraft

ISBN 978-3-8325-2006-9 43.00 EUR

87 Marita Schmidt: Kompetenzmodellierung und -diagnostik im Themengebiet Energie der Sekundarstufe I. Entwicklung und Erprobung eines Testinventars ISBN 978-3-8325-2024-3 37.00 EUR

88 Gudrun Franke-Braun: Aufgaben mit gestuften Lernhilfen. Ein Aufgabenformat zur Förderung der sachbezogenen Kommunikation und Lernleistung für den naturwissenschaftlichen Unterricht

ISBN 978-3-8325-2026-7 38.00 EUR

89 Silke Klos: Kompetenzförderung im naturwissenschaftlichen Anfangsunterricht. Der Einfluss eines integrierten Unterrichtskonzepts

ISBN 978-3-8325-2133-2 37.00 EUR 
90 Ulrike Elisabeth Burkard: Quantenphysik in der Schule. Bestandsaufnahme, Perspektiven und Weiterentwicklungsmöglichkeiten durch die Implementation eines Medienservers

ISBN 978-3-8325-2215-5 43.00 EUR

91 Ulrike Gromadecki: Argumente in physikalischen Kontexten. Welche Geltungsgründe halten Physikanfänger für überzeugend?

ISBN 978-3-8325-2250-6 41.50 EUR

92 Jürgen Bruns: Auf dem Weg zur Förderung naturwissenschaftsspezifischer Vorstellungen von zukünftigen Chemie-Lehrenden ISBN 978-3-8325-2257-5 43.50 EUR

93 Cornelius Marsch: Räumliche Atomvorstellung. Entwicklung und Erprobung eines Unterrichtskonzeptes mit Hilfe des Computers

ISBN 978-3-8325-2293-3 82.50 EUR

94 Maja Brückmann: Sachstrukturen im Physikunterricht. Ergebnisse einer Videostudie ISBN 978-3-8325-2272-8 39.50 EUR

95 Sabine Fechner: Effects of Context-oriented Learning on Student Interest and Achievement in Chemistry Education

ISBN 978-3-8325-2343-5 36.50 EUR

96 Clemens Nagel: eLearning im Physikalischen Anfängerpraktikum ISBN 978-3-8325-2355-8 39.50 EUR

97 Josef Riese: Professionelles Wissen und professionelle Handlungskompetenz von (angehenden) Physiklehrkräften

ISBN 978-3-8325-2376-3 39.00 EUR

98 Sascha Bernholt: Kompetenzmodellierung in der Chemie. Theoretische und empirische Reflexion am Beispiel des Modells hierarchischer Komplexität ISBN 978-3-8325-2447-0 40.00 EUR

99 Holger Christoph Stawitz: Auswirkung unterschiedlicher Aufgabenprofile auf die Schülerleistung. Vergleich von Naturwissenschafts- und Problemlöseaufgaben der PISA 2003-Studie

ISBN 978-3-8325-2451-7 37.50 EUR

100 Hans Ernst Fischer, Elke Sumfleth (Hrsg.): nwu-essen - 10 Jahre Essener Forschung zum naturwissenschaftlichen Unterricht ISBN 978-3-8325-3331-1 40.00 EUR

101 Hendrik Härtig: Sachstrukturen von Physikschulbüchern als Grundlage zur Bestimmung der Inhaltsvalidität eines Tests

ISBN 978-3-8325-2512-5 34.00 EUR

102 Thomas Grüß-Niehaus: Zum Verständnis des Löslichkeitskonzeptes im Chemieunterricht. Der Effekt von Methoden progressiver und kollaborativer Reflexion ISBN 978-3-8325-2537-8 40.50 EUR

103 Patrick Bronner: Quantenoptische Experimente als Grundlage eines Curriculums zur Quantenphysik des Photons 
ISBN 978-3-8325-2540-8 36.00 EUR

104 Adrian Voßkühler: Blickbewegungsmessung an Versuchsaufbauten. Studien zur Wahrnehmung, Verarbeitung und Usability von physikbezogenen Experimenten am Bildschirm und in der Realität

ISBN 978-3-8325-2548-4 47.50 EUR

105 Verena Tobias: Newton'sche Mechanik im Anfangsunterricht. Die Wirksamkeit einer Einführung über die zweidimensionale Dynamik auf das Lehren und Lernen ISBN 978-3-8325-2558-3 54.00 EUR

106 Christian Rogge: Entwicklung physikalischer Konzepte in aufgabenbasierten Lernumgebungen

ISBN 978-3-8325-2574-3 45.00 EUR

107 Mathias Ropohl: Modellierung von Schülerkompetenzen im Basiskonzept Chemische Reaktion. Entwicklung und Analyse von Testaufgaben

ISBN 978-3-8325-2609-2 36.50 EUR

108 Christoph Kulgemeyer: Physikalische Kommunikationskompetenz. Modellierung und Diagnostik

ISBN 978-3-8325-2674-0 44.50 EUR

109 Jennifer Olszewski: The Impact of Physics Teachers' Pedagogical Content Knowledge on Teacher Actions and Student Outcomes

ISBN 978-3-8325-2680-1 33.50 EUR

110 Annika Ohle: Primary School Teachers' Content Knowledge in Physics and its Impact on Teaching and Students' Achievement

ISBN 978-3-8325-2684-9 36.50 EUR

111 Susanne Mannel: Assessing scientific inquiry. Development and evaluation of a test for the low-performing stage

ISBN 978-3-8325-2761-7 40.00 EUR

112 Michael Plomer: Physik physiologisch passend praktiziert. Eine Studie zur Lernwirksamkeit von traditionellen und adressatenspezifischen Physikpraktika für die Physiologie

ISBN 978-3-8325-2804-1 34.50 EUR

113 Alexandra Schulz: Experimentierspezifische Qualitätsmerkmale im Chemieunterricht. Eine Videostudie

ISBN 978-3-8325-2817-1 40.00 EUR

114 Franz Boczianowski: Eine empirische Untersuchung zu Vektoren im Physikunterricht der Mittelstufe

ISBN 978-3-8325-2843-0 39.50 EUR

115 Maria Ploog: Internetbasiertes Lernen durch Textproduktion im Fach Physik ISBN 978-3-8325-2853-9 39.50 EUR 
116 Anja Dhein: Lernen in Explorier- und Experimentiersituationen. Eine explorative Studie zu Bedeutungsentwicklungsprozessen bei Kindern im Alter zwischen 4 und 6 Jahren

ISBN 978-3-8325-2859-1

45.50 EUR

117 Irene Neumann: Beyond Physics Content Knowledge. Modeling Competence Regarding Nature of Scientific Inquiry and Nature of Scientific Knowledge

ISBN 978-3-8325-2880-5 37.00 EUR

118 Markus Emden: Prozessorientierte Leistungsmessung des naturwissenschaftlichexperimentellen Arbeitens. Eine vergleichende Studie zu Diagnoseinstrumenten zu Beginn der Sekundarstufe I

ISBN 978-3-8325-2867-6 38.00 EUR

119 Birgit Hofmann: Analyse von Blickbewegungen von Schülern beim Lesen von physikbezogenen Texten mit Bildern. Eye Tracking als Methodenwerkzeug in der physikdidaktischen Forschung

ISBN 978-3-8325-2925-3 59.00 EUR

120 Rebecca Knobloch: Analyse der fachinhaltlichen Qualität von Schüleräußerungen und deren Einfluss auf den Lernerfolg. Eine Videostudie zu kooperativer Kleingruppenarbeit ISBN 978-3-8325-3006-8 36.50 EUR

121 Julia Hostenbach: Entwicklung und Prüfung eines Modells zur Beschreibung der Bewertungskompetenz im Chemieunterricht

ISBN 978-3-8325-3013-6 38.00 EUR

122 Anna Windt: Naturwissenschaftliches Experimentieren im Elementarbereich. Evaluation verschiedener Lernsituationen

ISBN 978-3-8325-3020-4 43.50 EUR

123 Eva Kölbach: Kontexteinflüsse beim Lernen mit Lösungsbeispielen ISBN 978-3-8325-3025-9 38.50 EUR

124 Anna Lau: Passung und vertikale Vernetzung im Chemie- und Physikunterricht ISBN 978-3-8325-3021-1 36.00 EUR

125 Jan Lamprecht: Ausbildungswege und Komponenten professioneller Handlungskompetenz. Vergleich von Quereinsteigern mit Lehramtsabsolventen für Gymnasien im Fach Physik

ISBN 978-3-8325-3035-8 38.50 EUR

126 Ulrike Böhm: Förderung von Verstehensprozessen unter Einsatz von Modellen ISBN 978-3-8325-3042-6 41.00 EUR

127 Sabrina Dollny: Entwicklung und Evaluation eines Testinstruments zur Erfassung des fachspezifischen Professionswissens von Chemielehrkräften

ISBN 978-3-8325-3046-4 37.00 EUR 
128 Monika Zimmermann: Naturwissenschaftliche Bildung im Kindergarten. Eine integrative Längsschnittstudie zur Kompetenzentwicklung von Erzieherinnen ISBN 978-3-8325-3053-2 54.00 EUR

129 Ulf Saballus: Über das Schlussfolgern von Schülerinnen und Schülern zu öffentlichen Kontroversen mit naturwissenschaftlichem Hintergrund. Eine Fallstudie ISBN 978-3-8325-3086-0 39.50 EUR

130 Olaf Krey: Zur Rolle der Mathematik in der Physik. Wissenschaftstheoretische Aspekte und Vorstellungen Physiklernender

ISBN 978-3-8325-3101-0 46.00 EUR

131 Angelika Wolf: Zusammenhänge zwischen der Eigenständigkeit im Physikunterricht, der Motivation, den Grundbedürfnissen und dem Lernerfolg von Schülern ISBN 978-3-8325-3161-4 45.00 EUR

132 Johannes Börlin: Das Experiment als Lerngelegenheit. Vom interkulturellen Vergleich des Physikunterrichts zu Merkmalen seiner Qualität

ISBN 978-3-8325-3170-6 45.00 EUR

133 Olaf Uhden: Mathematisches Denken im Physikunterricht. Theorieentwicklung und Problemanalyse

ISBN 978-3-8325-3170-6 45.00 EUR

134 Christoph Gut: Modellierung und Messung experimenteller Kompetenz. Analyse eines large-scale Experimentiertests

ISBN 978-3-8325-3213-0 40.00 EUR

135 Antonio Rueda: Lernen mit ExploMultimedial in kolumbianischen Schulen. Analyse von kurzzeitigen Lernprozessen und der Motivation beim länderübergreifenden Einsatz einer deutschen computergestützten multimedialen Lernumgebung für den naturwissenschaftlichen Unterricht

ISBN 978-3-8325-3218-5 45.50 EUR

136 Krisztina Berger: Bilder, Animationen und Notizen. Empirische Untersuchung zur Wirkung einfacher visueller Repräsentationen und Notizen auf den Wissenserwerb in der Optik

ISBN 978-3-8325-3238-3 41.50 EUR

137 Antony Crossley: Untersuchung des Einflusses unterschiedlicher physikalischer Konzepte auf den Wissenserwerb in der Thermodynamik der Sekundarstufe I ISBN 978-3-8325-3275-8 40.00 EUR

138 Tobias Viering: Entwicklung physikalischer Kompetenz in der Sekundarstufe I. Validierung eines Kompetenzentwicklungsmodells für das Energiekonzept im Bereich Fachwissen

ISBN 978-3-8325-3277-2 37.00 EUR

139 Nico Schreiber: Diagnostik experimenteller Kompetenz. Validierung technologiegestützter Testverfahren im Rahmen eines Kompetenzstrukturmodells ISBN 978-3-8325-3284-0 39.00 EUR 
140 Sarah Hundertmark: Einblicke in kollaborative Lernprozesse. Eine Fallstudie zur reflektierenden Zusammenarbeit unterstützt durch die Methoden Concept Mapping und Lernbegleitbogen

ISBN 978-3-8325-3251-2 43.00 EUR

141 Ronny Scherer: Analyse der Struktur, Messinvarianz und Ausprägung komplexer Problemlösekompetenz im Fach Chemie. Eine Querschnittstudie in der Sekundarstufe I und am Übergang zur Sekundarstufe II ISBN 978-3-8325-3312-0 43.00 EUR

142 Patricia Heitmann: Bewertungskompetenz im Rahmen naturwissenschaftlicher Problemlöseprozesse. Modellierung und Diagnose der Kompetenzen Bewertung und analytisches Problemlösen für das Fach Chemie ISBN 978-3-8325-3314-4 37.00 EUR

143 Jan Fleischhauer: Wissenschaftliches Argumentieren und Entwicklung von Konzepten beim Lernen von Physik ISBN 978-3-8325-3325-0 35.00 EUR

144 Nermin Özcan: Zum Einfluss der Fachsprache auf die Leistung im Fach Chemie. Eine Förderstudie zur Fachsprache im Chemieunterricht ISBN 978-3-8325-3328-1 36.50 EUR

145 Helena van Vorst: Kontextmerkmale und ihr Einfluss auf das Schülerinteresse im Fach Chemie ISBN 978-3-8325-3321-2 38.50 EUR

146 Janine Cappell: Fachspezifische Diagnosekompetenz angehender Physiklehrkräfte in der ersten Ausbildungsphase ISBN 978-3-8325-3356-4 38.50 EUR

147 Susanne Bley: Förderung von Transferprozessen im Chemieunterricht ISBN 978-3-8325-3407-3 40.50 EUR

148 Cathrin Blaes: Die übungsgestützte Lehrerpräsentation im Chemieunterricht der Sekundarstufe I. Evaluation der Effektivität ISBN 978-3-8325-3409-7 43.50 EUR

149 Julia Suckut: Die Wirksamkeit von piko-OWL als Lehrerfortbildung. Eine Evaluation zum Projekt Physik im Kontext in Fallstudien ISBN 978-3-8325-3440-0 45.00 EUR

150 Alexandra Dorschu: Die Wirkung von Kontexten in Physikkompetenztestaufgaben ISBN 978-3-8325-3446-2 37.00 EUR

151 Jochen Scheid: Multiple Repräsentationen, Verständnis physikalischer Experimente und kognitive Aktivierung: Ein Beitrag zur Entwicklung der Aufgabenkultur ISBN 978-3-8325-3449-3 49.00 EUR

152 Tim Plasa: Die Wahrnehmung von Schülerlaboren und Schülerforschungszentren ISBN 978-3-8325-3483-7 35.50 EUR 
153 Felix Schoppmeier: Physikkompetenz in der gymnasialen Oberstufe.Entwicklung und Validierung eines Kompetenzstrukturmodells für den Kompetenzbereich Umgang mit Fachwissen

ISBN 978-3-8325-3502-5 36.00 EUR

154 Katharina Groß: Experimente alternativ dokumentieren. Eine qualitative Studie zur Förderung der Diagnose- und Differenzierungskompetenz in der Chemielehrerbildung ISBN 978-3-8325-3508-7 43.50 EUR

155 Barbara Hank: Konzeptwandelprozesse im Anfangsunterricht Chemie. Eine quasiexperimentelle Längsschnittstudie

ISBN 978-3-8325-3519-3 38.50 EUR

156 Katja Freyer: Zum Einfluss von Studieneingangsvoraussetzungen auf den Studienerfolg Erstsemesterstudierender im Fach Chemie ISBN 978-3-8325-3544-5 38.00 EUR

157 Alexander Rachel: Auswirkungen instruktionaler Hilfen bei der Einführung des (Ferro-)Magnetismus. Eine Vergleichsstudie in der Primar- und Sekundarstufe ISBN 978-3-8325-3548-3 43.50 EUR

158 Sebastian Ritter: Einfluss des Lerninhalts Nanogrößeneffekte auf Teilchen- und Teilchenmodellvorstellungen von Schülerinnen und Schülern

ISBN 978-3-8325-3558-2 36.00 EUR

159 Andrea Harbach: Problemorientierung und Vernetzung in kontextbasierten Lernaufgaben

ISBN 978-3-8325-3564-3 39.00 EUR

160 David Obst: Interaktive Tafeln im Physikunterricht. Entwicklung und Evaluation einer Lehrerfortbildung ISBN 978-3-8325-3582-7 40.50 EUR

161 Sophie Kirschner: Modellierung und Analyse des Professionswissens von Physiklehrkräften

ISBN 978-3-8325-3601-5 35.00 EUR

162 Katja Stief: Selbstregulationsprozesse und Hausaufgabenmotivation im Chemieunterricht

ISBN 978-3-8325-3631-2 34.00 EUR

163 Nicola Meschede: Professionelle Wahrnehmung der inhaltlichen Strukturierung im naturwissenschaftlichen Grundschulunterricht. Theoretische Beschreibung und empirische Erfassung

ISBN 978-3-8325-3668-8 37.00 EUR

164 Johannes Maximilian Barth: Experimentieren im Physikunterricht der gymnasialen Oberstufe. Eine Rekonstruktion übergeordneter Einbettungsstrategien

ISBN 978-3-8325-3681-7 39.00 EUR

165 Sandra Lein: Das Betriebspraktikum in der Lehrerbildung. Eine Untersuchung zur Förderung der Wissenschafts- und Technikbildung im allgemeinbildenden Unterricht ISBN 978-3-8325-3698-5 40.00 EUR 
166 Veranika Maiseyenka: Modellbasiertes Experimentieren im Unterricht. Praxistauglichkeit und Lernwirkungen

ISBN 978-3-8325-3708-1 38.00 EUR

167 Christoph Stolzenberger: Der Einfluss der didaktischen Lernumgebung auf das Erreichen geforderter Bildungsziele am Beispiel der W- und P-Seminare im Fach Physik ISBN 978-3-8325-3708-1 38.00 EUR

168 Pia Altenburger: Mehrebenenregressionsanalysen zum Physiklernen im Sachunterricht der Primarstufe. Ergebnisse einer Evaluationsstudie.

ISBN 978-3-8325-3717-3 37.50 EUR

169 Nora Ferber: Entwicklung und Validierung eines Testinstruments zur Erfassung von Kompetenzentwicklung im Fach Chemie in der Sekundarstufe I

ISBN 978-3-8325-3727-2 39.50 EUR

170 Anita Stender: Unterrichtsplanung: Vom Wissen zum Handeln.

Theoretische Entwicklung und empirische Überprüfung des Transformationsmodells der Unterrichtsplanung

ISBN 978-3-8325-3750-0 41.50 EUR

171 Jenna Koenen: Entwicklung und Evaluation von experimentunterstützten Lösungsbeispielen zur Förderung naturwissenschaftlich-experimenteller Arbeitsweisen ISBN 978-3-8325-3785-2 43.00 EUR

172 Teresa Henning: Empirische Untersuchung kontextorientierter Lernumgebungen in der Hochschuldidaktik. Entwicklung und Evaluation kontextorientierter Aufgaben in der Studieneingangsphase für Fach- und Nebenfachstudierende der Physik ISBN 978-3-8325-3801-9 43.00 EUR

173 Alexander Pusch: Fachspezifische Instrumente zur Diagnose und individuellen Förderung von Lehramtsstudierenden der Physik ISBN 978-3-8325-3829-3 38.00 EUR

174 Christoph Vogelsang: Validierung eines Instruments zur Erfassung der professionellen Handlungskompetenz von (angehenden) Physiklehrkräften. Zusammenhangsanalysen zwischen Lehrerkompetenz und Lehrerperformanz

ISBN 978-3-8325-3846-0 50.50 EUR

175 Ingo Brebeck: Selbstreguliertes Lernen in der Studieneingangsphase im Fach Chemie ISBN 978-3-8325-3859-0 37.00 EUR

176 Axel Eghtessad: Merkmale und Strukturen von Professionalisierungsprozessen in der ersten und zweiten Phase der Chemielehrerbildung. Eine empirisch-qualitative Studie mit niedersächsischen Fachleiter_innen der Sekundarstufenlehrämter ISBN 978-3-8325-3861-3 45.00 EUR

177 Andreas Nehring: Wissenschaftliche Denk- und Arbeitsweisen im Fach Chemie. Eine kompetenzorientierte Modell- und Testentwicklung für den Bereich der Erkenntnisgewinnung

ISBN 978-3-8325-3872-9 39.50 EUR 
178 Maike Schmidt: Professionswissen von Sachunterrichtslehrkräften. Zusammenhangsanalyse zur Wirkung von Ausbildungshintergrund und Unterrichtserfahrung auf das fachspezifische Professionswissen im Unterrichtsinhalt „Verbrennung“ ISBN 978-3-8325-3907-8 38.50 EUR

179 Jan Winkelmann: Auswirkungen auf den Fachwissenszuwachs und auf affektive Schülermerkmale durch Schüler- und Demonstrationsexperimente im Physikunterricht ISBN 978-3-8325-3915-3 41.00 EUR

180 Iwen Kobow: Entwicklung und Validierung eines Testinstrumentes zur Erfassung der Kommunikationskompetenz im Fach Chemie ISBN 978-3-8325-3927-6 34.50 EUR

181 Yvonne Gramzow: Fachdidaktisches Wissen von Lehramtsstudierenden im Fach Physik. Modellierung und Testkonstruktion ISBN 978-3-8325-3931-3 42.50 EUR

182 Evelin Schröter: Entwicklung der Kompetenzerwartung durch Lösen physikalischer Aufgaben einer multimedialen Lernumgebung ISBN 978-3-8325-3975-7 54.50 EUR

183 Inga Kallweit: Effektivität des Einsatzes von Selbsteinschätzungsbögen im Chemieunterricht der Sekundarstufe I. Individuelle Förderung durch selbstreguliertes Lernen ISBN 978-3-8325-3965-8 44.00 EUR

184 Andrea Schumacher: Paving the way towards authentic chemistry teaching. A contribution to teachers' professional development ISBN 978-3-8325-3976-4 48.50 EUR

185 David Woitkowski: Fachliches Wissen Physik in der Hochschulausbildung. Konzeptualisierung, Messung, Niveaubildung ISBN 978-3-8325-3988-7 53.00 EUR

186 Marianne Korner: Cross-Age Peer Tutoring in Physik. Evaluation einer Unterrichtsmethode

ISBN 978-3-8325-3979-5 38.50 EUR

187 Simone Nakoinz: Untersuchung zur Verknüpfung submikroskopischer und makroskopischer Konzepte im Fach Chemie ISBN 978-3-8325-4057-9 38.50 EUR

188 Sandra Anus: Evaluation individueller Förderung im Chemieunterricht.Adaptivität von Lerninhalten an das Vorwissen von Lernenden am Beispiel des Basiskonzeptes Chemische Reaktion

ISBN 978-3-8325-4059-3 43.50 EUR

189 Thomas Roßbegalle: Fachdidaktische Entwicklungsforschung zum besseren Verständnis atmosphärischer Phänomene. Treibhauseffekt, saurer Regen und stratosphärischer Ozonabbau als Kontexte zur Vermittlung von Basiskonzepten der Chemie ISBN 978-3-8325-4059-3 45.50 EUR

190 Kathrin Steckenmesser-Sander: Gemeinsamkeiten und Unterschiede physikbezogener Handlungs-, Denk- und Lernprozesse von Mädchen und Jungen ISBN 978-3-8325-4066-1 38.50 EUR 
191 Cornelia Geller: Lernprozessorientierte Sequenzierung des Physikunterrichts im Zusammenhang mit Fachwissenserwerb. Eine Videostudie in Finnland, Deutschland und der Schweiz ISBN 978-3-8325-4082-1 35.50 EUR

192 Jan Hofmann: Untersuchung des Kompetenzaufbaus von Physiklehrkräften während einer Fortbildungsmaßnahme ISBN 978-3-8325-4104-0 38.50 EUR

193 Andreas Dickhäuser: Chemiespezifischer Humor. Theoriebildung, Materialentwicklung, Evaluation ISBN 978-3-8325-4108-8 37.00 EUR

194 Stefan Korte: Die Grenzen der Naturwissenschaft als Thema des Physikunterrichts ISBN 978-3-8325-4112-5 57.50 EUR

195 Carolin Hülsmann: Kurswahlmotive im Fach Chemie. Eine Studie zum Wahlverhalten und Erfolg von Schülerinnen und Schülern in der gymnasialen Oberstufe ISBN 978-3-8325-4144-6 49.00 EUR

196 Caroline Körbs: Mindeststandards im Fach Chemie am Ende der Pflichtschulzeit ISBN 978-3-8325-4148-4 34.00 EUR

197 Andreas Vorholzer: Wie lassen sich Kompetenzen des experimentellen Denkens und Arbeitens fördern? Eine empirische Untersuchung der Wirkung eines expliziten und eines impliziten Instruktionsansatzes

ISBN 978-3-8325-4194-1 37.50 EUR

198 Anna Katharina Schmitt: Entwicklung und Evaluation einer Chemielehrerfortbildung zum Kompetenzbereich Erkenntnisgewinnung

ISBN 978-3-8325-4228-3 39.50 EUR

199 Christian Maurer: Strukturierung von Lehr-Lern-Sequenzen ISBN 978-3-8325-4247-4 36.50 EUR

200 Helmut Fischler, Elke Sumfleth (Hrsg.): Professionelle Kompetenz von Lehrkräften der Chemie und Physik

ISBN 978-3-8325-4523-9 34.00 EUR

201 Simon Zander: Lehrerfortbildung zu Basismodellen und Zusammenhänge zum Fachwissen

ISBN 978-3-8325-4248-1 35.00 EUR

202 Kerstin Arndt: Experimentierkompetenz erfassen.

Analyse von Prozessen und Mustern am Beispiel von Lehramtsstudierenden der Chemie

ISBN 978-3-8325-4266-5 45.00 EUR

203 Christian Lang: Kompetenzorientierung im Rahmen experimentalchemischer Praktika ISBN 978-3-8325-4268-9 42.50 EUR

204 Eva Cauet: Testen wir relevantes Wissen? Zusammenhang zwischen dem Professionswissen von Physiklehrkräften und gutem und erfolgreichem Unterrichten

ISBN 978-3-8325-4276-4 39.50 EUR 
205 Patrick Löffler: Modellanwendung in Problemlöseaufgaben. Wie wirkt Kontext? ISBN 978-3-8325-4303-7 35.00 EUR

206 Carina Gehlen: Kompetenzstruktur naturwissenschaftlicher Erkenntnisgewinnung im Fach Chemie ISBN 978-3-8325-4318-1 43.00 EUR

207 Lars Oettinghaus: Lehrerüberzeugungen und physikbezogenes Professionswissen. Vergleich von Absolventinnen und Absolventen verschiedener Ausbildungswege im Physikreferendariat ISBN 978-3-8325-4319-8 38.50 EUR

208 Jennifer Petersen: Zum Einfluss des Merkmals Humor auf die Gesundheitsförderung im Chemieunterricht der Sekundarstufe I.

Eine Interventionsstudie zum Thema Sonnenschutz ISBN 978-3-8325-4348-8 40.00 EUR

209 Philipp Straube: Modellierung und Erfassung von Kompetenzen naturwissenschaftlicher Erkenntnisgewinnung bei (Lehramts-) Studierenden im Fach Physik ISBN 978-3-8325-4351-8 35.50 EUR

210 Martin Dickmann: Messung von Experimentierfähigkeiten. Validierungsstudien zur Qualität eines computerbasierten Testverfahrens ISBN 978-3-8325-4356-3 41.00 EUR

211 Markus Bohlmann: Science Education. Empirie, Kulturen und Mechanismen der Didaktik der Naturwissenschaften ISBN 978-3-8325-4377-8 44.00 EUR

212 Martin Draude: Die Kompetenz von Physiklehrkräften, Schwierigkeiten von Schülerinnen und Schülern beim eigenständigen Experimentieren zu diagnostizieren ISBN 978-3-8325-4382-2 37.50 EUR

213 Henning Rode: Prototypen evidenzbasierten Physikunterrichts. Zwei empirische Studien zum Einsatz von Feedback und Blackboxes in der Sekundarstufe ISBN 978-3-8325-4389-1 42.00 EUR

214 Jan-Henrik Kechel: Schülerschwierigkeiten beim eigenständigen Experimentieren. Eine qualitative Studie am Beispiel einer Experimentieraufgabe zum Hooke'schen Gesetz

ISBN 978-3-8325-4392-1 55.00 EUR

215 Katharina Fricke: Classroom Management and its Impact on Lesson Outcomes in Physics. A multi-perspective comparison of teaching practices in primary and secondary schools

ISBN 978-3-8325-4394-5 40.00 EUR

216 Hannes Sander: Orientierungen von Jugendlichen beim Urteilen und Entscheiden in Kontexten nachhaltiger Entwicklung. Eine rekonstruktive Perspektive auf Bewertungskompetenz in der Didaktik der Naturwissenschaft ISBN 978-3-8325-4434-8 46.00 EUR 
217 Inka Haak: Maßnahmen zur Unterstützung kognitiver und metakognitiver Prozesse in der Studieneingangsphase. Eine Design-Based-Research-Studie zum universitären Lernzentrum Physiktreff ISBN 978-3-8325-4437-9

$$
\text { 46.50 EUR }
$$

218 Martina Brandenburger: Was beeinflusst den Erfolg beim Problemlösen in der Physik? Eine Untersuchung mit Studierenden ISBN 978-3-8325-4409-6 42.50 EUR

219 Corinna Helms: Entwicklung und Evaluation eines Trainings zur Verbesserung der Erklärqualität von Schülerinnen und Schülern im Gruppenpuzzle ISBN 978-3-8325-4454-6 42.50 EUR

220 Viktoria Rath: Diagnostische Kompetenz von angehenden Physiklehrkräften. Modellierung, Testinstrumentenentwicklung und Erhebung der Performanz bei der Diagnose von Schülervorstellungen in der Mechanik

ISBN 978-3-8325-4456-0 42.50 EUR

221 Janne Krüger: Schülerperspektiven auf die zeitliche Entwicklung der Naturwissenschaften

ISBN 978-3-8325-4457-7 45.50 EUR

222 Stefan Mutke: Das Professionswissen von Chemiereferendarinnen und -referendaren in Nordrhein-Westfalen. Eine Längsschnittstudie ISBN 978-3-8325-4458-4 37.50 EUR

223 Sebastian Habig: Systematisch variierte Kontextaufgaben und ihr Einfluss auf kognitive und affektive Schülerfaktoren

ISBN 978-3-8325-4467-6 40.50 EUR

224 Sven Liepertz: Zusammenhang zwischen dem Professionswissen von Physiklehrkräften, dem sachstrukturellen Angebot des Unterrichts und der Schülerleistung ISBN 978-3-8325-4480-5 34.00 EUR

225 Elina Platova: Optimierung eines Laborpraktikums durch kognitive Aktivierung ISBN 978-3-8325-4481-2 39.00 EUR

226 Tim Reschke: Lesegeschichten im Chemieunterricht der Sekundarstufe I zur Unterstützung von situationalem Interesse und Lernerfolg ISBN 978-3-8325-4487-4 41.00 EUR

227 Lena Mareike Walper: Entwicklung der physikbezogenen Interessen und selbstbezogenen Kognitionen von Schülerinnen und Schülern in der Übergangsphase von der Primar- in die Sekundarstufe. Eine Längsschnittanalyse vom vierten bis zum siebten Schuljahr

ISBN 978-3-8325-4495-9 43.00 EUR

228 Stefan Anthofer: Förderung des fachspezifischen Professionswissens von Chemielehramtsstudierenden ISBN 978-3-8325-4498-0 39.50 EUR

229 Marcel Bullinger: Handlungsorientiertes Physiklernen mit instruierten Selbsterklärungen in der Primarstufe. Eine experimentelle Laborstudie ISBN 978-3-8325-4504-8 44.00 EUR 
230 Thomas Amenda: Bedeutung fachlicher Elementarisierungen für das Verständnis der Kinematik

ISBN 978-3-8325-4531-4 43.50 EUR

231 Sabrina Milke: Beeinflusst Priming das Physiklernen?

Eine empirische Studie zum Dritten Newtonschen Axiom

ISBN 978-3-8325-4549-4 42.00 EUR

232 Corinna Erfmann: Ein anschaulicher Weg zum Verständnis der elektromagnetischen Induktion. Evaluation eines Unterrichtsvorschlags und Validierung eines Leistungsdiagnoseinstruments

ISBN 978-3-8325-4550-5 49.50 EUR

233 Hanne Rautenstrauch: Erhebung des (Fach-)Sprachstandes bei Lehramtsstudierenden im Kontext des Faches Chemie

ISBN 978-3-8325-4556-7 40.50 EUR

234 Tobias Klug: Wirkung kontextorientierter physikalischer Praktikumsversuche auf Lernprozesse von Studierenden der Medizin

ISBN 978-3-8325-4558-1 37.00 EUR

235 Mareike Bohrmann: Zur Förderung des Verständnisses der Variablenkontrolle im naturwissenschaftlichen Sachunterricht

ISBN 978-3-8325-4559-8 52.00 EUR

236 Anja Schödl: FALKO-Physik - Fachspezifische Lehrerkompetenzen im Fach Physik. Entwicklung und Validierung eines Testinstruments zur Erfassung des fachspezifischen Professionswissens von Physiklehrkräften ISBN 978-3-8325-4553-6 40.50 EUR

237 Hilda Scheuermann: Entwicklung und Evaluation von Unterstützungsmaßnahmen zur Förderung der Variablenkontrollstrategie beim Planen von Experimenten ISBN 978-3-8325-4568-0 39.00 EUR

238 Christian G. Strippel: Naturwissenschaftliche Erkenntnisgewinnung an chemischen Inhalten vermitteln. Konzeption und empirische Untersuchung einer Ausstellung mit Experimentierstation

ISBN 978-3-8325-4577-2 41.50 EUR

239 Sarah Rau: Durchführung von Sachunterricht im Vorbereitungsdienst. Eine längsschnittliche, videobasierte Unterrichtsanalyse

ISBN 978-3-8325-4579-6 46.00 EUR

240 Thomas Plotz: Lernprozesse zu nicht-sichtbarer Strahlung. Empirische Untersuchungen in der Sekundarstufe 2

ISBN 978-3-8325-4624-3 39.50 EUR 
241 Wolfgang Aschauer: Elektrische und magnetische Felder. Eine empirische Studie zu Lernprozessen in der Sekundarstufe II

ISBN 978-3-8325-4625-0 50.00 EUR

242 Anna Donhauser: Didaktisch rekonstruierte Materialwissenschaft. Aufbau und Konzeption eines Schülerlabors für den Exzellenzcluster Engineering of Advanced Materials

ISBN 978-3-8325-4636-6 39.00 EUR

243 Katrin Schüßler: Lernen mit Lösungsbeispielen im Chemieunterricht. Einflüsse auf Lernerfolg, kognitive Belastung und Motivation

ISBN 978-3-8325-4640-3 42.50 EUR

244 Timo Fleischer: Untersuchung der chemischen Fachsprache unter besonderer Berücksichtigung chemischer Repräsentationen

ISBN 978-3-8325-4642-7 46.50 EUR

245 Rosina Steininger: Concept Cartoons als Stimuli für Kleingruppendiskussionen im Chemieunterricht. Beschreibung und Analyse einer komplexen Lerngelegenheit ISBN 978-3-8325-4647-2 39.00 EUR

246 Daniel Rehfeldt: Erfassung der Lehrqualität naturwissenschaftlicher Experimentalpraktika

ISBN 978-3-8325-4590-1 40.00 EUR

247 Sandra Puddu: Implementing Inquiry-based Learning in a Diverse Classroom: Investigating Strategies of Scaffolding and Students' Views of Scientific Inquiry ISBN 978-3-8325-4591-8 35.50 EUR

248 Markus Bliersbach: Kreativität in der Chemie. Erhebung und Förderung der Vorstellungen von Chemielehramtsstudierenden ISBN 978-3-8325-4593-2 44.00 EUR

249 Lennart Kimpel: Aufgaben in der Allgemeinen Chemie. Zum Zusammenspiel von chemischem Verständnis und Rechenfähigkeit

ISBN 978-3-8325-4618-2 36.00 EUR

250 Louise Bindel: Effects of integrated learning: explicating a mathematical concept in inquiry-based science camps

ISBN 978-3-8325-4655-7 37.50 EUR

251 Michael Wenzel: Computereinsatz in Schule und Schülerlabor. Einstellung von Physiklehrkräften zu Neuen Medien

ISBN 978-3-8325-4659-5 38.50 EUR

252 Laura Muth: Einfluss der Auswertephase von Experimenten im Physikunterricht. Ergebnisse einer Interventionsstudie zum Zuwachs von Fachwissen und experimenteller Kompetenz von Schülerinnen und Schülern

ISBN 978-3-8325-4675-5 36.50 EUR 
253 Annika Fricke: Interaktive Skripte im Physikalischen Praktikum. Entwicklung und Evaluation von Hypermedien für die Nebenfachausbildung

ISBN 978-3-8325-4676-2 41.00 EUR

254 Julia Haase: Selbstbestimmtes Lernen im naturwissenschaftlichen Sachunterricht. Eine empirische Interventionsstudie mit Fokus auf Feedback und Kompetenzerleben ISBN 978-3-8325-4685-4 38.50 EUR

255 Antje J. Heine: Was ist Theoretische Physik? Eine wissenschaftstheoretische Betrachtung und Rekonstruktion von Vorstellungen von Studierenden und Dozenten über das Wesen der Theoretischen Physik

ISBN 978-3-8325-4691-5 46.50 EUR

256 Claudia Meinhardt: Entwicklung und Validierung eines Testinstruments zu Selbstwirksamkeitserwartungen von (angehenden) Physiklehrkräften in physikdidaktischen Handlungsfeldern

ISBN 978-3-8325-4712-7 47.00 EUR

257 Ann-Kathrin Schlüter: Professionalisierung angehender Chemielehrkräfte für einen Gemeinsamen Unterricht

ISBN 978-3-8325-4713-4 53.50 EUR

258 Stefan Richtberg: Elektronenbahnen in Feldern. Konzeption und Evaluation einer webbasierten Lernumgebung

ISBN 978-3-8325-4723-3 49.00 EUR

259 Jan-Philipp Burde: Konzeption und Evaluation eines Unterrichtskonzepts zu einfachen Stromkreisen auf Basis des Elektronengasmodells

ISBN 978-3-8325-4726-4 57.50 EUR

260 Frank Finkenberg: Flipped Classroom im Physikunterricht ISBN 978-3-8325-4737-4 42.50 EUR

261 Florian Treisch: Die Entwicklung der Professionellen Unterrichtswahrnehmung im Lehr-Lern-Labor Seminar

ISBN 978-3-8325-4741-4 41.50 EUR

Alle erschienenen Bücher können unter der angegebenen ISBN direkt online (http://www.logosverlag.de) oder per Fax (030 - 428510 92) beim Logos Verlag Berlin bestellt werden. 


\section{Studien zum Physik- und Chemielernen}

Herausgegeben von Hans Niedderer, Helmut Fischler und Elke Sumfleth

Die Reihe umfasst inzwischen eine große Zahl von wissenschaftlichen Arbeiten aus vielen Arbeitsgruppen der Physik- und Chemiedidaktik und zeichnet damit ein gültiges Bild der empirischen physik- und chemiedidaktischen Forschung in Deutschland.

Die Herausgeber laden daher Interessenten zu neuen Beiträgen ein und bitten sie, sich im Bedarfsfall an den Logos-Verlag oder an ein Mitglied des Herausgeberteams zu wenden.

\section{Kontaktadressen:}

Prof. Dr. Hans Niedderer

Institut für Didaktik der Naturwissenschaften,

Abt. Physikdidaktik, FB Physik/Elektrotechnik,

Universität Bremen,

Postfach 3304 40, 28334 Bremen

Tel. 0421-218 2484/4695, e-mail:

niedderer@physik.uni-bremen.de

Prof. Dr. Helmut Fischler

Didaktik der Physik, FB Physik, Freie Universität Berlin,

Arnimallee 14, 14195 Berlin

Tel. 030-838 56712/55966, e-mail:

fischler@physik.fu-berlin.de

Prof. Dr. Elke Sumfleth

Didaktik der Chemie,

Fachbereich Chemie,

Universität Duisburg-Essen,

Schützenbahn 70, 45127 Essen

Tel. 0201-183 3757/3761, e-mail:

elke.sumfleth@uni-essen.de 
Die Vorstellung vieler Schülerinnen und Schüler von elektrischen Stromkreisen ist maßgeblich vom Stromkonzept geprägt, während die Spannung oft lediglich als Eigenschaft des elektrischen Stroms wahrgenommen wird. In der Folge verfügen viele Lernende nach der Sekundarstufe I oftmals über kein angemessenes konzeptionelles Verständnis von einfachen Stromkreisen.

Gegenstand der vorliegenden Design-Based-Research-Arbeit ist deshalb die theoriegeleitete Entwicklung und empirische Evaluation eines Unterrichtskonzepts zu einfachen Stromkreisen auf Grundlage eines didaktisch elementarisierten Elektronengasmodells für die Sekundarstufe I. Ein wesentliches Ziel besteht darin, den Lernenden ein qualitatives, konzeptionelles Verständnis der Grundbegriffe Spannung, Stromstärke und Widerstand sowie ihrer wechselseitigen Beziehung in einfachen Stromkreisen zu vermitteln. Hierzu wird das elektrische Potenzial mit dem Luftdruck bzw. dem sog. „elektrischen Druck“ in einem Leiter verglichen und die elektrische Spannung dementsprechend als „elektrischer Druckunterschied“ noch vor dem elektrischen Strom eingeführt.

Im Rahmen der empirischen Evaluation mit 790 Schülerinnen und Schülern zeigte sich, dass das neue Unterrichtskonzept verglichen mit dem traditionellen Physikunterricht zu einem deutlich besseren konzeptionellen Verständnis elektrischer Stromkreise führt, was sich u.a. in einem signifikant höheren Lernzuwachs widerspiegelt. Darüber hinaus geben die unterrichtenden Lehrkräfte an, das Konzept als wesentliche Verbesserung ihres Unterrichts wahrgenommen zu haben.

\section{Logos Verlag Berlin}

ISBN 978-3-8325-4726-4 